

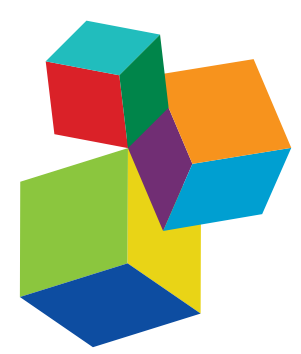

Frontiers eBook Copyright Statement

The copyright in the text of individual articles in this eBook is the property of their respective authors or their respective institutions or funders. The copyright in graphics and images within each article may be subject to copyright of other parties. In both cases this is subject to a license granted to Frontiers.

The compilation of articles constituting this eBook is the property of Frontiers.

Each article within this eBook, and the eBook itself, are published under the most recent version of the Creative Commons CC-BY licence. The version current at the date of publication of this eBook is CC-BY 4.0. If the CC-BY licence is updated, the licence granted by Frontiers is automatically updated to the new version.

When exercising any right under the CC-BY licence, Frontiers must be attributed as the original publisher of the article or eBook, as applicable.

Authors have the responsibility of ensuring that any graphics or other materials which are the property of others may be included in the

CC-BY licence, but this should be checked before relying on the

CC-BY licence to reproduce those materials. Any copyright notices relating to those materials must be complied with.

Copyright and source acknowledgement notices may not be removed and must be displayed in any copy, derivative work or partial copy which includes the elements in question.

All copyright, and all rights therein, are protected by national and international copyright laws. The above represents a summary only.

For further information please read Frontiers' Conditions for Website

Use and Copyright Statement, and the applicable CC-BY licence.

ISSN 1664-8714

ISBN 978-2-88966-940-0

DOI 10.3389/978-2-88966-940-0

\section{About Frontiers}

Frontiers is more than just an open-access publisher of scholarly articles: it is a pioneering approach to the world of academia, radically improving the way scholarly research is managed. The grand vision of Frontiers is a world where all people have an equal opportunity to seek, share and generate knowledge. Frontiers provides immediate and permanent online open access to all its publications, but this alone is not enough to realize our grand goals.

\section{Frontiers Journal Series}

The Frontiers Journal Series is a multi-tier and interdisciplinary set of open-access, online journals, promising a paradigm shift from the current review, selection and dissemination processes in academic publishing. All Frontiers journals are driven by researchers for researchers; therefore, they constitute a service to the scholarly community. At the same time, the Frontiers Journal Series operates on a revolutionary invention, the tiered publishing system, initially addressing specific communities of scholars, and gradually climbing up to broader public understanding, thus serving the interests of the lay society, too.

\section{Dedication to Quality}

Each Frontiers article is a landmark of the highest quality, thanks to genuinely collaborative interactions between authors and review editors, who include some of the world's best academicians. Research must be certified by peers before entering a stream of knowledge that may eventually reach the public - and shape society; therefore, Frontiers only applies the most rigorous and unbiased reviews.

Frontiers revolutionizes research publishing by freely delivering the most outstanding research, evaluated with no bias from both the academic and social point of view. By applying the most advanced information technologies, Frontiers is catapulting scholarly publishing into a new generation.

\section{What are Frontiers Research Topics?}

Frontiers Research Topics are very popular trademarks of the Frontiers Journals Series: they are collections of at least ten articles, all centered on a particular subject. With their unique mix of varied contributions from Original Research to Review Articles, Frontiers Research Topics unify the most influential researchers, the latest key findings and historical advances in a hot research area! Find out more on how to host your own Frontiers Research Topic or contribute to one as an author by contacting the Frontiers Editorial Office: frontiersin.org/about/contact 


\title{
PRESCRIBING PSYCHOTROPICS: MISUSE, ABUSE, DEPENDENCE, WITHDRAWAL AND ADDICTION
}

\author{
Topic Editors: \\ Fabrizio Schifano, University of Hertfordshire, United Kingdom \\ Giovanni Martinotti, University of Studies G. d'Annunzio Chieti and Pescara, Italy \\ Stefania Chiappini, University of Hertfordshire, United Kingdom
}

Citation: Schifano, F., Martinotti, G., Chiappini, S., eds. (2021). Prescribing Psychotropics: Misuse, Abuse, Dependence, Withdrawal and Addiction. Lausanne: Frontiers Media SA. doi: 10.3389/978-2-88966-940-0 


\section{Table of Contents}

05 Editorial: Prescribing Psychotropics: Misuse, Abuse, Dependence, Withdrawal and Addiction

Stefania Chiappini, Fabrizio Schifano and Giovanni Martinotti

08 Ranking the Harm of Psychoactive Drugs Including Prescription Analgesics to Users and Others-A Perspective of German Addiction Medicine Experts

Udo Bonnet, Michael Specka, Michael Soyka, Thomas Alberti, Stefan Bender, Torsten Grigoleit, Leopold Hermle, Jörg Hilger, Thomas Hillemacher, Thomas Kuhlmann, Jens Kuhn, Christian Luckhaus, Christel Lüdecke, Jens Reimer, Udo Schneider, Welf Schroeder, Markus Stuppe, Gerhard A. Wiesbeck, Norbert Wodarz, Heath McAnally and Norbert Scherbaum

17 High-Dose Dependence and Cognitive Side Effects to Medical Prescription of Etizolam

Stefano Tamburin, Elisa Mantovani, Anna Bertoldi, Angela Federico, Rebecca Casari and Fabio Lugoboni

22 Prescription Drug Misuse in "Clubbers" and Disco Goers in Ibiza Massimo di Giannantonio, Attilio Negri, Stefania Schiavone, Chiara Vannini, Mauro Pettorruso, Fabio De-Giorgio, Valeria Verrastro, Luigia Trabace, Mariangela Corbo, Rossella Gottardo, Cristian Camuto, Monica Mazzarino, Andrea Barra, Domenico De Berardis, Juan Iglesias Lopez, Cristina Merino Del Villar, Fabrizio Schifano and Giovanni Martinotti

31 Psychiatry Trainees' Attitudes, Knowledge, and Training in Addiction Psychiatry-A European Survey

Laura Orsolini, Irena Rojnić Palavra, Gabriele Duccio Papanti, Matej Potočan, Diego Quattrone, Matis Martens, Sandra Sklenářová, Jonna Levola, Leslie Grichy, Sean Naughton, Indre Kotryna Grinevičiene, Jelly Petra Kuiters, Tomasz M. Gondek, Anca-Livia Panfil, Milica M. Borovcanin, Alberto San Roman Uria, Ewelina Biskup, Ekin Sönmez Güngör, Marisa Casanova Dias, Sonila Tomori, Visnja Banjac,

Petra Marinova-Djambazova and Mariana Pinto da Costa

43 Gabapentinoid Abuse in France: Evidence on Health Consequences and New Points of Vigilance

Marine Tambon, Camille Ponté, Emilie Jouanjus, Nathalie Fouilhé, Joelle Micallef, Maryse Lapeyre-Mestre and the French Addictovigilance Network (FAN)

58 Continuous Infusion of Flumazenil in the Management of Benzodiazepines Detoxification

Anna Benini, Rossella Gottardo, Cristiano Chiamulera, Anna Bertoldi, Lorenzo Zamboni and Fabio Lugoboni

65 Case Report: Treatment of Kratom Use Disorder With a Classical Tricyclic Antidepressant

Alessandro E. Vento, Simone de Persis, Sergio De Filippis, Fabrizio Schifano, Flavia Napoletano, John M. Corkery and Georgios D. Kotzalidis 
74 The Severity of Dependence Scale (SDS) for Codeine: Preliminary Investigation of the Psychometric Properties of the SDS in an Online Sample of Codeine Users From the UK

Paolo Deluca, Michelle Foley, Jacklyn Dunne and Andreas Kimergård

82 Personality to Prescription Drug Misuse in Adolescents: Testing Affect

Regulation, Psychological Dysregulation, and Deviance Proneness Pathways

Sherry H. Stewart, Annie Chinneck, Kara Thompson, Mohammad H. Afzali, Raquel Nogueira-Arjona, Ioan T. Mahu and Patricia J. Conrod

97 Non-medical Use of Prescription Gabapentinoids (Gabapentin and Pregabalin) in Five European Countries

Francina Fonseca, William Lenahan, Richard C. Dart, Esther Papaseit, Paul I. Dargan, David M. Wood, Marilena Guareschi, Icro Maremmani, Marc Auriacombe, Magí Farré, Norbert Scherbaum and Marta Torrens

107 Focus on Over-the-Counter Drugs' Misuse: A Systematic Review on Antihistamines, Cough Medicines, and Decongestants

Fabrizio Schifano, Stefania Chiappini, Andrea Miuli, Alessio Mosca,

Maria Chiara Santovito, John M. Corkery, Amira Guirguis, Mauro Pettorruso, Massimo Di Giannantonio and Giovanni Martinotti

120 Early Detection of Prescription Drug Abuse Using Doctor Shopping Monitoring From Claims Databases: Illustration From the Experience of the French Addictovigilance Network

Thomas Soeiro, Clémence Lacroix, Vincent Pradel, Maryse Lapeyre-Mestre and Joëlle Micallef

132 Assessment of the Safety Signal for the Abuse Potential of Pregabalin and Gabapentin Using the FAERS Database and Big Data Search Analytics

Georgios Papazisis, Dimitrios Spachos, Spyridon Siafis, Niki Pandria,

Eleni Deligianni, Ioannis Tsakiridis and Antonios Goulas 


\title{
Editorial: Prescribing Psychotropics: Misuse, Abuse, Dependence, Withdrawal and Addiction
}

\author{
Stefania Chiappini ${ }^{1,2 * \dagger}$, Fabrizio Schifano ${ }^{1 \dagger}$ and Giovanni Martinotti ${ }^{1,2+}$ \\ ${ }^{1}$ Psychopharmacology, Drug Misuse and Novel Psychoactive Substances Research Unit, School of Life and Medical \\ Sciences, University of Hertfordshire, Hatfield, United Kingdom, ${ }^{2}$ Department of Neuroscience, Imaging and Clinical \\ Sciences, "G. D’Annunzio" University, Chieti, Italy
}

Keywords: drug abuse, drug misuse, prescription drug misuse, pharming, drug diversion, addiction, pharmacovigilance

\section{Editorial on the Research Topic}

\section{Prescribing Psychotropics: Misuse, Abuse, Dependence, Withdrawal, and Addiction}

Over the last decade, the trend of drug consumption has changed dramatically. The advent of a high number of new psychoactive substances (NPS) has contributed to the appearance and growth of a new "drug scenario" $(1,2)$ characterized by an increasing number of molecules with unknown effects; poor safety profiles and acute drug toxicity presentations; and psychiatric consequences (3-6). In this context, medications' misuse appears to be an increasingly concerning phenomenon, specifically driven by the already recorded rise in the opioid use, benzodiazepines, and other Central Nervous System (CNS) depressants (including sedatives and hypnotics), and prescription stimulants, e.g., amphetamines, methamphetamines, methylphenidate $(7,8)$. However, a range of remaining molecules have been reported as being misused; diverted; and recorded by drug users' online websites suggesting new trends and experimentations specifically with medicinal compounds (9-11). An increasing awareness regarding these issues has been contributing to the development of pharmacovigilance studies regarding the possible potential of misuse/abuse/dependence and withdrawal of both prescription (e.g., quetiapine, gabapentinoids, olanzapine, bupropion, etc.) and over-the-counter (OTC) drugs (e.g., loperamide, dextromethorphan, promethazine, benzydamine etc.) (12-17). Indeed, pharmacovigilance studies have helped in identifying signals of misuse associated with these molecules (18). For instance, whilst both pregabalin and gabapentin are approved treatments for epilepsy and neuropathic pain disorders, with pregabalin being prescribed as well in some countries for the treatment of generalized anxiety disorder (19), they have increasingly been reported for their misusing potential, especially when used in combination with opioids and sedatives (12). In 2018, after safety warnings following an increase in deaths related to their use, the UK Advisory Council on the Misuse of Drugs (ACMD) recommended that both had to be controlled under the Misuse of Drugs Act 1971 as Class C substances and scheduled under the Misuse of Drugs Regulations 2001 as Schedule 3 , so as not to preclude legitimate use on prescription (20). Conversely, a range of factors are thought to contribute to the non-medical use of prescription/OTC drugs, such as the perception of these molecules being more socially acceptable/less stigmatizing; likely lack of detection in standard drug screens; and safer than remaining illicit substances as well. "Pharming"; "pharmparties"; and "doctor-shopping” attitudes, involving high-/mega-dosage prescription drugs' intake, are trends which are increasingly being reported among young adult populations $(9,21,22)$. In parallel with this, increasing levels of access to the web over the past 15 years or so may have boosted the current scenario of prescribed drugs' misuse and abuse, with social networks playing 
a role in medications' aggressive marketing/distribution from rogue "pharmacy" websites $(10,23-25)$. Moreover, the web has been contributing to the diffusion of new synthetic compounds, such as designer benzodiazepines and illicit fentanyl analogs, which are associated with a high abuse potential and severe adverse effects including coma and death (26-28). Finally, since the beginning of 2020, due to drug shortage issues resulting to the COVID-19 pandemic, a shift in misusing behavior relating to both prescription and OTC medicines has been recorded (10, 13, 29-33).

Consistent with these issues, the current Research Topic has focussed on the assessment of the misuse, abuse, dependence, withdrawal, diversion, and addiction potential of prescribing and OTC drugs. A range of original research papers, systematic reviews, meta-analysis, reviews, and case reports are here made available. This Research Topic will hopefully shed further light on the harms associated with medications' misuse and abuse, highlighting the importance of this field for clinicians; prescribers; and health professionals in general. Indeed, 13 original articles of excellent quality and likely broad impact are here offered to the Frontiers in Psychiatry readers. A description of prescription drugs' misuse in "clubbers" and disco goers in Ibiza showed that current trends of such phenomenon may not be limited to subjects with psychiatric disorders, as prescription drugs may be used an alternative to classic and novel psychoactive compounds and/or may be used to tamper and self-medicate the effects determined by the use of substances. Considering prescription drugs misused, the diversion of the benzodiazepine etizolam was here recorded, being characterized by high-dosage intake and resulting dependence issues; also, the misuse and diversion of several OTCs, including antihistamines (e.g., diphenhydramine, promethazine, chlorpheniramine, and dimenhydrinate); dextromethorphan- and codeine-based cough medicines; and the nasal decongestant pseudoephedrine have here been reported. Furthermore, a few surveys are here being collected; the first one is a European survey investigating psychiatry trainees' attitudes, knowledge and training in addiction psychiatry, while a second paper evaluated the German addiction medicine physicians' knowledge of both health and psychosocial harms of 33 psychoactive substances, including opioids and non-opioid prescription analgesics, e.g., gabapentinoids. Finally, using data from the RADARS ${ }^{\circledR}$ survey on the non-medical use of prescription drugs conducted in five European countries, the non-medical use of gabapentinoids resulted to have the highest prevalence in Germany and UK compared with Spain, Italy, and France. Data related to gabapentinoids as recorded by the French Addictovigilance

\section{REFERENCES}

1. Corazza O, Prilutskaya M. Handbook of Novel Psychoactive Substances: What Clinicians Should Know about NPS, 1st ed. New York, NY: Routledge (2018). doi: 10.4324/9781315158082

2. Schifano F. Recent changes in drug abuse scenarios: the New/Novel Psychoactive Substances (NPS) phenomenon. Brain Sci. (2018) 8:221. doi: 10.3390/brainsci81 20221
Network confirmed the importance of pharmacovigilance monitoring for gabapentinoids due to their abuse potential and their related health harms, including hospitalization for serious neurologic, psychiatric or cardiac effects; requests for specific support; and deaths. Similarly, the analysis of the FDA Adverse Event Reporting System (FAERS) database, using big data search analytics as a supplementary tool to detect drug abuserelated safety signals, supported these issues. In parallel, within a multidimensional monitoring of prescription drug abuse, the early detection and quantification of "doctor shopping" practices may well need to be considered essential. Moreover, the identification of specific personality traits (e.g., hopelessness, anxiety sensitivity, sensation seeking, and impulsivity) and psychometric indicators (e.g., the Severity of Dependence ScaleSDS) might be useful in providing drug abusers with personalized interventions and strategies. Finally, the treatment of drug intoxication, as in a case of kratom use disorder, and of drug withdrawal through the continuous infusion of flumazenil in the management of benzodiazepines detoxification were here described.

In conclusion, the abuse of prescription and OTC drugs has become an issue of increasing public concern across the globe (34). Whilst health services are already under unprecedented levels of strain, the current drug scenarios have further modified, in parallel with the current pandemicrelated goods' and people local; national; and international restrictions of movements. At these challenging times, healthcare professionals are recommended to both be vigilant and develop strategies to ensure continuity of care for people who use drugs and people with drug use disorders, whilst preventing as well possible medicines' misuse and diversion issues (9).

\section{AUTHOR CONTRIBUTIONS}

All authors listed have made a substantial, direct and intellectual contribution to the work, and approved it for publication.

\section{ACKNOWLEDGMENTS}

The Topic Editors express their gratitude to all the contributors for submitting their work to this Research Topic, to the Review Editors and external Reviewers who participated in the review process, and to the Editorial and Production teams of Frontiers for their valuable assistance through the various stages of the publication process.
3. Schifano F, Orsolini L, Papanti D, Corkery J. NPS: medical consequences associated with their intake. Curr Top Behav Neurosci. (2017) 32:35180. doi: 10.1007/7854_2016_15

4. Schifano F, Napoletano F, Chiappini S, Guirguis A, Corkery JM, Bonaccorso S, et al. New/emerging psychoactive substances and associated psychopathological consequences. Psychol Med. (2019) 22:1-13. doi: 10.1017/S0033291719001727

5. Schifano F, Chiappini S, Miuli A, Corkery JM, Scherbaum N, Napoletano F, et al. New psychoactive substances (NPS) and 
serotonin syndrome onset: a systematic review. Exp Neurol. (2021) 339:113638. doi: 10.1016/j.expneurol.2021.113638

6. Martinotti G, De Risio L, Vannini C, Schifano F, Pettorruso M, Di Giannantonio M. Substance-related exogenous psychosis: a postmodern syndrome. CNS Spectr. (2021) 26:84-91. doi: 10.1017/S10928529200 01479

7. Lessenger JE, Feinberg SD. Abuse of prescription and overthe-counter medications. J Am Board Fam Med. (2008) 21:4554. doi: 10.3122/jabfm.2008.01.070071

8. Lipari RN, Williams M, Van Horn SL. Why do Adults Misuse Prescription Drugs? The CBHSQ Report: July 27, 2017. Center for Behavioral Health Statistics and Quality, Substance Abuse and Mental Health Services Administration. Rockville, MD (2017). Available online at: https://www. samhsa.gov/data/sites/default/files/report_3210/ShortReport-3210.html (accessed December 9, 2020).

9. Schifano F. Coming off prescribed psychotropic medications: insights from their use as recreational drugs. Psychother Psychosomat. (2020) 89:27482. doi: 10.1159/000507897

10. Chiappini S, Guirguis A, Corkery J, Schifano F. Understanding the use of prescription and OTC drugs in obtaining highs and the pharmacist role in preventing abuse. Pharm J. (2020) 305:7943. doi: 10.1211/PJ.2020.20208538

11. Schifano F, Chiappini S, Corkery JM, Guirguis A. Abuse of prescription drugs in the context of Novel Psychoactive Substances (NPS): a systematic review. Brain Sci. (2018) 8:73. doi: 10.3390/brainsci8040073

12. Chiappini S, Schifano F. A decade of gabapentinoid misuse: an analysis of the european medicines agency's 'suspected adverse drug reactions' database. CNS Drugs. (2016) 30:647-54. doi: 10.1007/s40263-016-0359-y

13. Chiappini S, Schifano F, Corkery JM, Guirguis A. Focus on clozapine withdrawal- and misuse-related cases as reported to the European Medicines Agency (EMA) pharmacovigilance database. Brain Sci. (2020) 10:105. doi: 10.3390/brainsci10020105

14. Chiappini S, Schifano F, Corkery JM, Guirguis A. Beyond the "purple drank": Study of promethazine abuse according to the European Medicines Agency adverse drug reaction reports. J Psychopharmacol. (2021). doi: 10.1177/0269881120959615. [Epub ahead of print].

15. Chiappini S, Miuli A, Mosca A, Pettorruso M, Guirguis A, Corkery JM, et al. The benzydamine experience: a systematic review of benzydamine abuse. Curr Neuropharmacol. (2021). doi: 10.2174/1570159X19666210113151136. [Epub ahead of print].

16. Schwan S, Sundström A, Stjernberg E, Hallberg E, Hallberg P. A signal for an abuse liability for pregabalin-results from the Swedish spontaneous adverse drug reaction reporting system. Eur J Clin Pharmacol. (2010) 66:94753. doi: 10.1007/s00228-010-0853-y

17. Vickers-Smith R, Sun J, Charnigo RJ, Lofwall MR, Walsh SL, Havens JR. Gabapentin drug misuse signals: a pharmacovigilance assessment using the FDA adverse event reporting system. Drug Alcohol Depend. (2020) 206:107709. doi: 10.1016/j.drugalcdep.2019.107709

18. WHO. WHO Policy Perspectives on Medicines. Looking at the Pharmacovigilance: Ensuring the Safe Use of Medicines. Geneva: World Health Organization (2014). Available online at: http://digicollection.org/hss/ en/d/Js6164e/3.1.html (accessed April 21, 2021).

19. Schifano F. Misuse and abuse of pregabalin and gabapentin: cause for concern? CNS Drugs. (2014) 28:491-46. doi: 10.1007/s40263-0140164-4

20. Advisory Council on the Misuse of Drugs (ACMD). Addendum to Advice on the Anticonvulsant Drugs Pregabalin and Gabapentin. (2018). Available online at: https://www.gov.uk/government/publications/advice-on-theanticonvulsant-drugs-pregabalin-and-gabapentin/addendum-to-adviceon-the-anticonvulsant-drugs-pregabalin-and-gabapentin-october-2018 (accessed December 9, 2020).

21. Levine DA. "Pharming": the abuse of prescription and overthe-counter drugs in teens. Curr Opin Pediatr. (2007) 19:2704. doi: 10.1097/MOP.0b013e32814b09cf
22. Simeone R. Doctor shopping behavior and the diversion of prescription opioids. Subst Abuse. (2017) 11:1178221817696077. doi: 10.1177/1178221817696077

23. Festinger DS, Dugosh KL, Clements N, Flynn AB, Falco M, McLellan AT, et al. Use of the internet to obtain drugs without a prescription among treatmentinvolved adolescents and young adults. J Child Adolesc Subst Abuse. (2016) 25:480-6. doi: 10.1080/1067828X.2015.1103345

24. Orsolini L, Francesconi G, Papanti D, Giorgetti A, Schifano F. Profiling online recreational/prescription drugs' customers and overview of drug vending virtual marketplaces. Hum Psychopharmacol. (2015) 30:30218. doi: 10.1002/hup.2466

25. Orsolini L, Papanti D, Corkery J, Schifano F. An insight into the deep web; why it matters for addiction psychiatry? Hum Psychopharmacol. (2017) 32:e2573. doi: 10.1002/hup.2573

26. Pichini S, Solimini R, Berretta P, Pacifici R, Busardò FP. Acute intoxications and fatalities from illicit fentanyl and analogues: an update. Ther Drug Monit. (2018) 40:38-51. doi: 10.1097/FTD.0000000000000465

27. Orsolini L, Corkery JM, Chiappini S, Guirguis A, Vento A, De Berardis D, et al. 'New/Designer Benzodiazepines': an analysis of the literature and psychonauts' trip reports. Curr Neuropharmacol. (2020) 18:80937. doi: 10.2174/1570159X18666200110121333

28. Zawilska JB, Wojcieszak J. An expanding world of new psychoactive substances-designer benzodiazepines. NeuroToxicology. (2019) 73:8-16. doi: 10.1016/j.neuro.2019.02.015

29. Chiappini S, Guirguis A, John A, Corkery JM, Schifano F. COVID-19: The hidden impact on mental health and drug addiction. Front Psychiatry. (2020) 11:767. doi: 10.3389/fpsyt.2020.00767

30. Clay JM, Parker MO. Alcohol use and misuse during the COVID-19 pandemic: a potential public health crisis? Lancet Public Health. (2020) 5:e259. doi: 10.1016/S2468-2667(20)30088-8

31. Dores AR, Carvalho IP, Burkauskas J, Simonato P, De Luca I, Mooney R, et al. Exercise and use of enhancement drugs at the time of the COVID-19 pandemic: a multicultural study on coping strategies during self-isolation and related risks. Front Psychiatry. (2021) 12:648501doi: 10.3389/fpsyt.2021.648501

32. Public Health Institute. Direct and Indirect Impacts of COVID-19 on Health and Wellbeing. Rapid Evidence Review (2020). Available online at: https:// www.nwppn.nhs.uk/attachments/article/2797/2020-07-direct-and-indirectimpacts-of-covid19-on-health-and-wellbeing.pdf (accessed April 21, 2021).

33. Zaami S, Marinelli E, Varì MR. New trends of substance abuse during COVID-19 pandemic: an international perspective. Front Psychiatry. (2020) 11:700. doi: 10.3389/fpsyt.2020.00700

34. Chiappini S, Schifano F. What about "Pharming?" Issues regarding the misuse of prescription and over-the-counter drugs. Brain Sci. (2020) 10:736. doi: 10.3390/brainsci10100736

Conflict of Interest: FS was a member of the UK Advisory Council on the Misuse of Drugs (ACMD; 2011-2019) and is currently a member of the EMA Advisory Board (Psychiatry). GM has been a consultant and/or a speaker and/or has received research grants from Angelini, Doc Generici, Janssen-Cilag, Lundbeck, Otsuka, Pfizer, Servier, Recordati.

The remaining author declares that the research was conducted in the absence of any commercial or financial relationships that could be construed as a potential conflict of interest.

Copyright (C) 2021 Chiappini, Schifano and Martinotti. This is an open-access article distributed under the terms of the Creative Commons Attribution License (CC BY). The use, distribution or reproduction in other forums is permitted, provided the original author(s) and the copyright owner(s) are credited and that the original publication in this journal is cited, in accordance with accepted academic practice. No use, distribution or reproduction is permitted which does not comply with these terms. 


\section{Ranking the Harm of Psychoactive Drugs Including Prescription Analgesics to Users and Others-A Perspective of German Addiction Medicine Experts}

\section{OPEN ACCESS}

Edited by:

Giovanni Martinotti,

University of Studies G. D'Annunzio

Chieti and Pescara, Italy

Reviewed by:

Fabrizio Schifano,

University of Hertfordshire,

United Kingdom

Mariangela Corbo,

Università Degli Studi G. D'Annunzio

Chieti e Pescara, Italy

Fabio De Giorgio,

Catholic University of the Sacred

Heart, Piacenza, Italy

${ }^{*}$ Correspondence:

Udo Bonnet

udo.bonnet@uni-due.de

Specialty section:

This article was submitted to

Addictive Disorders,

a section of the journal

Frontiers in Psychiatry

Received: 06 August 2020 Accepted: 22 September 2020

Published: 26 October 2020

Citation:

Bonnet U, Specka M, Soyka M,

Alberti T, Bender S, Grigoleit T,

Hermle L, Hilger J, Hillemacher T,

Kuhlmann T, Kuhn J, Luckhaus C,

Lüdecke C, Reimer J, Schneider U,

Schroeder W, Stuppe M,

Wiesbeck GA, Wodarz N, McAnally H and Scherbaum N (2020) Ranking the Harm of Psychoactive Drugs Including Prescription Analgesics to Users and

Others-A Perspective of German Addiction Medicine Experts.

Front. Psychiatry 11:592199.

doi: 10.3389/fpsyt.2020.592199
Udo Bonnet ${ }^{1,2 *}$, Michael Specka ${ }^{2}$, Michael Soyka ${ }^{3}$, Thomas Alberti $^{4}$, Stefan Bender ${ }^{5}$, Torsten Grigoleit ${ }^{6}$, Leopold Hermle ${ }^{7}$, Jörg Hilger ${ }^{8}$, Thomas Hillemacher ${ }^{9,10}$, Thomas Kuhlmann ${ }^{11}$, Jens Kuhn ${ }^{4,12}$, Christian Luckhaus ${ }^{13}$, Christel Lüdecke ${ }^{14}$, Jens Reimer ${ }^{15,16}$, Udo Schneider ${ }^{17}$, Welf Schroeder ${ }^{18}$, Markus Stuppe ${ }^{19}$, Gerhard A. Wiesbeck ${ }^{20}$, Norbert Wodarz ${ }^{21}$, Heath McAnally ${ }^{22,23}$ and Norbert Scherbaum ${ }^{2}$

${ }^{1}$ Department of Psychiatry, Psychotherapy and Psychosomatic Medicine, Evangelisches Krankenhaus Castrop-Rauxel, Academic Teaching Hospital of the University of Duisburg/Essen, Castrop-Rauxel, Germany, ${ }^{2}$ Department of Psychiatry and Psychotherapy, Faculty of Medicine, Landschaftsverband Rheinland-Hospital Essen, University of Duisburg-Essen, Essen, Germany, ${ }^{3}$ Department of Psychiatry and Psychotherapy University Hospital, Ludwig-Maximilians-Universität München, Munich, Germany, ${ }^{4}$ Department of Psychiatry, Psychotherapy and Psychosomatic, Johanniter Hospital Oberhausen, Oberhausen, Germany, ${ }^{5}$ Psychiatry, Psychotherapy and Psychosomatics, Landschaftsverband Westfalen-Lippe-Hospital Marsberg, Marsberg, Germany, ${ }^{6}$ Psychiatry and Psychotherapy, Landschaftsverband Rheinland-Hospital Langenfeld, Langenfeld, Germany, ${ }^{7}$ Clinic for Psychiatry and Psychotherapy, Christophsbad, Göppingen, Germany, ${ }^{8}$ Clinic for Psychiatry, Psychotherapy, Psychosomatics and Neurology, Evangelische Stiftung Tannenhof, Remscheid, Germany, ${ }^{9}$ Department of Psychiatry, Socialpsychiatry and Psychotherapy, Hannover Medical School, Hanover, Germany, ${ }^{10}$ Department of Psychiatry and Psychotherapy, Paracelsus Medical University, Nuremberg, Germany, ${ }^{11}$ Clinic for Psychosomatics Bergisch-Gladbach, Bergisch Gladbach, Germany, ${ }^{12}$ Faculty of Medicine and University Hospital Cologne, University of Cologne, Cologne, Germany, ${ }^{13}$ Division of Cognitive Neuropsychiatry and Psychiatric Preventive Medicine, Department of Psychiatry, Landschaftsverband Westfalen-Lippe University Hospital Bochum, Ruhr University Bochum, Bochum, Germany, ${ }^{14}$ Lower Saxonian Psychiatric Hospital, Asklepios Hospital, Göttingen, Germany, ${ }^{15}$ Center for Interdisciplinary Addiction Medicine, University Medical Center Hamburg-Eppendorf, Hamburg, Germany, ${ }^{16}$ Health North Hospital Group Bemen, Bremen, Germany, ${ }^{17}$ Department of Psychiatry and Psychotherapy, Ruhr-University Bochum, Campus Ostwestfalen-Lippe, Luebbecke, Germany, ${ }^{18}$ MEDIAN Clinics Wied, Wied, Germany, ${ }^{19}$ Department of Addiction Medicine, Helios Medical Center Schwerin, Carl-Friedrich-Flemming-Clinic, Schwerin, Germany, ${ }^{20}$ Psychiatric Hospital, University of Basel, Basel, Switzerland, ${ }^{21}$ Department of Psychiatry and Psychotherapy, University of Regensburg, Regensburg, Germany, ${ }^{22}$ Northern Anesthesia \& Pain Medicine, Limited Liability Company, Eagle River, AK, United States, ${ }^{23}$ Department of Anesthesiology and Pain Medicine, University of Washington School of Medicine, Seattle, WA, United States

Background: Over the past 15 years, comparative assessments of psychoactive substance harms to both users and others have been compiled by addiction experts. None of these rankings however have included synthetic cannabinoids or non-opioid prescription analgesics (NOAs, e.g., gabapentinoids) despite evidence of increasing recreational use. We present here an updated assessment by German addiction medicine experts, considering changing Western consumption trends-including those of NOAs.

Methods: In an initial survey, 101 German addiction medicine physicians evaluated both physical and psychosocial harms (in 5 dimensions) of 33 psychoactive substances including opioids and NOAs, to both users and others. In a second survey, 36 addiction medicine physicians estimated the relative weight of each health and social harm 
dimension to determine the overall harm rank of an individual substance. We compared our ranking with the most recent European assessment from 2014.

Results: Illicit drugs such as methamphetamine, heroin, cocaine and also alcohol were judged particularly harmful, and new psychoactive drugs (cathinones, synthetic cannabinoids) were ranked among the most harmful substances. Cannabis was ranked in the midrange, on par with benzodiazepines and ketamine-somewhat more favorable compared to the last European survey. Prescribed drugs including opioids (in contrast to the USA, Canada, and Australia) were judged less harmful. NOAs were at the bottom end of the ranking.

Conclusion: In Germany, alcohol and illicit drugs (including new psychoactive substances) continue to rank among the most harmful addictive substances in contrast to prescribed agents including opioid analgesics and NOAs. Current laws are incongruent with these harm rankings. This study is the first of its kind to include comparative harm rankings of several novel abused substances, both licit/prescribed and illicit.

Keywords: gabapentinoids, psychoropic drugs use, alcohol, illicit abused substance, new psychoactive drugs

\section{KEY POINTS}

Illicit drugs such as methamphetamine, heroin, and cocaine, and also alcohol were judged particularly harmful.

Prescribed drugs including opioids (in contrast to the USA, Canada and Australia) and non-opioid analgesics including gabapentinoids were judged less harmful.

Current laws are somewhat incongruent with these harm rankings.

\section{INTRODUCTION}

Abuse of addictive psychoactive substances is characterized by negative health and social consequences not only for the user, but also for non-users in the community or society $(1,2)$. The DSM-5 has defined various specific substance-related dependence and addiction conditions (3), and ICD-10 coding reflects distinct mental and behavioral disorders related to alcohol, tobacco, opiates, cocaine, stimulants, hallucinogens, sedatives and hypnotics, cannabis and cannabinoids, and volatile solvents (4).

Over the past 15 years, the relative health and social harms potential of various addictive substances has been determined in England (5), the Netherlands (6), Scotland (7), France (8), and most recently in Australia (9) by medical and non-medical addiction experts. The average overall harm of various substances is usually reported in relative rankings, based upon multidecision analyses $(5,9)$ or relying on "ad-hoc" assessments (6-8) using validated health and social dimensions (5). These rankings do not necessarily display congruence with legislative and law enforcement priorities in terms of relative regulation and control of substances, with alcohol being a prime example of dissonance between overall harms and control efforts (5-9). Nutt et al. were the first to demonstrate this incongruity (5).
In 2014, a group of 40 medical and non-medical addiction experts from $21 \mathrm{EU}$ countries came to the same conclusion (10). This survey included 20 substances (10). In the interim, as in other Western countries, there have been shifting patterns of substance abuse trends as well as political framework conditions in Germany, especially

- Increasing abuse of methamphetamine mainly in regions bordering the Czech Republic (11-13).

- Increasing occurrence of new psychoactive substances (NPS), in particular a plethora of synthetic cannabinoids and stimulants (mostly cathinones) (12-14).

- Increasing fatal overdoses with heroin/morphine, opioidcontaining, and non-opioid analgesics, synthetic opioids, narcotics, amphetamine, amphetamine derivatives, methamphetamine, and NPS, accompanied by a decrease in overdose deaths through opioid dependence treatment drugs such as methadone and buprenorphine $(11,15)$.

- Increasing availability of highly potent cannabis products with increased risk for psychosis and addiction $(11,13,16,17)$.

- Legalization of medicinal marijuana and cannabinoids for medical prescription (18).

Given these developments, we sought to update the assessment of the health and social harms from substances that are commonly misused in Germany and elsewhere and also of substances less frequently abused in our country, but already emerging $(11,12)$. In this context, synthetic cannabinoids (14) were included into harms rankings for the first time. We also included index surveys of harms rankings for propofol, an intravenous anesthetic (19), and some non-opioid analgesics (NOA), i.e., gabapentinoids, non-steroidal anti-inflammatory drugs (NSAIDs), flupirtine, and triptans (20-24). We decided to include NOAs together with opioid analgesics into our ratings because gabapentin and pregabalin (gabapentinoids) have recently entered the focus of addiction medicine. In the last 
decade, several pharmacovigilance databases, population-based studies and case reports have warned of their potential abuse liabilities and putative contribution to fatal overdoses especially in combination with opioids $(22,23)$. Even though NSAIDs are commonly thought to be non-addictive, there are recent case reports $(25,26)$ and epidemiologic $(27,28)$ as well as clinical data (24) that are raising some safety concerns about this traditional view. Other NOAs have also shown potential abuse and dependence liability e.g., flurpirtine (21) or triptans

TABLE 1 | Participants' characteristics.

\begin{tabular}{|c|c|c|c|}
\hline \multicolumn{2}{|l|}{ Surveys } & \multirow{2}{*}{$\begin{array}{l}\text { Cohort } 1 \\
(n=101)\end{array}$} & \multirow{2}{*}{$\begin{array}{c}\begin{array}{c}\text { Cohort } 2 \\
(n=36)\end{array} \\
52.9(6.9)\end{array}$} \\
\hline Age (years old) & Mean (SD) & & \\
\hline & Median & 50 & 55 \\
\hline \multirow[t]{2}{*}{ Gender } & Female $(n, \%)$ & 26 (25.7\%) & $10(27.8 \%)$ \\
\hline & Male $(n, \%)$ & 75 (74.3\%) & 26 (72.2\%) \\
\hline $\begin{array}{l}\text { Years of professional } \\
\text { experience }\end{array}$ & $\begin{array}{l}\text { Mean (SD) } \\
\text { Median }\end{array}$ & $\begin{array}{l}21.6(9.5) \\
20\end{array}$ & $\begin{array}{c}24.9(8.2) \\
26\end{array}$ \\
\hline \multirow[t]{2}{*}{ Years of tertiary care of SUD } & Mean (SD) & $16.3(8.4)$ & $17.6(7.4)$ \\
\hline & Median & 15 & 16,5 \\
\hline \multirow[t]{2}{*}{$\begin{array}{l}\text { Main focus of professional } \\
\text { work }\end{array}$} & $\begin{array}{l}\text { Acute care } \\
\text { hospital }(n, \%)\end{array}$ & 76 (75.2\%) & 26 (72.2\%) \\
\hline & $\begin{array}{l}\text { Rehabilitation } \\
\text { hospital }(n, \%)\end{array}$ & 25 (24.8\%) & 10 (27.8\%) \\
\hline
\end{tabular}

(20). Therefore, we felt it prudent to include the aforementioned NOAs for the first time in a study of this kind, too. This study is the first of its kind to include comparative harm rankings of several novel abused substances, both licit/prescribed and illicit.

\section{METHODS}

This cross-sectional questionnaire-study comprised two consecutive steps (survey 1 and survey 2, see below), in which quantitative questionnaires were distributed in written form among German addiction medicine experts. These experts were recruited at German addiction congresses and conferences. Additionally, the questionnaires were sent via email to 40 heads of German drug addiction treatment centers who were asked to distribute them in their zone of influence among other addiction medicine experts. Only those questionnaires which had been filled out by physicians who (i) were specialists, i.e., had extra expertise in at least one medical specialty and (ii) had been working longer than 5 years in tertiary care hospitals in the field of substance use disorders (SUD) treatment were included in the analysis. The experts' identity was kept anonymous with the exception of information about their age, gender, specialties, years of professional experience, years of work in tertiary care of SUD, and main focus of professional work (acute care or rehabilitation hospital) (Table $\mathbf{1}$ ).

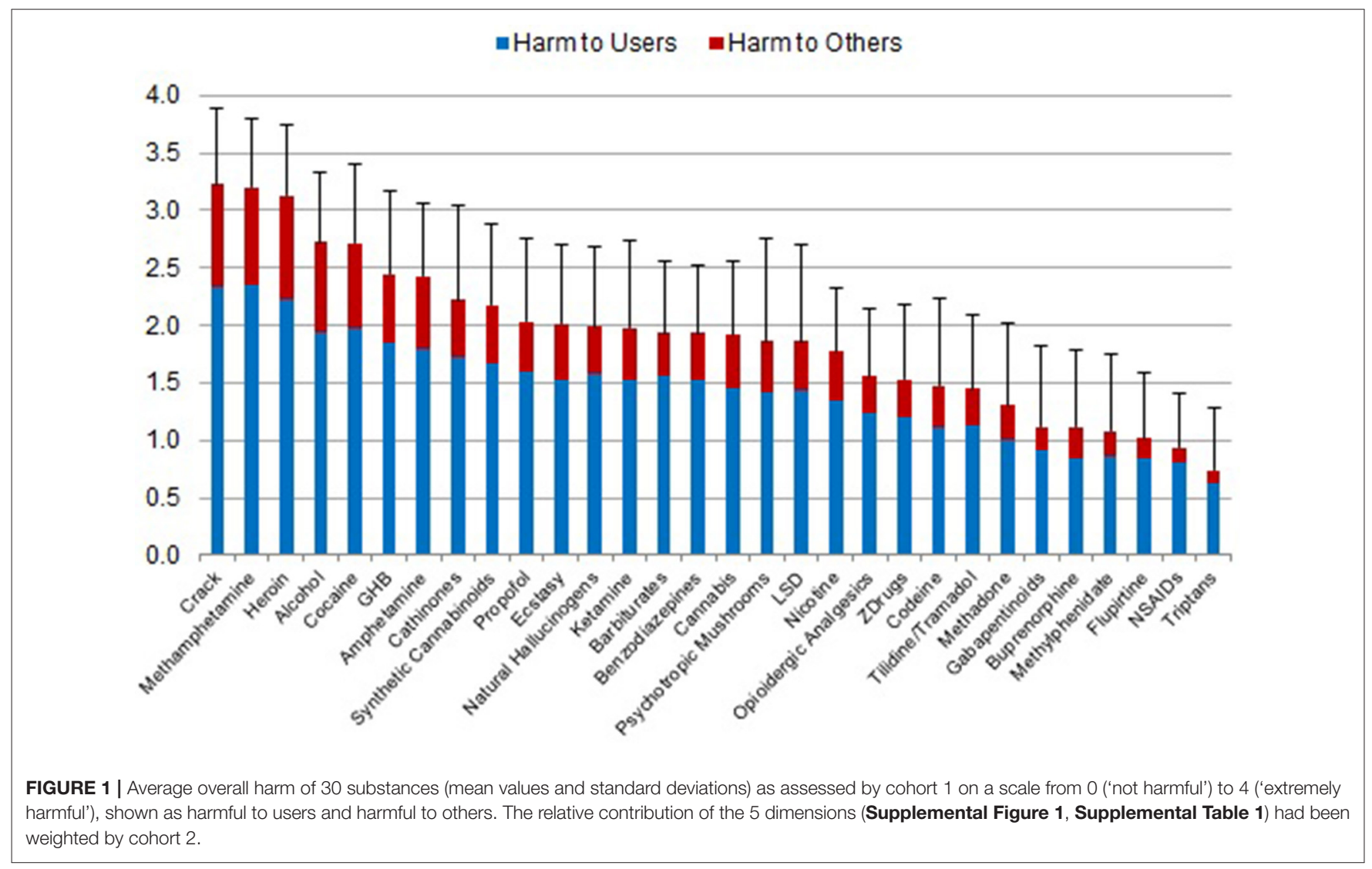


The first survey was conducted from March 2016 to September 2017 and assessed the average harm of 33 substances in in 5 dimensions (physical harm to users, psychological harm to users, social harm to users, physical and psychological harm to others, and social harm to others). As shown in Supplementary Figure 1, these dimensions were defined by 16 criteria, which have been validated in several studies of this type $(5,9,10)$ (see Supplementary Materials-Methods Section). Overall harm to users and overall harm to others comprised 3 (physical, psychological, social) dimensions and 2 (physical \& psychological, social) dimensions, respectively (for details see Supplementary Figure 1). The assessments were carried out using 5-point scales (from "not harmful" to "extremely harmful").

The questionnaire was returned by 122 physicians and from those 101 were evaluated since 21 experts did not meet the inclusion criteria. The physicians were allowed to decide for themselves whether to rate a substance or not, and they were instructed to estimate their professional experience ("no/little" or "moderate" or "a lot") with each substance they had rated. This information was needed to assess the validity of the ratings and to verify defined exclusion criteria, i.e., a substance with $<60 \%$ ratings or more than $60 \%$ "no/little experience" ratings was excluded from further analysis. Consequently, the substances ayahuasca, khat, and kratom had to be excluded from the harmevaluation (Supplementary Figures 2 and 3 ).

The second survey (weighting of the dimensions to determine the overall harm in Figure 1) was conducted from September 2017 to May 2018 by cohort 2, which were recruited only from the emails to the aforementioned 40 heads of German drug addiction treatment centers. This follow-up survey was administered subsequently because the first survey was quite comprehensive, and combining the two surveys was deemed likely to overburden cohort 1 respondents, reducing the return quota. The second survey asked participants to estimate the relative weight (as a proportion between 0 and 1) of each of the 5 dimensions

TABLE 2 | Plausibility check of the overall harm ranks.

\begin{tabular}{|c|c|c|c|c|c|c|c|c|}
\hline Substances/Rank in dimension & PHU & PSHU & SHU & PPHO & SHO & $\mathrm{OH}$ & LD- & LD+ \\
\hline Crack & 2 & 2 & 2 & 1 & 2 & 1 & 0 & 1 \\
\hline Methamphetamine & 1 & 1 & 3 & 3 & 3 & 2 & 1 & 1 \\
\hline Heroin & 5 & 4 & 1 & 2 & 1 & 3 & 2 & 2 \\
\hline Alcohol & 4 & 8 & 5 & 4 & 4 & 4 & 0 & 4 \\
\hline Cocaine & 7 & 3 & 4 & 5 & 5 & 5 & 2 & 2 \\
\hline $\mathrm{GHB}$ & 6 & 5 & 7 & 7 & 7 & 6 & 1 & 1 \\
\hline Amphetamines & 11 & 6 & 6 & 6 & 6 & 7 & 1 & 4 \\
\hline Cathinones & 9 & 10 & 10 & 9 & 8 & 8 & 0 & 2 \\
\hline Synthetic cannabinoids & 13 & 7 & 9 & 8 & 11 & 9 & -2 & 3 \\
\hline Propofol & 10 & 18 & 11 & 13 & 14 & 10 & 0 & 8 \\
\hline Ecstasy & 15 & 16 & 12 & 10 & 9 & 11 & -2 & 5 \\
\hline Natural hallucinogens & 8 & 14 & 18 & 15 & 17 & 12 & -4 & 5 \\
\hline Ketamine & 14 & 15 & 13 & 11 & 12 & 13 & -2 & 2 \\
\hline Barbiturates & 12 & 12 & 17 & 19 & 20 & 14 & -2 & 6 \\
\hline Benzodiazepines & 16 & 9 & 15 & 18 & 16 & 15 & -6 & 3 \\
\hline Cannabis & 21 & 13 & 8 & 17 & 10 & 16 & -8 & 5 \\
\hline Psychotropic mushrooms & 18 & 17 & 16 & 14 & 13 & 17 & -3 & 5 \\
\hline LSD & 20 & 11 & 14 & 16 & 15 & 18 & -7 & 2 \\
\hline Nicotine & 3 & 25 & 24 & 12 & 18 & 19 & -16 & 6 \\
\hline Opioidergic Analgesics & 19 & 19 & 19 & 23 & 22 & 20 & -1 & 3 \\
\hline ZDrugs & 22 & 20 & 22 & 22 & 23 & 21 & -1 & 2 \\
\hline Codeine & 23 & 22 & 20 & 20 & 19 & 22 & -3 & 1 \\
\hline Tilidine/Tramadol & 24 & 21 & 21 & 21 & 24 & 23 & -2 & 1 \\
\hline Methadone & 26 & 24 & 23 & 24 & 21 & 24 & -3 & 2 \\
\hline Gabapentinoids & 27 & 23 & 27 & 27 & 27 & 25 & -2 & 2 \\
\hline Buprenorphine & 30 & 27 & 25 & 25 & 25 & 26 & -1 & 4 \\
\hline Methylphenidate & 28 & 26 & 26 & 26 & 26 & 27 & -1 & 1 \\
\hline Flupirtine & 26 & 28 & 28 & 28 & 28 & 28 & -2 & 0 \\
\hline NSAIDs & 17 & 29 & 29 & 29 & 29 & 29 & -12 & 0 \\
\hline Triptans & 29 & 30 & 30 & 30 & 30 & 30 & -1 & 0 \\
\hline
\end{tabular}

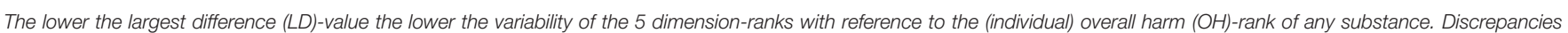

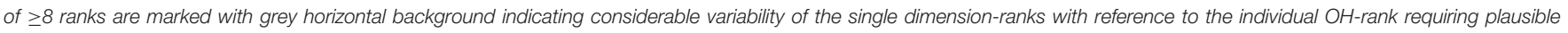

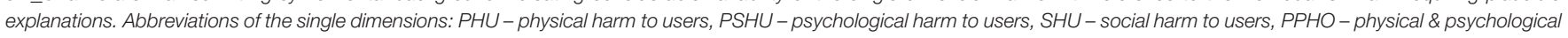

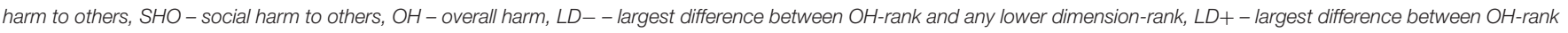
and any higher dimension-rank. 
used in the first survey for the constitution of overall harm of psychotropic substances. All of the 36 returned questionnaires were included. We used the mean relative weight given by the 36 experts to each dimension for calculating the overall harm of each substance (Figure 1). Further details of the overall harm calculation of the remaining 30 substances and related data analyses including the comparison with the previous EU-ranking (Figure 3) are presented in the Supplementary Materials.

Validation of rankings was performed first by evaluating the magnitude of variability between the overall harm rating and any of the five component dimensions. A difference between the overall harm rating and any of the 5 separate ratings in the dimensions $\geq 8$ ranks was considered significant and requires plausibility explanation (Table 2). An additional validation/sensitivity test was performed by substituting our survey-derived mean weights with the consensus-based weights of the previous EU-study (Supplementary Table 1) and comparing the resulting substance-ranks of Supplementary Figure 9 with those of Figure 1 (Supplementary Table 2).

\section{RESULTS}

\section{Sample and Participants' Experience}

The specialist physicians had worked for a median of 15 years (cohort 1) and 16.5 years (cohort 2) in the tertiary care of patients with SUD. Approximately three out of four participants worked in acute care hospitals, with the remainder working in rehabilitation clinics (Table 1).

\section{Average Overall Harm}

Experts' ratings in the 5 separate dimensions are shown in the (Supplementary Figures 4-8). Regarding overall harm, traditional drugs of abuse, i.e., cocaine (including "crack"), methamphetamine, heroin, and alcohol were ranked as being most harmful. The NPS, i.e., cathinones and synthetic cannabinoids, had subordinate positions in the top harm-level group. Ketamine, benzodiazepines, cannabis, psychotropic mushrooms, LSD, nicotine, and opioid analgesics were in the midrange. Methadone and buprenorphine (both preferred in Germany for maintenance therapy of opioid dependence) fell into the lower ranges, while methylphenidate (in Germany the preferred medication for ADHD-treatment), and NOAs were at the lowest ranges of the harm-ranking. Among the NOAs, gabapentin and pregabalin (gabapentinoids) were regarded as more harmful than flupirtine, NSAIDs and triptans (Figure 1).

\section{Difference Between Acute and Rehabilitation Hospital Raters?}

The assessments of the specialists from acute and rehabilitation hospitals were very similar as shown in Figure 2.

\section{Comparison With the Last European Analysis}

This updated German survey assessed methadone, nicotine, cannabis and alcohol as less harmful than did the EU-raters in

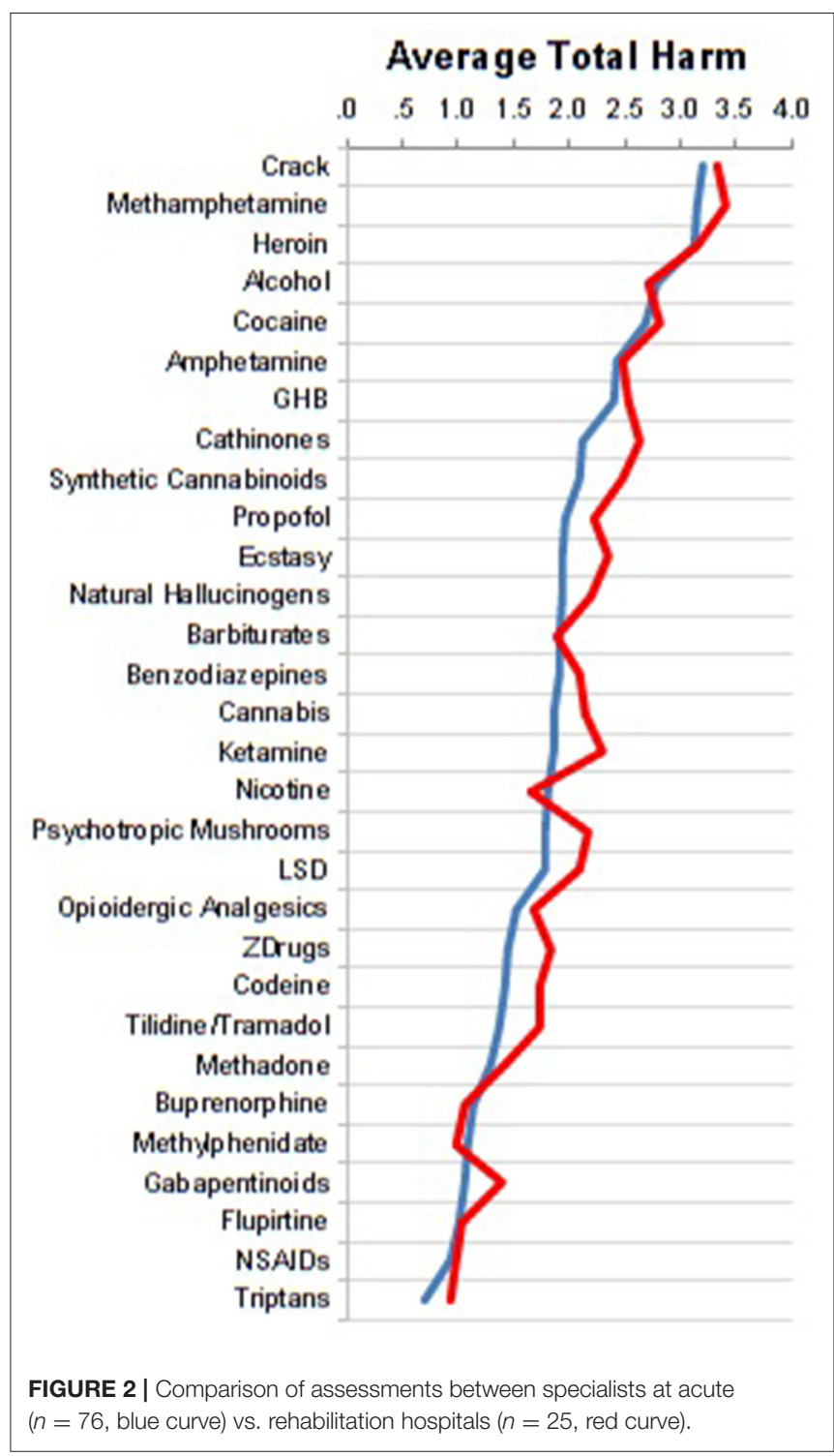

2014 (10), while psychotropic mushrooms, cathinones, ecstasy, $\mathrm{GHB}$, methamphetamine, and crack were judged to be more harmful-see Figure 3.

\section{Plausibility Check and Sensitivity Test}

The lowest discrepancies between the average overall harm-rank and the 5 health and social dimension-ranks were found for the traditional illegal drugs crack (and other cocaine), heroin, methamphetamine, and also for alcohol, which were also ranked at the top positions in terms of harms. The same applied to GHB and NPS ranking near the top, ketamine in the midrange, opioids at lower ranges, and most NOAs (gabapentinoids, flupirtine, triptans) at the lowest ranks. Striking discrepancies were seen for propofol, cannabis, nicotine and NSAIDs (Table 2). In case of nicotine and NSAIDs disproportionate physical harm concerns (e.g., cancer, stroke, coronary disease, COPD for the former, and GI bleeds, renal and cardiovascular disease for the latter) likely account for most of the discrepancy for those substances. 


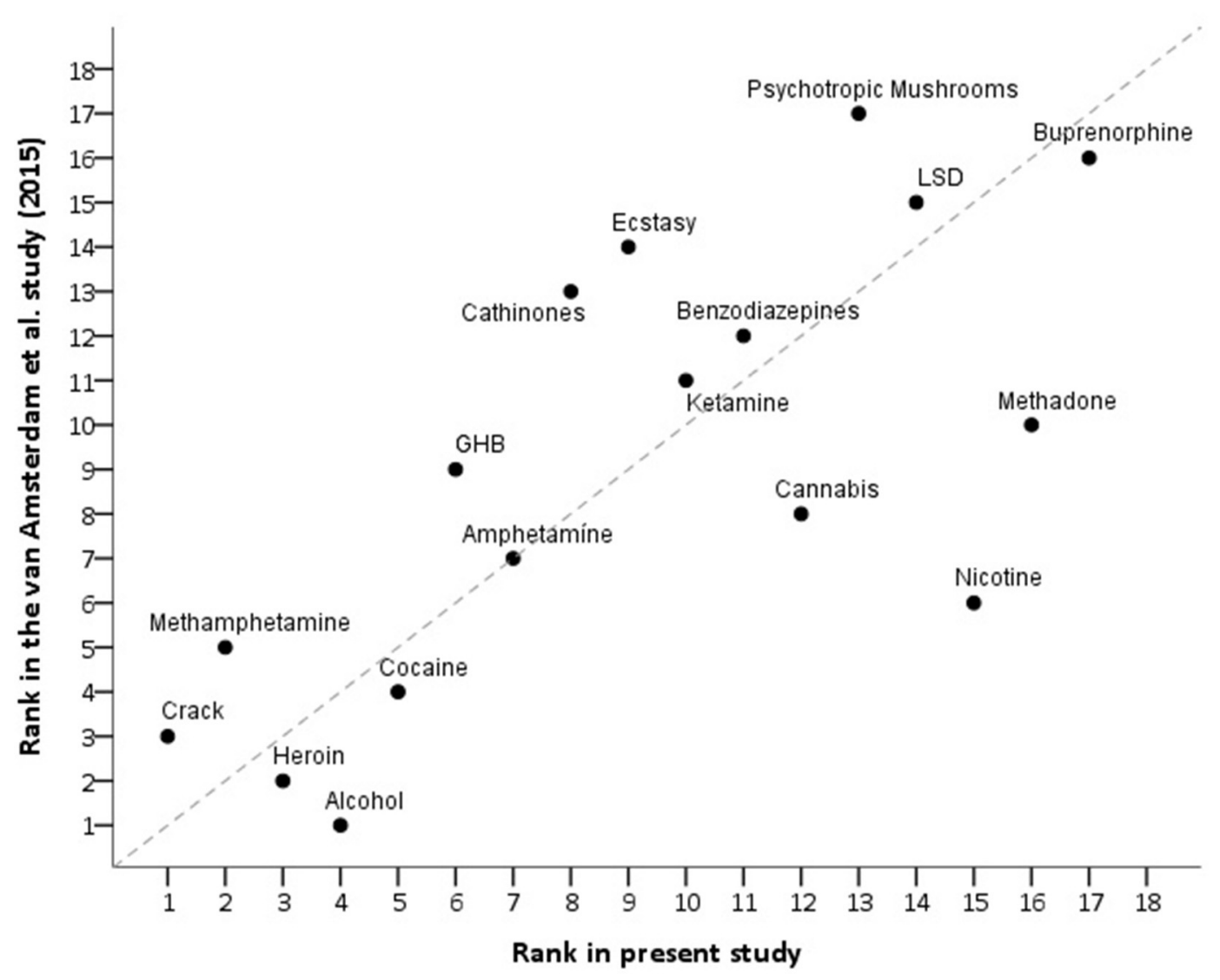

FIGURE 3 | Correlation of the present assessment and the last EU-assessment (10) of the overall harm of drugs of abuse ( $r$ s $=0.73)$. For better orientation, the bisector indicates perfect correlation ( $r s=1)$.

In the case of cannabis, the German literature currently reflects a general perception of relatively low physical harms and conversely a perception of elevated psychosocial harms to users, which dichotomy serves to corroborate the discrepancy here (29-31). The discrepancy for nicotine (and perhaps also for propofol to some extent) may be owing in part to an unexpectedly low ranking of psychological harm to users which diverges from empiric evidence. This potential underestimation may therefore threaten the validity of the overall harm-ranks of these specific substances.

When alternatively, we used the consensus-based weights of the EU-rating study (10) as a comparison sensitivity test, we found that the resulting ranking of overall harms (Supplementary Figure 9) was very similar to our survey-derived weighted rankings shown in Figure 1 (see Supplementary Table 2 for comparison). This suggests that the outlier/skewed weightings of individual dimensions (Supplementary Table 1) do not critically influence the resulting overall harm rankings in our study.

\section{DISCUSSION}

Our data corroborate the situation in many other countries (5-10) of discordance between expert harm rankings of popular drugs of abuse and their regulation by narcotic laws, as evidenced most strikingly by the assessment of alcohol-judged to be among the most harmful substances abused in our country. The relatively high prevalence of alcohol use/abuse (compared to that of less-frequently abused but perhaps more dangerous substances) likely contributes to its dimension-specific ratings, e.g., harm to others, as well as to its overall position. Similarly, the decreasing prevalence of nicotine use in Germany (as tobacco smoking has been banned from many public areas such as hospitals, educational establishments, public transport, restaurants, pubs, and discos during the last 10 years or so) may contribute to a lower-than-expected harm ranking. In addition it should be mentioned that nicotine use, despite its ability to produce considerable behavioral dependence is hardly associated with dramatic psychiatric effects, e.g., in contrast to alcohol or hallucinogen use. This study was the first to compare the harms of various NOAs with harms of well-characterized substances of abuse, and as expected identifies the harms of NOAs to be considerably lower than those of the traditional substances of abuse. The present study was also the first to include synthetic cannabinoids and propofol in an overall-harm ranking schema, which may be beneficial for the psychoeducation of users, for regulatory considerations, or for defining fields of political action for health promotion. 
NPS (cathinones and synthetic cannabinoids) have been assigned to the top harm-level group here. Policy-makers and clinicians would benefit from further data about the NPSphenomenon, e.g., associated morbidity $(32,33)$ and mortality which are on the rise (33).

Compared with the EU-rating from 2014 (10), cannabis, methadone and nicotine were assessed as less harmful, while crack, methamphetamine, GHB, cathinones, ecstasy, and psychotropic mushrooms were seen as more harmful (Figure 3). Cannabis and hallucinogens (i.e., ketamine, psychotropic mushrooms and LSD) were considered to be on the harm level of benzodiazepines or barbiturates. It should be mentioned that psilocybin (in Figure 1 listed as psychotropic mushrooms) and LSD have both enjoyed re-emerging therapeutic potential in psychiatric diseases and appear to show low abuse potential in that context (34).

It is interesting to note that opioid analgesics were not within the top ranks of harmful drugs. This could perhaps be related to the fact that an "opioid epidemic" (such as that in the USA, Canada and Australia), is yet not apparent in Germany or in Western Europe (35-38). The relatively low harm rankings of prescription opioids in our study stand in stark contrast to the high level of stigmatization of illicit opioids. These findings are congruent with the multi-decision analysis of nine experts ( 8 from the United Kingdom and 1 from the Netherlands) suggesting that the overall harms of non-medically used prescription opioids are less than half that of injected street heroin (39).

Methadone was assessed as less harmful than standard opioid analgesics, which viewpoint might be biased by addiction medicine physicians' conception of methadone primarily as a standard opioid dependence maintenance treatment, which in this context has been repeatedly shown to reduce morbidity and mortality (15). In the context of illicit use and abuse, methadone's harms (e.g., apneic and torsades-de-pointe deaths, addiction, and diversion) are obviously considerably higher than those of several other drugs ranked above it. This exposes a major limitation of drug harm-ranking studies based upon subjective assessments as they may not allow for clear differentiation between the harms of a drug with therapeutic indication in a medical context vs. illicit use/misuse outside of that context. These discrepancies in ranking of analgesics among other agents suggest that perhaps raters' experience in pain medicine should have been surveyed as well.

It cannot be excluded that our ratings may be biased toward metropolitan rather than rural perception of substance use harms; clarifying this would require further study in larger samples. Also, a possible gender influence on drug harm perceptions was not explicitly investigated here $(40,41)$. As we had sent out the questionnaires without tracking all recipients, requesting forwarding to other German addiction medicine experts, we are unable to provide information about the exact number of experts who finally received our questionnaires. However, such modus operandi is not unusual for studies of this kind (5). Other limitations, similar to previous studies (5-10) include the fact that the present work cannot claim to meet strict requirements for representativeness. We aimed to reduce subjectivity biases by recruiting a large and homogeneous study group (all physicians specializing in addiction medicine). However, no official statistic exists for how many specialists with more than 5 years of experience in tertiary care of SUD were working in Germany at the time of the study. We estimate that number to be somewhere between 250 and 500 physicians, thus our sample may yield a minority viewpoint. In Germany, addiction medicine experts usually are psychiatrists or general practitioners. Unlike the English (5), EU (10) and Australian (9) studies, we used no consensus-feedbacks. While this additional step may have increased the likelihood of survey participants' agreement (42), we decided against this course, because consensus-based decisions per se do not eliminate subjectivity (43) and there exists no "one-size-fits-all-method" for benefit-risk assessment (44). Furthermore, prior consensusbased studies utilized smaller samples comprising addiction experts from different professions $(5,9,10)$, whose heterogeneity of experiences in the treatment of SUD more likely needed a consensus-based decision strategy than did our homogeneous group. Similar to the Netherlands (6), the Scottish (7), and the French research groups (8) we performed an "ad-hoc" assessment, using validated health and social dimensions, which have been utilized in previous $(5,10)$ and recent $(9)$ empirical studies. This decision to use an "ad-hoc" format maximized the return of completed questionnaires.

Apart from the novel inclusion of NOAs, synthetic cannabinoids and propofol, there are a few strengths of the present study: (i) the utilization of one of the largest samples in this type of study; (ii) the considerable multidimensional addiction medicine experience of the participants, including that of rehabilitation clinic specialists (Figure 2), which in Germany focuses heavily upon psychosocial dimensions and outcomes; (iii) comparison with the previous EU-rating (Figure 3); and (iv) the addition of comparisons of illicit and licit drug rankings to the current literature.

The results of this cross-sectional questionnaire-study update the average overall harm (with component harms from various health and social dimensions) arising from use/misuse of various psychoactive substances (including prescription analgesics) from the perspective of German addiction medicine specialists. It should be emphasized however that these relative overall rankings apply to population-level risks, and depending on the individual and situational context as well as on the intensity of the individual misuse, nearly every psychoactive substance can be used in a very dangerous and harmful way.

\section{CONCLUSION}

This study provides an updated German addiction medicine expert ranking of the average overall harms as well as harms in specific health and social dimensions of various psychoactive substances, including analgesics. Alcohol was estimated to be among the most harmful addictive substances, along with heroin, cocaine, methamphetamine, GHB, and NPS (i.e., synthetic cannabinoids, cathinones). The elevated risks of alcohol are 
somewhat discordant with the German narcotic law, similar to most countries. Cannabis and ketamine were ranked in midrange on par with benzodiazepines. Therapeutically used drugs such as non-opioid analgesics, methylphenidate, and opioids were estimated to be on the whole to be the least harmful at present. Such relative safety perception however is certainly subject to change should misuse and abuse patterns change over time (45).

\section{DATA AVAILABILITY STATEMENT}

The raw data supporting the conclusions of this article will be made available by the authors, without undue reservation.

\section{ETHICS STATEMENT}

The studies involving human participants were reviewed and approved by Ethik-Kommission der Medizinischen Fakultät der Universität Duisburg-Essen. Written informed consent for participation was not required for this study in accordance with the national legislation and the institutional requirements.

\section{REFERENCES}

1. Korpi ER, den Hollander B, Farooq U, Vashchinkina E, Rajkumar R, Nutt DJ, et al. Mechanisms of action and persistent neuroplasticity by drugs of abuse. Pharmacol Rev. (2015) 67:872-1004. doi: 10.1124/pr.115. 010967

2. Morgen K. Counseling and Professional Identity in the 21st Century. Substance use Disorders and Addictions. Thousand Oaks, CA: Sage Publications, Inc (2017).

3. APA American Psychiatric Association. Diagnostic and Statistical Manual of Mental Disorders. 5th ed. Washington, DC: APA (2013).

4. Dilling H, Freyberger H. Taschenführer zur ICD-10-Klassifikation psychischer Störungen. Bern: Huber Verlag (2006).

5. Nutt DJ, King LA, Phillips LD. Independent Scientific Committee on Drugs. Drug harms in the UK: a multicriteria decision analysis. Lancet. (2010) 376:1558-65. doi: 10.1016/S0140-6736(10)61462-6

6. van Amsterdam J, Opperhuizen A, Koeter M, van den Brink W. Ranking the harm of alcohol, tobacco and illicit drugs for the individual and the population. Eur Addict Res. (2010) 16:202-7. doi: 10.1159/00031 7249

7. Taylor M, Mackay K, Murphy J, McIntosh A, McIntosh C, Anderson S, et al. Quantifying the RR of harm to self and others from substance misuse: results from a survey of clinical experts across Scotland. BMJ Open. (2012) 2:e000774. doi: 10.1136/bmjopen-2011-000774

8. Bourgain C, Falissard B, Blecha L, Benyamina A, Karila L, Reynaud M. A damage/benefit evaluation of addictive product use. Addiction. (2012) 107:441-50. doi: 10.1111/j.1360-0443.2011.03675.x

9. Bonomo Y, Norman A, Biondo S, Bruno R, Daglish M, Dawe S, et al. The Australian drug harms ranking study. J Psychopharmacol. (2019) 33:759-68. doi: $10.1177 / 0269881119841569$

10. van Amsterdam J, Nutt D, Phillips L, van den Brink W. European rating of drug harms. J Psychopharmacol. (2015) 29:655-60. doi: $10.1177 / 0269881115581980$

11. DSB (Drogen und Suchtbericht 2018). Die Drogenbeauftragte der Bundesregierung. (2018). Available online at: https://www.drogenbeauftragte.de/fileadmin/dateien-dba/Drogenbeauftragte/ Drogen_und_Suchtbericht/pdf/DSB-2018.pdf (accessed January 6, 2020).

\section{AUTHOR CONTRIBUTIONS}

UB: conception and design. MSp: analysis of the data. UB and MSp: collection and interpretation of data. UB: drafting the article. All authors: revising it critically for important intellectual content.

\section{ACKNOWLEDGMENTS}

We cordially thank Jennifer Haverkemper, MSc (Psychology), for her assistance in developing the questionnaire for Cohort 1, and Ann-Christin Kanti, MD, for data entry and maintenance. We also thank Gabriele Lührmann, chief secretary of the Department for Psychiatry, Psychotherapy, and Psychosomatics at the EVK Castrop-Rauxel for organizing correspondence with colleagues. We also thank the many physicians who kindly took the time to participate in this project.

\section{SUPPLEMENTARY MATERIAL}

The Supplementary Material for this article can be found online at: https://www.frontiersin.org/articles/10.3389/fpsyt. 2020.592199/full\#supplementary-material

12. Atzendorf J, Rauschert C, Seitz N, Lochbühler K, Kraus L. The use of alcohol, tobacco, illegal drugs and medicines. An estimate of consumption and substance-related disorders in Germany. Dtsch Arztebl Int. (2019) 116:577-84. doi: 10.3238/arztebl.2019.0577

13. Seitz NN, Lochbühler K, Atzendorf J, Rauschert C, Pfeiffer-Gerschel T, Kraus L. Trends in substance use and related disorders-analysis of the epidemiological survey of substance abuse 1995 to 2018. Dtsch Arztebl Int. (2019) 116:585-91. doi: 10.3238/arztebl.2019.0585

14. Scherbaum N, Schifano F, Bonnet U. New psychoactive substances (NPS) a challenge for the addiction treatment services. Pharmacopsychiatry. (2017) 50:116-22. doi: 10.1055/s-0043-102059

15. Dematteis M, Auriacombe M, D'Agnone O, Somaini L, Szerman N, Littlewood R, et al. Recommendations for buprenorphine and methadone therapy in opioid use disorder: a European consensus. Expert Opin Pharmacother. (2017) 18:1987-99. doi: 10.1080/14656566.2017.1409722

16. Chandra S, Radwan MM, Majumdar CG, Church JC, Freeman TP, ElSohly MA. New trends in cannabis potency in USA and Europe during the last decade (2008-2017). Eur Arch Psychiatry Clin Neurosci. (2019) 269:5-15. doi: 10.1007/s00406-019-00983-5

17. Di Forti M, Quattrone D, Freeman TP, Tripoli G, Gayer-Anderson C, Quigley $\mathrm{H}$, et al. The contribution of cannabis use to variation in the incidence of psychotic disorder across Europe (EU-GEI): a multicentre case-control study. Lancet Psychiatry. (2019) 6:427-36. doi: 10.1016/S2215-0366(19)30048-3

18. EMCDDA European Monitoring Centre for Drugs and Drug Addiction. 2018 Medical use of Cannabis and Cannabinoids: Questions and Answers for Policymaking. Luxembourg: Publications Office of the European Union (2018). Available online at: http://www.emcdda.europa.eu/system/files/publications/10171/20185584_TD 0618186ENN_PDF.pdf (accessed January 6, 2020).

19. Bonnet U. Assessment of the addictive risk of propofol. Fortschr Neurol Psychiatry. (2011) 79:442-52. doi: 10.1055/s-0031-1273411

20. Beau-Salinas F, Jonville-Béra AP, Cissoko H, Bensouda-Grimaldi L, Autret-Leca E. Drug dependence associated with triptans and ergot derivatives: a case/non-case study. Eur J Clin Pharmacol. (2010) 66:413-7. doi: 10.1007/s00228-009-0769-6

21. Gahr M, Freudenmann RW, Connemann BJ, Hiemke C, SchönfeldtLecuona C. Abuse liability of flupirtine revisited: implications of spontaneous 
reports of adverse drug reactions. J Clin Pharmacol. (2013) 53:1328-33. doi: $10.1002 /$ jcph.164

22. Evoy KE, Morrison MD, Saklad SR. Abuse and misuse of pregabalin and gabapentin. Drugs. (2017) 77:403-26. doi: 10.1007/s40265-017-0700-x

23. Bonnet $U$, Scherbaum N. How addictive are gabapentin and pregabalin? A systematic review. Eur Neuropsychopharmacol. (2017) 27:1185-2015. doi: 10.1016/j.euroneuro.2017.08.430

24. Bonnet U, Strasser JC, Scherbaum N. Screening for physical and behavioral dependence on non-opioid analgesics in a German elderly hospital population. Addict Behav. (2019) 90:265-71. doi: 10.1016/j.addbeh.2018.11.009

25. Etcheverrigaray F, Grall-Bronnec M, Blanchet M, Jolliet P, Victorri-Vigneau C. Ibuprofen dependence: a case report. Pharmacopsychiatry. (2014) 47:115-7. doi: $10.1055 / \mathrm{s}-0034-1371868$

26. Godersky ME, Vercammen LK, Ventura AS, Walley AY, Saitz R. Identification of non-steroidal anti-inflammatory drug use disorder: a case report. Addict Behav. (2017) 70:61-4. doi: 10.1016/j.addbeh.2017.02.008

27. Cryer B, Barnett MA, Wagner J, Wilcox CM. Overuse and misperceptions of nonsteroidal anti-inflammatory drugs in the United States. Am J Med Sci. (2016) 352:472-80. doi: 10.1016/j.amjms.2016.08.028

28. Wójta-Kempa M, Krzyzanowski DM. Correlates of abusing and misusing over-the-counter pain relievers among adult population of Wrocław (Poland). Adv Clin Exp Med. (2016) 25:349-60. doi: 10.17219/acem/58887

29. Hölscher F, Bonnet U, Scherbaum N. Use of an outpatient treatment center for cannabis abuse. Nervenarzt. (2008) 79:571-6. doi: 10.1007/s00115-008-2412-7

30. Bonnet U, Specka M, Scherbaum N. Frequent non-medical cannabis use: health sequelae and effectiveness of detoxification treatment. Dtsch Med Wochenschr. (2016) 141:126-31. doi: 10.1055/s-0041-106313

31. Schneider D. Konsumfolgen und Behandlungsbedarf von CannabisIntensivkonsumenten/innen im ambulanten setting. Sucht. (2016) 62:23-30. doi: 10.1024/0939-5911/a000405

32. Foti F, Marti M, Ossato A, Bilel S, Sangiorgi E, Botrè F, et al. Phenotypic effects of chronic and acute use of methiopropamine in a mouse model. Int J Legal Med. (2019) 133:811-20. doi: 10.1007/s00414-018-1891-8

33. Corkery JM, Schifano F, Martinotti G. How deaths can help clinicians and policy-makers understand the risks of novel psychoactive substances. Br J Clin Pharmacol. (2020) 86:482-98. doi: 10.1111/bcp.14183

34. Chi T, Gold JA. A review of emerging therapeutic potential of psychedelic drugs in the treatment of psychiatric illnesses. J Neurol Sci. (2020) 411:116715. doi: 10.1016/j.jns.2020.116715

35. Häuser W, Schug S, Furlan AD. The opioid epidemic and national guidelines for opioid therapy for chronic noncancer pain: a perspective from different continents. Pain Rep. (2017) 2:e599. doi: 10.1097/PR9.000000000 0000599

36. Kraus L, Seitz NN, Schulte B, Cremer-Schaeffer P, Braun B, Verthein U, et al. Estimation of the number of people with opioid addiction in Germany. Dtsch Arztebl Int. (2019) 116:137-43. doi: 10.3238/arztebl.2019.0137

37. Rosner B, Neicun J, Yang JC, Roman-Urrestarazu A. Opioid prescription patterns in germany and the global opioid epidemic: systematic review of available evidence. PLoS ONE. (2019) 14: e0221153. doi: 10.1371/journal.pone.0221153

38. Meyer A, LeClair C, McDonald JV. Prescription opioid prescribing in Western Europe and the United States. R I Med J. (2020) 103:45-8.

39. van Amsterdam J, Phillips L, Henderson G, Bell J, Bowden-Jones O, Hammersley R, et al. Ranking the harm of non-medically used prescription opioids in the UK. Regul Toxicol Pharmacol. (2015) 73:999-1004. doi: 10.1016/j.yrtph.2015.09.014
40. Bodnar RJ, Kest B. Sex differences in opioid analgesia, hyperalgesia, tolerance and withdrawal: central mechanisms of action and roles of gonadal hormones. Horm Behav. (2010) 58:72-81. doi: 10.1016/j.yhbeh.2009.09.012

41. Di Nicola M, Ferri VR, Moccia L, Panaccione I, Strangio AM, Tedeschi $\mathrm{D}$, et al. Gender differences and psychopathological features associated with addictive behaviors in adolescents. Front Psychiatry. (2017) 8:256. doi: 10.3389/fpsyt.2017.00256

42. Phillips LD, Banae Costa CA. Transparent prioritisation, budgeting and resource allocation with multi-criteria decision analysis and decision conferencing. Ann Operat Res. (2007) 154:51-68. doi: 10.1007/s10479-007-0183-3

43. Rolles S, Measham F. Questioning the method and utility of ranking drug harms in drug policy. Int J Drug Policy. (2011) 22:243-6. doi: 10.1016/j.drugpo.2011.04.004

44. Mt-Isa S, Hallgreen CE, Wang N, Callréus T, Genov G, Hirsch I, Hobbiger SF, et al. Balancing benefit and risk of medicines: a systematic review and classification of available methodologies. Pharmacoepidemiol Drug Saf. (2014) 23:667-78. doi: 10.1002/pds.3636

45. Martinotti G, de Risio L, Vannini C, Schifano F, Pettorruso M, Di Giannantonio M. Substance related exogenous psychosis: a post-modern syndrome. CNS Spectr. (2020) 1-8. doi: 10.1017/S1092852920001479

Conflict of Interest: NS has received honoraria for several activities (e.g., advisory board membership, lectures, manuscripts) from AbbVie, Camurus, Hexal, Janssen-Cilag, MSD, Medice, Mundipharma, Reckitt-Benckiser/Indivior, and Sanofi-Aventis. During the last 3 years he has participated in clinical trials financed by the pharmaceutical industry. TA has received honoraria (e.g., advisory board membership) and/or educational grants from Janssen-Cilag, Medice, and Otsuka-Lundbeck NW has received honoraria for (not product-related) lectures (Janssen-Cilag, mundipharma, and Reckitt-Benckiser/Indivior), During the last 3 years he has participated in clinical trials financed by the pharmaceutical industry and received public funding (BayStMGP) for the evaluation of Take-Home Naloxone. TH has received honoraria for several activities (e.g., advisory board membership, lectures) from Janssen-Cilag, Amomed, Shire, Takeda, Servier MSo has been working as a consultant or has Received speakers freut from Ammomed, Indivior, Camurus for the past 3 years. JR has received honoraria for participation in advisory boards, consulting and lectures from AbbVie, Camurus, Gilead, Hexal, Indivior, and Sanofi-Aventis. JK has received honoraria from Bayer, Janssen, Lundbeck, Neuraxpharm, Otsuka Pharma, Schwabe, and Servier for lecturing at conferences and financial support to travel. He has received financial support for Investigator initiated trials from Medtronic GmbH. HM is also affiliated with a private praxis (Northern Anesthesia; Pain Medicine, LLC, Eagle River, AK, USA), which has no bearing on this study.

The remaining authors declare that the research was conducted in the absence of any commercial or financial relationships that could be construed as a potential conflict of interest.

Copyright (๑) 2020 Bonnet, Specka, Soyka, Alberti, Bender, Grigoleit, Hermle, Hilger, Hillemacher, Kuhlmann, Kuhn, Luckhaus, Lüdecke, Reimer, Schneider, Schroeder, Stuppe, Wiesbeck, Wodarz, McAnally and Scherbaum. This is an open-access article distributed under the terms of the Creative Commons Attribution License (CC BY). The use, distribution or reproduction in other forums is permitted, provided the original author(s) and the copyright owner(s) are credited and that the original publication in this journal is cited, in accordance with accepted academic practice. No use, distribution or reproduction is permitted which does not comply with these terms. 
OPEN ACCESS

Edited by:

Fabrizio Schifano,

University of Hertfordshire,

United Kingdom

Reviewed by:

Antonio Mirijello,

Casa Sollievo della Sofferenza

(IRCCS), Italy

Mei Yang,

Shenzhen Mental Health

Centre, China

*Correspondence:

Stefano Tamburin

stefano.tamburin@univr.it orcid.org/0000-0002-1561-2187

Specialty section:

This article was submitted to

Addictive Disorders,

a section of the journal

Frontiers in Psychiatry

Received: 01 September 2020

Accepted: 12 October 2020

Published: 04 November 2020

Citation:

Tamburin S, Mantovani E, Bertoldi $A$

Federico A, Casari $R$ and Lugoboni $F$ (2020) High-Dose Dependence and

Cognitive Side Effects to Medical

Prescription of Etizolam

Front. Psychiatry 11:601827.

doi: 10.3389/fpsyt.2020.601827

\section{High-Dose Dependence and Cognitive Side Effects to Medical Prescription of Etizolam}

\author{
Stefano Tamburin ${ }^{*}$, Elisa Mantovani ${ }^{1}$, Anna Bertoldi ${ }^{2}$, Angela Federico ${ }^{1}$, \\ Rebecca Casari ${ }^{2}$ and Fabio Lugoboni ${ }^{2}$
}

${ }^{1}$ Department of Neurosciences, Biomedicine and Movement Sciences, University of Verona, Verona, Italy, ${ }^{2}$ Addiction

Medicine Unit, Department of Medicine, Verona University Hospital, Verona, Italy

Introduction: The use of novel designer drugs has increased worldwide over the years. Etizolam is a designer benzodiazepine (BZD) that has raised concern because of its growing non-medical use, liability to tolerance and dependence, and related harms. Studies exploring the abuse liability and cognitive effects of etizolam outside the therapeutic doses are lacking.

Aims: To explore the abuse liability of etizolam and the characteristics of patients affected by etizolam high-dose dependence in a nationwide tertiary referral addiction unit. To document the cognitive changes to etizolam high-dose use.

Design and Methods: Sociodemographic and clinical data on subjects with etizolam high-dose use were retrospectively collected from a database of 1,293 patients consecutively admitted to the Addiction Medicine Unit, Verona University Hospital, Italy for detoxification from high-dose BZDs or Z-drugs dependence. Thorough neuropsychological testing explored the cognitive side effects of high-dose etizolam use.

Results: We found eleven etizolam high-dose users, of which eight used etizolam only, and three used etizolam with other BZDs/zolpidem. All the patients were prescribed etizolam for medical reasons, i.e., anxiety and/or insomnia. Neuropsychological evaluation showed deficits of working memory, visuospatial memory and executive function in a 27-year-old woman who used etizolam $15 \mathrm{mg}$ daily.

Discussion: Our findings suggest that abuse and dependence liability of etizolam should be considered a public health and social problem. They offer preliminary evidence on the cognitive side effects of etizolam high-dose use.

Conclusions: This report offers new information on the potential harms of etizolam in patients who are prescribed this drug for medical reasons.

Keywords: benzodiazepine, BZD, cognition, dependence, etizolam, substance use disorder (SUD), neuropsychology 


\section{INTRODUCTION}

Benzodiazepines (BDZs) are gamma-amino-butyric acid type A (GABA-A) receptor positive allosteric modulators widely prescribed for anxiety, insomnia and other conditions (1).

The increasing use of novel designer BZD derivatives has been recently reported in countries, where these chemical compounds do not have marketing authorization as medicinal products (2).

Etizolam is a short-acting (half-life 5-7 h) thienodiazepine designer BZD with high affinity for the GABA-A receptor and anxiolytic and sedative properties (3). Etizolam is currently approved for therapeutic use and marketed in three countries, namely India, Italy and Japan, but available from the Internet for research purposes worldwide (4). The few available comparative studies reported that etizolam may induce less tolerance than lorazepam and may have lesser sedative effects than alprazolam and diazepam (4). The lower allosteric potency at the $\alpha 1$ subunit of the GABA-A receptor has been proposed as one reason for the reduced liability of etizolam to tolerance and dependence (5).

A consistent increase in the non-medical use and the illicit drug market of etizolam has been reported since 2014, being this drug implicated in several deaths in Scotland, United Kingdom, and to a lesser extent in the United States and Sweden $(4,6)$. A recent review concluded that few harms are documented with the therapeutic use of etizolam, being predominantly related to its non-medical use in illicitly manufactured pills and in the context of mixed-drug toxicity, in particular in combination with opioids (7). The World Health Organization Expert Committee on Drug Dependence (WHO-ECDD) considered etizolam abuse or dependence liability as an effectively public health and social problem (4). Some questions on etizolam side-effects profile are still unanswered. Evidence on etizolam safety is based on preclinical studies and case reports. Common adverse effects of etizolam include drowsiness, sedation and slurred speech, but this drug is considered generally welltolerated in terms of cognitive side effects (4). The auditory P300 was found to be prolonged with etizolam, but this slowed brain response showed habituation, while attention and memory appeared to be unaffected by etizolam (8). Therapeutic etizolam doses $(0.25-1 \mathrm{mg})$ had no effect on cognition in patients with anxiety (9) and on psychomotor performance and vigilance (10). Cognitive effects to higher doses of etizolam are still unexplored.

High-dose dependence of BZDs or related Z-drugs (e.g., zolpidem, zopiclone, eszopiclone, zaleplon), i.e., daily intake $\geq 5$ times the recommended maximum daily dosage (1) is an emerging substance use disorder estimated to affect $0.16 \%$ of the adult population in Switzerland (11), associated to poor quality of life (12), and cognitive dysfunction (13). Data on etizolam high-dose dependence are lacking.

This report is aimed to (a) explore the liability of etizolam to abuse and the characteristics of patients affected by etizolam high-dose dependence in a nationwide tertiary referral addiction unit; (b) document the cognitive changes to etizolam in a high-dose user who underwent thorough neuropsychological evaluation.

\section{METHODS}

Subjects with high-dose etizolam were retrospectively collected from a database of 1,293 patients (650 men, 643 women) aged $>18$ years and admitted (January 2003-December 2019) to the Addiction Medicine Unit, Verona University Hospital, Italy, a nationwide tertiary referral center for detoxification from high-dose BZD/Z-drug dependence with slow flumazenil infusion (14).

High-dose BZD/Z-drug dependence was defined according to DSM-IV-TR criteria (15) with use lasting $>6$ months, daily dosage exceeding at least 5 times the recommended maximum intake (i.e., $>50 \mathrm{mg}$ of daily diazepam dose equivalent, DDDE), otherwise problematic use of BZD/Z-drug, such as mixing different molecules, escalating dosage, obtaining them by illegal means, and using them to enhance the effect of other substances (14).

We collected socio-demographic and clinical variables of the patients. The dosage of BZD/Z-drugs was based on self-report. DDDE (mg) was calculated according to conversion tables (14).

TABLE 1 | Sociodemographic and clinical characteristics of the patients.

\begin{tabular}{|c|c|}
\hline Characteristics & \\
\hline \multicolumn{2}{|l|}{ Sociodemographic variables } \\
\hline Gender (men/women) & $4 / 7$ \\
\hline $\mathrm{Age}^{\mathrm{a}}$ & $41.4 \pm 7.7 ; 41 ; 27-52$ \\
\hline $\begin{array}{l}\text { Education (grade school/high } \\
\text { school/university) }\end{array}$ & $4 / 3 / 4$ \\
\hline Employment (unemployed/employed) & $3 / 8$ \\
\hline Marital status (single/engaged or married) & $6 / 5$ \\
\hline \multicolumn{2}{|l|}{ Clinical variables } \\
\hline Etizolam daily dosage & $27.3 \pm 29.3 ; 15 ; 5-100$ \\
\hline Etizolam formulation (tablet/drops/both) & $3 / 7 / 1$ \\
\hline $\begin{array}{l}\text { Concomitant abuse of other BZD/Z-drugs } \\
\text { (yes/no) }\end{array}$ & $3 / 8$ \\
\hline $\operatorname{DDDE}(m g)^{\mathrm{a}}$ & $272.7 \pm 263.5 ; 150 ; 70-1,000$ \\
\hline BZD/Z-drug use duration (mos) ${ }^{\mathrm{a}}$ & $36.6 \pm 26.0 ; 24 ; 10-84$ \\
\hline Age of first BZD/Z-drug intake ${ }^{a}$ & $24.6 \pm 8.3 ; 21 ; 14-37$ \\
\hline $\begin{array}{l}\text { Reason for prescription (anxiety/sleep } \\
\text { disorders/both) }\end{array}$ & $5 / 1 / 5$ \\
\hline Poly-drug use (yes/no) & $7 / 4$ \\
\hline Alcohol (yes/no) & $5 / 6$ \\
\hline Opioids (yes/no) & $1 / 10$ \\
\hline Cocaine (yes/no) & $2 / 9$ \\
\hline Cannabinoids (yes/no) & $2 / 9$ \\
\hline Tobacco & $5 / 6$ \\
\hline Psychiatric disorders (yes/no) & $9 / 2$ \\
\hline Anxiety disorders (yes/no) & $6 / 5$ \\
\hline Major depression (yes/no) & $4 / 7$ \\
\hline Other psychoses (yes/no) & $1 / 10$ \\
\hline Personality disorders (yes/no) & $2 / 9$ \\
\hline
\end{tabular}

${ }^{a}$ Mean $\pm S D$; median; range. BZD, benzodiazepine; DDDE, daily diazepam dose equivalent (sum of DDDEs for all BZDs and $Z$ drugs in case of concomitant abuse of other BZD/Z-drugs); Mos, months. 


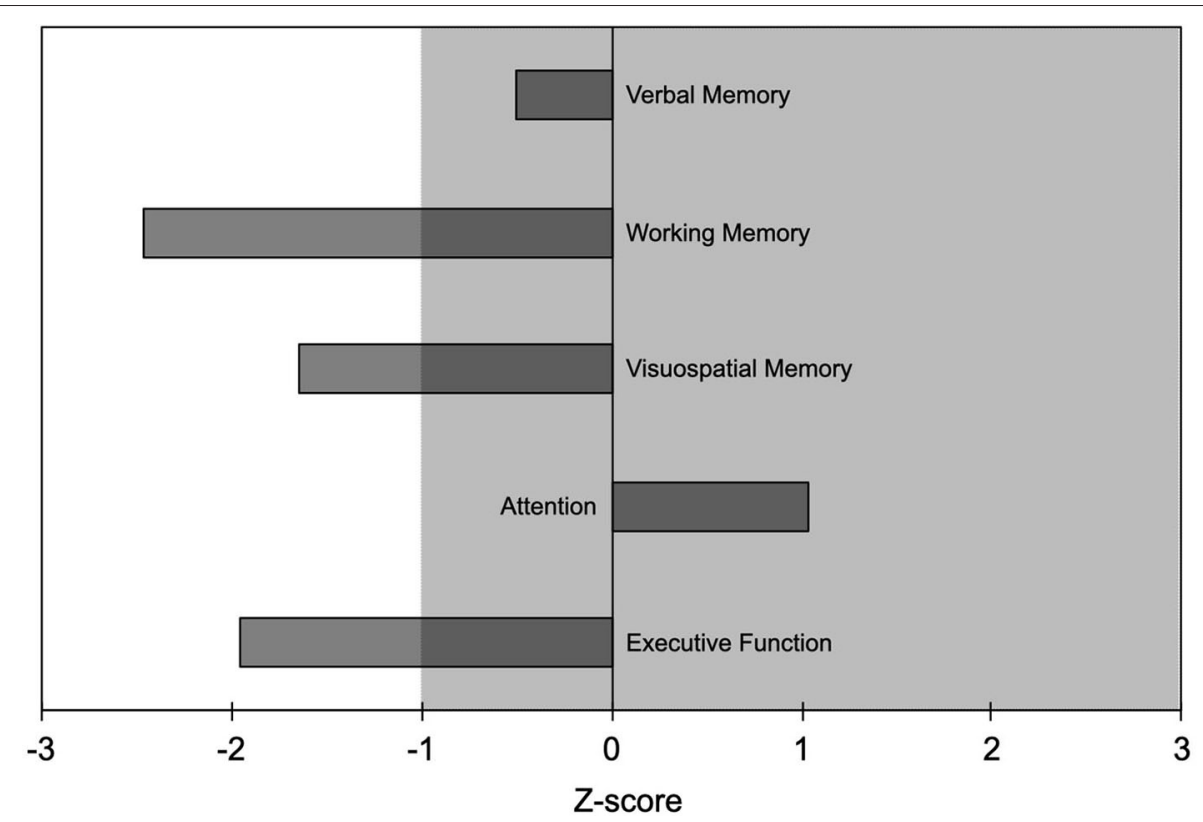

FIGURE 1 | Results of the neuropsychological evaluation from one patient (woman, age 27 years, education 8 years) who took etizolam 15 mg daily. Neuropsychological findings are reported as Z-scores with negative values indicating worse performance and positive values indicating better performance than the average value of the normal population. Abnormal values are worse than mean-1 SD; i.e., Z-scores <-1 indicate abnormal values. Shaded area indicates normal range of values.

The diagnosis of psychiatric disorders was based on screening tests, diagnostic interviews, and previous psychiatric assessments or evaluations, when available.

Neuropsychological evaluation explored verbal memory, working memory, visuospatial memory, attention and executive function. Verbal memory was assessed by means of the Italian versions of the Verbal Paired Association (VPA) (16) and the Digit Span Forward Test (DSFT) (17). Working memory was evaluated with the Digit Span Backward Test (DSBT) (17) and Paced Auditory Serial Addition Test (PASAT-3) (18). Visuospatial memory was explored with the 10/36 Spatial Recall Test (SPART) (18). Attention was measured with the Trail Making Test Part A (TMT-A) and the Symbol Digit Modalities Test (SDMT) (18). Executive function was evaluated by means of Trail Making Test Part B (TMT-B) (18) and the Stroop test (19). Results were standardized as Z-scores. Cognitive testing was performed 1 month before detoxification treatment.

All patients underwent a detoxification protocol with slow subcutaneous flumazenil infusion $(40.5 \mu \mathrm{g} / \mathrm{h}$ for $24 \mathrm{~h} /$ day for 7 days) with a prophylactic antiepileptic treatment (14).

The study was conducted according to the Declaration of Helsinki and approved by the ethics committee of the Verona University Hospital. All the patients gave written informed consent to the study.

\section{RESULTS}

Among the patients admitted from January 2003 to December 2019, we found 11 patients ( 4 men, 7 women) who used highdose etizolam either as the only BZD (8 patients) or with other
BZDs or Z-drugs (3 patients; bromazepam: 1; lorazepam and zolpidem: 1, clonazepam and triazolam: 1). All the patients were prescribed etizolam for medical use (anxiety: 5 patients; sleep disorders: 1; both reasons: 5) and obtained the drug through a prescription and a pharmacy. The number of patients was stable across years (2003-2007: 2 patients; 2008-2011: 3; 2012-2015: 4; 2016-2019: 2). Sociodemographic and clinical features of patients are reported in Table $\mathbf{1 .}$

The remaining 1,282 patients used other BZD/Z-drugs highdoses [for further details see $(12,13)]$.

One patient (woman, 27 years, education 8 years; $15 \mathrm{mg}$ of etizolam daily) underwent neuropsychological evaluation that showed working memory, visuospatial memory, and executive function to be outside normal values (i.e., $>1$ SD worse than normal controls; Figure 1). No medical conditions or other substance use disorder that could have contributed to the cognitive deficits were reported.

\section{DISCUSSION}

To the best of our knowledge, this is the first report of etizolam high-dose dependence in 11 patients who received etizolam for medical reasons (anxiety and/or sleep disorders). Our patients received on average $27.3 \pm 29.3 \mathrm{mg}$ etizolam daily, which is nearly ten times the maximum recommended daily dosage (i.e., $3 \mathrm{mg}$ ), with one patient taking $100 \mathrm{mg}$ daily, i.e., $>30$ times the maximum daily dosage. These findings support the conclusion of the recent WHO-ECDD report that abuse/dependence liability of etizolam should be considered as a public health and social problem (4). They are also in keeping with individual users 
reports on forums like Bluelight.org (20) and Erowid.org (21) that describe tolerance, craving and withdrawal to etizolam (7).

Etizolam high-dose users represented $0.9 \%$ of our whole sample and did not increase during the nearly 20 years covered by our large database, suggesting some concern, but stable figures across time. The big size difference between etizolam and other BZDs/Z-drugs high-dose users hampered a reasonable statistical comparison. However, when compared to high-dose users of lormetazepam, i.e., the most common BZD of high-dose use in Italy (22), etizolam high-dose users appear to be more frequently women, younger, more frequently employed, with lower DDDE, shorter BZD/Z-drug use duration, smaller age of first BZD/Zdrug intake and more frequent poly-drug use. These findings suggest that some populations of patients might be more prone to non-medical use and dependence of etizolam. In particular, they confirm the risk of etizolam harms in patients with other substance use disorders (4).

The small number of etizolam high-dose users in our large sample is likely related to the number of prescriptions in the general population. Etizolam does not stand among the ten most prescribed BZD active principles in Italy, in that its defined daily dose (DDD) in 2018 was $<0.5 / 1,000$, while that of the most commonly ones ranged from 13.2 for lormetazepam to 0.7 for flurazepam (23). The absence of data on etizolam prescription in Italian population, however, hampers the estimation of the conversion-rate from etizolam prescription to addiction.

Etizolam negatively influenced most of the cognitive domains in the patient who underwent neuropsychological testing, in particular working memory, visuospatial memory, and executive function, some of them being $<2$ SDs worse than normal values. This finding, despite being preliminary since stemming from a single patient, extends the notion that high doses of BZDs have an impact on cognition, even in younger patients $(13,24)$. BZDs cognitive side effects have been suggested to be related to the function of the GABA-A receptor $\alpha 1$ (responsible for anterograde amnesia) and the $\alpha 5$ subunits, which are involved in cognition, learning and memory (25). Based on animal studies showing etizolam lower affinity for the GABA-A receptor $\alpha 1$ subunit than

\section{REFERENCES}

1. Janhsen K, Roser P, Hoffmann K. The problems of long-term treatment with benzodiazepines and related substances. Prescribing practice, epidemiology and the treatment of withdrawal. Dtsch Arztebl Int. (2015) 112:1-7. doi: 10.3238/arztebl.2015.0001

2. Carpenter JE, Murray BP, Dunkley C, Kazzi ZN, Gittinger MH. Designer benzodiazepines: a report of exposures recorded in the national poison data system, 2014-2017. Clin Toxicol. (2019) 57:282-6. doi: 10.1080/15563650.2018.1510502

3. Altamura AC, Moliterno D, Paletta S, Maffini M, Mauri MC, Bareggi S. Understanding the pharmacokinetics of anxiolytic drugs. Expert Opin Drug Metab Toxicol. (2013) 9:423-40. doi: 10.1517/17425255.2013.759209

4. World Health Organization. Expert Committee on Drug Dependence: fortysecond report. Geneva. World Health Organization (2020). Available online at: https://apps.who.int/iris/handle/10665/331486 (accessed April 24, 2020).

5. Sanna E, Busonero F, Talani G, Mostallino MC, Mura ML, Pisu MG, et al. Low tolerance and dependence liabilities of etizolam: molecular, fuctional, the $\alpha 5$ one (5), we speculate that the cognitive effects of etizolam high-dose intake in our patient might be mainly mediated by the interaction with the $\alpha 5$ subunit.

The strengths of this study are that it represents the first series of etizolam high-dose users, and offers new information on the harms of this BZD derivative from a nationwide referral center in one of the few countries where it is marketed. The limitations are the retrospective design, the absence of systematic quantitative BZD measures to verify self-reported data, and neuropsychological data from a single patient that suggest caution in generalizing our findings on cognitive side effects of etizolam.

In conclusion, a small number of patients who use etizolam for therapeutic reasons appear to transition to high-dose use requiring specialist care. This report offers new information on the potential harms related to etizolam and extends them to patients who are prescribed this drug for medical reasons. Future studies should confirm our findings in larger populations and in other countries where etizolam is marketed.

\section{DATA AVAILABILITY STATEMENT}

The raw data supporting the conclusions of this article will be made available by the authors, without undue reservation.

\section{ETHICS STATEMENT}

The studies involving human participants were reviewed and approved by Ethics Committee of the Verona University Hospital. The patients/participants provided their written informed consent to participate in this study.

\section{AUTHOR CONTRIBUTIONS}

ST, EM, AB, AF, RC, and FL designed the study and gathered the data. ST analyzed the data. ST, EM, AB, AF, and RC drafted the manuscript. FL and ST revised the manuscript. All authors approved the final version of the manuscript. and pharmacological correlates. Eur J Clin Pharmacol. (2005) 519:3-42. doi: 10.1016/j.ejphar.2005.06.047

6. O'Connell CW, Sadler CA, Tolia VM, Ly BT, Saitman AM, Fitzgerald RL. Overdose of etizolam: the abuse and rise of a benzodiazepine analog. Ann Emerg Med. (2015) 65:465-6. doi: 10.1016/j.annemergmed.2014.12.019

7. Nielsen S, McAuley A. Etizolam: a rapid review on pharmacology, non-medical use and harms. Drug Alcohol Rev. (2020) 39:330-6. doi: 10.1111/dar.13052

8. Fukami G, Hashimoto T, Shirayama Y, Hasegawa T, Watanabe H, Fujisaki $\mathrm{M}$, et al. Effects of etizolam and ethyl loflazepate on the P300 eventrelated potential in healthy subjects. Ann Gen Psychiatry. (2010) 9:37. doi: 10.1186/1744-859X-9-37

9. De Candia MP, Di Sciascio G, Durbano F, Mencacci C, Rubiera M, Aguglia E, et al. Effects of treatment with etizolam $0.5 \mathrm{mg}$ BID on cognitive performance: a 3-week, multicenter, randomized, double-blind, placebo-controlled, two-treatment, three- period, noninferiority crossover study in patients with anxiety disorder. Clin Ther. (2009) 31:2851-9. doi: 10.1016/j.clinthera.2009.12.010 
10. Busardo FP, Di Trana A, Montanari E, Mauloni S, Tagliabracci A, Giorgetti R. Is etizolam a safe medication? Effects on psychomotor perfomance at therapeutic dosages of a newly abused psychoactive substance. Forensic Sci Int. (2019) 301:137-41. doi: 10.1016/j.forsciint.2019.05.018

11. Petitjean S, Ladewig D, Meier CR, Amrein R, Wiesbeck GA. Benzodiazepine prescribing to the Swiss adult population: results from a national survey of community pharmacies. Int Clin Psychopharmacol. (2007) 22:292-8. doi: 10.1097/YIC.0b013e328105e0f2

12. Tamburin S, Federico A, Faccini M, Casari R, Morbioli L, Sartore V, et al. Determinants of quality of life in high-dose benzodiazepine misusers. Int J Environ Res Public Health. (2017) 14:38. doi: 10.3390/ijerph14010038

13. Federico A, Tamburin S, Maier A, Faccini M, Casari R, Morbioli L, et al. Multifocal cognitive dysfunction in high-dose benzodiazepine users: a crosssectional study. Neurol Sci. (2017) 38:137-42. doi: 10.1007/s10072-016-2732-5

14. Tamburin S, Faccini M, Casari R, Federico A, Morbioli L, Franchini E, et al. Low risk of seizures with slow flumazenil infusion and routine anticonvulsant prophylaxis for high-dose benzodiazepine dependence. J Psychopharmacol. (2017) 31:1369-73. doi: 10.1177/0269881117714050

15. American Psychiatric Association, APA. Diagnostic and Statistical Manual of Mental Disorders. 4th ed. Washington, DC: American Psychiatric Press (2000).

16. Zappalà G, Measso G, Cavarzeran F, Grigoletto F, Lebowitz B, Pirozzolo F, et al. Aging and memory: corrections for age, sex and education for three widely used memory tests. Ital J Neurol Sci. (1995) 16:177-84. doi: 10.1007/BF02282985

17. Orsini A, Grossi D, Capitani E, Laiacona M, Papagno C, Vallar G. Verbal and spatial immediate memory span: normative data from 1355 adults and 1112 children. Ital J Neurol Sci. (1987) 8:539-48. doi: 10.1007/BF023 33660

18. Goretti B, Patti F, Cilia S, Mattioli F, Stampatori C, Scarpazza C, et al. The rao's brief repeatable battery version $\mathrm{B}$ : normative values with age, education and gender corrections in an Italian population. Neurol Sci. (2014) 35:79-82. doi: 10.1007/s10072-013-1558-7

19. Brugnolo A, De Carli F, Accardo J, Amore M, Bosia LE, Bruzzaniti C, et al. An updated Italian normative dataset for the stroop color word test (SCWT). Neurol Sci. (2016) 37:365-72. doi: 10.1007/s10072-015-2428-2
20. Bluelight.org. Benzos (thienodiazepine) Etizolam Megathread V2. (2020) Available online at: https://www.bluelight.org/xf/threads/thienodiazepineetizolam-megathread-v2.660685/page-97\#post-14600229 (accessed April 24, 2020)

21. Erowid.org. Etizolam. (2020). Available online at: https://erowid.org/pharms/ etizolam/ (accessed April 24, 2020).

22. Faccini M, Tamburin S, Casari R, Morbioli L, Lugoboni F. High-dose lormetazepam dependence: strange case of Dr. Jekyll and Mr. Hyde. Intern Emerg Med. (2019) 14:1271-8. doi: 10.1007/s11739-019-02101-8

23. Agenzia Italiana del Farmaco (AIFA). The Medicines Utilisation Monitoring Centre. National Report on Medicines use in Italy. Year 2018. Rome: Italian Medicines Agency. (2019). Available online at: https://www.aifa.gov.it/ documents/20142/0/Rapporto_OsMed_2018.pdf/c9eb79f9-b791-2759-4a9ee56e1348a976 (accessed April 24, 2020).

24. Federico A, Lugoboni F, Mantovani E, Martini A, Morbioli L, Casari $\mathrm{R}$, et al. Detoxification improves multidomain cognitive dysfunction in high-dose benzodiazepine abusers. Front Neurosci. (2020) 14:747. doi: $10.3389 /$ fnins.2020.00747

25. Mohler H. The legacy of the benzodiazepine receptor: from flumazenil to enhancing cognition in down syndrome and social interaction in autism. $A d v$ Pharmacol. (2015) 72:1-36. doi: 10.1016/bs.apha.2014.10.008

Conflict of Interest: The authors declare that the research was conducted in the absence of any commercial or financial relationships that could be construed as a potential conflict of interest.

The reviewer, AM, declared a past co-authorship with one of the authors, FL, to the handling Editor.

Copyright (c) 2020 Tamburin, Mantovani, Bertoldi, Federico, Casari and Lugoboni. This is an open-access article distributed under the terms of the Creative Commons Attribution License (CC BY). The use, distribution or reproduction in other forums is permitted, provided the original author(s) and the copyright owner(s) are credited and that the original publication in this journal is cited, in accordance with accepted academic practice. No use, distribution or reproduction is permitted which does not comply with these terms. 


\section{OPEN ACCESS}

Edited by:

Liana Fattore,

National Research Council (CNR), Italy

Reviewed by:

Daria Piacentino,

National Institutes of Health $(\mathrm{NIH})$,

United States

Amira Guirguis,

Swansea University, United Kingdom

*Correspondence:

Attilio Negri

ngrtt/@gmail.com

tThese authors have contributed equally to this work and share first authorship

Specialty section:

This article was submitted to Addictive Disorders,

a section of the journal

Frontiers in Psychiatry

Received: 07 August 2020 Accepted: 16 November 2020 Published: 15 December 2020

Citation:

di Giannantonio M, Negri $A$

Schiavone S, Vannini C, Pettorruso M, De-Giorgio F, Verrastro V, Trabace L, Corbo M, Gottardo R, Camuto $C$ Mazzarino M, Barra A, De Berardis D, Lopez Jl, Del Villar CM, Schifano F and Martinotti G (2020) Prescription Drug Misuse in "Clubbers" and Disco Goers in Ibiza. Front. Psychiatry 11:592594.

doi: 10.3389/fpsyt.2020.592594

\section{Prescription Drug Misuse in "Clubbers" and Disco Goers in Ibiza}

\author{
Massimo di Giannantonio ${ }^{1 \dagger}$, Attilio Negri ${ }^{2,3+*}$, Stefania Schiavone ${ }^{4}$, Chiara Vannini ${ }^{1}$, \\ Mauro Pettorruso ${ }^{1}$, Fabio De-Giorgio ${ }^{5,6}$, Valeria Verrastro ${ }^{7}$, Luigia Trabace ${ }^{4}$, \\ Mariangela Corbo ${ }^{1}$, Rossella Gottardo ${ }^{8}$, Cristian Camuto ${ }^{5,9}$, Monica Mazzarino ${ }^{9}$, \\ Andrea Barra ${ }^{10}$, Domenico De Berardis ${ }^{11}$, Juan Iglesias Lopez ${ }^{12}$, \\ Cristina Merino Del Villar ${ }^{12}$, Fabrizio Schifano ${ }^{13}$ and Giovanni Martinotti ${ }^{1,13}$ \\ ${ }^{1}$ Department of Neuroscience, Imaging, Clinical Sciences, University G.d'Annunzio, Chieti-Pescara, Italy, ${ }^{2}$ Department of \\ Clinical, Pharmaceutical and Biological Sciences, School of Life \& Medical Sciences, University of Hertfordshire, Hatfield, \\ United Kingdom, ${ }^{3}$ Postgraduate School of Clinical Pharmacology and Toxicology, University of Milan, Milan, Italy, \\ ${ }^{4}$ Department of Clinical and Experimental Medicine, University of Foggia, Foggia, Italy, ${ }^{5}$ Department of Health Care \\ Surveillance and Bioethics, Section of Legal Medicine, University Cattolica del Sacro Cuore, Rome, Italy, ${ }^{6}$ Fondazione \\ Policlinico Universitario A. Gemelli IRCCS, Rome, Italy, ${ }^{7}$ Department of Medical and Surgical Sciences, Magna Graecia \\ University of Catanzaro, Catanzaro, Italy, ${ }^{8}$ Unit of Forensic Medicine, Department of Diagnostics and Public Health, University \\ of Verona, Verona, Italy, ${ }^{9}$ Laboratorio Antidoping FMSI, Rome, Italy, ${ }^{10}$ Azienda Sanitaria Locale Potenza, Potenza, Italy, \\ ${ }^{11}$ NHS, Department of Mental Health, Psychiatric Service for Diagnosis and Treatment, Hospital "G. Mazzini," ASL 4, Teramo, \\ Italy, ${ }^{12}$ Can Misses Hospital, Ibiza, Spain, ${ }^{13}$ Psychopharmacology, Drug Misuse \& Novel Psychoactive Substances Research \\ Unit, School of Life \& Medical Sciences, University of Hertfordshire, Hatfield, United Kingdom
}

Background: Prescription drug misuse and its related risks are considered a worldwide public health issue. Current trends show that the extent of such phenomenon may not be limited to subjects with psychiatric disorders, as it also spreads to dance party and nightclub attendees, who often consume prescription drugs in combination with alcohol and psychoactive substances. This study aims to report the sociodemographic data and the psychiatric and clinical features of a sample of clubbers reporting prescription drugs use.

Methods: Patients admitted to the psychiatry ward of the Can Misses Hospital in Ibiza were recruited for the study during a span of four consecutive years (2015-2018). The inclusion criteria were age 18-75 years old and the intake of psychoactive substances or more than five alcohol units during the previous $24 \mathrm{~h}$. Substance use habits, psychopathological features, and use of unprescribed pharmaceuticals were investigated. Urine samples were collected and analyzed using gas chromatography/mass spectrometry.

Results: A total of 110 subjects with psychoactive substance intoxication were recruited for the study. Among these, 37 (40\%) disclosed the use of prescription drugs without medical supervision. The most common compounds were benzodiazepines (66\%), antiepileptic drugs (8\%), antidepressants (6\%), opioids (6\%), antipsychotics (6\%), stimulants (6\%), and non-steroidal anti-inflammatory drugs (NSAIDs, 2\%). Prescription drug misuse was negatively associated with the use of psychodysleptics (two-tailed Fisher's exact test $p=0.018, \rho=-0.262$ ).

Conclusions: The use of prescription drugs is also common among clubbers, usually characterized by low propensity to be prescribed benzodiazepines, antipsychotics, or antidepressants. Prescription drugs may be an alternative to classic and novel 
psychoactive compounds or may be used to tamper and self-medicate the effects determined by the use of substances. Party goers should be adequately informed about possible risks of co-intake of psychoactive substances and prescription drugs to prevent serious medical and psychiatric consequences.

Keywords: prescription drugs, novel psychoactive substance (NPS), club drugs, psychopathology, substance usage disorders (SUDs)

\section{BACKGROUND}

Prescription drug misuse and related risks, including coingestion with recreational drugs, have recently risen as a worldwide public health phenomenon. They may involve a variety of medical and social consequences that require effective public health policies to counteract such habit, as well as continuous updates for health professionals to promote education and harm reduction $(1,2)$. Prescription medicine misuse or non-medical use is commonly defined as the use of medications without a prescription or in a manner other than prescribed (3). This includes a number of conditions, such as using these compounds for purposes other than the medical condition they were prescribed for (i.e., recreational use or selfharm), consuming at larger doses or higher frequencies than intended, using an alternative route of administration (e.g., intravenous), or co-using with alcohol or recreational drugs (4). Studies report that the prevalence of misuse of any prescription drug in the United States increased by 67\% from 1991-1992 to 2001-2002, while treatment-seeking for prescription drug use disorders increased by 53\% (2). In 2017, 14 countries in EU reported on the non-medical use of such compounds (5). Among the 10,956 drug-related acute toxicity emergency room (ER) presentations in the Euro-DEN Plus dataset, approximately $29 \%$ involved at least one prescription medicine (most commonly benzodiazepines and opioids), and $45 \%$ of these involved only prescription drugs, with no illicit compounds involved (6).

Current trends show that the extent of prescription drug misuse is not limited to subjects with psychiatric disorders or co-occurring substance use disorders (SUDs). Admissions to ER and psychiatric intensive care units due to psychotropic pharmaceutical intoxications involve a heterogeneous cohort of users, including traditional drug users, "psychonauts" [from

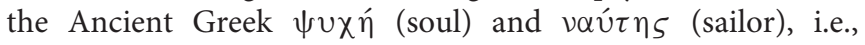
subjects who define themselves as explorers of the human soul through the use of psychoactive substances], clubbers, students, marginalized populations, and individuals with patterns of non-habitual recreational drug consumption (7). In this context, the phenomenon of co-ingesting prescription drugs in order to imitate, potentiate, modulate, or counteract the effects of prohibited psychoactive substances has been increasingly reported (8). This trend involves not only novel highly potent opioid, such as fentanyl and its derivatives, or designer benzodiazepines but also antipsychotics, antidepressants, stimulants, performance-enhancing drugs (PEDs), hormones, vitamins, beta-blockers, gabapentinoids and over-the-counter (OTC) drugs (8).
For example, students and workers may consume attention deficit hyperactivity disorder (ADHD) medications such as methylphenidate to improve their academic performance or working tasks (1). Gamma hydroxybutyrate (GHB), a drug used for many conditions, has been increasingly associated with practices such as "chemsex" (9). Furthermore, compounds such as benzodiazepines (e.g., diazepam and alprazolam) or atypical antipsychotics (e.g., quetiapine and risperidone) are often used by club goers to counteract the effects of psychostimulant drugs, such as cocaine or methylenedioxymethamphetamine (MDMA) (10, 11). Venlafaxine, a selective noradrenaline reuptake inhibitor, has been associated with recreational use at high dosages, earning for itself the name of "baby ecstasy" (i.e., MDMA) (8). With regard to the nightlife and clubbing scene, the situation shows peculiar characteristics. The growing offer of novel and traditional prescription drugs has found a fertile ground in this scenario. Summer holiday periods in popular resorts have historically represented an opportunity for excesses and experimentation, especially among young people who find an environment in which hedonistic partying is socially accepted and drugs are typically easily available (12). Alcohol use, particularly during binge drinking, and psychoactive substance use are commonly reported among festival-goers and clubbers in holiday resorts; practices such as poly-substance abuse and prescription drug misuse have also been reported (13-16). The use of a variety of pharmaceuticals including benzodiazepines $(17,18)$, stimulants $(19,20)$, opioids $(21)$, antidepressants $(8)$, and sedatives such as GHB (22) has been associated to dance music party attendees. Such heterogeneous cohort of compounds, presented in different forms and with various ways of intake (e.g., ingested, snorted, or intravenous), may lead to potential negative medical outcomes, including acute intoxications, SUD, and other psychiatric disorders. Nevertheless, pharmaceuticals are often perceived as less harmful and less stigmatizing than illicit drugs, particularly among young people, partly due to these substances' legitimate medical purposes $(23,24)$. Moreover, information on the actions of these drugs is widely available in package inserts, advertisements, and on the internet; therefore, their effects (including adverse reactions) and dosages are considered more predictable (25).

Such phenomenon is further complicated by the rise on the nightlife market of novel psychoactive substances (NPS). A number of these substances were originally developed as research chemicals and diverted for recreational purposes, as they often mimic the pharmacological effect of traditional drugs of abuse or popular prescription drugs (4). Their effects and related risks are often unknown to both users and health professionals, 
due to the scarcity of evidence-based information regarding their toxicological profiles and to the ever-changing nature of this market $(7,26-28)$. Nevertheless, growing evidence reported potential acute and chronic psychiatric risks associated to NPS consumption, including confusion; paranoid thoughts; auditory and visual hallucinations; dissociation; delusions of reference, persecution, grandeur, and jealousy; cognitive impairment; hypomanic states; aggressiveness and irritability; violence; and suicidal thoughts $(8,29-31)$.

The current dynamic of recreational substance use is a serious matter of concern for public health institutions worldwide. In particular, the threats posed by psychoactive compounds and concomitant prescription drug misuse require updated policies provided by local and supranational regulatory agencies, as well as appropriate approaches by health professional, to prevent negative outcomes and reduce associated harms (32), including deaths (33). In such context, Ibiza and the Balearic Islands, two of the most popular destinations with nightlife resorts for summer holidays in Europe, may be considered as an interesting real-life scenario to explore such phenomenon. Previous studies confirmed a higher prevalence of risky behaviors for both residents and tourists in Ibiza, including problematic alcohol use, substance use, and sexual disinhibition (34-36). Moreover, it has been reported that traffickers and dealers have introduced NPS and pharmaceuticals into the Ibiza drug market to test new compounds and drug combinations on unaware customers (36).

This study aimed to assess patients admitted to the psychiatric ward of the Can Misses Hospital in Ibiza for psychoactive substance intoxication, in order to (1) identify which psychotropic prescription drugs are mostly involved in cases of concomitant psychoactive substance use and (2) report the psychopathological features and patterns of consumption associated to prescription drug use in a nightlife resort setting.

\section{MATERIALS AND METHODS}

Patients admitted to the psychiatry ward of the Can Misses Hospital in Ibiza during summer when nightclubs are open (May-October) were recruited for the study during a span of four consecutive years (2015-2018). The subjects were evaluated according to the DSM-5 diagnostic classification. The inclusion criteria were age 18-75 years old and the intake of psychoactive substances or more than five alcohol units (i.e., $10 \mathrm{ml}$ or $8 \mathrm{~g}$ of pure alcohol) during the previous $24 \mathrm{~h}$. Clinical conditions such as delirium tremens, epilepsy, liver encephalopathy, dementia, and other neurological diseases, severe cardiac failure, diabetes mellitus, severe liver impairment, kidney failure, or neoplastic diseases were among the exclusion criteria, as the presence of such conditions could present a confounding factor. Demographic (age, gender, family, and nationality) and socioeconomic data (living status, job status, and level of education) were collected, as well as recent and past medical and psychiatric history, current pharmacological treatment, and alcohol and substance use habits (including NPS), with a specific focus on prescription drugs misuse. Among these, recent and lifetime use of benzodiazepines (e.g., diazepam, alprazolam, and lorazepam), ADHD medications (e.g., amphetamine/dextroamphetamine and methylphenidate), and opioid painkillers (e.g., morphine, methadone, oxycodone, and fentanyl), as well as other popular prescription drugs (e.g., GHB and gabapentinoids) was investigated.

To explore the different psychopathological aspects related to substance use, such as depressive or manic symptoms, anxiety, psychosis negative and positive symptoms, somatic disorders, aggressiveness, and suicidality, the following psychodiagnostic tests were administered to patients during their hospitalization: Timeline Follow-Back (TLFB) for psychoactive substances and alcohol; Brief Psychiatric Rating Scale (BPRS); Positive and Negative Symptoms Scale (PANSS); Mania Rating Scale (MRS); Hamilton Depression Scale (HAM-D); Hamilton Anxiety Scale (HAM-A); and Modified Overt Aggression Scale (MOAS). TLFB was used to identify the main substance of abuse for each patient. The subjects were divided in three macrogroups according to the TLFB and the results of the urinalysis: psychostimulants (e.g., cocaine, amphetamines, and synthetic cathinones), depressors (e.g., opioids, alcohol, and benzodiazepines), and psychodysleptics (e.g., cannabinoids, psychedelics, and dissociatives). This classification was derived from our previous reports on the topic $(7,36)$.

Data collection was carried out in an anonymous and confidential way; all participants received a detailed explanation of the design of the study and a written informed consent was systematically obtained from every subject, according to the Declaration of Helsinki. Ethics approval was granted by the University of Hertfordshire Health and Human Sciences ECDA, protocol no. aPHAEC1042(03); by the CEI Illes Balears, protocol no. IB 2561/15 PI; and by the University "G. d'Annunzio" of Chieti-Pescara, no. 7/09-04-2015. Majorcan local ethics committee also gave approval to the study.

\section{Urine Sample Analysis}

A urine sample was collected at admission, stored at $-30^{\circ} \mathrm{C}$, and subsequently analyzed at the laboratory of the Department of Forensic Toxicology of the Università Politecnica delle Marche, at the FMSI Antidoping of Rome, and at the University of Verona, Italy. The urine samples were analyzed at the FMSI Antidoping of Rome using a routine screening test for drugs of abuse. The urine samples were extracted with a solid-phase cartridge (Oasis MCX), and the obtained solution was evaporated until dry and reconstituted with mobile phase. An Agilent 1290 Infinity II UHPLC with a binary gradient system and an automatic injector (Agilent Technologies, Cernusco sul Naviglio, Milano, Italy) was used for the chromatographic separation. The instrument was equipped with an Agilent ZORBAX Eclipse Plus C18 column $(100 \times 2.1 \mathrm{~mm}$ i.d., particle size $1.8 \mu \mathrm{m})(37)$. The detector was an Orbitrap Q Exactive (Thermo Fisher Scientific) with an ESI source. The method was validated according to WADA guidelines and for a screening method in antidoping test defining selectivity, limit of detection (LOD), recovery, carry over, and repeatability (38). The method showed no interference or carry over, $\mathrm{LOD}<1 \mathrm{ng} / \mathrm{ml}$, recovery $>70 \%$, and repeatability estimated as $\mathrm{CV} \%<1 \%$ for all the analytes. 
A comprehensive screening of urine samples was performed at both the Unit of Forensic Medicine of the University of Verona and at the Politecnico of Ancona, by using a Toxtyper ${ }^{\mathrm{TM}}$ LC/IT-MS platform (Bruker Daltonics, Bremen, Germany) consisting of an ultra high performance liquid chromatography (UHPLC) coupled to a high-speed ion trap mass analyzer (IT-MS). The instrument applied the analytical protocols provided by the manufacturer, and compound identification was provided by using the Maurer/Wissenbach/Weber (MWW) library containing as many as 4,500 therapeutic, toxic/illicit drugs and their metabolites (including NPS) (39). Prior to injection, urine sample were diluted $1 / 10(\mathrm{v} / \mathrm{v})$ with water (40).

\section{Data Analysis}

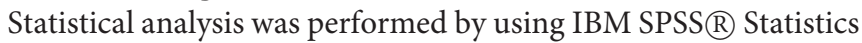
software, version 20 and GraphPad 5.0 software for Windows (La Jolla, CA, USA). Fisher's exact test was used to determine whether or not there was a significant association between the categorical variables "abuse of prescription drugs" and "use of distinct categories of psychoactive substances." Spearman's correlation value $(\rho)$ was calculated to determine if variables (abuse of prescription drugs and categories of substances) were positively or negatively correlated. Independent samples $t$-test was used to determine whether or not there was a significant difference in scale scores between subjects who abused and subjects who did not abuse prescription drugs. One-way analysis of variance (ANOVA) followed by Tukey's post-hoc test was used to assess whether or not there was a significant difference in scale scores among subjects who abused different classes of prescription drugs. For all tests, a two-tailed $p$-value $<0.05$ was considered statistically significant.

\section{RESULTS}

A total of 110 subjects were recruited for the study, with most of them being of European nationality $(n=76,71.8 \%)$. Age ranged from 19 to 63 years old, with the majority of patients $(n=57$, $51.8 \%)$ under 30 years old. The median age of the 110 patients was 32.57 years. A higher percentage of males $(n=76,69.1 \%)$ was reported in our sample. Nine patients were full-time or parttime students (8.1\%), 52 (47.3\%) were employees, and 40 (36.4\%) were unemployed.

All the subjects of the sample were diagnosed with substance intoxication at admission. Although the majority of patients declared multiple substance use $(n=77,70.0 \%)$ and $33 \%$ of them reported the use of more than two substance, the participants were divided in three macro-groups according to their responses to the TLFB test and their urinalysis results to identify a category of substances "of choice" for each patient. Thus, 17 (15\%) depressors users, 44 (40\%) stimulant users, and 49 (45\%) psychodysleptics users were identified.

When asked about lifetime use of specific groups of substances, stimulant use was disclosed by 74 patients (32\%) and cannabinoid use by 68 patients (29\%). These were followed by depressors $(n=32,14 \%)$, empathogens-entactogens $(n=$ $28,12 \%)$, dissociatives $(n=15,6 \%)$, opioids $(n=9,4 \%)$, and psychedelic drugs $(n=7,3 \%)$. Almost half of the participants
TABLE 1 | The most common substances used by patients who declared prescription drug misuse.

\begin{tabular}{lcc}
\hline Prescription Drug & N & $\%$ \\
\hline Benzodiazepines (e.g., diazepam and alprazolam) & 32 & 66 \\
NSAIDs (e.g., paracetamol) & 1 & 2 \\
Antidepressants (e.g., paroxetine and clomipramine) & 3 & 6 \\
Antipsychotics (e.g., risperidone and clotiapine) & 3 & 6 \\
Anticonvulsants (e.g., valproate and pregabalin) & 4 & 8 \\
Opioid derivatives and synthetic opioids (e.g., methadone and fentanyl) & 3 & 6 \\
Stimulants (e.g., methylphenidate) & 3 & 6
\end{tabular}

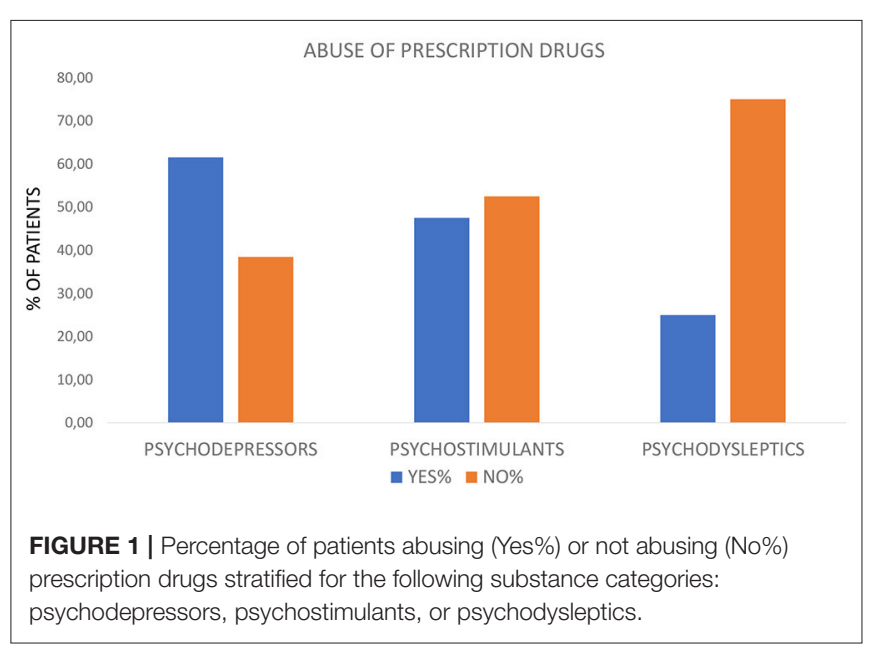

(46\%) declared to have used a substance without knowing what it was at least once in their life. These results will be described in a separate manuscript (31).

In our sample, 37 patients (40\%) disclosed a lifetime misuse of prescription drugs. The most commonly reported compounds were benzodiazepines, which were used by 32 subjects. Table 1 presents the complete information on the type of pharmaceuticals reported by users.

Prescription drug misuse was reported for 8 psychodepressor (e.g., non-prescription opioids and alcohol) users, 19 psychostimulant (e.g., cocaine and amphetamines) users, and 10 psychodysleptic (e.g., cannabis and dissociatives) users. The percentage for each group of substance users is reported in Figure 1. Abuse of unprescribed pharmaceuticals was negatively associated with the use of psychodysleptics (two-tailed Fisher's exact test $p=0.018, \rho=-0.262$ ).

According to their lifetime use of specific compounds, prescription drug consumption without medical supervision was reported by 31 stimulant users, 21 cannabinoid users, 10 depressor users, 7 opioid users, 7 empathogen-entactogen users, 5 dissociative users, and 1 psychedelic user.

The severity of psychiatric symptoms according to HAM-A Psychotic Anxiety scale, PANNS BPRS, and MRS was comparable among users and non-users of unprescribed pharmaceuticals. Patients who disclosed prescription drug misuse tended to report 

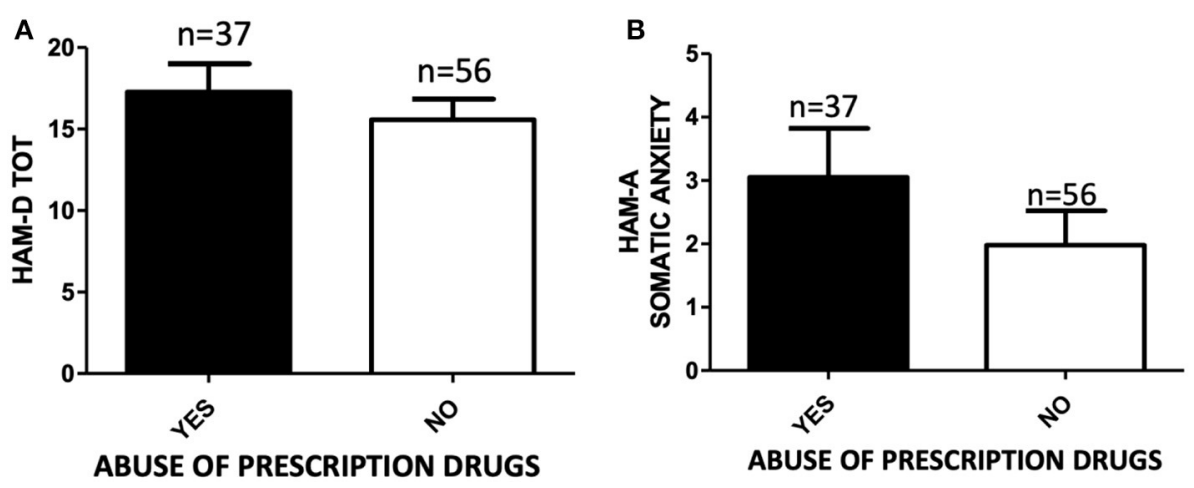

FIGURE 2 | (A) HAM-D total score of subjects abusing (YES, $n=37$ ) or not abusing (NO, $n=56)$ prescription drugs. Independent samples $t$-test, $p$ > 0.05; (B) HAM-A Somatic Anxiety scale score of subjects abusing (YES, $n=37$ ) or not abusing (NO, $n=56$ ) prescription drugs. Student's $t$-test, $p>0.05$.

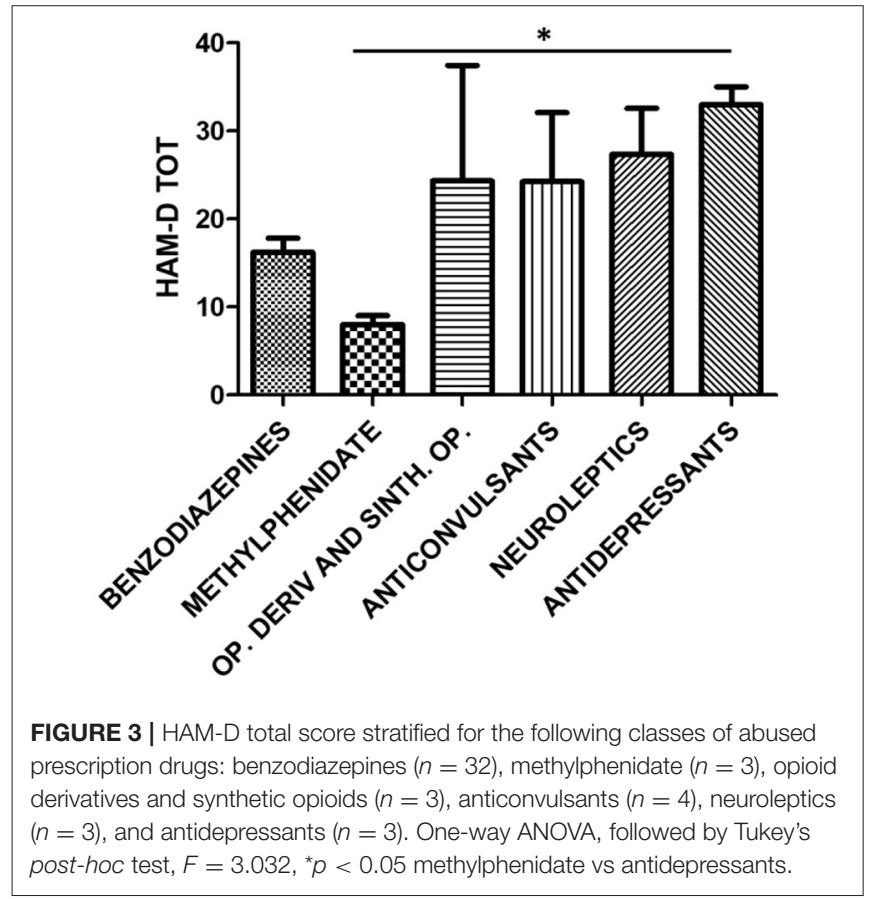

higher scores in HAM-D and HAM-A Somatic Anxiety, although this tendency did not reach the statistical significance (Figure 2).

One-way ANOVA for HAM-A total score $(F=0.6808, p>$ $0.05)$, PANNS $(F=1.487, p>0.05)$, MRS $(F=0.4402, p>0.05)$, and BPRS $(F=3.094, p>0.05)$ did not report any statistically significant difference among users of benzodiazepines, methylphenidate, prescription opioids, anticonvulsants, antipsychotics, and antidepressants. A statistical difference was found for HAM-D scores between methylphenidate and antidepressant users (one-way ANOVA, followed by Tukey's post-hoc test, $F=3.032,{ }^{*} p<0.05$ methylphenidate vs. antidepressants) (Figure 3), with higher scores of depression in the group of patients taking antidepressants.
The most common diagnosis at discharge among the patients who disclosed prescription drug use was substance or alcohol use disorder ( $n=26,48 \%)$, followed by schizophrenia spectrum disorders $(n=10,18 \%)$ (Figure 4).

\section{DISCUSSION}

Our study evaluated the use of prescription drugs among a sample of clubbers, who were mainly composed of young subjects (more than $50 \%$ of the participants being aged under 30) with a medium-high socioeconomic status. Many subjects (40\%) reported the use of prescription drugs. Therefore, our results show that such use is not only limited to subjects with psychiatric disorders and co-occurring SUD but can also involve subjects who are usually not considered as typical psychoactive substance users. This data pave the way for serious considerations on the possible pharmacological interactions with alcohol and other substances, as well as on other shortand long-term consequences, both physical and psychiatric. As users may concomitantly consume various prescription drugs and substances of abuse, an increased risk of drugdrug interactions may be observed, both pharmacokinetic (e.g., between prescription opioids and heroin) and pharmacodynamic (e.g., between opioids of abuse and benzodiazepines or other CNS sedative drugs) (41). This involves not only depressors, such as benzodiazepines, opioids, and alcohol, but also stimulant drugs commonly used by clubbers. For example, metabolic pathways of synthetic cathinones, antidepressants, and ADHD medications have been shown to overlap, including metabolism via cytochrome P450 enzymes and their inhibition (42).

Benzodiazepines were the most prevalent class of prescription drugs reported in our sample. This result may be explained by the use of benzodiazepines as a "trip terminator" to calm down the strong experience caused by the use of multiple substances. This confirms the data from Messina et al. (10), who showed that benzodiazepines and atypical antipsychotics are often used by club goers to counteract the effects of psychostimulant drugs, such as cocaine or MDMA. In terms of preventive strategies, the use of benzodiazepines in the context of a multiple substance use 


\title{
ABUSE OF PRESCRIPTION DRUGS AND DISCHARGE DIAGNOSIS
}

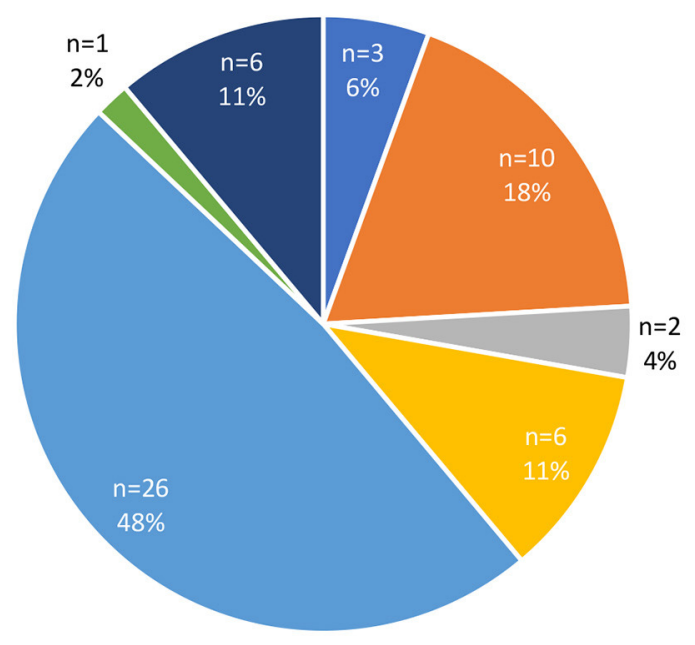

\author{
- Bipolar Spectrum Disorders \\ - Schizophrenia Spectrum Disorders \\ - Depressive Disorders \\ - Cluster B Personality Disorders \\ - Substance and Alcohol Use Disorders \\ - Adjustment Disorders \\ - Behaviour Disorders
}

FIGURE 4 | Discharge diagnosis (expressed both as raw number and \%) of patients abusing prescription drugs.

could be dangerous as it causes respiratory depression and risk of overdoses, specifically in combination with opiates, alcohol, ketamine and derivatives, and inhalants (18, 43, 44). Specific policies and harm-reduction approaches should be advised for these potentially lethal combinations, particularly with the intake of large amounts of long half-life compounds, such as diazepam. Furthermore, a number of novel designer benzodiazepines, with undisclosed toxicological profiles and variable potencies, have recently been made available in the drug market. They are developed in order to mimic prescription benzodiazepines and Z-drugs, but they may lead users to adverse events of various severities, particularly if used in combination with other substances $(4,45,46)$.

Among the different categories of substances, psychodepressors were the most commonly associated with the use of prescription drugs, whereas only a small percentage of psychodysleptic users reported such habit. The typology of subject using psychodysleptics such as LSD, psilocybin, MDMA, ayahuasca, and other plants, is characterized by the search for a strong inner experience, spirituality, and high level of emotionality $(47,48)$. The use of benzodiazepines and antipsychotics can inhibit or temper the perception of these experiences and therefore may not be chosen by users. With regard to antidepressants, which can determine affective blunting and enhance the distance from emotional experiences, the same consideration can be reported.

Interestingly, patients who disclosed prescription drug misuse tended to report higher scores in HAM-D and HAM-A Somatic Anxiety. This finding emphasizes how those patients are the most vulnerable in terms of psychopathological load. In this regard, those who report taking prescription drugs may actually be the subjects with a psychiatric history. A prescription drug may have already been tested for therapeutic purposes and therefore may have made the patient more accustomed to its use out of indication. Moreover, the high level of depression is an issue that needs to be considered and can represent a significant suicidal risk factor in people who misuse alcohol and psychoactive substances. In fact, the use of psychotropics can represent an additional risk factor, given the possibility of a consistent increase in the levels of impulsivity, violence, and self-directed aggression due to such drugs. Therefore, it is very relevant to evaluate these patients and to put specific strategies in place to manage these psychopathological manifestations, with a specific focus on the prevention of anti-conservative behaviors.

A further point of interest, although expected, is the presence of high levels of depressive symptoms on the Hamilton scale in relation to the use of antidepressants without a specific medical prescription. This fact suggests how sometimes the use of prescription drugs may be related not only to the goal to "get high" or to the management of an intoxication but also to the self-medication need of patients who perceive a subleveling of their mood. For this reason, a shared strategy could be justified, even more than in other types of patients with dual disorders. Conversely, methylphenidate use was associated with lower scores at the Hamilton depression scale. This prescription drug with stimulant properties $(49,50)$, usually indicated for attention deficit hyperactivity disorder, can probably be chosen by users of psychostimulants as a cheaper alternative to cocaine and amphetamine. In the short run, it could also show some antidepressant properties, thus explaining the data observed at the HAM-D. The detection of methylphenidate among the prescription drugs reported in our sample may indicate some level of comorbidity between adult ADHD and SUD, as recently reported (51).

In terms of the role of the discharge psychiatric diagnosis, alcohol or substance use disorder showed a high prevalence, 
although the diagnoses of schizophrenia and bipolar spectrum disorder were also significantly reported. In some cases, the presence of a psychiatric comorbidity could justify the use of prescription drugs such as antidepressants, mood stabilizers, and benzodiazepines. However, the presence of a relevant percentage of addiction diagnoses (alcohol use disorder and/or substance use disorder) further confirms that these patients do not typically represent pure psychiatric patients who increase their dosages of prescribed drugs but are instead classical party-goers who use prescription drugs for other purposes.

Limitations of this study are represented by a low and heterogeneous sample size, with a high prevalence of benzodiazepine as the main prescription drug. Moreover, although the target of the study is that of young clubbers, a significant subgroup of participants were middle-aged adults.

In conclusion, in this study, we have highlighted how the use of prescription drugs is common also among clubbers and disco-goers. These subjects usually do not have a previous psychiatric history and share a low propensity to be prescribed with benzodiazepines, antipsychotics, and antidepressants by a mental health professional. These data confirm that prescription drugs may be an alternative for classic and novel psychoactive compounds, may be used to modulate and temper the experience, and, in some cases, may be used to reduce the negative effects determined by the use of substances. From the treatment prospective and as a useful preventive strategy, a specific psycho-education process should be indicated for subjects at risk. Party-goers should be adequately informed about the possible risks of co-intake of NPS, classical substances, and prescription drugs to prevent serious medical and psychiatric consequences.

\section{REFERENCES}

1. Young AM, Glover N, Havens JR. Nonmedical use of prescription medications among adolescents in the United States: a systematic review. J Adolesc Health. (2012) 51:6-17. doi: 10.1016/j.jadohealth.2012.01.011

2. Blanco C, Alderson D, Ogburn E, Grant BF, Nunes EV, Hatzenbuehler ML, et al. Changes in the prevalence of non-medical prescription drug use and drug use disorders in the United States: 1991-1992 and 2001-2002. Drug Alcohol Depend. (2007) 90:252-60. doi: 10.1016/j.drugalcdep.2007.04.005

3. National Institute on Drug Abuse (NIDA), National Institute of Health, USA. Misuse of prescription drugs. Available online at: https://www.drugabuse. gov/publications/research-reports/misuse-prescription-drugs/summary (accessed May 25, 2020).

4. Bersani FS, Imperatori C. Misuse, recreational use and addiction in relation to prescription medicines. In: Corazza O, Roman-Urrestarazu A, editors. Handbook of Novel Psychoactive Substances: What Clinicians Should Know About NPS. New York, NY: Routledge (2018).

5. United Nations Office for Drug and Crime. World Drug Report 2019. United Nations (2019).

6. European Monitoring Center for Drugs and Drugs Addiction (EMCDDA). Hospital Emergency Presentations and Acute Drug Toxicity in Europe: Update From the Euro-DEN Plus Research Group and the EMCDDA. (2016). doi: $10.2810 / 894142$

7. Martinotti G, Merino del Villar C, Giorgetti R, Schifano F, Di Giannantonio M. Novel and traditional club substances are associated to psychopathological and medical sequelae: the Ibiza project. In: Corazza O, Roman-Urrestarazu A,

\section{DATA AVAILABILITY STATEMENT}

The raw data supporting the conclusions of this article will be made available by the authors, without undue reservation.

\section{ETHICS STATEMENT}

The studies involving human participants were reviewed and approved by University of Hertfordshire CEI Illes Balears University G. d'Annunzio of Chieti-Pescara. The patients/participants provided their written informed consent to participate in this study.

\section{AUTHOR CONTRIBUTIONS}

MdG and AN wrote the manuscript. CV, JL, MC, CD, AN, and GM recruited patients inside the Can Misses Hospital of Ibiza. SS and LT performed the statistical analysis. MP, VV, FS, and GM elaborated the study protocol and performed the translation for scales and questionnaire. FD-G, RG, CC, and MM executed the urine analysis in the different centers. $A B$ and DD performed literature search about the topic and elaborated all the ethical procedures required for the study approval in both countries. GM coordinated all the study processes. All authors contributed to the article and approved the submitted version.

\section{ACKNOWLEDGMENTS}

This study was partly funded by the European Project entitled Analysis, Knowledge dissemination, Justice implementation and Special Testing of Novel Synthetic Opioids-JUST-2017AG-DRUG.

editors. Handbook of Novel Psychoactive Substances, What Clinicians Should Know About NPS. New York, NY: Routledge (2018).

8. Schifano F, Chiappini S, Corkery JM, Guirguis A. Abuse of prescription drugs in the context of novel psychoactive substances (NPS): a systematic review. Brain Sci. (2018) 8:73. doi: 10.3390/brainsci8040073

9. Edmundson C, Heinsbroek E, Glass R, Hope V, Mohammed H, White M, et al. Sexualised drug use in the United Kingdom (UK): a review of the literature. Int J Drug Policy. (2018) 55:131-48. doi: 10.1016/j.drugpo.2018. 02.002

10. Messina BG, Dutta NM, Silvestri MM, Diulio AR, Garza KB, Murphy JG, et al. Modeling motivations for non-medical use of prescription drugs. Addict Behav. (2016) 52:46-51. doi: 10.1016/j.addbeh.2015. 07.024

11. Vento AE, Kotzalidis GD, Cacciotti M, Papanti GD, Orsolini L, Rapinesi C, et al. Quetiapine abuse fourteen years later: where are we now? A systematic review. Subst Use Misuse. (2020) 55:304-13. doi: 10.1080/10826084.2019.1668013

12. Kelly D, Hughes K, Bellis MA. Work hard, party harder: drug use and sexual behaviour in young British casual workers in Ibiza, Spain. Int J Environ Res Public Health. (2014) 11:10051-61. doi: 10.3390/ijerph1110 10051

13. Esser MB, Guy GP Jr, Zhang K, BrewerRD. Binge drinking and prescription opioid misuse in the U.S., 2012-2014. Am J Prev Med. (2019) 57:197-208. doi: 10.1016/j.amepre.2019.02.025

14. Busto Miramontes A, Moure-Rodríguez L, Díaz-Geada A, Rodríguez-Holguín $\mathrm{S}$, Corral M, Cadaveira F, et al. Heavy drinking and non-medical use of 
prescription drugs among university students: a 9-year follow-up. Int J Environ Res Public Health. (2019) 16:2939. doi: 10.3390/ijerph16162939

15. Grigg J, Barratt MJ, Lenton S. Double dropping down under: correlates of simultaneous consumption of two ecstasy pills in a sample of Australian outdoor music festival attendees. Drug Alcohol Rev. (2018) 37:851-5. doi: 10.1111/dar.12843

16. Martins D, Barratt MJ, Pires CV, Carvalho H, Vilamala MV, Espinosa IF, et al. The detection and prevention of unintentional consumption of DOx and 25xNBOMe at Portugal's boom festival. Hum Psychopharmacol. (2017) 32:e2608. doi: $10.1002 /$ hup. 2608

17. Kurtz SP, Inciardi JA, Surratt HL, Cottler L. Prescription drug abuse among ecstasy users in Miami. J Addict Dis. (2005) 24:1-16. doi: 10.1300/J069v24n04_01

18. Kurtz SP, Buttram ME, Surratt HL. Benzodiazepine dependence among young adult participants in the club scene who use drugs. J Psychoact Drugs. (2017) 49:39-46. doi: 10.1080/02791072.2016.1269978

19. Butler R, Sheridan J. Innocent parties or devious drug users: the views of primary healthcare practitioners with respect to those who misuse prescription drugs. Harm Reduct J. (2010) 7:21. doi: 10.1186/1477-7517-7-21

20. León KS, Martínez DE. To Study, to party, or both? Assessing risk factors for non-prescribed stimulant use among middle and high school students. $J$ Psychoact Drugs. (2017) 49:22-30. doi: 10.1080/02791072.2016.1260187

21. Palamar JJ. Use of "lean" among electronic dance music party attendees. Am J Addict. (2019) 28:347-52. doi: 10.1111/ajad.12897

22. Brennan R, van Hout MC. Gamma-hydroxybutyrate (GHB): a scoping review of pharmacology, toxicology, motives for use, and user groups. J Psychoactive Drugs. (2014) 46:243-51. doi: 10.1080/02791072.2014.921746

23. Hernandez SH, Nelson LS. Prescription drug abuse: insight into the epidemic. Clin Pharmacol Ther. (2010) 88:307-17. doi: 10.1038/clpt.2010.154

24. Kelly BC, Vuolo M. Social network ties to nightlife and healthcare professionals and prescription drug misuse among young adults. Int J Drug Policy. (2019) 66:48-56. doi: 10.1016/j.drugpo.2019.01.007

25. United Nations Office on Drugs and Crime (UNODC). The non-medical use of prescription drugs, policy direction issues. United Nations Office on Drugs and Crime, 96. (2010). Available online at: http://www.unodc.org/unodc/en/ drug-prevention-and-treatment/non-medical-use-prescription-drugs.html. (accessed March 25, 2020).

26. Schifano F, Leoni M, Martinotti G, Rawaf S, Rovetto F. Importance of cyberspace for the assessment of the drug abuse market: preliminary results from the Psychonaut 2002 project. Cyberpsychol Behav. (2003) 6:405-10. doi: 10.1089/109493103322278790

27. Corazza O, Assi S, Simonato P, Corkery J, Bersani FS, Demetrovics Z, et al. Promoting innovation and excellence to face the rapid diffusion of novel Psychoactive substances in the EU: the outcomes of the reDNet project. Hum Psychopharmacol. (2013) 28:317-23. doi: 10.1002/hup.2299

28. Simonato P, Corazza O, Santonastaso P, Corkery J, Deluca P, Davey Z, et al. Novel psychoactive substances as a novel challenge for health professionals: results from an Italian survey. Hum Psychopharm Clin Exp. (2013) 28:324-31. doi: 10.1002/hup. 2300

29. Kehr J, Ichinose F, Yoshitake S, Goiny M, Sievertsson T, Nyberg F, et al. Mephedrone, compared with MDMA (ecstasy) and amphetamine, rapidly increases both dopamine and 5-HT levels in nucleus accumbens of awake rats. Br J Pharmacol. (2011) 164:1949-58. doi: 10.1111/j.1476-5381.2011.0 1499.x

30. Lovrecic B, Lovrecic M, Gabrovec B, Carli M, Pacini M, Maremmani AGI, et al. Non-medical use of novel synthetic opioids: a new challenge to public health. Int J Environ Res Public Health. (2019) 16:E177. doi: 10.3390/ijerph16020177

31. Martinotti G, De Risio L, Vannini C, Schifano F, Pettorruso M, Di Giannantonio M. Substance related exogenous psychosis: a post-modern syndrome. CNS Spectr. (2020) 25:1-20. doi: 10.1017/S1092852920001479

32. Santacroce R, Ruiz Bennasar C, Sancho Jaraiz JR, Fiori F, Sarchione F, Angelini F, et al. A matter of life and death: substance-caused and substance-related fatalities in Ibiza in 2015. Hum Psychopharmacol Clin Exp. (2017) 32:e2592. doi: 10.1002/hup. 2592

33. Corkery JM, Schifano F, Martinotti, G. How deaths can help clinicians and policy-makers understand the risks of novel psychoactive substances. Br J Clin Pharmacol. (2020) 86:482-98. doi: 10.1111/bcp.14183
34. Bellis MA, Hale G, Bennett A, Chaudry M, Kilfoyle M. Ibiza uncovered: changes in substance use and sexual behaviour amongst young people visiting an international night-life resort. Int J Drug Policy. (2000) 11:235-44. doi: 10.1016/s0955-3959(00)00053-0

35. Bellis MA, Hughes $\mathrm{K}$, Lowey $\mathrm{H}$. Healthy nightclubs and recreational substance use. From a harm minimisation to a healthy settings approach. Addict Behav. (2002) 27:1025-35. doi: 10.1016/s0306-4603(02) 00271-x

36. Martinotti G, Cinosi E, Santacroce R, Papanti D, Pasquini A, Mancini V, et al. (2017). Substance-related psychopathology and aggressiveness in a nightlife holiday resort: Results from a pilot study in a psychiatric inpatient unit in Ibiza. Hum Psychopharmacol. 32:e2586. doi: 10.1002/hup. 2586

37. Chieffi C, Camuto C, De-Giorgio F, de la Torre X, Diamanti F, Mazzarino $\mathrm{M}$, et al. Metabolic profile of the synthetic drug 4,4'-dimethylaminorex in urine by, LC-MS- based techniques: selection of the most suitable markers of its intake. Forensic Toxicol. (2020). doi: 10.1007/s11419-020-0 0544-9. [Epub ahead of print].

38. Camuto C, Pellegrini S, De-Giorgio F, de la Torre X, Marti M, Mazzarino $\mathrm{M}$, et al. Urinary excretion profile of methiopropamine in mice following intraperitoneal administration: a liquid chromatography-tandem mass spectrometry investigation. Drug Test Anal. (2020) 1-10. doi: 10.1002/dt a. 2900

39. Gottardo R, Murari M, Bertaso A, Bortolotti F, Tagliaro F. Drug screening by using the Toxtyper ${ }^{\mathrm{TM}}$ LC-ion trap MS: optimization of its application on serum samples in DUID context. Clinica Chimica Acta. (2020) 510:537-43. doi: 10.1016/j.cca.2020.08.009

40. van der Schaar JA, Attema-de Jonge ME, Gresnigt FM, Franssen EJ. Toxicological screening in the Amsterdam acute setting becomes more relevant if the standard panel of the drug-of-abuse point-of-care test is expanded with GHB ketamine. Toxicol Rep. (2020) 7:539-46. doi: 10.1016/j.toxrep.2020.04.007

41. Pérez-Mañá C, Papaseit E, Fonseca F, Farré A, Torrens M, Farré M. Drug interactions with new synthetic opioids. Front Pharmacol. (2018) 9:1145. doi: 10.3389/fphar.2018.01145

42. Contrucci RR, Brunt TM, Inan F, Franssen EJF, Hondebrink L. Synthetic cathinones and their potential interactions with prescription drugs. Ther Drug Monit. (2020) 42:75-82. doi: 10.1097/FTD.0000000000000682

43. Riley ED, Evans JL, Hahn JA, Briceno A, Davidson PJ, Lum PJ, et al. A longitudinal study of multiple drug use and overdose among young people who inject drugs. Am J Public Health. (2016) 106:915-7. doi: 10.2105/AJPH.2016.303084

44. Andersson JA, Brekke M, Vallersnes OM. Acute poisoning from substance abuse of benzodiazepines. Akutt forgiftning ved rusrelatert bruk av benzodiazepiner. Tidsskr Nor Laegeforen. (2020) 10. doi: 10.4045/tidsskr.20.0035

45. Orsolini L, Corkery JM, Chiappini S, Guirguis A, Vento A, De Berardis $\mathrm{D}$, et al. 'New/designer benzodiazepines': an analysis of the literature and psychonauts' trip reports. Curr Neuropharmacol. (2020) 18:809-37. doi: 10.2174/1570159x18666200110121333

46. Batisse A, Eiden C, Peyriere H, Djezzar S. French Addictovigilance Network. Use of new psychoactive substances to mimic prescription drugs: The trend in France. Neurotoxicology. (2020) 79:20-4. doi: 10.1016/j.neuro.2020. 03.015

47. Hupli A, Berning M, Zhuparris A, Fadiman J. Descriptive assemblage of psychedelic microdosing: netnographic study of Youtube ${ }^{\mathrm{TM}}$ videos and on-going research projects. Perfor Enhanc Health. (2019) 6:129-38. doi: 10.1016/j.peh.2019.01.001

48. Orsolini L, Ciccarese M, Papanti D, De Berardis D, Guirguis A, Corkery JM, et al. Psychedelic fauna for psychonaut hunters: a mini-review. Front Psychiatry. (2018) 9:153. doi: 10.3389/fpsyt.2018. 00153

49. Guthrie SK, Teter CJ, McCabe SE, Boyd CJ. Illicit methylphenidate use in an undergraduate student sample: prevalence and risk factors. Pharmacotherapy. (2003) 23:609-17. doi: 10.1592/phco.23.5.609.32210

50. Sussman S, Pentz MA, Spruijt-Metz D, Miller T. Misuse of "study drugs:" prevalence, consequences, and implications for policy. Subst Abuse Treat Prev Policy. (2006) 1:15. doi: 10.1186/1747-597X-1-15 
51. Özgen H, Spijkerman R, Noack M, Holtmann M, Schellekens ASA, van de Glind G, et al. International consensus statement for the screening, diagnosis, and treatment of adolescents with concurrent attention-deficit/hyperactivity disorder and substance use disorder. Eur Addict Res. (2020) 26(Suppl 45):223-232. doi: 10.1159/000508385

Conflict of Interest: The authors declare that the research was conducted in the absence of any commercial or financial relationships that could be construed as a potential conflict of interest.
Copyright (๔ 2020 di Giannantonio, Negri, Schiavone, Vannini, Pettorruso, De-Giorgio, Verrastro, Trabace, Corbo, Gottardo, Camuto, Mazzarino, Barra, De Berardis, Lopez, Del Villar, Schifano and Martinotti. This is an open-access article distributed under the terms of the Creative Commons Attribution License (CC BY). The use, distribution or reproduction in other forums is permitted, provided the original author(s) and the copyright owner(s) are credited and that the original publication in this journal is cited, in accordance with accepted academic practice. No use, distribution or reproduction is permitted which does not comply with these terms. 


\section{Psychiatry Trainees' Attitudes, Knowledge, and Training in Addiction Psychiatry-A European Survey}

\section{OPEN ACCESS}

\section{Edited by: \\ Fabrizio Schifano, \\ University of Hertfordshire,}

United Kingdom

Reviewed by:

Amira Guirguis,

Swansea University, United Kingdom

Diana Martinez,

Columbia University, United States

${ }^{*}$ Correspondence:

Laura Orsolini

laura.orsolini01@gmail.com

Specialty section:

This article was submitted to Addictive Disorders,

a section of the journal

Frontiers in Psychiatry

Received: 21 July 2020 Accepted: 29 October 2020 Published: 08 January 2021

Citation:

Orsolini L, Rojnić Palavra I,

Papanti GD, Potočan M, Quattrone $D$, Martens M, Sklenáŕová S, Levola J, Grichy L, Naughton S, Grinevičiene IK,

Kuiters JP, Gondek TM, Panfil A-L, Borovcanin MM, San Roman Uria A,

Biskup E, Sönmez Güngör E, Casanova Dias M, Tomori S, Banjac V, Marinova-Djambazova $P$ and Pinto da Costa M (2021) Psychiatry Trainees' Attitudes, Knowledge, and Training in Addiction Psychiatry - A European

Survey. Front. Psychiatry 11:585607. doi: 10.3389/fpsyt.2020.585607

\author{
Laura Orsolini ${ }^{1,2 \star}$, Irena Rojnić Palavra ${ }^{3}$, Gabriele Duccio Papanti ${ }^{4}$, Matej Potočan 5 , \\ Diego Quattrone ${ }^{6}$, Matis Martens ${ }^{7}$, Sandra Sklenářová ${ }^{8,9}$, Jonna Levola ${ }^{10}$, Leslie Grichy ${ }^{11}$, \\ Sean Naughton ${ }^{12}$, Indre Kotryna Grinevičiene ${ }^{13}$, Jelly Petra Kuiters ${ }^{14}$, \\ Tomasz M. Gondek ${ }^{15}$, Anca-Livia Panfil ${ }^{16}$, Milica M. Borovcanin ${ }^{17}$, \\ Alberto San Roman Uria ${ }^{18}$, Ewelina Biskup ${ }^{19,20}$, Ekin Sönmez Güngör ${ }^{21}$, \\ Marisa Casanova Dias ${ }^{22,23}$, Sonila Tomori ${ }^{24}$, Visnja Banjac ${ }^{25}$, \\ Petra Marinova-Djambazova ${ }^{26}$ and Mariana Pinto da Costa $27,28,29$
}

${ }^{1}$ Unit of Clinical Psychiatry, Department of Clinical Neurosciences/DIMSC, School of Medicine, Polytechnic University of Marche, Ancona, Italy, ${ }^{2}$ Psychopharmacology, Drug Misuse and Novel Psychoactive Substances Research Unit, School of Life and Medical Sciences, University of Hertfordshire, Hatfield, United Kingdom, ${ }^{3}$ Psychiatric Hospital Sveti Ivan, Zagreb, Croatia, ${ }^{4}$ Mental Health Department, Friuli Venezia Giulia, Italy, ${ }^{5}$ Psychiatric Hospital Begunje, Begunje na Gorenjskem, Slovenia, ${ }^{6}$ Social, Genetic and Developmental Psychiatry Centre, Institute of Psychiatry, Psychology and Neuroscience, King's College London, London, United Kingdom, ${ }^{7}$ Vaasa Central Hospital, Psychiatry Outpatient Clinic, Vaasa, Finland, ${ }^{8}$ Department of Child Psychiatry, Charles University Second Faculty of Medicine, Motol University Hospital, Prague, Czechia, ${ }^{9}$ Institute of Neuropsychiatric Care, Prague, Czechia, ${ }^{10}$ Psychiatry, Hospital District of Helsinki and Uusimaa, Helsinki, Finland, ${ }^{11}$ Adult Partial Hospitalization Program, Department of Psychiatry, Universitary Hospital Louis Mourier, Colombes, France, ${ }^{12}$ Health Service Executive, Dublin, Ireland, ${ }^{13}$ Vilnius City Mental Health Center, Vilnius, Lithuania, ${ }^{14}$ Lentis, Zuidlaren, Netherlands, ${ }^{15}$ European Psychiatric Association-Early Career Psychiatrists Committee, Wroclaw, Poland, ${ }^{16}$ County Emergency Clinical Hospital, Pius Brînzeu, Liaison Psychiatry, Timisoara, Romania, ${ }^{17}$ Department of Psychiatry, Faculty of Medical Sciences, University of Kragujevac, Kragujevac, Serbia, ${ }^{18}$ Psychiatric Inpatient Unit, Department of Psychiatry, Hospital Universitario Nuestra Señora de Valme, Seville, Spain, ${ }^{19}$ Department of Advanced Biomedical Sciences, Federico II University of Naples, Naples, Italy, ${ }^{20}$ School of Clinical Medicine, Shanghai University of Medicine and Health Sciences, Shanghai, China, ${ }^{21}$ University of Health Sciences, Erenköy Mental Health and Neurological Diseases Training and Research Hospital, Istanbul, Turkey, ${ }^{22}$ National Centre for Mental Health, MRC Centre for Neuropsychiatric Genetics and Genomics, Cardiff University, Cardiff, United Kingdom, ${ }^{23}$ Section of Women's Mental Health, King's College, Institute of Psychiatry, Psychology and Neuroscience, London, United Kingdom, ${ }^{24}$ University Hospital Center "Mother Theresa", Tirana, Albania, ${ }^{25}$ Clinic of Psychiatry, University Clinical Center of the Republic of Srpska, Banjaluka, Bosnia and Herzegovina, ${ }^{26}$ Department of Psychiatry in Medical University, University Hospital "Aleksandrovska", Sofia, Bulgaria, ${ }^{27}$ Unit for Social and Community Psychiatry, World Health Organization (WHO) Collaborating Centre for Mental Health Services Development, Queen Mary University of London, London, United Kingdom, ${ }^{28}$ Institute of Biomedical Sciences Abel Salazar, University of Porto, Porto, Portugal, ${ }^{29}$ Hospital de Magalhães Lemos, Porto, Portugal

Background: Although psychoactive substance use disorders (PSUDs) are a domain of mental health, addiction psychiatry is only formally recognized as a subspecialty in a few European countries, and there is no standardized training curriculum.

Methods: A 76-item questionnaire was developed and disseminated through an online anonymous data-collecting system and hand-to-hand amongst psychiatric trainees from the 47 European countries of the Council of Europe plus Israel and Belarus.

Results: 1,049/1,118 psychiatric trainees from 30 European countries completed the questionnaire. Fifty-nine-point nine percent of trainees stated to have training in addictions. Amongst the trainees who described having training in addictions, 43\% documented a not well-structured training and $37 \%$ an unsatisfactory training, mainly due to poor acquired knowledge. Overall, $97 \%$ of trainees stated that addiction represents a 
core curriculum for their training. Overall, general adult psychiatric trainees reported a better knowledge in addictions, compared to trainees in child and adolescent psychiatry.

Conclusion: Despite a growing spread of PSUDs in European countries, addiction psychiatry is a relatively poorly trained field within psychiatry training programs. Further research should investigate reasons for poor training and timings of the educational activities to optimize experiential education training in addiction psychiatry.

\section{Keywords: addiction psychiatry, addiction, EFPT, psychiatry trainees, psychiatry training}

\section{INTRODUCTION}

According to the Global Burden Disease study (1), alcohol, tobacco, and illicit substance use significantly determine the global burden of disability, morbidity and mortality, being considered amongst the top four health burdens across many upper-middle and high-income countries. Mental and behavioral disorders due to psychoactive substance use include different conditions caused by the intake of medically or not medically prescribed psychoactive substances (2). Psychoactive substance use disorder (PSUD) was firstly coded as a discrete diagnostic category both in the American Psychiatric Association (APA) Diagnostic and Statistical Manual (DSM)-3rd edition (DSM-III) and in the World Health Organization (WHO) International Classification of Diseases and Related Health Problems (ICD)9th edition of the (2-4). The current Diagnostic and Statistical Manual-5th edition (DSM-5) (5) amalgamated the abuse and dependence under a single category named "Substance Use Disorder" whilst the ICD-11 beta draft (6) described substance dependence (not substance use) (ICD: F10.xx to F19.xx) as a "disorder of regulation of the use of a psychoactive substance arising from repeated or continuous use of the substance $[\ldots]$ ]" $(5,6)$. Overall, PSUD may largely differ in severity and intensity in their psychopathological and clinical manifestation, i.e., ranging from an uncomplicated intoxication to the development of clinically significant psychotic disorders or other psychopathological and/or clinical manifestations) (2).

People with PSUD, including those classified as affected with a dual disorder, have been considered, compared to the general population, at higher risk of developing a range of medical and psychiatric disorders in comorbidity (7-9). Overall, PSUD subjects, particularly those with concurrent mental disorders, are overall associated with poorest outcomes, higher psychopathological severity and an increased rate of risky behaviors (i.e., hypersexuality, syringes/needles sharing, etc.) which can predispose them to an increased occurrence of serious infection diseases like Human Immunodeficiency Virus/Acquired Immune Deficiency Syndrome (HIV/AIDS) and Hepatitis C Virus (HCV), compared to the general population (10). Moreover, people with PSUD display a worsen psychosocial impairment (e.g., unemployment and homelessness) and they can more likely be involved in criminal and antisocial behaviors, compared to people affected by other mental disorders with a concurrent substance and/or alcohol use disorder $(8,11,12)$.

However, although the PSUDs are fully considered among the mental and behavioral disorders, the contribution of psychiatrists, early career psychiatrists (ECPs) and psychiatry trainees into this clinical and research field, should be better developed. For instance, addiction psychiatry (sometimes named as addiction medicine) appears not to be adequately and homogeneously incorporated within the psychiatric training, across all European countries. Furthermore, psychiatry trainees' levels of knowledge and experiences in addiction psychiatry may greatly vary across European countries and cultures. As already documented by the 2014 WHO Global Survey on Resources for Prevention and Treatment of Substance Use Disorders, around $37 \%$ of the 155 responding countries do not provide adequate access to the post-graduate training programme for professionals working in PSUD treatment (13). Globally almost $30 \%$ of countries did not report a dedicated training programme for the treatment and the management of PSUD patients (52\% of low-income countries vs. $16 \%$ of high-income countries), being mainly included in a short cycle tertiary education programme (48\%). Overall, $95 \%$ of countries documented that psychiatrists are commonly involved in the treatment of people with PSUD, followed by psychologists, who are involved in PSUD treatment and management in around $86 \%$ of the countries. Furthermore, more than $80 \%$ of European countries reported the availability of a post-graduate training programme for the treatment and management of PSUD for psychiatrists (14).

Contextually, psychiatrists and psychiatry trainees' attitudes toward PSUD patients largely differ across different countries and cultures, where people with PSUD are generally more exposed to psychiatrists' and health professionals negative attitudes/perception as well stigmatizing behaviors, and language (15). Stigmatizing behaviors and attitudes displayed by both psychiatrists and other physicians may lead to an inadequate and inhomogeneous physical, mental health care and treatment, including prescribing non evidence-based pharmacological/not pharmacological treatments, prescribing an inadequate/insufficient posology and duration of therapy. Moreover, use of potentially stigmatizing language may lead mental health professionals to a poor/inadequate communication with their PSUD patients, displaying an overall judgmental and unempathetic attitude, and other problematic and potentially stigmatizing behaviors (16-19).

The present study aimed at evaluating the organization of the addiction psychiatry training, trainees' satisfaction, trainees' attitudes toward people who use psychoactive substances and addiction psychiatry, and how psychiatric trainees manage psychopharmacology and pharmacotherapy in the most common clinical presentations of people with PSUD and 
their levels of confidence/perceived competence in the field of addiction psychiatry.

\section{METHODS}

\section{Study Design}

The EFPT-PSUD Study has been an international cross-sectional survey of European psychiatry trainees carried out in the context of the European Federation of Psychiatric Trainees (EFPT), the umbrella organization of the national trainees' associations in psychiatry in Europe $(20,21)$. Among the framework of the EFPT, a working group specifically dedicated to the PSUD developed a self-administered survey that was disseminated at European level, by involving both Child and Adolescent Psychiatry (CAP) and General Adult Psychiatric (GAP) trainees.

\section{Pilot Phase}

All active members of the EFPT-PSUD Working Group, constituted during the 2014 EFPT Forum in London (22) and initially comprising national representatives from 5 countries (Italy, Croatia, Lithuania, Denmark, and Estonia), firstly conducted a preliminary overview about the current state-of-theart regarding the training in addiction psychiatry in the European CAP/GAP training programs, and subsequently developed the survey. The survey was initially piloted amongst the members of the EFPT-PSUD Working Group.

\section{Full Study Phase}

The previously developed survey was circulated at the European level both to CAP and GAP trainees. The survey was circulated to the national representatives of each 47 European countries of the Council of Europe plus Israel and Belarus.

The European countries not represented in the survey were those not able to identify a National Coordinator who would take over the responsibility of the study or those unable to collect at least 10 completed questionnaires from their own country.

\section{Instrument}

The questionnaire was a 76-item self-report survey (Appendix 1 in the Supplementary Material). The questionnaire consisted of: (a) single answer and/or multiple answer questions (i.e., for evaluating trainees' knowledge in a specific field); (b) an increasing five-item Likert scale (i.e., for evaluating attitudes and interests toward the addiction medicine and psychiatry); and, (c) a series of open-ended questions (i.e., asking for further specification and/or clarification of the provided answers). In particular, the section on general knowledge on addiction consists of 36 items in which each question correctly answered gave 1 point (range score: $0-36$ ). This section was developed by GDP, following the evidence-based practices of the Substance Abuse and Mental Health Services Administration (SAMHSA) (https://www.samhsa.gov/ebp-resource-center).

For the present article, we have focused on the following sections of the survey:

- General socio-demographic section;
- General information about training in GAP (General Adult Psychiatry) or Child and Adolescent Psychiatry (CAP), including experiences (if any) on addiction psychiatry;

- General attitudes and interest toward addictions, addiction psychiatry;

- Level of knowledge about addictions, addictive disorders, including treatment.

\section{Data Collection}

One national coordinator per each of 47 European countries of the Council of Europe plus Israel and Belarus facilitated the delivery of the survey through an online data collecting system (https://www.surveymonkey.com/r/EFPT-PSUDstudy) and/or, if necessary, delivering the questionnaire hand-by-hand, in a paper form (Appendix 1). The questionnaire was circulated in English across all European countries (in French language in France) and no translation in other languages was deemed necessary, as psychiatric trainees were deemed by their national coordinators to have sufficient command of English to reliably answer the questions (i.e., this was preliminarily evaluated by each national coordinator). Data were collected from 15th August 2015 to 15th October 2016. Annual EFPT forum as well as European and national congresses or educational events were chosen to reach out to all CAP/GAP trainees in each country or to involve national coordinators, needed for those countries still not represented in the sample of the survey. Moreover, European contact e-mail databases were periodically used to disseminate the link for the online survey (https://www.surveymonkey.com/r/ EFPT-PSUDstudy). All hand-to-hand questionnaires completed were subsequently entered into the online study database by the National Coordinator via the online survey tool Survey Monkey. The online survey link was only accessible by invitation.

\section{Inclusion Criteria}

The inclusion criteria were: (i) being a CAP/GAP trainee, defined as a fully qualified medical doctor enrolled in a nationally recognized specialist training programme in CAP or GAP; (ii) belonging to one of the 47 European countries of the Council of Europe plus Israel and Belarus.

The participant countries included in the present analysis were those countries of whom each CAP/GAP National Coordinator was able to collect at least 10 completed questionnaires [not considering the last section regarding Novel Psychoactive Substances (NPS)]. Those countries with a National Coordinator who took responsibility to take part in the study but did not reach an enough minimum number of completed questionnaires were excluded in the present analysis (Greece, Belgium, Germany, Slovakia, Ukraine, Sweden, Denmark, and Israel). Amongst these, the following European countries participated in the present survey with a valid number of filled questionnaires: Albania, Austria, Azerbaijan, Belarus, Bosnia and Herzegovina, Bulgaria, Croatia, Czech Republic, Estonia, Finland, France, Hungary, Ireland, Italy, Kosovo, Latvia, Lithuania, Moldova, Netherlands, Norway, Poland, Portugal, Romania, Russia, Serbia, Slovenia, Spain, Switzerland, Turkey, and UK. 


\section{Ethics Approval and Consent}

The survey was conducted according to the principles of good scientific practice, which was supported by previous EFPTsponsored psychiatry trainees' surveys (23). Ethical approval for the study has been sought and granted by the School of Pharmacy Ethics Committee at the University of Hertfordshire (December 15, 2010, reference code PHAEC/10-42), with a further extension of the approval granted in November 2013. The patients/participants provided their written online informed consent to participate in this study.

Before filling out the survey which was self-administered anonymously, all participants were asked to give written online informed consent before, as legally and ethically required.

\section{Statistical Analysis}

Data was analyzed using the Software Package for Social Sciences for Windows v. 24.0 (SPSS 24) (IBM Corp, Armonk NY). Categorical variables were summarized as $n(\%)$, and continuous variables as means [standard deviation (SD)]. Pearson's $\chi^{2}$-tests were used to compare demographic and categorical variables, such as the trainees' attitudes toward addiction psychiatry. Student's $t$-tests and one-way analysis of variance (ANOVA)-tests were used to compare continuous variables, including comparisons of training experiences. Ordinal regression was used to model the predictors of trainee satisfaction. Variables added to the model included trainees' sub-specialty and percentage of training completed. The significance level was set a priori at $p \leq 0.05$, and all hypotheses were two-tailed.

\section{RESULTS}

\section{Sampling and Sample Characteristics}

The total number of questionnaires correctly filled during the collection process and afterwards included in the analysis was of 1,118, amongst all trainees in Europe who took part in the survey. However, after excluding missing data (i.e., including only complete questionnaires) only 1,049 responses were included (Table 1). There were differences in the gender distribution, being most of them women (68.7\%) and this difference is statistically significant when we stratified the sample by subspecialty $\left(\chi^{2}=25, p<0.001\right)$, being $84.6 \%$ of the CAP sample represented by women, whilst in the GAP sample, a percentage of $64 \%$ was represented by women, by reaching a total amount of GAP and CAP trainees of 936 (after excluding those trainees in forensic psychiatry or others with an unspecified other psychiatry training). The mean age of respondents was $30.48( \pm 4.84)$ years, without any statistically significant differences between GAP and CAP samples. The majority (73.8\%) were GAP trainees, whereas $15.4 \%$ were CAP trainees, whilst around $10.6 \%$ of the sample did not specify if they are GAP or CAP trainees. Amongst the respondents, the total number of years required to complete GAP and CAP training programs may largely differ across European countries. To adjust the analysis for this confounder, it was calculated the percentage of progression/completeness of individual training for each country, in order to measure the most reliable and objective variable. This variable reported that in an average of $67.4 \%$ of the total sample, CAP/GAP trainees were in the last quantile of their training programme, without any statistically significant difference between GAP and CAP (see Figure 1). The CAP/GAP trainees overall belong to 30 different countries, with the highest proportion of respondents amongst those training in France $(16.3 \%)$, followed by Italy $(5.7 \%)$, Spain (5.1\%) and the UK (5.0\%). See Table 1 for further demographic features.

\section{Trainees' Experience, Satisfaction, and Training in Addiction Psychiatry}

Amongst those who answered the question "Have you performed part of your psychiatric training in the treatment of patient with substance use disorder?", only 59.9\% of trainees reported to have spent part of their training in addiction psychiatry settings, with a statistically significant difference between GAP and CAP trainees $(p=0.018)$. Amongst those trainees who declared to have received training in addiction psychiatry during their psychiatry training, only $43 \%$ described that the PSUD training was not well-structured due to several reasons. First, the addiction training program is often too short to allow trainees to deepen knowledge on all theoretical and practical aspects of addiction psychiatry; second, during the addiction training program, CAP/GAP trainees are often alone in the management of PSUD patients (often without a dedicated supervisor/mentor); third, the addiction training program usually consists in a mere observership experience (without a practical frontline experience). Amongst those trainees who had training in addictions only $37 \%$ of them declared that they were not satisfied about the level of training offered, mainly stating lack of enough acquired skills and knowledge in the field, largely below their initial expectations. There was no significant difference in the percentage of training completed amongst those trainees who reported being satisfied with their addiction psychiatry training, compared to those trainees who did not document an enough level of satisfaction $\left[\mathrm{F}_{(1,555)}=2.244, p=0.135\right]$. Trainees with larger caseloads had generally progressed further in their training, compared to those trainees with smaller caseloads $\left[\mathrm{F}_{(5,551)}=6.487, p<0.001\right]$. Most of the sample $(97 \%)$ agreed or strongly agreed that addiction represents a core curriculum for training. Subspecialty was a significant predictor of satisfaction with training $(\beta=1.713 ; p=0.042)$, being GAP trainees overall more satisfied, compared to CAP trainees, even though this finding is not strictly correlated by the percentage of training completed $(\beta=1.005 ; p=0.176)$.

\section{Trainees' Attitudes Towards People Who Use Psychoactive Substances and Addiction Psychiatry}

Approximately one third of the sample (33.27\%) agreed or strongly agreed to be confident with their basic skills needed/requested necessary to work in addiction settings after their training $\left(\chi^{2}=82.864 ; p<0.001\right)$. Interestingly, on the other hand, around $66.9 \%$ of the trainees agreed or strongly agreed that "Addiction psychiatrists are usually less skilled than their 
TABLE 1 | Demographic characteristics.

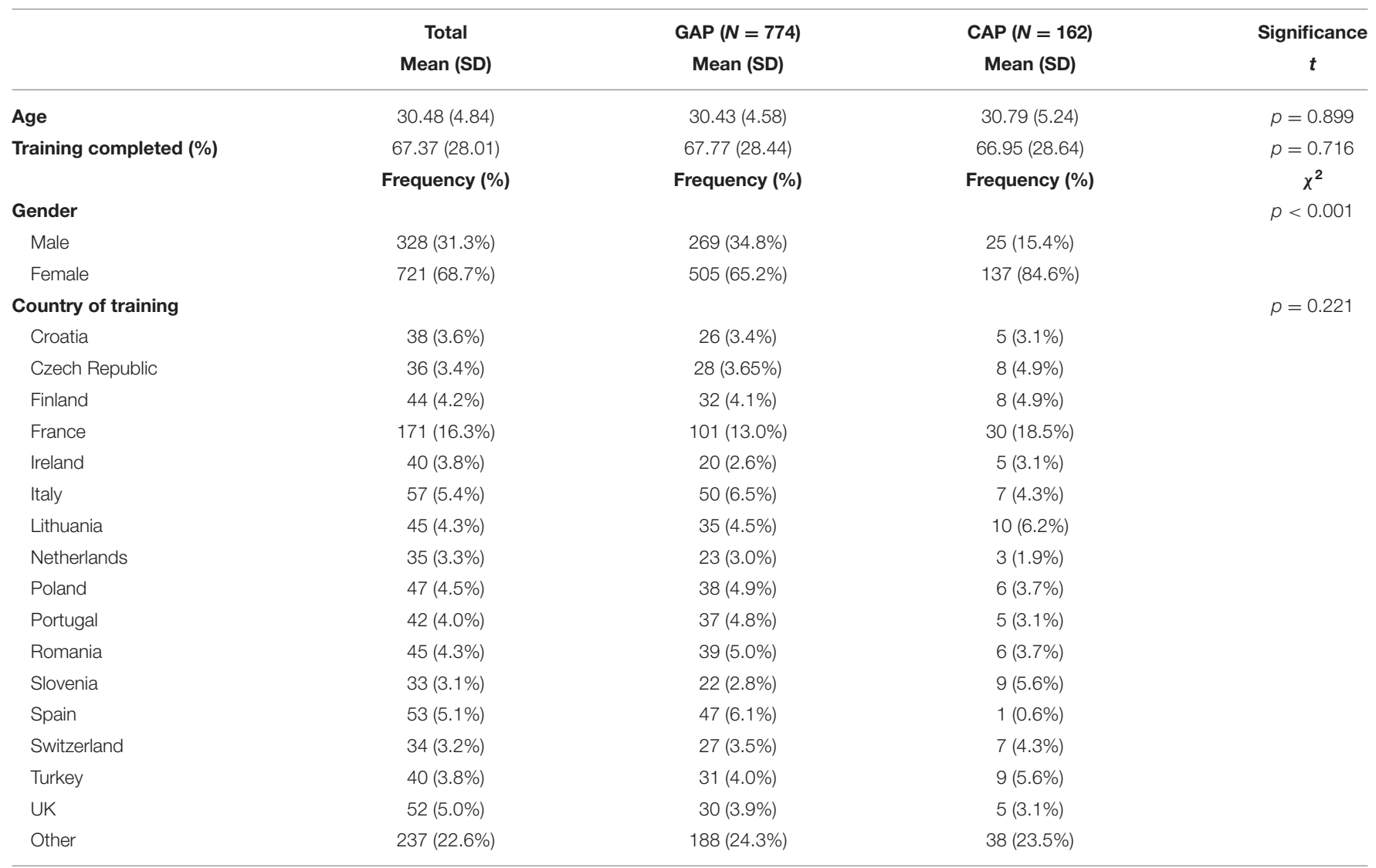

GAP, General Adult Psychiatry; CAP, Child and Adolescent Psychiatry; SD, Standard Deviation; UK, United Kingdom.

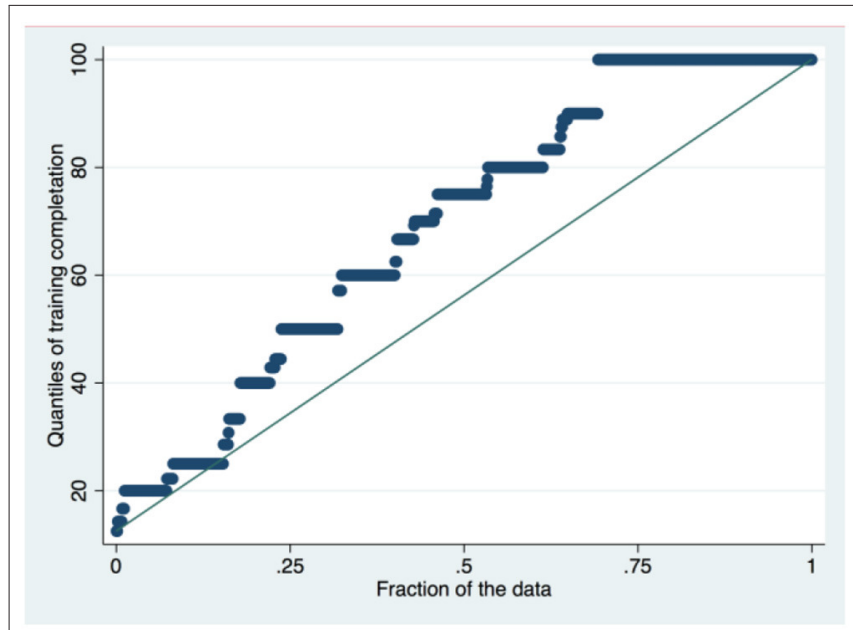

FIGURE 1 | Distribution of training completion.

colleagues working in GAP/CAP" (Table 2). Moreover, around $75.7 \%$ disagreed or strongly disagreed that addictions are mental disorders; similarly, $77.8 \%$ of the sample agreed or strongly agreed that people with drug addiction cannot be recovered (Table 2).

Over three-quarters of respondents (76.1\%) knew/had previously known someone outside of their workplace with an addiction-related problem (Table 3). The findings showed that those who knew/had known someone with addiction related problems were significantly associated with a stronger desire to work in the addictions after their training $\left[\chi^{2}(4)=16.311, p=\right.$ 0.003] (Figure 2).

\section{Trainees' Basic Knowledge and Confidence/Perceived Competence in Addiction Psychiatry}

Respondents who had treated someone with an addiction-related condition significantly declared to have almost completed their training, compared to those trainees who had not $\left[\mathrm{F}_{(1,991)}=\right.$ 99.155, $p<0.001$ ] (Figure 3). Figure 4 represents the graphical distribution of the knowledge score, by indicating that most trainees responded correctly to most of the questions regarding their general and specific knowledge of addiction psychiatry (mean average $25.77 \pm$ SD 3.59), with a minimum score of 7 and a maximum score of 34 (skewness $=-0.956$ ). There 
TABLE 2 | Attitude of trainees who have/haven't performed part of their training in the treatment of a patient with addiction.

\begin{tabular}{|c|c|c|c|c|}
\hline & & \multicolumn{2}{|c|}{$\begin{array}{l}\text { Have you performed part of your psychiatric } \\
\text { training in the treatment of patients with addiction? }\end{array}$} & \\
\hline & & Yes & No & \\
\hline \multirow{5}{*}{$\begin{array}{l}\text { Illicit drugs (e.g., heroin) addicted } \\
\text { are good people }\end{array}$} & Strongly agree & 9 & 3 & \multirow{5}{*}{$\begin{array}{l}\chi^{2}=8.773 \\
p=0.067\end{array}$} \\
\hline & Agree & 33 & 22 & \\
\hline & Neither agree or disagree & 303 & 236 & \\
\hline & Disagree & 101 & 52 & \\
\hline & Strongly disagree & 62 & 60 & \\
\hline \multirow{5}{*}{$\begin{array}{l}\text { I don't feel confident with my } \\
\text { skills to work in addiction }\end{array}$} & Strongly agree & 36 & 22 & \multirow{5}{*}{$\begin{array}{c}\chi^{2}=82.864 \\
p<0.001\end{array}$} \\
\hline & Agree & 178 & 44 & \\
\hline & Neither agree or disagree & 125 & 84 & \\
\hline & Disagree & 146 & 181 & \\
\hline & Strongly disagree & 23 & 42 & \\
\hline \multirow{5}{*}{$\begin{array}{l}\text { I think that people with drug } \\
\text { addiction cannot recover }\end{array}$} & Strongly agree & 138 & 88 & \multirow{5}{*}{$\begin{array}{r}\chi^{2}=3.872 \\
p=0.424\end{array}$} \\
\hline & Agree & 257 & 188 & \\
\hline & Neither agree or disagree & 87 & 75 & \\
\hline & Disagree & 25 & 19 & \\
\hline & Strongly disagree & 1 & 3 & \\
\hline \multirow[t]{5}{*}{ Addiction is a mental disorder } & Strongly agree & 5 & 4 & \multirow{5}{*}{$\begin{aligned} \chi^{2} & =6.263 \\
p & =0.180\end{aligned}$} \\
\hline & Agree & 25 & 15 & \\
\hline & Neither agree or disagree & 59 & 61 & \\
\hline & Disagree & 262 & 198 & \\
\hline & Strongly disagree & 15 & 95 & \\
\hline \multirow{5}{*}{$\begin{array}{l}\text { Addiction psychiatrists are } \\
\text { usually less skilled than their } \\
\text { colleagues working in general } \\
\text { adult and child adolescent } \\
\text { psychiatry }\end{array}$} & Strongly agree & 159 & 130 & \multirow{5}{*}{$\begin{array}{r}\chi^{2}=6.565 \\
p=0.161\end{array}$} \\
\hline & Agree & 181 & 140 & \\
\hline & Neither agree or disagree & 104 & 73 & \\
\hline & Disagree & 57 & 24 & \\
\hline & Strongly disagree & 7 & 6 & \\
\hline
\end{tabular}

was no significant difference in terms of the most prevalent addiction-related condition that was treated/observed during their addiction psychiatry training $\left[\mathrm{F}_{(4,479)}=1.523, p=0.194\right]$. However, those trainees who had treated alcohol withdrawal syndrome, delirium tremens, opioid withdrawal syndrome, or substance induced-psychosis were significantly more senior in their level of training completeness, compared to those trainees who had not treated these addiction-related conditions who were more junior (all $p$-values $<0.001$ ). Similarly, those trainees prescribing acamprosate, naltrexone, methadone, and buprenorphine were also significantly further in their training than those who did not prescribe a medication for an addiction (all $p$-values $<0.001$ ). In addition, GAP trainees more likely reported to have treated a person affected with an addiction during their training, compared to CAP trainees $\left[\chi^{2}{ }_{(1)}=8.328\right.$, $p=0.004]$. Likewise, GAP trainees more likely reported to have prescribed medication for an addiction-related condition, compared to CAP trainees $\left[\chi^{2}{ }_{(1)}=9.482, p=0.002\right]$. Furthermore, GAP trainees reached higher scores, compared to those undergoing CAP training, when questioned about their general and specific knowledge of addictions $\left[\mathrm{F}_{(1,802)}=14.181, p\right.$ $<0.001]$. Moreover, GAP trainees were more likely aware of the existance of legal highs/smart drugs/novel substances, compared to CAP trainees $\left[\chi^{2}(2)=25.663, p<0.001\right]$. However, when the knowledge score includes in the analysis also those questions about legal highs/smart drugs/novel substances, there was no significant difference in the total score between GAP and CAP trainees $\left[\mathrm{F}_{(1,531)}=0.524, p=0.470\right]$.

\section{DISCUSSION}

\section{Key Findings and Comparison With the Literature}

PSUD have been historically perceived as personal, family, social, moral, or criminal issues rather than a health condition (24). Therefore, subjects with PSUD have been supposed to be better managed at the individual, family or justice level (i.e., through existing social infrastructure or civil and criminal justice interventions) (24). Indeed, criminalization of people with PSUD exacerbated their perceived and experienced stigma, avoidant attitudes and behaviors of contempt, by worsening their marginalization and poor access to adequate treatment and care (24). People with PSUD tend to be stigmatized due to their use of drugs and drug-seeking behaviors (24). Moreover, other PSUD-related risky behaviors, such as speeding/dangerous driving, violence, aggressiveness, 
TABLE 3 | Attitude of trainees who have/haven't known someone outside their workplace with addiction related problems.

\begin{tabular}{|c|c|c|c|c|}
\hline & & \multicolumn{2}{|c|}{$\begin{array}{c}\text { I know/had known someone outside my workplace } \\
\text { (family, friends, relatives, neighborhood) who } \\
\text { has/had addiction related problems }\end{array}$} & \\
\hline & & Yes & No & \\
\hline \multirow{5}{*}{$\begin{array}{l}\text { I am afraid to work with persons } \\
\text { with cocaine addiction }\end{array}$} & Strongly agree & 204 & 39 & \multirow{5}{*}{$\begin{aligned} \chi^{2} & =14.623 \\
p & =0.006\end{aligned}$} \\
\hline & Agree & 309 & 101 & \\
\hline & Neither agree or disagree & 92 & 42 & \\
\hline & Disagree & 62 & 20 & \\
\hline & Strongly disagree & 7 & 5 & \\
\hline \multirow{5}{*}{$\begin{array}{l}\text { I am afraid to work with persons } \\
\text { with alcohol }\end{array}$} & Strongly agree & 283 & 68 & \multirow{5}{*}{$\begin{aligned} \chi^{2} & =8.305 \\
p & =0.081\end{aligned}$} \\
\hline & Agree & 298 & 97 & \\
\hline & Neither agree or disagree & 64 & 30 & \\
\hline & Disagree & 25 & 11 & \\
\hline & Strongly disagree & 4 & 1 & \\
\hline \multirow[t]{5}{*}{ Addiction is a mental disorder } & Strongly agree & 8 & 1 & \multirow{5}{*}{$\begin{aligned} \chi^{2} & =14.525 \\
p & =0.006\end{aligned}$} \\
\hline & Agree & 34 & 6 & \\
\hline & Neither agree or disagree & 83 & 37 & \\
\hline & Disagree & 339 & 121 & \\
\hline & Strongly disagree & 210 & 42 & \\
\hline \multirow{5}{*}{$\begin{array}{l}\text { Individual psychotherapy should } \\
\text { be preferred in treating addiction }\end{array}$} & Strongly agree & 14 & 1 & \multirow{5}{*}{$\begin{aligned} \chi^{2} & =12.680 \\
p & =0.013\end{aligned}$} \\
\hline & Agree & 125 & 45 & \\
\hline & Neither agree or disagree & 236 & 93 & \\
\hline & Disagree & 238 & 52 & \\
\hline & Strongly disagree & 61 & 16 & \\
\hline
\end{tabular}

and impulse dysregulation, are barely seen as part of a complex disorder, so that people with PSUD are usually rejected by the society due to the supposed moral valence of these behaviors $(24,25)$. These patients may also be seen as a burden for the healthcare system, by indeed increasing the disparities of cares, the risk to not adequately provide evidence-based and effective treatments $(19,25)$. Due to this disadvantageous framework, patients with PSUD may develop a self-stigmatizing attitude as well (e.g., a subjective process characterized by negative feelings about own self, maladaptive behaviors, stereotype endorsement resulting from individual's experiences/perceptions/feelings and anticipation of negative social reactions) (26-29). In fact, potentially "stigmatized" attitudes and behaviors, overly provided by healthcare professionals, including psychiatrists and psychiatry trainees, may be potentially trigger and maintain these self-stigmatizing attitudes, as already reported in the literature and confirmed by our findings (16, 19, 26-29). Furthermore, subjects with PSUD are symbolically associated with a range of other stigmatized health conditions, including HIV/AIDS, $\mathrm{HCV}$, risk and disinhibiting behaviors such as impaired driving, prodigality, criminality, risky sexual behaviors, and social issues $(30,31)$. Stigmatizing beliefs and behaviors about PSUD may be influenced by the level of knowledge (and education) about these mental health conditions and the personal experience with people affected with PSUD. Furthermore, it has been reported that media portrayal of people with PSUD and media coverage/level of news disseminated about significant and impactful related events, mainly occurring due to a drug intoxication and/or drug dependence/abuse/misuse, can significantly increase these stigmatizing beliefs and attitudes $(29,32)$.

Furthermore, addictions have not been historically recognized as conditions requiring a medical, psychological and psychopharmacological treatment (19). This is in line with our findings in which most GAP and CAP trainees declared that the addictions are not mental disorders. In fact, as previously documented in the literature, this overall consideration regrading PSUD appears to be widely spread not only at the general population level but also amongst mental health professionals who overall reported negative and pessimistic views about PSUD, people with PSUD and do not routinely screen patients in daily practice for addictions $(15,26,33,34)$.

However, the individual perceptions and attitudes towards people with PSUD may largely vary according to different factors. For instance, people are less likely to endorse the stereotype of violence together with a negative connotation of addiction disorders, if they have had direct contact with people (or also family members or close friends) who were affected with PSUD or did not experience violent acts by people affected with PSUD (35). This is comparable with our findings which demonstrated that those trainees who have/had experience with people with a PSUD significantly declared to have a stronger desire to work in the addiction field and with subjects with PSUD after their training. 


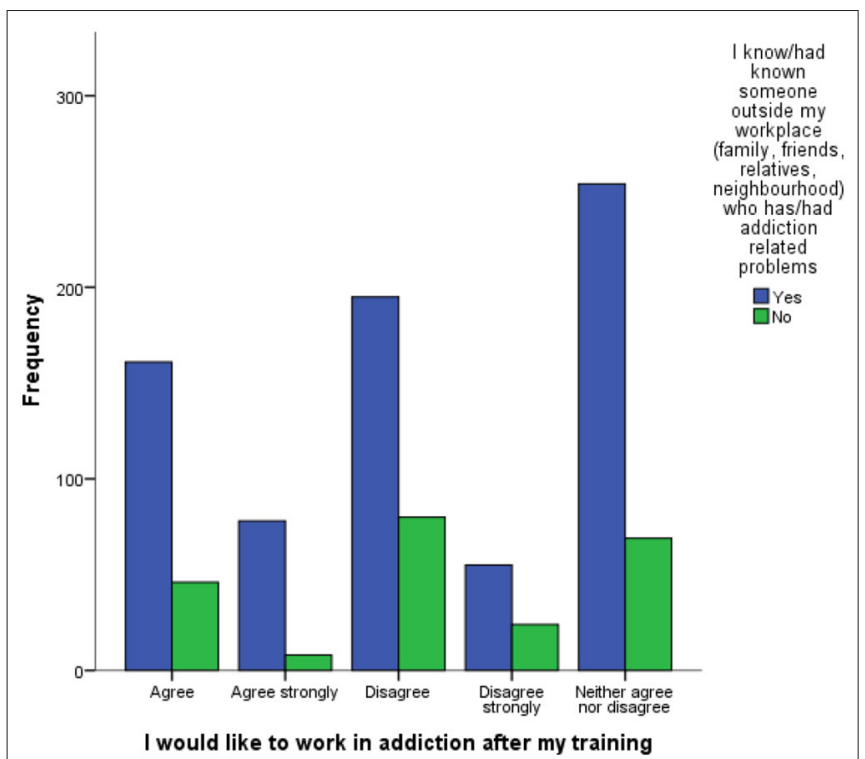

FIGURE 2 | Frequency of trainees who would like to work in addiction following completion (by those who have/haven't known someone outside the workplace who has had an addiction related problem).

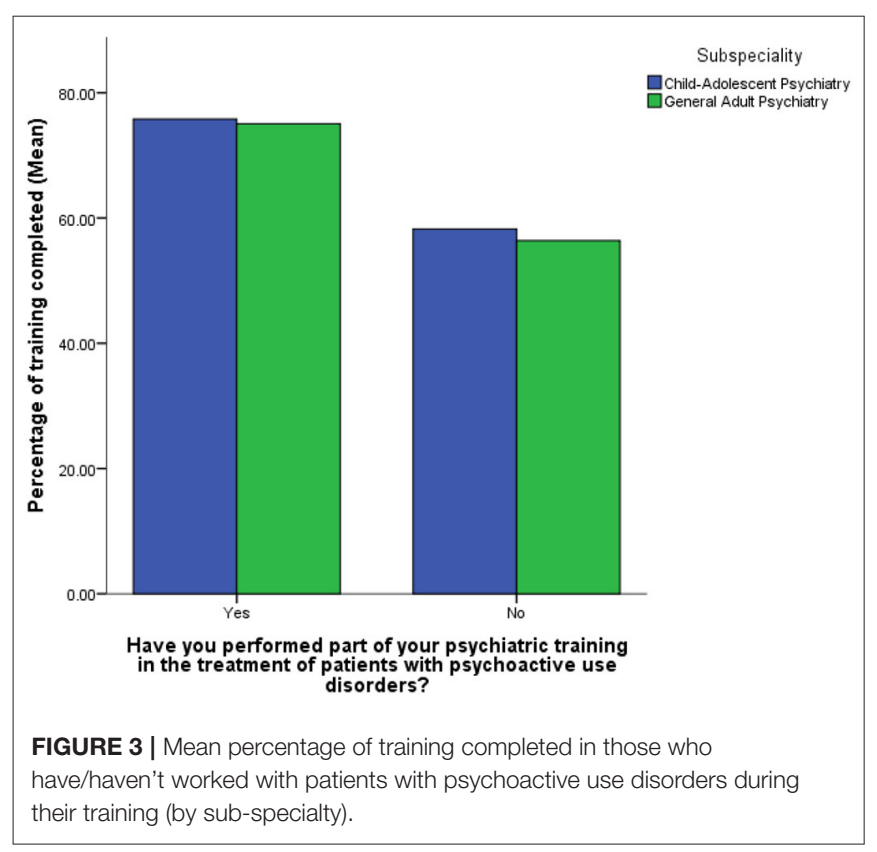

Furthermore, despite a compelling need for PSUD treatment in Europe, mental healthcare professionals (including psychiatry trainees) overall appear poorly or neither trained, nor especially eager to accept/tolerate patients with PSUD $(15,33,34,36)$. In general, psychiatrists do not feel competent/confident in treating addiction disorders, do not like working with patients affected with PSUD and do not find rewarding treating patients with PSUD (33, 37, 38). A lack of (practical) experience and/or an inadequate (theoretical and practical) training in

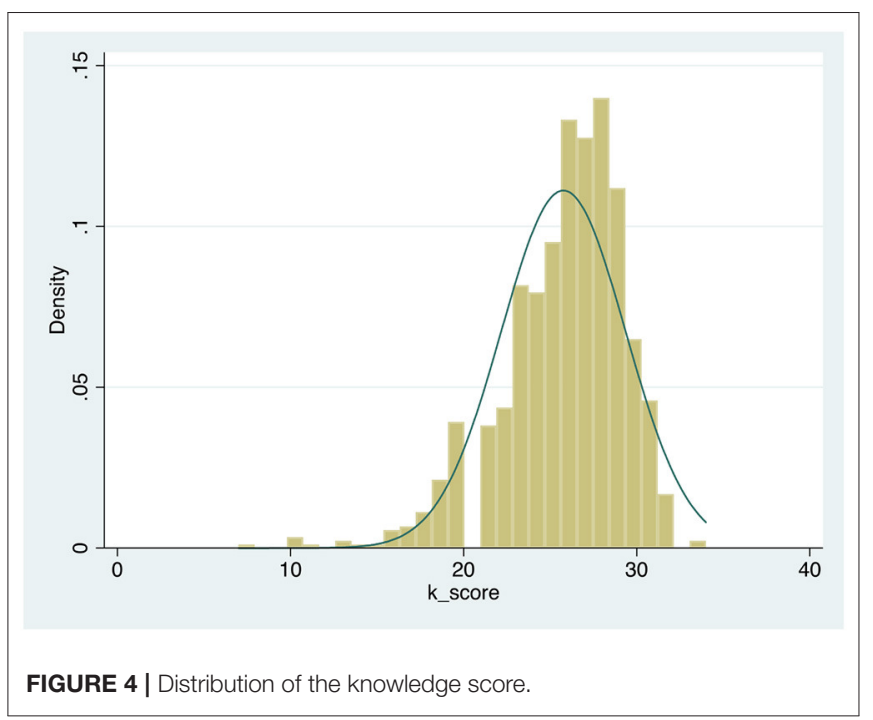

addiction psychiatry may indeed result in an endless loop of incompetence and neglect regarding the addiction psychiatry, amongst mental health care professionals. However, despite the evidence demonstrating the need to improve addiction medicine's training not only amongst psychiatry trainees but also amongst all physician trainees, most medical students and $\mathrm{CAP} / \mathrm{GAP}$ trainees generally receive an inadequate (practical and theoretical) training in the field of addiction medicine/psychiatry $(33,39,40)$. Moreover, most CAP/GAP trainees generally display lacking core clinical and therapeutic competences, as required for working with patients with PSUD $(33,39$, 40). Although formal addiction training within the medical field has been closely tied to psychiatry, psychiatric training generally provides a poor improvement and a limited level of knowledge over medical school, about addictions (39, 40). These considerations are particularly significant in the European countries, whereas there are several inequalities and heterogeneous training levels in addiction psychiatry, as documented by our findings. Furthermore, most CAP and GAP trainees reported to be less skilled in the addiction field, compared to other fields of psychiatry. Interestingly, there are not statistically significant differences between GAP and CAP trainees regarding this finding. This appears particularly relevant if we consider that CAP trainees should possess a comprehensive experience including behavioral, psychosocial and addiction problems particularly amongst youngsters/adolescents who have been well-demonstrated to be those patients more frequently exposed to drugs and/or other addictive behaviors, but also those patients more vulnerable toward the new onset of mental disorders associated with a PSUD (41).

Furthermore, an insufficient training experience with patients with PSUD, along with the lack of a highly-specialized faculty (i.e., short addiction training experience, lack of a supervisor/mentor during the addiction training, and poor quality of addiction training), may overall lead to a discouraging training experience amongst CAP/GAP trainees, as reported 
in our study. Overall, one could argue that this general psychiatry trainees' attitudes and perceptions towards the addiction psychiatry might discourage trainees' interest and willingness to deepen the management and therapy of patients with PSUD, independently by their level of psychiatry training, as well as their interests in working in addiction psychiatry (38). Renner et al. (38) described the following main predictors of poor perception of careers in addiction medicine by GAP trainees: (a) the poor/not enough/lacking experience with patients with PSUD; (b) the perceived sensation and feeling to work with "difficult" patients; (c) the lack of a competent training in the addiction; (d) an overemphasis, during psychiatry training, about the detoxification process rather than a longterm rehabilitative and care program for the addiction-related conditions. Miller et al. (33) identified the following hypothesized barriers/determinants explaining the different attitudes and practices of medical students, trainees and physicians towards addiction psychiatry: (a) lack of acceptance of a medical model for addictive disorders; (b) lack of positive and/or optimistic attitudes about patients with PSUD, by accepting the prevalent stereotype of subjects with PSUD as those patients whose social and medical prognosis is poor; (c) curricula deficits throughout the Continuum Medical Education (CME) in the field of the addiction psychiatry/medicine, particularly the total time devoted to addictive disorders during the medical school and psychiatry training; (d) lack of parity and physician advocacy in medical education; (e) prejudices and misunderstandings about addictive disorders, along with ungrounded fears of huge costs connected with addiction treatment and the perception that addiction treatment owns a low ratio of benefits to costs; (f) personal and/or family history of drug and/or alcohol disorders. Conversely, Rush et al. (42) found that the factors associated with more positive attitudes towards the treatment of addictive disorders and subjects with PSUD may be represented by: (a) the number of subjects with PSUD treated/visited; (b) the physicians' perceived effectiveness in the management of the addictive disorders; and, (c) the numbers of hours of CME specifically addressed on the addictive disorders.

However, as widely reported in the literature, the level of knowledge and education about PSUD and addiction psychiatry can positively influence mental health professionals' attitudes and interests towards the field of addictions, limit the misdiagnosis and potentially reduce improper and inadequate treatment regimens for these disorders (43-45), even though other studies demonstrated a deterioration in attitudes throughout medical school years and suggested a continued decline throughout the years of training, mainly due to time and resources spent for those subjects with PSUD (19, 46-49). The enhancement of these beliefs appears to be more significant when we compared those subjects with PSUD with those with AUD (49). As proposed by Miller et al. (33), to achieve an adequate level of education and training in addiction psychiatry, it should be ensured that all trainees reach an enough and adequate knowledge and skills in the diagnosis and treatment of the addictive disorders, by favoring the development of curricula for the addictive disorders in all medical schools, residency training programs and CME; by supporting the research and revising all discriminatory policies that create barriers to the implementation of curricula in addictive disorders; by providing the detection and intervention for students, trainees and physicians who have addictive disorders; and, by supporting the parity between the addictive disorders and other medical and psychiatric diseases.

\section{Main Strengths and Limitations}

To the best of our knowledge, this has been the only study specifically investigating the levels of training, experiences, attitudes and perceptions as well as the level of perceived confidence and capacity in the management of people with a PSUD, carried out amongst European CAP/GAP trainees. The present survey also included a large sample size of CAP/GAP trainees in Europe $(n=1,118)$ which comprises many European countries $(n=30)$. Furthermore, collecting data from different European countries might lend strength to the generalization of these findings also to other WHO Regions, beyond European Region. Moreover, our study identifies gaps in knowledge by demonstrating that addiction psychiatry appears not to be adequately and homogeneously incorporated within the psychiatric training, across all European countries. Moreover, a key finding is represented by the significant number of recruited psychiatry's trainees who do not consider addiction as a psychiatric disorder.

Despite its original and poorly investigated topic, there are several limitations that should be here drawn up. Firstly, being a self-report questionnaire and partly online administered, potential recall, social desirability, and reporting biases may occur. Secondly, the sampling method may be hugely affected both by the fact that not in all European countries we reached an enough number of completed questionnaires or reached an available official national coordinator. In fact, some European countries initially included have been a posteriori excluded in our analysis as they did not reach an enough number of completed questionnaires (cut-off of 10 for each country), like Greece, Belgium, Germany, Slovakia, Ukraine, Sweden, Denmark, and Israel. Furthermore, sampling rates largely vary within different European countries, being some countries (i.e., Croatia, Finland, France, Ireland, Italy, Lithuania, Poland, Portugal, Romania, Slovenia, Spain, Turkey, and UK) most represented in our sample compared to Albania, Bosnia, Bulgaria, Czech Republic, Estonia, The Netherlands, Serbia, and Switzerland. The level of perceived confidence and knowledge in addiction psychiatry, being mainly based on a set of questionnaires, may also be susceptible to the updated information and new available and emerging pharmacological and not pharmacological treatments, may not completely reflect the current situation occurring at the time of writing of the present study. Moreover, the present study does not examine what happens once GAP/CAP residency is completed and the GAP/CAP enters career's practice. It should be relevant to document further data particularly regarding the level of attitude or perception of PSUD patients with added experiences and added continuing educational opportunities during their clinical career. Finally, the present study does not specifically define whether psychiatry trainees' attitudes differ towards caring for subjects with AUD and/or SUD. 
Relevance of the Findings and Implications for Practice, Policies, and Research

The present study provides significant and valuable information on the current European CAP/GAP trainees' level of experiences, training, perceived knowledge/competence, and subjective attitudes/perceptions towards the addiction psychiatry. These findings not only serve to investigate the current European situation in terms of level of subspecialty offered in the addiction psychiatry as well as the potential differences across all analyzed European countries, but they might also investigate those situations which should be implemented/enhanced as lacking in providing opportunities both in terms of internship (practical training) and knowledge (theoretical training) in the field of the addictions. Moreover, addressing the identified reasons/factors determining a different level of training in addiction psychiatry as well as a different level of interest CAP/GAP trainees, in strenghtening knowledge in this field might be a way to modulate and act on these factors, to improve the CAP/GAP training conditions in the field of addiction psychiatry (50). Regarding the need to improve all CAP/GAP training programmes, the standardization of curricula would be important to produce both GAP and CAP trainees able and capable (self-confident) in the management and correct identification of both physical and mental/behavioral PSUDrelated conditions. This should be part of the essential core knowledge that should be indispensable for all psychiatric practice. In terms of the enhancement of GAP/CAP trainees' education/knowledge in the addiction medicine and psychiatry, an implementation of a mandatory addiction rotation during the CAP and GAP training program, could greatly improve the level of trainees' confidence and competence in identifying and dealing with all different addictive disorders. Furthermore, in CAP and GAP training, the need to develop and satisfy objective measurable educational criteria must be balanced with the acquisition of subjective skills needed to treat subjects with PSUD effectively (e.g., increasing empathy and not judgmental approach as well as addressing stigma), as well as reaching an enough comfort in working with PSUD patients and obtaining a minimum sense of mastery in the field of the addictions. Finally, it might be suggested to all European GAP and CAP training programmes to administer to all psychiatry trainees at the end of their training program, validated tools for assessing addiction psychiatry training and early identify potential deficits, such as the Addiction Training Scale (ATS) (51).

These findings may assist the decision-makers to implement strategies to adapt their national diversities in CAP/GAP training programmes and make them homogenous especially at the European level. The need for psychiatry trainees' education and experience in treating patients with addiction problems has been outlined. Lastly, although these preliminary findings may help in mapping the reality of this field of psychiatry, further studies are needed to focus on the main motivations underpinning the existing differences across European countries in terms of level of training in addiction psychiatry (i.e., cultural and/or religious factors, epidemiological motivations, etc.) and consequences of different experiences/training in the level of knowledge of a CAP/GAP trainees as well as their attitude/perception towards addictions in general and people who use psychoactive substances. Moreover, it would also be of interest to repeat the present survey with identical methodology every 4 or 5 years (being the average duration of CAP/GAP European training) to assess potential trends in these findings and attitudes/opinions of psychiatry trainees over time and evaluate if any enhancing intervention has been provided at European and national level concerning addiction psychiatry training and evaluate if any positive/neutral/negative impact was reached amongst psychiatry trainees' attitudes and knowledge.

\section{CONCLUSIONS}

Despite the growing dissemination of addictive disorders across all European countries, addiction psychiatry seems to be an underdeveloped part of psychiatry within psychiatry training programmes. However, we found substantial consensus among all European psychiatry trainees that more education and experience in treating patients with addictive disorders should be guaranteed and be part of the core curricula in GAP and CAP training. Further research needs to be directed towards the causes of poor training as well as timings of these educational activities to optimize experiential education programs to be implemented within GAP and CAP training programs.

\section{DATA AVAILABILITY STATEMENT}

The raw data supporting the conclusions of this article will be made available by the authors, without undue reservation.

\section{ETHICS STATEMENT}

The studies involving human participants were reviewed and approved by Ethical approval for the study has been sought and granted by the School of Pharmacy Ethics Committee at the University of Hertfordshire (December 15, 2010, Reference Code PHAEC/10-42), with a further extension of the approval Granted in November 2013. The patients/participants provided their written informed consent to participate in this study.

\section{AUTHOR CONTRIBUTIONS}

LO, IR, GDP, MPo, DQ, and MM conceived and conceptualized the study. GDP, LO, and IR performed the survey, the methodology and the ways to disseminate the survey across all European countries. IR and GDP mainly dealt with data curation, collection and analysis. DQ performed formal analysis of data collected reported in this article. A preliminary draft was written by GDP, LO, IR, and MPi. LO wrote, revised and edited the final draft. MPi supervised the work and contributed to the final editing of this manuscript. All other co-authors equally collected data from their respective countries and provided further final feedback to the draft. 


\section{ACKNOWLEDGMENTS}

We would like to thank the European Federation of Psychiatric Trainees (EFPT) for their support to this research project. We would like to thank Tove Mogren (Sweden), Tor Svensson (Sweden), Orest Suvalo (Ukraine), Livia de Picker (Belgium), Kfir Feffer (Israel), and Katharina Schönegger (Austria) for their support in the data collection in their countries. We would also like to thank Lauren Piper for providing support with the initial exploratory statistical analysis of this study; Andrew

\section{REFERENCES}

1. GBD 2016 Alcohol and Drug Use Collaborators. The Global Burden of Disease Attributable to Alcohol and Drug Use in 195 Countries and Territories, 1990-2016: A Systematic Analysis for the Global Burden of Disease Study 2016. Lancet Psychiatry. (2018) 5:987-1012. doi: 10.1016/S2215-0366(18) 30337-7

2. Basu D, Ghosh A. Substance use and other addictive disorders in international classification of Diseases-11, and their relationship with diagnostic and statistical Manual-5 and international classification of Diseases-10. Indian J Soc Psychiatry. (2018) 34(Suppl. S1):54-62. doi: 10.4103/ijsp.ijsp_83_17

3. American Psychiatric Association. Diagnostic and Statistical Manual of Mental Disorders. Revised. 3rd ed. Washington, DC: American Psychiatric Association (1987)

4. World Health Organization. The ICD-9 Classification of Mental and Behavioral Disorders: Clinical Descriptions and Diagnostic Guidelines. Geneva: World Health Organization (1979).

5. American Psychiatric Association. Diagnostic and Statistical Manual of Mental Disorders. 5th ed. Washington, DC: American Psychiatric Association (2013).

6. World Health Organization. ICD-11 Beta Draft. Geneva: World Health Organization (2016). Available online at: http://www.apps.who.int/ classifications/icd11/browse/l-m/en (accessed June 30, 2020).

7. EMCDDA. Comorbidity of Substance Use and Mental Disorders in Europe. (2015). Available online at: http://www.emcdda.europa.eu/attachements.cfm/ att_245096_EN_TDXD15019ENN.pdf (accessed June 30, 2020).

8. EMCDDA. Perspectives on Drugs. Comorbidity of Substance Use and Mental Health Disorders in Europe. (2016). Available online at: http://www. emcdda.europa.eu/system/files/attachments/2639/Comorbidity_POD2016. pdf (accessed June 30, 2020).

9. Bahorik AL, Satre DD, Kline-Simon AH, Weisner CM, Campbell CI. Alcohol, cannabis, and opioid use disorders, and disease burden in an integrated health care system. J Addict Med. (2017) 11:3-9. doi: 10.1097/ADM.0000000000000260

10. Kirsch D, Nemeroff CM, Lippard ETC. Early life stress and substance use disorders: underlying neurobiology and pathways to adverse outcomes. Adv Res Sci. (2020) 1:29-47. doi: 10.1007/s42844-020-00005-7

11. Ayu AP, Schellekens AF, Iskandar S, Pinxten L, De Jong CA. Effectiveness and organization of addiction medicine training across the globe. Eur Addict Res. (2015) 21:223-39. doi: 10.1159/000381671

12. Peacock. Global Statistics on Alcohol, Tobacco and Illicit Drug Use: 2017 Status Report. Peacock (2018). doi: 10.1111/add.14234

13. WHO (2018). Available online at: https://www.who.int/gho/substance_abuse/ human_resources/en/ (accessed June 30, 2020).

14. WHO (2017). WHO ATLAS on Substance Use 2017: Resources for the Prevention and Treatment of Substance Use Disorders. Available online at: https://www.who.int/substance_abuse/activities/fadab/msb_adab_ 2017_atlas_su_25June2017.pdf?ua=1 (accessed June 30, 2020).

15. Van Boekel LC, Brouwers EP, Van Weeghel J, Garretsen HF. Stigma among health professionals toward patients with substance use disorders and its consequences for healthcare delivery: systematic review. Drug Alcohol Depend. (2013) 131:23-35. doi: 10.1016/j.drugalcdep.2013.02.018

16. Livingston JD, Boyd JE. Correlates and consequences of internalized stigma for people living with mental illness: a systematic review and meta-analysis. Soc Sci Med. (2010) 71:2150-61. doi: 10.1016/j.socscimed.2010.09.030
Putskys and Tibor Jager for their collaboration in the beginning of organization of the PSUD Working group. We wish to thank all co-authors for contribuiting to paying for the publication fees of the Journal.

\section{SUPPLEMENTARY MATERIAL}

The Supplementary Material for this article can be found online at: https://www.frontiersin.org/articles/10.3389/fpsyt. 2020.585607/full\#supplementary-material

17. Gilchrist G, Moskalewicz J, Slezakova S, Okruhlica L, Torrens M, Vajd R, et al. Staff regard towards working with substance users: a European multi-centre study. Addiction. (2011) 106:1114-25. doi: 10.1111/j.1360-0443.2011.03407.x

18. Klimas, J. Training in addiction medicine should be standardised and scaled up. BMJ. (2015) 351:h4027. doi: 10.1136/bmj.h4027

19. Avery J, Zerbo E. Improving psychiatry residents' attitudes toward individuals diagnosed with substance use disorders. Harv Rev Psychiatry. (2015) 23:296-300. doi: 10.1097/HRP.0000000000000047

20. Schulze TG, Treichel KC. The European Federation of Psychiatric Trainees (EFPT)-an integral part of the European harmonisation of psychiatric education and practise. Eur Psychiatry. (2002) 17:300-5. doi: 10.1016/S0924-9338(02)00671-5

21. Beezhold J. Twelve years old: the European Federation of Psychiatric Trainees (EFPT). Int Psychiatry. (2005) 2:20-1. doi: 10.1192/S1749367600 007128

22. Casanova Dias M, Ryland H, Harrison J. Evaluation of the European Federation of Psychiatric Trainees' Forum 2014. Eur Psychiatry. (2015) 30:1. doi: 10.1016/S0924-9338(15)31455-3

23. Pinto da Costa M, Giurgiuca A, Holmes K, Biskup E, Mogren T, Tomori S, et al. To which countries do European psychiatric trainees want to move to and why? Eur Psychiatry. (2017) 45:174-81. doi: 10.1016/j.eurpsy.2017.06.010

24. White W. Slaying the Dragon: The History of Addiction Treatmen and Recovery in America. 2nd ed. Bloomington, IL: Chestnut Health Systems (2014).

25. Weiss MG, Ramakrishna J, Somma D. Health-related stigma: rethinking concepts and interventions. Psychol Health Med. (2006) 11:277-87. doi: 10.1080/13548500600595053

26. Karam-Hage M, Nerenberg L, Brower KJ. Modifying residents' professional attitudes about substance abuse treatment and training. Am J Addict. (2001) 10:40-7. doi: 10.1080/105504901750160466

27. Corrigan P, Kerr A, Knudsen L. The stigma of mental illness: explanatory models and methods for change. Appl Prev Psychol. (2005) 11:179-90. doi: 10.1016/j.appsy.2005.07.001

28. Rao H, Mahadevappa H, Pillay P, Sessay M, Abraham A, Luty J. A study of stigmatized attitudes towards people with mental health problems among health professionals. J Psychiatr Ment Health Nurs. (2009) 16:279-84. doi: 10.1111/j.1365-2850.2008.01369.x

29. Schomerus G, Lucht M, Holzinger A, Matschinger H, Carta MG, Angermeyer MC. The stigma of alcohol dependence compared with other mental disorders: a review of population studies. Alcohol Alcohol. (2011) 46:105-12. doi: 10.1093/alcalc/agq089

30. Habib SE, Adorjany LV. Hepatitis $\mathrm{C}$ and injecting drug use: the realities of stigmatization and discrimination. Health Educ J. (2003) 62:256-65. doi: 10.1177/001789690306200307

31. Rasinski KA, Woll P, Cooke A. Stigma and substance use disorders. In: Corrigan PW, editor. On the Stigma of Mental Illness: Practical Strategies for Research and Social Change. Washington, DC: American Psychological Association (2005). p. 219-36.

32. Swanson JW, McGinty EE, Fazel S, Mays VM. Mental illness and reduction of gun violence and suicide: bringing epidemiologic research to policy. Ann Epidemiol. (2015) 25:366-76. doi: 10.1016/j.annepidem.2014.03.004

33. Miller NS, Sheppard LM, Colenda CC, Magen J. Why physicians are unprepared to treat patients who have alcohol- and drug-related disorders. Acad Med. (2001) 76:410-8. doi: 10.1097/00001888-200105000-00007 
34. Meltzer EC, Suppes A, Burns S, Shuman A, Orfanos A, Sturiano CV, et al. Stigmatization of substance use disorders among internal medicine residents. Subst Abuse. (2013) 34:356-62. doi: 10.1080/08897077.2013.815143

35. Jorm AF, Reavley NJ. Public belief that mentally ill people are violent: is the USA exporting stigma to the rest of the world? Aust N Z J Psychiatry. (2014) 48:213-5. doi: 10.1177/0004867413509697

36. Das S, Roberts LW. Addiction training: striving to fill an unmet need. Acad Psychiatry. (2016) 40:451-3. doi: 10.1007/s40596-016-0535-x

37. Ewan CE, Whaite A. Training health professionals in substance abuse: a review. Int J Addictions. (1982) 17:1211-29. doi: 10.3109/10826088209056350

38. Renner JA Jr., Karam-Hage M, Levinson M, Craig T, Eld B. What do psychiatric residents think of addiction psychiatry as a career? Acad Psychiatry. (2009) 33:139-42. doi: 10.1176/appi.ap.33.2.139

39. Rasyidi E, Wilkins JN, Danovitch I. Training the next generation of providers in addiction medicine. Psychiatr Clin North Am. (2012) 35:461-80. doi: 10.1016/j.psc.2012.04.001

40. Ram A, Chisolm MS. The time is now: improving substance abuse training in medical schools. Acad Psychiatry. (2016) 40:454-60. doi: 10.1007/s40596-015-0314-0

41. Orsolini L, Chiappini S, Corkery JM, Guirguis A, Papanti D, Schifano F. The use of new psychoactive substances (NPS) in young people and their role in mental health care: a systematic review. Exp Rev Neurotherap. (2019) 12:1253-64. doi: 10.1080/14737175.2019.1666712

42. Rush BR, Bass M, Stewart M, McCraken E, Labreque M, Bondy S. Detecting, preventing, and managing patients' alcohol problems. Can Fam Physician. (1994) 40:1557-66.

43. Wang PS, Demler O, Kessler RC. Adequacy of treatment for serious mental illness in the United States. Am J Public Health. (2002) 92:92-8. doi: 10.2105/AJPH.92.1.92

44. Giacco D, Matanov A, Priebe S. Providing mental healthcare to immigrants: current challenges and new strategies. Curr Opin Psychiatry. (2014) 27:282-8. doi: 10.1097/YCO.0000000000000065

45. Koyi MB, Nelliot A, MacKinnon D, Rastegar DA, Fingerhood M, Alvanzo A, et al. Change in medical student attitudes toward patients with substance use disorders after course exposure. Acad Psychiatry. (2018) 42:283-7. doi: 10.1007/s40596-017-0702-8
46. Fisher JC, Mason RL, Keeley KA, Fisher JV. Physicians and alcoholics: the effects of medical training on attitudes toward alcoholics. J Stud Alcohol. (1975) 36:949-55. doi: 10.15288/jsa.1975.36.949

47. Geller G, Levine DM, Mamon JA, Moore RD, Bone LR, Stokes EJ. Knowledge, attitudes, and reported practices of medical house staff regarding the diagnosis and treatment of alcoholism. JAMA. (1989) 261:3115-20. doi: 10.1001/jama.1989.03420210063017

48. Gopalan R, Santora P, Stokes EJ, Moore RD, Levine DM. Evaluation of a model curriculum on substance abuse at the Johns Hopkins University School of Medicine. Acad Med. (1992) 62:260-6. doi: 10.1097/00001888-199204000-00014

49. Lindberg M, Vergara C, Wild-Wesley R, Gruman C. Physicians-intraining attitudes toward caring for and working with patients with alcohol and drug abuse diagnoses. South Med J. (2006) 99:28-35. doi: 10.1097/01.smj.0000197514.83606.95

50. Casanova Dias M, Riese F, Tasman A. Curriculum development for psychiatric training. In: Fiorillo A, Volpe U, Bhugra D, editors. Psychiatry in Practice: Education, Experience and Expertise. Oxford: Oxford University Press (2016).

51. Sattar SP, Madison J, Markert RJ, Bhatia SC, Petty F. Addiction training scale: pilot study of a self-report evaluation tool for psychiatry residents. Acad Psychiatry. (2004) 28:204-8. doi: 10.1176/appi.ap.28.3.204

Conflict of Interest: The authors declare that the research was conducted in the absence of any commercial or financial relationships that could be construed as a potential conflict of interest.

Copyright (C) 2021 Orsolini, Rojnić Palavra, Papanti, Potočan, Quattrone, Martens, Sklenárová, Levola, Grichy, Naughton, Grinevičiene, Kuiters, Gondek, Panfil, Borovcanin, San Roman Uria, Biskup, Sönmez Güngör, Casanova Dias, Tomori, Banjac, Marinova-Djambazova and Pinto da Costa. This is an open-access article distributed under the terms of the Creative Commons Attribution License (CC BY). The use, distribution or reproduction in other forums is permitted, provided the original author(s) and the copyright owner(s) are credited and that the original publication in this journal is cited, in accordance with accepted academic practice. No use, distribution or reproduction is permitted which does not comply with these terms. 
OPEN ACCESS

Edited by:

Fabrizio Schifano,

University of Hertfordshire,

United Kingdom

Reviewed by:

Amira Guirguis,

Swansea University, United Kingdom

Georgios Demetrios Kotzalidis,

Sapienza University of Rome, Italy

*Correspondence: Camille Ponté

ponte.c@chu-toulouse.fr

these authors have contributed equally to this work and share first

authorship

¥ORCID:

Camille Ponté

orcid.org/0000-0001-8616-5313

Emilie Jouanjus

orcid.org/0000-0002-3510-2475

Nathalie Fouilhé

orcid.org/0000-0002-5620-6804

Joelle Micallef

orcid.org/0000-0002-7172-7835

Maryse Lapeyre-Mestre orcid.org/0000-0002-5494-5873

Specialty section:

This article was submitted to

Addictive Disorders,

a section of the journal

Frontiers in Psychiatry

Received: 09 December 2020

Accepted: 06 January 2021

Published: 03 February 2021

Citation:

Tambon M, Ponté C, Jouanjus E, Fouilhé N, Micallef $\mathrm{J}$,

Lapeyre-Mestre $M$ and

the French Addictovigilance Network (FAN) (2021) Gabapentinoid Abuse in

France: Evidence on Health

Consequences and New Points of

Vigilance.

Front. Psychiatry 12:639780. doi: 10.3389/fpsyt.2021.639780

\section{Gabapentinoid Abuse in France: Evidence on Health Consequences and New Points of Vigilance}

\author{
Marine Tambon ${ }^{1 \dagger}$, Camille Ponté ${ }^{1 * t \neq}$, Emilie Jouanjus ${ }^{1,2 \neq}$, Nathalie Fouilhé ${ }^{3 \neq}$, \\ Joelle Micallef $f^{4,5 \neq}$, Maryse Lapeyre-Mestre ${ }^{1,2,6 \neq}$ and \\ the French Addictovigilance Network (FAN) \\ ${ }^{1}$ Centre d'Evaluation et Information sur la Pharmacodépendance-Addictovigilance, Service de Pharmacologie Médicale et \\ Clinique, CHU de Toulouse, Toulouse, France, ${ }^{2}$ Unité Mixte de Recherche, 1027 Inserm-Université, Pharmacoépidémiologie, \\ Université de Toulouse, Toulouse, France, ${ }^{3}$ Centre d'Evaluation et Information sur la Pharmacodépendance-Addictovigilance, \\ Service de Pharmacologie-Pharmacosurveillance, CHU Grenoble-Alpes, Grenoble, France, ${ }^{4}$ Aix Marseille Univ, AP-HM, \\ INSERM, Inst Neurosci Syst, Service de Pharmacologie Clinique et Pharmacovigilance, Marseille, France, \\ ${ }^{5}$ CEIP-Addictovigilance PACA Corse, Marseille, France, ${ }^{6}$ Centre d'Investigation Clinique 1436, CHU de Toulouse, Toulouse, \\ France
}

Introduction: Gabapentinoid drugs (gabapentin and pregabalin) are widely used worldwide for epileptic and pain disorders. First signals of gabapentinoid abuse occurred in the last decade. This study aims to describe clinical characteristics of gabapentinoid use related disorders and health consequences in France.

Materials and Methods: We designed a multisource investigation reviewing data reported to the French Addictovigilance Network (FAN) with pregabalin and gabapentin from 2010 to 2019. Information was obtained through the analysis of Spontaneous Reports (SRs) notified by health professionals and the pharmacoepidemiological surveys OSIAP (suspicious prescriptions forms indicators of potential abuse), OPPIDUM (observation of illicit drugs and misuse of psychotropic medications), DRAMES (death related to prescription drugs and other substances), and DTA (toxic deaths due to analgesics).

Results: Over 2010-2019 period, were collected: (i) 265 SRs (258 pregabalin; 7 gabapentin); (ii) 816 forged prescription forms (805 pregabalin, 10 gabapentin, 1 involving both drugs); (iii) 145 cases of gabapentinoid use in people who use drugs (121 pregabalin; 24 gabapentin) and (iv) 31 cases of gabapentinoid-related deaths (25 pregabalin; 6 gabapentin). Risk factors of gabapentinoid abuse were opioid use disorders or psychiatric history, but cases of primary abuse in subjects without any substance abuse history were observed. Adverse outcomes concern almost exclusively pregabalin, with coma, dyspnea, convulsion, and conduction disorders. Treatment demands increased from 10.6\% in 2018 to $23.1 \%$ in 2019, with pregabalin cited as the first substance leading to addictological care in the 2019 OPPIDUM survey. Gabapentinoid-related deaths increased over time. Pregabalin has become the first drug mentioned in forged prescriptions in 2019 (23.8\% of OSIAP), while it ranked at the 15th position in 2017 (2.6\%). 
Discussion: This study shows the importance of addictovigilance monitoring for gabapentinoids. Addictovigilance data helped to make visible the gabapentinoid-abuse related health harms (hospitalization for serious neurologic, psychiatric or cardiac effects, requests for addictological support and deaths) and to confirm the intrinsic abuse potential of pregabalin. These data highlight new points of vigilance considering observed primary abuse. At this point in France, the risk of abuse and related complications is very apparent with pregabalin. Still, it is identical to that observed elsewhere with gabapentin.

Keywords: addictovigilance, gabapentinoids, psychotropic adverse effects, addiction, prescription drug abuse

\section{INTRODUCTION}

Gabapentin and pregabalin are two pharmacologically closely related drugs, belonging to the class of gabapentinoids [mirogabalin, only available in Japan, represents the third member (1)]. This class present structural similarities with gamma-amino-butyric acid (GABA) without acting on its receptor. The mechanism of action of gabapentinoids is generally described as binding on the alpha2-voltage-dependent calcium channels in the central nervous system, reducing central neuronal excitability. This action is believed to contribute to the antinociceptive, anticonvulsant and anxiolytic properties of these drugs. Gabapentin (approved in the early 1990s) and pregabalin (approved in 2004) are widely used for epilepsy and neuropathic pain (gabapentin is indicated for post zoster pain). Pregabalin is also approved for generalized anxiety disorder and for fibromyalgia and gabapentin for restless leg syndrome only in the US. The European commercial success of pregabalin since its marketing authorization in 2004 has led to the expansion of its use in off-label indications [any type of pain or to manage benzodiazepines or alcohol withdrawal $(2,3)]$. In 2010, toxicology and pharmacovigilance data as clinician reporting in Europe [Scandinavian countries, Germany and Southern Europe (4-7)] first reported involvement of pregabalin in deaths related to substance abuse. Since then, an increasing number of reviews have been published on the subject, arguing the evidence of gabapentinoid misuse and abuse. A minority of these reviews concluded that gabapentinoids has no appearing addictive potential themselves and may lead to abuse only by persons with opioid use disorders $(8,9)$. It should be noted that subjects with a history of psychiatric or substance use disorders are overall more at risk of such behaviors. Most of these reviews suggest that misuse and abuse occur more frequently in users of pregabalin compared with gabapentin (10-12). In France, only a few cases of gabapentin misuse and abuse have been reported until 2014 (13-15). In 2011, a first case of recreational use of pregabalin has been reported by a general practitioner in 2011 and received particular attention by the French Addictovigilance Network (FAN) as an early signal for pregabalin abuse potential. Data have been since collected leading to further evidence that pregabalin misuse and abuse is now widespread in France, with visible harmful consequences in terms of treatment demands, somatic complications, and even risk of death.
Based on data collected through the French addictovigilance system from 2010 to 2019, this study aims to describe clinical characteristics of pregabalin and gabapentin use related disorders and their health consequences, focusing on primary dependence potential, life-threatening complications and management of abuse and dependence.

\section{MATERIALS AND METHODS}

We designed a multisource investigation reviewing data reported to the French Addictovigilance Network (FAN). The FAN is made up of 13 Addictovigilance Centers, it was set up in 1990 under the supervision of the French Medicines Agency ("ANSM" for Agence Nationale de Sécurité des Médicaments et des Produits de Santé) to monitor the abuse potential of psychoactive substances (with the exclusion of tobacco and alcohol) (16-18).

\section{Data Related to Spontaneous Reports (SRs) Notified by Health Professionals}

All cases of pregabalin/gabapentin-related disorders reported between 2010 and 2019 were analyzed with data on individual features (age, gender, past medical history) and clinical features (clinical signs related to substance use, patterns of substance use). All psychoactive substances included, over the 2010-2019 period, the FAN has recorded more than 41,500 SRs.

\section{Data Related to Forged/Falsified Prescriptions Forms Reported by Community Pharmacists (OSIAP Survey)}

This survey aims to identify drugs liable to be diverted from their medical use or at risk of abuse or dependence. Prescription forms recorded from 2010 to 2019 including citations of pregabalin and gabapentin were analyzed. All prescription drugs included, over the 2010-2019 period, the FAN has recorded about 11,000 prescription forms $(19,20)$.

\section{Data Related to Patterns of Psychoactive Drug Use Reported by People Who Use Drugs (PWUD) Visiting Specialized Addiction Care Centers (OPPIDUM Survey)}

This annual, cross-sectional survey aims to collect information on self-reported drug use by PWUD. Data of individuals 
reporting pregabalin and gabapentin use between 2010 and 2019 were analyzed. All psychoactive substances included, over the 2010-2019 period, the FAN has recorded data on around 52,000 individuals (21).

\section{Data Related to Drug-Related Deaths From Toxicological and Medico-Legal Data (DRAMES and DTA Surveys)}

These surveys aim to identify cases of death related to prescription drugs and other substances (DRAMES survey) or toxic deaths due to analgesics (DTA survey, since 2013). For a given case, each substance identified in the blood is subjected to a causality assessment, establishing the link between the substance and the cause of death. The strength of causal connection is determined by a score, from high (level 1) to low (level 4). The causal link is made on blood concentrations (or other matrices if no blood) quantification and relies on analysis of toxicology experts and different published references (22). For pregabalin, the retained therapeutic concentration is from 2 to 5 $\mathrm{mg} / \mathrm{L}$, toxic concentration is at $10 \mathrm{mg} / \mathrm{L}$ and lethal concentration at $25 \mathrm{mg} / \mathrm{L}$ and above (23), whether pregabalin is alone or in combination with other drugs. Cases of death for which pregabalin and gabapentin were confirmed and quantified, were analyzed, over the 2010-2018 period for DRAMES survey and over the 2013-2018 period for DTA survey. All psychoactive substances included, over the 2010-2018 period, the FAN has collected data on almost 4,000 deaths. The 2019 DRAMES/DTA data were not complete at the time of our study (because delay for forensic context); available information was analyzed.

Other data used into the multisource approach include the level of drug exposure in the French general population from the French Health Insurance System (Système National des Données de Santé, SNDS https://www.snds.gouv.fr/SNDS/Accueil) and the French Pharmacovigilance database for all reports of any adverse drug reaction (including misuse and abuse). Figure 1 presents the partnership involved in the network providing field/post-marketing data and the sources of addictovigilance data used in this study (16). The level of exposure to pregabalin and gabapentin in the French general population for the 20102019 period was computed as the number of people living in France who received at least one prescription of these drugs each year.

To describe gabapentinoid use related disorders, the following terms and definitions were used in the manuscript:

- Misuse: use in a manner that is non consistent with the summary of the product characteristics (regarding therapeutic indications, route of administration or posology) or a nontherapeutic use of prescription drug (24)

- Abuse: misuse or illicit drug use leading to health harms (somatic or psychiatric, hospitalization, death, etc)

- Dependence: condition according to which, upon cessation, a withdrawal syndrome (somatic or psychiatric symptoms) emerges

- Substance use disorder: defined by the DSM-5 (25), when the level of available information is sufficient to conclude this or reported as such by a specialist in addiction.
As this study was performed retrospectively using routinely collected anonymous data, it did not require any ethics committee approval, in line with the French regulations for mandatory reporting of addiction cases by health professionals.

\section{RESULTS}

Over the 2010-2019 period, the following data were collected: (i) $265 \mathrm{SRs}$ of gabapentinoid abuse (258 with pregabalin and 7 with gabapentin); (ii) 816 forged/falsified prescription forms (805 involving pregabalin, 10 gabapentin and 1 involving both drugs) from OSIAP survey; (iii) 145 cases of gabapentinoid use in people who use drugs (PWUD) (121 with pregabalin and 24 with gabapentin) from OPPIDUM survey; and (iv) 31 cases of gabapentinoid-related deaths (25 with pregabalin and 6 with gabapentin) from DRAMES and DTA surveys.

\section{Evolution of Gabapentinoid Abuse Phenomenon in France From 2010 to 2019}

During the study period, the consumption of both pregabalin and gabapentin increased significantly, with gabapentin level remaining about four times lower compared to pregabalin (Figure 2). In contrast, the proportion of falsified prescriptions with pregabalin increased sharply from 2018 onwards with a citation rate (number of pregabalin citations among all forged prescriptions collected) below $3.0 \%$ up to 2017 and increased to $11.9 \%$ in 2018 and $23.8 \%$ in 2019 (Figure 2). A similar pattern has been observed in other surveys (Figure 3). From 2010 to 2017, a gabapentinoid abuse has been reported in 24 cases $(<0.5 \%$ of total of SRs per year). In 2018 and 2019, this figure increased significantly to 71 in 2018 (1.2\% of total SRs) and $117(2.0 \%)$ in 2019. In 2013, the first gabapentinoid-related deaths were reported with one case involving pregabalin. The number of reported deaths was at its maximum in $2018(n=10$, data for 2019 being not completely collected at the time of the study). The number of gabapentinoid users among PWUD reached the highest level in 2019 with 40 ( $0.7 \%$ of the surveyed population) users, i.e., 2.6 times higher than in 2018. The gabapentinoid abuse phenomenon involved almost exclusively pregabalin and remained marginal for gabapentin.

\section{Socio-Demographic Profiles of Problematic Users of Gabapentinoids}

According to SRs, a total of 258 individuals with pregabalin abuse and 7 with gabapentin were reported. This population mainly consisted of men $(72.5 \%)$. The median age was 30 years old over the period but dropped to 24 years old in 2019 . The proportion of subjects under the age of eighteen was of $22.3 \%$. Psychiatric history was reported in $70(26.4 \%)$ patients, chronic pain in 69 (26.0\%) and epilepsy in $6(2.3 \%)$ patients. An existing substance use disorder was documented in 143 patients (54.0\%, missing data $45.7 \%$ ); but one reported case confirmed the absence of any substance use disorder for this patient. Substance use disorder data were available for 88 (61.5\%) patients, with $61.3 \%$ of them $(N=54)$ having opioid use disorder. 
A

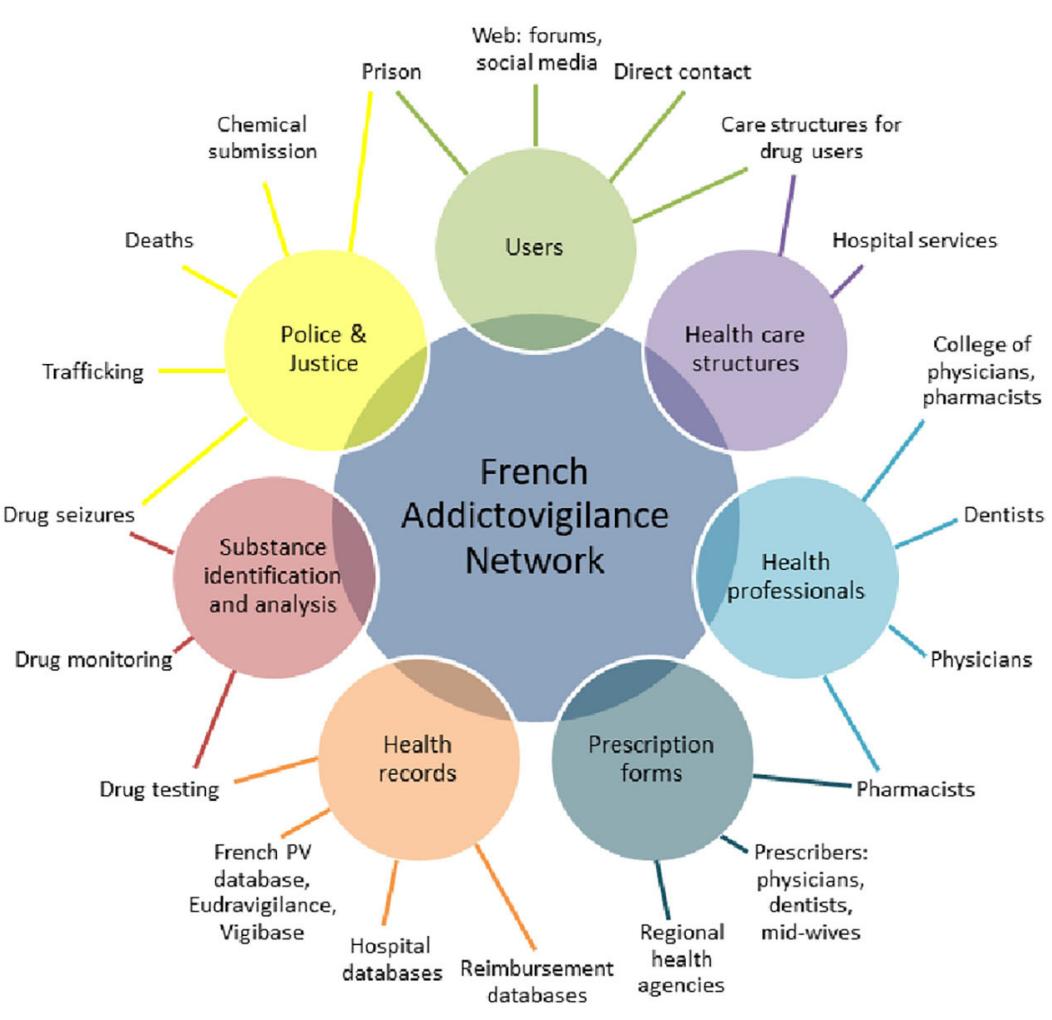

B

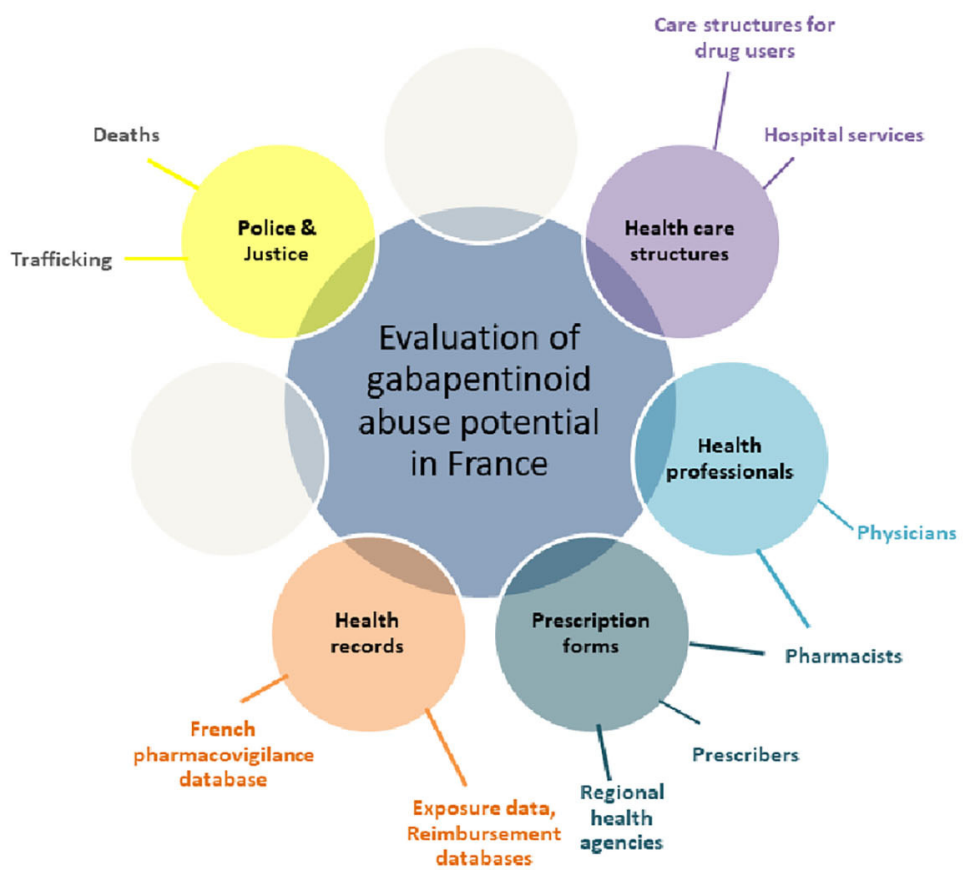

FIGURE 1 | The multidimensional French Addictovigilance Network: collaborative relationships and data sources involved in the national system (A) and the data sources used for the evaluation of gabapentinoid abuse potential (B). 


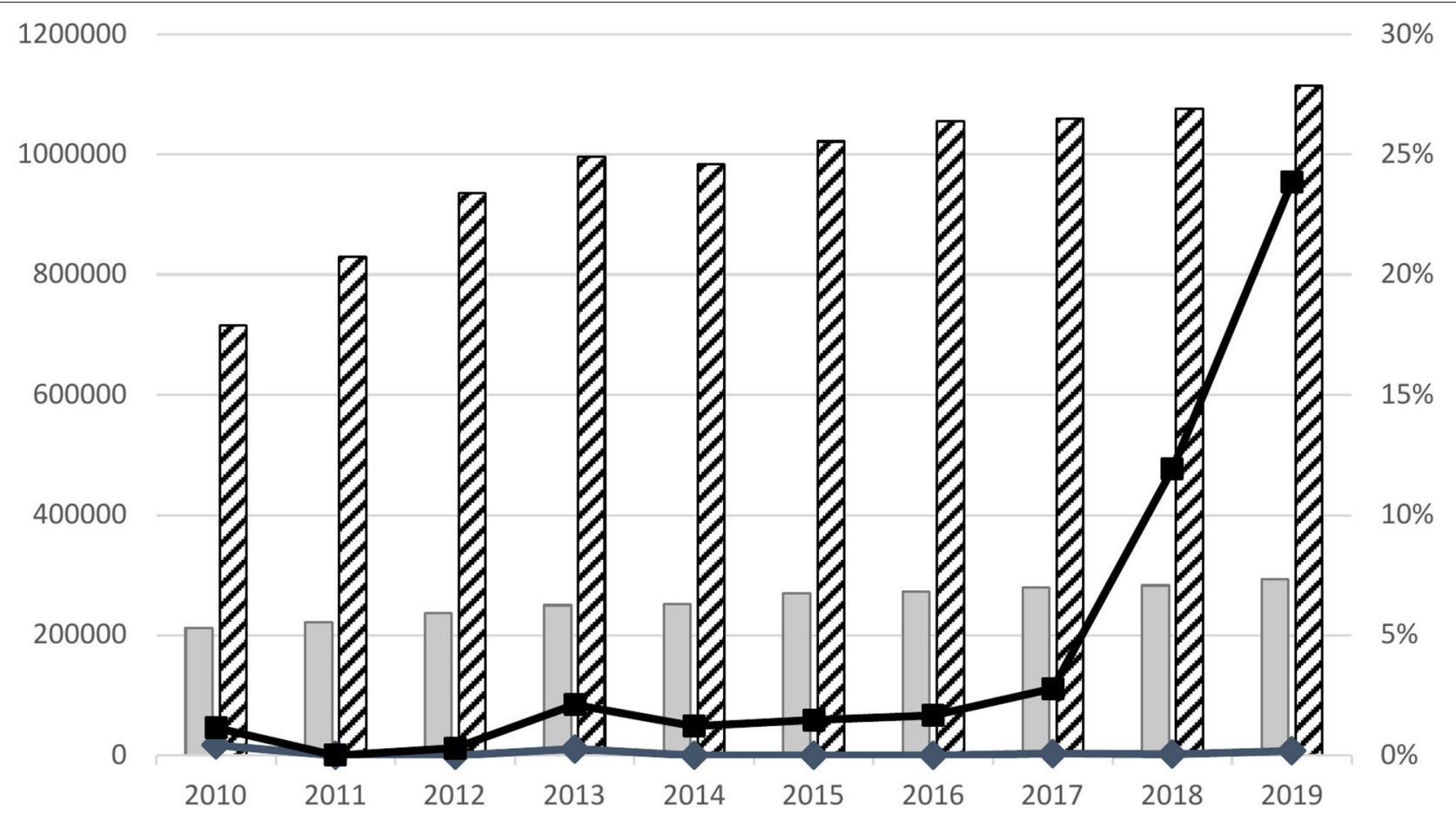

Gabapentin

ZZZPregabalin

$\longrightarrow$ Gabapentin citation rate in forged prescriptions (\%) $\rightarrow$ Pregabalin citation rate in forged prescriptions (\%)

FIGURE 2 | Gabapentinoid abuse phenomenon in France from 2010 to 2019 through the monitoring of forged/falsified prescription forms (OSIAP survey) with regard to number of gabapentinoid users.

Given the limited data collected with gabapentin compared to pregabalin, the results presented in the following paragraphs 3.3 and 3.4 focus on pregabalin cases (gabapentin cases are excluded) (Table 1).

\section{How Pregabalin Is Used in the Context of Abuse}

According to the 258 SRs, pregabalin was use in combiation with other psychoactive substance (including alcohol) by 69 (26.7\%) individuals (Figure 4). Among the desired non-therapeutic effects, euphoria ranked first reported by $28(10.9 \%)$ individuals. It was followed by research of high in 23 (8.9\%) individuals. Criteria related to substance use disorders were found: 20 (7.8\%) individuals continued taking pregabalin to prevent the occurrence of withdrawal symptoms, and $3(1.2 \%)$ took it by craving or routinely. Pregabalin was either used as a substitute or to prevent alternative drug use by $12(4.7 \%)$ subjects [mainly benzodiazepines (5/12), opioids (5/12) and cocaine $(2 / 12)$ ] or to potentiate effects of other drugs [opioids $(2 / 3)$ and cocaine (1/3)]. Pregabalin was used in the context of a drug experimentation for $2(0.8 \%)$ subjects, including one by intranasal route. Oral administration was preferred but intranasal use was reported occasionally. Also, one subject inhaled ("smoked") pregabalin by a process similar to that used to prepare free-base cocaine. Regarding frequency intake, 138 (53.5\%) individuals used pregabalin daily. From detailed cases (68.8\%) the median dose was of $900 \mathrm{mg}$ per day [Q1 $=450$; Q3 $=1,200$ ], with a maximum reported dose of 12.6 grams per day after 4 months of pregabalin exposure in a context of substance addiction transfer from buprenorphine to pregabalin. There were $71(27.5 \%)$ cases relative to acute exposure of pregabalin; in these cases, the maximum reported dose (out of deliberate selfpoisoning contexts) was 3.6 grams per intake to reach high and hypnotic effects. In $20(7.8 \%)$ cases, the subjects consumed pregabalin occasionally or over a few days. The information on frequency or doses consumed was missing in 29 (11.2\%) cases. Pregabalin was obtained illegally by 94 (57.7\%, 95 missing data) subjects through illicit market, forged/falsified prescriptions or medical/pharmaceutical nomadism. In $70(42.9 \%)$ cases, a valid prescription form was used. In one case, pregabalin was purchased in pharmacy without prescription (outside France). Data from 2019 OSIAP survey have shown that pregabalin has become the first drug mentioned in forged/falsified prescriptions forms presented in pharmacy (citation rate of $23.8 \%$ ), while 


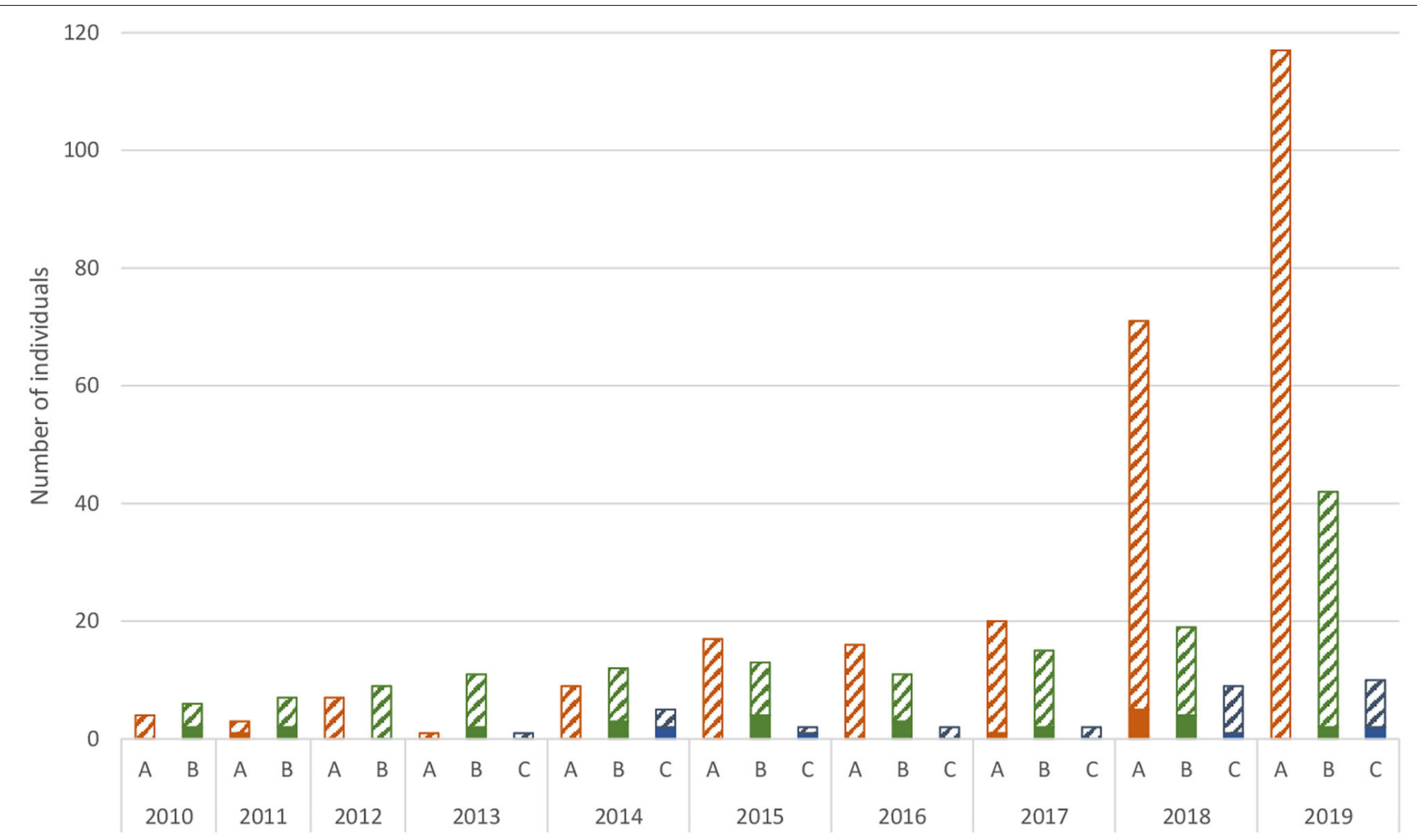

Gabapentin $\quad$ Pregabalin

FIGURE 3 | Number of individuals affected by gabapentinoid related disorders through addictovigilance data sources (A) (orange): number of gabapentinoid related disorders from Spontaneous Reports (SRs). (B) (green): number of gabapentinoid users in people who use drugs (OPPIDUM survey). (C) (blue): number of gabapentinoid-related deaths (DRAMES and DTA surveys) - data collection uncompleted for 2019.

TABLE 1 | Main characteristics based on the 258 NotS (spontaneous reports) of pregabalin use disorders collected by the French Addictovigilance Network from 2010 to 2019.

1 The dynamics of pregabalin problematic use phenomenon intensified in France from 2018 and still growing in 2019. Among the 258 collected NotS, 183 (70.9\%) occurred in 2018 and 2019.

2 Subjects were mainly men (72.5\%), young (median age of 24 years in 2019). An existing substance use disorder was documented in $54 \%$ patients, including subjects with no opioid use disorder, and one reported case confirmed the lack of any substance use disorder for this patient.

3 Pregabalin was preferentially misused by the oral route, at high dose [median daily dose at $900 \mathrm{mg}$ (Q1: 450; Q3: 1,200)]; occasional intakes until (3.6 grams) and illegally obtained (false prescription forms and street market). Among desired non-therapeutic effects, euphoria ranked first cited by 28 (10.9\%) individuals followed by research of high in 23 (8.9\%) of them.

$4 \quad$ Pregabalin abuse frequently led to neurological (81.6\%) and psychiatric (34.4\%) complications alone or in combination. A convulsive episode and a cardiac serious complication (atrioventricular block) occurred with pregabalin alone.

5 Ninety patients (34.9\%) presented criteria of pregabalin use disorder, whether the subjects used it to obtain therapeutic effects or not. Between 2018 and 2019 , the proportion of individuals demanding for specialized addiction care have increased from 10.6 to $23.1 \%$. Withdrawal strategies were instituted by health professionals (hospitalization, gradual tapering off, medication support).

it ranked at the 15th position in the 2017 survey (citation rate of $2.6 \%$ ) (Figure 2). The $300 \mathrm{mg}$ dosage was the most concerned $(67.3 \%$ of citations of pregabalin, missing data in 20.1\%). Pregabalin street names have been reported: "l'extase" (bliss), "saroukh," "fusée" (rocket) or "taxi," and street prices: for the $150 \mathrm{mg}$ dosage, 10 euros per 14 capsules, for the $300 \mathrm{mg}$ dosage, 1-2 euros each capsule or 30 euros the box of 56 capsules.

\section{Pregabalin-Related Complications in the Context of Abuse Clinical Symptoms}

Among the 258 patients presenting a problematic use of pregabalin, a hospital based care was needed in 100 (38.8\%) cases and $125(48.4 \%)$ have presented clinical complications: 106 in a context of polydrug use and 19 with pregabalin alone (Table 2). 


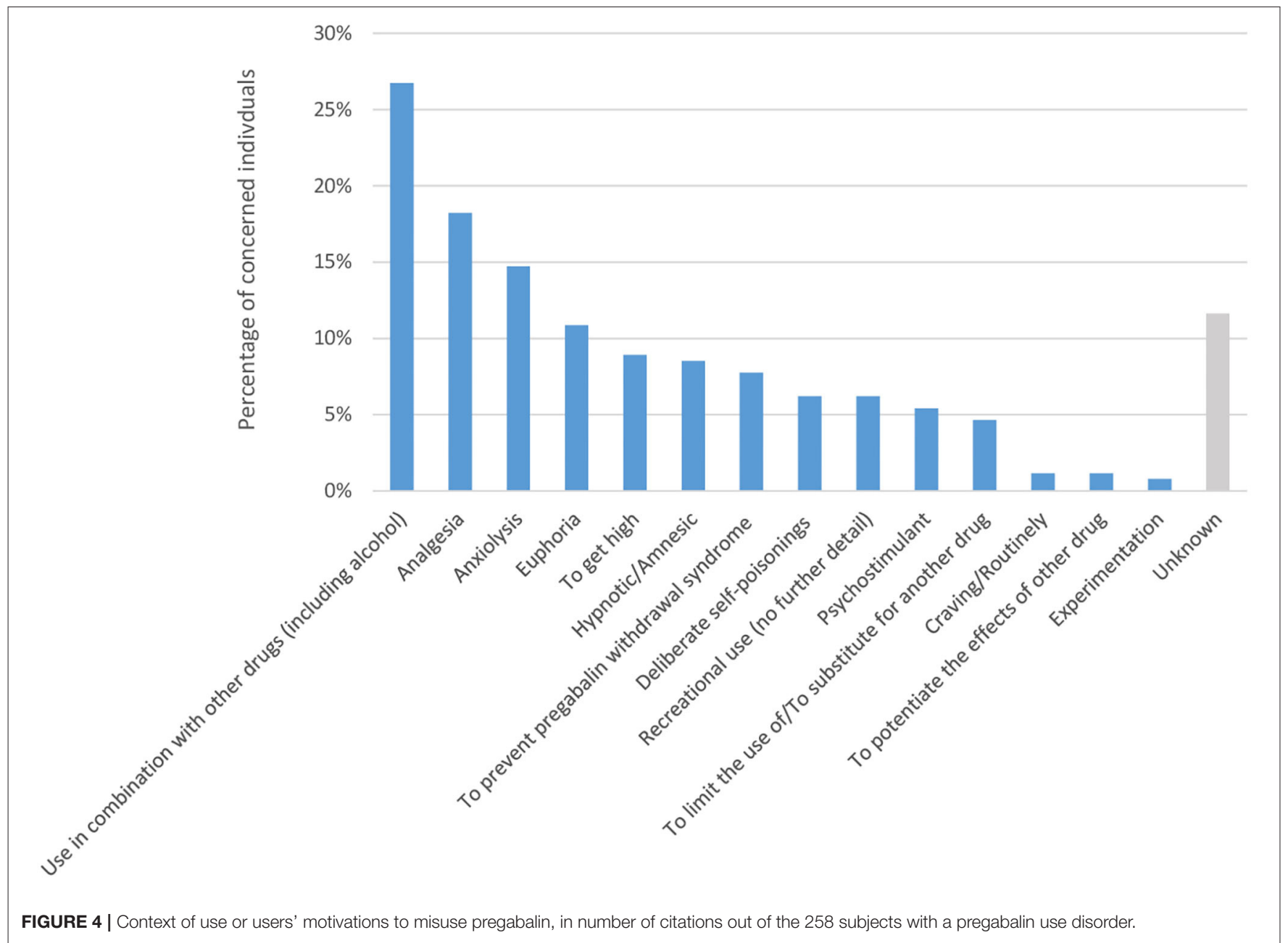

Among complications with pregabalin alone as in combination, neurological complications ranked first, concerned $81.6 \%$ of patients, mainly represented by consciousness impairment. Coma occurred in 12 patients in polydrug use context only, with benzodiazepine being co-ingested in 10/12 cases. The convulsive episode with pregabalin alone occurred in a 15year-old girl without any history of epilepsy after an intake of $1,200 \mathrm{mg}$. Psychiatric complications came second with pregabalin alone as in-combination, concerned $34.4 \%$ of patients, and particularly behavioral issues such as agitation, aggressiveness, impulsiveness or disinhibition. Among psychotic symptoms, hallucinations were reported three times, all occurred with pregabalin combinations ( 1 case with alcohol after occasional pregabalin intake of $400 \mathrm{mg}, 1$ case with buprenorphine and oxazepam and 1 case with cannabis), in subjects without any psychotic history. Euphoria was reported with pregabalin alone (after an intake of $600 \mathrm{mg}$ by oral route). Clinical presentations of opioid overdose (not included in impaired consciousness/miosis/dyspnea categories) concerned 11 (8.8\%) patients, exclusively in the context of polyconsumption but not exclusively with opioid substances. Dyspnea (out of opioid overdose presentation) concerned $4(3.2 \%)$ patients who have used pregabalin with other drugs, mainly opioids (3/4 cases). Two serious cardiac complications have been reported: an atrioventricular block in a male aged 35 using pregabalin by intranasal route for several months and hypertrophic cardiomyopathy in a male aged 17 who regularly used clonazepam and cannabis.

\section{Addictological Complications and Demands for Specialized Care}

Among the 258 pregabalin abuse SRs, 90 (34.9\%) presented criteria of a pregabalin use disorder, whether the subjects used it to obtain therapeutic effects or not. Time to onset was specified in $48(53.3 \%)$ cases, and the shortest was about 2 months. Over the 2010-2019 period, 49 (19.0\%) subjects have requested addictological support due to pregabalin problematic use or were referred to specialized addiction care. Between 2018 and 2019, the proportion of subjects demanding for specialized addiction care have increased from 10.6 to $23.1 \%$. Withdrawal strategies consisted in hospitalization to stop using pregabalin [12 (24.5\%) subjects] or ambulatory, in gradual tapering off (9, $18.4 \%$ ) or by introducing medication such as benzodiazepine, sedative antipsychotic or antidepressant (5, 10.2\%). A prior 


\begin{tabular}{|c|c|c|c|c|c|c|c|}
\hline & $\begin{array}{c}\text { All } \\
\text { pregabalin } \\
\text { exposures }\end{array}$ & $(\%)$ & $\begin{array}{l}\text { Pregabalin- } \\
\text { only }\end{array}$ & $(\%)$ & $\begin{array}{l}\text { Pregabalin } \\
\text { in co- } \\
\text { consumption }\end{array}$ & (\%) & Co-consumed reported substances $(n)$ \\
\hline Number of patients & 258 & & 73 & & 185 & & \\
\hline $\begin{array}{l}\text { Number of patients with } \\
\text { reported clinical } \\
\text { complications }\end{array}$ & 125 & $48.4 \%$ & 19 & $26.0 \%$ & 106 & $57.3 \%$ & \\
\hline Neurological complications & 102 & $81.6 \%$ & 16 & $84.2 \%$ & 86 & $81.1 \%$ & \\
\hline $\begin{array}{l}\text { Impaired consciousness (out of } \\
\text { clinical "triad" of opioid } \\
\text { overdose }^{*} \text { ) }\end{array}$ & 68 & $66.7 \%$ & 11 & $68.8 \%$ & 57 & $66.3 \%$ & $\begin{array}{l}\text { Benzodiazepine (33); Opioids (17); Psychostimulants (19); Alcohol (18); Other } \\
\text { psychotropic drugs (15); Cannabis (14) }\end{array}$ \\
\hline Incl. Coma $(G S C<9)$ & 12 & $11.8 \%$ & 0 & & 12 & $14.0 \%$ & $\begin{array}{l}\text { Benzodiazepine (10); Other psychotropic drugs (6); Opioids (5); Psychostimulants (5); } \\
\text { Alcohol (5); Cannabis (3) }\end{array}$ \\
\hline $\begin{array}{l}\text { Psychomotor retardation - } \\
\text { Dizziness - Ataxia }\end{array}$ & 19 & $18.6 \%$ & 5 & $31.3 \%$ & 14 & $16.3 \%$ & $\begin{array}{l}\text { Opioids (5); Cannabis (5); Benzodiazepine (4); Psychostimulants (3); Alcohol (3); Other } \\
\text { psychotropic drugs (2) }\end{array}$ \\
\hline $\begin{array}{l}\text { Involuntary/abnormal } \\
\text { movements (dyskinesia, tremor, } \\
\text { nystagmus, chorea) }\end{array}$ & 8 & $7.8 \%$ & 0 & & 8 & $9.3 \%$ & $\begin{array}{l}\text { Cannabis (3); Alcohol (3); Benzodiazepine (2); Opioids (2); Psychostimulants (2); Other } \\
\text { psychotropic drugs (2) }\end{array}$ \\
\hline Convulsion & 7 & $6.9 \%$ & 1 & $6.3 \%$ & 6 & $7.0 \%$ & $\begin{array}{l}\text { Opioids (3); Psychostimulants (3); Other psychotropic drugs (3); Alcohol (3); } \\
\text { Benzodiazepine (2); Cannabis (2) }\end{array}$ \\
\hline $\begin{array}{l}\text { Miosis (out of clinical 'triad' of } \\
\text { opioid overdose) }\end{array}$ & 10 & $9.8 \%$ & 0 & & 10 & $11.6 \%$ & $\begin{array}{l}\text { Cannabis (6); Benzodiazepine (5); Psychostimulants (4); Other psychotropic drugs (4); } \\
\text { Alcohol (4); Opioids (3) }\end{array}$ \\
\hline Mydriasis & 7 & $6.9 \%$ & 1 & $6.3 \%$ & 6 & $7.0 \%$ & Alcohol (4); Psychostimulants (3); Cannabis (3); Benzodiazepine (2); Opioids (2) \\
\hline Psychiatric complications & 43 & $34.4 \%$ & 5 & $26.3 \%$ & 38 & $35.8 \%$ & \\
\hline $\begin{array}{l}\text { Behavioral issues (agitation, } \\
\text { aggressiveness, impulsiveness, } \\
\text { disinhibition) }\end{array}$ & 27 & $62.8 \%$ & 3 & $60.0 \%$ & 24 & $63.2 \%$ & $\begin{array}{l}\text { Benzodiazepine (11); Psychostimulants (8); Cannabis (8); Opioids (7); Alcohol (5); } \\
\text { Other psychotropic drugs (4) }\end{array}$ \\
\hline Depressed mood, dysthymia & 7 & $16.3 \%$ & 1 & $20.0 \%$ & 6 & $15.8 \%$ & $\begin{array}{l}\text { Benzodiazepine (4); Psychostimulants (3); Alcohol (2); Opioids (2); Other psychotropic } \\
\text { drugs (1) }\end{array}$ \\
\hline $\begin{array}{l}\text { Psychotic symptoms (delirium, } \\
\text { hallucinations) }\end{array}$ & 6 & $14.0 \%$ & 0 & & 6 & $15.8 \%$ & $\begin{array}{l}\text { Opioids (2); Alcohol (2); Cannabis (2); Psychostimulants (1); Other psychotropic drugs } \\
\text { (1); Benzodiazepine (1) }\end{array}$ \\
\hline Anxiety & 4 & $9.3 \%$ & 1 & $20.0 \%$ & 3 & $7.9 \%$ & Psychostimulants (2); Benzodiazepine (1); Cannabis (1); Other psychotropic drugs (1) \\
\hline Euphoria & 2 & $4.7 \%$ & 1 & $20.0 \%$ & 1 & $2.6 \%$ & Opioids (1) \\
\hline $\begin{array}{l}\text { Clinical presentation of opioid } \\
\text { overdose (uncounted }^{*} \\
\text { elsewhere) }\end{array}$ & 11 & $8.8 \%$ & 0 & & 11 & $10.4 \%$ & $\begin{array}{l}\text { Benzodiazepine (8); Opioids (8); Psychostimulants (7); Cannabis (6); Other } \\
\text { psychotropic drugs (2); Alcohol (1) }\end{array}$ \\
\hline Respiratory complications & 4 & $3.2 \%$ & 0 & & 4 & $3.8 \%$ & \\
\hline $\begin{array}{l}\text { Dyspnea (out of clinical 'triad' of } \\
\text { opioid overdose) }\end{array}$ & 4 & $100.0 \%$ & 0 & & 4 & $100.0 \%$ & $\begin{array}{l}\text { Opioids (3); Psychostimulants (2); Benzodiazepine (1); Cannabis (1); Other } \\
\text { psychotropic drugs (1) }\end{array}$ \\
\hline Cardiac complications & 2 & $1.6 \%$ & 1 & $5.3 \%$ & 1 & $0.9 \%$ & \\
\hline Atrioventricular block & 1 & $50.0 \%$ & 1 & $100.0 \%$ & 0 & & \\
\hline Hypertrophic cardiomyopathy & 1 & $50.0 \%$ & 0 & & 1 & $100.0 \%$ & Benzodiazepine (1); Cannabis (1) \\
\hline Others & 3 & $2.4 \%$ & 1 & $5.3 \%$ & 2 & $1.9 \%$ & \\
\hline Hyperglycemia & 1 & $33.3 \%$ & 0 & & 1 & $50.0 \%$ & Opioids (1); Psychostimulants (1); Cannabis (1); Alcohol (1) \\
\hline Hypoglycemia & 2 & $66.7 \%$ & 1 & & 1 & $50.0 \%$ & Benzodiazepine (1); Psychostimulants (1); Cannabis (1) \\
\hline
\end{tabular}

\section{Number of patien}

reported clinical

overdose*

Psychomotor retardation -

movements (dyskinesia, tremor,

Miosis (out of clinical 'triad' of

opioid overdose)

\section{Psychiatric complications}

Psychotic symptoms (delirium, hallucinations)

Anxiety

Clinical presentation of opioid

Cardiac complications

Atrioventricular block

*impaired consciousness and myosis and bradypnea. Bold values are indicates in main titles. Italic values are i 
withdrawal attempt was reported in 13 (26.5\%) subjects. Data from 2019 OPPIDUM survey have shown the highest level of pregabalin consumption in individuals seeking addiction care (Figure 3). In 2019, for the first time since the beginning of the OPPIDUM investigation, two subjects reported pregabalin as the first psychoactive substance that leads to dependence.

\section{Pregabalin Related Deaths}

From 2010 to 2018, pregabalin was detected and quantified in 51 cases of death. Pregabalin was responsible for death (level 1 of causal connection) in 17 cases (Table 3); alone (case 12) or in combination with other drugs (all other cases). The most frequently detected drugs assessed as co-responsible for death were opioids involved in 12/16 cases (with tramadol and methadone, respectively, involved in 5 and 4 cases). The blood concentrations were lethal in 8 cases, ranged from 26 to 154 $\mathrm{mg} / \mathrm{L}$ (cases 1-8) and toxic in 9 cases (cases 9-17), ranged from 9 to $21 \mathrm{mg} / \mathrm{L}$. The first pregabalin-related death was recorded in 2013. From 2013 to 2017, one to three pregabalin related deaths were reported each year, whereas the year 2018 counted 8 deaths (which represents $1.4 \%$ of all deaths due to drugs in 2018). Among the 17 cases, 9 have been reported in a context of substance use disorders and exclusively concerned men with a median age of 34 years old while the remaining 8 cases without substance use disorder context concerned mainly women $(6 / 8$ cases) with a median age of 50 years old. In the same period, only 4 cases of gabapentin-related deaths were reported over the 2010-2018 period. The data of the year 2019 were not completely available at the time of this study (because delay for forensic context), but 8 cases involving pregabalin and 2 cases involving gabapentin had already been reported in that year (Figure 3).

\section{DISCUSSION}

This paper aims to describe gabapentinoid use related disorders and their health consequences in France using multi-sourced information and pharmacological expertise. From 2010 to 2019, the general French population has been increasingly exposed to gabapentinoids and particularly pregabalin, which has led to an expanding risk level of adverse events including substance use disorders. The dynamics of gabapentinoid, particularly pregabalin, abuse phenomenon is recent, intensified from 2018 and still grown in 2019. Indeed, over the 2010-2019 period, $70.9 \%$ of abuse cases reported to the FAN occurred in 2018 and 2019. Health indicators were reflecting this growth: hospital based care for serious neurologic, psychiatric or cardiac complications, demands for addiction care and deaths. Over a year (between 2018 and 2019), the proportion of subjects demanding for addiction care increased from 10.6 to $23.1 \%$. French practitioners are currently facing the management of gabapentinoid withdrawals and have initiated strategies (hospitalization, tapering off, introducing medication), despite having proper guidelines (26). Based on the rise of pregabalin involved in overdose deaths worldwide (27-33), the FAN worked jointly with the French Society of Analytical Toxicology (Société Française de Toxicologie Analytique) to include gabapentinoids in toxicological investigations in clinical situations involving new psychoactive substances and deaths encountered in the practice of forensic toxicology (34). Such awareness could have explained the increase of reported gabapentinoid-related deaths. It certainly helped to better assess gabapentinoid use disorders related harms (35). Experimental studies have shown that the combination of pregabalin with opioids has an additive effect or reverse tolerance to depress respiration and therefore increases the risk of acute overdose death (36); this was also observed in observational studies with gabapentin and pregabalin in patients exposed to opioids (for maintenance therapy) or for pain (37-40).

Gabapentinoids (gabapentin and pregabalin) exhibit calcium channel antagonism and attenuate calcium influx, which can explain unwanted electrophysiological effects. By this way, they present a similar spectrum of adverse drug reactions, which are dose-dependent. Some studies highlighted the implication of gabapentin and pregabalin in cardiac conduction disorders $(15,41)$. These deleterious cardiac outcomes were observed in case of misuse and abuse of high doses of pregabalin in our study even if rarely reported in the literature (42). We also cannot exclude the implication of gabapentinoids in overdose death not only through exacerbating respiratory depression but also through dysrhythmic disorders.

Along with the population approach, published data and those collected in this study have demonstrated that pregabalin presents a true abuse potential by its own. Clinical and experimental studies have shown the "drug-liking" and reinforcing effects of pregabalin $(37,43)$, not correlated with the mesolimbic dopaminergic system but potentially mediated through a possible glutamatergic mechanism (44-46). This rewarding effect is supported by data collected by the FAN through spontaneous reports with $10.9 \%$ of problematic users searching for euphoria, $8.9 \%$ to get high, $5.4 \%$ searching for psychostimulation and $1.2 \%$ feeling a craving for pregabalin or routinely use it. Of note, two subjects used pregabalin in the context of drug experimentation. These elements are in favor of an intrinsic attractiveness of pregabalin. Concerning gabapentin, experimental studies have shown that gabapentin induced drug-seeking behavior but only with the highest dose (47). This has also been demonstrated with mirogabalin (48). Published literature has shown that the risk of gabapentinoid abuse increased in subjects with a history of substance use disorder, particularly in those with opioid use disorder $(8,9,49-$ 54). The present data suggest that abuse could be observed in subjects without any opioid abuse history; within the 265 spontaneous reports of gabapentinoid abuse collected, 38.7\% of subjects with a substance abuse disorder had no opioid use disorder. In addition, a case of pregabalin abuse concerned a patient without any substance use disorder, which constitutes an early signal given the well-known under-reporting phenomenon $(14,55)$. Other elements are in favor of the possible occurrence of pregabalin de novo dependence; in the 2019 OPPIDUM survey, for the first time, two subjects cited pregabalin as the first psychoactive substance that led to dependence (implying that the pregabalin use disorder was at the cause of the demand for addiction care), which is also an emerging signal. Some international studies in the general population have shown that from 8 to $12 \%$ of subjects initiating prescribed pregabalin 
TABLE 3 | Pregabalin-attributed deaths: data from DRAMES and DTA surveys.

\begin{tabular}{|c|c|c|c|c|c|c|c|c|c|}
\hline Case & $\begin{array}{l}\text { Gender, } \\
\text { Age (y) }\end{array}$ & & Drug responsik & for death an & od concentra & & $\begin{array}{l}\text { Other drugs } \\
\text { detected }\end{array}$ & Autopsy data & $\begin{array}{l}\text { Context of death, individual } \\
\text { health history }\end{array}$ \\
\hline 1 & $M, 43$ & $\begin{array}{l}\text { Pregabalin } \\
154 \mathrm{mg} / \mathrm{L}\end{array}$ & $\begin{array}{l}\text { Cocaine } \\
1,750 \mu \mathrm{g} / \mathrm{L}\end{array}$ & & & & $\begin{array}{l}\text { Cyamemazine, } \\
\text { venlafaxine, } \\
\text { cannabis }\end{array}$ & $\begin{array}{l}\text { Toxic death. Drug } \\
\text { use by intravenous } \\
\text { administration. }\end{array}$ & $\begin{array}{l}\text { Context of abuse/dependence. } \\
\text { Found at his home, injection } \\
\text { drug use equipment next to him. } \\
\text { Prescribed pregabalin. }\end{array}$ \\
\hline 2 & M, 35 & $\begin{array}{l}\text { Pregabalin } 76 \\
\mathrm{mg} / \mathrm{L}\end{array}$ & $\begin{array}{l}\text { Cocaine } 128 \\
\mu \mathrm{g} / \mathrm{L}\end{array}$ & & & & $\begin{array}{l}\text { Diazepam, } \\
\text { nordiazepam }\end{array}$ & $\begin{array}{l}\text { Acute heart } \\
\text { rhythm disorder or } \\
\text { ischemia. Possible } \\
\text { complication of } \\
\text { body packing. }\end{array}$ & $\begin{array}{l}\text { Context of abuse/dependence. } \\
\text { Psychiatric history, found at his } \\
\text { home in a state of putrefaction. } \\
\text { Notion of alcohol abuse. } \\
\text { Prescribed pregabalin. }\end{array}$ \\
\hline 3 & $\mathrm{M}, 40$ & $\begin{array}{l}\text { Pregabalin } \\
59.5 \mathrm{mg} / \mathrm{L}\end{array}$ & $\begin{array}{l}\text { Tramadol } \\
12,800 \mu \mathrm{g} / \mathrm{L}\end{array}$ & & & & & $\begin{array}{l}\text { Toxic death } \\
\text { secondary to } \\
\text { pulmonary edema. }\end{array}$ & $\begin{array}{l}\text { History of chronic back pain. } \\
\text { Died at his home. Prescribed } \\
\text { pregabalin. }\end{array}$ \\
\hline 4 & M, 32 & $\begin{array}{l}\text { Pregabalin } 46 \\
\mathrm{mg} / \mathrm{L}\end{array}$ & $\begin{array}{l}\text { Methadone } \\
723 \mu \mathrm{g} / \mathrm{L}\end{array}$ & $\begin{array}{l}\text { Olanzapine } \\
650 \mu \mathrm{g} / \mathrm{L}\end{array}$ & & & $\begin{array}{l}\text { Diazepam, } \\
\text { nordiazepam, } \\
\text { mianserine, } \\
\text { zopiclone, ethanol }\end{array}$ & $\begin{array}{l}\text { Asphyxia probably } \\
\text { toxic. }\end{array}$ & $\begin{array}{l}\text { Context of abuse/dependence. } \\
\text { History of methadone abuse } \\
\text { (intranasal use and doctor } \\
\text { shopping behavior), ongoing } \\
\text { drug withdrawal, found in his } \\
\text { vehicle, alcohol, methadone } \\
\text { packages and intranasal } \\
\text { equipment next to him. }\end{array}$ \\
\hline 5 & $\mathrm{M}, 44$ & $\begin{array}{l}\text { Pregabalin } \\
45.3 \mathrm{mg} / \mathrm{L}\end{array}$ & $\begin{array}{l}\text { Buprenorphine } \\
0.37 \mu \mathrm{g} / \mathrm{L}\end{array}$ & $\begin{array}{l}\text { Oxazepam } \\
2,860 \mu \mathrm{g} / \mathrm{L}\end{array}$ & & & $\begin{array}{l}\text { Diazepam, } \\
\text { nordiazepam, } \\
\text { levetiracetam, } \\
\text { zopiclone }\end{array}$ & $\begin{array}{l}\text { Toxic death } \\
\text { secondary to } \\
\text { pulmonary edema. }\end{array}$ & $\begin{array}{l}\text { Context of abuse/dependence. } \\
\text { On buprenorphine maintenance } \\
\text { therapy. Increased drug use in } \\
\text { the context of traumatic pain } 6 \\
\text { months ago with doctor } \\
\text { shopping behavior. }\end{array}$ \\
\hline 6 & $F, 40$ & $\begin{array}{l}\text { Pregabalin } \\
40.6 \mathrm{mg} / \mathrm{L}\end{array}$ & $\begin{array}{l}\text { Tramadol } \\
6,880 \mu \mathrm{g} / \mathrm{L}\end{array}$ & $\begin{array}{l}\text { Amitriptyline } \\
3,400 \mu \mathrm{g} / \mathrm{L}\end{array}$ & & & $\begin{array}{l}\text { Oxazepam, } \\
\text { venlafaxine, } \\
\text { lamotrigine }\end{array}$ & $\begin{array}{l}\text { Pink foam on lips } \\
\text { and nose. }\end{array}$ & $\begin{array}{l}\text { History of bipolar disorder and } \\
\text { alcohol addiction. Context of } \\
\text { suicide with drug medications } \\
\text { next to her and a suicide note. }\end{array}$ \\
\hline 7 & $M, 45$ & $\begin{array}{l}\text { Pregabalin } \\
29.8 \mathrm{mg} / \mathrm{L}\end{array}$ & $\begin{array}{l}\text { Buprenorphine } \\
4.39 \mu \mathrm{g} / \mathrm{L}\end{array}$ & $\begin{array}{l}\text { Olanzapine } \\
460 \mu \mathrm{g} / \mathrm{L}\end{array}$ & & & $\begin{array}{l}\text { Clonazepam, } \\
\text { cannabis }\end{array}$ & $\begin{array}{l}\text { Toxic death } \\
\text { secondary to } \\
\text { nervous system } \\
\text { and respiratory } \\
\text { depression. } \\
\text { Cirrhotic subject. }\end{array}$ & $\begin{array}{l}\text { Context of abuse/dependence. } \\
\text { Psychiatric, epileptic and drug } \\
\text { addiction history, died at his } \\
\text { home. Medication drugs next to } \\
\text { him. }\end{array}$ \\
\hline 8 & M, 29 & $\begin{array}{l}\text { Pregabalin } 26 \\
\text { mg/L }\end{array}$ & $\begin{array}{l}\text { Methadone } \\
43 \mu \mathrm{g} / \mathrm{L}\end{array}$ & & & & $\begin{array}{l}\text { Phenobarbital, } \\
\text { fluoxetine, ethanol }\end{array}$ & Not known & $\begin{array}{l}\text { Context of abuse/dependence. } \\
\text { Found in a state of putrefaction } \\
\text { with drug medications next to } \\
\text { him. }\end{array}$ \\
\hline 9 & $F, 38$ & $\begin{array}{l}\text { Pregabalin } 21 \\
\mathrm{mg} / \mathrm{L}\end{array}$ & $\begin{array}{l}\text { Lorazepam } \\
440 \mu \mathrm{g} / \mathrm{L}\end{array}$ & $\begin{array}{l}\text { Quetiapine } \\
4,420 \mu \mathrm{g} / \mathrm{L}\end{array}$ & $\begin{array}{l}\text { Tramadol } \\
4,650 \mu \mathrm{g} / \mathrm{L}\end{array}$ & $\begin{array}{l}\text { Venlafaxine } 3,360 \\
\mu \mathrm{g} / \mathrm{L}\end{array}$ & $\begin{array}{l}\text { Dosulepine, } \\
\text { duloxetine }\end{array}$ & $\begin{array}{l}\text { Toxic death } \\
\text { secondary to } \\
\text { cardio-respiratory } \\
\text { decompensation. }\end{array}$ & $\begin{array}{l}\text { Context of suicide. Pregabalin } \\
\text { used by oral route. }\end{array}$ \\
\hline
\end{tabular}


TABLE 3 | Continued

\begin{tabular}{|c|c|c|c|c|c|c|c|c|}
\hline Case & Gender, & & Drug responsible & for death and & od concentrations & Other drugs & Autopsy data & Context of death, individual \\
\hline 10 & $F, 76$ & $\begin{array}{l}\text { Pregabalin } \\
19.4 \mathrm{mg} / \mathrm{L}\end{array}$ & $\begin{array}{l}\text { Tramadol } \\
1,300 \mu \mathrm{g} / \mathrm{L}\end{array}$ & $\begin{array}{l}\text { Flecainide } \\
2,100 \mu \mathrm{g} / \mathrm{L}\end{array}$ & & Ethanol & $\begin{array}{l}\text { Possible toxic } \\
\text { death. }\end{array}$ & History of cardiac issues. \\
\hline 11 & $F, 47$ & $\begin{array}{l}\text { Pregabalin } 17 \\
\mathrm{mg} / \mathrm{L}\end{array}$ & $\begin{array}{l}\text { Hydroxyzine } \\
660 \mu \mathrm{g} / \mathrm{L}\end{array}$ & $\begin{array}{l}\text { Codeine } \\
1,538 \mu \mathrm{g} / \mathrm{L}\end{array}$ & $\begin{array}{l}\text { Tramadol } \\
1,300 \mu \mathrm{g} / \mathrm{L}\end{array}$ & $\begin{array}{l}\text { Paracetamol, } \\
\text { cyamemazine, } \\
\text { zopiclone, } \\
\text { oxazepam }\end{array}$ & $\begin{array}{l}\text { Acute pulmonary } \\
\text { edema. }\end{array}$ & $\begin{array}{l}\text { Died at her home. Prescribed } \\
\text { pregabalin. Pregabalin used by } \\
\text { oral route. }\end{array}$ \\
\hline 12 & $F, 63$ & $\begin{array}{l}\text { Pregabalin } 17 \\
\mathrm{mg} / \mathrm{L}\end{array}$ & & & & $\begin{array}{l}\text { Tramadol, } \\
\text { cyamemazine }\end{array}$ & $\begin{array}{l}\text { Possible toxic } \\
\text { death secondary } \\
\text { to bronchial } \\
\text { inhalation due to } \\
\text { coma. }\end{array}$ & $\begin{array}{l}\text { History of depressive syndrome. } \\
\text { Prescribed pregabalin. }\end{array}$ \\
\hline 13 & $\mathrm{M}, 33$ & $\begin{array}{l}\text { Pregabalin } 17 \\
\mathrm{mg} / \mathrm{L}\end{array}$ & $\begin{array}{l}\text { Methadone } \\
333 \mu \mathrm{g} / \mathrm{L}\end{array}$ & & & $\begin{array}{l}\text { Buprenorphine, } \\
\text { cyamemazine, } \\
\text { diazepam, } \\
\text { mianserine, } \\
\text { paroxetine, } \\
\text { cannabis }\end{array}$ & No & $\begin{array}{l}\text { Context of abuse/dependence. } \\
\text { On buprenorphine, died } \\
\text { in detention. }\end{array}$ \\
\hline 14 & $M, 25$ & $\begin{array}{l}\text { Pregabalin } \\
12.6 \mathrm{mg} / \mathrm{L}\end{array}$ & $\begin{array}{l}\text { Methadone } \\
46.7 \mu \mathrm{g} / \mathrm{L}\end{array}$ & & & $\begin{array}{l}\text { Diazepam, } \\
\text { nordiazepam, } \\
\text { temazepam, THC }\end{array}$ & Not known & $\begin{array}{l}\text { Context of abuse/dependence. } \\
\text { History of cannabis and alcohol } \\
\text { abuse. No information available } \\
\text { on methadone use. }\end{array}$ \\
\hline 15 & $F, 53$ & $\begin{array}{l}\text { Pregabalin } \\
11.7 \mathrm{mg} / \mathrm{L}\end{array}$ & $\begin{array}{l}\text { Amitriptyline } \\
16.6 \mu \mathrm{g} / \mathrm{L}\end{array}$ & $\begin{array}{l}\text { Bromazepam } \\
1,140 \mu \mathrm{g} / \mathrm{L}\end{array}$ & $\begin{array}{l}\text { Venlafaxine } \\
840 \mu \mathrm{g} / \mathrm{L}\end{array}$ & Nordiazepam & $\begin{array}{l}\text { Possible toxic } \\
\text { death or natural } \\
\text { cardiac death }\end{array}$ & $\begin{array}{l}\text { History of depression, found at } \\
\text { his home, alcohol and } \\
\text { medication drugs next to her. No } \\
\text { notion of pregabalin treatment. }\end{array}$ \\
\hline 16 & M, 29 & $\begin{array}{l}\text { Pregabalin } 11 \\
\mathrm{mg} / \mathrm{L}\end{array}$ & $\begin{array}{l}\text { Benzoylecgonine } \\
930 \mu \mathrm{g} / \mathrm{L}\end{array}$ & & & & $\begin{array}{l}\text { Mechanical } \\
\text { asphyxiation by } \\
\text { false food route } \\
\text { due to toxic } \\
\text { overdose. }\end{array}$ & $\begin{array}{l}\text { Context of abuse/dependence. } \\
\text { History of depressive syndrome } \\
\text { and cocaine use, died at his } \\
\text { home. Notion of alcohol use the } \\
\text { day before. }\end{array}$ \\
\hline 17 & $F, 53$ & $\begin{array}{l}\text { Pregabalin } 9 \\
\mathrm{mg} / \mathrm{L}\end{array}$ & $\begin{array}{l}\text { Amitriptyline } \\
290 \mu \mathrm{g} / \mathrm{L}\end{array}$ & $\begin{array}{l}\text { Morphine } 125 \\
\mu \mathrm{g} / \mathrm{L}\end{array}$ & $\begin{array}{l}\text { Oxazepam } \\
6,170 \mu \mathrm{g} / \mathrm{L}\end{array}$ & Zolpidem & $\begin{array}{l}\text { Organ damages } \\
\text { due to multiple } \\
\text { pathologies. }\end{array}$ & Prescribed pregabalin. \\
\hline
\end{tabular}

M, Male; F, Female; $Y$, years. 
TABLE 4 | International regulatory status, amendments or monitoring of dispensing for pregabalin and gabapentin.

\begin{tabular}{|c|c|c|c|}
\hline Year & Month & Country & Measure \\
\hline 2005 & July & $\begin{array}{l}\text { United States of } \\
\text { America (USA) }\end{array}$ & Pregabalin: Drug Schedule V Controlled Substances (Federal law). \\
\hline & October & Russia & Pregabalin: Listed as controlled medicine \\
\hline & December & United Arab Emirates & $\begin{array}{l}\text { Pregabalin and Gabapentin: List of Controlled Medicines and Medications, Narcotic and Controlled Prescriptions. } \\
\text { Limited prescription to } 3 \text { days for general practioners, } 2 \text { weeks for specialists, } 4 \text { weeks in hospital. Prescription } \\
\text { validity: once (no possible renewal). Register for prescribers and pharmacies and specific prescription support } \\
\text { provided by the Ministry of Health. }\end{array}$ \\
\hline \multirow[t]{3}{*}{2016} & August & Minnesota (USA) & Gabapentin: Mandated reporting to a PDMP \\
\hline & December & Argentina & Pregabalin: Listed as Other Substance for Special Control \\
\hline & & Ohio (USA) & Gabapentin: Mandated reporting to a PDMP \\
\hline \multirow[t]{6}{*}{2017} & February & Virginia (USA) & Gabapentin: Mandated reporting to a PDMP \\
\hline & May & Wyoming (USA) & Gabapentin: Mandated reporting to a PDMP \\
\hline & August & Massachusetts (USA) & Gabapentin: Mandated reporting to a PDMP \\
\hline & & North Dakota (USA) & Gabapentin: Mandated reporting to a PDMP \\
\hline & November & Turkey & $\begin{array}{l}\text { Pregabalin: Prescription validity for } 1 \text { year. Specialized opinion (neurologist or psychiatrist) for chronic prescription. } \\
\text { Electronic prescription since January } 2018 .\end{array}$ \\
\hline & November & Jordan & $\begin{array}{l}\text { Pregabalin: Listed as controlled substance, second table (Drugs, Psychotropic substances and Precursor } \\
\text { chemicals appended to the Narcotic Drugs and Psychotropic Substances Law no. } 23 \text { of 2016). Limited packaging } \\
\text { to } 64 \text { tablets. Precribing and dispensing register. }\end{array}$ \\
\hline \multirow[t]{4}{*}{2018} & January & Nebraska & Gabapentin: Mandated reporting to a PDMP \\
\hline & April & Norway & Pregabalin: Schedule B (alongside benzodiazepine) \\
\hline & May & New Jersey (USA) & Gabapentin: Mandated reporting to a PDMP \\
\hline & June & West Virginia (USA) & Gabapentin: Drug Schedule V Controlled Substances (State law) \\
\hline
\end{tabular}

PDMP, Prescription Drug Monitoring Program.

presented a misuse (56-58). In the French cohort study, a possible evolution toward a primary addiction was found for $11 \%$ of the gabapentinoid misusers without previous any history of drug use disorder before drug initiation, whereas it was 1.6 times lower for duloxetine misusers (56).

At this stage in France, the risk of abuse of pregabalin is indisputable, and its harmful consequences are becoming problematic on a population scale. The potential of gabapentin abuse exists and has been observed elsewhere, in the USA and the $\operatorname{UK}(8,11,12,38,39,59)$. It is still not very apparent in France (13); this discrepancy could be due to the level of use, which is about four times lower for gabapentin than pregabalin. Moreover, geographical variations must be interpreted with caution and could be partly explained by the health professionals' awareness regarding the abuse potential of these drugs (37-39, 59-61).
This paper shows the importance of specific post-marketing monitoring on substance use related disorders (that is addictovigilance). The isolated analysis of pregabalin exposure data could not have revealed the suspected misuse behaviors to obtain this drug highlighted by OSIAP survey. Along with spontaneous reports, these data support the growing ease of access to pregabalin through street market with falsified or valid prescription forms. Moreover, at the time of pregabalin marketing approval, pre-clinical and clinical studies on abuse potential were limited, and states decisions were different in the USA and Europe. Based on a clinical abuse liability study showing that pregabalin $(450 \mathrm{mg})$ could be as attractive as diazepam (30 mg) leading subjective effects of "drug-liking" and higher reported euphoria as an adverse reaction in clinical trials compared to placebo ( 4 vs. $1 \%$ of patients), the FDA scheduled pregabalin as a controlled substance (Schedule V) indicating that 
it had abuse potential, while the EMA did not at once, even in 2006 when extending market approval to generalized anxiety disorder was submitted and concluded to a low abuse potential in analogy with gabapentin $(62,63)$. Since, the phenomenon of abuse of gabapentinoids has spread to an international level (Europe, Australia, USA). Since 2015, a dozen countries around the world have regulated the prescription and dispensing procedures for pregabalin, and several have extended these restrictions to gabapentin (Table 4) (64). In France, proposals for regulatory measures have been made and are currently being considered by the French Medicines Agency. Health damages due to gabapentinoid abuse are to balance with their clinical efficacy. Precisely, after a growing enthusiasm for the multiform therapeutic virtues claimed by various promoters of this drug, a growing number of publications highlight the insufficient or unproven effectiveness of pregabalin in neuropathic pain and fibromyalgia (65), as well as in the management of substance use disorders $(66,67)$ or long-term beneficial impact in posttraumatic stress disorder (68). Finally, recent observations from population-based studies, and animal models, have demonstrated that association of gabapentinoids and opioids (analgesics, maintenance drugs, or illicit opioids) significantly increase the risk of opioid death, with the reversibility of tolerance for opioid respiratory depression $(36-38,40)$.

In clinical practice, based on available guides $(69,70)$, results of this study and published data, some recommendations may be proposed at different steps. Before prescribing gabapentinoids, the medical questioning should search for possible psychiatric or substance abuse (including alcohol and tobacco) history. To consider other drugs taken, whenever prescribed or not, should avoid potentially dangerous drug-drug interactions such as gabapentinoid-opioid interaction on respiratory distress. Or, at least get to know the patients/users of respiratory distress symptoms and the first emergency actions. During a gabapentinoid medication, the minimal effective dose should be taken and the benefits/risks balance evaluated at each prescription and refill. Considering substance disorders-related risks, the following signs should be monitored and raised prescriber's attention: tolerance (that is, the reduction of effects as exposure continues at constant dose, or the corollary of this, the need to increase doses to achieve the desired effects), searching for psychoactive effects other than those of the initial indication, drug-seeking behavior (71) or the occurrence of withdrawal symptoms during discontinuation/between gabapentinoid intakes. If possible, due to withdrawal syndrome, gabapentinoid discontinuation may be planned and used schedules. A hospitalization could be proposed if experiencing withdrawal difficulties or existing substance use disorder or psychiatric co-morbidities. The absence of a substance use transfer at the time of discontinuation should be monitored (68). To improve the knowledge on the evaluation of drugs in real life, at any time of management, to report any adverse event, including those related to substance use disorders, to the territorial vigilance systems.

The strength of this study is to cross results of different data sources collected over a recent 10 years' period, for both pregabalin and gabapentin drugs. However, there are limitations related to the four addictovigilance data sources used. The level of reported information in SRs could be different from one case to another, on individuals or clinical features, depending on the person filling the reporting form and available/patientprovided information at time of reporting. Moreover, it could exist an awareness bias with pregabalin compared to gabapentin, with first specific sensitizations of French health professionals on pregabalin misuse since 2016 (72). The pharmacoepidemiological studies OSIAP, OPPIDUM, and DRAMES/DTA could presented bias related to participation and reported information. It has to be note that the results of DRAMES/DTA surveys should not be considered as an exhaustive description of drug-related deaths in France. They are based on voluntary participation of expert toxicologists, requested toxicological analysis carried out by judicial authorities and the spectrum of substances analyzed (73). Besides these limits, DRAMES/DTA surveys are currently references for the assessment of drug-related deaths in France.

\section{DATA AVAILABILITY STATEMENT}

The data analyzed in this study is subject to the following licenses/restrictions: According to the French laws (Articles R.5132-113 and R.5132-114), each case was recorded in the French Addictovigilance database, in an anonymous way. All authors had full access to all the data in the study and took responsibility for the integrity of the data and the accuracy of the data analysis. Requests to access these datasets should be directed to Camille Ponté, ponte.c@chu-toulouse.fr.

\section{AUTHOR CONTRIBUTIONS}

MT and CP analyzed and interpreted data. EJ, NF, and JM, respectively, managed the national OSIAP, DRAMES/DTA, and OPPIDUM database and extracted the data. MLM was responsible for the study conception and design. MT, CP, EJ, NF, $\mathrm{JM}$, and MLM took part in drafting the manuscript and critical revision, and all authors approved the final version.

\section{ACKNOWLEDGMENTS}

The authors wish to thank Olivia Ponté for her valuable assistance in writing English language.

\section{LIST OF THE FAN INVESTIGATORS}

Amélie Daveluy (Addictovigilance Center, Bordeaux), Reynald Le Boisselier (Addictovigilance Center, Caen), Christine Fournier-Choma (Addictovigilance Center, Clermont-Ferrand), Bruno Revol (Addictovigilance Center, Grenoble), Sylvie Deheul (Addictovigilance Center, Lille), Cécile Chevallier (Addictovigilance Center, Lyon), Liselotte Pochard (Addictovigilance Center, Marseille), Hélène Peyrière (Addictovigilance Center, Montpellier), Valérie Gibaja (Addictovigilance Center, Nancy), Marylène Guerlais (Addictovigilance Center, Nantes), Julie Heredia (Addictovigilance Center, Paris), Marie-Christine Perault Pochat (Addictovigilance Center, Poitiers), Florence Fabre (Addictovigilance Center, Toulouse) 


\section{REFERENCES}

1. Calandre EP, Rico-Villademoros F, Slim M. Alpha2delta ligands, gabapentin, pregabalin and mirogabalin: a review of their clinical pharmacology and therapeutic use. Expert Rev Neurother. (2016) 16:1263-77. doi: 10.1080/14737175.2016.1202764

2. Bramness JG, Sandvik P, Engeland A, Skurtveit S. Does pregabalin (Lyrica ${ }^{\circledR}$ ) help patients reduce their use of benzodiazepines? A comparison with gabapentin using the Norwegian Prescription Database. Basic Clin Pharmacol Toxicol. (2010) 107:883-6. doi: 10.1111/j.1742-7843.2010.00590.x

3. Oulis P, Konstantakopoulos G. Pregabalin in the treatment of alcohol and benzodiazepines dependence. CNS Neurosci Ther. (2010) 16:4550. doi: 10.1111/j.1755-5949.2009.00120.x

4. European Monitoring Centre for Drugs and Drug Addiction. Annual Report 2010: the State of the Drugs Problem in Europe. Lisbon: Publications Office of the European Union (2010).

5. Schwan S, Sundström A, Stjernberg E, Hallberg E, Hallberg P. A signal for an abuse liability for pregabalin-results from the Swedish spontaneous adverse drug reaction reporting system. Eur J Clin Pharmacol. (2010) 66:94753. doi: 10.1007/s00228-010-0853-y

6. Carrus D, Schifano F. Pregabalin misuse-related issues; intake of large dosages, drug-smoking allegations, and possible association with myositis: two case reports. J Clin Psychopharmacol. (2012) 32:839-40. doi: 10.1097/JCP.0b013e318272864d

7. Gahr M, Freudenmann RW, Hiemke C, Kölle MA, Schönfeldt-Lecuona C. Pregabalin abuse and dependence in Germany: results from a database query. Eur J Clin Pharmacol. (2013) 69:1335-42. doi: 10.1007/s00228-012-1464-6

8. McAnally H, Bonnet $\mathrm{U}$, Kaye AD. Gabapentinoid benefit and risk stratification: mechanisms over myth. Pain Ther. (2020) 9:441-52. doi: 10.1007/s40122-020-00189-x

9. Bonnet U, Scherbaum N. How addictive are gabapentin and pregabalin? A systematic review. Eur Neuropsychopharmacol. (2017) 27:1185-215. doi: 10.1016/j.euroneuro.2017.08.430

10. Chiappini S, Schifano F. A decade of gabapentinoid misuse: an analysis of the European medicines agency's 'suspected adverse drug reactions' database. CNS Drugs. (2016) 30:647-54. doi: 10.1007/s40263-016-0359-y

11. Hägg $S$, Jönsson $A K$, Ahlner J. Current evidence on abuse and misuse of gabapentinoids. Drug Saf. (2020) 43:123554 doi: 10.1007/s40264-020-00985-6

12. Schifano F, D’Offizi S, Piccione M, Corazza O, Deluca P, Davey Z, et al. Is there a recreational misuse potential for pregabalin analysis of anecdotal online reports in comparison with related gabapentin and clonazepam data. Psychother Psychosom. (2011) 80:118-22. doi: 10.1159/000321079

13. Victorri-Vigneau C, Guerlais M, Jolliet P. Abuse, dependency and withdrawal with gabapentin: a first case report. Pharmacopsychiatry. (2007) 40:434. doi: 10.1055/s-2006-958522

14. Bossard JB, Ponté C, Dupouy J, Lapeyre-Mestre M, Jouanjus E. Disproportionality analysis for the assessment of abuse and dependence potential of pregabalin in the French pharmacovigilance database. Clin Drug Investig. (2016) 36:735-42. doi: 10.1007/s40261-016-0421-z

15. Fuzier R, Serres I, Guitton E, Lapeyre-Mestre M, Montastruc JL, The French Network of Pharmacovigilance Centres. Adverse drug reactions to gabapentin and pregabalin: a review of the French pharmacovigilance database. Drug Saf. (2013) 36:55-62. doi: 10.1007/s40264-012-0006-6

16. Lapeyre-Mestre M, Dupui M. Drug abuse monitoring: which pharmacoepidemiological resources at the European level? Therapie. (2015) 70:147-65. doi: 10.2515/therapie/2015010

17. Jouanjus E, Micallef J, Mallaret M, Lapeyre-Mestre M. Comment on: an insight into Z-drug abuse and dependence: an examination of reports to the European medicines agency database of suspected adverse drug reactions. Int J Neuropsychopharmacol. (2019) 22:528-30. doi: 10.1093/ijnp/pyz033

18. Jouanjus E, Gibaja V, Kahn JP, Haramburu F, Daveluy A. Signal identification in addictovigilance: the functioning of the French system. Thérapie. (2015) 70:123-31. doi: 10.2515/therapie/2015011

19. Boeuf O, Lapeyre-Mestre M. Survey of forged prescriptions to investigate risk of psychoactive medications abuse in France. Drug Saf. (2007) 30:26576. doi: 10.2165/00002018-200730030-00007
20. Jouanjus E, Guernec G, Lapeyre-Mestre M, French Addictovigilance Network. Medical prescriptions falsified by the patients: a twelve-year national monitoring to assess prescription drug diversion. Fundam Clin Pharmacol. (2018) 32:306-322. doi: 10.1111/fcp.12356

21. Frauger E, Moracchini C, Le Boisselier R, Braunstein D, Thirion X, Micallef J, et al. OPPIDUM surveillance program: 20 years of information on drug abuse in France. Fundam Clin Pharmacol. (2013) 27:672-82. doi: 10.1111/fcp.12024

22. Hiemke C, Bergemann N, Clement HW, Conca A, Deckert J, Domschke $\mathrm{K}$, et al. Consensus guidelines for therapeutic drug monitoring in neuropsychopharmacology: update 2017. Pharmacopsychiatry. (2018) 51:962. doi: $10.1055 / \mathrm{s}-0043-116492$

23. Button J, Berry D, Holt DW. Two fatalities involving pregabalin. Toxichem Krimtech. (2010) 77:247-8.

24. Singier A, Noize P, Berdaï D, Daveluy A, Arnaud M, Molimard M, et al. Medicine misuse: a systematic review and proposed hierarchical terminology. Br J Clin Pharmacol. (2020) 1-10. doi: 10.22541/au.159177426.69495466

25. Hasin DS, O’Brien CP, Auriacombe M, Borges G, Bucholz K, Budney A, et al. DSM-5 criteria for substance use disorders: recommendations and rationale. Am J Psychiatry. (2013) 170:834-51. doi: 10.1176/appi.ajp.2013.12060782

26. Roche S, Blaise M. Pregabalin and risk of addiction: a new care issue? L'Encephale. (2020) 46:372-81. doi: 10.1016/j.encep.2020.02.008

27. Häkkinen M, Vuori E, Kalso E, Gergov M, Ojanperä I. Profiles of pregabalin and gabapentin abuse by postmortem toxicology. Forensic Sci Int. (2014) 241:1-6. doi: 10.1016/j.forsciint.2014.04.028

28. Guerrieri D, Rapp E, Roman M, Druid H, Kronstrand R. Postmortem and toxicological findings in a series of furanylfentanyl-related deaths. J Anal Toxicol. (2017) 41:242-9. doi: 10.1093/jat/bkw129

29. Elliott SP, Burke $T$, Smith C. Determining the toxicological significance of pregabalin in fatalities. J Forensic Sci. (2017) 62:169-73. doi: 10.1111/1556-4029.13263

30. Haukka J, Kriikku P, Mariottini C, Partonen T, Ojanperä I. Non-medical use of psychoactive prescription drugs is associated with fatal poisoning. Addict Abingdon Engl. (2018) 113:464-72. doi: 10.1111/add.14014

31. Thompson A, Morey S, Griffiths A. Pregabalin and its involvement in coronial cases. J Anal Toxicol. (2020) 44:29-35. doi: 10.1093/jat/bkz041

32. Lynn E, Cousins G, Lyons S, Bennett KE. A repeated cross-sectional study of factors associated with pregabalin-positive poisoning deaths in Ireland. Drug Alcohol Depend. (2020) 206:107741. doi: 10.1016/j.drugalcdep.2019.107741

33. Nahar LK, Murphy KG, Paterson S. Misuse and mortality related to gabapentin and pregabalin are being under-estimated: a twoyear post-mortem population study. J Anal Toxicol. (2019) 43:564-70. doi: 10.1093/jat/bkz036

34. Société Française de Toxicologie Analytique. SFTA Guidelines for the Achievement of Toxicological Analyzes Involving NPS - 2020 Version. (2020). Available online at: https://sfta.org/articles/view/recommandations-de-lasfta-pour-la-realisation-des-analyses-toxicologiques-impliquant-des-npsversion-2020 (accessed December 9, 2020).

35. Société Française de Toxicologie Analytique. Actes du congrès 2020. Toxicol Anal Clin. (2020) 32:S33-7. doi: 10.1016/S2352-0078(20)30271-7

36. Hill R, Dewey WL, Kelly E, Henderson G. Oxycodone-induced tolerance to respiratory depression: reversal by ethanol, pregabalin and protein kinase $\mathrm{C}$ inhibition. Br J Pharmacol. (2018) 175:2492-503. doi: 10.1111/bph.14219

37. Lyndon A, Audrey S, Wells C, Burnell ES, Ingle S, Hill R, et al. Risk to heroin users of poly-drug use of pregabalin or gabapentin. Addict Abingdon Engl. (2017) 112:1580-9. doi: 10.1111/add.13843

38. Gomes T, Juurlink DN, Antoniou T, Mamdani MM, Paterson JM, van den Brink W. Gabapentin, opioids, and the risk of opioid-related death: a population-based nested case-control study. PLoS Med. (2017) 14:e1002396. doi: 10.1371/journal.pmed.1002396

39. Torrance N, Veluchamy A, Zhou Y, Fletcher EH, Moir E, Hebert HL, et al. Trends in gabapentinoid prescribing, co-prescribing of opioids and benzodiazepines, and associated deaths in Scotland. Br J Anaesth. (2020) 125:159-67. doi: 10.1016/j.bja.2020.05.017

40. Abrahamsson T, Berge J, Öjehagen A, Håkansson A. Benzodiazepine, zdrug and pregabalin prescriptions and mortality among patients in opioid maintenance treatment-A nation-wide register-based open cohort study. Drug Alcohol Depend. (2017) 174:58-64. doi: 10.1016/j.drugalcdep.2017.01.013 
41. de Landaluce O, Carbonell P, Asensio C, Escoda N, López P, Laporte JR. Gabapentin and pregabalin and risk of atrial fibrillation in the elderly: a population-based cohort study in an electronic prescription database. Drug Saf. (2018) 41:1325-31. doi: 10.1007/s40264-018-0695-6

42. Dahan A, Tournoud C, Muller C, Gibaja V, Lapeyre-Mestre M, Bayle E, et al. Toxicomanie à la prégabaline par voie intranasale et troubles de la conduction cardiaque. Toxicol Anal Clin. (2017) 29:S37-8. doi: 10.1016/j.toxac.2017.03.046

43. Vashchinkina E, Piippo O, Vekovischeva O, Krupitsky E, Ilyuk R, Neznanov $\mathrm{N}$, et al. Addiction-related interactions of pregabalin with morphine in mice and humans: reinforcing and inhibiting effects. Addict Biol. (2018) 23:94558. doi: $10.1111 / \mathrm{adb} .12538$

44. Coutens B, Mouledous L, Stella M, Rampon C, Lapeyre-Mestre M, Roussin A, et al. Lack of correlation between the activity of the mesolimbic dopaminergic system and the rewarding properties of pregabalin in mouse. Psychopharmacology. (2019) 236:2069-82. doi: 10.1007/s00213-019-05198-Z

45. Althobaiti YS, Almalki A, Alsaab H, Alsanie W, Gaber A, Alhadidi Q, et al. Pregabalin: potential for addiction and a possible glutamatergic mechanism. Sci Rep. (2019) 9:15136. doi: 10.1038/s41598-019-51556-4

46. Asaoka Y, Kato T, Ide S, Amano T, Minami M. Pregabalin induces conditioned place preference in the rat during the early, but not late, stage of neuropathic pain. Neurosci Lett. (2018) 668:133-7. doi: 10.1016/j.neulet.2018.01.029

47. Althobaiti YS, Alghorabi A, Alshehri FS, Baothman B, Almalki AH, Alsaab $\mathrm{HO}$, et al. Gabapentin-induced drug-seeking-like behavior: a potential role for the dopaminergic system. Sci Rep. (2020) 10:10445 doi: 10.1038/s41598-020-67318-6

48. Mendell J, Levy-Cooperman N, Sellers E, Vince B, Kelsh D, Lee J, et al. Abuse potential of mirogabalin in recreational polydrug users. Ther Adv Drug Saf. (2019) 10:2042098619836032. doi: 10.1177/2042098619836032

49. Evoy KE, Morrison MD, Saklad SR. Abuse and misuse of pregabalin and gabapentin. Drugs. (2017) 77:403-26. doi: 10.1007/s40265-017-0700-x

50. Wilens T, Zulauf C, Ryland D, Carrellas N, Catalina-Wellington I. Prescription medication misuse among opioid dependent patients seeking inpatient detoxification. Am J Addict. (2015) 24:173-7. doi: 10.1111/ajad.12159

51. Grosshans M, Lemenager T, Vollmert C, Kaemmerer N, Schreiner R, Mutschler J, et al. Pregabalin abuse among opiate addicted patients. Eur J Clin Pharmacol. (2013) 69:2021-5. doi: 10.1007/s00228-013-1578-5

52. Wagner E, Raabe F, Martin G, Winter C, Plörer D, Krause DL, et al. Concomitant drug abuse of opioid dependent patients in maintenance treatment detected with a multi-target screening of oral fluid. Am J Addict. (2018) 27:407-12. doi: 10.1111/ajad.12737

53. Baird CRW, Fox P, Colvin LA. Gabapentinoid abuse in order to potentiate the effect of methadone: a survey among substance misusers. Eur Addict Res. (2014) 20:115-8. doi: 10.1159/000355268

54. Lancia M, Gambelunghe A, Gili A, Bacci M, Aroni K, Gambelunghe C. Pregabalin abuse in combination with other drugs: monitoring among methadone patients. Front Psychiatry. (2020) 10:1022. doi: 10.3389/fpsyt.2019.01022

55. Driot D, Chicoulaa B, Jouanjus E, Dupouy J, Oustric S, LapeyreMestre M. Pregabalin use disorder and secondary nicotine dependence in a woman with no substance abuse history. Therapie. (2016) 71:5758. doi: 10.1016/j.therap.2016.04.006

56. Driot D, Jouanjus E, Oustric S, Dupouy J, Lapeyre-Mestre M. Patterns of gabapentin and pregabalin use and misuse: results of a populationbased cohort study in France. Br J Clin Pharmacol. (2019) 85:12609. doi: 10.1111/bcp.13892

57. Schjerning O, Pottegård A, Damkier P, Rosenzweig M, Nielsen J. Use of pregabalin - a nationwide pharmacoepidemiological drug utilization study with focus on abuse potential. Pharmacopsychiatry. (2016) 49:15561. doi: 10.1055/s-0042-101868

58. Bodén R, Wettermark B, Brandt L, Kieler H. Factors associated with pregabalin dispensing at higher than the approved maximum dose. Eur J Clin Pharmacol. (2014) 70:197-204. doi: 10.1007/s00228-013-1594-5

59. Buttram ME, Kurtz SP, Dart RC, Margolin ZR. Law enforcement-derivated data on gabapentin diversion and misuse, 2002-2015: diversion rates and qualitative research findings. Pharmacoepidemiol Drug Saf. (2017) 26:10836. doi: $10.1002 / p d s .4230$
60. Vickers-Smith R, Sun J, Charnigo RJ, Lofwall MR, Walsh SL, Havens JR. Gabapentin drug misuse signals: a pharmacovigilance assessment using the FDA adverse event reporting system. Drug Alcohol Depend. (2020) 206:107709. doi: 10.1016/j.drugalcdep.2019.1 07709

61. Martinez GM, Olabisi J, Ruekert L, Hasan S. A call for caution in prescribing gabapentin to individuals with concurrent polysubstance abuse: a case report. J Psychiatr Pract. (2019) 25:308-12. doi: 10.1097/PRA.00000000000 00403

62. Food and Drug Administration (FDA), Center for Drug Evaluation and Research. Scheduling Recommendation for Pregabalin. (2004). Available online at: https://www.accessdata.fda.gov/drugsatfda_docs/nda/2004/021446_Lyrica \%20Capsules_medr.pdf (accessed December 9, 2020).

63. European Medicines Agency. EPAR - Scientific Discussion - Variation Lyrica-H-C-546-II-0004. (2006). Available online at: https://www.ema. europa.eu/en/documents/scientific-discussion-variation/lyrica-h-c-546-ii0004-epar-scientific-discussion-variation_en.pdf (accessed December 9, 2020).

64. Peckham AM, Ananickal MJ, Sclar DA. Gabapentin use, abuse, and the US opioid epidemic: the case for reclassification as a controlled substance and the need for pharmacovigilance. Risk Manag Healthc Policy. (2018) 11:10916. doi: 10.2147/RMHP.S168504

65. Wiffen PJ, Derry S, Moore RA, Aldington D, Cole P, Rice AS, et al. Antiepileptic drugs for neuropathic pain and fibromyalgia - an overview of cochrane reviews. Cochrane Database Syst Rev. (2013) 11:CD010567. doi: 10.1002/14651858.CD010567.pub2

66. Caniff K, Telega E, Bostwick JR, Gardner KN. Pregabalin as adjunctive therapy in benzodiazepine discontinuation. Am J Health Syst Pharm. (2018) 75:67-71. doi: 10.2146/ajhp160712

67. Baandrup L, Ebdrup BH, Rasmussen JØ, Lindschou J, Gluud C, Glenthøj BY. Pharmacological interventions for benzodiazepine discontinuation in chronic benzodiazepine users. Cochrane Database Syst Rev. (2018) 3:CD011481. doi: 10.1002/14651858.CD011481.pub2

68. Galliot G, Ponté C, Schmitt L, Hakimi Y, Sergent S, Lapeyre-Mestre M, et al. Case report: the comorbidity of pregabalin-use disorder and post- traumatic stress disorder: clinical and pharmacological issues. Int J Ment Health Addict. (2020) 1-10. doi: 10.1007/s11469-020-00347-4

69. Parsons G. Guide to the management of gabapentinoid misuse. Prescriber. (2018) 29:25-30. doi: 10.1002/psb.1664

70. Nicholls G, Samios P, Hampton S. Management of pregabalin and gabapentin prescribing and use in NSW prisons. Med J Aust. (2019) 211:474474.e1. doi: 10.5694/mja2.50398

71. James J. Dealing with drug-seeking behaviour. Aust Prescr. (2016) 39:96100. doi: 10.18773/austprescr.2016.022

72. Agence Nationale de Sécurité du Médicament et des Produits de Santé (ANSM). Risques d'abus, de mésusage et de pharmacodépendance liés à l'utilisation de la prégabaline (Lyrica et génériques) - Point d'Information. (2016). Available online at: https://www.ansm.sante.fr/S-informer/Pointsd-information-Points-d-information/Risques-d-abus-de-mesusage-etde-pharmacodependance-lies-a-l-utilisation-de-la-pregabaline-Lyrica-etgeneriques-Point-d-Information (accessed December 9, 2020).

73. Boulat T. Identification des obstacles à la surveillance et à la connaissance des décès par surdose en France. Paris: Observatoire Français des Drogues et des Toxicomanies (OFDT) (2017).

Conflict of Interest: The authors declare that the research was conducted in the absence of any commercial or financial relationships that could be construed as a potential conflict of interest.

Copyright (c) 2021 Tambon, Ponté, Jouanjus, Fouilhé, Micallef, Lapeyre-Mestre and the French Addictovigilance Network (FAN). This is an open-access article distributed under the terms of the Creative Commons Attribution License (CC BY). The use, distribution or reproduction in other forums is permitted, provided the original author(s) and the copyright owner(s) are credited and that the original publication in this journal is cited, in accordance with accepted academic practice. No use, distribution or reproduction is permitted which does not comply with these terms. 


\title{
Continuous Infusion of Flumazenil in the Management of Benzodiazepines Detoxification
}

\author{
Anna Benini ${ }^{1}$, Rossella Gottardo ${ }^{2}$, Cristiano Chiamulera ${ }^{1}$, Anna Bertoldi ${ }^{3}$, \\ Lorenzo Zamboni $^{3 *}$ and Fabio Lugoboni ${ }^{3}$ \\ ${ }^{1}$ Department of Diagnostics and Public Health, University of Verona, Verona, Italy, ${ }^{2}$ Forensic Toxicology Laboratory, \\ Department of Diagnostics and Public Health, University of Verona, Verona, Italy, ${ }^{3}$ Department of Internal Medicine, Addiction \\ Unit, Verona University Hospital, Verona, Italy
}

\section{OPEN ACCESS}

Edited by:

Giovanni Martinotti,

University of Studies G. d'Annunzio

Chieti and Pescara, Italy

Reviewed by:

Antonio Mirijello,

Casa Sollievo della Sofferenza

(IRCCS), Italy

Francesco Di Carlo,

University of Studies G. d'Annunzio

Chieti and Pescara, Italy

*Correspondence:

Lorenzo Zambon

lorenzo.zamboni88@gmail.com

Specialty section: This article was submitted to

Addictive Disorders,

a section of the journa

Frontiers in Psychiatry

Received: 24 December 2020 Accepted: 15 February 2021

Published: 18 March 2021

Citation:

Benini A, Gottardo R, Chiamulera C, Bertoldi A, Zamboni $L$ and Lugoboni F

(2021) Continuous Infusion of

Flumazenil in the Management of Benzodiazepines Detoxification.

Front. Psychiatry 12:646038. doi: 10.3389/fpsyt.2021.646038
An effective approach in the treatment of benzodiazepine (BZD) overdosing and detoxification is flumazenil (FLU). Studies in chronic users who discontinued BZD in a clinical setting suggested that multiple slow bolus infusions of FLU reduce BZD withdrawal symptoms. The aim of this study was to confirm FLU efficacy for reducing BZD withdrawal syndrome by means of continuous elastomeric infusion, correlated to drugs plasma level and patients' compliance.

Methods: Seven-day FLU $1 \mathrm{mg} /$ day subcutaneously injected through an elastomeric pump and BZDs lormetazepam, clonazepam, and lorazepam were assessed by HPLC-MS/MS in serum of patients before and after 4 and 7 days of FLU continuous infusion treatment. Changes in withdrawal severity were assessed by using the BZD Withdrawal Scale (BWS).

Results: Fourteen patients (mean age \pm SD $42.5 \pm 8.0$ years, 5 male and 9 female), admitted to the hospital for high-dose BZD detoxification, were enrolled in the study. Serum FLU concentrations significantly decreased from $0.54 \pm 0.33 \mathrm{ng} / \mathrm{ml}$ (mean \pm SD) after 4 days of treatment to $0.1 \pm 0.2 \mathrm{ng} / \mathrm{ml}$ at the end of infusion. Lormetazepam concentrations were $502.5 \pm 610.0 \mathrm{ng} / \mathrm{ml}$ at hospital admission, $26.2 \pm 26.8 \mathrm{ng} / \mathrm{ml}$ after 4 days, and 0 at the end of treatment. BWS values decreased during FLU treatment temporal period. FLU was well-tolerated by patients.

Conclusions: Elastomeric FLU infusion for BZD detoxification is a feasible administration device to maintain adequate, constant, and tolerated FLU concentrations for reducing BZD withdrawal symptoms.

\section{Keywords: benzodiazepine, flumazenil, withdrawal, high dose, detoxifcation}

\section{INTRODUCTION}

Although benzodiazepines (BZDs) constitute one of the most broadly prescribed drug classes worldwide, the frequent and often inappropriate use is a problem that remains considerably underestimated by practitioners and most regulatory agencies (1). BZD can produce tolerance and dependence; thus, their use is recommended for a limited time (2). Surveys carried out in the 1990s in France, Germany, Italy, and the United Kingdom showed that 3.9\% of hypnotic drug users and $3.2 \%$ of anxiolytic drug users had been taking a dose exceeding the recommended 
one (2-4). In Italy, about $7.5-10 \%$ of adult population are BDZ users, half of these being long-term users (LTU) with a diagnosis of BZD use disorder (5). Another study conducted in Italy showed that $14.0 \%$ of patients visiting general practitioners were taking BZDs, with $4.7 \%$ of the total sample being LTU, using BZDs daily for at least 12 months (6).

BZD tolerance was first reported in 1961 (7), but this phenomenon has been often obscured by the enthusiastic use of these drugs, which were able to replace barbiturates. The low toxicity coupled to a high potential of tolerance can lead to very high-dose misuse (8). From a clinical point of view, the only proposed solution of a gradual reduction of BZD is too simplistic. For long-term users, in general, if properly applied, gradually reducing the dosage works, but it is much less effective for high-dose users $(2,8,9)$. This is worth mentioning because withdrawing from high doses of BZD carries significant risk for the health of the patient $(2,10)$.

It is in this area of HDUs that the use of flumazenil (FLU), used worldwide to treat the overdose of BZD, has been demonstrated as effective $(9,11-13)$. Experimental findings have shown that FLU acts as a BZD partial agonist with a weak intrinsic activity, when administered by slow intravenous infusion. While withdrawal symptoms may be brought on by the use of FLU, BZD-tolerant patients only reported mild symptoms $(14,15)$.

BZDs positively modulate $\gamma$-aminobutyric acid (GABA) through distinct binding sites on $\mathrm{GABA}_{\mathrm{A}}$ receptors, and there is little variation among BZDs in pharmacodynamical factors such as selectivity and efficacy. Consequently, the choice of a particular BZD for clinical use is primarily based on pharmacokinetic features. Only one drug, flumazenil (FLU), is currently approved to reverse the effects of BZDs. FLU is a BZD partial agonist commonly used in the treatment of BZD overdose. Studies in chronic users who have discontinued BZDs suggested that multiple slow bolus infusions of FLU reduce the symptoms of BZD withdrawal when compared to placebo (9). The mechanism of FLU action remains, however, unclear: its action may facilitate the coupling of $\mathrm{GABA}_{\mathrm{A}}$ and $\mathrm{BZD}$ receptor complexes, presumably by reversing the down-regulation/uncoupling that occurs with long-term BZD use (16). This mechanism is supposed to underlie FLU's weak agonist action and may explain its ability to attenuate BZD withdrawal symptoms (9). FLU does not antagonize the effects of other CNS sedative-hypnotics, such as ethanol, opioids, or general anesthetics (17).

FLU owns a rapid and extensive distribution phase with high volume of distribution and a second phase with fast metabolic elimination and short half-life (18). Its brief BZD-antagonism duration is due to a rapid hepatic elimination, determining its short half-life (60-90 min) and high plasma clearance (31-78 $1 / \mathrm{h}$ ). The low plasma protein binding of FLU (about 50\%) does not limit its wide distribution (apparent distribution volume $0.6-1.6 \mathrm{l} / \mathrm{kg}$ ) or its partly flow-dependent hepatic elimination $(19,20)$. Pharmacokinetic parameters of FLU do not change whether the drug is administered alone or in combination with other BZDs (18). For BZD detoxification, a viable method is the intravenous administration of FLU by using multiple bolus infusions either alone $(14,21)$ or in combination with tapering doses of BZDs (11).
The pharmacodynamical mechanisms of FLU are therefore crucial to determine its clinical effect, which could be achieved thanks to specific FLU infusion parameters in order to guarantee timing and extent of receptor occupancy (14). Thus, the choice of the most appropriate mode of delivery must be based on the correlation between FLU infusion parameters, plasma levels, and clinical endpoint. Our addiction unit has been employing FLU for high-dose BZD detoxifications since 2003, initially by means of endovenous continuous infusion administered by day. Such mode of delivery was both inconstant at maintaining adequate serum levels, being unfeasible for the night, and uncomfortable for the patient. In order to maintain constant serum concentration of FLU and to reduce modality of administration from multiple to single, we aimed to deliver FLU by slow subcutaneous infusion by using an elastomeric infusion pump at constant flow. In this study, we correlated the efficacy of continuous elastomeric FLU infusion on BZD withdrawal clinical endpoint to both drugs' (FLU and BZDs) plasma levels and, of equal importance, to patients' compliance and tolerance to treatment.

\section{MATERIALS AND METHODS}

This study was approved by the Ethical Review Board of the University Hospital (protocol number: 50771; prog. n. 683CESC). Informed consent was obtained from each subject.

\section{Subjects}

Five male and nine female patients (mean age \pm SD $42.5 \pm$ 8.0 years), admitted to the hospital for BZD detoxification, were enrolled in the study (see Table 1 for patients' characteristics). The BZD use was stopped on day 1 of admission. The therapy with antidepressants, if any (Table 1), was maintained and continued after discharge.

All patients reported a history of BZD dependence according to the Diagnostic and Statistical Manual of Mental Disorders, Fifth Edition (DSM-5) criteria (22). Before hospitalization, all patients were interviewed by a physician to assess degree of BZD dependence and general health conditions. All patients had voluntarily contacted the Addiction Unit of Verona University Hospital and were aware of their BZD dependence.

Inclusion criteria were as follows: (i) age older than 18 years; (ii) diagnosis of BZD use disorder according to the DSM-5 criteria; (iii) BZD abuse lasting more than 6 months; and (iv) high dose of BZD abuse, meaning BZD intake exceeding at least five times the recommended daily amount (e.g., $>50 \mathrm{mg}$ in diazepam equivalents). Individuals were excluded if presenting the following: (i) current substance use disorder, defined as a history of illicit drug dependence or abuse within the previous 6 months; (ii) active medical illnesses or psychosis; and (iii) previous history of seizures, but not due to BZD withdrawal.

\section{Elastomeric Pump}

Patients were treated with a solution containing $7 \mathrm{mg}$ of flumazenil (Anexate ${ }^{\circledR}$, Roche), available commercially in 0.5 $\mathrm{mg} / 5 \mathrm{ml}$ vials at $\mathrm{pH}=4$. The elastomeric pump (Infusor LV 1.5, code $2 \mathrm{C} 1087 \mathrm{~K}$, Baxter S.p.A., Rome, Italy) was arranged with a 
TABLE 1 | Patients' characteristics.

\begin{tabular}{|c|c|c|c|c|}
\hline $\begin{array}{l}\text { Patient } \\
\text { number }\end{array}$ & $\begin{array}{l}\text { Gender } \\
\text { (M/F) }\end{array}$ & $\begin{array}{l}\text { Age } \\
\text { (years) }\end{array}$ & $\begin{array}{l}\text { Reported BDZ dosage } \\
\text { at admission }\end{array}$ & $\begin{array}{l}\text { Antide } \\
\text { pressant }\end{array}$ \\
\hline 1 & M & 38 & $\begin{array}{l}\text { LRZ } 25 \text { mg/day } \\
\text { CLO } 2 \text { mg/day }\end{array}$ & Agomelatine \\
\hline 2 & M & 44 & LRM 75 mg/day & None \\
\hline 3 & $\mathrm{~F}$ & 42 & LRM 75 mg/day & Duloxetine \\
\hline 4 & $\mathrm{~F}$ & 55 & ALP 35 mg/day & Escitalopram \\
\hline 5 & $\mathrm{~F}$ & 47 & CLO 12 mg/day & Mirtazapine \\
\hline 6 & $\mathrm{~F}$ & 52 & $\begin{array}{l}\text { LRM } 25 \text { mg/day } \\
\text { LRM } 12 \text { mg/day } \\
\text { DZP } 100 \text { mg/day } \\
\text { DZP } 30 \text { mg/day } \\
\text { FLZ } 180 \text { mg/day } \\
\text { TRZ } 1.5 \text { mg/day } \\
\text { LRZ } 15 \mathrm{mg} / \text { day } \\
\text { ALP } 4 \text { mg/day } \\
\text { CLO } 12 \text { mg/day }\end{array}$ & $\begin{array}{l}\text { Venlafaxine } \\
\text { plus } \\
\text { agomelatine }\end{array}$ \\
\hline 7 & $\mathrm{~F}$ & 47 & $\begin{array}{l}\text { LRM } 400 \text { mg/day } \\
\text { DLZ } 12 \text { mg/day }\end{array}$ & Agomelatine \\
\hline 8 & $\mathrm{~F}$ & 37 & LRM 40 mg/day & Agomelatine \\
\hline 9 & M & 36 & LRM 150 mg/day & None \\
\hline 10 & $\mathrm{~F}$ & 43 & LRZ 50 mg/day & Citalopram \\
\hline 11 & $\mathrm{~F}$ & 31 & LRM 100 mg/day & Paroxetine \\
\hline 12 & $\mathrm{~F}$ & 30 & LRM 75 mg/day & Sertraline \\
\hline 13 & M & 38 & ALP 15 mg/day & Escitalopram \\
\hline 14 & M & 32 & LRM 150 mg/day & Citalopram \\
\hline
\end{tabular}

ALP, aprazolam; CLO, clonazepam; DLZ, delorazepam; DZP, diazepam; F, female; FLZ, flurazepam; $L R M$, lormetazepam; $L R Z$, lorazepam; $M$, male; $T R Z$, triazolam.

maximum capacity of $250 \mathrm{ml}$ and constant release of $1.5 \mathrm{ml} / \mathrm{h}$ for 7 days. The pump was connected to the patient's anterior abdominal wall via a butterfly needle inserted subcutaneously. The pump, releasing $1 \mathrm{mg}$ of flumazenil every $24 \mathrm{~h}$, was then placed in a small bag that could be carried attached to the belt or on the shoulder. Patients' tolerance for the infusion device was investigated on a daily basis, through clinical examination and interview.

Throughout the detoxification, FLU subcutaneous infusion (FLU-SI) was associated with therapeutic doses of clonazepam, orally administered every day in the evening and gradually tapered from $6 \mathrm{mg}$ on the 1 st day to $0.5-2.0 \mathrm{mg}$ on the last day of treatment. The different speed in the tapering of clonazepam was due to clinical criteria, in particular we considered the quality of sleep and the intensity of withdrawal symptoms. In this way, at the end of hospitalization, $3 / 14$ patients were discharged with no clonazepam, and 11/14 (78.6\%) patients were discharged with a low dose of clonazepam ranging from 0.5 to $2.0 \mathrm{mg} /$ day; these patients were recommended to gradually taper it in a few weeks (8). Unfortunately, patients were not followed-up as outpatients, and we cannot be sure whether they succeeded in tapering and eventually stopping clonazepam.

Ten days prior to the admission, anti-epileptic prophylaxis (1 $\mathrm{g} /$ day valproic acid or levetiracetam) was given to all patients in order to prevent seizures during treatment. Anti-epileptic treatment was maintained during the hospital stay and for further 20-40 days after discharge.

Patients under concurrent treatment with antidepressant (12/14 patients, see Table 1) were maintained under this pharmacotherapy.

\section{Sampling Protocol}

Blood samples were collected without anticoagulant at the moment of admission, after 4 days of FLU treatment, and at the end of the 7 days of treatment, before discharge from the addiction unit.

Samples were centrifuged (3,000 rpm, $10 \mathrm{~min})$ and sera were frozen at $-80^{\circ} \mathrm{C}$ until HPLC-MS analysis.

\section{Flumazenil and Main BZD Concentration Analysis}

Blank serum samples, used for the development and validation of the procedure, were obtained from healthy volunteers abstinent from any drug during the week before sampling. A 250- $\mu$ l aliquot of serum was added to an equal volume of $0.1 \mathrm{M}$ phosphate solution ( $\mathrm{pH} 8.8$ ), and the mixture was spiked with the IS (diazepam-D5) to have a final concentration of $40 \mathrm{ng} / \mathrm{ml}$. The mixtures were added with $1.5 \mathrm{ml}$ of ethyl acetate, then extracted by vortex-mixing for $1 \mathrm{~min}$, and centrifuged at 4,000 rpm for $15 \mathrm{~min}$. The organic phase was then evaporated to dryness under nitrogen stream and the residue dissolved in $50 \mu \mathrm{l}$ of ultrapure water.

The determination of FLU and lormetazepam was obtained by using a model 1290 UHPLC coupled to a model 6450 triple quadruple mass spectrometer (Agilent Technologies, Waldbronn, Germany) operating in positive ionization mode. Gradient elution was performed on a UHPLC ZORBAX Eclipse reversed-phase column (RRHD $2.1 \mathrm{~mm} \times 100 \mathrm{~mm}$, $1.8 \mu \mathrm{m}$ ) (Agilent) by mixing $5 \mathrm{mM}$ aqueous ammonium formate containing $0.01 \%$ formic acid (eluent $\mathrm{A}$ ) and acetonitrile added with $0.01 \%$ formic acid at a flow rate of $0.5 \mathrm{ml} / \mathrm{min}$ (eluent B) from 10 to $95 \% \mathrm{~B}$ in $7 \mathrm{~min}$. The analyses were performed in multiple reaction ion monitoring (MRM) mode using the following ion transitions: FLU 304 217, 232, and 258 (collision energy: $20 \mathrm{eV}$ ); lormetazepam 335 317, 289, and 177.0 (collision energy: $20 \mathrm{eV}$ ); and diazepam-D5 290262 (collision energy: $27 \mathrm{eV})$.

Method was linear in the concentration range of $78-5,000$ $\mathrm{pg} / \mathrm{ml}$ for FLU and of 3-200 ng/ml for lormetazepam. Lower limit of quantification (LLOQ) corresponded to $78 \mathrm{pg} / \mathrm{ml}$ for FLU and $3 \mathrm{ng} / \mathrm{ml}$ for lormetazepam.

Precision (\% CV) of the assay was $\leq 9.8 \%$ for both the analytes, whereas the inter-assay accuracy was $\leq 3.8$ and $\leq 4.7 \%$, respectively. The accuracy and CVs for day-to-day tests resulted always below $7.93 \%$.

\section{Withdrawal Assessment}

A Benzodiazepine Withdrawal Scale (BWS) form exploring withdrawal symptoms (33 items each with a score of 0-4 from best to worst) was given to each patient for daily report (23). 


\section{Statistical Analysis}

Statistical analysis was performed using the software Graph Pad PRISM version 6.0. The results were expressed as mean \pm standard error of the mean (SEM). Student's $t$-test was utilized for statistical analysis by comparing different treatment times of the same group of patients.

\section{RESULTS}

Drug plasma levels are shown in Figure 1. Plasma FLU concentrations were $0.54 \pm 0.089 \mathrm{ng} / \mathrm{ml}$ (mean $\pm \mathrm{SEM}$ ) at $\mathrm{T} 1$ after 4 days of continuous subcutaneous infusion, ranging from 0.14 to $1.4 \mathrm{ng} / \mathrm{ml}$. Values recorded at T2 (end of therapy) were $0.09 \pm 0.05 \mathrm{ng} / \mathrm{ml}$, with FLU concentrations below limits of detection in 10 patients out of 14 .

Lormetazepam (LRM) levels were $502.5 \pm 163.0 \mathrm{ng} / \mathrm{ml}$ at T0 baseline. A significant decrease $(11.2 \pm 5.7 \mathrm{ng} / \mathrm{ml} ; p=0.008)$ in LRM levels was recorded at T1 and $0.43 \pm 0.43 \mathrm{ng} / \mathrm{ml}$ at T2. High LRM plasma levels recorded at T0 are in agreement with patients' self-report of BZD use at admission, whereas low T1 and T2 levels confirmed compliance to detoxification treatment.

Lorazepam (LRZ) levels showed a similar pattern, with high initial plasma concentrations $(83.1 \pm 27.4 \mathrm{ng} / \mathrm{ml})$, then a significant decrease to $20.4 \pm 11.4 \mathrm{ng} / \mathrm{ml}(p=0.01)$ at $\mathrm{T} 1$ and $9.4 \pm 5.6 \mathrm{ng} / \mathrm{ml}$ at T2 after 7 days of FLU administration.

Clonazepam (CLN) plasma levels were low at T0 (14.0 \pm $8.6 \mathrm{ng} / \mathrm{ml}), 35.5 \pm 5.0 \mathrm{ng} / \mathrm{ml}$ at T1, and $25.4 \pm 3.9 \mathrm{ng} / \mathrm{ml}$ at T2. Note that three patients were treated with CLN before hospital admission (see Table $\mathbf{1}$ ).

According to different BZD behaviors, BWS showed a decrease from 26.4 to 17.7 points, as portrayed in Figure 2. During the treatment, 10/14 subjects (71.4\%) completed the Benzodiazepine Withdrawal Scale (BWS) with scores ranging from 0 to 132 on a daily basis, in order to subjectively assess their withdrawal symptoms. Four out of 14 patients could not complete the BWS. As shown in Figure 2, BWS improved significantly during FLU treatment in all subjects. No major event (i.e., convulsive crisis) occurred.

The elastomeric pump was well tolerated by patients. Since FLU is further diluted in a saline solution inside the device, no skin irritation around the insertion of the needle was noticed. Since elastomeric pumps are light and compact, patients appreciated the freedom of movement and rated them as painless, safe, and comfortable, with no bound to the pump and respecting the privacy about the therapy, whereas nurses acknowledged they required less time to manage them.

\section{DISCUSSION}

BZD represents a class of drugs characterized by low acute toxicity even at high doses in the absence of any concurrent drug abuse such as alcohol and opioids (2). Literature data on the toxicity of high-dose BZD are old and mostly based on anecdotal case reports. The lack of clinical studies and the high tolerability of these drugs have produced the erroneous perception that the administration of high doses of BZD for a prolonged time, although not recommended, could be not harmful. However, several complications have been associated to chronic BZD consumption, such as memory and attention deficit, inability to learn, increased risk of falls, road accidents, depression, and reduced quality of life (Lugoboni DAD 2014). Thus, although the prolonged use of high dose of BZD seems not to induce liver toxicity, it remains a serious health concern (24). The severe discomfort experienced by patients stopping longterm BZD use led to the development of treatment strategies for discontinuing these medications $(1,10)$. The common management of BZD withdrawal syndrome includes, either individually or in combination: (i) a gradual tapering of the drug; (ii) switching to an equivalent dose of a long half-life BZD before tapering withdrawal $(10,25)$; and (iii) adding medications prior to detoxification and continuing those medications after BZD discontinuation $(1,10)$. A potential approach is the abrupt discontinuation of the medication and a rapid BZD detoxification using FLU. FLU is commonly used in the treatment of BZD overdose; it is usually considered a BZD antagonist (9). When compared to placebo, bolus infusion of flumazenil (1 mg in $5 \mathrm{~min}$ ) produced effects similar to BZD withdrawal in BZD users $(23,26)$. Nonetheless, results of studies in chronic BZD users who have discontinued BZD use suggest that multiple slow bolus infusions of flumazenil reduce the symptoms of withdrawal $(9,11,21,27)$.

Subcutaneous route of FLU administration was previously described only in three patients (14), suggesting the usefulness of this route for its excellent tolerability, efficacy, and improvement on measure of psychological distress. According to these data, we decided to administer FLU by subcutaneous route utilizing elastomeric pumps normally used for pain control in cancer patients or, more recently, for continuous infusion of antibiotics (28) or for treatment of idiopathic hypersomnia (29).

To our knowledge, the results present in this paper are the first data of FLU serum concentrations following subcutaneous infusion by elastomeric pump described in literature. FLU serum concentrations were low, but consistent with data of FLU administered by i.v. route (14).

FLU is characterized by short half-life $(0.8-1.2 \mathrm{~h})$ (30) and requires repeated doses or continuous infusion to reverse BZD overdose. In spite of its low lipophilicity, FLU has a large volume of distribution, and its weak binding to plasma proteins explains its rapid distribution. Moreover, FLU is extensively metabolized by hepatic cytochromes $\mathrm{P} 450$ 3A4, 3A5, and 2C9 and readily eliminated. Maximum brain concentrations are reached 5 to 8 min after i.v. administration (31).

Subcutaneous administration of flumazenil eliminates some problems with first-pass hepatic metabolism observed orally and is likely to facilitate better absorption. Subcutaneous administration also provides continuous dosing, which would be hard to achieve with oral or sublingual administration, and the slow absorption may abrogate side effects related to high serum concentrations. The subcutaneous route is easier to establish than the intravenous administration, and there is no risk for patient's veins. Study data suggested that flumazenil administered by the s.c. route might have equitable clinical benefits to i.v. administration, but it might be superior in that it requires less 

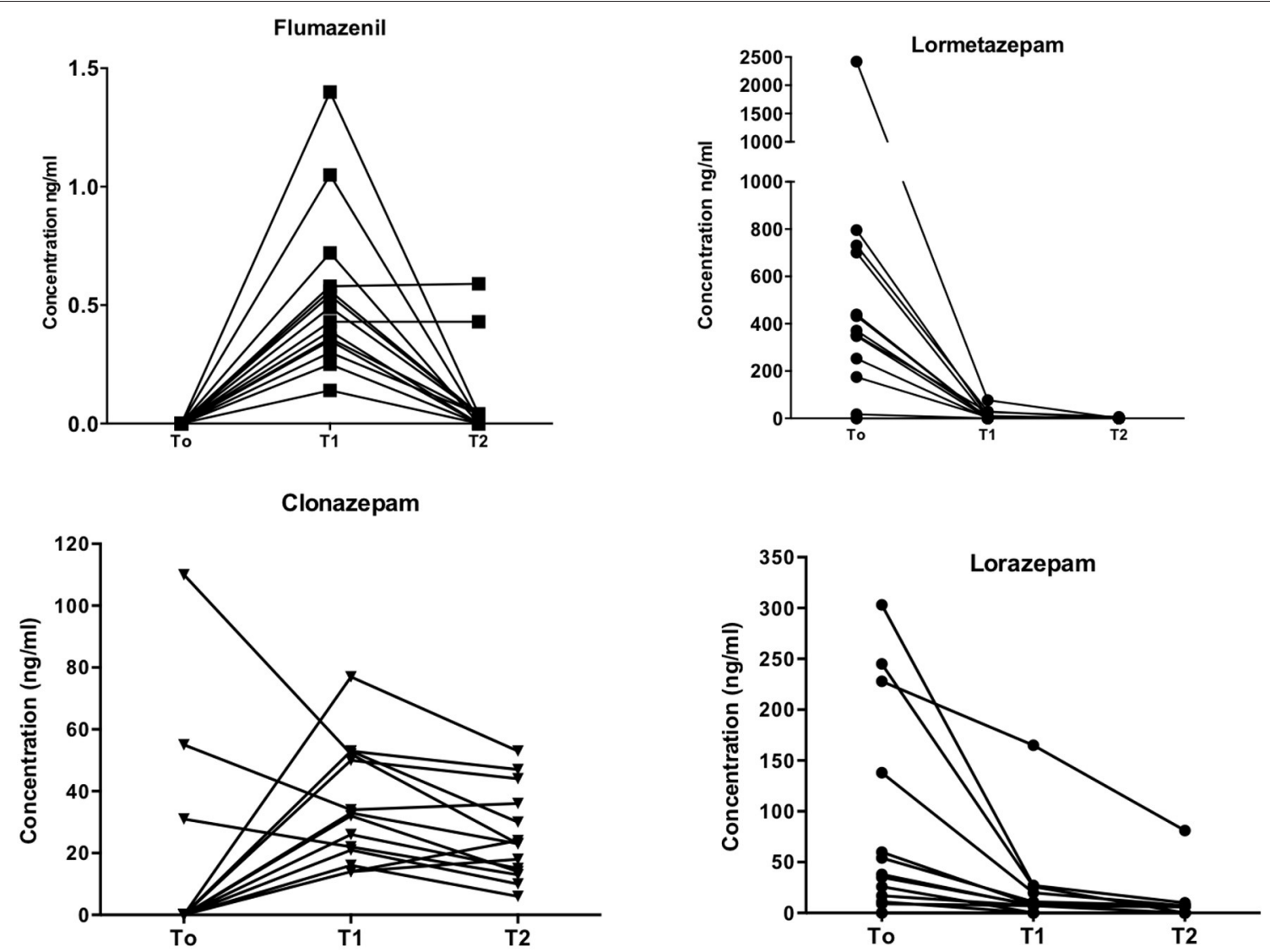

FIGURE 1 | Drugs plasma levels. Individual plasma levels ( $\mathrm{ng} / \mathrm{ml}$; ordinates) for flumazenil, lormetazepam, clonazepam, and lorazepam at different time-points (abscissa), i.e., at admission (TO), four (T1) and seven days after flumazenil start of elastomeric infusion.

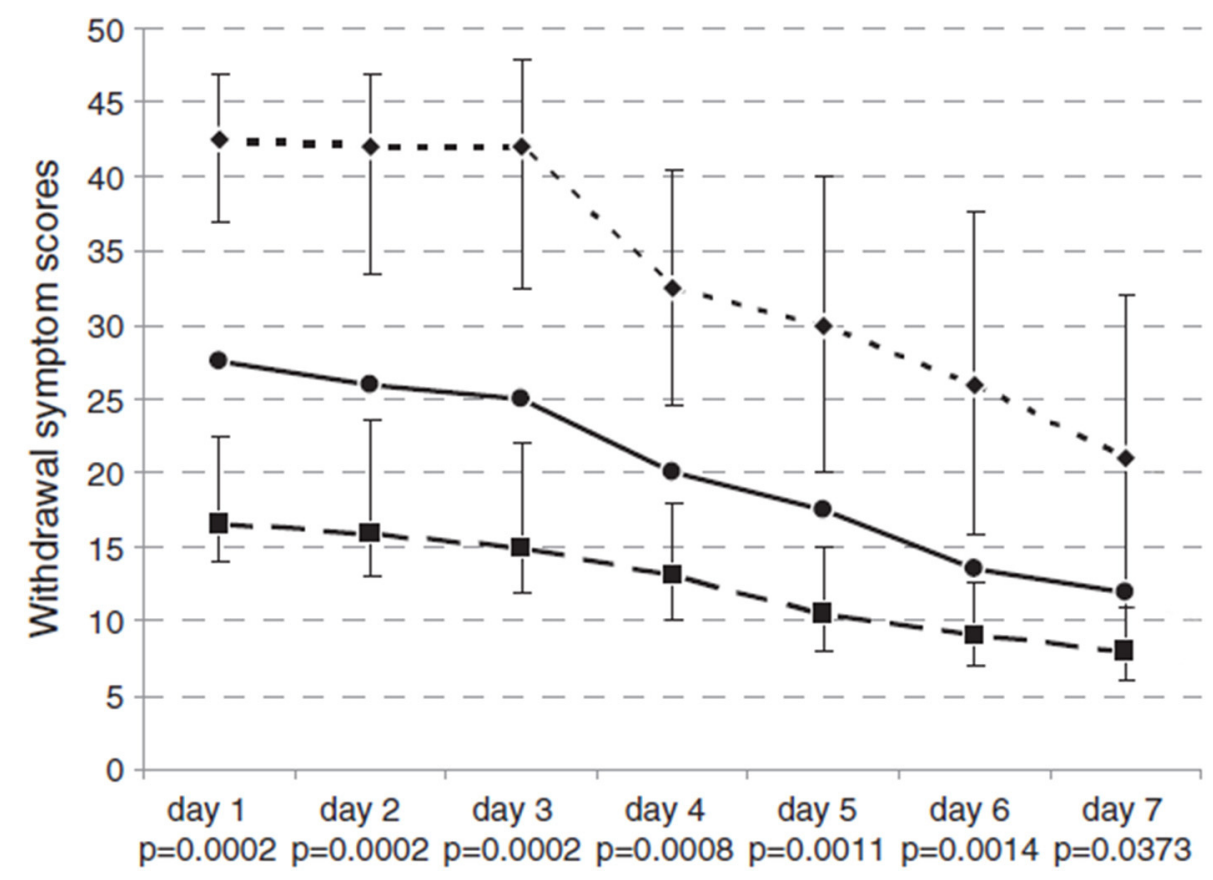

FIGURE 2 | Withdrawal Symptom Scores from day 1 to day 7 of FLU-SI treatment. 
clinical monitoring and is likely associated with less equipment problems (i.e., dislodged or blocked i.v. needle/line) and adverse events (i.e., venous tissue irritation). These advantages, as well as an improved patient mobility over the treatment period, will also likely result in increased patient satisfaction $(9,14)$.

The subcutaneous route of administration may be associated to the absence of adverse events associated with i.v. FLU administration. In fact, our patients did not report any kind of adverse events such as those frequently reported during or after FLU administration $(8,14,32)$.

Our results demonstrated low and constant serum concentrations during all treatment and a prompt decrease nearly to 0 at the end of treatment, protecting patients from peak serum levels. We utilized an elastomeric infusion pump mostly utilized in our hospital for analgesic purposes.

Several elastomeric pumps are commercially available, and they are calibrated in different conditions, including operating temperature and pressure, viscosity of fluid, backpressure, and time recommended between filling of the device and beginning of the infusion. All of these factors affect the infusion rate of pumps. Elastomeric infusion pumps are feasible to use and less bed bounding for patients, although a little less precise than other pumps.

Moreover, Höjer et al. (33) studied the stability of infusion solutions of flumazenil in concentrations of 1.0 and $5.0 \mu \mathrm{g} / \mathrm{ml}$ stored for periods of up to 9 months and concluded that the stability of flumazenil in infusion solution was satisfactory.

Importantly, serum levels of other BZDs (such as LRM and LRZ) are 0 after 4 days of FLU administration, proving both the efficacy of FLU and patients' compliance despite the elevated BZD plasma levels measured at the beginning of the treatment. The good patients' compliance was confirmed by CLN concentrations in serum that showed a trend to decrease after 7 days. Most interestingly, during the detoxification process, all patients reported low levels of craving for $\mathrm{BZD}$, which might represent a rarely seen feature in the spectrum of drug detoxification. According to previous studies, high-dose BZD chronic use determines a severe impairment of psychological, physical, and social functioning, along with a significant reduction of quality of life $(34,35)$.

\section{REFERENCES}

1. Soyka M. Treatment of benzodiazepine dependence. N Engl J Med. (2017) 376:2399-400. doi: 10.1056/NEJMc1705239

2. Lader M. Benzodiazepines revisited: will we ever learn? Addiction. (2011) 106:2086-2109. doi: 10.1111/j.1360-0443.2011.03563.x

3. Petitjean S, Ladewig D, Meier CR, Amrein, R, Wiesbeck GA. Benzodiazepine prescribing to the Swiss adult population: results from a national survey of community pharmacies. Int Clin Psychopharmacol. (2007) 22:292-8. doi: 10.1097/YIC.0b013e328105e0f2

4. Ohayon MM, Lader MH. Use of psychotropic medication in the general population of France, Germany, Italy, and the United Kingdom. J Clin Psychiatry. (2002) 63:817-25. doi: 10.4088/JCP.v63n0912

5. Magrini N, Vaccheri A, Parma E, D’Alessandro R, Bottoni A, Occhionero M, et al. Use of benzodiazepines in the Italian general population. Prevalence, patterns of use and risk factors for use. Eur J Clin Pharmacol. (1996) 50:19-25. doi: $10.1007 /$ s002280050063
The main limitation to this study is the lack of a follow-up phase to determine whether all patients were successfully able to taper and suspend clonazepam and to assess the relapse rate. Another limitation of the study is its monocentric design. The problem is not new. Although more than 30 years have passed since the first studies of the efficacy of FLU in the treatment of addiction to high doses of BZD, to our knowledge, there are no more than five centers worldwide offering this treatment. This continues to represent a major obstacle to the definition of more shared and standardized protocols. Currently, FLU protocol is the same for all patients, regardless of sex, age, BMI, and BZD daily intake. Future prospects should include further investigations of the individual variables and clinical outcomes in order to individualize the detoxification therapy.

\section{DATA AVAILABILITY STATEMENT}

The raw data supporting the conclusions of this article will be made available by the authors, without undue reservation.

\section{ETHICS STATEMENT}

The studies involving human participants were reviewed and approved by CE 292CESC. The patients/participants provided their written informed consent to participate in this study.

\section{AUTHOR CONTRIBUTIONS}

ABen and RG equally contributed for the analytes'plasma measurements. ABen, $\mathrm{CC}$, and ABer contributed to the manuscript's draft and proofreading. LZ processed the statistical analysis and FL followed the clinical part and designed the study. All authors contributed to the article and approved the submitted version.

\section{ACKNOWLEDGMENTS}

The authors would like to remember the figure of Malcolm Lader (1936-2020), our mentor in pharmacological research on benzodiazepines and their misuse.

6. Barbui C, Gregis M, Zappa M. A cross-sectional audit of benzodiazepine use among general practice patients. Acta Psychiatr Scand. (1998) 97:153-6. doi: 10.1111/j.1600-0447.1998.tb09979.x

7. Hollister LE, Motzenbecker FP, Degan RO. Withdrawal reactions from chlordiazepoxide ("Librium"). Psychopharmacologia. (1961) 2:63-8. doi: 10.1007/BF00429621

8. Faccini M, Leone R, Opri S, Casari R, Resentera C, Morbioli L, et al. Slow subcutaneous infusion of flumazenil for the treatment of long-term, highdose benzodiazepine users: a review of 214 cases. J Psychopharmacol. (2016) 30:1047-53. doi: 10.1177/0269881116647505

9. Hood SD, Norman A, Hince DA, Melichar JK, Hulse GK. Benzodiazepine dependence and its treatment with low dose flumazenil. Br J Clin Pharmacol. (2014) 77:285-94. doi: 10.1111/bcp.12023

10. Denis C, Fatséas M, Lavie E, Auriacombe M. Pharmacological interventions for benzodiazepine mono-dependence management in outpatient settings. Cochrane Database Syst Rev. (2006) (3):CD005194. doi: 10.1002/14651858.CD005194.pub2 
11. Gerra G, Zaimovic A, Giusti F, Moi G, Brewer C. Intravenous flumazenil versus oxazepam tapering in the treatment of benzodiazepine withdrawal: a randomized, placebo-controlled study. Addict Biol. (2002) 7:385-95. doi: 10.1080/1355621021000005973

12. Faccini M, Tamburin S, Casari R, Morbioli L, Lugoboni F. High-dose lormetazepam dependence: strange case of Dr. Jekyll and Mr Hyde. Intern Emerg Med. (2019) 14:1271-8. doi: 10.1007/s11739-019-02101-8

13. Gallo AT, Hulse G. Pharmacological uses of flumazenil in benzodiazepine use disorders: a systematic review of limited data. J Psychopharmacol. (2021) 9:269881120981390. doi: 10.1177/0269881120981390

14. Hulse GK, O’Neil G, Morris N, Bennett K, Norman A, Hood SD. Withdrawal and psychological sequelae, and patient satisfaction associated with subcutaneous flumazenil infusion for the management of benzodiazepine withdrawal: a case series. J Psychopharmacol. (2013) 27:222-7. doi: 10.1177/0269881112446532

15. Tamburin S, Mantovani E, Bertoldi A, Federico A, Casari R, Lugoboni F. High-dose dependence and cognitive side effects to medical prescription of etizolam. Front Psychiatry. (2020) 11:601827. doi: 10.3389/fpsyt.2020.601827

16. Pericić D, Jazvinsćak Jembrek M, Svob Strac D, Lazić J, Spoljarić IR. Enhancement of benzodiazepine binding sites following chronic treatment with flumazenil. Eur J Pharmacol. (2005) 507:7-13. doi: 10.1016/j.ejphar.2004.10.057

17. Mascia MP, Ledda G, Orrù A, Marongiu A, Loriga G, Maciocco E, et al. Differential modulation of GABA(A) receptor function by aryl pyrazoles. Eur J Pharmacol. (2014) 15:1-6. doi: 10.1016/j.ejphar.2014.03.039

18. Weinbroum AA, Flaishon R, Sorkine P, Szold O, Rudick V. A risk-benefit assessment of flumazenil in the management of benzodiazepine overdose. Drug Saf. (1997) 17:181-96. doi: 10.2165/00002018-199717030-00004

19. Whithwam JG, Amrein R. Pharmacology of flumazenil. Acta Anaesthesiol Scand. (1995) 59 (Suppl. 108):3-14. doi: 10.1111/j.1399-6576.1995.tb04374.x

20. Klotz U, Kanto J. Pharmacokinetics and Clinical Use of Flumazenil (Ro 15-1788). Clin Pharmacokinet. (1988) 14:1-12. doi: 10.2165/00003088-198814010-00001

21. Saxon L, Hjemdahl P, Hiltunen AJ, Borg S. Effects of flumazenil in the treatment of benzodiazepine withdrawal-a double-blind pilot study. Psychopharmacology (Berl). (1997). 131:153-60. doi: 10.1007/s002130050278

22. American Psychiatric Association. American Psychiatric Association Diagnostic and Statistical Manual of Mental Disorders DSM-5, 5th edn. Washington, DC: American Psychiatric Publishing (2013).

23. Mintzer MZ, Stoller KB, Griffiths RR. A controlled study of flumazenilprecipitated withdrawal in chronic low-dose benzodiazepine users. Psychopharmacology (Berl). (1999) 147:200-9. doi: 10.1007/s0021300 51161

24. Lugoboni F, Mirijello A, Morbioli L, Arzenton E, Leone R, Faccini M, et al. Does high-dose benzodiazepine abuse really produce liver toxicity? Results from a series of 201 benzodiazepine monoabusers. Expert Opin Drug Saf. (2018) 17:451-456. doi: 10.1080/14740338.2018.1457646
25. Lader M, Tylee A, Donoghue J. Withdrawing benzodiazepines in primary care. CNS Drugs. (2009) 23:19-34. doi: 10.2165/0023210-200923010-00002

26. Mintzer MZ, Griffiths RR. Flumazenil-precipitated withdrawal in healthy volunteers following repeated diazepam exposure. Psychopharmacology. (2005) 178:259-67. doi: 10.1007/s00213-004-2009-1

27. Federico A, Lugoboni F, Mantovani E, Martini A, Morbioli L, Casari $\mathrm{R}$, et al. Detoxification Improves Multidomain Cognitive Dysfunction in High-Dose Benzodiazepine Abusers. Front Neurosci. (2020) 14:747. doi: 10.3389 /fnins. 2020.00747

28. Mujal A, Sola J, Hernandez M, Villarino MA, Baylina M, Tajan J, et al. Safety and effectiveness of outpatient parenteral antimicrobial therapy in older people. J Antimicrob Chemother. (2016) 71:1402-1407. doi: $10.1093 / j a c / d k v 478$

29. Kelty E, Martyn V, O’Neil G, Hulse GK. Use of subcutaneous flumazenil preparations for the treatment of idiopathic hypersomnia: a case report. $J$ Psychopharmacol. (2014) 28:703-6. doi: 10.1177/0269881114523865

30. Bentue-Ferrer D, Bureau M, Patat A, Allain H. Flumazenil. CNS Drug Rev. (1996) 2:390-414. doi: 10.1111/j.1527-3458.1996.tb00308.x

31. Roncari G, Ziegler WH, Guentert TW. Pharmacokinetics of the new benzodiazepine antagonist Ro 15-1788 in man following intravenous and oral administration. Br J Clin Pharmacol. (1986) 22:421-8. doi: 10.1111/j.1365-2125.1986.tb02912.x

32. Penninga EI, Graudal N, Ladekarl MB, Jürgens G. Adverse events associated with flumazenil treatment for the management of suspected benzodiazepine Intoxication - A Systematic Review with Meta-Analyses of Randomised Trials. Basic Clin Pharmacol Toxicol. (2016) 118:37-44. doi: 10.1111/bcpt.12434

33. Höjer J, Baehrendtz S, Forsström A, Lundqvist T, Jansson B. The stability of flumazenil in infusion solution. Acta Pharm Nordica. (1990) 2:101-4.

34. Lugoboni F, Mirijello A, Addolorato G, Faccini M, Casari R, Cossari A, et al. Quality of life in a cohort of high-dose benzodiazepine dependent patients. Drug Alcohol Depend. (2014) 142:105-9. doi: 10.1016/j.drugalcdep.2014.06.020

35. Tamburin S, Federico A, Faccini M, Casari R, Morbioli L, Sartore V, et al. Determinants of quality of life in high-dose benzodiazepine misusers. Int J Environ Res Public Health. (2017) 14:38. doi: 10.3390/ijerph14010038

Conflict of Interest: The authors declare that the research was conducted in the absence of any commercial or financial relationships that could be construed as a potential conflict of interest.

Copyright (C) 2021 Benini, Gottardo, Chiamulera, Bertoldi, Zamboni and Lugoboni. This is an open-access article distributed under the terms of the Creative Commons Attribution License (CC BY). The use, distribution or reproduction in other forums is permitted, provided the original author(s) and the copyright owner(s) are credited and that the original publication in this journal is cited, in accordance with accepted academic practice. No use, distribution or reproduction is permitted which does not comply with these terms. 


\section{OPEN ACCESS}

Edited by:

Liana Fattore,

National Research Council (CNR), Italy

Reviewed by:

Oliver Grundmann,

University of Florida, United States

Walter Prozialeck,

Midwestern University, United States

Susruta Majumdar,

St. Louis College of Pharmacy,

United States

*Correspondence:

Georgios D. Kotzalidis

giorgio.kotzalidis@uniroma1.it

Specialty section: This article was submitted to

Addictive Disorders,

a section of the journal

Frontiers in Psychiatry

Received: 10 December 2020 Accepted: 05 March 2021

Published: 31 March 2021

Citation:

Vento AE, de Persis S, De Filippis S, Schifano F, Napoletano F, Corkery JM and Kotzalidis GD (2021) Case Report: Treatment of Kratom Use Disorder With a Classical Tricyclic Antidepressant.

Front. Psychiatry 12:640218. doi: 10.3389/fpsyt.2021.640218

\section{Case Report: Treatment of Kratom Use Disorder With a Classical Tricyclic Antidepressant}

\author{
Alessandro E. Vento ${ }^{1,2}$, Simone de Persis ${ }^{3}$, Sergio De Filippis ${ }^{4}$, Fabrizio Schifano ${ }^{5}$, \\ Flavia Napoletano ${ }^{5}$, John M. Corkery ${ }^{5}$ and Georgios D. Kotzalidis ${ }^{4,6 *}$ \\ ${ }^{1}$ ASL (Azienda Sanitaria Locale) Roma 2, Rome, Italy, ${ }^{2}$ Addictions' Observatory (ODDPSS), Rome, Italy, ${ }^{3}$ ASL, Rieti, Italy, \\ ${ }^{4}$ Villa von Siebenthal Neuropsychiatric Clinic and Hospital, Genzano di Roma, Italy, ${ }^{5}$ Psychopharmacology, Drug Misuse \& \\ Novel Psychoactive Substances Research Unit, School of Life and Medical Sciences, University of Hertfordshire, Hatfield, \\ United Kingdom, ${ }^{6}$ NESMOS Department (Neurosciences, Mental Health, and Sensory Organs), School of Medicine and \\ Psychology, Sant'Andrea Hospital, Sapienza University, Rome, Italy
}

Kratom or Mitragyna speciosa (Korth.) is an evergreen tree of the coffee family native to South-East Asia and Australasia. It is used by locals recreationally to induce stimulant and sedative effects and medically to soothe pain and opiate withdrawal. Its leaves are smoked, chewed, or infused, or ground to yield powders or extracts for use as liquids. It contains more than 40 alkaloids; among these, mitragynine and 7-hydroxymitragynine are endowed with variable mu, delta, and kappa opioid stimulating properties (with 7-hydroxymitragynine having a more balanced affinity), rhynchophylline, which is a non-competitive NMDA glutamate receptor antagonist, but is present in negligible quantities, and raubasine, which inhibits $\alpha_{1}$-adrenceptors preferentially over $\alpha_{2}$-adrenceptors, while the latter are bound by 7-hydroxymitragynine, while mitragynine counters 5- $\mathrm{HT}_{2 \mathrm{~A}}$ receptors. This complexity of neurochemical mechanisms may account for kratom's sedative-analgesic and stimulant effects. It is commonly held that kratom at low doses is stimulant and at higher doses sedative, but no cut-off has been possible to define. Long-term use of kratom may produce physical and psychological effects that are very similar to its withdrawal syndrome, that is, anxiety, irritability, mood, eating, and sleep disorders, other than physical symptoms resembling opiate withdrawal. Kratom's regulatory status varies across countries; in Italy, both mitragynine and the entire tree and its parts are included among regulated substances. We describe the case of a patient who developed anxiety and dysphoric mood and insomnia while using kratom, with these symptoms persisting after withdrawal. He did not respond to a variety of antidepressant combinations and tramadol for various months, and responded after 1 month of clomipramine. Well-being persisted after discontinuing tramadol.

Keywords: kratom, mitragynine, substance use disorder, clomipramine, withdrawal syndrome

\section{INTRODUCTION}

The interest of the medical world in Mitragyna speciosa Korthals (MsK) dates back to the 1950s (1-6). MsK (kratom) was first described by the Dutch colonial botanist Pieter Korthals in 1839 and is indigenous to Thailand, Indonesia, Malaysia, Myanmar, and Papua New Guinea, where it has been used in traditional medicine and religious (7) contexts since at least the $19^{\text {th }}$ Century, as well as a voluptuary substance 
use (a surrogate to opium) well before Korthals' description $(8,9)$. In these countries, leaves of MsK are first dried and then chewed or consumed as smoke in long pipes, extract, or powder, or brewed into a tea (10). Mixtures with other substances are also made, thus increasing dangerousness of consumption. Some of them are confectioned into pills (11). Concern over its use was not raised until recently, when it became largely available in Western countries and its toxic potential realized. A first Malaysian report found kratom consumers to develop addiction and psychiatric symptoms (12), while its psychoactive properties were detailed in the late 1980s (13).

MsK alkaloids were quantitatively determined in its leaves after separation by thin-layer chromatography, with ultraviolet spectrophotometry (14), with colorimetry (15), densitometry (16); indoles and oxindoles were identified in the first place (17). Since then, more than 25 significant alkaloids were identified (11). The corynanthe-type indole mitragynine contributes $66 \%$ to MsK alkaloids, paynantheine $9 \%$, 7-hydroxymitragynine $2 \%$, and speciociliatine $1 \%$; other alkaloids contribute $<1 \%$ each (11). However, their contribution varies across locations (18) and products sold across the world might not always contain MsK at all (19). The first whose structure was determined in 1958 was mitraphylline (20), with many other alkaloids following suit (18). The MsK alkaloids may differ in their brain accessibility and crossing of the blood-brain barrier; for example, mitragynine penetrates in the brain significantly more than 7 hydroxymitragynine, at least in the rat (21). However, the latter is held to be responsible for almost all kratom effects on opioid receptors, and despite low content, it is produced by cytochrome P450 (CYP3A4 isoenzyme) conversion from mitragynine (22).

Other biochemically and neurochemically interesting compounds include rhynchophylline derivatives (23, 24), which down-regulate NMDA-mediated responses in animals (25-27), and a yohimbine and mitragynine analog, ajmalicine or raubasine (28), which differently from mitragynine (29), inhibits $\alpha_{2}$-adrenoceptors, although less than $\alpha_{1}$-adrenoceptors (30-32). Of note, kratom alkaloids closely interact with $\alpha_{2}$ adrenoceptors, and mitragynine and 7-hydroxymitragynine bind them (33). Furthermore, mitragynine inhibits the activity of $5-\mathrm{HT}_{2 \mathrm{~A}}$ receptors, although indirectly so $(29,34,35)$, as it shows a $K_{\mathrm{i}}>10 \mu \mathrm{M}$ for the $5-\mathrm{HT}_{2 \mathrm{~A}}$ receptor (36). It is possible that the interplay between these receptor effects and between MsK alkaloids underpin the different effects of kratom at low vs. high doses.

Although kratom was reportedly used to substitute for opiate addiction and cure it (37), the demonstration of their binding by MsK had to await the discovery of opioid receptors (38, 39). Mitragynine and other kratom alkaloids were shown to be possibly allosteric (40) agonists to opioid receptors (41-44), to possess analgesic properties thanks to their binding to brain $\mu$ and $\delta$-opioid receptors, and to induce ileal and vas deferens distention through the same receptors at peripheral sites $(43,45)$. These properties were long harnessed by traditional healers in the countries where kratom grows. In South-East Asia kratom is used to alleviate muscle aches, and sometimes to heal wounds and cure worm infections, while some users support they assume it to increase resistance to fatigue and to stimulate sexuality
(46). Indeed, mitragynine and kratom alkaloids are likely to be associated with "dependence" signs and symptoms which are less severe than those usually associated to opiates and they may be used to alleviate classical opiate withdrawal $(47,48)$. This is not surprising, since they act as agonists on opioid mu receptors.

Legislations concerning kratom varies across countries. In Europe it is illegal in Denmark, Finland, Ireland, Latvia, Lithuania, Poland, Romania, and Sweden (49), while in the UK it has been included in the Psychoactive Substances Bill 2015 (50), hence it is illegal since March 2016, being regulated through the Psychoactive Substances Act 2016 (51). In Italy, it became illegal in 2016. In Canada and Australia, kratom is illegal, while in New Zealand it is a regulated substance. In the United States it is forbidden in some States and not in other (49). Many US state legislations are likely to change their attitude toward kratom in the near future. Similarly, Thailand, one of kratom's major producers, which prohibits since 1943 the cultivation of new plants and mandates the abatement of the existing ones, while restricting possession and use and establishing sanctions for quantities superior or inferior to $10 \mathrm{Kg}$, is on the verge of changing its legislation. In Malaysia, Bhutan and Myanmar kratom is illegal, and in Indonesia it will be banned by 2022 (52). Discrepancies among the various legislations internationally, as well as the increase in the use of internet and globalization have resulted in an increased use of kratom for voluptuary purposes (53) indicating the need for international coordination of scientists and legislators (54). That kratom could induce an opioid-like withdrawal syndrome, therefore it can be included among addictive substances, is shown by the fact that it may be present in neonates exposed to the substance due to their mothers' heavy use during pregnancy (55-61). In fact, the World Anti-Doping Agency placed mitragynine on its Monitoring List since 2014, and 1 year later, four cases of mitragynine use among strength sportsmen were detected (62). Kratom use has been also reported in fitness settings (63).

The effects of the use of kratom are variable and may depend on the cultural and genetic background of the user as well as on differences in product composition. Product conservation and transport factors may also be involved, as are co-administered sedative or multisubstance use. In a US-Thailand comparison, for example, symptoms were more severe and mortality higher in the US sample, with drowsiness, irritability-agitation and tachycardia being the most common in order of increasing frequency (64). Kratom may be used according to users' taste and adjusted according to the desired effects, with low doses producing stimulant and activating effects and high doses sedative and tranquilizing effects (65-67), although these dose-related effects were not confirmed in a recent study and was unrelated to the amount and duration of kratom use (68). Many people, especially in South-East Asia, get to use kratom after being addicted to opioids and in the attempt to quit; others are prompted to use kratom due to its anxiolytic and mood enhancing effects (69-71). It is expected that upon discontinuing, rebound mood and anxiety symptoms emerge. In regular users, withdrawal symptoms may occur which are more intense in long-time users or after stopping heavy use, and involve usually moderate anxiety and depression (72), as well as aching and disordered sleep (73). 
However, kratom withdrawal syndromes are usually mild and transient (72-74), similar to but milder than those of opiate withdrawal $(74,75)$, but may be complicated in some users $(54)$.

We here report the case of an adult man who used kratom and developed withdrawal symptoms while trying to quit. He did well on clompipramine just 1 month after initiation and, 9 months later, is currently symptom-free.

\section{CASE REPORT}

A 44-year-old man, married to a 44-year-old, currently pregnant woman, with a 5-year-old son, a graduate in economy and employed as a researcher at a University, sought help at a community psychiatric service for symptoms of kratom withdrawal and elevated anxiety.

The patient was collaborative at interview, appropriately dressed and well-oriented in time and space; he showed free-floating and somatic anxiety, with tachycardia, profuse sweating, psychomotor agitation, insomnia, dysphoric mood, and emotional lability. His thoughts were focused on anxious experiences and hopelessness. He reported being treated during the last few months with various benzodiazepines and selective serotonin reuptake inhibitors (SSRIs), first paroxetine $40 \mathrm{mg} / \mathrm{Day}$ and then sertraline $200 \mathrm{mg} /$ day, to which he associated cognitivebehavioral therapy, with no clear benefit.

The patient had experienced two important major depressive episodes coinciding with stressful life events, which he overcame through the use of SSRIs and long-term psychotherapy. When he was young, he had engaged in polysubstance use, while in his adult life he first used cannabis and alcohol, but later turned to benzodiazepines, alcohol, and kratom, which he obtained through dark internet sites. The patient has been vague as to when and how he started consumption, and also very unclear regarding dosing. His internet-related kratom sources varied, so we are not in a position to determine the purity of the samples he received. During the last 10 months preceding the visit, he had scheduled daily kratom infusions, but had discontinued quite sharply during the last 2 months. The patient used to continue drinking the infusion until he reached the desired effect. Having realized in the last 2 months he was becoming severely dependent, he decided to quit kratom and to no longer seek it on the internet.

Urinary drug testing was positive for benzodiazepines. Blood chemistry showed no abnormal values. However, kratom could not be quantified due to the unavailability of routine laboratory tests. The electrogram (ECG) showed no abnormalities, with a QTc of $385 \mathrm{~ms}$ and a heart rate of 60 beats $/ \mathrm{min}$. We established treatment with pregabalin $25 \mathrm{mg}$ b.i.d., gradually tapering off sertraline and substituting it with $150 \mathrm{mg}$ /day bupropion, taken in the morning, and $300 \mathrm{mg}$ controlled-release trazodone, administered in the evening. His next visit was scheduled after 2 weeks.

During the second visit, his clinical conditions were unchanged. The patient was restless, anxious, agitated, insomniac, dysphoric, with frequent cry spells and unstructured ideation of self-harm. He craved for benzodiazepines and alcohol and often abused them. Bupropion was increased to $300 \mathrm{mg} /$ day and pregabalin, $75 \mathrm{mg}$ b.i.d. was initiated.

During his third visit, after further 15 days, the above clinical picture persisted. The patient reported to be able to relax, but observed no symptom improvement. We agreed to add 50 $\mathrm{mg}$ /day tramadol in the evening. He noted since the first days of tramadol addition a mild reduction in craving and restlessness, with disappearance of self-harm ideas, while anxiety, which the patient reported as paralyzing, dysphoric mood, cry spells, and avolition remained unchanged. The patient asked for a medical certificate to abstain from work, since he considered teaching at the University a complex and stressful activity. We agreed to increase tramadol to $100 \mathrm{mg}$ b.i.d., gradually introducing clomipramine to a target dose of $75 \mathrm{mg} /$ day, while gradually discontinuing bupropion.

Three months after the first visit and about 1 month after introducing clomipramine, the clinical picture was on the way to resolution; the patient himself asked to discontinue tramadol. Free-floating and somatic anxiety had subsided and craving for all substances, including alcohol, benzodiazepines, and kratom, was significantly attenuated, while mood was stable and in the normal range (euthymic).

In the following months, given the clear clinical improvement and the remission of withdrawal symptoms, it was possible to gradually discontinue both pregabalin and trazodone.

Currently, the clinical picture is stable; the patient continues on clomipramine $75 \mathrm{mg}$ /day and about 9 months after its introduction reports to have resumed normal life.

The patient signed free informed consent for the publication of his case and all treatments received.

\section{DISCUSSION}

In this report we presented the case of an adult Italian man in his forties, who deliberately used kratom to soothe his anxiety symptoms. The patient was well-educated and upper socioeconomic class. He had started kratom after engaging in multisubstance use and psychotherapy, while completing steps toward reaching a high social status. He used the internet to obtain kratom, but had no available supplies when he came to our attention, so we could not analyse any kratom specimen he used. After trying several therapeutic strategies, including pharmacotherapy, he was unable to resolve his anxiety symptoms, either during kratom use or during abstinence, and was switched to low-dose clomipramine eventually discontinuing all other psychotherapeutic drugs; 1 month after initiating clomipramine, his symptoms had resolved and so were his anxiety symptoms that had originated psychiatric visits.

There have been several case reports of kratom use toxicity and withdrawal in literature, but clomipramine treatment had not been reported to date. Cases vary in severity and symptom presentation. One of the first described cases of mitragynine toxicity was of severe seizures and come occurring in a 64-yearold man, that resolved soon with symptomatic treatment, that is, intubation to preserve airway integrity (76). In another case, 
seizures occurred when a 43-year-old man tried to self-treat his opiate dependence with a kratom-modafinil combination (69); the case resolved with few kratom-related withdrawal symptoms. A further case presenting with seizures occurred in a 18-year old man and treated with antiepileptic drugs. Magnetic resonance imaging showed bilateral alterations in the striatum, cerebral peduncles, and subthalamic nuclei in this chronic kratom user, indicating possible permanent effects of kratom in brain structure (77). Finally, a 27 -year-old nab with history of anxiety, attention-deficit/hyperactivity disorder, substance use disorders (benzodiazepines and opioids) developed seizures while using kratom and opioids and recovered with anti-anxiety agents (78). Another 36-year-old man was unresponsive to external stimuli and near comatose, did not respond to naloxone and was treated with respiratory support and symptomatic management (79). Another kratom overdose case occurred in a 38-year-old woman who resented with respiratory depression at the emergency department and resolved with naloxone (80). A 33-year-old male polysubstance user exhibited cardiovascular shock features and high procalcitonin levels promptly treated with vasopressors (81). An otherwise healthy 35-year-old man suffered a cardiac arrest after using kratom alone and was found with small brain infarcts, but recovered spontaneously (82). Finally, a 62-year-old woman who used kratom for the first time to soothe traumatic pain presented at the emergency room with intractable vomiting and nausea that responded to ondansetron, promethazine, and famotidine (83).

Cases of kratom-related deaths are usually linked to simultaneous assumption of kratom with other drugs, as in the above described case. Initial death reports regarded associations, but more recent cases show that people who only take kratom are at risk. One case of death of a 20 -year-old man occurred with propylhexedrine and kratom; the latter was not determined to have caused the death, which has been associated to accidental propylhexedrine (84). Nine cases of death occurring in one year were described in Sweden in 2011 with the simultaneous intake of mitragynine and $\mathrm{O}$-desmethyltramadol. Decedents' age ranged 22-35; seven were men and two were women (85). The authors concluded that mitragynine-related herbal mixes are not so safe as per internet propaganda. Another death case in which mitragynine was involved, but the death was attributed to quetiapine overdose, has been described in a 27-year-old man succumbing to hyperthermia associated with seizures. One case of a 17-year adolescent male who was trying to quit opioid use by self-medicating with kratom, points to kratom being occasionally toxic; the boy was found dead with pulmonary congestion and oedema, as well as urinary bladder distension, which are typical of opiate intoxication (86). The case was labeled as "probable kratom toxicity." Another case of kratom intoxication-related death was found to be associated with high blood amounts of mitragynine and 7hydroxymitragynine and unremarkable pathological finding at autopsy in a middle-aged man with psychiatric history and illicit drug use disorder (87). Another report of death related to kratom use was one of a 24-year man with opiate and alcohol use disorders, who was found dead with high peripheral alkaloid concentrations, pulmonary oedema and congestion, and urinary retention, compatible with opioid intoxication; the patient was using several psychiatric medications that were found at therapeutic blood levels (88). Further two cases of young men could not be attributed to the documented kratom use, despite high mitragynine levels in femoral blood (89). One of the patients had attempted suicide just after taking kratom with prescription drugs, while the other took a mix of drugs. Another fatality was due to 3-methoxyphencyclidine, and mitragynine was just one of many other substances the 58-yearold man had taken (90). An emergency case presenting with cardiorespiratory arrest could not be rescued despite the use of intralipid, that nevertheless improved somehow the conditions of a 26-year-old man, but proved ineffective in avoiding exitus, attributed to cardiorespiratory failure and hypoxic brain damage (91). A Canadian 56-year-old woman with chronic obstructive pulmonary disease, after skipping her medication and consuming kratom purchased from Indonesia, died due to respiratory failure (92). Her mitragynine levels in the femoral vein were found to be substantial but sublethal (under the reporting laboratory's threshold of fatality, which was $0.21 \mathrm{mg} / \mathrm{L}$ ). Another case of multiple drug use ensuing in death has been related to mitragynine due to the very high doses found in inferior cava blood of a 33-year-old man (93). In general, fatality case studies suffer from heterogeneity in kratom alkaloid detection methods and sites.

Cases of chronic kratom used followed by withdrawal symptoms have been reported to resolve with gabapentin in a 26-year-old woman and gabapentin and clonidine in a 27year-old man (94). A case similar to ours has been described in a 44-year-old man with a history of alcohol use and anxiety; gradually tapered-off dihydrocodeine and lofexidine were followed by rapid withdrawal symptom resolution (95). In our case, the psychiatric symptoms of our patient were more prominent and stubborn, and briefly trialed clonidine in the past had sorted no effect. Hence the need for something more specific for anxiety disorders. Cases of kratom withdrawal in a 47-year-old woman (96) and in a 24-year-old man with an autism spectrum disorder (97) have been treated with clonidine and hydroxyzine, similarly to ordinary opiate withdrawal syndromes. The latter case and four other cases of kratom withdrawal were treated successfully with buprenorphinenaloxone maintenance (98-100). A further withdrawal from combined kratom-tilidine addiction has been successfully treated with retarded morphine (101). Finally, a recent paper reported an unusual presentation of obsessive-compulsive disorderlike syndrome during kratom withdrawal that responded to lorazepam (102). We did not use treatments aimed at treating patient's kratom withdrawal, since the syndrome was mild despite being obstinate, but rather focused on the anxiety disorder, which usually responds to antidepressants. By treating our patient's background psychological symptoms, we were successful in reducing withdrawal symptomatology.

Kratom use has been often linked to liver toxicity. Kratom has been associated with biliary cholangitis and cholestasis in several cases $(67,103-110)$ and with one case of hepatomegaly (111), but also with acute hepatitis (112). Mitragynine inhibits hepatic and intestinal cytochrome P450 3A activities (113) and 
hepatic microsomal CYP2D6 (114), thus increasing blood levels of other concomitantly administered drugs that are metabolized by these isoenzymes, that is, most psychiatric drugs. This may expose to further hepatotoxicity $(115,116)$. Our patient did not develop liver abnormalities during his kratom use period, despite the fact he was concurrently using alcohol. We did not perform kratom quantification analyses in our patient throughout the treatment period. This was because the patient refused to provide organic specimens or leaves for forensic analyses. There are reliable methods for detecting mitragynine and its derivatives in the urine (117) and in plant and extracts (118) for forensic purposes, but these are not currently routine practice. There is need for standardizing methods of kratom alkaloid detection in reported users.

A limitation of the current review is that the supposed benefits-to-risk ratio of kratom use cannot be currently addressed adequately. There is insufficient epidemiological documentation as to the extent of kratom use worldwide and in specific countries (119), so to estimate how many people use it and how many develop unwanted effects. Besides this, risks may increase, as many kratom users have concurrent other substance use (120), and this is difficult to disentangle. The most recent estimates in indicate kratom use in the adult US population is $0.8 \%$ for the past year and $1.3 \%$ lifetime (120). The debate on epidemiological issues is strong and ongoing, and points to the evergreen "more studies are needed" $(121,122)$. The advocates of kratom use to ease opioid dependence and harness its effects on strength and endurance while involved in work activities do not publish in scientific literature, but put forward their uncontrolled views and opinions in sites of their own property. Hence, it is an impervious task to try to respond to the question whether kratom use is relatively safe, but it appears it is not (123). Currently, there is not sufficient evidence to recommend changes in kratom regulation, nor to recommend the use of clomipramine in cases of kratom withdrawal.

Our patient showed while withdrawing from kratom mitigated signs and symptoms typical of opiate withdrawal, which were mixed with other psychiatric symptoms presumably linked to his background psychopathology. Knowing that the withdrawal is generally time-limited and mild, we chose to use an anxiety-specific agent, clomipramine, with preference for serotonin transporter over noradrenaline transporter inhibition, which is a tricyclic antidepressant used in anxiety disorders and obsessive-compulsive disorder and has shown good evidence in these disorders. We used it at $75 \mathrm{mg} /$ day, which is on the lower range of clinical effectiveness for these disorders. Mitragynine counters serotonin $5-\mathrm{HT}_{2 \mathrm{~A}}$ receptors (29) and clomipramine downregulates the same receptors after chronic treatment $(124,125)$; furthermore, it has pain supressing effects even at low doses through spinal mechanisms (126). Hence, it is possible that some mitragynine withdrawal symptoms were alleviated concomitantly with clomipramine's anxiety relieving effects.
However, this is not the most likely mechanism whereby clomipramine reduced our patient's symptomatology. In fact, clomipramine may obviate for the opiate-like mitragynine withdrawal syndrome through interference with opioid receptors, which it was shown to bind (127); chronic, but not subacute clomipramine administration, induced a mu receptor down-regulation in the rat (128). In this case, clomipramine could reduce the quantity of opioid receptors in the need for occupation, as it occurs in opiate withdrawal. However, the response of human opioid receptors to chronic clomipramine appears to be weak (129). We are unsure about how improvement was obtained, but the timeline appears to match the usual onset of clomipramine antidepressant effects.

\section{CONCLUDING REMARKS}

Summarizing the above evidence, we may conclude that kratom may induce addiction, acute toxicity which may be sometimes lethal and, upon discontinuation, it induces a withdrawal syndrome, which may vary in intensity. In many instances that appeared in literature, kratom was regularly used by patients with psychiatric history and/or substance use disorders. Legislations should take very seriously peer-reviewed published evidence and regulate the substance. In parallel, we need to enforce kratom detection methods in consent-providing users for forensic purposes. International drug policies should be coordinated and inform the public about kratom and other novel addictive drugs.

\section{DATA AVAILABILITY STATEMENT}

The original contributions presented in the study are included in the article/supplementary material, further inquiries can be directed to the corresponding author/s.

\section{ETHICS STATEMENT}

The patient signed free informed consent for the publication of his case and all treatments received.

\section{AUTHOR CONTRIBUTIONS}

$\mathrm{AV}, \mathrm{SP}$, and SD saw the patient and wrote the first draft. FS, FN, and JC supervised the case and the writing of the manuscript. GK wrote the last draft and performed literature searches. All authors saw and approved the final version of the manuscript.

\section{ACKNOWLEDGMENTS}

We thank Librarians of the Library of the Sapienza University of Rome, Faculty of Medicine and Psychology Mimma Ariano, Ales Casciaro, Teresa Prioreschi, and Susanna Rospo for rendering precious bibliographical material accessible. 


\section{REFERENCES}

1. Raymond-Hamet A. Les alcaloïdes du Mitragyna speciosa Korthals [The alkaloids of Mitragyna speciosa Korthals-French]. Ann Pharm Fr. (1950) 8:482-90.

2. Ongley PA. Les alcaloïdes des Mitragyna [The alkaloids of Mitragyna]. Ann Pharm Fr. (1953) 11:594-602.

3. Shellard EJ, Lees MD. The Mitragyna species of Asia. V The anatomy of the leaves of Mitragyna speciosa. Korth Planta Med. (1965) 13:280-90. doi: 10.1055/s-0028-1100122

4. Beckett AH, Shellard EJ, Phillipson JD, Lee CM. Alkaloids from Mitragyna speciosa (Korth.). J Pharm Pharmacol. (1965) 17:753-735. doi: 10.1111/j.2042-7158.1965.tb07599.x

5. Beckett AH, Shellard EJ, Phillipson JD, Lee CM. The Mitragyna species of Asia. VII Indole alkaloids from the leaves of Mitragyna speciosa Korth. Planta Med. (1966) 14:277-88. doi: 10.1055/s-0028-1100055

6. Beckett AH, Shellard EJ, Phillipson JD, Lee CM. The Mitragyna species of Asia. VI. Oxindole alkaloids from the leaves of Mitragyna speciosa Korth. Planta Med. (1966) 14:266-76. doi: 10.1055/s-0028-1100054

7. Singh D, Narayanan S, Vicknasingam B. Traditional and non-traditional uses of Mitragynine (Kratom): a survey of the literature. Brain Res Bull. (2016) 126(Pt 1):41-6. doi: 10.1016/j.brainresbull.2016.05.004

8. Holmes EM. Some medicinal products from the straits settlements. Pharm J. (1895) 54:1095-6.

9. Burkill IH. A Dictionary of the Economic Products of the Malay Peninsula, Vol. II. London: Crown Agents for the Colonies (1935). p. 1480-3.

10. Wray I. Notes on the anti-opium remedy. Pharm J. (1907) 78:453.

11. Hassan Z, Muzaimi M, Navaratnam V, Yusoff NH, Suhaimi FW, Vadivelu $\mathrm{R}$, et al. From Kratom to mitragynine and its derivatives: physiological and behavioural effects related to use, abuse, and addiction. Neurosci Biobehav Rev. (2013) 37:138-51. doi: 10.1016/j.neubiorev.2012.11.012

12. Suwanlert S. A study of kratom eaters in Thailand. Bull Narc. (1975) 27:21-7.

13. Jansen KL, Prast CJ. Psychoactive properties of mitragynine (kratom). J Psychoactive Drugs. (1988) 20:455-7. doi: 10.1080/02791072.1988.10472519

14. Shellard EJ, Alam MZ. The quantitative determination of some mitragyna oxindole alkaloids after separation by thin-layer chromatography. I Ultraviolet spectrophotometry. J Chromatogr. (1968) 32:472-88. doi: 10.1016/s0021-9673(01)80520-3

15. Shellard EJ, Alam MZ. The quantitative determination of some mitragyna oxindole alkaloids after separation by thin-layer chromatography. II Colorimetry, using the Vitali-Morin reaction. J Chromatogr. (1968) 32:489501. doi: 10.1016/s0021-9673(01)80521-5

16. Shellard EJ, Alam MZ. The quantitative determination of some mitragyna oxindole alkaloids after separation by thin layer chromatography. I. Ultraviolet spectrophotometry. J Chromatogr. (1968) 33:347-69. doi: 10.1016/s0021-9673(00)98661-8

17. Beckett AH, Dwuma-Badu D, Haddock RE. Some new mitragyna-type indoles and oxindoles the influence of stereochemistry on mass spectra. Tetrahedron. (1969) 25:5961-9. doi: 10.1016/s0040-4020(01)83103-3

18. Shellard EJ. The alkaloids of Mitragyna with special reference to those of Mitragyna speciosa, Korth. Bull Narc. (1974) 26:41-55.

19. Ogata J, Kawamura M, Hakamatsuka T, Kikura-Hanajiri R. 改良 PCRRFLP 法によるKratom 品の [Discrimination of kratom products by an improved PCR-RFLP method-Japanese]. Yakugaku Zasshi. (2020) 140:15018. doi: 10.1248/yakushi.20-00170

20. Seaton JC, Tondeur R, Marion L. The structure of mitraphylline. Canad J Chem. (1958) 36:1031-8.

21. Yusof SR, Mohd Uzid M, Teh EH, Hanapi NA, Mohideen M, Mohamad Arshad AS, et al. Rate and extent of mitragynine and 7-hydroxymitragynine blood-brain barrier transport and their intra-brain distribution: the missing link in pharmacodynamic studies. Addict Biol. (2019) 24:935-45. doi: $10.1111 / \mathrm{adb} .12661$

22. Kruegel AC, Uprety R, Grinnell SG, Langreck C, Pekarskaya EA, Le Rouzic $\mathrm{V}$, et al. 7-Hydroxymitragynine is an active metabolite of mitragynine and a key mediator of its analgesic effects. ACS Cent Sci. (2019) 5:992-1001. doi: 10.1021/acscentsci.9b00141

23. Sinou V, Fiot J, Taudon N, Mosnier J, Martelloni M, Bun SS, et al. High-performance liquid chromatographic method for the quantification of
Mitragyna inermis alkaloids in order to perform pharmacokinetic studies. $J$ Sep Sci. (2010) 33:1863-9. doi: 10.1002/jssc.201000008

24. Flores-Bocanegra L, Raja HA, Graf TN, Augustinović M, Wallace ED, Hematian S, et al. The chemistry of kratom [Mitragyna speciosa]: Updated characterization data and methods to elucidate indole and oxindole alkaloids. J Nat Prod. (2020) 83:2165-77. doi: 10.1021/acs.jnatprod.0c00257

25. Zhou JY, Mo ZX, Zhou SW. Rhynchophylline down-regulates NR2B expression in cortex and hippocampal CA1 area of amphetamine-induced conditioned place preference rat. Arch Pharm Res. (2010) 33:557-65. doi: 10.1007/s12272-010-0410-3

26. Shao H, Yang Y, Mi Z, Zhu GX, Qi AP, Ji WG, et al. Anticonvulsant effect of Rhynchophylline involved in the inhibition of persistent sodium current and NMDA receptor current in the pilocarpine rat model of temporal lobe epilepsy. Neuroscience. (2016) 337:355-69. doi: 10.1016/j.neuroscience.2016.09.029

27. Yang Y, Ji WG, Zhu ZR, Wu YL, Zhang ZY, Qu SC. Rhynchophylline suppresses soluble $A \beta 1-42$-induced impairment of spatial cognition function via inhibiting excessive activation of extrasynaptic NR2Bcontaining NMDA receptors. Neuropharmacology. (2018) 135:100-12. doi: 10.1016/j.neuropharm.2018.03.007

28. León F, Habib E, Adkins JE, Furr EB, McCurdy CR, Cutler SJ. Phytochemical characterization of the leaves of Mitragyna speciosa grown in U.S.A. Nat Prod Commun. (2009) 4:907-10.

29. Matsumoto K, Mizowaki M, Takayama H, Sakai S, Aimi N, Watanabe H. Suppressive effect of mitragynine on the 5-methoxy-N,Ndimethyltryptamine-induced head-twitch response in mice. Pharmacol Biochem Behav. (1997) 57:319-23. doi: 10.1016/s0091-3057(96)00314-0

30. Demichel P, Gomond P, Roquebert J. alpha-Adrenoceptor blocking properties of raubasine in pithed rats. Br J Pharmacol. (1982) 77:449-54. doi: 10.1111/j.1476-5381.1982.tb09317.x

31. Roquebert J, Demichel P. Inhibition of the alpha 1 and alpha 2adrenoceptor-mediated pressor response in pithed rats by raubasine, tetrahydroalstonine and akuammigine. Eur J Pharmacol. (1984) 106:203-5. doi: 10.1016/0014-2999(84)90698-8

32. Roquebert J. Selectivity of raubasine stereoisomers for alpha 1- and alpha 2-adrenoceptors in the rat. Arch Int Pharmacodyn Ther. (1986) 282:252-61

33. Obeng S, Kamble SH, Reeves ME, Restrepo LF, Patel A, Behnke M, et al. Investigation of the adrenergic and opioid binding affinities, metabolic stability, plasma protein binding properties, and functional effects of selected indole-based kratom alkaloids. J Med Chem. (2020) 63:433-9. doi: 10.1021/acs.jmedchem.9b01465

34. Chang-Chien GC, Odonkor CA, Amorapanth P. Is kratom the new 'legal high' on the block?: the case of an emerging opioid receptor agonist with substance abuse potential. Pain Physician. (2017) 20:E195-E198.

35. Johnson LE, Balyan L, Magdalany A, Saeed F, Salinas R, Wallace S, et al. The potential for kratom as an antidepressant and antipsychotic. Yale J Biol Med. (2020) 93:283-9.

36. Váradi A, Marrone GF, Palmer TC, Narayan A, Szabó MR, Le Rouzic V, et al. Mitragynine/Corynantheidine pseudoindoxyls as opioid analgesics with mu agonism and delta antagonism, which do not recruit $\beta$-arrestin-2. J Med Chem. (2016) 59:8381-97. doi: 10.1021/acs.jmedchem.6b00748

37. Ridley HN. Malay plant names. J Straits Branch of the Royal Asiatic Society. (1897) 30:31-283.

38. Pert CB, Snyder SH. Opiate receptor: demonstration in nervous tissue. Science. (1973) 179:1011-4. doi: 10.1126/science.179.4077.1011

39. Terenius L. Stereospecific interaction between narcotic analgesics and a synaptic plasm a membrane fraction of rat cerebral cortex. Acta Pharmacol Toxicol. (1973) 32:317-20. doi: 10.1111/j.1600-0773.1973. tb01477.x

40. Zhou Y, Ramsey S, Provasi D, El Daibani A, Appourchaux K, Chakraborty $\mathrm{S}$, et al. Predicted mode of binding to and allosteric modulation of the $\mu$-opioid receptor by kratom's alkaloids with reported antinociception in vivo. Biochemistry. (2020). doi: 10.1021/acs.biochem.0c00658. [Epub ahead of print].

41. Matsumoto K, Mizowaki M, Suchitra T, Takayama H, Sakai S, Aimi N, et al. Antinociceptive action of mitragynine in mice: evidence for the involvement of supraspinal opioid receptors. Life Sci. (1996) 59:1149-55. doi: 10.1016/0024-3205(96)00432-8 
42. Matsumoto K, Mizowaki M, Suchitra T, Murakami Y, Takayama H, Sakai S, et al. Central antinociceptive effects of mitragynine in mice: contribution of descending noradrenergic and serotonergic systems. Eur J Pharmacol. (1996) 317:75-81. doi: 10.1016/s0014-2999(96)00714-5

43. Yamamoto LT, Horie S, Takayama H, Aimi N, Sakai S, Yano $\mathrm{S}$, et al. Opioid receptor agonistic characteristics of mitragynine pseudoindoxyl in comparison with mitragynine derived from Thai medicinal plant Mitragyna speciosa. Gen Pharmacol. (1999) 33:73-81. doi: 10.1016/s0306-3623(98)00265-1

44. Takayama H, Ishikawa H, Kurihara M, Kitajima M, Aimi N, Ponglux $\mathrm{D}$, et al. Studies on the synthesis and opioid agonistic activities of mitragynine-related indole alkaloids: discovery of opioid agonists structurally different from other opioid ligands. J Med Chem. (2002) 45:1949-56. doi: 10.1021/jm010576e

45. Thongpradichote S, Matsumoto K, Tohda M, Takayama H, Aimi N, Sakai S, et al. Identification of opioid receptor subtypes in antinociceptive actions of supraspinally-administered mitragynine in mice. Life Sci. (1998) 62:1371-8. doi: 10.1016/s0024-3205(98)00075-7

46. Warner ML, Kaufman NC, Grundmann O. The pharmacology and toxicology of kratom: from traditional herb to drug of abuse. Int J Legal Med. (2016) 130:127-38. doi: 10.1007/s00414-015-1279-y

47. Wilson LL, Harris HM, Eans SO, Brice-Tutt AC, Cirino TJ, Stacy HM, et al. Lyophilized kratom tea as a therapeutic option for opioid dependence. Drug Alcohol Depend. (2020) 216:108310. doi: 10.1016/j.drugalcdep.2020.108310

48. Wilson LL, Chakraborty S, Eans SO, Cirino TJ, Stacy HM, Simons CA, et al. Kratom alkaloids, natural and semi-synthetic, show less physical dependence and ameliorate opioid withdrawal. Cell Mol Neurobiol. (2021). doi: 10.1007/s10571-020-01034-7. [Epub ahead of print].

49. Veltri C, Grundmann O. Current perspectives on the impact of Kratom use. Subst Abuse Rehabil. (2019) 10:23-31. doi: 10.2147/SAR.S164261

50. Barber S. The Psychoactive Substances Bill 2015. Briefing Paper Number CBP 7334. London: House of Commons Library (2015).

51. Review of the Psychoactive Substances Act 2016 Presented to Parliament pursuant to Section 58 of the Psychoactive Substances Act 2016, November 2018. London: APS Group on behalf of the Controller of Her Majesty's Stationery Office (2018).

52. Kratom Herald. Indonesian Kratom Ban Has Been Moved Up From 2024 To 2022, And This Would Cut Off The Entire Supply Of Kratom In The United States, But There Is Some Hope. (2021). Available online at: https:// kratomherald.com/indonesian-kratom-ban-has-been-moved-up-from2024-to-2022-and-this-would-cut-off-the-entire-supply-of-kratom-inthe-united-states-but-there-is-some-hope/ (accessed on February 24, 2021).

53. Prozialeck WC, Avery BA, Boyer EW, Grundmann O, Henningfield JE, Kruegel AC, et al. Kratom policy: the challenge of balancing therapeutic potential with public safety. Int J Drug Policy. (2019) 70:70-7. doi: 10.1016/j.drugpo.2019.05.003

54. Swogger MT, Walsh Z. Kratom use and mental health: a systematic review. Drug Alcohol Depend. (2018) 183:134-40. doi: 10.1016/j.drugalcdep.2017.10.012

55. Eldridge WB, Foster C, Wyble L. Neonatal abstinence syndrome due to maternal kratom use. Pediatrics. (2018) 142:e20181839. doi: $10.1542 /$ peds.2018-1839

56. Mackay L, Abrahams R. Novel case of maternal and neonatal kratom dependence and withdrawal. Can Fam Physician. (2018) 64:121-2.

57. Chomchai S, Phuditshinnapatra J, Mekavuthikul P, Chomchai C. Effects of unconventional recreational drug use in pregnancy. Semin Fetal Neonatal Med. (2019) 24:142-8. doi: 10.1016/j.siny.2019.01.010

58. Davidson L, Rawat M, Stojanovski S, Chandrasekharan P. Natural drugs, not so natural effects: Neonatal abstinence syndrome secondary to 'kratom'. J Neonatal Perinatal Med. (2019) 12:109-12. doi: 10.3233/NPM-1863

59. Murthy P, Clark D. An unusual cause for neonatal abstinence syndrome. Paediatr Child Health. (2019) 24:12-4. doi: 10.1093/pch/pxy084

60. White CM. Pharmacologic and clinical assessment of kratom: An update. Am J Health Syst Pharm. (2019) 76:1915-25. doi: 10.1093/ajhp/zxz221

61. Bin Abdullah MFIL. Kratom dependence and treatment options: a comprehensive review of the literature. Curr Drug Targets. (2020) 21:156679. doi: 10.2174/1389450121666200719011653
62. Guddat S, Görgens C, Steinhart V, Schänzer W, Thevis M. Mitragynine (Kratom) - monitoring in sports drug testing. Drug Test Anal. (2016) 8:11148. doi: 10.1002/dta.1970

63. Nacca N, Schult RF, Li L, Spink DC, Ginsberg G, Navarette K, et al. Kratom adulterated with phenylethylamine and associated intracerebral hemorrhage: Linking toxicologists and public health officials to identify dangerous adulterants. J Med Toxicol. (2020) 16:71-4. doi: 10.1007/s13181-019-00741-y

64. Davidson C, Cao D, King T, Weiss ST, Wongvisavakorn S, Ratprasert N, et al. A comparative analysis of kratom exposure cases in Thailand and the United States from 2010-2017. Am J Drug Alcohol Abuse. (2020) 24:1-10. doi: 10.1080/00952990.2020.1836185

65. Babu KM, McCurdy CR, Boyer EW. Opioid receptors and legal highs: Salvia divinorum and Kratom. Clin Toxicol. (2008) 46:146-52. doi: 10.1080/15563650701241795

66. Burillo-Putze G, López Briz E, Climent Díaz B, Munné Mas P, Nogue Xarau $\mathrm{S}$, Pinillos MA, et al. Drogas emergentes (III): plantas y hongos alucinógenos [Emergent drugs (III): hallucinogenic plants and mushrooms-Spanish]. An Sist Sanit Navar. (2013) 36:505-18. doi: 10.4321/s1137-66272013000300015

67. Gandhi D, Ahuja K, Quade A, Batts KP, Patel L. Kratom induced severe cholestatic liver injury histologically mimicking primary biliary cholangitis: A case report. World J Hepatol. (2020) 12:863-9. doi: 10.4254/wjh.v12.i10.863

68. Singh D, Narayanan S, Grundmann O, Dzulkapli EB, Vicknasingam B. Effects of kratom (Mitragyna speciosa Korth.) use in regular users. Subst Use Misuse. (2019) 54:2284-9. doi: 10.1080/10826084.2019.1645178

69. Boyer EW, Babu KM, Adkins JE, McCurdy CR, Halpern JH. Self-treatment of opioid withdrawal using kratom (Mitragynia speciosa Korth). Addiction. (2008) 103:1048-50. doi: 10.1111/j.1360-0443.2008.02209.x

70. Smith KE, Lawson T. Prevalence and motivations for kratom use in a sample of substance users enrolled in a residential treatment program. Drug Alcohol Depend. (2017) 180:340-8. doi: 10.1016/j.drugalcdep.2017. 08.034

71. Ismail I, Wahab S, Sidi H, Das S, Lin LJ, Razali R. Kratom and future treatment for the opioid addiction and chronic pain: Periculo beneficium? Curr Drug Targets. (2019) 20:166-72. doi: 10.2174/1389450118666170425154120

72. Singh D, Narayanan S, Müller CP, Swogger MT, Rahim AA, Leong Bin Abdullah MFI, et al. Severity of kratom (Mitragyna speciosa Korth.) psychological withdrawal symptoms. J Psychoactive Drugs. (2018) 50:445-0. doi: 10.1080/02791072.2018.1511879

73. Singh D, NarayananS, Vicknasingam BK, Prozialeck WC, Ramanathan $\mathrm{S}$, Zainal H, et al. Severity of pain and sleep problems during Kratom (Mitragyna speciosa Korth.) cessation among regular kratom users. J Psychoactive Drugs. (2018) 50:266-74. doi: 10.1080/02791072.2018.1443234

74. Halpenny GM. Mitragyna speciosa: Balancing potential medical benefits and abuse. ACS Med Chem Lett. (2017) 8:897-9. doi: 10.1021/acsmedchemlett.7b00298

75. Saingam D, Assanangkornchai S, Geater AF, Lerkiatbundit S. Factor analytical investigation of Krathom (Mitragyna speciosa Korth.) withdrawal syndrome in Thailand. J Psychoactive Drugs. (2016) 48:76-85. doi: 10.1080/02791072.2016.1156791

76. Nelsen JL, Lapoint J, Hodgman MJ, Aldous KM. Seizure and coma following Kratom (Mitragynina speciosa Korth) exposure. J Med Toxicol. (2010) 6:4246. doi: 10.1007/s13181-010-0079-5

77. Tatum WO, Hasan TF, Coonan EE, Smelick CP. Recurrent seizures from chronic kratom use, an atypical herbal opioid. Epilepsy Behav Case Rep. (2018) 10:18-20. doi: 10.1016/j.ebcr.2018.04.002

78. Afzal H, Esang M, Rahman S. A case of kratom-induced seizures. Cureus. (2020) 12:e6588. doi: 10.7759/cureus.6588

79. Palasamudram Shekar S, Rojas EE, D’Angelo CC, Gillenwater SR, Martinez Galvis NP. Legally lethal kratom: a herbal supplement with overdose potential. J Psychoactive Drugs. (2019) 51:28-30. doi: 10.1080/02791072.2018.1562591

80. Overbeek DL, Abraham J, Munzer BW. Kratom (Mitragynine) ingestion requiring naloxone reversal. Clin Pract Cases Emerg Med. (2019) 3:24-6. doi: 10.5811/cpcem.2018.11.40588

81. Zuberi M, Guru PK, Bansal V, Diaz-Gomez J, Grieninger B, Alejos D. Undifferentiated shock and extreme elevation of procalcitonin 
related to kratom use. Indian J Crit Care Med. (2019) 23:239-41. doi: 10.5005/jp-journals-10071-23170

82. Abdullah HMA, Haq I, Lamfers R. Cardiac arrest in a young healthy male patient secondary to kratom ingestion: is this 'legal high' substance more dangerous than initially thought? BMJ Case Rep. (2019) 12:e229778. doi: 10.1136/bcr-2019-229778

83. Singh V, Mulla N, Wilson JL, Umansky A, Lee J, Stead T, et al. Intractable nausea and vomiting in naïve ingestion of kratom for analgesia. Int J Emerg Med. (2020) 13:42. doi: 10.1186/s12245-020-00301-0

84. Holler JM, Vorce SP, McDonough-Bender PC, Magluilo JJr, Solomon CJ, Levine B. A drug toxicity death involving propylhexedrine and mitragynine. J Anal Toxicol. (2011) 35:54-9. doi: 10.1093/anatox/35.1.54

85. Kronstrand R, Roman M, Thelander G, Eriksson A. Unintentional fatal intoxications with mitragynine and $O$-desmethyltramadol from the herbal blend Krypton. J Anal Toxicol. (2011) 35:242-7. doi: 10.1093/anatox/35.4.242

86. Neerman MF, Frost RE, Deking J. A drug fatality involving Kratom. J Forensic Sci. (2013) 58(Suppl 1):S278-S279. doi: 10.1111/1556-4029.12009

87. Karinen R, Fosen JT, Rogde S, Vindenes V. An accidental poisoning with mitragynine. Forensic Sci Int. (2014) 245:e29-32. doi: 10.1016/j.forsciint.2014.10.025

88. McIntyre IM, Trochta A, Stolberg S, Campman SC. Mitragynine 'Kratom' related fatality: a case report with postmortem concentrations. J Anal Toxicol. (2015) 39:152-5. doi: 10.1093/jat/bku137

89. Domingo O, Roider G, Stöver A, Graw M, Musshoff F, Sachs H, et al. Mitragynine concentrations in two fatalities. Forensic Sci Int. (2017) 271:e1e7. doi: 10.1016/j.forsciint.2016.12.020

90. Mitchell-Mata C, Thomas B, Peterson B, Couper F. Two fatal intoxications involving 3-methoxyphencyclidine. J Anal Toxicol. (2017) 41:503-7. doi: $10.1093 / \mathrm{jat} / \mathrm{bkx} 048$

91. Aggarwal G, Robertson E, McKinlay J, Walter E. Death from Kratom toxicity and the possible role of intralipid. J Intensive Care Soc. (2018) 19:61-3. doi: $10.1177 / 1751143717712652$

92. Wang C, Walker AE. Fatal mitragynine-associated toxicity in Canada: A case report and review of the literature. Acad Forensic Pathol. (2018) 8:340-6. doi: $10.1177 / 1925362118782076$

93. Matson M, Schenk N. Fatality of 33-year-old man involving kratom toxicity. J Forensic Sci. (2019) 64:1933-5. doi: 10.1111/1556-4029.14082

94. Stanciu CN, Gnanasegaram SA, Ahmed S, Penders T. Kratom withdrawal: A systematic review with case series. J Psychoactive Drugs. (2019) 51:12-8. doi: 10.1080/02791072.2018.1562133

95. McWhirter L, Morris S. A case report of inpatient detoxification after kratom (Mitragyna speciosa) dependence. Eur Addict Res. (2010) 16:229-31. doi: $10.1159 / 000320288$

96. Galbis-Reig D. A case report of kratom addiction and withdrawal. WMJ. (2016) 115:49-52. quiz 53.

97. Diep J, Chin DT, Gupta S, Syed F, Xiong M, Cheng J. Kratom, an emerging drug of abuse: a case report of overdose and management of withdrawal. $A A$ Pract. (2018) 10:192-4. doi: 10.1213/XAA.0000000000000658

98. Buresh M. Treatment of kratom dependence with buprenorphinenaloxone maintenance. $J$ Addict Med. (2018) 12:481-3. doi: 10.1097/ADM.0000000000000428

99. Schmuhl KK, Gardner SM, Cottrill CB, Bonny AE. Home induction and outpatient treatment of kratom use disorder with buprenorphinenaloxone: A case report in a young adult. Subst Abus. (2020) 41:311-4. doi: 10.1080/08897077.2019.1671945

100. Bowe A, Kerr PL. A complex case of kratom dependence, depression, and chronic pain in opioid use disorder: effects of buprenorphine in clinical management. J Psychoactive Drugs. (2020) 17:1-6. doi: 10.1080/02791072.2020.1773586

101. Müller E, Hillemacher T, Müller CP. Kratom instrumentalization for severe pain self-treatment resulting in addiction - A case report of acute and chronic subjective effects. Heliyon. (2020) 6:e04507. doi: 10.1016/j.heliyon.2020.e04507

102. Sablaban IM, Gautam M. The diagnosis of severe obsessions in the setting of kratom withdrawal and treatment with lorazepam: case report. J Addict Dis. (2020) 12:1-2. doi: 10.1080/10550887.2020.1813357

103. Kapp FG, Maurer HH, Auwärter V, Winkelmann M, Hermanns-Clausen M. Intrahepatic cholestasis following abuse of powdered kratom (Mitragyna speciosa). J Med Toxicol. (2011) 7:227-31. doi: 10.1007/s13181-011-0155-5
104. Dorman C, Wong M, Khan A. Cholestatic hepatitis from prolonged kratom use: a case report. Hepatology. (2015) 61:1086-7. doi: 10.1002/hep.27612

105. Drago JZ, Lane B, Kochav J, Chabner B. The harm in kratom. Oncologist. (2017) 22:1010-1. doi: 10.1634/theoncologist.2017-0279

106. Riverso M, Chang M, Soldevila-Pico C, Lai J, Liu X. Histologic characterization of kratom use-associated liver injury. Gastroenterology Res. (2018) 11:79-82. doi: 10.14740/gr990e

107. Osborne CS, Overstreet AN, Rockey DC, Schreiner AD. Drug-induced liver injury caused by kratom use as an alternative pain treatment amid an ongoing opioid epidemic. J Investig Med High Impact Case Rep. (2019) 7:2324709619826167. doi: 10.1177/2324709619826167

108. Fernandes CT, Iqbal U, Tighe SP, Ahmed A. Kratom-induced cholestatic liver injury and its conservative management. J Investig Med High Impact Case Rep. (2019) 7:2324709619836138. doi: 10.1177/2324709619836138

109. Aldyab M, Ells PF, Bui R, Chapman TD, Lee H. Kratom-induced cholestatic liver injury mimicking anti-mitochondrial antibody-negative primary biliary cholangitis: a case report and review of literature. Gastroenterology Res. (2019) 12:211-5. doi: 10.14740/gr1204

110. Antony A, Lee TP. Herb-induced liver injury with cholestasis and renal injury secondary to short-term use of kratom (Mitragyna speciosa). Am J Ther. (2019) 26:e546-e547. doi: 10.1097/MJT.0000000000000802

111. Griffiths CL, Gandhi N, Olin JL. Possible kratom-induced hepatomegaly: a case report. J Am Pharm Assoc (2003). (2018) 58:561-3. doi: 10.1016/j.japh.2018.05.006

112. Mousa MS, Sephien A, Gutierrez J, O'Leary C. N-acetylcysteine for acute hepatitis induced by kratom herbal tea. Am J Ther. (2018) 25:e550-e551. doi: 10.1097/MJT.0000000000000631

113. Tanna RS, Tian DD, Cech NB, Oberlies NH, Rettie AE, Thummel KE, et al. Refined prediction of pharmacokinetic kratom-drug interactions: timedependent inhibition considerations. J Pharmacol Exp Ther. (2020) 376:6473. doi: $10.1124 /$ jpet. 120.000270

114. Kamble SH, Sharma A, King TI, Berthold EC, León F, Meyer PKL, et al. Exploration of cytochrome P450 inhibition mediated drug-drug interaction potential of kratom alkaloids. Toxicol Lett. (2020) 319:148-54. doi: 10.1016/j.toxlet.2019.11.005

115. Hughes RL. Fatal combination of mitragynine and quetiapine - a case report with discussion of a potential herb-drug interaction. Forensic Sci Med Pathol. (2019) 15:110-3. doi: 10.1007/s12024-018-0049-9

116. Schimmel J, Dart RC. Kratom (Mitragyna Speciosa) liver injury: a comprehensive review. Drugs. (2020) 80:263-83. doi: 10.1007/s40265-019-01242-6

117. Philipp AA, Meyer MR, Wissenbach DK, Weber AA, Zoerntlein SW, Zweipfenning PG, et al. Monitoring of kratom or Krypton intake in urine using GC-MS in clinical and forensic toxicology. Anal Bioanal Chem. (2011) 400:127-35. doi: 10.1007/s00216-010-4464-3

118. Parthasarathy S, Ramanathan S, Murugaiyah V, Hamdan MR, Said MI, Lai CS, et al. A simple HPLC-DAD method for the detection and quantification of psychotropic mitragynine in Mitragyna speciosa (ketum) and its products for the application in forensic investigation. Forensic Sci Int. (2013) 226:1837. doi: $10.1016 /$ j.forsciint.2013.01.014

119. Henningfield JE, Grundmann O, Babin JK, Fant RV, Wang DW, Cone EJ. Risk of death associated with kratom use compared to opioids. Prev Med. (2019) 128:105851. doi: 10.1016/j.ypmed.2019.105851

120. Schimmel J, Amioka E, Rockhill K, Haynes CM, Black JC, Dart RC, et al. Prevalence and description of kratom (Mitragyna speciosa) use in the United States: a cross-sectional study. Addiction. (2021) 116:176-81. doi: $10.1111 /$ add. 15082

121. Grundmann O, Babin JK, Henningfield JE, Garcia-Romeu A, Kruegel AC, Prozialeck WC, et al. Kratom use in the United States: a diverse and complex profile. Addiction. (2021) 116:202-3. doi: 10.1111/add.15173

122. Schimmel J, Amioka E, Rockhill K, Haynes CM, Black JC, Dart RC, et al. Kratom use in the United States: Response to Grundmann et al. Addiction. (2021) 116:203-4. doi: 10.1111/add.15170

123. Eggleston W, Stoppacher R, Suen K, Marraffa JM, Nelson LS. Kratom use and toxicities in the United States. Pharmacotherapy. (2019) 39:775-7. doi: $10.1002 /$ phar. 2280

124. Todd KG, McManus DJ, Baker GB. Chronic administration of the antidepressants phenelzine, desipramine, clomipramine, or maprotiline decreases binding to 5-hydroxytryptamine2A receptors without affecting 
benzodiazepine binding sites in rat brain. Cell Mol Neurobiol. (1995) 15:36170. doi: $10.1007 / \mathrm{BF} 02089946$

125. Attar-Lévy D, Martinot J-L, Blin J, Dao-Castellana M-H, Crouzel C, Mazoyer B, et al. The cortical serotonin2 receptors studied with positronemission tomography and $\left[{ }^{18} \mathrm{~F}\right]$-setoperone during depressive illness and antidepressant treatment with clomipramine. Biol Psychiatry. (1999) 45:1806. doi: 10.1016/s0006-3223(98)00007-9

126. Kostadinov ID, Delev DP, Kostadinova II. Antinociceptive effect of clomipramine through interaction with serotonin $5-\mathrm{HT}_{2}$ and 5- $\mathrm{HT}_{3}$ receptor subtypes. Folia Med (Plovdiv). (2012) 54:69-77. doi: 10.2478/v10153-012-0008-2

127. Carydakis C, Bourhim N, Giraud P, Cantau P, Oliver C, Castanas E. Les antidépresseurs tricycliques interagissent directement avec les sites de liaison opiacés dans la médullosurrénale bovine [Direct interaction of tricyclic antidepressants with opiate binding sites in the bovine adrenal medullaFrench]. C R Acad Sci III. (1986) 302:419-22.

128. Benkelfat C, Aulakh CS, Bykov V, Rice KC, De Costa BR, Rothman RB. Apparent down-regulation of rat brain mu- and kappa-opioid binding sites labelled with $\left[{ }^{3} \mathrm{H}\right]$ cycloFOXY following chronic administration of the potent 5-hydroxytryptamine reuptake blocker, clomipramine. J Pharm Pharmacol. (1989) 41:865-7. doi: 10.1111/j.2042-7158.1989.tb06390.x

129. Naber D, Jungkunz G. Opiate receptor sensitivity in depressed patients before and after clomipramine treatment. J Affect Disord. (1986) 11:59-62. doi: 10.1016/0165-0327(86)90060-1

Conflict of Interest: The authors declare that the research was conducted in the absence of any commercial or financial relationships that could be construed as a potential conflict of interest.

Copyright (๑ 2021 Vento, de Persis, De Filippis, Schifano, Napoletano, Corkery and Kotzalidis. This is an open-access article distributed under the terms of the Creative Commons Attribution License (CC BY). The use, distribution or reproduction in other forums is permitted, provided the original author(s) and the copyright owner(s) are credited and that the original publication in this journal is cited, in accordance with accepted academic practice. No use, distribution or reproduction is permitted which does not comply with these terms. 


\section{OPEN ACCESS}

Edited by:

Fabrizio Schifano,

University of Hertfordshire,

United Kingdom

Reviewed by:

Amira Guirguis,

Swansea University, United Kingdom

Melanie L. Schwandt,

National Institutes of Health $(\mathrm{NIH})$

United States

${ }^{*}$ Correspondence:

Andreas Kimergård

andreas.kimergard@kcl.ac.uk

Specialty section:

This article was submitted to

Addictive Disorders,

a section of the journal

Frontiers in Psychiatry

Received: 17 August 2020 Accepted: 11 March 2021

Published: 01 April 2021

Citation:

Deluca P, Foley M, Dunne $J$ and Kimergård A (2021) The Severity of Dependence Scale (SDS) for Codeine:

Preliminary Investigation of the

Psychometric Properties of the SDS in an Online Sample of Codeine Users

From the UK

Front. Psychiatry 12:595706

doi: 10.3389/fpsyt.2021.595706
The Severity of Dependence Scale (SDS) for Codeine: Preliminary Investigation of the Psychometric Properties of the SDS in an Online Sample of Codeine Users From the UK

\author{
Paolo Deluca ${ }^{1}$, Michelle Foley ${ }^{2}$, Jacklyn Dunne ${ }^{1}$ and Andreas Kimergård ${ }^{1 *}$ \\ ${ }^{1}$ National Addiction Centre, King's College London, Institute of Psychiatry, Psychology and Neuroscience, London, \\ United Kingdom, ${ }^{2}$ School of Health Sciences, Waterford Institute of Technology, Waterford, Ireland
}

Objective: Investigate the psychometric properties of the Severity of Dependence Scale (SDS) for codeine and its association with aberrant codeine related behaviors.

Design: A voluntary and uncompensated cross-sectional online survey.

Setting: Online population ( $\geq 18$ years).

Respondents: Two hundred and eighty-six respondents (66\% women) who had used codeine containing medicines in the last 3 months and were living in the UK.

Results: Of the respondents (mean age $=35.4$ years, $S D=12.5$ ), more than half were employed. Only 3.5\% respondents reported no income. The majority of respondents (45.1\%) primarily obtained prescription-only codeine from a consultation with a health professional, whilst $40.9 \%$ mainly purchased "over-the-counter" codeine containing medicines in a pharmacy without a medical prescription. Principal component analysis indicated a single factor solution accounting for $75 \%$ of the variance. Factor loadings ranged from 0.83 to 0.89 . Cronbach's Alpha was high $(\alpha=0.92)$. Several behaviors relating to codeine use were found to significantly predict probable codeine dependence. These included: daily codeine use in the last 3 months (OR $=66.89$, $95 \% \mathrm{Cl}=15.8-283.18)$; tolerance to codeine $(\mathrm{OR}=32.14,95 \% \mathrm{Cl}=13.82-74.75)$; problems with role responsibility due to intoxication $(\mathrm{OR}=9.89,95 \% \mathrm{Cl}=4.95-19.78)$; having sought advice on the internet to manage codeine use $(O R=9.56,95 \%$ $\mathrm{Cl}=4.5-20.31)$; history of alcohol or drug treatment $(\mathrm{OR}=3.73,95 \% \mathrm{Cl}=1.88-7.43)$.

Conclusions: The SDS was acceptable and feasible to use to assess probable psychological codeine dependence in an online sample of people using codeine containing medicines. SDS scores were associated with behaviors known to be indicators of codeine dependence. Studies are needed in well-defined populations of people who use codeine to test the different aspects of psychometry of the scale compared against "gold standard" criterion [a diagnosis according to the Diagnostic and Statistical Manual of Mental Disorders (DSM-5)].

Keywords: psychometric validation, Severity of Dependence Scale, opioid misuse, codeine, addiction 


\section{STRENGHTS AND LIMITATIONS OF THIS STUDY}

- Advances the understanding of the use of screening tools and scales to assess dependence on codeine containing medicines for research purposes.

- The study recruited a broad cross-section of codeine users in the UK, providing an initial investigation of the psychometric properties of the Severity of Dependence Scale for codeine.

- Online purposive samples have unknown population characteristics which must be recognized when interpreting the findings of the present study.

- Studies in well-defined populations of people using codeine are needed to test different aspects of psychometry of the scale compared against independent "gold standard" criterion.

\section{INTRODUCTION}

In the UK, the use of codeine containing medicines and the resulting possibility of dependence and severe health outcomes (1) pose a burden on primary and secondary care, specialized addiction treatment (2) and mortality (3). Codeine is used in form of codeine-based Prescription-Only Medicines (POM) or Pharmacy medicines $(\mathrm{P})$, which contain a lower amount of codeine and may be sold under the supervision of a pharmacist without a medical prescription (sold "over-the-counter") (4). Codeine is currently controlled under the Misuse of Drugs Regulations 2001 classified as Schedule 5 (Controlled drugs excepted from the prohibition on importation, exportation and possession) (5).

Many codeine containing medicines include a combination of codeine and a non-opioid analgesic such as ibuprofen or paracetamol (6). In 2014, the UK accounted for nearly onesixth of the global consumption of codeine (7). Sales of codeine containing "over-the-counter" products in packs of 32 tablets more than doubled in the period of 2006 to 2008 from 5.3 to 11.1 $\mathrm{mn}(8)$.

During 2007 to 2016, the number of registered drug-related deaths involving codeine increased from 60 to 131 in England and Wales (9). In Scotland, codeine or a codeine-containing compound was implicated in an average of 19 deaths per year between 2003 and 2007, 27 deaths per year between 2008 and 2012, and 33 deaths per year between 2011 and 2015 (10). The accessibility to codeine is under scrutiny in many countries, including the UK, due to concerns of dependence and severe harm from excessive use and overdose of accompanying paracetamol and ibuprofen (11-13). The recent indicators of an emerging "codeine problem" in the UK expose the need for reliable and accurate instruments to identify and treat early signs of codeine dependence to reduce long-term use, mortality, and the economic burden of addiction treatment.

The Severity of Dependence Scale (SDS) is a simple and practical 5-item, 15-point scale used to assess the degree of psychological dependence across several substance classes (14). In research to date, the psychometric properties of the SDS have been investigated in populations using illicit drugs (1416), alcohol (17), and nicotine (18). Optimal cut-off scores on the SDS for probable psychological dependence, when measured against the presence of a diagnosis obtained from the Diagnostic and Statistical Manual of Mental Disorders (DSM-5), have been determined for amphetamine (19), cocaine $(20,21)$, benzodiazepines (22), alcohol (23), and cannabis (24). The scale has previously been used to determine the level of probable codeine dependence amongst adults in Australia reporting use of "over-the-counter" codeine (25). Further investigation of the psychometric properties of the SDS for codeine would add understanding and value to the use of the scale for research purposes and possibly in clinical settings.

Using data collected from a cross-sectional, self-completed, online survey of adults who used codeine, the article presents a preliminary investigation of (i) the psychometric properties of the SDS for people living in the UK and (ii) the relation between the scale and behaviors known to be indicators of codeine dependence. Scales to identify people who are codeine dependent which are reliable and simple to administer are currently needed to promote public health.

\section{METHODS}

\section{Ethics}

The study received ethics approval granted by the Psychiatry, Nursing, and Midwifery Research Ethics Subcommittee (PNM RESC), King's College London. REC Reference Number: PNM/14/15-110.

\section{Recruitment}

The survey was advertised on Facebook, Twitter, health and drug related websites and e-mail circulars to include a broad sample of people using codeine resembling the general population. Recruitment lasted between July 2015 and March 2016. The main inclusion criterion was use of codeine containing medicines, prescribed or "over-the-counter," on at least one occasion in the last 3 months. Only respondents over the age of 18 were asked to participate. Participation in the study was voluntary, anonymous and uncompensated. A more detailed account of the survey has previously been published (26).

\section{Sample}

The survey was embedded within the CODEMISUSED collaboration aiming to carry out national and international studies to estimate levels of codeine use, misuse and dependence in partner countries (Ireland, South Africa and the UK) (27). For this reason, the online survey was open to respondents from all countries. However, in these re-analyses of the data in the present study, it was decided to only include respondents living the UK for several reasons: (i) There is great disparity between levels of codeine consumption, availability of codeine as "over-the-counter" medicines or POM, the amount of codeine included in codeine containing medicines and regulation of advertising of codeine containing medicines across countries around the world $(11,28-30)$ which may affect aberrant codeine behavior differently. By limiting the sample to the 
UK, respondents completing the SDS were sourcing and using codeine under similar conditions and regulation; (ii) conducting analysis of the SDS according to nationality was not feasible as some nations were represented by very few respondents; and (iii) the survey was only available in English. Limiting the analysis to respondents living in the UK presumably reduces the risk of misunderstanding due to potential language barriers.

\section{Procedure}

The online survey was developed in Bristol Online Surveys (BOS) and consisted of 49 questions about demographic information, codeine use, codeine dependence, social factors, treatment history, and other substance use (26).

The SDS was included as part of this larger study questionnaire, with the scale items included as questions 28-32 out of a total of 49 questions. The wording of each item of the scale was adapted to enquire about the use of codeine in the last 3 months. Respondents were asked:

(i) In the last 3 months did you think your use of codeine was out of control? (Responses: "Never/almost never" $=0$; "Sometimes" = 1; "Often" = 2; "Always/nearly always" = 3).

(ii) In the last 3 months did the prospect of not taking codeine make you anxious? (Responses: "Never/almost never" $=0$; "Sometimes" = 1; "Often" = 2; "Always/nearly always" = 3).

(iii) In the last 3 months did you worry about your use of codeine? (Responses: "Never/almost never" = 0 ; "Sometimes" = 1; "Often" = 2; "Always/nearly always" = 3).

(iv) In the last 3 months did you wish you could stop taking codeine? (Responses: "Never/almost never" = 0; "Sometimes" = 1; "Often" = 2; "Always/nearly always" = 3).

(v) In the last 3 months how difficult did you find it to stop using codeine? (Responses: "Not difficult" = 0; "Quite difficult" $=1$; "Very difficult" $=2$; "Impossible" $=3$ ).

Responses were scored from 0 to 3 for a total score between 0 and 15.

Likely indicators of codeine dependence included frequency of use in the last 3 months, reported as a dichotomous variable (daily or non-daily use). Additional questions were asked about tolerance to codeine and withdrawal symptoms after the use of codeine. Respondents were asked to report if they had sought help to control their use of codeine from (i) a community pharmacist, (ii) a general medical practitioner (GP), or (iii) from the internet. Respondents were also asked about past treatment for alcohol and illicit drug use. The survey included items from the Alcohol, Smoking and Substance Involvement Screening Test (ASSIST) to investigate problems with role responsibility due to the use of codeine (31). Questions from a scale designed to measure reasons for substance use were included to investigate use of codeine for anxiety (32). Several questions about tampering of codeine containing medicines were developed for the study, including about extraction of codeine (otherwise known as "cold water extraction") (33) and drinking codeine cough syrups mixed with soft drinks or with alcohol. A question about life-time use of illicit drugs, such as cannabis, amphetamines, ecstasy, cocaine, and heroin was included in the survey.
The complete survey was reviewed by experts in codeine misuse and dependence and piloted amongst addiction treatment service users. The survey took between 15 and $20 \mathrm{~min}$ to complete.

\section{Data Analysis}

Data were downloaded from the online questionnaires and imported to SPSS. All data analyses were conducted using SPSS, version 24. Before undertaking analyses, all respondents living in countries other than the UK were removed from the dataset. Principal Component Analysis (PCA) was conducted for the five SDS items as proposed by Gossop et al. (14). PCA was applied to determine the number of dimensions and item loading structure. The Cronbach's Alpha coefficient was used to assess the internal consistency of the scale. Monovariate logistic regression analyses were conducted to estimate the associations between the presence of codeine dependence and individual behaviors relating to codeine use. Comparisons were made between a baseline comparison group consisting of non-dependent codeine users and codeine dependent users. For this part of the analysis, a SDS score of five or above indicated probable psychological dependence on codeine, consistent with previous use of the scale to assess dependence to codeine $(25,34)$. A score of below five indicated non-dependence. Independent variables were demographic characteristics, frequency of codeine use, tolerance, seeking help to manage codeine, past treatment for alcohol or drug use, social problems, codeine use for emotional distress, tampering of codeine containing medicines, and other substance use.

\section{Missing Data}

To reduce the amount of missing data, most items were mandatory in the computerized survey and respondents could not proceed to the next question without providing an answer. Missing data was therefore uncommon. However, data was missing for amount of codeine consumed on last occasion of use precluding an analysis of this item in the logistic regression model.

\section{RESULTS}

Between July 2015 and March 2016, 472 respondents using codeine in the last 3 months and over the age of 18 completed the survey online. Respondents from outside the UK were removed, leaving a total of 286 respondents in the final analysis. As Table $\mathbf{1}$ shows, $66.4 \%$ of these were female. The mean age of the sample was 35.4 years $(\mathrm{SD}=12.5)$ with a range of $18-71$ years. More than half of the respondents were employed full or part-time $(60.5 \%)$. The main source of obtaining codeine containing medicines was prescribed following a face-to-face consultation with a doctor (45.1\% of respondents). The second most common source was purchased "over-the-counter" in a pharmacy without a medical prescription ( $40.9 \%$ of respondents). In the 3 months prior to completing the survey, $39.2 \%(n=112)$ of the respondents had consumed codeine daily. A majority of 219 respondents (76.6\%) took less or equal to the maximum recommended daily dose of 
TABLE 1 | Respondent characteristics $(n=286)$.

\begin{tabular}{lc}
\hline Characteristic & \\
\hline Age, mean (SD) & $35.4(\mathrm{SD}=12.5)$ \\
Gender, $\boldsymbol{n}$ (\%) ${ }^{\mathrm{a}}$ & \\
Female & $190(66.4 \%)$ \\
Male & $92(32.1 \%)$ \\
Income, $\boldsymbol{n}$ (\%) & \\
Employment & $173(60.5 \%)$ \\
Student allowance & $38(13.3 \%)$ \\
Dependent on other & $17(5.9 \%)$ \\
Disability allowance & $17(5.9 \%)$ \\
No income & $10(3.5 \%)$ \\
Temporary benefit & $8(2.8 \%)$ \\
Pension & $7(2.4 \%)$ \\
Other & $8(2.8 \%)$ \\
Main type of codeine, $\boldsymbol{n}$ (\%) & \\
Prescription codeine & $129(45.1 \%)$ \\
"Over-the-counter" codeine & $117(40.9 \%)$ \\
Otherc & $40(14 \%)$ \\
\hline SD, Standard Deviation. & \\
${ }^{c}$ Eight respondents did not say (2.8\%). & \\
${ }^{c}$ Purchased online and obtained from friends and family. & \\
& \\
\hline
\end{tabular}

TABLE 2 | Factor loadings and percentage of variance accounted for.

\begin{tabular}{lc}
\hline & $\begin{array}{c}\text { Respondents (use of codeine in past } \mathbf{3} \text { months) } \\
\boldsymbol{n}=\mathbf{2 8 6}\end{array}$ \\
\hline SDS score, mean (SD) & $2.2(\mathrm{SD}=3.5)$ \\
Range & $0-15$ \\
Principal components analysis & \\
Number of factors & 1 \\
Factor loadings & \\
Item 1 & 0.89 \\
Item 2 & 0.88 \\
Item 3 & 0.87 \\
Item 4 & 0.87 \\
Item 5 & 0.83 \\
Item \% variance accounted for & 75 \\
Cronbach's alpha & 0.92 \\
\hline
\end{tabular}

codeine (240 mg), 31 (10.8\%) took more and 36 (12.6\%) did not provide this information or answered the question incorrectly.

\section{Variance and Consistency of the SDS}

The respondents answered all required questions for the SDS. The responses to the scale produced a full range of scores from 0 to 15 (Mean score $=2.2, \mathrm{SD}=3.5$ ). Principal component analysis (PCA) was undertaken, indicating a single factor solution which accounted for $75 \%$ of the variance in codeine dependence (Table 2). The SDS had high internal consistency (Cronbach's alpha $=0.92$ ).

\section{Associations With Aberrant Codeine Use}

Mono-variate logistic regression analyses were used to investigate the relation between codeine dependence and aberrant behaviors in themselves indicating codeine dependence. Non-dependent codeine users were the reference category (Table 3 ).

Compared with those who were not dependent on codeine, the group of people with probable codeine dependence were significantly more likely to report daily use of codeine (96.1 vs. $26.8 \%, p<0.01)$. In relation to experiences of physical dependence, there was a significantly higher proportion of codeine dependent respondents $(58.8 \%)$ who reported tolerance to codeine in comparison with non-dependent $(4.3 \%)(p<0.01)$.

SDS scores were investigated in relation to seeking help to control the use of codeine and specialized addiction treatment history. In the logistic regression model, independent variables that were found to significantly predict probable codeine dependence were having sought help on the Internet $(\mathrm{OR}=9.56$, 95\% CI $=4.5-20.31)$ and having sought help from a GP $(\mathrm{OR}=9.31,95 \% \mathrm{CI}=3.21-27.01)$. Codeine dependent respondents were more likely to have received treatment to manage alcohol and illicit drug use than non-dependent users (35.3\% compared to $12.8 \%, p<0.01)$.

Those who were dependent on codeine were more likely to report problems with role responsibility, such as missing appointments at work or at home due to intoxication, compared to those who were not dependent (52.9 vs. 10.2\%, p < 00.1). SDS scores were investigated in relation to whether a friend or relative or anyone else had expressed concern about the respondents' use of codeine, which was found to significantly predict codeine dependence $(\mathrm{OR}=8.74,95 \% \mathrm{CI}=4.39-17.38)$.

Non-medical use of codeine relating to depression and anxiety were found to significantly predict probable codeine dependence, including using codeine to stop worrying about a problem $(\mathrm{OR}=6.03,95 \% \mathrm{CI}=2.83-12.83)$ and using codeine to feel better when down or depressed $(\mathrm{OR}=5.41,95 \% \mathrm{CI}=2.77$ 10.55).

The group of people with probable codeine dependence had a high proportion of respondents who had consumed codeine cough syrups mixed with soft drinks, juice or alcohol (25.5\%) compared to the group of non-dependent respondents reporting this behavior $(9.4 \%)(p<0.01)$.

There was no significant association between probable codeine dependence and consuming codeine extracted from codeine containing medicines or life-time illicit drug use.

\section{DISCUSSION}

This study demonstrates the feasibility of screening 286 respondents to an online cross-sectional survey for probable codeine dependence using the SDS. Pilot testing of the survey indicated that the five SDS items were easy to understand and the assessment easy to complete. The high questionnaire completion rate to the scale (all items of the scale were completed by all 286 respondents) shows that the SDS was acceptable to use as part of a larger survey study. PCA showed a single factor solution accounting for $75 \%$ of the variance. The alpha value 
TABLE 3 | SDS score and its association with aberrant codeine related behaviors.

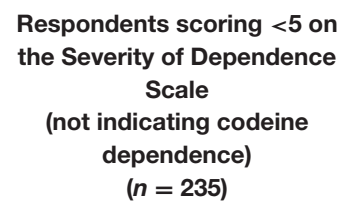

Codeine consumption

Daily use in last 3 months

Physical dependence

Codeine tolerance

Sought advice to manage the use of codeine

On the Internet

From a GP

Drug addiction treatment

Received treatment to help control alcohol or drug use

Impact on social life

Problems with role responsibility due to codeine

Others expressed concern about use of codeine

Emotional distress

Used codeine to feel better when down or depressed

Used codeine to stop worrying about a problem

Codeine tampering

Consumed codeine extracted from codeine containing medicines

Drinking codeine cough syrups mixed with soft drink, juice or alcohol

Illicit drug use

Life-time substance use
$\%$

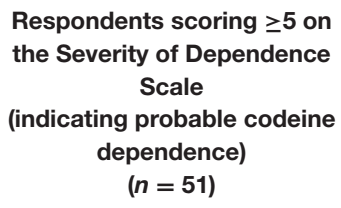

$\%$

OR $(95 \% \mathrm{Cl})$

\begin{tabular}{|c|c|c|}
\hline $26.8 \%$ & $96.1 \%$ ** & $66.89(15.8-283.18)$ \\
\hline $4.3 \%$ & $58.8 \%$ ** & $32.14(13.82-74.75)$ \\
\hline $6.8 \%$ & $41.2 \%$ ** & $9.56(4.5-20.31)$ \\
\hline $2.6 \%$ & $19.6 \%$ ** & $9.31(3.21-27.01)$ \\
\hline $12.8 \%$ & $35.3 \% \%^{\star \star}$ & $3.73(1.88-7.43)$ \\
\hline $10.2 \%$ & $52.9 \% \%^{\star *}$ & 9.89 (4.95-19.78) \\
\hline $10.6 \%$ & $51 \%$ ** & 8.74 (4.39-17.38) \\
\hline $13.2 \%$ & $45.1 \%$ ** & $5.41(2.77-10.55)$ \\
\hline $7.7 \%$ & $33.3 \%$ ** & 6.03 (2.83-12.83) \\
\hline $14.5 \%$ & $25.5 \%$ & $1.78(0.86-3.7)$ \\
\hline $.4 \%$ & $25.5 \%$ ** & $3.33(1.55-7.14)$ \\
\hline $.8 \%$ & $64.7 \%$ & $1.54(0.85-2.79)$ \\
\hline
\end{tabular}

${ }^{* *} \mathrm{P}<0.01 ; \mathrm{OR}$, odds ratio; $\mathrm{Cl}$, confidence interval.

was high (Cronbach's alpha $=0.92)$. Using a score of five and above to indicate probable psychological dependence to codeine, the study demonstrated associations between SDS scores and measures in themselves indicating probable codeine dependence, including daily consumption, tolerance, and problems with role responsibility due to codeine intoxication $(25,35)$. This compares favorably with a previous study using a similar online research design where probable codeine dependence (indicated by a cutoff score $\geq 5$ ) was associated with past alcohol and drug addiction treatment, chronic pain, and exceeding medical guidance for dose consumption (25). Online purposive samples have unknown population characteristics (36), but have in this study provided useful preliminary data and indication of using the SDS to assess probable codeine dependence.

\section{PCA and Consistency of the Scale}

PCA has been used to investigate the dimensionality of the SDS for heroin, cocaine, amphetamine and cannabis $(14,37)$. In this study, PCA indicated a single factor solution accounting for $75 \%$ of the variance, suggesting that the five SDS items are suitable as a single measure of psychological dependence. Previous research on the SDS, comparable to findings presented here, found single factor solutions accounting for a range of $45.5-80 \%$ of the variance $(14,17,37)$.
Cronbach's alpha was used as a measure of internal consistency. According to previous research, values of $\geq 0.70$ were considered adequate (38). An alpha value of 0.92 in the study is equal to or slightly higher than in previous investigations of the scale $(14,24,37)$. In addition to the PCA analyses, a high alpha value is also a necessary condition for unidimensionality (14).

While the conducted analyses, including the PCA, satisfy a number of criteria to account for the SDS as a single measure of psychological dependence on codeine, they do not account for how well the SDS determines if respondents have the condition or not. As such the diagnostic properties of the SDS are unclear until further analyses can be completed comparing SDS scores against indicators of codeine dependence from the DSM-5.

\section{External Validation}

Using a cut-off score of five or above, the validity of the SDS score is supported by the association with codeine related behaviors known to be related to the severity of codeine dependence. These include exceeding dose recommendations, daily use, chronic pain, psychological distress, past alcohol and drug addiction treatment, and codeine use to prevent withdrawal symptoms $(25,34,35)$. The results obtained in this study show that probable codeine dependence was associated with daily use over the past 3 months, having sought advice and treatment to manage dependence, drinking codeine cough syrup mixed with juice and 
alcohol, having experienced that other expressed concern about codeine use and using codeine for emotional distress.

A well-known limitation of the SDS is that it was designed to measure psychological elements of dependence, such as compulsion or craving, whilst excluding components relating to physical dependence like tolerance and withdrawal caused by neuroadaptation (14). It is notable in this respect that respondents who were codeine dependent according to the SDS were significantly more likely to report tolerance to codeine than those who were not codeine dependent, supporting the validity of the SDS by its association with this central component of physical dependence.

\section{Limitations}

Whilst, to our knowledge, this is the first study to report on the psychometric properties of the SDS for codeine, the sample size restricts inference of these results to wider populations of people who are using codeine. The sample size is relatively small when considering the time during which the survey was open for recruitment. Lack of data and understanding of codeine dependent populations in the UK impede the construction of a sampling frame and make the representativeness of our sample difficult to measure. Furthermore, it must be noted that online purposive sampling has biases due to unknown characteristics of people who participate in online communities and forums (36). Using online recruitment potentially excludes those with no immediate access to the Internet and may restrict respondents to those with a certain income, social situation and level of education. The differences between levels of codeine dependence and associated problems in online and non-online populations are currently unclear. Missing data precluded an analysis of codeine dose consumption amongst non-dependent and dependent respondents, although dose is a well-known indicator of problematic medicine use (25). Though our findings suggest that a score of 5 and above is an acceptable indicator of probable codeine dependence, the SDS was not designed as a screening tool to decide categorically between non-dependence and dependence (14). Further research is therefore required to compare the adopted cut-off score of 5 against a validated screening tool diagnosing substance dependence. Further research should also explore the use of the SDS compared against a validated diagnostic assessment in different age groups and according to gender.

\section{Implications for Research}

Further studies are needed in well-defined populations to test the different aspects of psychometry of the SDS for codeine to determine its feasibility and validity in research settings. Studies should also investigate the validity of the SDS within different settings, such as primary care, community pharmacies and specialized addiction services. The test-retest reliability of the SDS for codeine is not known. Data that provides an indication of the stability of SDS scores across occasions (39) would add additional value to the scale.

\section{Implications in Practice}

Previous studies have determined a cut-off point on the SDS that discriminates between the presence and absence of a DSM-5 diagnosis for substance dependence suggesting its implementation and usefulness in clinical settings. These studies found a SDS score of 3 or above optimal for characterizing a DSM-5 diagnosis of alcohol dependence (23), whereas a cut-off score of 7 was found to be the appropriate threshold for dependence to benzodiazepines (22). In this study, several factors relating to aberrant codeine use were associated with probable codeine dependence when using a cut-off score of 5 . Research with people attending specialized drug addiction treatment for codeine would enable a comparison between SDS scores and DSM-5 diagnosis, possibly enabling its use in clinical settings as a quick way of determining possible psychological dependence on codeine. Obtaining good assessment amongst people presenting with substance use typically improves care and use of screening, assessment and monitoring tools is recommended (40). This study demonstrated that the SDS is useful as a screening tool for research purposes, which can be included in larger study questionnaires with an excellent response rate presumably due to its short length.

\section{DATA AVAILABILITY STATEMENT}

The raw data supporting the conclusions of this article will be made available by the authors, without undue reservation.

\section{ETHICS STATEMENT}

The study involving human participants was reviewed, approved and received ethics approval granted by the Psychiatry, Nursing, and Midwifery Research Ethics Subcommittee (PNM RESC), King's College London. REC Reference Number: PNM/14/15-110. The patients/participants provided their written informed consent online to participate in this study.

\section{AUTHOR CONTRIBUTIONS}

$\mathrm{AK}, \mathrm{MF}$, and $\mathrm{PD}$ developed the survey used with people who had recently used codeine. The ongoing monitoring of the survey and recruitment was managed by AK. Data analysis was conducted by AK, MF, and JD. All authors contributed to the writing of the paper, with writing and analyses led by PD and $\mathrm{AK}$.

\section{FUNDING}

This work was supported by the European Community's Seventh Framework Programme FP7/2007-2013 under grant agreement no. 611736. AK and PD, King's College London, are supported by the National Institute for Health Research (NIHR) Applied Research Collaboration South London (NIHR ARC South London) at King's College 
Hospital NHS Foundation Trust. PD was also supported by the National Institute for Health Research (NIHR) Maudsley Biomedical Research Center (BRC). The views expressed are those of the author and not necessarily those of the NIHR or the Department of Health and Social Care. JD was funded by a grant from Guy's and St Thomas' Charity.

\section{REFERENCES}

1. Frei MY, Nielsen S, Dobbin MDH, Tobin CL. Serious morbidity associated with misuse of over-the-counter codeine-ibuprofen analgesics: a series of 27 cases. Med J Aust. (2010) 193:294-6. doi: 10.5694/j.1326-5377.2010.tb03911.x

2. Parry CDH, Deluca P, Cooper R, Van Hout MC. Do we have sufficient information to optimally inform regulatory or other policy decisions about medications containing codeine? Addiction. (2015) 110:1690-1. doi: 10.1111/add.13047

3. Office for National Statistics. Deaths Related to Drug Poisoning in England and Wales: 2018 Registrations. London: Office for National Statistics (2019).

4. King LA. Forensic Chemistry of Substance Misuse. Cambridge: The Royal Society of Chemistry (2009).

5. Statutory Instruments. 2001 No. 3998 Dangerous Drugs. The Misuse of Drugs Regulations. (2001). Available online at: https://www.legislation.gov.uk/uksi/ 2001/3998/pdfs/uksi_20013998_en.pdf(accessed March 9, 2021).

6. Foley M, Breindahl T, Hindersson P, Deluca P, Kimergård A. Misuse of "overthe-counter" codeine analgesics: does formulation play a role? Public Health. (2016) 130:95-6. doi: 10.1016/j.puhe.2015.10.006

7. International Narcotics Control Board. Estimated World Requirements for 2016: Statistics for 2014. Vienna: United Nations (2015).

8. Reed DK, Witton MJ, Cornish R, Hickman PM, Strang PJ. The Changing Use of Prescribed Benzodiazepines and z-Drugs and of Over-the-Counter CodeineContaining Products in England: A Structured Review of Published English and International Evidence and Available Data to Inform Consideration of the Extent of Dependence and Harm. London and Bristol: The National Addiction Centre, Kings College London and School of Social and Community Medicine, University of Bristol, United Kingdom (2011).

9. Office for National Statistics. Deaths Related to Drug Poisoning in England and Wales: 2016 registrations. London: Office for National Statistics (2017).

10. National Records of Scotland. Drug-Related Deaths in Scotland in 2015. Edinburgh: National Records of Scotland (2016).

11. Tobin CL, Dobbin M, McAvoy B. Regulatory responses to over-the-counter codeine analgesic misuse in Australia, New Zealand and the United Kingdom. Austr N Zeal J Public Health. (2013) 37:483-8. doi: 10.1111/1753-6405.12099

12. Harris K, Jiang A, Knoeckel R, Isoardi KZ. Rescheduling codeine-containing analgesics reduced codeine-related hospital presentations. Med J Aust. (2020) 212:328. doi: 10.5694/mja2.50400

13. Therapeutic Goods Administration. Final Decisions and Reasons for Decisions by Delegates of the Secretary to the Department of Health. (2017). Available online at: https://www.tga.gov.au/sites/default/files/scheduling-delegatesfinal-decision-codeine-december-2016.pdf (accessed March 9, 2021).

14. Gossop M, Darke S, Griffiths P, Hando J, Powis B, Hall W, et al. The Severity of Dependence Scale (SDS): psychometric properties of the SDS in English and Australian samples of heroin, cocaine and amphetamine users. Addiction. 90:607-14. doi: 10.1111/j.1360-0443.1995.tb02199.x

15. Bruno R, Matthews AJ, Topp L, Degenhardt L, Gomez R, Dunn M. Can the severity of dependence scale be usefully applied to "ecstasy?" Neuropsychobiology. (2009) 60:137-47. doi: 10.1159/000253550

16. Kassim S, Islam S, Croucher R. Validity and reliability of a Severity of Dependence Scale for khat (SDS-khat). J Ethnopharmacol. (2010) 132:5707. doi: 10.1016/j.jep.2010.09.009

17. Gossop M, Marsden J, Stewart D. Dual dependence: assessment of dependence upon alcohol and illicit drugs, and the relationship of alcohol dependence among drug misusers to patterns of drinking, illicit drug use and health problems. Addiction. (2002) 97:169-78. doi: 10.1046/j.1360-0443.2002.00028.x

\section{ACKNOWLEDGMENTS}

We thank the individuals and organizations assisting with recruitment, in particularly Bluelight, Action on Addiction, and the Painkiller Addiction Information Network. We thank Ian Russell for constructive criticisms and valuable comments on the manuscript.

18. Grassi MC, Enea D, Ferketich AK, Culasso F, Nencini P. Validity of the Italian version of the severity of dependence scale (SDS) for nicotine dependence in smokers intending to quit. Psychol Rep. (2014) 114:113. doi: 10.2466/18.15.PR0.114k16w7

19. Topp L, Mattick RP. Choosing a cut-off on the Severity of Dependence Scale (SDS) for amphetamine users. Addiction. (1997) 92:839-45. doi: 10.1111/j.1360-0443.1997.tb02953.x

20. González-Sáiz F, Domingo-Salvany A, Barrio G, Sánchez-Niubó A, Brugal MT, de la Fuente L, et al. Severity of dependence scale as a diagnostic tool for heroin and cocaine dependence. Eur Addict Res. (2009) 15:8793. doi: $10.1159 / 000189787$

21. Kaye S, Darke S. Determining a diagnostic cut-off on the Severity of Dependence Scale (SDS) for cocaine dependence. Addiction. (2002) 97:72731. doi: 10.1046/j.1360-0443.2002.00121.x

22. Cuevas CDL, Sanz EJ, Fuente JADL, Padilla J, Berenguer JC. The Severity of Dependence Scale (SDS) as screening test for benzodiazepine dependence: SDS validation study. Addiction. (2000) 95:245-50. doi: 10.1046/j.1360-0443.2000.95224511.x

23. Lawrinson P, Copeland J, Gerber S, Gilmour S. Determining a cut-off on the Severity of Dependence Scale (SDS) for alcohol dependence. Addict Behav. (2007) 32:1474-9. doi: 10.1016/j.addbeh.2006.09.005

24. Martin G, Copeland J, Gates P, Gilmour S. The Severity of Dependence Scale (SDS) in an adolescent population of cannabis users: reliability, validity and diagnostic cut-off. Drug Alcohol Depend. (2006) 83:903. doi: 10.1016/j.drugalcdep.2005.10.014

25. Nielsen S, Cameron J, Lee N. Characteristics of a non-treatment-seeking sample of over-the-counter codeine users: implications for intervention and prevention. J Opioid Manag. (2011) 7:363-70. doi: 10.5055/jom.2011. 0077

26. Kimergård A, Foley M, Davey Z, Dunne J, Drummond C, Deluca P. Codeine use, dependence and help-seeking behaviour in the UK and Ireland: an online cross-sectional survey. QJM. (2017) 110:559-64. doi: 10.1093/qjmed/hcx076

27. European Commission. Final Report Summary - CODEMISUSED (Codeine Use, Misuse and Dependence). Brussels. Available online at: https://cordis. europa.eu/project/id/611736/reporting (accessed March 9, 2021).

28. Foley M, Kelly P, Deluca P, Kimergård A. Advertising of over-the-counter codeine-containing medicines in the EU: differences in the regulation of advertising between member states. Pharm Med. (2018) 32:32733. doi: 10.1007/s40290-018-0245-7

29. Foley M, Harris R, Rich E, Rapca A, Bergin M, Norman I, et al. The availability of over-the-counter codeine medicines across the European Union. Public Health. (2015) 129:1465-70. doi: 10.1016/j.puhe.2015.06.014

30. International Narcotics Control Board. Narcotic Drugs: Estimated World Requirements for 2019. Vienna: United Nations (2018).

31. World Health Organization. The Alcohol, Smoking and Substance Involvement Screening Test (ASSIST): Manual for Use in Primary Care. Geneva: World Health Organization (2010).

32. Boys A. Understanding reasons for drug use amongst young people: a functional perspective. Health Educ Res. (2001) 16:457-69. doi: 10.1093/her/16.4.457

33. Kimergård A, Deluca $\mathrm{P}$, Hindersson $\mathrm{P}$, Breindahl T. How resistant to tampering are codeine containing analgesics on the market? assessing the potential for opioid extraction. Pain Ther. (2016) 5:187-201. doi: 10.1007/s40122-016-0053-2

34. Van Hout MC, Horan A, Santlal K, Rich E, Bergin M. "Codeine is my companion:" misuse and dependence on codeine containing medicines in Ireland. Ir J Psychol Med. (2018) 35:275-88. doi: 10.1017/ipm.2015.60 
35. Sproule BA, Busto UE, Somer G, Romach MK, Sellers EM. Characteristics of dependent and non-dependent regular users of codeine. J Clin Psychopharmacol. (1999) 19:36772. doi: 10.1097/00004714-199908000-00014

36. Barratt MJ, Ferris JA, Lenton S. Hidden populations, online purposive sampling, and external validity: taking off the blindfold. Field Methods. (2015) 27:3-21. doi: 10.1177/1525822X14526838

37. van der Pol P, Liebregts N, de Graaf R, Korf DJ, van den Brink W, van Laar M. Reliability and validity of the Severity of Dependence Scale for detecting cannabis dependence in frequent cannabis users: the severity of dependence scale in frequent cannabis users. Int J Methods Psychiatr Res. (2013) 22:138-43. doi: 10.1002/mpr.1385

38. Streiner DL. Starting at the beginning: an introduction to coefficient alpha and internal consistency. $J$ Pers $\begin{array}{llr}\text { Assess. (2003) 80:99-103. doi: 10.1207/S15327752JPA8 } & \end{array}$ 001_18

39. Gossop M, Best D, Marsden J, Strang J. Test-retest reliability of the severity of dependence scale. Addiction. (1997) 92:353-4. doi: 10.1080/096521497 38439
40. Clinical Guidelines on Drug Misuse and Dependence Update 2017 Independent Expert Working Group. Drug Misuse and Dependence: UK Guidelines on Clinical Management. London: Department of Health (2017).

Conflict of Interest: AK was appointed to the list of experts for 2020-2023 used by the EU drug agency, the EMCDDA, for the assessment of risks posed by NPS.

The remaining authors declare that the research was conducted in the absence of any commercial or financial relationships that could be construed as a potential conflict of interest.

Copyright (c) 2021 Deluca, Foley, Dunne and Kimergård. This is an open-access article distributed under the terms of the Creative Commons Attribution License (CC $B Y)$. The use, distribution or reproduction in other forums is permitted, provided the original author(s) and the copyright owner(s) are credited and that the original publication in this journal is cited, in accordance with accepted academic practice. No use, distribution or reproduction is permitted which does not comply with these terms. 


\section{OPEN ACCESS}

Edited by:

Fabrizio Schifano,

University of Hertfordshire,

United Kingdom

Reviewed by:

Georgios Demetrios Kotzalidis,

Sapienza University of Rome, Italy

Laura Orsolini

University of Hertfordshire,

United Kingdom

*Correspondence: Sherry H. Stewart

sstewar!@dal.ca

Specialty section: This article was submitted to

Addictive Disorders,

a section of the journal

Frontiers in Psychiatry

Received: 12 December 2020 Accepted: 17 March 2021

Published: 27 April 2021

Citation:

Stewart SH, Chinneck $A$,

Thompson K, Afzali MH,

Nogueira-Arjona R, Mahu IT and

Conrod PJ (2021) Personality to

Prescription Drug Misuse in

Adolescents: Testing Affect

Regulation, Psychological

Dysregulation, and Deviance

Proneness Pathways.

Front. Psychiatry 12:640766.

doi: 10.3389/fpsyt.2021.640766
Personality to Prescription Drug Misuse in Adolescents: Testing Affect Regulation, Psychological Dysregulation, and Deviance Proneness Pathways

\author{
Sherry H. Stewart ${ }^{1,2 *}$, Annie Chinneck ${ }^{2}$, Kara Thompson ${ }^{3}$, Mohammad H. Afzali ${ }^{4}$, \\ Raquel Nogueira-Arjona ${ }^{2}$, Ioan T. Mahu ${ }^{2}$ and Patricia J. Conrod ${ }^{4}$
}

${ }^{1}$ Department of Psychiatry, Dalhousie University, Halifax, NS, Canada, ${ }^{2}$ Department of Psychology \& Neuroscience, Dalhousie University, Halifax, NS, Canada, ${ }^{3}$ Department of Psychology, St. Francis Xavier University, Antigonish, NS, Canada, ${ }^{4}$ Department of Psychiatry, Université de Montréal, Montréal, $P Q$, Canada

Background: Fifteen to 25-year-olds are the age group most likely to misuse prescription drugs. Few studies have tested theory-driven models of adolescent risk for prescription drug misuse. Moreover, rarely are distinct pathways to different forms of prescription drug misuse considered.

Methods: We tested mediational paths from personality to mental health symptoms to prescription drug misuse, informed by etiological models of addiction. We specified pathways from particular personality traits to unique forms of prescription drug misuse via specific mental health symptoms. We used semi-longitudinal data collected across two waves of the Co-Venture Trial. Our sample included students from 31 Canadian high schools tested in Grade $9(n=3,024)$ and again in Grade $10(n=2,869 ; 95 \%$ retention). Personality (hopelessness, anxiety sensitivity, impulsivity, sensation seeking) was assessed in Grade 9. Mental health symptoms (depression, anxiety, ADHD, conduct disorder) and prescription drug misuse (opioids, sedatives/tranquilizers, stimulants) were assessed at both time points.

Results: Consistent with the negative affect regulation model, hopelessness was specifically associated with opioid misuse via depressive symptoms, and anxiety sensitivity was specifically associated with sedative/tranquilizer misuse via anxiety symptoms. Consistent with positive affect regulation, sensation seeking was directly associated with stimulant misuse. Consistent with the psychological dysregulation model, impulsivity was associated with stimulant misuse via ADHD symptoms. And consistent with the deviance proneness model, impulsivity was also associated with unconstrained (i.e., all three forms of) prescription drug misuse via conduct disorder symptoms.

Conclusions: Screening for adolescents high in hopelessness, anxiety sensitivity, sensation seeking, or impulsivity and providing them with personality-matched 
cognitive-behavioral interventions may be helpful in preventing or mitigating prescription drug misuse. Our results point to the specific mental health symptoms that are important to target in each of these personality-matched interventions.

Keywords: adolescents, personality risk, prescription drug misuse, anxiety sensitivity, hopelessness, sensation seeking, impulsivity, mental health symptoms

\section{INTRODUCTION}

The National Survey on Drug Use and Mental Health defines prescription drug (PD) misuse as use of PDs "in any way that a doctor did not direct you to use them" including (a) use without a prescription of one's own; (b) use in greater amounts, for longer, or more often than prescribed; or (c) use in any other way that was not prescribed by a physician (1). Many young people consider PDs to be less harmful than illicit drugs (2). Due to their potency, potential for addiction, and overdose potential, however, PD misuse can be injurious or even fatal (3).

Of any age group, 15-25-year-olds are the most likely to misuse PDs (1). After cannabis, PDs are the drugs most commonly misused by North American adolescents (1, 4). One study showed that among adolescents aged 12-17, 5\% reported past year PD misuse (5). PDs are readily accessible to adolescents through legitimate medical prescriptions (6), diversion $(7,8)$, and online pharmacies $(9,10)$. These trends are concerning for several reasons. First, prescription opiate misuse increases risk for serious injury (11), respiratory depression, and death (12). Moreover, the prevalence of adolescent misuse of sedatives/tranquilizers, including novel designer benzodiazepines, is significantly increasing (13-15), couse with opioids is common (16), and sedative/tranquilizerrelated deaths increased by 137\% from 2007 to 2016 (17). Stimulant misuse is associated with adverse short-term (e.g., headaches, sleep problems, academic difficulties) and long-term effects [e.g., decreased likelihood of college graduation; (18)]. Adolescent-onset PD misuse is linked with elevated substance use disorder rates in adulthood (18, 19).

While several risk and protective factors for adolescent PD misuse have been identified [see review by (20)], few studies have tested theoretical models of adolescent risk for PD misuse (21). And although the predictors of PD misuse may vary considerably by drug class (22), little work has examined unique pathways to specific forms of PD misuse. One potential risk factor that may help fill both these identified gaps is personality: specific traits may present risk for particular classes of $\mathrm{PD}$ misuse via unique theory-informed pathways.

\section{Personality as a Risk Factor}

Personality is a robust predictor of addictive behavior [e.g., (23)]. Internalizing and externalizing traits have been reliably associated with an increased susceptibility for alcohol and illicit substance misuse in adolescence (24). Pihl and Peterson (25) developed a model that delineates four such traits. The first two traits in this model are internalizing. Hopelessness (HOP) involves the trait-like tendency to expect aversive events but not desirable ones $(26,27)$. Anxiety sensitivity (AS) involves the fear of anxiety-related sensations, due to an unrealistic expectation that such sensations will have catastrophic consequences (28). In adolescents, both HOP and AS are associated with coping motives for substance use (29). Young people high in these traits tend to preferentially misuse depressant drugs $(30,31)$. In adults, HOP uniquely predicts opioid dependence and AS uniquely predicts anxiolytic dependence $(30,32)$. The specificity of these paths has yet to be tested in adolescents.

The remaining two traits in Pihl and Peterson's (25) model are externalizing. Impulsivity (IMP), or impulsiveness, is the tendency to act without sufficient forethought (33). IMP has been associated with a pattern of polysubstance use $(34,35)$. Deficits in response inhibition make high IMP teens more susceptible to early experimentation and to later compulsive substance use (36). Sensation seeking (SS), or novelty seeking (37), involves the desire for novel and intense stimulation (38). High SS substance users are sensitive to the rewarding properties of drugs (39) and tend to specifically misuse stimulants (40) to study, stay awake/alert, "get high," "party," and experiment (41).

Traits from Pihl and Peterson's (25) four-factor personality vulnerability model have proven useful in predicting adolescent alcohol $(42)$ and illicit drug use $(43,44)$, emerging adult PD use $(31,45)$, and adult PD use (30). This model has yet to be applied to adolescent PD misuse.

\section{Etiological Models of Addiction}

Theoretically, these four traits exert their influence on substance use via negative and positive affect regulation, deviance proneness, and/or psychological dysregulation processes (39). The models most relevant to linking HOP, AS, SS, and IMP with PD misuse are described below (see also Table 1). These theoretical models have informed the mediators in the hypothesized paths from personality to PD misuse.

\section{Affect Regulation Models}

Affect regulation models theorize that drugs are taken to regulate emotions-either for negative reinforcement (i.e., a drug's ability to relieve negative affect) or positive reinforcement (i.e., a drug's hedonic effects) (31). Negative affect regulation involves PD use to avoid or control negative emotional states whereas positive affect regulation involves $\mathrm{PD}$ use to increase positive emotional states. This dichotomy is in keeping with McCabe et al.'s (46) work on PD misuse motives, which suggests that PDs are misused for self-medication (negative affect regulation) or recreation (positive affect regulation).

\section{Negative Affect Regulation}

Individuals high in HOP or AS are theoretically most prone to PD misuse for negative affect regulation (29). First, those high in 
TABLE 1 | Summary of theories and hypotheses.

\begin{tabular}{|c|c|c|}
\hline $\begin{array}{l}\text { Personality } \\
\text { trait }\end{array}$ & $\begin{array}{l}\text { Relevant } \\
\text { etiological } \\
\text { model }\end{array}$ & Derived hypotheses \\
\hline HOP, AS & $\begin{array}{l}\text { Negative affect } \\
\text { regulation }\end{array}$ & $\begin{array}{l}H 1: \mathrm{HOP} \rightarrow \text { depressive symptoms } \rightarrow \text { opioid } \\
\text { misuse } \\
\mathrm{H} 2: \mathrm{AS} \rightarrow \text { anxiety symptoms } \rightarrow \\
\text { sedative/tranquilizer misuse }\end{array}$ \\
\hline SS & $\begin{array}{l}\text { Positive affect } \\
\text { regulation }\end{array}$ & H3: SS $\rightarrow$ stimulant misuse \\
\hline IMP & $\begin{array}{l}\text { Deviance } \\
\text { proneness }\end{array}$ & $\begin{array}{l}H 4: \mathrm{IMP} \rightarrow \mathrm{CD} \text { symptoms } \rightarrow \text { opioid misuse } \\
\mathrm{IMP} \rightarrow \mathrm{CD} \text { symptoms } \rightarrow \\
\text { sedative/tranquilizer misuse } \\
\mathrm{IMP} \rightarrow \mathrm{CD} \text { symptoms } \rightarrow \text { stimulant misuse }\end{array}$ \\
\hline IMP & $\begin{array}{l}\text { Psychological } \\
\text { dysregulation }\end{array}$ & $\begin{array}{l}\text { H5: IMP } \rightarrow \text { ADHD symptoms } \rightarrow \text { stimulant } \\
\text { misuse }\end{array}$ \\
\hline
\end{tabular}

HOP are thought to misuse opioids to control or avoid symptoms of depression. High HOP adults preferentially misuse opioids over other substances (30-32). HOP also predicts adolescent depression (47), and depression increases risk of PD misuse (21). The negative affect regulation model suggests that depressive symptoms should mediate HOP's specific effect on opioid misuse.

Those high in AS are also theoretically prone to PD misuse for negative affect regulation but through a distinct pathway. Specifically, they are thought to misuse sedatives/tranquilizers to control or avoid anxiety symptoms. High AS adults preferentially misuse anxiolytics over other substances (30, 31). AS incrementally predicts anxiety disorder symptoms in children and adolescents $(48,49)$ and anxiety disorders are associated with increased risk for sedative/tranquilizer misuse (50). In sum, the negative affect regulation model supports two distinct and specific pathways: HOP to opioid misuse via depressive symptoms vs. AS to sedative/tranquilizer misuse via anxiety symptoms.

\section{Positive Affect Regulation}

Stimulants activate mesolimbic dopamine activity and increase positive mood (51). High SS individuals are theoretically most prone to stimulant misuse for positive affect regulation. SS is robustly related to sensitivity to drug reward (39) and to enhancement motivated substance use (31). High SS individuals preferentially misuse stimulants $(32,40)$. The positive affect regulation model suggests this is because SS underlies sensitivity to stimulant reinforcement (52). The positive affect regulation model suggests a direct pathway from SS to stimulant misuse that is not mediated through mental health symptoms.

\section{Deviance Proneness Model}

Another model relevant to understanding PD misuse is the deviance proneness model (53). High IMP individuals are thought to be prone to a broad, unconstrained pattern of PD misuse (opioid, sedative/tranquilizer, and stimulant), occurring amidst other "deviant" or antisocial behaviors. IMP is associated with comorbid addictive and antisocial behaviors (54). IMP in elementary school students is concurrently and prospectively associated with conduct problems (55). Conduct disorder (CD) symptom severity is associated with greater substance involvement (56), including unconstrained PD misuse (57), in adolescence. The deviance proneness model suggests that $\mathrm{CD}$ symptoms mediate IMP's effect on unconstrained PD misuse (i.e., all three types of PD misuse).

\section{Psychological Dysregulation Model}

The psychological dysregulation model is an alternative model for explaining the specific link of IMP to stimulant misuse. Individuals high in IMP are most prone to PD misuse resulting from an adverse environment triggering a heritable tendency toward psychological dysregulation (58). ADHD is an externalizing disorder characterized by high IMP (59). Individuals with ADHD (60) or high IMP levels (24) are more likely to misuse stimulants. While only $4 \%$ of $10-18$-year-olds endorse past-month stimulant misuse (61), $14 \%$ of $4-17$-yearolds with ADHD endorse past-2-week stimulant misuse (62). IMP's effect on stimulant misuse may be attributable, at least in part, to an inability to inhibit pre-potent responses (63). ADHD symptoms are associated with stimulant misuse even after controlling for prescribed use (64). The psychological dysregulation model suggests that symptoms of ADHD mediate IMP's specific effect on stimulant misuse.

\section{Objectives}

Nargiso et al. (20) reviewed 50 articles on adolescent PD misuse and identified the following limitations. First, most studies were cross-sectional. Second, non-demographic risk factors (e.g., personality, mental health symptoms) were understudied. Third, there was a lack of specificity regarding predictors of misuse across PD classes. The present study sought to address these limitations by examining predictors of different forms of PD misuse (i.e., opioid, sedative/tranquilizer, stimulant) in a large sample of Canadian adolescents, tested prospectively in Grades 9 and 10 through a "semi-longitudinal design." In this design, one part is longitudinal (i.e., tests of personality to mental health symptoms and personality to PD misuse) and the other part is cross-sectional (i.e., tests of mental health symptoms to PD misuse). We used a broad definition of PD misuse in the present study, involving use of a PD in any way not directed by a physician (1).

See Table 1 for a summary of our hypotheses. Based on the theories described above, we hypothesized that: in keeping with the negative affect regulation model, (H1) Grade 9 HOP would specifically predict Grade 10 opioid misuse via Grade 10 depressive symptoms, and (H2) Grade 9 AS would specifically predict Grade 10 sedative/tranquilizer misuse via Grade 10 anxiety symptoms; in keeping with the positive affect regulation model, (H3) Grade 9 SS would directly predict Grad 10 stimulant misuse; in keeping with the deviance proneness model, (H4) Grade 9 IMP would predict Grade 10 opioid misuse, sedative tranquilizer misuse, and stimulant use, all via Grade $10 \mathrm{CD}$ symptoms; and in keeping with the psychological dysregulation 
model, (H5) Grade 9 IMP would also predict Grade 10 stimulant misuse via Grade 10 ADHD symptoms.

\section{METHODS}

The present study's data was archival. It was collected as part of the Co-Venture Trial (65) examining the longer-term efficacy of personality-targeted substance misuse prevention. Assenting students from 31 high schools (public and private; English and French) in Montreal, Canada participated. Data was collected annually (during the fall and spring terms) beginning in September 2012. A web-based platform (Delosis Ltd., London, U.K.) was used to survey students during regular class times. At baseline, students were in Grade 7. The present study used data collected prospectively in Grade 9 (September 2014May 2015) and Grade 10 (September 2015-May 2016). Risk increases as adolescents transition from middle to high school (66). In Canada, high school normally runs from Grades 912 (67). We therefore excluded Grade 7-8 (i.e., middle school) data. Ethical approval was granted by Sainte-Justine Hospital's Research Ethics Board (approval number $=2012-396,3427$ ) and by each administrative school board.

\section{Participants}

Sample sizes were $n=3,024$ in Grade 9 and $n=2,869$ of these same students in Grade 10 (5\% attrition). See Table 2 for sample characteristics.

\section{Measures \\ Personality}

The 23-item Substance Use Risk Profile Scale (SURPS; 30) was used to assess personality as part of the Co-Venture Trial. The SURPS has four subscales: HOP (7 items; "I feel that I'm a failure"), AS (5 items; "It is frightening to feel dizzy or faint"), SS (6 items; "I like doing things that frighten me a little"), and IMP (5 items; "I usually act without stopping to think"). Participants responded using a 5-point Likert scale (1 strongly disagree to 5 strongly agree). Following reverse scoring of certain negatively keyed items, subscale scores were generated by summing component items. The SURPS was chosen for use in the large-scale Co-Venture survey given its brevity and its strong psychometric properties in both English (43) and French (73). These include acceptable to good internal consistency, factorial validity, convergent and discriminant validity (e.g., with similar personality measures), and concurrent, predictive, and incremental validity in relation to substance use and substancerelated problems in youth [e.g., $(31,43,74)]$. In the present sample, the subscales were internally consistent (see Table 2).

\section{Internalizing Symptoms}

The 18-item Brief Symptom Inventory-18 [BSI-18; (70)] was used to assess depression and anxiety symptoms. It measures pastweek psychological distress. In this study, only the Depression (6 items; "feeling blue") and Anxiety (6 items; "nervousness or shakiness inside") subscales were used. Participants responded using a 5-point Likert Scale (0 not at all to 4 extremely often). Subscale scores were generated by summing component items.
The BSI-18 has strong psychometric properties in both English (75) and French (76). In our sample, the subscales were internally consistent (see Table 2).

\section{Externalizing Symptoms}

The 25-item Youth Self-Report Strengths and Difficulties Questionnaire (SDQ; 73) was used to assess ADHD and CD symptoms. It measures symptoms over the past 6-months. In this study, only the Hyperactivity/Inattention (5 items; "restless, cannot sit still for long") and Conduct Problems (5 items; "often accused of lying or cheating") subscales were used (77). The remaining subscales were excluded as they pertain instead to prosocial (Prosocial Behavior) and internalizing (Emotional Symptoms, Peer Relationship Problems) behaviors (77). Participants responded using a 3-point Likert Scale (0 not true to 2 certainly true). Following reverse scoring of certain items, subscale scores were generated by summing component items. The SDQ has strong psychometric properties in both English (78) and French (79). In our sample, the subscales were internally consistent (see Table 2).

\section{Prescription Drug Misuse}

A modified and validated version of the Detection of Alcohol and Drug Problems in Adolescents (DEP-ADO; 77) assessed lifetime PD misuse for: (1) Opioids: e.g., "Codeine, Demerol, Morphine, Percodan, Methadone, Darvon, Opium, Dilaudid, or Talwin"; (2) sedatives: e.g., "barbiturates, sedatives, downers, or sleeping pills like Seconal and Quaaludes"; (3) tranquilizers: e.g., "Valium, Librium, or Ativan"; and (4) stimulants: e.g., "stimulants, speed, methamphetamine, amphetamine, or Benzedrine." Participants responded using a 6-point frequency scale ( 0 never to 5 every day). To deal with zero-inflation, items were scored dichotomously (i.e., $1=$ had used that $P D$ class, $0=$ had not). In keeping with our previous research (45), sedatives and tranquilizers were collapsed into a single category. The DEP-ADO has strong psychometrics and is available in both English (69) and French (80). It was developed for and validated with adolescents aged 14-17 years (i.e., Grades 9-11). It has a strong test-retest reliability $(r=0.94)$, acceptable to good internal consistency (Cronbach's alpha $=0.61-0.86$ ), and content, convergent, and criterion-related validity (sensitivity $=0.84$; specificity $=0.91)(69)$.

\section{Alcohol Misuse}

Alcohol misuse was also assessed using the modified DEP-ADO (69). This scale includes 10 yes/no items that pertain to lifetime issues with: physical health, psychological health, familial relationships, intimate relationships, academics, finances, delinquency, risky behavior, alcohol tolerance, and treatment seeking, attributable to one's alcohol use. This sole focus on alcohol was a change from the original DEP-ADO which asked these items for alcohol and other drugs combined (69). Items were summed to create a $0-10$ total score. Only those indicating a frequency of drinking greater than or equal to "weekends or once or twice during the week" on a previous DEP-ADO item were asked these alcohol misuse items; the others were skipped over these items and automatically assigned an alcohol misuse 
TABLE 2 | Frequencies and descriptive statistics.

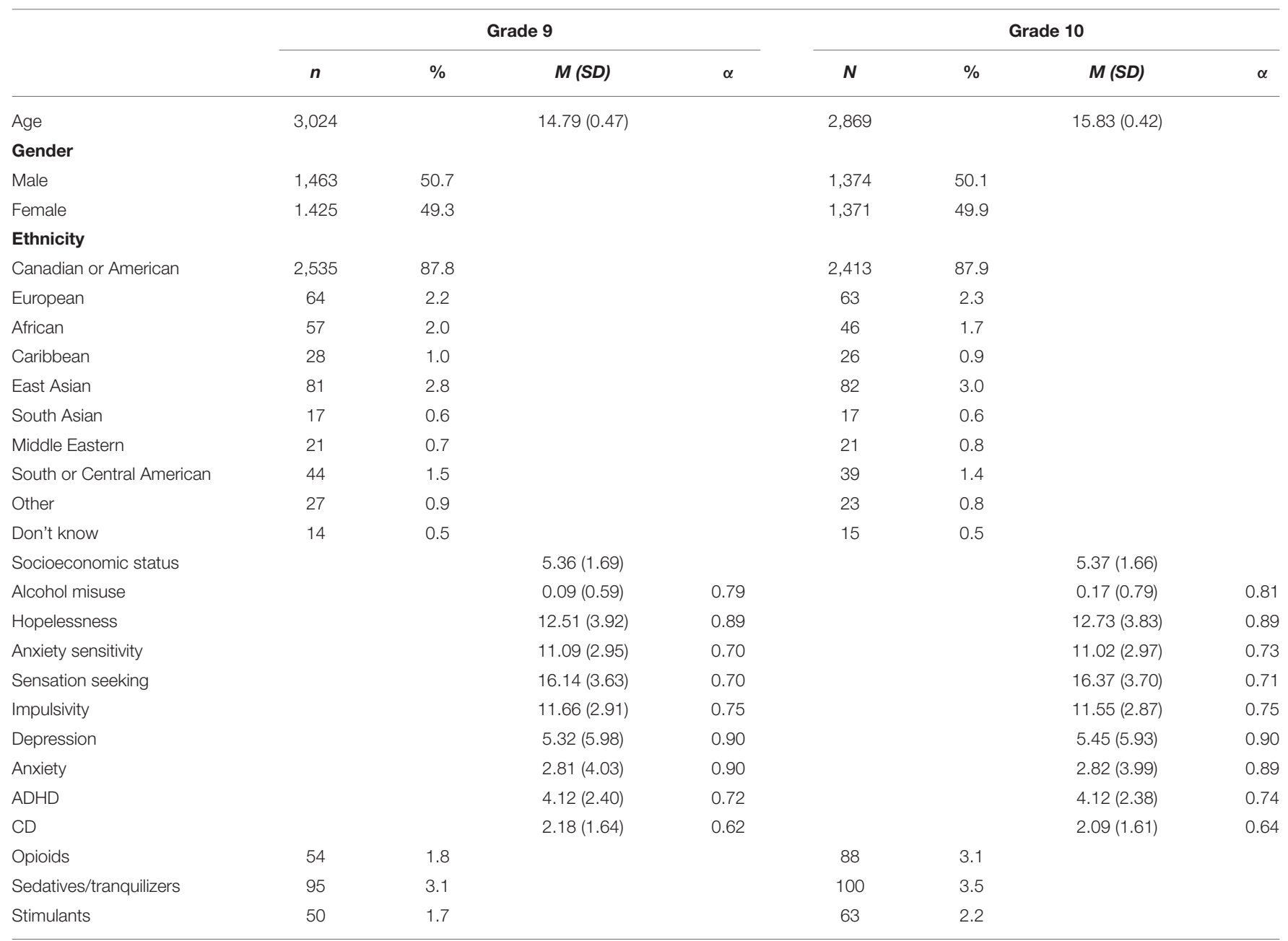

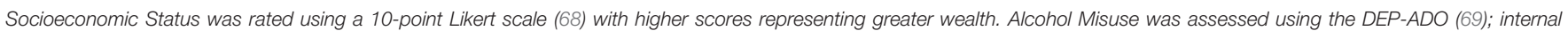

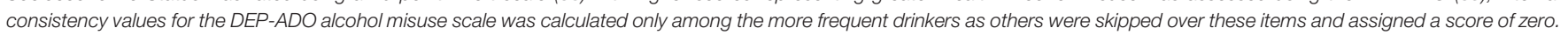

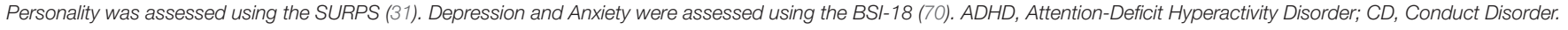

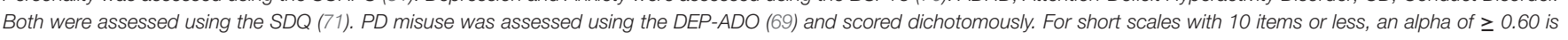
considered acceptable (72).

score of 0 . In the present sample, the alcohol misuse scale was internally consistent (see Table 2).

\section{Statistical Analyses}

Sample descriptive statistics were first calculated in SPSS 20.0. Ttests and chi square tests were used to compare baseline (Grade $9)$ characteristics of those retained $(n=2,869)$ vs. lost to followup $(n=155)$ in Grade 10. Correlations were specified between the personality, mental health, and PD misuse variables. The hypothesized model was then run in MPlus 7.11 (81). Because our dependent variables were categorical, a robust weighted least squares approach was used [ESTIMATOR = WLSMV; (82)]. Missing data was handled using pairwise deletion such that only those with data at both timepoints were used in hypothesis testing. We controlled for school and for Grade 9 mental health and PD misuse. Our model therefore accounts for new users. We also controlled for age, sex, ethnicity, and socioeconomic status (68), given their known effects on PD misuse $(20,83)$. Because high-intensity drinking is associated with PD misuse (84), we controlled for alcohol misuse as assessed on the DEP-ADO. These covariates were regressed onto all the outcome variables.

Standard indices were used to assess model fit. RMSEA $\leq$ 0.05 and CFI/TLI $\geq 0.95$ indicate good fit. RSMEA $\leq 0.08$ and CFI/TLI $\geq 0.90$ indicate adequate fit (85). Since chi-square values are often significant when the sample size is large (86), we did not interpret the chi-square as a fit statistic. Instead, we used the $\chi^{2} / d f$ ratio where a value $<3.0$ indicates good fit. Significant effects were detected at a 95\% confidence interval. Bootstrapped confidence intervals were used to determine the significance of indirect effects (i.e., significant if the confidence intervals did not cross zero). 


\section{RESULTS}

\section{Sociodemographic Features}

On average, students were $14.8(S D=0.5)$ years of age in Grade 9. There was a relatively equal split of the sample across gender at both waves. Most students were middle class and of Canadian or American descent (see Table 2).

\section{Personality}

Sample mean scores on the four subscales of the SURPS were relatively consistent with norms on the measure from a previously tested sample of adolescents (31). Scores remained relatively stable from Grade 9 to Grade 10 (see Table 2).

\section{Mental Health}

Sample mean scores on the BSI-18 measure of internalizing mental health symptoms indicated that levels of anxiety and depression symptoms were both relatively low, on average, in our non-clinical sample at baseline (Grade 9), with depression symptom scores somewhat higher than anxiety symptom scores overall. Sample mean scores on the SDQ measure of externalizing mental health symptoms similarly indicated that levels of ADHD and $\mathrm{CD}$ symptoms were both relatively low, on average, in our non-clinical sample at baseline (Grade 9), with ADHD symptom scores somewhat higher than $\mathrm{CD}$ symptoms scores overall. Scores remained relatively stable on all four measures of mental health symptoms from Grade 9 to Grade 10 (see Table 2).

\section{Substance Misuse}

In Grade 10, lifetime PD misuse rates were: 3\% for opioids, $4 \%$ for sedatives/tranquilizers, and 2\% for stimulants (see Table 2). Rates of misuse of each type of PD rose between Grade 9 and Grade 10 with the sharpest increase observed for opioid misuse. Levels of alcohol misuse also rose between Grade 9 and Grade 10 (see Table 2).

\section{Comparison of Students Retained vs. Lost to Follow-Up}

$T$-tests and chi-square tests suggested that, at baseline (Grade 9), those who were later lost to follow-up (Grade 10) were older, more likely to attend certain schools, and endorsed more personality vulnerability (HOP, SS, IMP), mental health symptoms (depression, $\mathrm{CD}, \mathrm{ADHD}$ ), alcohol misuse, and PD misuse.

\section{Correlations}

Bivariate correlations between study variables are displayed in Table 3. With respect to correlations between Grade 9 personality and Grade 10 mental health symptoms, HOP was most strongly associated with depressive symptoms, AS was most strongly associated with anxiety symptoms, and IMP and SS were most strongly associated with ADHD and CD symptoms (with IMP showing much stronger associations than SS in this regard). With respect to correlations between Grade 10 mental health symptoms and Grade 10 PD misuse, the strongest correlations were between CD symptoms with all three forms of PD misuse, anxiety and depressive symptoms with sedative/tranquilizer misuse, and ADHD symptoms with stimulant misuse. Grade
9 alcohol misuse was significantly associated with all Grade 9 personality factors save AS, with all four measures of Grade 10 mental health symptoms, and with all three forms of PD misuse in Grade 10, underlining the importance of alcohol misuse as a covariate.

\section{Hypothesis Tests}

Our hypothesized model (see Figure 1) showed good fit across fit indices: $\chi^{2}(71)=158.07, p<0.001 ; \chi^{2} / d f=2.23$; RMSEA $=0.02,90 \% \mathrm{CI}[0.02,0.03] ; \mathrm{CFI}=0.98 ; \mathrm{TLI}=0.96$. Indirect effects are reported in Table 4.

Grade 9 HOP significantly predicted Grade 10 depressive symptoms which in turn were significantly associated with Grade 10 opioid misuse. Consistent with $H 1$, the indirect effect was statistically significant $(p<0.05)$. Grade 9 AS significantly predicted Grade 10 anxiety symptoms which in turn were significantly associated with Grade 10 sedative/tranquilizer misuse. Consistent with $\mathrm{H} 2$, the indirect effect was statistically significant $(p<0.01)$.

Consistent with $\mathrm{H3}$, the direct path from Grade 9 SS to Grade 10 stimulant misuse was statistically significant. Grade 9 IMP significantly predicted Grade $10 \mathrm{CD}$ symptoms which in turn were significantly associated with Grade 10 opioid and sedative/tranquilizer misuse and marginally associated with Grade 10 stimulant misuse $(p=0.06)$. Consistent with $H 4$, all three indirect effects were statistically significant $(p<0.05$ for opioid and stimulant misuse; $p<0.01$ for sedative/tranquilizer misuse). Grade 9 IMP also significantly predicted Grade 10 ADHD symptoms which were in turn associated with Grade 10 stimulant misuse. Consistent with $\mathrm{H} 5$, the indirect effect was statistically significant $(p<0.05)$.

\section{Tests of Pathway Specificity}

To determine the specificity of the HOP to opioid misuse pathway via depression symptoms [H1] and the AS to sedative/tranquilizer misuse pathway via anxiety symptoms [H2], we examined modification indices (MIs). These indicated that the inclusion of paths from AS to depression (MI: 0.23) and HOP to anxiety (MI: 2.47) did not improve model fit (values $>3.84$ indicate that the model would be improved). Thus, for the sake of model parsimony, these were not added to the model.

\section{DISCUSSION}

\section{Main Findings}

In the present study, we sought to address the limitations of the extant literature on adolescent PD misuse, as outlined by Nargiso et al. (20). We applied the four-factor personality vulnerability model (25) to understanding risk for misuse of specific classes of PDs in adolescents. Moreover, we applied different theoretical models of addiction (39) to understanding specific pathways from personality to adolescents' future PD misuse, as mediated through specific sets of mental health symptoms.

Different personality traits showed effects on specific types of PD misuse through unique sets of mental health symptoms, consistent with different theoretical models of addiction, namely the negative and positive affective regulation, 
TABLE 3 | Correlation matrix.

\begin{tabular}{|c|c|c|c|c|c|c|c|c|c|c|c|c|}
\hline & 1 & 2 & 3 & 4 & 5 & 6 & 7 & 8 & 9 & 10 & 11 & 12 \\
\hline \multicolumn{13}{|l|}{ Grade 9} \\
\hline 1. Hopelessness & 1.00 & 0.27 & -0.03 & 0.32 & 0.11 & 0.46 & 0.35 & 0.33 & 0.22 & 0.07 & 0.11 & 0.09 \\
\hline 2. Anxiety sensitivity & & 1.00 & -0.12 & 0.19 & 0.02 & 0.23 & 0.33 & 0.14 & 0.05 & -0.03 & -0.01 & -0.01 \\
\hline 3. Sensation seeking & & & 1.00 & 0.25 & 0.14 & -0.01 & -0.02 & 0.12 & 0.16 & 0.13 & 0.11 & 0.11 \\
\hline 4. Impulsivity & & & & 1.00 & 0.11 & 0.22 & 0.18 & 0.45 & 0.41 & 0.11 & 0.11 & 0.12 \\
\hline 5. Alcohol harms & & & & & 1.00 & 0.08 & 0.06 & 0.08 & 0.13 & 0.12 & 0.17 & 0.20 \\
\hline \multicolumn{13}{|l|}{ Grade 10} \\
\hline 6. Depression & & & & & & 1.00 & 0.73 & 0.29 & 0.24 & 0.07 & 0.16 & 0.10 \\
\hline 7. Anxiety & & & & & & & 1.00 & 0.29 & 0.20 & 0.06 & 0.11 & 0.07 \\
\hline 8. ADHD & & & & & & & & 1.00 & 0.40 & 0.09 & 0.09 & 0.10 \\
\hline 9. $C D$ & & & & & & & & & 1.00 & 0.14 & 0.14 & 0.14 \\
\hline 10. Opioids & & & & & & & & & & 1.00 & 0.21 & 0.30 \\
\hline 11. Sedatives/tranquilizers & & & & & & & & & & & 1.00 & 0.17 \\
\hline 12. Stimulants & & & & & & & & & & & & 1.00 \\
\hline
\end{tabular}

$A D H D$ is attention-deficit hyperactivity disorder; $C D$ is conduct disorder. Bold correlations are significant at $p<0.05$.

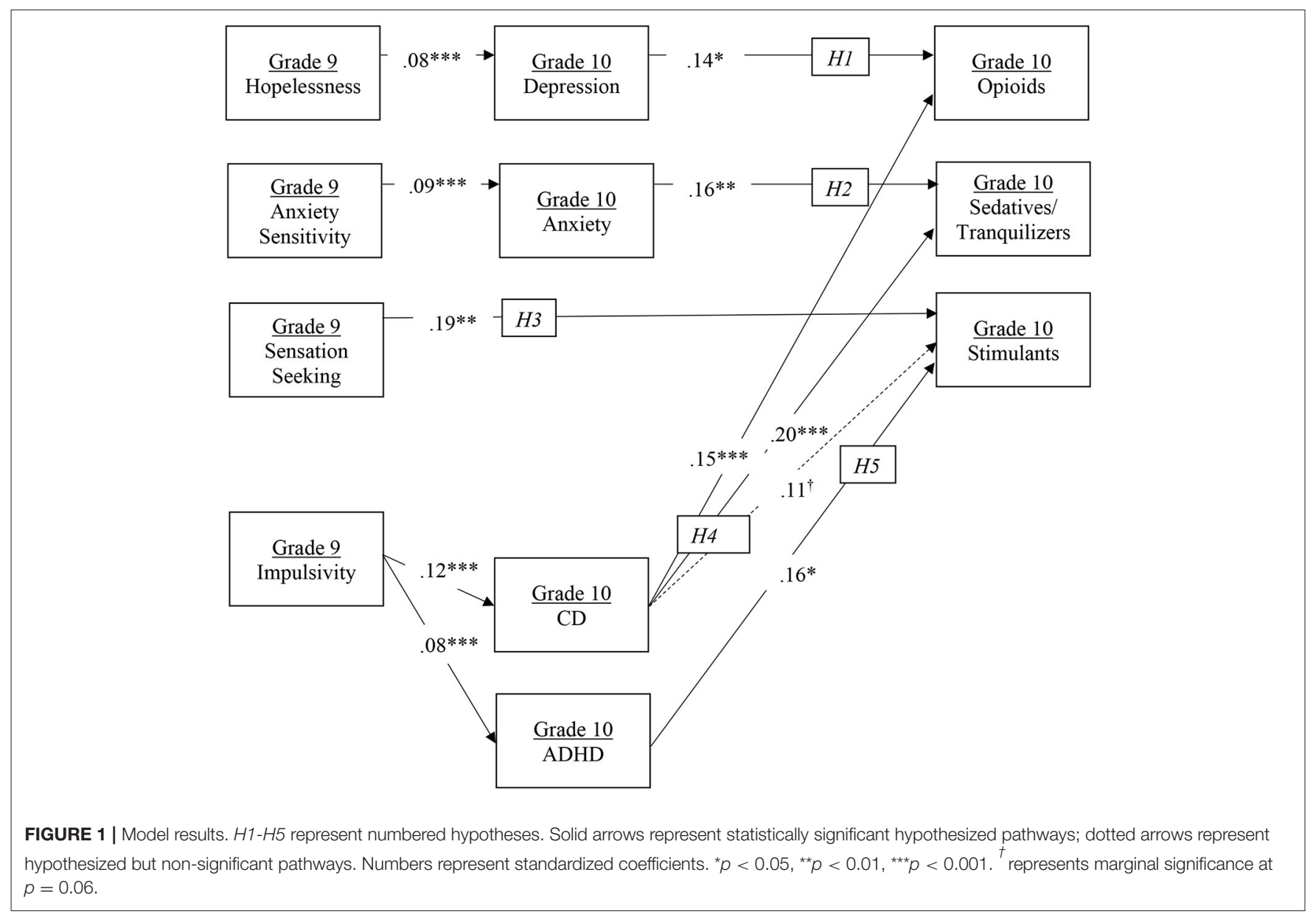

deviance proneness, and psychological dysregulation models. Two internalizing personality traits (HOP and AS) followed a negative affect regulation model for predicting specific $\mathrm{PD}$ misuse, while SS (an externalizing trait) followed a positive affect regulation model. First, depressive symptoms mediated the relationship between HOP and future opiate misuse. Second, anxiety symptoms mediated the relationship between AS and future tranquilizer misuse. While both these paths are 
TABLE 4 | Tests of hypothesized indirect effects.

\begin{tabular}{|c|c|c|c|c|c|}
\hline Hypothesis & Predictor & Mediator & Outcome & Indirect effect & $95 \%$ confidence interval \\
\hline$H 1$ & Hopelessness & Depression & Opioids & 0.003 & {$[0.000,0.007]^{*}$} \\
\hline $\mathrm{H} 2$ & Anxiety sensitivity & Anxiety & Sedatives/tranquilizers & 0.005 & {$[0.002,0.012]^{\star *}$} \\
\hline \multirow[t]{3}{*}{$\mathrm{H} 4$} & Impulsivity & $\mathrm{CD}$ & Opioids & 0.005 & {$[0.001,0.010]^{*}$} \\
\hline & Impulsivity & $\mathrm{CD}$ & Sedatives/tranquilizers & 0.008 & {$[0.004,0.014]^{\star \star}$} \\
\hline & Impulsivity & $C D$ & Stimulants & 0.005 & {$[0.000,0.014]^{\star}$} \\
\hline H5 & Impulsivity & ADHD & Stimulants & 0.005 & {$[0.000,0.012]^{\star}$} \\
\hline
\end{tabular}

$A D H D$ is attention-deficit hyperactivity disorder; $C D$ is conduct disorder. ${ }^{\star} p<0.05,{ }^{\star \star} p<0.01$.

consistent with negative affect regulation, they suggest that high HOP adolescents may be using opiates to self-medicate their depressive symptoms-while high AS teens may be using tranquilizers to self-medicate their anxiety symptoms. Third, SS was predictive of future stimulant misuse suggesting high SS adolescents may be using stimulants to enhance positive affect. This suggests that adolescents high in HOP and AS are prone to PD misuse via negative affect regulation pathways while those high in SS are prone to PD misuse via a positive affect regulation pathway. Fourth, CD symptoms mediated the relationship between IMP and future opiate, sedative/tranquilizer, and stimulant misuse, consistent with a deviance proneness pathway. Unlike the other three traits, IMP therefore seems to be a more general predictor of $\mathrm{PD}$ misuse, rather than a specific predictor of a particular form of PD misuse. Higher IMP adolescents appear to more prone to misusing PDs indiscriminately-in the same way that they are prone to engaging in broadband antisocial behaviors. Finally, ADHD symptoms also mediated IMP's effect in the case of future stimulant misuse. We have suggested that this unique personality-to-PD misuse pathway may represent self-medication of psychological dysregulation. In the next section, we look at each of these main findings in relation to the extant literature.

\section{Comparison With the Literature}

H1 predicted that HOP would specifically predict future opioid misuse via depressive symptoms. This hypothesis, informed by the negative affect regulation model, was supported through a significant indirect effect from Grade 9 HOP to Grade 10 opioid misuse 1 year later as mediated through Grade 10 depressive symptoms. Depression has been identified as the mental health issue most strongly related to opioid misuse (odds ratios from 1.2 to 4.3 ) (87). Zullig and Divin (88) found that students who endorsed HOP, depression, and suicidality were 1.18-1.43 times more likely to misuse opioids. Opioids possess psychic painnumbing properties (89), which may make them particularly attractive to high HOP adolescents-who are prone to depression and may be looking to dull their psychological pain. Our mediational findings are consistent with a mechanism where HOP confers risk for opioid misuse in adolescence via negative affect regulation. More specifically, high HOP adolescents may be self-medicating their depressive symptoms by misusing opioids. Given that opioids are prescribed for the management of physical pain (89) but not for the management of depression (90), any use of opioids for depression self-medication would be considered opioid misuse since it would involve taking the medication for a non-prescribed purpose (91). To help establish the specificity of this HOP risk pathway to opioid use, we tested an additional personality to $\mathrm{PD}$ misuse pathway informed by the negative affect regulation model involving AS (i.e., H2).

H2 predicted that AS would specifically predict future sedative/tranquilizer misuse via anxiety symptoms. This hypothesis, also informed by the negative affect regulation model, was supported through a significant indirect effect from Grade 9 AS to Grade 10 sedative/tranquilizer misuse 1 year later as mediated through Grade 10 anxiety symptoms. While sedatives/tranquilizers are commonly prescribed for anxiety (92), the relevant DEP-ADO items (69) specify use "without a prescription," suggesting that high AS adolescents may be taking non-prescribed sedatives/tranquilizers that they have obtained from family, friends, dealers, or online pharmacies (15) to self-medicate their anxiety symptoms. Taken together, support for H1-2 suggests that there are two distinct negative affect regulation paths from personality to $\mathrm{PD}$ misuse. The first is specific to opioid misuse through HOP and the self-medication of depression, and the second specific to sedative/tranquilizer misuse through AS and the self-medication of anxiety. Furthermore, modification indices indicated that the inclusion of paths from AS-to-depression and HOP-to-anxiety did not improve model fit, providing further evidence of the specificity of these pathways.

Informed by the positive affect regulation model, $H 3$ predicted that SS would lead to future stimulant misuse. This hypothesis was supported through a direct path from Grade 9 SS to Grade 10 stimulant misuse. SS is strongly related to sensitivity to positive reinforcement and enhancement motives (31). It predicts substance misuse (93) that is driven by a need for positive affect and psycho-stimulation (29). Previously, we found that SS predicted undergraduate stimulant misuse (45). Other studies also support a robust association between SS and adolescent alcohol misuse (74). Finn et al. (94) found that SS was both directly linked to alcohol problems as well as indirectly linked through alcohol use and positive alcohol expectancies. Castellanos-Ryan et al. (95) concluded that SS's effect on binge drinking was mediated by a reward response bias. Thus, SS likely confers risk for adolescent stimulant misuse as well as excessive drinking via a positive affect regulation pathway. Taken together, the support for $H 1-H 3$ suggests that three distinct 
affect regulation paths predict PD misuse in adolescence: two involving negative affect regulation (i.e., HOP to depression to opioid misuse and AS to anxiety to sedative/tranquilizer misuse) and one involving positive affect regulation (i.e., SS to stimulant misuse).

Unlike the specific associations of each of HOP, AS and SS with particular forms of PD misuse, we expected IMP to have a more general association with $\mathrm{PD}$ misuse, including future opioid, sedative/tranquilizer, and stimulant misuse. $\mathrm{H} 4$ predicted that Grade 9 IMP would be associated with all three forms of PD misuse in Grade 10 via Grade $10 \mathrm{CD}$ symptoms. These hypotheses, informed by the deviance proneness model, and IMP's centrality as a characteristic of CD $(59,96)$, were all supported in tests of indirect effects. This pattern is in keeping with previous research with other substances. Mackie et al. (93), for instance, found that IMP predicted adolescent alcohol use via $\mathrm{CD}$ symptoms. This result also replicates and extends prior research linking $\mathrm{CD}$ symptoms to unconstrained PD misuse in adolescents, including misuse of opioids (97) and stimulants (64). IMP's relationship with substance misuse is motivationally undefined (31) in that it is more reflective of a general inability to inhibit behavior (98). IMP is associated with deficits in response execution and inhibition (95). Poor response inhibition is a risk factor for both CD (99) and substance misuse (100). Paths from IMP to both CD and alcohol problems are also partially mediated by deficient response inhibition $(94,95)$. In sum, we know that high IMP adolescents struggle to regulate and inhibit their impulses. This makes them more vulnerable to deviance (including $\mathrm{CD}$ and $\mathrm{PD}$ misuse). Our results are consistent with the idea that IMP confers risk for broadband PD misuse (including all three types of PD misuse) via a general proneness toward deviance in adolescence.

In addition to these general IMP to CD symptoms to PD misuse pathways, $H 5$ predicted a second indirect pathway specifically linking IMP to later stimulant misuse via ADHD symptoms. This hypothesis, informed by the psychological dysregulation model, was supported by a significant indirect effect from Grade 9 IMP to Grade 10 stimulant misuse via Grade 10 ADHD symptoms. IMP is a prominent symptom of ADHD (101) for which stimulants are prescribed (102). Previously, we showed that IMP was concurrently associated with both medically sanctioned stimulant use and stimulant misuse in university students (45). Prescription stimulants are classified as Schedule III under the Canadian Controlled Drugs and Substances Act (S.C. 1996, C. 19) due to their high potential for misuse (103). Their use is legal only when prescribed by a licensed practitioner and taken by the person for whom they were prescribed. For those high in IMP, availability is the best motivational predictor of misuse (34). Adolescents who report symptoms of ADHD are more likely to have stimulant prescriptions, which they can then misuse [e.g., by taking their stimulants in greater amounts or more often than prescribed, via non-intended routes, for non-prescribed reasons, and/or with contraindicated substances; (91)]. While rates of stimulant misuse are relatively low in general adolescent samples, rates are much higher among adolescents who: have symptoms of $\mathrm{ADHD}$, have $\mathrm{ADHD}$ diagnoses, are receiving treatment for
ADHD, or have stimulant prescriptions (104). Interestingly, some research suggests that the young people most likely to misuse prescription stimulants are those with markers of a possible mental health disorder (e.g., ADHD) but without a formal diagnosis or prescription (105). Our results suggest that some young people may misuse stimulants to cope with their ADHD-related disorganization, poor time management, forgetfulness, and distractibility (64). Thus, in adolescence, IMP may confer risk for stimulant misuse, in part, via self-medication of psychological dysregulation-a form of self-medication that is theoretically distinct from the self-medication of negative affect pathways described above for AS and HOP.

\section{Strengths and Limitations}

Our study has several important strengths. These include the large sample size, inclusion of both French- and English-speaking students, the longitudinal component (personality to mental health symptoms and personality to PD misuse paths) over a 1year follow-up across the developmentally challenging transition to high school, the excellent retention rate (95\%), the control of baseline levels of mediators and outcomes in all models, and the theoretically driven hypotheses. Moreover, the topic of the paper is likely to be of interest to both a general and specialty audience of mental health professionals, particularly those that work with youth.

These findings should be interpreted in the context of several potential study limitations. First, we measured personality in Grade 9-and mental health symptoms and PD misuse in Grade 10. As such, the final pathways in our semi-longitudinal model (from mental health symptoms to PD misuse) were crosssectional. Methodologically, we set up our semi-longitudinal model in this manner because $H 1-H 3$ pertain to self-medication. We considered assessing PD misuse in Grade 11, in a threewave design, but this would have meant testing whether students misused PDs to cope with the mental health symptoms they had reported a year earlier. We wanted to measure mental health symptoms and PD misuse in closer proximity. Self-medication models posit that the mental health-to-PD misuse relationship is unidirectional (50). There are data, however, that suggest that it may be bidirectional. PD misuse, for example, has been shown to exacerbate students' mental health symptoms (106). Our data do not allow us to compare these possibilities and our model does not allow for causal inference. Nonetheless, mediation analyses with even partially cross-sectional data can be a useful starting point (107) and our model had the advantage of being semi-longitudinal (i.e., where part of the design was longitudinal-specifically personality to mental health symptoms and personality to $\mathrm{PD}$ misuse). To demonstrate reliability and address these limitations, however, our model should be replicated in a fully longitudinal design that uses shorter (e.g., 6 month) lags between waves. Future research could also use ecological momentary assessment to examine these relationships day-to-day [e.g., (108)].

A second potential limitation pertains to our measure of PD misuse. The DEP-ADO was chosen because it is standardized, has been demonstrated reliable and valid in the measurement of Canadian high school students' substance use (69), and 
can be use with both English- and French-speaking Québécois adolescents (73). Despite these strengths, the DEP-ADO has some shortcomings. For example, we assessed each type of PD misuse with a single item, introducing measurement error. It also provides little information about students' means of access (e.g., diversion sources, online pharmacies), administration routes, or motives for use. Moreover, different definitions of PD misuse abound (109), and it has been suggested that none of the instruments published to date can adequately assess PD misuse (110). When improved PD misuse measurement tools become available, our model should be replicated. This would reduce measurement error, allowing for a more accurate and refined test of personality's effects on PD misuse generally and on specific classes of PD misuse specifically.

Third, our sampling was limited. While our study was bolstered by its large sample size, this increases the likelihood that small effects will be statistically significant. And some of our effects were relatively small in magnitude, calling for evaluation of their clinical significance (see below). In addition, the students who did not complete our Grade 10 measures were more likely to report Grade 9 personality vulnerability, mental health symptoms, and alcohol and PD misuse, and were more likely to come from specific schools. Some of these results are in keeping with previous studies, in which adolescents lost to follow up were more likely to be involved in drug use and other deviant behavior (111-113). Moreover, we controlled effects of school in our analyses. It still bears noting, however, as samples and findings can be biased when the individuals who drop out differ substantially from those who are retained (114).

Finally, while the use of our brief personality measure [SURPS; (31)] allowed for brevity in the context of a large-scale survey, it did not allow for nuanced assessment of the components of each of our traits. For example, the longer Childhood Anxiety Sensitivity Index (115) would have allowed for examination of the relative contributions of the AS Physical, Social/Control, and Psychological concerns dimensions (116) to the anxiety symptom mediated pathway to sedative/tranquilizer misuse observed in the present study. Similarly, the longer Barratt Impulsiveness Scale (117) would have allowed for examination of the relative contributions of the Attentional, Motor, and Non-planning Impulsiveness components (118) to the $\mathrm{CD}$ and $\mathrm{ADHD}$ symptom mediated pathways to PD misuse observed in the present study.

\section{Future Research Directions}

The present study focused on the mediating effects of mental health symptoms. Motives for PD misuse were not assessed. Bennett and Holloway (119) have concluded that opioids, sedatives/tranquilizers, and stimulants tend to be misused in one of two ways. PDs are misused for self-medication of mental health (e.g., more sleep, less anxiety) or physical health (e.g., to manage a pre-existing illness) problems. They are also misused for pleasure (e.g., to party, get high, or experiment). Boyd et al. (22) and McCabe et al. (46) have published measures of motives for PD misuse. Negatively and positively reinforcing motives are both associated with increased PD misuse frequency (120). Follow-up studies might test whether personality predicts specific motives for PD misuse just as personality predicts specific motives for alcohol use (121). Previously, in the alcohol field, we found chained mediation from personality to mental health symptoms, to drinking motives, to alcohol outcomes (122). The results of the present study suggest that a four-variable chained mediational model might be equally applicable to PD misuse. For example, HOP may predict opioid misuse via symptoms of depression and in turn self-medication motives.

There are also several other areas of future research that are worthy of investigation in the field of personality and PD misuse risk more broadly. First, given that online marketplaces are an accessible source of PDs for young people [e.g., largely uncontrolled, not requiring a prescription, allowing for anonymous access; $(123,124)]$, and thus a significant public health concern, we need more information on the types of adolescents who are accessing PDs via these sites. While the demographic characteristics of the typical customers of such online marketplaces have been identified [i.e., young, male, Caucasian; (125)], we have not yet identified their personality or mental health characteristics, which would be helpful for targeting prevention efforts. Second, given the well-established role of social influence in young people's drug misuse [e.g., (126)] and emerging data concerning online drug forums and social networking sites where those experimenting with psychotropics, including PDs, share drug-related information (9), it would be interesting to study whether involvement in such communities might be related to personality. For example, are these experimenters or "psychonauts" higher in sensation seeking? Finally, personality and mental health factors may be relevant when it comes to pre-marketing assessment trials of the abuse liability of new prescription drugs. Current practices in this regard have been criticized for excluding those with a previous history of drug misuse or addiction [e.g., (8)]. Given the present findings of significant links of four factor personality model traits and mental health symptoms to different forms of PD misuse, there could be utility to testing a new compound's abuse potential using these more substance-misuse prone individuals in pre-marketing assessment trials to get at the compound's truer abuse liability.

\section{Clinical Implications}

Our model suggests that treatment of opioid misuse in adolescents might benefit from a specific targeting of HOP and IMP youth. Cognitive-behavioral therapy (CBT) could benefit teens high in HOP, by teaching them to better cope with their symptoms of depression (127). Motivational approaches could benefit antisocial teens high in IMP, by increasing their future-oriented thinking and teaching them to weigh the short vs. long term consequences of their behavior (128). Because we substantiated paths from IMP to CD symptoms, to opioid, sedative/tranquilizer, and stimulant misuse-a focus on this personality factor would theoretically reduce misuse of a variety of types of PDs. The results of our specificity tests further suggest that treatments of sedative/tranquilizer misuse be targeted toward youth high in AS and include techniques drawn from CBT for anxiety (128). To treat stimulant misuse, our model suggests we should be targeting adolescents high in externalizing traits. Those 
high in SS could be encouraged to pursue other stimulating yet prosocial activities (129). "Alternate rebellions" including hair dyeing, getting a tattoo, or getting a piercing (130) are safer activities that might meet these adolescents' need for excitement. In contrast, psychologically dysregulated, highIMP teens could be trained in behavioral ADHD-management techniques (131).

Treating PD misuse is, of course, important. But, given the ongoing PD crisis in North America (132), preventing it is critical. Adolescent overdoses from prescription opioids rose 95\% from 1999 to 2016 (133). The likelihood of reporting PD misuse during adolescence, increases with age (83), as we saw across each PD type from Grade 9 to 10 in our sample. Research has shown that PD misuse rates rise consistently between Grade 8-12 and ages 12-17 (134). Thus, prevention efforts geared toward at-risk youth are especially vital. Our results suggest that identifying high personality-risk adolescents (i.e., those high in HOP, AS, SS, or IMP) would benefit both early intervention and targeted prevention strategies for PD misuse.

Personality-matched interventions have effectively reduced illicit drug use in adolescence (135) and PD misuse in adulthood (136). The present study was embedded within a larger trial, which evaluated the longer-term efficacy of the Preventure Program (65). This personality-matched prevention program targets teens with elevated four-factor trait scores (25). It is rooted in the cognitive-behavioral model and incorporates psycho-educational and motivational interviewing components. When applied to alcohol and illicit drug use, the Preventure Program has resulted in delayed onset and reduced escalation of misuse (65). Our study suggests that personality is related to $\mathrm{PD}$ misuse in a similar manner to its relations with alcohol and illicit drug use, through mental health symptoms. Thus, personality-matched interventions may have the potential to reduce PD misuse and even prevent PD uptake, if administered prior to PD misuse onset. Our results suggest that the Preventure Program should next be investigated in relation to its utility in targeting adolescent PD misuse.

\section{REFERENCES}

1. Substance Abuse and Mental Health Services Administration (SAMHSA) (2015). Results From the 2014 National Survey on Drug Use and Health: Summary of National Findings. Rockville, MD: Department of Health and Human Services.

2. Manchikanti L. Prescription drug abuse: what is being done to address this new drug epidemic? Testimony before the subcommittee on criminal justice, drug policy, human resources. Pain Phys. (2006) 9:287-321.

3. National Advisory Committee on Prescription Drug Misuse. First Do No Harm: Responding to Canada's Prescription Drug Crisis. Ottawa, ON: Canadian Centre on Substance Abuse (2013).

4. Centre for Addiction and Mental Health. Drug use Among Ontario Students: Highlights from the Ontario Student Drug Use and Health Survey (1977-2017). Toronto, ON: Centre for Addition and Mental Health (2017).

5. Miech R, Schulenberg J, Johnston L, Bachman J, O’Malley P, Patrick M. Monitoring the Future National Adolescent Drug Trends in 2017: Findings

\section{DATA AVAILABILITY STATEMENT}

The raw data supporting the conclusions of this article will be made available by the authors, without undue reservation.

\section{ETHICS STATEMENT}

Ethical approval was granted by Sainte-Justine Hospital's Research Ethics Board and each administrative school board. The consent process varied across schools. Some schools opted for active consent where written informed consent to participate in the study was provided by the participants' legal guardian/next of kin. Other schools opted for a passive consent procedure where legal guardians/next of kin were fully informed about the study and they declined if they did not consent for their adolescent to participate. All adolescents provided their assent prior to participating.

\section{AUTHOR CONTRIBUTIONS}

SS and AC wrote the manuscript with input from all co-authors. $\mathrm{KT}$ ran the statistical analyses. MA assisted with database management. The data collection was coordinated by PC as part of the CoVenture trial. All authors assisted with conceptualization of the model and interpretation of the results.

\section{FUNDING}

Data collection was funded by a grant to PC for the CoVenture trial from the Canadian Institutes of Health Research (Grant \#: FRN: 114887). Conduct of this study was also supported by a grant to PC for the CUSP Trial from the Canadian Institutes of Health Research (Grant \#: PJT155914). SS and PC are funded through Tier 1 Canada Research Chairs. AC conducted this work as a part of her dissertation research at Dalhousie under the supervision of SS and she was supported through a doctoral fellowship from the Social Sciences and Humanities Research Council of Canada. KT was funded through a Jules Leger Research Chair at Saint Francis Xavier University.

Released. Ann Arbor, MI: Institute for Social Research, The University of Michigan (2017).

6. Miech R, Johnston L, O'Malley PM, Keyes KM, Heard K. Prescription opioids in adolescence and future opioid misuse. Pediatrics. (2015) 136:e1169-77. doi: 10.1542/peds.2015-1364

7. Boyd CJ, McCabe SE, Cranford JA. Prescription drug abuse and diversion among adolescents in a southeast Michigan school district. Arch Pediatr Adolesc Med. (2007) 161:276-81. doi: 10.1001/archpedi.161.3.276

8. Schifano. F, Papanti GD, Orsolini L, Corkery JM. The consequences of drug misuse on post-marketing surveillance. Exp Rev Clin Pharmacol. (2016) 9:867-71. doi: 10.1080/17512433.2016.1178571

9. Chiappini S, Schifano F. What about "pharming": issues regarding the misuse of prescription and over-the-counter drugs. Brain Sci. (2020) 10:736. doi: 10.3390/brainsci10100736

10. Monteith S, Glenn T. Searching online to buy commonly prescribed psychiatric drugs. Psychiatry Res. (2018) 260:248-54. doi: $10.1016 /$ j.psychres.2017.11.037 
11. Pulver A, Davison C, Parpia A, Purkey E, Pickett W. Nonmedical use of prescription opioids and injury risk among youth. J Child Adolesc Substance Abuse. (2016) 25:522-9. doi: 10.1080/1067828X.2015.1115795

12. Compton WM, Volkow ND. Major increases in opioid abuse in the United States: concerns and strategies. Drug Alcohol Dependence. (2006) 81:103-7. doi: 10.1016/j.drugalcdep.2005.05.009

13. Carrasco-Garrodo P, Jimenez-Trujillo I, Hernandez-Barrera V, GarciaGomez-Heras S, Alonso-Fernandez N, Palacios-Cena D. Trends in the misuse of tranquilizers, sedatives, and sleeping pills by adolescents in Spain. J Adolesc Health. (2018) 63:709-16. doi: 10.1016/j.jadohealth.2018.04.003

14. Ford JA. The prescription drug problem we are missing: risks associated with the misuse of tranquilizers and sedatives. J Adolesc Health. (2018) 63:665-6. doi: 10.1016/j.jadohealth.2018.09.007

15. Orsolini L, Corkery JM, Chiappini S, Guirguis A, Vento A, DeBerandis $D$, et al. 'New/designer benzodiazepines': an analysis of the literature and psychonauts' trip reports. Curr Neuropharmacol. (2020) 18:809-37. doi: 10.2174/1570159X18666200110121333

16. Schepis TS, West BT, Teter CJ, McCabe SE. Prevalence and correlates of coingestion of prescription tranquilizers and other psychoactive substances by US high school seniors: results from a national survey. Addict Behav. (2016) 52:8-12. doi: 10.1016/j.addbeh.2015.08.002

17. Centres for Disease Control and Prevention, National Centre for Health Statistics. Multiple cause of death 1999-2018. CDC WONDER Online Database. Centres for Disease Control and Prevention, National Centre for Health Statistics (2020).

18. McCabe SE, Veliz P, Wilens TE, Schulenberg JE. Adolescents' prescription stimulant use and adult functional outcomes: a national prospective study. J Am Acad Child Adolesc Psychiatry. (2017) 56:226-33. doi: 10.1016/j.jaac.2016.12.008

19. McCabe SE, Veliz P, Boyd CJ, Schulenberg JE. Medical and nonmedical use of prescription sedatives and anxiolytics: adolescents' use and substance use disorder symptoms in adulthood. Addict Behav. (2017) 65:296-301. doi: 10.1016/j.addbeh.2016.08.021

20. Nargiso JE, Ballard EL, Skeer MR. A systematic review of risk and protective factors associated with nonmedical use of prescription drugs among youth in the United States: a social ecological perspective. J Stud Alcohol Drugs. (2015) 76:5-20. doi: 10.15288/jsad.2015.76.5

21. Ford JA, Reckdenwald A, Marquardt B. Prescription drug misuse and gender. Subst Use Misuse. (2014) 49:842-51. doi: 10.3109/10826084.2014.880723

22. Boyd CJ, McCabe SE, Cranford JA, Young A. Adolescents' motivations to abuse prescription medications. Pediatrics. (2006) 118:2472-80. doi: 10.1542/peds.2006-1644

23. Cloninger CR, Bohman M, Sigvardsson S. Childhood personality predicts alcohol abuse in young adults. Alcohol Clin Experi Res. (1988) 12:494-505. doi: 10.1111/j.1530-0277.1988.tb00232.x

24. Conrod PJ. Personality-targeted interventions for substance use and misuse. Curr Addiction Rep. (2016) 3:426-36. doi: 10.1007/s40429-016-0127-6

25. Pihl RO, Peterson JB. Alcoholism: the role of different motivational systems. J Psychiatry Neurosci. (1995) 20:372-96.

26. Abramson LY, Metalsky GI, Alloy LB. Hopelessness depression: a theory-based subtype of depression. Psychol Rev. (1989) 96:358-72. doi: 10.1037/0033-295X.96.2.358

27. Beck AT. Beck Hopelessness Scale. San Antonio, TX: The Psychological Corporation (1988).

28. Taylor S. Anxiety Sensitivity: Theory, Research, and Treatment of the Fear of Anxiety. New York, NY: Routledge (2014). doi: 10.4324/9781410603326

29. Comeau N, Stewart SH, Loba P. The relations of trait anxiety, anxiety sensitivity, and sensation seeking to adolescents' motivations for alcohol, cigarette, marijuana use. Addict Behav. (2001) 26:803-25. doi: 10.1016/S0306-4603(01)00238-6

30. Conrod PJ, Pihl RO, Stewart SH, Dongier M. Validation of a system of classifying female substance abusers on the basis of personality and motivational risk factors for substance abuse. Psychol Addict Behav. (2000) 14:243. doi: 10.1037/0893-164X.14.3.243

31. Woicik PA, Stewart SH, Pihl RO, Conrod PJ. The substance use risk profile scale: a scale measuring traits linked to reinforcement-specific substance use profiles. Addict Behav. (2009) 34:1042-55. doi: 10.1016/j.addbeh.2009. 07.001
32. Mahu IT, Conrod PJ, Barrett SP, Sako A, Swansburg J, Lawrence M, et al. Specificity of personality relationships to particular forms of concurrent substance use among opiate agonist therapy clients. Addict Behav. (2019) 98:106056. doi: 10.1016/j.addbeh.2019.106056

33. Dawe S, Loxton NJ. The role of impulsivity in the development of substance use and eating disorders. Neurosci Biobehav Rev. (2004) 28:343-51. doi: 10.1016/j.neubiorev.2004.03.007

34. Hecimovic K, Barrett SP, Darredeau C, Stewart SH. Cannabis use motives and personality risk factors. Addict Behav. (2014) 39:729-32. doi: 10.1016/j.addbeh.2013.11.025

35. Moody L, Franck C, Hatz L, Bickel WK. Impulsivity and polysubstance use: a systematic comparison of delay discounting in mono-, dual, and tri-substance use. Exp Clin Psychopharmacol. (2016) 24:30-7. doi: $10.1037 /$ pha0000059

36. Castellanos-Ryan N, Struve M, Whelan R, Banaschewski T, Barker GJ, Bokde AL, et al. Neural and cognitive correlates of the common and specific variance across externalizing problems in young adolescence. Am J Psychiatry. (2014) 171:1310-9. doi: 10.1176/appi.ajp.2014.13111499

37. Cloninger CR, Svrakic DM, Pryzbeck TR. A psychobiological model of temperament and character. Arch Gene Psychiatry. (1993) 50:975-90. doi: 10.1001/archpsyc.1993.01820240059008

38. Zuckerman M. Biological Expressions and Biosocial Bases of Sensation Seeking. New York, NY: Press Syndicate of the University of Cambridge (1994).

39. Castellanos-Ryan N, Conrod P. Personality and substance misuse: evidence for a four-factor model of vulnerability. In: Vester JC, Brady K, Galanter M, Conrod P, editors. Drug Abuse and Addiction in Medical Illness: Causes, Consequences, Treatment. New York, NY: Springer (2012). p. 47-62. doi: 10.1007/978-1-4614-3375-0_4

40. Herman-Stahl MA, Krebs CP, Kroutil LA, Heller DC. Risk and protective factors for nonmedical use of prescription stimulants and methamphetamine among adolescents. J Adolesc Health. (2006) 39:374-80. doi: 10.1016/j.jadohealth.2006.01.006

41. Yudko E, Lozhkina O, Fouts A. A comprehensive review of the psychometric properties of the drug abuse screening test. J Subst Abuse Treatment. (2007) 32:189-98. doi: 10.1016/j.jsat.2006.08.002

42. Stewart SH, McGonnell M, Wekerle C, Adlaf E. Associations of personality with alcohol use behaviour and alcohol problems in adolescents receiving child welfare services. Int J Mental Health Addict. (2011) 9:492-506. doi: 10.1007/s11469-011-9339-0

43. Castellanos-Ryan N, O'Leary-Barrett M, Sully L, Conrod P. Sensitivity and specificity of a brief personality screening instrument in predicting future substance use, emotional, behavioural problems. Alcohol Clin Experi Res. (2013) 37:E281-90. doi: 10.1111/j.1530-0277.2012.01931.x

44. Mahu IT, Doucet C, O'Leary-Barrett M, Conrod PJ. Can cannabis use be prevented by targeting personality risk in schools? Twenty-four-month outcome of the Adventure trial on cannabis use: a cluster-randomized controlled trial. Addiction. (2015) 110:1625-33. doi: 10.1111/add.12991

45. Chinneck A, Thompson K, Mahu IT, Davis-MacNevin P, Dobson $\mathrm{K}$, Stewart SH. Personality and prescription drug use/misuse among first year undergraduates. Addict Behav. (2018) 87:122-30. doi: 10.1016/j.addbeh.2018.07.001

46. McCabe SE, Boyd CJ, Teter CJ. Subtypes of nonmedical prescription drug misuse. Drug Alcohol Depend. (2009) 102:63-70. doi: 10.1016/j.drugalcdep.2009.01.007

47. Joiner TR. A test of the hopelessness theory of depression in youth psychiatric inpatients. J Clin Child Psychol. (2000) 19:231-42. doi: 10.1207/S15374424jccp2902_3

48. Knapp AA, Blumenthal H, Mischel ER, Badour CL, Leen-Feldner EW. Anxiety sensitivity and its factors in relation to generalized anxiety disorder among adolescents. J Abnormal Child Psychol. (2015) 44:233-44. doi: 10.1007/s10802-015-9991-0

49. McLaughlin EN, Stewart SH, Taylor S. Childhood anxiety sensitivity index factors predict unique variance in DSM-IV anxiety disorder symptoms. Cognit Behav Ther. (2007) 36:210-9. doi: 10.1080/16506070701 499988

50. Becker WC, Fiellin DA, Desai RA. Non-medical use, abuse, and dependence on sedatives and tranquilizers among U.S. adults: psychiatric 
and socio-demographic correlates. Drug Alcohol Depend. (2007) 90:280-7. doi: 10.1016/j.drugalcdep.2007.04.009

51. Seeman P, Madras B. Methylphenidate elevates resting dopamine which lowers the impulse-triggered release of dopamine: a hypothesis. Behav Brain Res. (2002) 130:79-83. doi: 10.1016/S0166-4328(01)00435-1

52. Miller ME, Badger GJ, Heil SH, Higgins ST, Sigmon SC. Associations between sensation seeking and d-amphetamine reinforcement. Am J Addict. (2015) 24:435-42. doi: 10.1111/ajad.12226

53. Sher KJ, Slutske WS. Disorders of impulse control. In: Stricker GA, Widiger T, editors. Handbook of Psychology. New York, NY: Wiley (2003). p. 195-228. doi: 10.1002/0471264385.wei0808

54. Castellanos-Ryan N, Conrod P. Personality correlates of the common and unique variance across conduct disorder and substance misuse symptoms in adolescence. J Abnormal Child Psychol. (2011) 39:563-76. doi: 10.1007/s10802-010-9481-3

55. López-Romero L, Romero E, Andershed H. Conduct problems in childhood and adolescence: developmental trajectories, predictors, and outcomes in a six-year follow-up. Child Psychiatry Human Dev. (2015) 46:762-73. doi: 10.1007/s10578-014-0518-7

56. Costello EJ, Mustillo S, Erkanli A, Keeler G, Angold A. Prevalence and development of psychiatric disorders in childhood and adolescence. Arch Gene Psychiatry. (2003) 60:837-44. doi: 10.1001/archpsyc.60.8.837

57. Khoddam R, Leventhal AM. Alternative and complementary reinforcers as mechanisms linking adolescent conduct problems and substance use. Exp Clin Psychopharmacol. (2016) 24:376-89. doi: 10.1037/pha0000088

58. Tarter RE, Kirisci L, Mezzich A, Cornelius JR, Pajer K, Vanyukov M, et al. Neurobehavioral disinhibition in childhood predicts early age at onset of substance use disorder. Am J Psychiatry. (2003) 160:1078-85. doi: 10.1176/appi.ajp.160.6.1078

59. American Psychiatric Association (APA). Diagnostic and Statistical Manual of Mental Disorders: DSM-5. Arlington, VA: American Psychiatric Association (2013). doi: 10.1176/appi.books.9780890425596

60. Cassidy TA, Varughese S, Russo L, Budman SH, Eaton TA, Butley SF. Nonmedical use and diversion of ADHD stimulants among U.S. adults ages 18-49: a national internet survey. J Attent Disord. (2015) 19:630-40. doi: $10.1177 / 1087054712468486$

61. Cottler LB, Striley CW, Lasopa SO. Assessing prescription stimulant use, misuse, and diversion among youth 10-18 years of age. Curr Opin Psychiatry. (2013) 26:511-9. doi: 10.1097/YCO.0b013e3283642cb6

62. Sawyer MG, Reece CE, Sawyer ACP, Johnson S, Lawrence D, Zubrick SR. The prevalence of stimulant and antidepressant use by Australian children and adolescents with attention-deficit/hyperactivity disorder and major depressive disorder: a national survey. J Child Adolesc Psychopharmacol. (2017) 27:177-84. doi: 10.1089/cap.2016.0017

63. Logan GD. On the ability to inhibit thought and action: a user guide to the stop signal paradigm. In: Dagenback D, Carr HT, editors. Inhibitory Processes in Attention, Memory, Language. San Diego, CA: Academic Press (1994). p. $189-239$.

64. Van Eck K, Markle RS, Flory K. Do conduct problems and sensation seeking moderate the association between ADHD and three types of stimulant use in a college population? Psychol Addict Behav. (2012) 26:939-47. doi: $10.1037 / \mathrm{a} 0027431$

65. O’Leary-Barrett M, Mâsse R, Pihl R, Stewart SH, Séguin JR, Conrod P. A cluster-randomised controlled trial evaluating the effects of delaying onset of adolescent substance abuse on cognitive development and addiction following a selective, personality-targeted intervention program: the CoVenture trial. Addiction. (2017) 112:1871-81. doi: 10.1111/add.13876

66. McIntosh K, Flannery KB, Sugai G, Braun DH, Cochrane KL. Relationships between academics and problem behaviour in the transition from middle school to high school. J Positive Behav Intervent. (2008) 10:243-55. doi: 10.1177/1098300708318961

67. Our Kids. Canadian Provincial Grade Levels: Comparing the Education and Grade Levels of Provinces in Canada. (2019). Available online at: https:// www.ourkids.net/school/canadian-province-grade-levels (accessed April 13, 2021).

68. Currie CE, Elton RA, Todd J, Platt S. Indicators of socioeconomic status for adolescents: the WHO health behaviour in school-aged children survey. Health Educ Res. (1997) 12:385-97. doi: 10.1093/her/12.3.385
69. Landry M, Tremblay J, Guyon L, Bergeron J, Brunelle N. La grille de dépistage de la consommation problématique d'alcool et de drogues chez les adolescents et les adolescents (DEP-ADO): développement et qualités psychométriques. Drogues Santé Soc. (2004) 3:20-37. doi: 10.7202/010517ar

70. Derogatis LR. BSI 18, Brief Symptom Inventory 18: Administration, Scoring and Procedures Manual. Minneapolis, MN: National Computer Systems (2001). doi: 10.1037/t07502-000

71. Goodman R. The strengths and difficulties questionnaire: a research note. J Child Psychol Psychiatry. (1997) 38:581-6. doi: 10.1111/j.1469-7610.1997.tb01545.x

72. Lowenthal KM. An Introduction to Psychological Tests and Scales. 2nd ed. Hove: Psychology Press (2004).

73. Castonguay-Jolin L, Perrier-Ménard E, Castellanos-Ryan N, Parent S, Vitaro F, Tremblay RE, et al. Validation de la traduction française de la SURPS pour une population d'adolescents québécois. Canad J Psychiatry. (2013) 58:538-45. doi: 10.1177/070674371305800909

74. Krank M, Stewart SH, O'Connor R, Woicik PB, Wall AM, Conrod PJ. Structural, concurrent, and predictive validity of the substance use profile scale in early adolescence. Addict Behav. (2011) 36:37-46. doi: 10.1016/j.addbeh.2010.08.010

75. Lancaster MA, McCrea MA, Nelson LD. Psychometric properties and normative data for the Brief Symptom Inventory-18 (BSI-18) in high school and collegiate athletes. Clin Neuropsychol. (2016) 31:321-33. doi: 10.1080/13854046.2016.1138504

76. Perrudet-Badoux A. Évaluation des désordres psychologiques dans une population d'asmathiques à l'aide du Brief Symptom Inventory (DSI). Psychol Med. (1987) 19:2457-60.

77. Goodman A, Lamping DL, Ploubidis GB. When to use broader internalizing and externalizing subscales instead of the hypothesized five subscales on the Strengths and Difficulties Questionnaire (SDQ): Data from British parents, teachers, and children. J Abnormal Child Psychol. (2010) 38:1179-91. doi: 10.1007/s10802-010-9434-x

78. He JP, Burstein M, Schmitz A, Merikangas KR. The Strengths and Difficulties Questionnaire (SDQ): the structure and scale validation in U.S. adolescents. $J$ Abnormal Child Psychol. (2013) 41:583-95. doi: 10.1007/s10802-012-9696-6

79. Capron C, Thérond C, Duyme M. Psychometric properties of the French version of the self-report and teacher Strengths and Difficulties Quesionnaire (SDQ). Eur J Psychol Assessment. (2007) 23:79-88. doi: 10.1027/1015-5759.23.2.79

80. Bernard M, Bolognini M, Plancherel B, Chinet L, Laget J, Stephan P, et al. French validity of two substance-use screening tests among adolescents: a comparison of the CRAFT and DEP-ADO. J Substance Use. (2005) 10:38595. doi: 10.1080/14659890412331333050

81. Muthén LK, Muthén BO. MPlus User's Guide. 7th ed. Los Angeles, CA: Muthén and Muthén (1998).

82. Muthén B. Bayesian Analysis in MPlus: A Brief Introduction. (2010). Available online at: www.statmodel.com/download/IntroBayesVersion (accessed April 13, 2021).

83. Milner LA, Ham LS, Zamboanga BL. Adolescents misusing prescription drugs: who's the riskiest users of them all? J Substance Use. (2014) 19:68-74. doi: 10.3109/14659891.2012.734541

84. McCabe SE, Veliz P, Patrick ME. High-intensity drinking and nonmedical use of prescription drugs: results from a national survey of $12^{\text {th }}$ grade students. Drug Alcohol Depend. (2017) 178:372-9. doi: 10.1016/j.drugalcdep.2017.05.038

85. Hu L, Bentler PM. Cutoff criteria for fit indexes in covariance structure analysis: conventional criteria versus new alternatives. Struct Eq Model A Multidiscipl J. (1999) 6:1-55. doi: 10.1080/10705519909540118

86. Curran PJ, West SG, Finch JF. The robustness of test statistics to nonnormality and specification error in confirmatory factor analysis. Psychol Methods. (1996) 1:16-29. doi: 10.1037/1082-989X.1.1.16

87. Amari E, Rehm J, Goldner E, Fischer B. Nonmedical prescription opioid use and mental health and pain comorbidities: a narrative review. Canad J Psychiatry. (2011) 56:495-501. doi: 10.1177/070674371105 600808

88. Zullig KJ, Divin AL. The association between non-medical prescription drug use, depressive symptoms, and suicidality among college students. Addict Behav. (2012) 37:890-9. doi: 10.1016/j.addbeh.2012.02.008 
89. Smith K, Mattick RP, Bruno R, Nielsen S, Cohen M, Campbell G, et al. Factors associated with the development of depression in chronic non-cancer pain patients following the onset of opioid treatment for pain. J Affect Disord. (2015) 184:72-80. doi: 10.1016/j.jad.2015.05.049

90. Singh N, Reece J. Psychotherapy, pharmacotherapy, and their combination for adolescents with major depressive disorder: a meta-analysis. Austr Educat Dev Psychol. (2014) 31:47-65. doi: 10.1017/edp.2013.20

91. Haydon E, Monga B, Rehm J, Adlah E, Fischer B. Prescription drug abuse in Canada and the diversion of prescription drugs into the illicit drug market. Canad J Public Health. (2006) 96:459-61. doi: 10.1007/ BF03405190

92. Bisaga A, Mariani JJ. Benzodiazepines and other sedatives and hypnotics. In: Galanter M, Kleber HD, Brady TK, editors. The American Psychiatric Publishing Textbook of Substance Abuse Treatment. 5th edn. Arlington, VA: American Psychiatric Publishing Inc. (2015). p. 239-62. doi: 10.1176/appi.books.9781615370030.mg17

93. Mackie CJ, Castellanos-Ryan N, Conrod PJ. Personality moderates the longitudinal relationship between psychological symptoms and alcohol in adolescents. Alcohol Clin Experi Res. (2011) 35:703-16. doi: 10.1111/j.1530-0277.2010.01388.x

94. Finn PR, Sharkansky EJ, Brandt KM, Turcotte N. The effects of familial risk, personality, and expectancies on alcohol use and abuse. J Abnormal Psychol. (2000) 109:122-33. doi: 10.1037/0021-843X.109.1.122

95. Castellanos-Ryan N, Rubia K, Conrod PJ. Response inhibition and reward response bias mediate the predictive relationships between impulsivity and sensation seeking and common and unique variance in conduct disorder and substance misuse. Alcohol Clin Experi Res. (2011) 35:140-55. doi: 10.1111/j.1530-0277.2010.01331.x

96. Thompson LL, Whitmore EA, Raymond KM, Crowley TJ. Measuring impulsivity in adolescents with serious substance and conduct problems. Assessment. (2006) 13:3-15. doi: 10.1177/1073191105 282247

97. Morioka CK, Howard DE, Caldeira KM, Wang MQ, Arria AM. Affective dysregulation predicts incident non-medical prescription analgesic use among college students. Addict Behav. (2018) 76:328-34. doi: 10.1016/j.addbeh.2017.08.034

98. Finn PR, Mazas C, Jutus A, Steinmetz JE. Early-onset alcoholism with conduct disorder: Go/no-go learning deficits, working memory capacity, and personality. Alcohol Clin Experi Res. (2002) 26:186-206. doi: 10.1111/j.1530-0277.2002.tb02524.x

99. Herba CM, Tranah T, Rubia K, Yule W. Conduct problems in adolescence: three domains of inhibition and effect of gender. Dev Neurosci. (2006) 24:11017-22. doi: 10.1207/s15326942dn3002_2

100. Li CS, Huang C, Constable RT, Sinha R. Imaging response inhibition in a stop-signal task: neural correlates independent of signal monitoring and post-response processing. J Neurosci. (2006) 26:186-92. doi: 10.1523/JNEUROSCI.3741-05.2006

101. Barkley RA, Murphy KR, Fischer M. ADHD in Adults: What the Science Says. New York, NY: Guilford Press (2008).

102. Solanto MV, Arnsten AFT, Castellanos FX. (Eds.). Stimulant Drugs and ADHD: Basic and Clinical Neuroscience. New York, NY: Oxford University Press (2001).

103. Kollins SH. Abuse liability of medications used to treat attentiondeficit/hyperactivity disorder (ADHD). Am J Addict. (2007) 16:35-44. doi: 10.1080/10550490601082775

104. Weyandt L, Oster D, Marraccini ME, Gudmundsdottir B, Munro B, Mattinez-Zavras B, et al. Pharmacological interventions for adolescents and adults with ADHD: stimulant and nonstimulant medications and misuse of prescription stimulants. Psychol Res Behav Manage. (2014) 7:223-49. doi: 10.2147/PRBM.S47013

105. Arria AM, DuPont RL. Nonmedical prescription stimulant use among college students: why we need to do something and what we need to do. $J$ Addict Dis. (2010) 29:417-26. doi: 10.1080/10550887.2010.509273

106. Soule M, Connery HS. Co-occurring substance use disorders. In: Tse J, Volpp YS, editors. A Case-Based Approach to Public Psychiatry. New York, NY: Oxford University Press (2018). p. 155-61. doi: $10.1093 / \mathrm{med} / 9780190610999.003 .0020$
107. Hayes AF. Introduction to Mediation, Moderation, and Conditional Process Analysis: A Regression-Based Approach. New York, NY: Guilford Publications. (2013).

108. Serre F, Fatseas M, Debrabant R, Alexandre, J.-M., Auriacombe M, et al. Ecological momentary assessment in alcohol, tobacco, cannabis, and opiate dependence: a comparison of feasibility and validity. Drug Alcohol Depend. (2012) 126:118-23. doi: 10.1016/j.drugalcdep.2012. 04.025

109. Barrett SP, Meisner JR, Stewart SH. What constitutes prescription drug misuse? Problems and pitfalls of current conceptualizations. Curr Drug Abuse Rev. (2008) 1:255-62. doi: 10.2174/1874473710801030255

110. Smith SM, Paillard F, McKeown A, Burke LB, Edwards RR, Katz NP, et al. Instruments to identify prescription medication misuse, abuse, and related events in clinical trials: an ACTTION systematic review. J Pain. (2015) 16:389-441. doi: 10.1016/j.jpain.2015.01.009

111. Tebes JK, Snow DL, Arthur MW. Panel attrition and external validity in the short-term follow-up study of adolescent substance use. Evaluat Rev. (1992) 16:151-70. doi: 10.1177/0193841X9201600203

112. Snow DL, Tebes JK, Arthur MW. Panel attrition and external validity in adolescent substance use research. J Consult Clin Psychol. (1992) 60:804-7. doi: 10.1037/0022-006X.60.5.804

113. Brook JS, Cohen P, Gordon AS. Impact of attrition in a sample in a longitudinal study of adolescent drug use. Psychol Rep. (1983) 53:375-8. doi: $10.2466 /$ pro.1983.53.2.375

114. Boys A, Marsden J, Stillwell G, Hatchings K, Griffiths P, Farrell M. Minimizing attrition in longitudinal research: practical applications from a cohort of adolescent drinking. J Adolesc. (2003) 26:363-73. doi: 10.1016/S0140-1971(03)00011-3

115. Silverman WK, Fleisig W, Rabian B, Peterson RA. Childhood anxiety sensitivity index. J Clin Child Psychol. (1991) 20:162-8. doi: 10.1207/s15374424jccp2002_7

116. Walsh TM, Stewart SH, McLaughlin E, Comeau N. Gender differences in Childhood anxiety sensitivity index (CASI) dimensions. J Anxiety Disord. (2004) 18:695-706. doi: 10.1016/S0887-6185(03)00043-4

117. Barratt ES. Anxiety and impulsiveness related to psychomotor efficiency. Perceptual Motor Skills. (1959) 9:191-8. doi: 10.2466/pms.1959.9.3.191

118. Patton JH, Stanford MS, Barrett ES. Factor structure of the barratt impulsiveness scale. J Clin Psychol. (1995) 51:768-74. doi: 10.1002/1097-4679(199511)51:6<768::aid-jclp2270510607>3.0.co;2-1

119. Bennett T, Holloway K. Motives for illicit prescription drug use among university students: a systematic review and meta-analysis. Int J Drug Policy. (2017) 44:12-22. doi: 10.1016/j.drugpo.2017.02.012

120. Kelly BC, Rendina HJ, Vuolo M, Wells BE, Parsons JT. A typology of prescription drug misuse: a latent class approach to differences and harms. Drug Alcohol Rev. (2015) 34:211-20. doi: 10.1111/dar.12192

121. Cooper ML, Frone MR, Russell M, Mudar P. Drinking to regulate positive and negative emotions: a motivational model of alcohol use. J Personal Soc Psychol. (1995) 69:990-1005. doi: 10.1037/0022-3514.69.5.990

122. Chinneck A, Thompson K, Dobson KS, Stuart H, Teehan M, Stewart $\mathrm{SH}$, et al. Neurotic personality traits and risk for adverse alcohol outcomes: Chained mediation through emotional disorder symptoms and drinking to cope. Substance Use Misuse. (2018) 53:173-4. doi: 10.1080/10826084.2018.1432647

123. Orsolini L, Papanti D, Corkery J, Schifano F. An insight into the deep web: why it matters for addiction psychiatry? Human Psychopharmacol Clin Experi. (2017) 32:e2573. doi: 10.1002/hup.2573

124. Shifano F, Chiappini S, Corkery JM, Guirguis A. Abuse of prescription drugs in the context of novel psychoactive substances (NPS): a systematic review. Brain Sci. (2018) 8:73. doi: 10.3390/brainsci8040073

125. Orsolini L, Francesconi G, Papanti D, Giergetti A, Schifano F. Profiling online recreational/prescription drugs' customers and overview of drug vending virtual marketplaces. Hum Psychopharmacol Clin Exp. (2015) 30:302-18. doi: 10.1002/hup.2466

126. Bartel SJ, Sherry SB, Smith MM, Glowacka M, Speth TA, Stewart $\mathrm{SH}$. Social influences on binge drinking in emerging adults: which social network members matter most? Substance Abuse. (2020) 41:480-4. doi: 10.1080/08897077.2019.1709604 
127. Colognori DB, Herzig K, Reigada LC, Leiby A, Warner CM. Cognitivebehavioural therapy for youth with functional somatic and internalizing symptoms. In: Anbar RD, editor. Functional Symptoms in Pediatric Disease: A Clinical Guide. Berlin: Springer Science and Business Media (2014). p. 269-84. doi: 10.1007/978-1-4939-1974-1_19

128. Conrod PJ, Stewart SH, Comeau N, Maclean AM. Preventative efficacy of cognitive behavioural strategies to the motivational bases of alcohol misuse in at-risk youth. J Clin Child Adolesc Psychol. (2006) 35:490-504. doi: 10.1207/s15374424jccp3504_6

129. Herie MA, Watkin-Merek L. Structured Relapse Prevention: An outpatient Counselling Approach. 2nd ed. Toronto, ON: Centre for Addiction and Mental Health (2006).

130. Linehan MM. DBT Skills Training Manual. Module. 4: Distress Tolerance Skills. Handouts and Worksheets. 2nd ed. New York, NY: Guilford Press (2015).

131. Antshel KM, Olszewski AK. Cognitive behavioural therapy for adolescents with ADHD Child. Adolesc Psychiatric Clin North Am. (2014) 23:825-42. doi: 10.1016/j.chc.2014.05.001

132. Fischer B, Keates A, Bühringer G, Reimer J, Rehm J. Non-medical use of prescription opioids and prescription opioid-related harms: why so markedly higher in North America compared to the rest of the world? Addiction. (2014) 109:177-81. doi: 10.1111/add.12224

133. Gaither JR, Shabanova V, Leventhal JM. US national trends in pediatric deaths from prescription and illicit opioids, 1999-2016. JAMA Network Open. (2018) 1:e186558. doi: 10.1001/jamanetworkopen.2018.6558
134. Johnston LD, O'Malley PM, Bachman JG, Schulenberg JE. Monitoring the Future National Survey Results on Drug Use 1975-2009: Volume II. College Students and Adults Ages 19-50. Bethesda, MD: National Institute on Drug Abuse (2010).

135. Conrod PJ, Castellanos-Ryan N, Strang J. Brief, personalitytargeted coping skills intervention and survival as a non-drug user over a 2-year period during adolescence. Arch Gene Psychiatry. (2010) 67:85-93. doi: 10.1001/archgenpsychiatry.20 09.173

136. Conrod PJ, Stewart SH, Pihl RO, Côté S, Fontaine V, Dongier M. Efficacy of brief coping skills interventions that match personality profiles of female substance abusers. Psychol Addict Behav. (2000) 14:231-42. doi: 10.1037/0893-164X.14.3.231

Conflict of Interest: The authors declare that the research was conducted in the absence of any commercial or financial relationships that could be construed as a potential conflict of interest.

Copyright (C) 2021 Stewart, Chinneck, Thompson, Afzali, Nogueira-Arjona, Mahu and Conrod. This is an open-access article distributed under the terms of the Creative Commons Attribution License (CC BY). The use, distribution or reproduction in other forums is permitted, provided the original author(s) and the copyright owner(s) are credited and that the original publication in this journal is cited, in accordance with accepted academic practice. No use, distribution or reproduction is permitted which does not comply with these terms. 


\section{OPEN ACCESS}

Edited by:

Fabrizio Schifano,

University of Hertfordshire,

United Kingdom

Reviewed by:

Georgios Demetrios Kotzalidis,

Sapienza University of Rome, Italy

Amira Guirguis,

Swansea University, United Kingdom

*Correspondence:

Marta Torrens

mtorrens@imim.es

tThese authors have contributed equally to this work and share first

authorship

¥These authors share senior authorship

Specialty section:

This article was submitted to

Addictive Disorders,

a section of the journal

Frontiers in Psychiatry

Received: 04 March 2021

Accepted: 29 March 2021

Published: 28 April 2021

Citation:

Fonseca F, Lenahan W, Dart RC, Papaseit E, Dargan PI, Wood DM,

Guareschi M, Maremmani I,

Auriacombe M, Farré M,

Scherbaum N and Torrens M (2021)

Non-medical Use of Prescription

Gabapentinoids (Gabapentin and

Pregabalin) in Five European

Countries.

Front. Psychiatry 12:676224.

doi: 10.3389/fpsyt.2021.676224

\section{Non-medical Use of Prescription Gabapentinoids (Gabapentin and Pregabalin) in Five European Countries}

\begin{abstract}
Francina Fonseca ${ }^{1,2,3 \dagger}$, William Lenahan ${ }^{4 \dagger}$, Richard C. Dart ${ }^{4}$, Esther Papaseit ${ }^{5,6}$, Paul I. Dargan ${ }^{7,8}$, David M. Wood ${ }^{7,8}$, Marilena Guareschi ${ }^{9}$, Icro Maremmani ${ }^{10}$, Marc Auriacombe ${ }^{11,12,13}$, Magí Farré ${ }^{5,6 \neq}$, Norbert Scherbaum ${ }^{14 \neq}$ and Marta Torrens ${ }^{1,2,3 * \neq}$

${ }^{1}$ Hospital del Mar, Institut de Neuropsiquiatria i Addiccions (INAD), Barcelona, Spain, ${ }^{2}$ Grup de Recerca en Addiccions, Institut Hospital del Mar d'Investigacions Mèdiques (IMIM), Barcelona, Spain, ${ }^{3}$ Department of Psychiatry and Legal Medicine, Universitat Autònoma de Barcelona (UAB), Cerdanyola del Vallès, Spain, ${ }^{4}$ Rocky Mountain Poison and Drug Safety, Denver Health and Hospital Authority, Denver, CO, United States, ${ }^{5}$ Clinical Pharmacology Unit, Hospital Universitari Germans Trias i Pujol (HUGTP-Germans Trias i Pujol Research Institute), Badalona, Spain, ${ }^{6}$ Department of Pharmacology, Therapeutics and Toxicology, Universitat Autònoma de Barcelona (UAB), Cerdanyola del Vallès, Spain, ${ }^{7}$ Clinical Toxicology, Guy's and St Thomas' NHS Foundation Trust, London, United Kingdom, ${ }^{8} \mathrm{Clinical}$ Toxicology, Faculty of Life Sciences and Medicine, King's College London, London, United Kingdom, ${ }^{9}$ Association for the Application of Neuroscientific Knowledge to Social Aims (AU-CNS), Lucca, Italy, ${ }^{10}$ Vincent P. Dole Research Group, Santa Chiara University Hospital, University of Pisa, Pisa, Italy, ${ }^{11}$ Addiction Psychiatry Department, University of Bordeaux, Bordeaux, France, ${ }^{12}$ Addiction Team, Sanpsy Centre National de la Recherche Scientifique (CNRS) USR 3413, Bordeaux, France, ${ }^{13}$ Pôle Addictologie et Filière Régionale, Centre Hospitalier (CH) Charles Perrens and Centre Hospitalier Universitaire (CHU) de Bordeaux, Bordeaux, France, ${ }^{14}$ LVR-Hospital Essen, Department of Psychiatry and Psychotherapy, Medical Faculty, University of Duisburg-Essen, Essen, Germany
\end{abstract}

Background: Non-medical use (NMU) of prescription GABA analogs (pregabalin and gabapentin) has been reported especially in opiate dependent persons. However, by now the prevalence of NMU of gabapentinoids in the general population has not been sufficiently evaluated. The aim of this research paper is to determine the prevalence of prescription GABA analog NMU and associated demographics in five European countries with special detail of Spain.

Methods: The RADARS Survey of Non-Medical Use of Prescription Drugs Program (NMURx) is a harmonized series of contemporaneous cross-sectional surveys of adults conducted in multiple countries. NMURx collects data from the general population in each participating country about NMU of prescription drugs, illicit drugs, and associated demographics. NMU was defined as "using a medication without a doctor's prescription or for any reason other than what was recommended by their doctor." Responses from Spain (4Q2017, $n=10,062$ ) were analyzed in detail. Comparative data were available from France, Germany, Italy, and UK. Responses were collected using non-probability quota sampling and post-stratification population weighting was applied to reflect the national distributions of adults, based on age, gender, and census region. Rates of NMU and associated demographics were reported as rate of past 90-day NMU per 100,000 adult population with 95\% confidence intervals.

Results: Germany (1,197 per 100,000 adult population [95\% Cl: 1,004.3-1,379.1]) and United Kingdom (1,067 per 100,000 adult population [95\% Cl: 851.3-1,283.2]) presented the highest prevalence of gabapentinoids NMU. In Spain the prevalence 
of past 90 days GABA analog NMU was: 344.4, 95\% (Cl 204.8-484.0), with male predominance. Those who non-medically use GABA analogs had a higher prevalence of lifetime chronic pain, lifetime illicit drug use, and previous substance abuse treatment. In Spain, 20\% of respondents who ever have used gabapentinoids, reported a lifetime $\mathrm{NMU}$; the prevalence was higher for pregabalin 624 (6.2\%) than for gabapentin 444 (4.4\%). The main reasons for use were to self-treat pain and other medical conditions.

Conclusions: The risk of NMU of gabapentinoids should not be neglected. Subjects with a history of chronic pain and lifetime substance use disorders had an increased risk of NMU of gabapentinoids.

Keywords: gabapentin, pregabalin, non-medical use, prescription drugs, misuse

\section{INTRODUCTION}

Gabapentinoids, pregabalin and gabapentin, are widely used for the treatment of neuropathic pain and epileptic disorders according to the United States (US) Food and Drug Administration (FDA). Both gabapentin and pregabalin have been approved by the European Medicine Agency (EMA) for neuropathic pain and generalized anxiety disorder, respectively. Additionally, some off-label uses of gabapentinoids include treatment for chronic lower back pain, insomnia, migraine, social phobia, panic disorder, mania, bipolar disorder, and alcohol withdrawal $(1,2)$.

Gabapentinoids are now among the most commonly prescribed medications in most countries (3). For instance, the overall rate of pregabalin prescriptions use increased from 1.0 per 1,000 individuals in 2013 to 22.0 per 1,000 individuals in 2014 in Ontario, Canada (4). Also, there has been a progressive increase in the reported cases of misuse and dependence to the European Medicines Agency's EudraVigilance database, specifically in subjects with previous history of substance use disorders (3).

At the pharmacological level, gabapentinoids selectively bind to the $\alpha 2 \delta$-subunit of voltage-gated calcium channels in central nervous system neuronal tissues. This in turn increases the GABA levels and decreases other excitatory neurotransmitters (5). This mechanism is associated with their antinociceptive, anticonvulsant, anxiolytic, and sleepmodulating effects (6). Gabapentinoids have significant risks despite their reputation as safe drugs. Sedation, dizziness, gait instability, and feeling of intoxication are quite common side effects; as many as one in three patients taking therapeutic doses experience dizziness or somnolence (7). Although, both substances share some mechanisms of action, they also have some pharmacokinetic differences that could explain differences in their abuse potential; for instance, pregabalin is absorbed more rapidly by oral route, with maximum plasma concentrations attained within $1 \mathrm{~h}$, whereas, maximum plasma concentrations of gabapentin are detected $3-4 \mathrm{~h}$ after oral administration. Pregabalin absorption is linear, and gabapentin absorption is saturable (non-linear -zero-order- process) with less predictable pharmacokinetics. Bioavailability is also different; pregabalin has a $90 \%$ bioavailability independently from the dosage, but gabapentin bioavailability changes with dosage, from $60 \%$ at 900 $\mathrm{mg} /$ day to $33 \%$ at 3,600 $\mathrm{mg} /$ day. On the other hand, similarities in pharmacokinetics are: both can be given without regard of meals, they do not bind to plasma proteins and both are excreted renally with an elimination half-live of $6 \mathrm{~h}(7)$. The linear pharmacokinetics of pregabalin and its greater potency explains its steep dose-response relationship and differences in abuse potential and severe adverse events as respiratory depression.

Evidence regarding misuse and diversion of gabapentinoids has grown in recent years $(8-10)$. The first description of their misuse and abuse were published in 2010 (11). Prevalence of misuse and abuse in the general population is an estimated 2.5\% (12) but, the rates in people suffering a substance use disorder (SUD) is higher (pregabalin: 3-68\%; gabapentin: 15-22\%) (1). In a systematic review aimed to evaluate the abuse liability of gabapentin and pregabalin, the authors found that pregabalin had a greater potential for addiction than gabapentin based on the magnitude of behavioral dependence symptoms, transitions from prescription to self-administration, and the durability of the self-administrations (8).

Current research suggests that the addictive potential of gabapentinoids is primarily a concern among patients with other substance use disorders, especially opioid use disorder (8). The reasons that motivate gabapentinoid misuse and abuse are not clearly described. Also, the subjective effects described by people who report non-medical use are multiple: self-treatment of pain and other medical conditions, pursuit of changes in states of consciousness, and "to get high" $(1,13)$. According to a recent systematic review (13), one of the most predictive factors associated with gabapentinoid use was the concomitant use of opioids.

The neurobiological mechanism involved in the abuse liability of gabapentinoids has not been yet clearly investigated. The usual increase in the dopamine levels at the mesolimbic brain circuits has not been proved in preclinical studies $(14,15)$. Gabapentinoids have been reported to produce alcohol/gamma hydroxybutyrate (GHB)/benzodiazepine-type effects mixed with euphoria. Rates of euphoria have been reported at between 1 and $12 \%$ but this has been for therapeutic doses. Other reported effects include dissociative feeling, improved sociability, relaxation and sense of calm, and psychedelic effects $(10,16)$. 
On the other hand, however, there are studies indicating that gabapentin could be an useful treatment for alcohol use disorder. For instance, a recent randomized controlled trial showed efficacy of gabapentin in the treatment of alcohol use disorder, improving the alcohol withdrawal syndrome, reducing the heavy drinking days and more total abstinence in the group treated with $1,200 \mathrm{mg}$ of gabapentin (17). Also, in a meta-analysis of seven studies, gabapentin showed efficacy in the treatment of alcohol use disorder, reducing the number of heavy drinking days (18).

In countries as United Kingdom gabapentinoids have been reclassified as Class $\mathrm{C}$ controlled drugs under the Misuse of Drugs Act, from 1 April 2019 (19). That means that it is illegal to dispense them without a signed prescription, but that they do not require safe custody in controlled drug cabinets. In Spain, Italy, Germany, and France, gabapentin and pregabalin are available both only under a medical prescription. Alternatively, in the USA, the Drug Enforcement Administration (DEA) classifies pregabalin as a Schedule V controlled substance, or the lowest abuse potential among controlled substances, and gabapentin as a non-controlled substance (20).

The aim of this study is to determine the prevalence of prescription gabapentinoids non-medical use and associated demographics in five European countries (France, Germany, Italy, Spain, and the United Kingdom) and to evaluate the main factors related with its misuse in Spain.

\section{MATERIALS AND METHODS}

\section{Design and Participants}

The data were obtained from the Researched, Abuse, Diversion and Addiction Related Surveillance (RADARS $($ ) System Survey of Non-Medical Use of Prescription Drugs (NMURx) Program that collects data on respondent demographics and the prevalence, reasons of use, routes of administration, and method of drug acquisition for NMU of prescription drugs across multiple countries. The methodology and the validity of this program is explained in its validation study $(21,22)$.

The whole program collects information from France, Germany, Italy, Spain, and the United Kingdom. Recruitment and data collection are delivered to country-based members through a global survey panel company, in the native language of the country where the survey is undertaken and in English. Each launch has a "soft launch" of around 500 participants to ensure proper data collection. The surveys were available during the following timeframes: In France: from 2017 December 13 to 2018, January, 7: in Germany: from 2017 December 12 to 2018, January, 16; in Italy: 2017, from December 14 to December 26; in Spain: From 2017 December 12 to 2018, January, 4; and in UK: 2017, from September 28 to December 1.

The inclusion criteria were: agree to be included and give informed consent at the beginning of the survey; adult age that was defined as ages 15-110 years in Spain, 16-110 years in the United Kingdom, and 18-110 in France, Germany, and Italy; in order to reflect the geographical and gender distribution of the country, surveys from different countries and regions have been included if region/sex sampling strata that has not yet met its sampling quota; and have completed the survey in its entirety.
Respondents and/or surveys were excluded from the analysis if the respondent met criteria for careless response as defined by the validation study (21).

Calibration weights were applied to the survey population to be representative of the distribution of the adult population of each of the countries included in the study based on geographic region, age, sex, limitations in daily activities, and smoking status (21). National data utilized for this weighting scheme was calculated from estimates from Eurostat and the European Social Survey; NMURx was approved by the Colorado Multiple Institutional Review Board (Protocol Number: 13-2394) and locally by the Parc de Salut Mar Ethics Committee (Protocol Number: $2017 / 7331 / \mathrm{I})$. Data used in this analysis is from the surveys launched in the second half of 2017 (17Q4).

\section{Measures}

Respondents were asked if they had ever used prescription gabapentin or pregabalin for any reason in their lifetime; a "yes" response classified lifetime use. If respondents reported lifetime use, they were asked about last 12- month use and last 12-month NMU, where NMU was defined as "in a way not directed by your healthcare provider."

Basic demographics (age and gender) were collated together with data on prevalence of last 12-month gabapentin/pregabalin use and NMU.

\section{Analyses}

The weighted proportion and 95\% confidence intervals (CIs) of select demographic and respondent characteristics were calculated to describe the population. Weighted prevalence estimates and 95\% CIs were calculated for last 12-month use and NMU of prescription gabapentin only, pregabalin only, and pregabalin and gabapentin. The prevalence of prescription or NMU in the last 12 months was estimated by gender and age. Differences in prevalence of prescription and were compared by gender and age range $(18-24,25-34,35-44,45-54,55-$ $64,65+$ years). Analyses were conducted in SPSS Version 25.0 (Armonk, NY).

\section{RESULTS}

\section{Survey Termination and Completion for the Five Countries}

In the last quarter of 2017, approximately 63,450 French panelists were invited to participate in the survey. Of the 16,903 who initiated the survey, the inclusion and exclusion criteria below were applied and a total of 10,072 respondents were included in the analysis (5,058 (50.2\%) females, $46.8 \pm 15.17$ years).

In Germany, $\sim 64,982$ German panelists were invited to participate in the survey. Of the 21,977 who initiated the survey, 15,051 completed it and fulfilled the inclusion criteria $(7,531$ (50.0\%) female, mean age $46.8 \pm 14.24$ years).

In Italy, 41,167 Italian panelists were invited to participate in the survey. Of the 12,766 who initiated the survey, the inclusion and exclusion criteria below were applied and 10,019 surveys were included (5,019 females (50.1\%), mean age $43.5 \pm$ 13.72 years). 


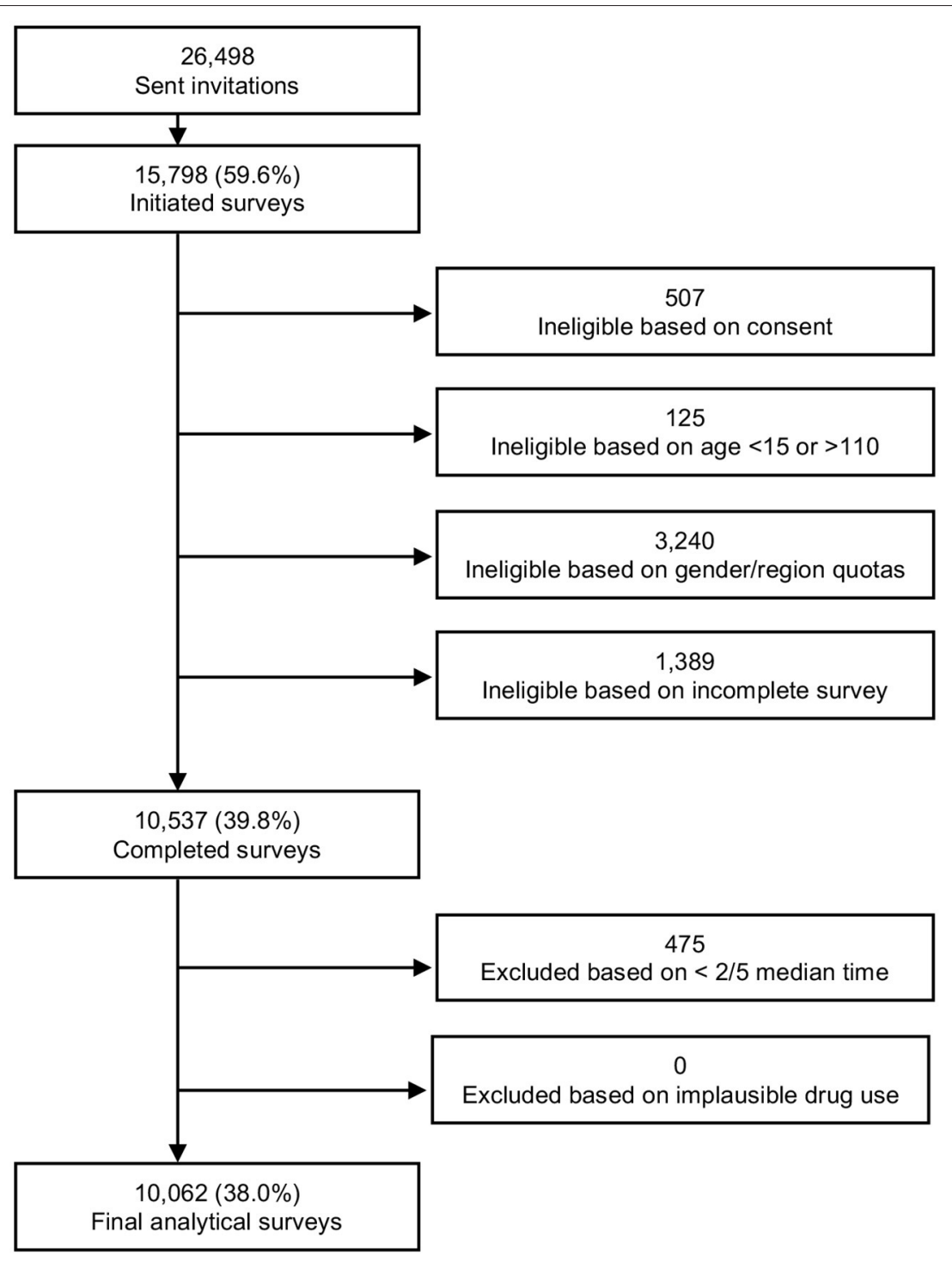

FIGURE 1 | Final analytic sample flowchart.

In Spain, 26,498 panelists were invited to participate in the survey. Of the 15,798 who initiated the survey, the inclusion and exclusion criteria below were applied (Figure 1). Finally, 10,062 people completed the survey $(5,030(50.0 \%)$ female, mean age: $41.6 \pm 12.74$ years).

In the United Kingdom, there were 108,633 panelists invited to participate in the survey, of which 13,036 initiated the survey and 10,004 were included in the analysis (5,003 (50.0\%) females, mean age $51.6 \pm 15.33$ years).

\section{Comparison of Five Countries}

Prevalence of past 90 day GABA analog NMU was highest in Germany (1,191.7 per 100,000 population, 95\% CI 1,004.31,379.1) and the UK (1,067.2, 95\% CI 851.3-1,283.2), and lowest in Spain (344.4, 95\% CI 204.8-484.0) and Italy (366.2, 95\% CI 207.7-524.6) (Table 1).
NMU was evenly distributed between genders except in Spain which showed a male predominance (Table 2). Those who non-medically use GABA analogs were estimated to have higher incidence of lifetime chronic pain, lifetime illicit drug use, and previous substance abuse therapy (Table 2).

\section{Spanish Respondents Characteristics}

Approximately 26,498 Spanish panelists were invited to participate in the survey. Of the 15,798 who initiated the survey, the inclusion and exclusion criteria below were applied (Figure 1). Finally, 10,062 people completed the survey $(5,030$ $(50.0 \%)$ female, mean age: $41.6+12.74$ years). The main characteristics (unweighted and weighted) of the respondents are described in Table 3. The responses are weighted to represent the population above 15 years old in Spain by region, gender and age. 
A total of $1,003(10.0 \%)$ respondents referred a lifetime use of gabapentinoids; after weighting the responses a 9.9\% (95\% CI: 9.2-10.6) (Table 4).

From the total Spanish sample, 444 (4.4\%) respondents have ever used gabapentin and $624(6.2 \%)$ pregabalin. Out of them, $84(18.9 \%)$, and $126(20.6 \%)$ reported non-medical use of gabapentin and pregabalin, respectively (cave: according to Table 4 the \% of respondents with NMU of gabapentinoids should be something higher $>>2.9$ out of $9.9 . \%=29.3 \%$. The others respondents were not sure $(40$ (9.0\%) for gabapentin and $33(5.3 \%)$ for pregabalin) about their NMU (that means, that they were not sure whether they followed the recommendations of the prescriber) or answered that they do not use for NMU (320 (72.1\%) for gabapentin and 465 (74.5\%) for pregabalin).

\section{Characteristics of Non-medical Use in Spain}

The main reasons for non-medical use were to self-treat pain and other medical condition different from pain (Table 5).

Respondents who declare NMU of gabapentinoids, usually used the oral route of administration (either swallowed or chewed and then swallowed). Those of them who used to get high, reported to inject gabapentin (41\%) and pregabalin (14.3\%) (Table 6).

TABLE 1 | Last 90 day non-medical use of GABA analogs by country.

\begin{tabular}{lrrrrr}
\hline & France & Germany & Italy & Spain & UK \\
\hline Rate $\mathbf{( 9 5 \% ~ C l )}$ & 574.2 & 1191.7 & 366.2 & 344.4 & 1067.2 \\
per 100,000 & $(424.4$, & $(1004.3$, & $(207.7$, & $(204.8$, & $(851.3$, \\
Adult & $724.0)$ & $1379.1)$ & $524.6)$ & $484.0)$ & $1283.2)$ \\
Population & & & & & \\
Rate $\mathbf{( 9 5 \% ~ C l ) ~}$ & 216.8 & 470.4 & 242.6 & 105.9 & 174.0 \\
per 100,000 & $(160.2$, & $(396.5$, & $(137.6$, & $(63.0$, & $(138.8$, \\
Standard Units & 273.4) & $544.4)$ & $347.6)$ & $148.9)$ & $209.2)$ \\
\hline
\end{tabular}

${ }^{a}$ Rates based on the weighted estimated number of adults who reported NMU of each drug class in the last 90 days per 100,000 adult population.

${ }^{b}$ Rates are based on the weighted estimated number of adults who reported NMU of each drug class in the last 90 days per 100,000 standard units sold.
Respondents said that they main method of drug acquisition in Spain was by a prescription of a doctor/dentist $(61.3 \%$ for gabapentin and $69.8 \%$ for pregabalin), however, they used several methods to acquire them including family or friends (either bought or given), taken from family, friends and other people, bought outside the country, by internet or to a dealer (Table 7).

Finally, in Table 8, is described the last purchase of gabapentin and pregabalin where respondents said that they have obtained the substances from a dealer of bought in internet. The median price paid for both was similar $(10 €)$.

\section{DISCUSSION}

The main finding of this study is that it confirms the potential abuse liability and then non-medical use of the gabapentinoids gabapentin and pregabalin. When comparing the five European countries, those who non-medically use gabapentinoids were estimated to have a higher likelihood of chronic pain, use of illicit substances, and history of substance abuse treatment compared to the general population. These results are in concordance with country surveys, reviews and metanalyses published previously $(8,23,24)$.

There are differences in the rate per 100,000 people among the five countries, with Germany and UK the countries having a higher rate compared to France, Italy and Spain. Reasons for these differences could be related to the availability of other sedative type substances in those countries. According to the European Drug Report of the same year that the information of this study was recorded (25), the prevalence of cannabis use in France, Italy, and Spain was higher than 15\%, whereas, in Germany and United Kingdom the prevalence was lower than $15 \%$. We can hypothesize that some reasons for using cannabis and gabapentinoids could be similar: to treat pain and anxiety symptoms; in countries with higher availability of cannabinoids and opioids, subjects could prefer them to gabapentinoids. Also, in some countries, gabapentinoids might replace partially benzodiazepines; in Spain, a general population survey performed every 2 years, showed data on life-time NMU of benzodiazepines about $3.0 \%$ in male and $3.1 \%$ in female (26).

TABLE 2 | Demographics of those who have non-medically used GABA Analogs in the last 90 days vs. the general adult population demographics.

\begin{tabular}{|c|c|c|c|c|c|c|c|c|c|c|}
\hline & \multicolumn{2}{|c|}{ France } & \multicolumn{2}{|c|}{ Germany } & \multicolumn{2}{|c|}{ Italy } & \multicolumn{2}{|c|}{ Spain } & \multicolumn{2}{|c|}{ UK } \\
\hline & $\begin{array}{c}\text { GABA } \\
\text { analog NMU }\end{array}$ & $\begin{array}{c}\text { General } \\
\text { population }\end{array}$ & $\begin{array}{c}\text { GABA } \\
\text { analog NMU }\end{array}$ & $\begin{array}{c}\text { General } \\
\text { population }\end{array}$ & $\begin{array}{c}\text { GABA } \\
\text { analog NMU }\end{array}$ & $\begin{array}{c}\text { General } \\
\text { population }\end{array}$ & $\begin{array}{c}\text { GABA } \\
\text { analog NMU }\end{array}$ & $\begin{array}{c}\text { General } \\
\text { population }\end{array}$ & $\begin{array}{c}\text { GABA } \\
\text { analog NMU }\end{array}$ & $\begin{array}{c}\text { General } \\
\text { population }\end{array}$ \\
\hline Male & $\begin{array}{c}56.8 \% \\
(48.19,65.36)\end{array}$ & $\begin{array}{c}47.6 \% \\
(46.59,48.68)\end{array}$ & $\begin{array}{c}54.0 \% \\
(48.13,59.90)\end{array}$ & $\begin{array}{c}48.6 \% \\
(47.79,49.49)\end{array}$ & $\begin{array}{c}45.0 \% \\
(33.54,56.38)\end{array}$ & $\begin{array}{c}49.1 \% \\
(47.89,50.21)\end{array}$ & $\begin{array}{c}65.7 \% \\
(53.60,77.79)\end{array}$ & $\begin{array}{c}48.6 \% \\
(47.47,49.79)\end{array}$ & $\begin{array}{c}46.8 \% \\
(39.82,53.83)\end{array}$ & $\begin{array}{c}48.8 \% \\
(47.61,49.94)\end{array}$ \\
\hline $\begin{array}{l}\text { Chronic pain } \\
\text { during lifetime }\end{array}$ & $\begin{array}{c}72.1 \% \\
(64.39,79.88)\end{array}$ & $\begin{array}{c}33.0 \% \\
(32.03,34.05)\end{array}$ & $\begin{array}{c}77.6 \% \\
(72.26,82.46)\end{array}$ & $\begin{array}{c}39.2 \% \\
(38.41,40.09)\end{array}$ & $\begin{array}{c}68.6 \% \\
(57.86,79.26)\end{array}$ & $\begin{array}{c}29.4 \% \\
(28.29,30.47)\end{array}$ & $\begin{array}{c}63.1 \% \\
(51.92,74.37)\end{array}$ & $\begin{array}{c}30.9 \% \\
(29.82,32.05)\end{array}$ & $\begin{array}{c}70.3 \% \\
(63.79,76.72)\end{array}$ & $\begin{array}{c}38.9 \% \\
(37.84,40.04)\end{array}$ \\
\hline $\begin{array}{l}\text { Lifetime illicit } \\
\text { drug use }\end{array}$ & $\begin{array}{c}33.9 \% \\
(25.71,42.05)\end{array}$ & $\begin{array}{c}18.2(17.39 \\
18.94)\end{array}$ & $\begin{array}{c}28.7 \% \\
(23.42,34.05)\end{array}$ & $\begin{array}{c}25.4 \% \\
(24.70,26.17)\end{array}$ & $\begin{array}{c}37.8 \% \\
(26.47,49.04)\end{array}$ & $\begin{array}{c}20.5 \% \\
(19.64,21.38)\end{array}$ & $\begin{array}{c}42.0 \% \\
(30.86,53.20)\end{array}$ & $\begin{array}{c}24.4 \% \\
(23.45,25.32)\end{array}$ & $\begin{array}{c}48.0 \% \\
(40.95,54.98)\end{array}$ & $\begin{array}{c}27.3 \% \\
(26.22,28.31)\end{array}$ \\
\hline $\begin{array}{l}\text { Previous } \\
\text { substance } \\
\text { abuse }\end{array}$ & $\begin{array}{c}11.5 \%(6.04 \\
16.89)\end{array}$ & $\begin{array}{c}1.7 \%(1.46 \\
1.97)\end{array}$ & $\begin{array}{c}7.7 \%(4.56 \\
10.91)\end{array}$ & $\begin{array}{c}1.8 \%(1.59 \\
2.05)\end{array}$ & $\begin{array}{c}3.8 \%(0.00 \\
8.40)\end{array}$ & $\begin{array}{c}0.6 \%(0.47 \\
0.80)\end{array}$ & $\begin{array}{c}13.3 \%(6.45 \\
20.21)\end{array}$ & $\begin{array}{c}2.2 \%(1.92 \\
2.55 \%)\end{array}$ & $\begin{array}{c}19.3 \% \\
(13.35,25.21)\end{array}$ & $\begin{array}{c}1.7 \%(1.41 \\
2.02)\end{array}$ \\
\hline
\end{tabular}

treatment 
TABLE 3 | Spanish survey respondents' demographics $(N=10,062)$.

\begin{tabular}{|c|c|c|}
\hline Variable & $\begin{array}{l}\text { Unweighted } \\
\qquad \boldsymbol{N}(\%)\end{array}$ & $\begin{array}{l}\text { Weighted } \\
\%(95 \% \mathrm{Cl})\end{array}$ \\
\hline \multicolumn{3}{|l|}{ Gender } \\
\hline Male & $5,032(50.0 \%)$ & $48.8(47.6,49.9)$ \\
\hline \multicolumn{3}{|l|}{ Age (years) } \\
\hline Mean (STD) & $41.6(12.74)$ & $45.7(0.2)$ \\
\hline Median (IQR) & $41.0(32.0,50.0)$ & $46.0(33.6,56.9)$ \\
\hline Range & $(15.0,90.0)$ & $(15.0,90.0)$ \\
\hline \multicolumn{3}{|l|}{ Age categories (years) } \\
\hline $15-24$ & $1,008(10.0 \%)$ & $0(0.0,0.0)$ \\
\hline $25-34$ & $2,021(20.1 \%)$ & $0(0.0,0.0)$ \\
\hline $35-44$ & 2,999 (29.8\%) & $14.4(13.8,15.0)$ \\
\hline $45-54$ & 2,391 (23.8\%) & $19.8(19.0,20.5)$ \\
\hline $55+$ & $1,643(16.3 \%)$ & $18.2(17.5,19.0)$ \\
\hline \multicolumn{3}{|l|}{ Territory of residence } \\
\hline Andalucía & $1,813(18.0 \%)$ & $18.0(17.1,18.9)$ \\
\hline Aragón & $570(5.7 \%)$ & $5.7(5.2,6.3)$ \\
\hline Canarias & 467 (4.6\%) & $4.7(4.2,5.1)$ \\
\hline Cantabria & $51(0.5 \%)$ & $0.5(0.3,0.7)$ \\
\hline Castilla y León & $617(6.1 \%)$ & $6.1(5.5,6.7)$ \\
\hline Castilla-La Mancha & $424(4.2 \%)$ & $4.1(3.6,4.6)$ \\
\hline Cataluña & $1,673(16.6 \%)$ & $17.1(16.3,18.0)$ \\
\hline Ciudad Autónoma de Ceuta & $2(0.0 \%)$ & $0.0(0.0,0.0)$ \\
\hline Ciudad Autónoma de Melilla & $10(0.1 \%)$ & $0.2(0.1,0.3)$ \\
\hline Comunidad de Madrid & $1,380(13.7 \%)$ & $13.7(12.9,14.5)$ \\
\hline Comunidad Foral de Navarra & $65(0.6 \%)$ & $0.6(0.5,0.8)$ \\
\hline Comunidad Valenciana & $1,105(11.0 \%)$ & $10.4(9.8,11.1)$ \\
\hline Extremadura & $188(1.9 \%)$ & $1.9(1.5,2.2)$ \\
\hline Galicia & $643(6.4 \%)$ & $6.2(5.6,6.7)$ \\
\hline Illes Balears & $131(1.3 \%)$ & $1.3(1.1,1.6)$ \\
\hline La Rioja & $39(0.4 \%)$ & $0.4(0.2,0.5)$ \\
\hline País Vasco & 290 (2.9\%) & $2.9(2.5,3.3)$ \\
\hline Principado de Asturias & $283(2.8 \%)$ & $3.0(2.6,3.5)$ \\
\hline Región de Murcia & $311(3.1 \%)$ & $3.1(2.7,3.5)$ \\
\hline \multicolumn{3}{|l|}{ Region of residence } \\
\hline Noroeste & 977 (9.7\%) & $9.7(9.0,10.5)$ \\
\hline Noreste & 964 (9.6\%) & $9.6(9.0,10.3)$ \\
\hline Comunidad de Madrid & $1,380(13.7 \%)$ & $13.7(12.9,14.5)$ \\
\hline Centro & $1,229(12.2 \%)$ & $12.1(11.3,12.9)$ \\
\hline Este & 2,909 (28.9\%) & $28.9(27.9,29.9)$ \\
\hline Sur & $2,136(21.2 \%)$ & $21.3(20.3,22.2)$ \\
\hline Canarias & $467(4.6 \%)$ & $4.7(4.2,5.1)$ \\
\hline \multicolumn{3}{|c|}{ Net monthly household income } \\
\hline Under $€ 499$ & $404(4.0 \%)$ & $4.0(3.5,4.4)$ \\
\hline Between $€ 500$ and $€ 799$ & $430(4.3 \%)$ & $4.6(4.1,5.1)$ \\
\hline Between $€ 800$ and $€ 999$ & $588(5.8 \%)$ & $5.9(5.3,6.4)$ \\
\hline Between $€ 1.000$ and $€ 1.499$ & $2,145(21.3 \%)$ & $20.5(19.6,21.4)$ \\
\hline Between $€ 1.500$ and $€ 1.999$ & $1,723(17.1 \%)$ & $16.6(15.8,17.5)$ \\
\hline Between $€ 2.000$ and $€ 2.499$ & $1,472(14.6 \%)$ & $14.4(13.5,15.2)$ \\
\hline Between $€ 2.500$ and $€ 2.999$ & $1,105(11.0 \%)$ & $11.1(10.4,11.9)$ \\
\hline Between $€ 3.000$ and $€ 4.999$ & $1,116(11.1 \%)$ & $11.5(10.7,12.2)$ \\
\hline Between $€ 5.000$ and $€ 6.999$ & $204(2.0 \%)$ & $2.1(1.8,2.5)$ \\
\hline
\end{tabular}

(Continued)
TABLE 3 | Continued

\begin{tabular}{|c|c|c|}
\hline Variable & $\begin{array}{c}\text { Unweighted } \\
\boldsymbol{N}(\%)\end{array}$ & $\begin{array}{l}\text { Weighted }^{\mathrm{a}} \\
\%(95 \% \mathrm{Cl})\end{array}$ \\
\hline$€ 7.000$ or more & $92(0.9 \%)$ & $0.8(0.7,1.0)$ \\
\hline Prefer not to say & 783 (7.8\%) & $8.5(7.8,9.2)$ \\
\hline \multicolumn{3}{|l|}{ Marital status } \\
\hline Single & 3,709 (36.9\%) & $32.3(31.3,33.3)$ \\
\hline Married & $5,463(54.3 \%)$ & $55.5(54.3,56.6)$ \\
\hline Separated/divorced & $760(7.6 \%)$ & $9.5(8.7,10.3)$ \\
\hline Widowed & $130(1.3 \%)$ & $2.7(2.2,3.2)$ \\
\hline \multicolumn{3}{|l|}{ Education achieved } \\
\hline No studies or incomplete primary studies & $25(0.2 \%)$ & $0.3(0.2,0.5)$ \\
\hline Comprehensive primary education & $174(1.7 \%)$ & $2.3(1.9,2.7)$ \\
\hline Secondary studies 1 st stage & $1,481(14.7 \%)$ & $15.8(14.9,16.7)$ \\
\hline Secondary studies 2nd stage & $3,544(35.2 \%)$ & $35.9(34.8,37.0)$ \\
\hline Middle University studies & $2,274(22.6 \%)$ & $22.0(21.0,22.9)$ \\
\hline Higher University studies & $2,564(25.5 \%)$ & $23.7(22.7,24.6)$ \\
\hline \multicolumn{3}{|l|}{ Student within the last 3 months } \\
\hline Yes & 1,403 (13.9\%) & $13.6(12.8,14.3)$ \\
\hline No & $8,659(86.1 \%)$ & $86.4(85.7,87.2)$ \\
\hline \multicolumn{3}{|c|}{ A member or former member of the armed forces } \\
\hline Yes & 479 (4.8\%) & $4.6(4.2,5.1)$ \\
\hline No & $9,583(95.2 \%)$ & $95.4(94.9,95.8)$ \\
\hline \multicolumn{3}{|l|}{ Currently a healthcare professional } \\
\hline Yes & $615(6.1 \%)$ & $5.6(5.1,6.1)$ \\
\hline No & 9,447 (93.9\%) & $94.4(93.9,94.9)$ \\
\hline \multicolumn{3}{|l|}{ Pregnancy status $^{b}$} \\
\hline Yes & $250(5.0 \%)$ & $3.3(2.9,3.8)$ \\
\hline No & $4,780(95.0 \%)$ & $96.7(96.2,97.1)$ \\
\hline \multicolumn{3}{|l|}{ Gestation $^{\mathrm{c}}$ (months) } \\
\hline Mean (STD) & $4.8(2.08)$ & $4.8(0.1)$ \\
\hline Median (IQR) & $5.0(3.0,6.0)$ & $4.4(2.6,5.9)$ \\
\hline Range & $(1.0,9.0)$ & $(1.0,9.0)$ \\
\hline \multicolumn{3}{|l|}{ Survey language } \\
\hline English & $197(2.0 \%)$ & $2.1(1.8,2.5)$ \\
\hline Spanish & 9,865 (98.0\%) & $97.9(97.5,98.2)$ \\
\hline
\end{tabular}

CI, Confidence Interval; STD, Standard deviation; IQR, Interquartile range.

${ }^{a}$ Responses are weighted to represent the distribution of adults (ages 15+) in Spain by region, gender, and age.

${ }^{b}$ Among females only $(n=5,030)$.

${ }^{c}$ Among pregnant females only $(n=250)$.

Some studies have tried to analyze the possible usefulness of pregabalin and gabapentin in the treatment of benzodiazepine use disorder, but there are no clear results regarding this $(27,28)$.

When evaluating the rates by drug, as described before, pregabalin has more endorsements than gabapentin, for example, in a recent paper describing data from addictovigilance monitoring for gabapentinoids (24). Some publications have described a higher abuse liability for pregabalin compared to gabapentin. One of the explanations of this difference could be the higher prevalence of euphoria in pregabalin compared to gabapentin. The studies that have described this effect reported that this is a dose-dependent effect and it is not related to treatment indication, nor previous abuse of substances; its 
prevalence varies among different studies from 1 to $40 \%(10,29)$. The theory of people taking pregabalin to experience euphoria and to get high it is not completely explained by our results, as the majority of the respondents used pregabalin as selftreatment. The differences between the two substances could also be explained by the different pharmacokinetic characteristics of both molecules; pregabalin has more rapid absorption than gabapentin; also, the peak plasma concentration is more rapidly achieved with pregabalin ( $1 \mathrm{~h}$ compared to $4-5 \mathrm{~h}$ ) and has a longer half-life (7).

In the subsample of Spanish population evaluated, about $20 \%$ of all persons ever using gabapentinoids report on NMU of these substances. A risk for NMU that should not be neglected. The main reason for non-medical use was in both medications for self-treat any pain, followed to treat other medical conditions; few respondents used them to get high or to come down; also, there were a percentage of people using them to prevent withdrawal symptoms. Another article, based on data of pharmavigilance (24), found that the use of pregabalin was not only related to the objective to get high, but also, to prevent withdrawal symptoms, as a substitute of other substances and to potentiate the effect of other drugs (mainly benzodiazepines and opioids). In our sample, the inhaled and intravenous route were mainly reported for those who use pregabalin and gabapentin to prevent withdrawal syndrome, to come down and to get high. It is important to consider the possibility of using the intravenous route, and asking patients about it to prevent the transmission of blood borne infections (Hepatitis B and C, and HIV).

TABLE 4 | Respondents that reported use of gabapentinoids (from total survey respondents $n=10,062$ ).

\begin{tabular}{lcc}
\hline & Unweighted $\mathbf{N}(\%)$ & Weighted $^{\mathbf{a}} \mathbf{\%} \mathbf{( 9 5 \% \mathbf { ~ C l } )}$ \\
\hline Lifetime use & $1,003(10.0 \%)$ & $9.9(9.2,10.6)$ \\
Lifetime non-medical use & $323(3.2 \%)$ & $2.9(2.6,3.3)$ \\
Last 12 month non-medical use & $169(1.7 \%)$ & $1.5(1.2,1.7)$ \\
Last 90 day non-medical use & $45(0.4 \%)$ & $0.4(0.3,0.6)$ \\
Last 30 day non-medical use & $42(0.4 \%)$ & $0.4(0.3,0.6)$ \\
Last 7 day non-medical use & $35(0.3 \%)$ & $0.4(0.2,0.5)$ \\
\hline
\end{tabular}

Cl, Confidence Interval.

${ }^{a}$ Responses are weighted to represent the distribution of adults (ages 15+) in Spain by region, gender, and age.
Another aspect to take into account may be the polymedication risk. Pregabalin and gabapentin are usually prescribed with other pain medications, mainly with opioids; among $50-70 \%$ were reported in a recent paper (23). This combination could increase the risk for overdose death (30). Otherwise, the usefulness of the combination of pregabalin and opioids for the treatment of some kind of pain is not clear, as some researchers have described that pregabalin plus opioids was associated with more pain severity and higher oral doses of opioids; furthermore, pregabalin use was not associated with improvements on mental health symptoms (31).

When prescribing these medications it is important to be aware and monitor for signs of misuse and overdosification, mainly in patients with risk factors for NMU (previous history of substance use disorder and chronic pain). It is important to remark that, although NMU of gabapentinoids is more frequent in patients with previous substance use disorder, there are described cases of a primary abuse in people without any of the known risk factors (24), for this reason, it is important to monitor for signs of NMU in all patients in treatment with gabapentinoids. The detection of NMU could be complicated as these medications are not detected in routine toxicology urine controls. Furthermore, prescribers should be aware of the risk of $\mathrm{NMU}$, when patients request for specific drugs of higher doses, when they obtain medications from different sources (doctor shopping), when the medications are lost or stolen frequently or they ask for new prescriptions too early (1).

The NMURx survey methodology is useful to identify underdocumented use and misuse of medication and can detect changes in trends of substance use and misuse; also, it permits to make comparisons among different countries. The large sample size and post-stratification weighting applied creates estimates that are representative of general populations. However, there are some limitations related to online surveys, in first place the reliance of participants to provide honest responses; also, another limitation of the study is that respondents who have acquired a gabapentinoid product from a family member, friend, or dealer may not be aware whether it was initially obtained with a prescription or from another source. However, these limitations will apply to all surveys so still allow for comparison across countries.

In conclusion, in spite of the risk of NMU, gabapentinoids are useful medications in the treatment of neuropathic pain, generalized anxiety disorder, and some forms of epilepsy.

TABLE 5 | Reasons for non-medical use in the Spanish sample.

\begin{tabular}{lcccccc}
\hline $\boldsymbol{N}^{\mathbf{a}}$ & $\begin{array}{c}\text { To self-treat } \\
\text { my pain } \\
\mathbf{N}(\%)\end{array}$ & $\begin{array}{c}\text { To treat a medical } \\
\text { condition, other than } \\
\text { pain } \\
\mathbf{N}(\%)\end{array}$ & $\begin{array}{c}\text { For enjoyment } \\
\text { to get high } \\
\mathbf{N}(\%)\end{array}$ & $\begin{array}{c}\text { To come } \\
\text { down } \\
\mathbf{N}(\%)\end{array}$ & $\begin{array}{c}\text { To prevent or } \\
\text { treat withdrawal } \\
\text { symptoms } \\
\mathbf{N}(\%)\end{array}$ & $\begin{array}{c}\text { Other } \\
\text { reason } \\
\boldsymbol{N}(\%)\end{array}$ \\
\hline Gabapentin & 124 & $65(52.4 \%)$ & $40(32.3 \%)$ & $12(9.7 \%)$ & $10(8.1 \%)$ & $13(10.5 \%)$ \\
Pregabalin & 159 & $76(47.8 \%)$ & $45(28.3 \%)$ & $14(8.8 \%)$ & $9(5.7 \%)$ & $9(5.7 \%)$ \\
\hline
\end{tabular}

${ }^{a}$ Includes all survey respondents who report non-medical use of the product.

Respondents may check multiple options, percentages may not sum to 100 . 
TABLE 6 | Route of administration by reason for non-medical use in Spain.

\begin{tabular}{|c|c|c|c|c|c|c|c|}
\hline Reason for NMU & $N^{a}$ & $\begin{array}{c}\text { Swallowed } \\
\qquad N(\%)\end{array}$ & $\begin{array}{c}\text { Chewed and } \\
\text { then swallowed } \\
N(\%)\end{array}$ & $\begin{array}{l}\text { Dissolved in mouth (e.g., } \\
\text { between cheek and gum, } \\
\text { under tongue) } \\
N(\%)\end{array}$ & $\begin{array}{l}\text { Inhaled } \\
\text { (snorted or } \\
\text { smoked) } \\
N(\%)\end{array}$ & $\begin{array}{c}\text { Injected (shot } \\
\text { it up) } \\
N(\%)\end{array}$ & $\begin{array}{c}\text { Other route } \\
N(\%)\end{array}$ \\
\hline \multicolumn{8}{|c|}{ Gabapentin (e.g., Gabatur, Neurontin®, or generic), tablets/capsules } \\
\hline To self-treat my pain & 65 & $47(72.3 \%)$ & $24(36.9 \%)$ & $19(29.2 \%)$ & $13(20.0 \%)$ & $12(18.5 \%)$ & $10(15.4 \%)$ \\
\hline To treat a medical condition, other than pain & 40 & $27(67.5 \%)$ & $13(32.5 \%)$ & $14(35.0 \%)$ & $7(17.5 \%)$ & $9(22.5 \%)$ & $3(7.5 \%)$ \\
\hline For enjoyment/to get high & 12 & $4(33.3 \%)$ & $5(41.7 \%)$ & $3(25.0 \%)$ & $1(8.3 \%)$ & $5(41.7 \%)$ & $2(16.7 \%)$ \\
\hline To come down & 10 & $3(30.0 \%)$ & $5(50.0 \%)$ & $6(60.0 \%)$ & $1(10.0 \%)$ & $3(30.0 \%)$ & $0(0.0 \%)$ \\
\hline To prevent or treat withdrawal symptoms & 13 & 5 (38.5\%) & $6(46.2 \%)$ & 7 (53.8\%) & $6(46.2 \%)$ & $4(30.8 \%)$ & $4(30.8 \%)$ \\
\hline Other reason & 20 & $10(50.0 \%)$ & $6(30.0 \%)$ & 5 (25.0\%) & $6(30.0 \%)$ & $3(15.0 \%)$ & $5(25.0 \%)$ \\
\hline \multicolumn{8}{|c|}{ Pregabalin (e.g., Lyrica $®$ or generic), tablets/capsules } \\
\hline To self-treat my pain & 76 & 53 (69.7\%) & $19(25.0 \%)$ & $13(17.1 \%)$ & $12(15.8 \%)$ & $8(10.5 \%)$ & $5(6.6 \%)$ \\
\hline To treat a medical condition, other than pain & 45 & $30(66.7 \%)$ & $21(46.7 \%)$ & $8(17.8 \%)$ & $4(8.9 \%)$ & $5(11.1 \%)$ & $1(2.2 \%)$ \\
\hline For enjoyment/to get high & 14 & $2(14.3 \%)$ & $6(42.9 \%)$ & $8(57.1 \%)$ & $4(28.6 \%)$ & $2(14.3 \%)$ & $1(7.1 \%)$ \\
\hline To come down & 9 & $1(11.1 \%)$ & $7(77.8 \%)$ & $3(33.3 \%)$ & $3(33.3 \%)$ & $2(22.2 \%)$ & $1(11.1 \%)$ \\
\hline To prevent or treat withdrawal symptoms & 9 & $5(55.6 \%)$ & $6(66.7 \%)$ & $4(44.4 \%)$ & $4(44.4 \%)$ & $3(33.3 \%)$ & $1(11.1 \%)$ \\
\hline Other reason & 28 & 17 (60.7\%) & 9 (32.1\%) & 7 (25.0\%) & 5 (17.9\%) & 4 (14.3\%) & 10 (35.7\%) \\
\hline
\end{tabular}

alncludes all survey respondents who report each reason for non-medical use of the product.

Respondents may check multiple options, percentages may not sum to 100.

TABLE 7 | Reported method of drug acquisition in Spain.

\begin{tabular}{|c|c|c|c|c|c|c|c|c|}
\hline & $N^{a}$ & $\begin{array}{l}\text { Was prescribed } \\
\text { it by a doctor or } \\
\text { dentist } \\
N(\%)\end{array}$ & $\begin{array}{l}\text { Bought it or was } \\
\text { given it by } \\
\text { friends or family } \\
\text { members } \\
N(\%)\end{array}$ & $\begin{array}{c}\text { Took it from } \\
\text { friends or family } \\
\text { members } \\
\text { without their } \\
\text { knowledge } \\
N(\%)\end{array}$ & $\begin{array}{c}\text { Took it from } \\
\text { someone other } \\
\text { than friends/ } \\
\text { family without } \\
\text { their knowledge } \\
N(\%)\end{array}$ & $\begin{array}{c}\text { Bought it abroad } \\
\text { (outside Spain) } \\
\text { without a Rx } \\
N(\%)\end{array}$ & $\begin{array}{c}\text { Bought it on the } \\
\text { internet without } \\
\text { a } \operatorname{Rx} N(\%)\end{array}$ & $\begin{array}{c}\text { Bought it from a } \\
\text { dealer } \\
N(\%)\end{array}$ \\
\hline Gabapentin & 124 & 76 (61.3\%) & 35 (28.2\%) & 30 (24.2\%) & 39 (31.5\%) & 33 (26.6\%) & 32 (25.8\%) & 38 (30.6\%) \\
\hline Pregabalin & 159 & 111 (69.8\%) & 51 (32.1\%) & $40(25.2 \%)$ & 33 (20.8\%) & 41 (25.8\%) & $39(24.5 \%)$ & 46 (28.9\%) \\
\hline
\end{tabular}

aIncludes all survey respondents who report non-medical use of the product.

Respondents may check multiple options, percentages may not sum to 100 .

TABLE 8 | Last purchase characteristics in Spain.

\begin{tabular}{|c|c|c|c|c|}
\hline & $N^{a}$ & $\begin{array}{l}\text { Number/volume } \\
\text { purchased }\end{array}$ & Strength $^{\mathbf{b}}$ & $\begin{array}{l}\text { Total price paid } \\
(€)\end{array}$ \\
\hline Gabapentin & 45 & $\begin{array}{l}\mathrm{N}: 45 \\
\text { Mean (STD): } 5.8 \\
(8.53) \\
\text { Median (IQR): } 2.0 \\
(1.0,6.0) \\
\text { Range: (0.0, 33.0) }\end{array}$ & $\begin{array}{l}\mathrm{N}: 12 \\
\text { Mean (STD): } 38.9 \\
(49.44) \\
\text { Median (IQR): } 8.0 \\
(2.0,100.0) \\
\text { Range: }(1.0,120.0)\end{array}$ & $\begin{array}{l}\text { N: } 45 \\
\text { Mean (STD): } 14.4 \\
\text { (15.42) } \\
\text { Median (IQR): } 10.0 \\
\text { (2.0, 20.0) } \\
\text { Range: (0.0, 55.0) }\end{array}$ \\
\hline Pregabalin & 52 & $\begin{array}{l}\text { N: } 52 \\
\text { Mean (STD): } 5.4 \\
(7.65) \\
\text { Median (IQR): } 2.0 \\
(1.0,6.0) \\
\text { Range: (0.0, 35.0) }\end{array}$ & $\begin{array}{l}\text { N: } 16 \\
\text { Mean (STD): } 26.3 \\
(61.40) \\
\text { Median (IQR): } 3.5 \\
(2.0,27.0) \\
\text { Range: }(1.0,250.0)\end{array}$ & $\begin{array}{l}\text { N: } 52 \\
\text { Mean (STD): } \\
185,204.8 \\
(1,302,466.10) \\
\text { Median (IQR): } 10.0 \\
\text { (2.0, 32.0) } \\
\text { Range: (0.0, } \\
\text { 9,393,939.0) }\end{array}$ \\
\hline
\end{tabular}

STD, Standard deviation; IQR, Interquartile Range.

aIncludes all survey respondents who report non-medical use of the product and "Bought it from a dealer" or "Bought it on the internet".

bStrength: MG per tablet/capsule, MCG/h per patch, MG per oral film, MG/ML per liquid, MCG per Iollipop, MCG per lozenge, MG per suppository; All non-numeric entries were excluded.

Respondents have option to check 'I'm not sure' under strength.
Professionals prescribing these medications should be aware and actively search for signs of misuse and diversion.

\section{DATA AVAILABILITY STATEMENT}

The raw data supporting the conclusions of this article will be made available by the authors, without undue reservation.

\section{ETHICS STATEMENT}

The studies involving human participants were reviewed and approved by the Colorado Multiple Review Board (Protocol Number: 13-2394) and locally by the Parc de Salut Mar Ethics Committee (Protocol Number: 2017/7331/I). Written informed consent to participate in this study was provided by the participants' legal guardian/next of kin.

\section{AUTHOR CONTRIBUTIONS}

FF, PD, DW, NS, MG, IM, MA, and RD were responsible to prepare and adapt the country protocols. MG and RD were 
responsible for the project concept and study design. FF, WL, EP, and MF contributed to drafting the manuscript. MF, NS, and MT were responsible for the final revision. All authors have read and approved the final submitted manuscript.

\section{FUNDING}

This work was supported by the Researched Abuse, Diversion and Addiction-Related Surveillance (RADARS) System. The RADARS System is supported by subscriptions from pharmaceutical manufacturers, government, and non-government agencies for surveillance, research, and

\section{REFERENCES}

1. Evoy KE, Morrison M, Saklad SR. Pregabalin and gabapentin abuse: a systematic review. Drugs. (2017) 77:403-26. doi: 10.1007/s40265-017-0700-x

2. Calandre EP, Rico-Villademoros F, Slim M. Alpha2delta ligands, gabapentin, pregabalin, and mirogabalin: a review of their clinical pharmacology and therapeutic use. Expert Rev Neurother. (2016) 16:1263-77. doi: 10.1080/14737175.2016.1202764

3. Chiappini S, Schifano F. A decade of gabapentinoid misuse: an analysis of the European medicines agency's 'suspected adverse drug reactions' database. CNS Drugs. (2016) 30:647-54. doi: 10.1007/s40263-016-0359-y

4. Kwok H, Khuu W, Fernandes K, Martins D, Tadrous M, Singh S, et al. Impact of unrestricted access to pregabalin on the use of opioids and other CNSactive medications: a cross-sectional time series analysis. Pain Med. (2017) 18:1019-26. doi: 10.1093/pm/pnw351

5. Tran-Van-Minh A, Dolphin AC. The alpha2delta ligand gabapentin inhibits the Rab11-dependent recycling of the calcium channel subunit alpha2delta-2. J Neurosci. (2010) 30:12856-67. doi: 10.1523/JNEUROSCI.2700-10.2010

6. Frampton JE. Pregabalin: a review of its use in adults with generalized anxiety disorder. CNS Drugs. (2014) 28:835-54. doi: 10.1007/s40263-014-0192-0

7. Bockbader HN, Wesche D, Raymond M, Chapel S, Janiczek N, Burger P. A comparison of thepharmacokinetics and pharmacodynamics of pregabalin and gabapentin. Clin. Pharmacokin. (2010) 49:661-9. doi: 10.2165/11536200-000000000-00000

8. Bonnet U, Scherbaum N. How addictive are gabapentin and pregabalin? A systematic review. Eur Neuropsychopharmacol. (2017) 27:1185-215. doi: 10.1016/j.euroneuro.2017.08.430

9. Mersfelder TL, Nichols WH. Gabapentin: abuse, dependence, and withdrawal. Ann Pharmacother. (2016) 50:229-33. doi: 10.1177/1060028015620800

10. Schjerning $\mathrm{O}$, Rosenzweig $\mathrm{M}$, Pottegård A, Damkier P, Nielsen J. Abuse potential of pregabalin: a systematic review. CNS Drugs. (2016) 30:9-25. doi: 10.1007/s40263-015-0303-6

11. Schwan S, Sundström A, Stjernberg E, Hallberg E, Hallberg P. A signal for an abuse liability for pregabalin-results from the Swedish spontaneous adverse drug reaction reporting system. Eur J Clin Pharmacol. (2010) 66:947-53. doi: 10.1007/s00228-010-0853-y

12. Kapil V, Green JL, Le Lait MC, Wood DM, Dargan PI. Misuse of the caminobutyric acid analogues baclofen, gabapentin and pregabalin in the UK. Br J Clin Pharmacol. (2014) 78:190-1. doi: 10.1111/bcp.12277

13. Evoy KE, Sadrameli S, Contreras J, Covvey JR, Peckham AM, Morrison MD. Abuse and misuse of pregabalin and gabapentin: a systematic review update. Drugs. (2021) 81:125-56. doi: 10.1007/s40265-020-01432-7

14. Coutens B, Mouledous L, Stella M, Rampon C, Lapeyre-Mestre M, Roussin A, et al. Lack of correlation between the activity of the mesolimbic dopaminergic system and the rewarding properties of pregabalin in mouse. Psychopharmacology. (2019) 236:2069-82. doi: 10.1007/s00213-019-05198-Z

15. Peng XQ, Li X, Li J, Ramachandran PV, Gagare PD, Pratihar D, et al. Effects of gabapentin on cocaine self-administration, cocaine-triggered relapse and cocaine-enhanced nucleus accumbens dopamine in rats. Drug Alcohol Depend. (2008) 97:207-15. doi: 10.1016/j.drugalcdep.2007.09.019 reporting services. RADARS System is the property of Denver Health and Hospital Authority, a political subdivision of the State of Colorado. Authors were employed by Denver Health and Hospital Authority during this work. Denver Health retains exclusive ownership of all data, databases and systems. No subscriber participated in the conception, analysis, drafting, or review of this manuscript. Instituto de Salud Carlos III-FEDER-Red de Trastornos Adictivos UE-FEDER 2016 (RD16/0017/0010 and RD16/0017/003); AGAUR-Suport Grups de Recerca (2017 SGR530) and Acció instrumental d'Intensificació de Professionals de la Salut - Facultatius especialistes (PERIS: SLT006/17/00014).

16. Ponton R. Pregabalin misuse: preventing potential problems in New Zealand. $N$ Z Med J. (2018) 131:50-54. Available online at: https://assets-global website-files.com/5e332a62c703f653182faf47/5e332a62c703f6a7b62fd37e_ Ponton-FINAL.pdf

17. Anton RF, Latham P, Voronin K, Book S, Hoffman M, Prisciandaro J, et al. Efficacy of gabapentin for the treatment of alcohol use disorder in patients with alcohol withdrawal symptoms: a randomized clinical trial. JAMA Intern Med. (2020) 180:728-36. doi: 10.1001/jamainternmed.202 0.0249

18. Kranzler HR, Feinn R, Morris P, Hartwell EE. A meta-analysis of the efficacy of gabapentin for treating alcohol use disorder. Addiction. (2019) 114:1547-55. doi: 10.1111/add.14655

19. Torjesen I. Pregabalin and gabapentin: what impact will reclassification have on doctors and patients? BMJ. (2019) 364:11107. doi: 10.1136/bmj. 11107

20. Peckham AM, Ananickal MJ, Sclar DA. Gabapentin use, abuse, and the US opioid epidemic: the case for reclassification as a controlled substance and the need for pharmacovigilance. Risk Manag Healthc Policy. (2018) 11:109-16. doi: 10.2147/RMHP. S168504

21. Black JC, Rockhill K, Forber A, Amioka E, May KP, Haynes CM, et al. An online survey for pharmacoepidemiological investigation (survey of nonmedical use of prescription drugs program): validation study. J Med Internet Res. (2019) 21:e15830. doi: 10.2196/15830

22. Ng FL, Rockhill K, Black J, May KP, Whittington MD, Wood DM, et al. UK survey of non-medical use of prescription drugs (NMURx) as a valuable source of general population illicit drug use data. Postgrad Med J. (2018) 94:627-33. doi: 10.1136/postgradmedj-2018-135798

23. Evoy KE, Covvey JR, Peckham AM, Reveles KR. Gabapentinoid misuse, abuse and non-prescribed obtainment in a United States general population sample. Int J Clin Pharm. (2021). doi: 10.1007/s11096-020-01217-8. [Epub ahead of print].

24. Tambon M, Ponté C, Jouanjus E, Fouilhé N, Micallef J, LapeyreMestre M, et al. Gabapentinoid abuse in France: evidence on health consequences and new points of vigilance. Front Psychiatry. (2021) 12:639780. doi: $10.3389 /$ fpsyt.2021.639780

25. European Monitoring Centre for Drugs and Drug Addiction. European Drug Report 2017: Trends and Developments. (2017). Publications Office of the European Union. Available online at: https://www.emcdda.europa.eu/system/ files/publications/4541/TDAT17001ENN.pdf (accessed February 22, 2021).

26. Observatorio Español de las Drogas y las Adicciones. Encuesta Sobre Alcohol y Otras Drogas en España, EDADES 2019/20. Madrid: Ministerio de Sanidad. Delegación del Gobierno para el Plan Nacional sobre Drogas (2021). p. 93. Available online at: https://pnsd.sanidad.gob.es/profesionales/ sistemasInformacion/sistemaInformacion/pdf/2019_Informe_EDADES.pdf (accessed February 27, 2021).

27. Baandrup L, Ebdrup BH, Rasmussen JØ, Lindschou J, Gluud C, Glenthøj BY. Pharmacological interventions for benzodiazepine discontinuation in chronic benzodiazepine users. Cochrane Database Syst Rev. (2018) 3:CD011481. doi: 10.1002/14651858.CD011481.pub2 
28. Caniff K, Telega E, Bostwick JR, Gardner KN. Pregabalin as adjunctive therapy in benzodiazepine discontinuation. Am J Health Syst Pharm. (2018) 75:67-71. doi: 10.2146/ajhp160712

29. Lang N, Sueske E, Hasan A, Paulus W, Tergau F. Pregabalin exerts oppositional effects on different inhibitory circuits in human motor cortex: a double-blind, placebo-controlled transcranial magnetic stimulation study. Epilepsia. (2006) 47:813-9. doi: 10.1111/j.1528-1167.2006.00544.x

30. Bykov K, Bateman BT, Franklin JM, Vine SM, Patorno E. Association of gabapentinoids with the risk of opioid-related adverse events in surgical patients in the United States. JAMA Netw Open. (2020) 3:e2031647. doi: 10.1001/jamanetworkopen.2020.31647

31. Nielsen S, Gisev N, Leung J, Clare P, Bruno R, Lintzeris N, et al. Clinical correlates and outcomes associated with pregabalin use among people prescribed opioids for chronic non-cancer pain: A five-year prospective cohort study. Br J Clin Pharmacol. (2020). doi: 10.1111/bcp.14715. [Epub ahead of print].

Conflict of Interest: FF has received during the last 3 years travel grants from Lundbeck, Otsuka, Indivior, Pfizer, Gilead, Angelini, and Servier; and she has received grant/research support from Indivior and Servier. MT has been consultant/advisor and/or speaker for Gilead Sciences, Merck Sharp and Dohme Corp, Indivior, Mundipharma Pharmaceutics, Servier, and Adamed. NS received honoraria for several activities (advisory boards, lectures, manuscripts) by the factories AbbVie, Camurus, Hexal, Janssen-Cilag, MSD, Medice, Mundipharma, Reckitt-Benckiser/Indivior, and Sanofi-Aventis. During the last 3 years he participated in clinical trials financed by the pharmaceutical industry. IM served as Board Member for Angelini, Camurus, CT Sanremo, D\&A Pharma, Gilead, Indivior, Lundbeck, Molteni, MSD, and Mundipharma. MA over the past 3 years has interacted directly or through the University of Bordeaux Foundation with Camurus, Mundipharma, Accord Healthcare, Indivior for expert advice and/or funding donation grants.

The remaining authors declare that conflicts of interest had no role in the design of the study, data collection, analyses, and interpretation, in the writing of the manuscript, or in the decision to publish the results.

Copyright (c) 2021 Fonseca, Lenahan, Dart, Papaseit, Dargan, Wood, Guareschi, Maremmani, Auriacombe, Farré, Scherbaum and Torrens. This is an open-access article distributed under the terms of the Creative Commons Attribution License (CC $B Y)$. The use, distribution or reproduction in other forums is permitted, provided the original author(s) and the copyright owner(s) are credited and that the original publication in this journal is cited, in accordance with accepted academic practice. No use, distribution or reproduction is permitted which does not comply with these terms. 


\section{OPEN ACCESS}

Edited by: Nicolas Simon,

Aix Marseille Université, France

Reviewed by: Nicolas Franchitto,

Université Toulouse III Paul

Sabatier, France

Oussama Kebir

Institut National de la Santé et de la

Recherche Médicale (INSERM), France

*Correspondence: Stefania Chiappin stefaniachiappini9@gmail.com

Specialty section:

This article was submitted to Addictive Disorders,

a section of the journal

Frontiers in Psychiatry

Received: 22 January 2021

Accepted: 15 March 2021

Published: 07 May 2021

Citation:

Schifano F, Chiappini S, Miuli A, Mosca A, Santovito MC, Corkery JM,

Guirguis A, Pettorruso M, Di

Giannantonio $M$ and Martinotti $G$ (2021) Focus on Over-the-Counter Drugs' Misuse: A Systematic Review on Antihistamines, Cough Medicines, and Decongestants.

Front. Psychiatry 12:657397.

doi: 10.3389/fpsyt.2021.657397

\section{Focus on Over-the-Counter Drugs' Misuse: A Systematic Review on Antihistamines, Cough Medicines, and Decongestants}

\author{
Fabrizio Schifano ${ }^{1}$, Stefania Chiappini ${ }^{1,2 *}$, Andrea Miuli ${ }^{2}$, Alessio Mosca ${ }^{2}$, \\ Maria Chiara Santovito ${ }^{2}$, John M. Corkery ${ }^{1}$, Amira Guirguis ${ }^{3}$, Mauro Pettorruso ${ }^{2}$, \\ Massimo Di Giannantonio ${ }^{2}$ and Giovanni Martinotti ${ }^{2}$
}

'Psychopharmacology, Drug Misuse and Novel Psychoactive Substances Research Unit, School of Life and Medical Sciences, University of Hertfordshire, Hatfield, United Kingdom, ${ }^{2}$ Department of Neuroscience, Imaging and Clinical Sciences, "G. D'Annunzio" University, Chieti, Italy, ${ }^{3}$ Swansea University Medical School, Institute of Life Sciences 2, Swansea University, Swansea, United Kingdom

Background: Over the past 20 years or so, the drug misuse scenario has seen the emergence of both prescription-only and over-the-counter (OTC) medications being reported as ingested for recreational purposes. OTC drugs such as antihistamines, cough/cold medications, and decongestants are reportedly the most popular in being diverted and misused.

Objective: While the current related knowledge is limited, the aim here was to examine the published clinical data on OTC misuse, focusing on antihistamines (e.g., diphenhydramine, promethazine, chlorpheniramine, and dimenhydrinate), dextromethorphan (DXM)- and codeine-based cough medicines, and the nasal decongestant pseudoephedrine.

Methods: A systematic literature review was carried out with the help of Scopus, Web of Science databases, and the related gray literature. For data gathering purposes, both the Preferred Reporting Items for Systematic Reviews and Meta-analyses (PRISMA) and PROSPERO guidelines were followed (PROSPERO identification code CRD42020209261).

Results: After completion of the selection, eligibility, and screening phases, some 92 articles were here taken into consideration; case reports, surveys, and retrospective case series analyses were included. Findings were organized according to the specific OTC recorded. Most articles focused here on DXM $(n=54)$ and diphenhydramine $(n=12)$. When specified, dosages, route(s) of administration, toxicity symptoms (including both physical and psychiatric ones), and outcomes were here reported.

Conclusion: Results from the systematic review showed that the OTC misusing issues are both widespread worldwide and popular; vulnerable categories include adolescents 
and young adults, although real prevalence figures remain unknown, due to a lack of appropriate monitoring systems. Considering the potential, and at times serious, adverse effects associated with OTC misusing issues, healthcare professionals should be vigilant, and ad hoc preventative actions should be designed and implemented.

Keywords: drug abuse, drug misuse, prescription drug misuse, pharming, drug diversion, over the counter drug misuse, addiction, OTC

\section{INTRODUCTION}

Since generally being considered safe, over-the-counter (OTC) medicines are available without a prescription and can be purchased directly from related pharmacies/stores $(1,2)$. OTC medicines are meant to treat a variety of illnesses and symptoms, including pain, coughs and colds, diarrhea, nausea, etc. OTC availability, while encouraging self-care, has contributed to a public perception of safety and a lack of awareness relating to their potential for misuse, dependence, and harm (3-6). Indeed, some OTC medicines have active ingredients possessing a misusing potential at higher-than-recommended dosages (7) and are becoming increasingly popular for the possibility of their diversion in order to reach central psychoactive effects $(8-11)$. Currently, there is minimal information about the prevalence of OTC misuse, abuse, and dependence (8-10, 12). Indeed, current lack of knowledge may partly be due to poor sales' monitoring because of OTCs' favorable legal status. However, the so-called "pharming" phenomenon (13-15) has been requiring attention at different levels because of increased treatment admissions, dangerous behavior, more emergency room visits, drug-related deaths, and overdoses $(11,16,17)$. Most implicated drugs include certain cough suppressants, sleep aids, and antihistamines, which can at times be ingested in combination with remaining recreational psychotropics and/or prescription drugs and/or alcohol $(17,18)$. Overall, the misuse of OTC drugs is considered as more socially acceptable, less stigmatizing, and safer than the intake of illicit substances, also due to their likely lack of detection in standard drug screens (16). OTC drugs' intake may involve snorting or injecting the crushed tablets' powder to amplify the effects of a drug or ingesting these molecules for a purpose different from the therapeutic one. This may be the case for dextromethorphan (DXM) and codeine-based cough mixtures, being possibly misused at high dosages for recreational or euphoric effects; conversely, loperamide is at times being ingested for self-medicating withdrawal symptoms $(7,16,18-20)$. OTC misuse has also been associated with notable drug interactions, physical and mental health effects, individual variation in responses, and significant socioeconomic impact for the users, their family, and the wider community (13-15). Currently, most OTC misusing data are obtained through clinical records (e.g., case reports and case series) and surveys.

\section{Aims of the Study}

Thus, the current review aimed at (i) examining the current literature on the misuse of OTC drugs, focusing on the following OTCs: among antihistamines, diphenhydramine (DPH), promethazine, chlorpheniramine, and dimenhydrinate (DH);
DXM- and codeine-based cough medicines; and the nasal decongestant pseudoephedrine; (ii) illustrating patterns of OTCs' misuse, psychopathological effects, and harms associated; and (iii) better understanding the psychotropic molecular mechanisms underlying their recreational use.

\section{METHODS}

\section{Systematic Review Procedures}

A systematic electronic search was conducted from October 2020 to December 2020 and was set without a timeframe on the following scientific search engines: PubMed, Scopus, and Web of Science (WoS). The gray literature was also checked for relevant information. The following search strategies were used, respectively, in PubMed: ("diphenhydramine" OR "promethazine" OR "chlorpheniramine" OR "dimenhydrinate" OR "dextromethorphan" OR "pseudoephedrine" OR codeinebased cough medicines) AND ("abuse" OR "misuse" OR "craving" OR "addiction") NOT review NOT (animal OR rat OR mouse) NOT "in vitro;" in Scopus: [TITLEABS-KEY ("Diphenhydramine") OR TITLE-ABS-KEY ("Promethazine") OR TITLE-ABS-KEY ("Chlorpheniramine") OR TITLE-ABS-KEY ("Dimenhydrinate") OR TITLEABS-KEY ("Dextromethorphan") OR TITLE-ABS-KEY ("Pseudoephedrine") OR TITLE-ABS-KEY (codeine-based cough medicines) AND TITLE-ABS-KEY ("Abuse”) OR TITLEABS-KEY ("Misuse") OR TITLE-ABS-KEY ("Craving”) OR TITLE-ABS-KEY (“Addiction”) AND NOT TITLE-ABS-KEY (Review) AND NOT TITLE-ABS-KEY (animal) OR TITLE-ABSKEY (rat) OR TITLE-ABS-KEY (mouse) AND NOT TITLEABS-KEY ("in vitro")]; and WoS: ("diphenhydramine" OR "promethazine" OR "chlorpheniramine" OR "dimenhydrinate" OR "dextromethorphan" OR "pseudoephedrine" OR codeinebased cough medicines) AND ("abuse" OR "misuse" OR "craving" OR "addiction") NOT Review NOT (animal OR rat OR mouse) NOT "in vitro." The systematic review was structured in accordance with the Preferred Reporting Items for Systematic Reviews and Meta-analyses (PRISMA) (21) and PROSPERO guidelines (22). All data collected were tabulated on an Excel sheet to enable easy comparison and analysis.

\section{Data Synthesis Strategy}

The selection and eligibility phase of the articles was carried out by three independent reviewers (AM, AMo, and MCS), who screened articles based on title and abstract; the first screening was followed by full text reviews, using predetermined criteria for inclusion and exclusion. Eligible articles were 
considered if the published studies met all the following criteria: (i) original articles (open-label or double-blind trials, prospective or retrospective observational studies, case series and case reports); (ii) studies involving all age individuals misusing the OTC drugs selected. There were no other restrictions on the type of study population or publication time period. Exclusion criteria were as follows: (i) nonoriginal research articles (e.g., review, letter, commentary, editorial, book chapter, professional or clients' opinions); (ii) non fulltext articles (e.g., meeting/conference abstracts); (iii) languages other than English; (iv) animal/in vitro studies; (v) articles mentioning OTC drugs only as an example in the context of OTC drugs misuse; and (vi) articles not dealing with the misuse of the OTC drugs selected (e.g., DPH, promethazine, chlorpheniramine, and DH; DXM- and codeine-based cough medicines; and pseudoephedrine). Individual studies were also manually searched to identify additional citations. A final, between reviewers, cross-check was carried out, supervised by SC and MP, with both doubtful cases and possible inclusion/exclusion disagreements resolved through discussion with GM, MDG, and FS.

\section{Protocol and Registration}

Current research methods were approved by PROSPERO (identification code CRD42020209261).

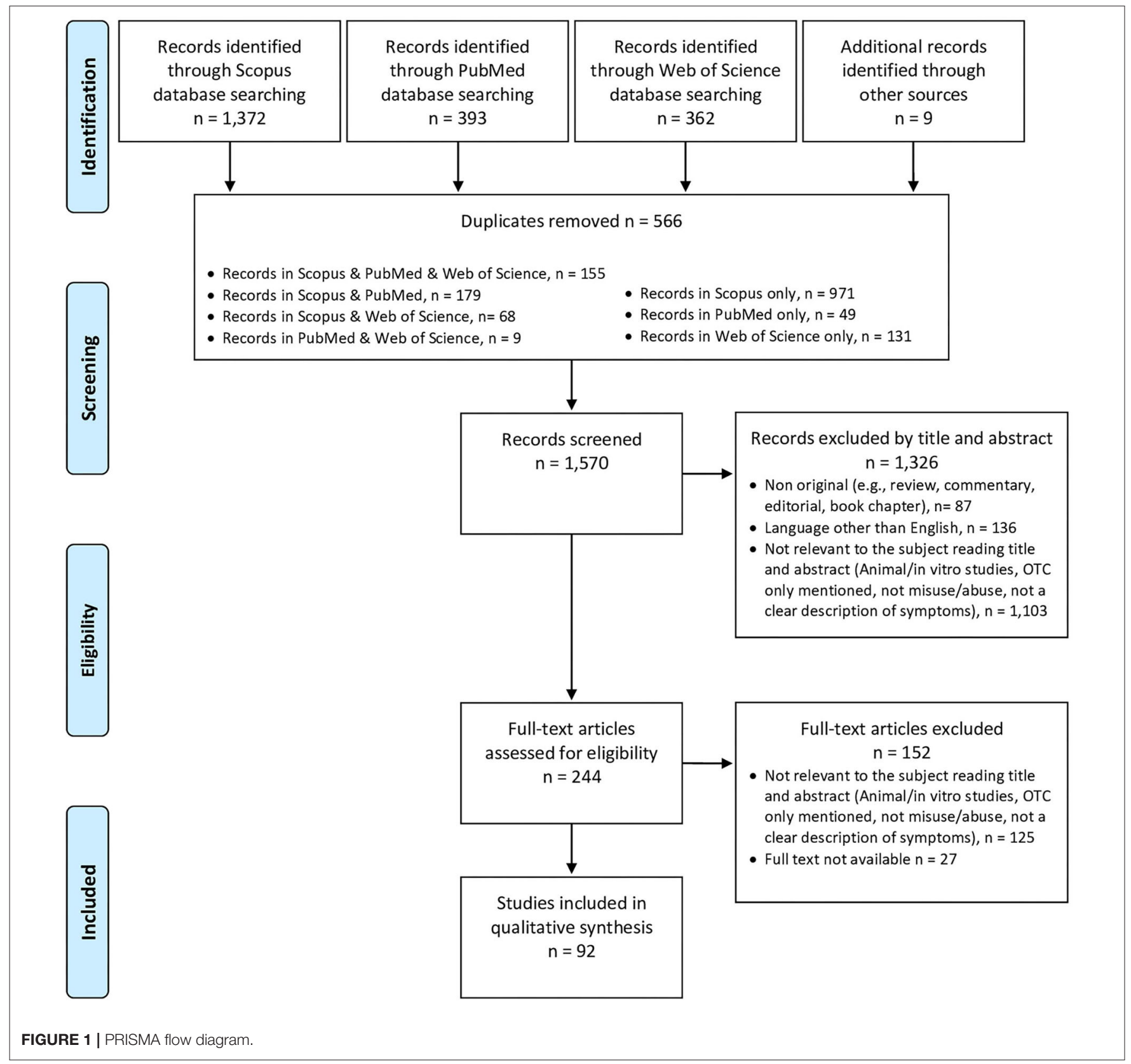




\section{Risk of Bias}

The assessment of risk of bias was made in accordance with the Cochrane risk of bias 2 ( $R o B 2$ ) tool (23).

\section{RESULTS}

In removing duplicate articles $(n=566)$ from a total of 2,136 papers $($ PubMed $=393$; Scopus $=1,372 ;$ WoS $=362$; additional sources =9), some 15,70 records resulted to be relevant for screening. Those considered not relevant to the subject while considering both the title and the abstract ( $n=1,103$; e.g., animal/in vitro studies; articles only mentioning OTC drugs, or not regarding OTC misuse/abuse, or not giving a clear description of related symptoms), those not written in English ( $n$ $=136)$, and those that were non-original articles $(n=87)$ were eliminated. Out of the 244 remaining full-text articles assessed for eligibility, some 125 papers did not match the inclusion criteria and 27 were not available. Hence, 92 articles were taken into consideration and properly analyzed (Figure 1). Findings were organized according to the specific OTC recorded, reported in alphabetical order in Supplementary Table 1; conversely, the most relevant characteristics of the misusing potential of the range of OTC drugs commented are summarized in Table $\mathbf{1 .}$

\section{Dextrometorphan}

DXM resulted to be the most reported misused drug, with $n=54$ related papers having been here identified (Supplementary Table 1). Indeed, it was recorded in two retrospective studies $(24,25)$, in 10 case series (26-35), and in several case reports $(24,25,36-77)$. Most represented users were male adolescent and young adults; DXM was mostly used alone $(28,36,37,40,44,45,54,57,66)$ or in DXM-containing cough mixtures $(26,29,30,39,41,42,47,50,52,53,62,64,68,71,72,74$, 76). Concomitant drugs included both licit and illicit substances, such as alcohol $(25,30,31,35,52,53,55,60,71,76)$; cannabis $(25,31,35,48,60)$; sedatives drugs, e.g., benzodiazepines (35); diethylamide lysergic acid (LSD) (35); opioids, e.g., morphine, heroin $(25,35,54)$; ecstasy (35); cocaine (35); and phencyclidine/ketamine $(34,35)$. Dosages varied among cases, up to super-high dosages (up to $4,920 \mathrm{mg})(31,35,36,61$ ). The only route of administration (ROA) here recorded was the oral one. Autonomic (e.g., mydriasis, tachycardia, palpitations) $(30,33,35,42,44,46,47,51,67,70,71)$, gastrointestinal (32, 35, 42, 47), neurological [e.g., amnesia, nystagmus, ataxia, seizures, and dystonia; $(24,26,29,30,32,34,35,39,43-$ $46,49,51-53,56,59,67)]$, and psychiatric symptoms, such as euphoria, agitation/irritability, confusion, hallucinations, and delusions, have been recorded $(24,25,27-31,33-38,40-$ $50,52-54,56,58,60,61,63,66,67,70-74,76)$. DXM misusers' psychiatric history frequently included alcohol and substance use disorders (SUD) $(25-27,29,31,32,34-$ 37, 40, 43, 45-48, 50, 53, 55-62, 64-67, 69, 76), mood disorders $(29,31,32,35,37,38,41,46,56-65,67,68,71)$, and schizophrenia $(37,53,69)$. Regarding the outcome, most cases required hospitalization with supportive treatments and antipsychotics [e.g., haloperidol $(43,47,71,73,75)$ ], risperidone
(74), and olanzapine $(54,61)$ administration. A DXM-related suicide has been recorded (31).

\section{Chlorpheniramine and Codeine}

Chlorpheniramine and codeine were recorded as having been misused in two papers (respectively, 68 and 69), as constituents of BRON, a Japanese codeine-based cough suppressant, together with methyl-ephedrine and caffeine $(78,79)$. BRON abuse has been associated with both psychotic/affective symptoms and dependence/withdrawal issues (78). Moreover, a case of severe intoxication of a codeine-based cough mixture determining a respiratory acidosis and requiring hospitalization was recorded (80) (Supplementary Table 1).

\section{Dimenhydrinate}

$\mathrm{DH}$ misuse was described in eight articles (Supplementary Table 1), including five case reports (8185) and three case series (86-88), mostly involving adults or adolescents (88). Most important psychiatric comorbidities described were represented by mood disorders $(82,84)$, SUD (83-87), and schizophrenia (85, 87). Massive dosages, up to $5,000 \mathrm{mg}$, of $\mathrm{DH}$ have been recorded in a few cases $(84,85,87)$. DH administration was always oral, except for one case where the molecule was administered intramuscularly in association with opiates and benzodiazepines (83). The symptoms recorded ranged from recreational stimulating effects (87) to emotional lability, agitation, anxiety, and druginduced delirium with paranoia, thought incoherence, and visual/auditory hallucinations $(81,86)$. The physical effects reported were mild and included mydriasis, tachycardia, hypertension, flushing, restlessness, dystonic reactions, and ataxia $(81,82,84-86,88)$, while one case reported generalized seizures (87). Withdrawal symptoms have been recorded after the abrupt interruption of chronic use and included irritability, anxiety, and craving $(82,84,87)$. When reported, treatment was almost supportive $(81-83,85,88)$; in two cases, benztropine was required to treat dyskinesia and related movement, muscle control, and balance symptoms $(81,84)$.

\section{Diphenhydramine}

DPH misuse was reported in 12 articles, including 10 case reports $(17,89-97)$; the remaining two included, respectively, a case series (98) and a retrospective review study (99) (Supplementary Table 1). Apart from the retrospective review study focusing on all Mandrax $®(\mathrm{DPH}+$ Methaqualone) abuse cases ( $n=67$, male) retrieved from the United States (US) Army during January-June 1972, users were here mostly represented by female (F/M, 9/6). A high number of users were adolescents, aged between 13 and 18 years (17, 94, 9698). Reported psychiatric comorbidities mostly included SUD (17, 89-92, 95), schizophrenia/psychotic symptoms (89, 91, 92), and mood disorders (17, 90, 91). DPH was taken in most cases orally, but both intramuscular (IM) (90) and intravenous (IV) (96-98) administrations were reported as well. Super-high dosages were recorded, up to 2,000 mg daily $(91-93,98)$. In a few cases, DPH was misused together with alcohol (91, 99), lorazepam (98), and cannabis (99). 
TABLE 1 | Drug classification and main characteristics of misuse of the selected OTC drugs.

$\begin{array}{lll}\begin{array}{l}\text { Drug/drug } \\ \text { classification }\end{array} & \begin{array}{l}\text { Administration Mechanism of action } \\ \text { path }\end{array} & \text { Effects }\end{array}$

\begin{tabular}{|c|c|c|}
\hline $\begin{array}{l}\text { Drug/drug } \\
\text { classification }\end{array}$ & $\begin{array}{l}\text { Administration } \\
\text { path }\end{array}$ & Mechanism of action \\
\hline Chlorpheniramir & Oral & - Chlorpheniramine acts primarily as \\
\hline
\end{tabular}

Chlorpheniramine acts primarily

(antihistamine)

- Moderate anticholinergic activity

- Chlorpheniramine has been found

to act as a serotonin

reuptake inhibitor

- ACUTE EFFECTS: psychiatric effects: (i) sedating and anxiolytic properties; (ii) its abuse has been related to pleasurable feelings such as euphoria and stimulating effects; (iii) it may be associated with psychotic symptoms in predisposed individuals (e.g., people with mental illnesses or individuals concomitantly abusing other drugs)

- CHRONIC EFFECTS: dependence

Codeine (opioid) Oral, IV

Dextromethorphan (DXM)

(non-competitive

NMDA receptor

antagonist and

sigma 1 agonist

antitussive)
Oral; IV and IN use also recorded in misuse cases
- It is a selective agonist of the mu-opioid receptor; it is a natural isomer of methylated morphine requiring metabolic activation by O-demethylation to morphine by CYP2D6

- ACUTE EFFECTS: psychiatric effects: euphoria, elation, analgesia, calmness; physical effects: respiratory depression, extreme somnolence progressing to stupor or coma, skeletal muscle flaccidity, cold and clammy skin, and sometimes bradycardia and hypotension. The triad of coma, pinpoint pupils, and respiratory depression is strongly suggestive of opiate poisoning. In severe overdosage, death may occur

- CHRONIC EFFECTS: dependence

- At high doses, acting as a NMDA receptor antagonist, DXM and its potent metabolite dextrorphan inhibit the excitatory amino acid and neurotransmitter glutamate causing hallucinogenic and

dissociative states

- DXM also exhibits binding activity at serotonergic receptors by hallucinations, paranoia, perceptual
- Neurobehavioural effects begin within 30-60 min of ingestion and persist for approximately $6 \mathrm{~h}$

- They are dose-related, starting from a mild to moderate stimulation with restlessness and euphoria (100-200 mg), to a state characterized distortions, delusional beliefs, ataxia, and out-of-body experiences (>1,000 mg)

- ACUTE EFFECTS: (i) psychiatric effects: euphoria, altered mental status, mania, mood lability, irritability, dysphoria, insomnia; (ii) physical effects: tachycardia, hypertension, vomiting, mydriasis,

diaphoresis, nystagmus, dystonia, loss of motor coordination;

- CHRONIC EFFECTS: (i) toxic psychosis and cognitive deterioration; (ii) folate deficiency and neuropathy; (iii) since DXM is produced as the crystalline hydrobromide salt, bromism is a rare consequence that has been identified in heavy chronic abusers of DXM (neurotoxic effects, resulting in somnolence, psychosis, seizures, and delirium
Does it cause dependence?

- Drug dependence is recorded after long-term use

- Withdrawal symptoms, including excessive irritability, anger outbursts, insomnia, sweating, and craving
Street names and brand

"Triple c" refers to Coricidin ${ }^{\circledR}$ cough and cold tablets; the combination of codeine, methyl ephedrine chlorpheniramine, and caffeine is marketed as $\mathrm{Bron}^{\circledR}$; Panado ${ }^{\circledR}$ is a combination of chlorpheniramine, paracetamo and pseudoephedrine; Advil ${ }^{\circledR}$ includes ibuprofen, chlorpheniramine and phenylephrine; other brand names: Polaramine ${ }^{\circledR}$, Chlortrimeton ${ }^{\circledR}$

- Codeine has an identified abuse liability potential, given its effect and development of tolerance within a short timeframe on regular or excessive use

- Codeine-dependence was here recorded, and associated with daily use of codeine

- Although DXM is not thought to have addictive properties, its chronic use might determine addiction due to

GABAergic/antiglutamatergic mechanisms, including substance-taking compulsive behaviors, tolerance, and autonomic withdrawal symptoms - EMCDDA: regarded as NPS

Street names: "Captain Cody," "Cody," "Little C," "Schoolboy," "Doors \& Fours." Common brand names for codeine and codeine containing combinations: Aspalgin ${ }^{\circledR}$ for aspirin and codeine; Nurofen Plus $^{\circledR}$ for ibuprofen and codeine; Panadeine Forte ${ }^{\circledR}$ for paracetamol and codeine

Street names: "Bromage," "Brome," "Candy," "Dex,"

"Dextro," "DM," "Drex," "DXM," "Red Devils," "Robo," "Rojo," "Skittles," "Triple C," "Tussin," "Velvet," and "Vitamin D," "Poo Man's Ecstasy"; the practice of using large amounts of DXM to achieve psychoactive effects is known as "robotrippin."

Common brand names are: Balminil DM ${ }^{\circledR}$, Benylin DM ${ }^{\circledR}$ Bronchophan ${ }^{\circledR}$, Buckleys $D^{\circledR}$, Calylin \#1, Delsym ${ }^{\circledR}$, Koffex $\mathrm{DM}^{\circledR}$, Novahistex $\mathrm{DM}^{\circledR}$, Robitussin $^{\circledR}$ 


\begin{tabular}{|c|c|c|c|c|c|}
\hline $\begin{array}{l}\text { Drug/drug } \\
\text { classification }\end{array}$ & $\begin{array}{l}\text { Administration } \\
\text { path }\end{array}$ & Mechanism of action & Effects & Does it cause dependence? & $\begin{array}{l}\text { Street names and brand } \\
\text { names }\end{array}$ \\
\hline $\begin{array}{l}\text { Diphenhydramine } \\
\text { (DPH) } \\
\text { (antihistamine } \\
\text { moiety of } \\
\text { dimenhydrinate/DH) }\end{array}$ & $\begin{array}{l}\text { Oral; IV and } \\
\text { IN use also } \\
\text { recorded in } \\
\text { misuse cases }\end{array}$ & $\begin{array}{l}\text { - It is a first generation } \\
\text { - H1-antihistamine } \\
\text { - Diphenhydramine also acts as a } \\
\text { potent anticholinergic agent } \\
\text { - It can acutely block the cell } \\
\text { membrane pump mechanism of } \\
\text { central } 5 \text {-hydroxytryptophane and } \\
\text { peripheral noradrenaline neurons }\end{array}$ & $\begin{array}{l}\text { - ACUTE EFFECTS: (i) psychiatric effects: euphoria, } \\
\text { altered mental status, hallucinations, and/or } \\
\text { psychosis; (ii) physical effects: tachycardia, } \\
\text { xerostomia, mydriasis, blurred vision, ileus, } \\
\text { urinary retention, CNS depression, agitation, and } \\
\text { hyperactivity } \\
\text { - CHRONIC EFFECTS: dependence }\end{array}$ & $\begin{array}{l}\text { Reported cases of DPH } \\
\text { dependence have resulted from } \\
\text { usage of large doses (often over } \\
1,000 \text { mg per day) over periods of } \\
\text { months or years. Withdrawal } \\
\text { symptoms include craving, } \\
\text { worsening of insomnia, rhinorrhoea, } \\
\text { nausea, irritability, restlessness, } \\
\text { abdominal cramps, sweating, and } \\
\text { diarrhea. Gradual tapering has } \\
\text { been the only described } \\
\text { detoxification treatment plan }\end{array}$ & 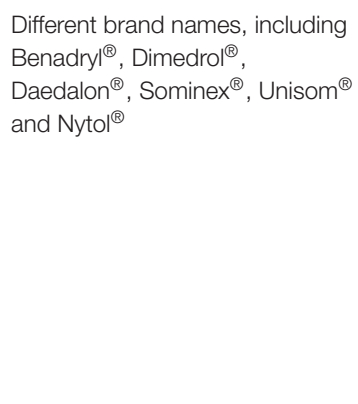 \\
\hline $\begin{array}{l}\text { Promethazine } \\
\text { (antihistamine) }\end{array}$ & Oral & $\begin{array}{l}\text { - It is a phenothiazine derivative and } \\
\text { a H1 receptor antagonist; It also } \\
\text { acts as a direct antagonist at } \\
\text { muscarinic (M1) and dopamine (D2) } \\
\text { receptors. It is classified as a } \\
\text { first-generation antihistamine } \\
\text { molecule which easily penetrates } \\
\text { the blood-brain barrier and is } \\
\text { associated with adverse effects } \\
\text { such as sedation }\end{array}$ & $\begin{array}{l}\text { - ACUTE EFFECTS: from mild sedation and CNS } \\
\text { depression to profound hypotension, respiratory } \\
\text { depression, unconsciousness, and sudden } \\
\text { death; overdosage might determine an } \\
\text { antimuscarinic delirium, agitation and neuroleptic } \\
\text { malignant syndrome } \\
\text { - it can be used to enhance effects of other } \\
\text { co-ingested substances, e.g., opioids } \\
\text { - CHRONIC EFFECTS: NR }\end{array}$ & $\begin{array}{l}\text { - EMCDDA: regarded as NPS } \\
\text { - Dependence might develop after } \\
\text { long-term use of promethazine } \\
\text { cough mixtures (containing opioids) }\end{array}$ & $\begin{array}{l}\text { Promethazine mixed with a soft } \\
\text { drink and/or alcohol is known as } \\
\text { "purple drank," "lean," "syzzurp," } \\
\text { "Texas tea"; Phenergan }{ }^{\circledR} \text { and } \\
\text { Phenadoz }{ }^{\circledR} \text { are common brand } \\
\text { names }\end{array}$ \\
\hline $\begin{array}{l}\text { Pseudoephedrine } \\
\text { (decongestant) }\end{array}$ & $\begin{array}{l}\text { Oral; IV use } \\
\text { also recorded } \\
\text { in misuse } \\
\text { cases }\end{array}$ & $\begin{array}{l}\text { - Sympathomimetic properties, } \\
\text { exerting a stimulating action on } \\
\text { alpha, beta1-, and } \\
\text { beta2-adrenergic receptors }\end{array}$ & $\begin{array}{l}\text { - ACUTE EFFECTS: stimulant effects, e.g., } \\
\text { euphoria, insomnia, diminished sense of fatigue, } \\
\text { anorexia, and accelerated thinking; psychotic } \\
\text { symptoms with auditory and visual hallucinations, } \\
\text { persecutory delusions, fear, disorganized behavior } \\
\text { might develop after high-dose consumption } \\
\text { - CHRONIC EFFECTS: dependence }\end{array}$ & $\begin{array}{l}\text { - Dependence might be developed } \\
\text { after long-term use } \\
\text { - Withdrawal symptoms include: } \\
\text { dysphoria, restlessness, abnormal } \\
\text { perceptions } \\
\text { - Due to the possibility to be used to } \\
\text { manufacture the class A controlled } \\
\text { drug methylamphetamine, } \\
\text { restrictions have been in place in } \\
\text { the UK to manage the risk of } \\
\text { products containing } \\
\text { pseudoephedrine and ephedrine; in } \\
\text { the US, a prescription is not } \\
\text { needed in most States, and in } \\
\text { remaining States there are limits on } \\
\text { how much an adult subject can } \\
\text { buy each month }\end{array}$ & $\begin{array}{l}\text { "Chalk," "Crank," "Meth," } \\
\text { "Speed"; 'Russian Cocktail' } \\
\text { includes pseudoephedrine } \\
\text { consumed together with } \\
\text { potassium permanganate and } \\
\text { acetylsalicylic acid diluted in } \\
\text { water; common brand names: } \\
\text { Sudafed®, Nexafed }{ }^{\circledR}, \\
\text { Zephrex- } D^{\circledR} \text {; Claritin } \\
\text { pseudoephedrine and loratadine }\end{array}$ \\
\hline
\end{tabular}

CNS, central nervous system; DH, Dimenhydrinate; DPH, Diphenhydramine; EMCDDA, European Monitoring Centre for Drugs and Drug Addiction; GABA, Gamma-Amino-Butyric Acid; H, Histamine; IN, Intranasal; IV, Intravenous; NMDA, N-Methyl-D-Aspartate; NPS, New Psychoactive Substance; OTC, Over-The-Counter; 5-HT, Serotonin. 
A polydrug overdose (e.g., DH together with bupropion, citalopram, acetaminophen, omeprazole, and naproxen) was recorded (94). DPH recreational use was associated with relaxation, calmness, and sleep improvement $(90,92,96,98,99)$. Acute intoxication was associated with psychotic symptoms, psychomotor agitation, restlessness, and disorientation $(89,92-$ 96, 98, 99). Withdrawal, consisting in both physical (e.g., bowel and bladder incontinence, hypertension, hypertonia, and extrapyramidal symptoms) and psychological (e.g., anxiety, irritability, rebound insomnia, and craving) symptoms have been recorded (17, 89, 90, 92, 95, 98, 99). DPH-induced intoxication was associated with signs and symptoms of anticholinergic toxicity, such as fever, mydriasis, flushed skin, dry mouth, dry eyes, decreased sweating, urinary retention, and dyskinesia (92-94, 98). A severe toxicity case was associated with cardiac conduction abnormalities and increased QT interval (90). On-drug cases of violent behavior, including suicide, have been reported $(97,99)$. Treatment required hospitalization and supportive care; drugs used were antipsychotics, such as fluphenazine and quetiapine, benzodiazepines, and benztropine $(89,90,92,93)$.

\section{Promethazine}

A few papers recorded here the misuse of promethazine; a retrospective analysis of data from the American Association of Poison Control Centres (AAPCC) National Poison Data System (NPDS) from January 2002 to December 2012 reported 354 promethazine intentional misuse/abuse cases (100) (Supplementary Table 1). All cases involved adolescents and young adults who misused promethazine orally. In most cases $(n=259)$ promethazine abuse was associated with other substances, such as DXM, codeine, phenylephrine, pseudoephedrine, caffeine, etc. Intoxication symptoms ranged from mild to severe effects, up to seizures and coma, but no fatalities have been reported. Agitation, confusion, slurred speech, and hallucinations were described as well. Promethazinealone abuse cases were mostly managed in healthcare facilities, while promethazine in coformulation mostly required emergency department (ED) care management (100). Moreover, further cases of nonmedical use of promethazine were here identified from (i) the Danish Poison and Information Centre (DPIC) and related registers used within the State Serum Institute of Denmark (SSI) (101); (ii) a prospective database of poisoning admissions (January 1987-May 2007) to a UK regional toxicology service (102); and (iii) a prospective study regarding patterns of misuse of heroin injectors (103). Druginduced delirium was the most represented psychiatric effects; this was managed with antipsychotics and benzodiazepines (101, 102). Interestingly, the use of promethazine injection in opioid users was reported as a substitute for heroin or to increase the effects of an inadequate heroin dosing (103). A case of drug-induced delirium deriving from the coingestion of high-dose promethazine, cyproheptadine, and fluvoxamine in a young girl was recorded (104). Finally, a case of promethazine dependence and withdrawal after 2year continuing use of a promethazine-cough mixture was described (105).

\section{Pseudoephedrine}

Seven articles, including six case reports (106-111) and one case series (112), described the misuse of pseudoephedrine (Supplementary Table 1). Cases mostly involved male adults (age range, 18-45 years) (F/M, 3/7) suffering from mood disorders (107, 109-111). One paper recorded an SUD [e.g., alcohol, cannabis, and heroin; (112)]. Massive dosages [e.g., 3,000-4,500 mg of pseudoephedrine/day; (107)] and IV administrations (108, 111, 112) have been associated with the misuse of pseudoephedrine. Physical symptoms associated with pseudoephedrine high dosage ingestion included stimulating effects such as decreased appetite, dry mouth, palpitations (106, 107, 112), and motor symptoms [e.g., gait and balance disorder, postural instability, generalized dystonia, hypokinesia, bradykinesia, psychomotor retardation; (106-108, 112)]. Pseudoephedrine effects were dose dependent and ranged from euphoria, insomnia, diminished sense of fatigue, and accelerated thinking, to psychotic symptoms with auditory and visual hallucinations, persecutory delusions, fear, and disorganized behavior (106, 109-111). Withdrawal symptoms have been recorded after the abrupt interruption of the longterm use $(106,107)$. Some cases required hospitalization and treatment with antipsychotics, e.g., haloperidol (106, 109-111); benzodiazepines (108); and antidepressants, e.g., amitriptyline $(106,108)$. No fatalities have been recorded.

\section{DISCUSSION}

This systematic review has illustrated a range of both themes and data regarding the misuse/abuse of some selected OTC drugs, including DXM, DPH, DH, codeine-based cough syrups, promethazine, and pseudoephedrine. Their misuse potential may be particularly significant in adolescents and young adults (10, 12, 113). OTC recreational intake appeared to be associated with high/very high dosages $(17,27,30,31,35,36,40,42,45$, $46,55,58,61,66,76,79,84,85,88,90-93,104,107,114)$; idiosyncratic routes of administration (e.g., snorting; IM; IV; 39, $69,88-90,100,103)$; and associated with ingestion of both licit [e.g., alcohol, prescription opioids, benzodiazepines, other OTCs; $(25,35,49,52-55,60,61,72,76,83,91,94,99,101,102)]$ and illicit (e.g., cannabis, cocaine, ketamine, etc.) drugs (30, 31, 34, $35,48,58,60,61,88,99)$. OTC drugs were obtained by various means (8-11), including family and friends (63), multiple doctor prescriptions $(27,36,63,90,93)$, illegal online pharmacies/shops $(36,42,70,77)$, and theft/burglary from hospitals, residences, and pharmacies $(27,105,110)$. DXM pills named "Snurf" were also reported to have been acquired online and in having been marketed as a legal high (70).

Overall, two main populations of OTC misusers were identified (11): (a) patients already suffering from a health condition and/or a psychiatric disorder who became dependent on their prescription/OTC drugs due to prolonged/high-dosage use (115), e.g., DXM-based cough mixtures started for sinusitis, cough, nasal congestion, and then continued for years at higher dosages $(27,58)$. Other examples have included $\mathrm{DH}$ prescribed for emesis in pregnancy and then continued for 
12 years at a higher dosage without a prescription (82), $\mathrm{DPH}$ use initiated to assist with initial insomnia and then continued for 6 months up to $1,600 \mathrm{mg}$ daily (92), and pseudoephedrine self-administered to lose weight then causing addiction (106); (b) individuals, including substance abusers, not in treatment for a medical disorder or illness who may have started to misuse/abuse with OTC medications for recreational purposes $(36,40,43,45,70,116)$.

Out of a total of $n=185$ OTC misusers described in case reports/series surveys $(24,25,77,78,99-103)$, male subjects were the most represented $(\mathrm{F} / \mathrm{M}=51 / 134)$, with an SUD history having been recorded in 53 of them (53/185 $=28.6 \%)$. A range of psychiatric diagnoses were reported (45/185 misusers, 24.3\%), including mood disorders (e.g., bipolar disorder, depression, dysthymia; $N=26$ ), anxiety disorders (e.g., adjustment disorder, anxiety; $N=5$ ), psychotic disorders (e.g., schizoaffective disorder, schizophrenia, psychosis, delusional disorder; $N=11$ ), attention deficit and hyperactivity disorder (ADHD, $N=1$ ), eating disorders (e.g., bulimia; $\mathrm{N}=1$ ), and personality disorders (e.g., dependent disorder; $N=1$ ). Regarding the outcome, most cases recorded were associated with a full recovery after hospitalization, with treatment having been either supportive $(32,44-46,65)$ or symptomatic, with the latter consisting of benzodiazepines and antipsychotics $(25,27$, $28,43,47,49,51,54,61,67,68,71,73-75,79,111,115)$. A full detoxification procedure was recorded in cases of dependence and withdrawal $(17,82,92,95,98,105,107,109,115)$; examples included buprenorphine $2 \mathrm{mg}$ /day to treat a sudden opiate (codeine) withdrawal symptoms (114), naltrexone as a relapse prevention agent for DXM dependence (63), and topiramate for DXM craving (56). Some cases required specific actions in the Emergency Unit (80). Finally, it has been suggested here that drug use treatment would benefit from counseling, behavioral therapies support, and rehabilitation treatment to better overcome drug craving $(11,18,27,28,34,36,46,48,53,59$, $60,78,84,110,117)$. OTC-related fatalities were here related to either cases characterized by unusually high dosages $(24,31,96)$ or to suicide/self-aggression (31).

The cough-suppressant DXM resulted here to be the most popular OTC being misused (Supplementary Table 1) due to its dose-dependent sedative, dissociative, and stimulant properties (16, 118-120). Indeed, DXM psychotropic effects are mostly related to its active metabolite dextrorphan, which, if used in large dosages, is able to antagonizes $\mathrm{N}$ methyl-D-aspartate (NMDA) receptors, hence modulating the excitatory neurotransmission; this results in the production of specific dissociative, ketamine-like, experiences (19, 25, 31, 56, 118-121) (Supplementary Table 1 and Table 1). The effects depend upon several factors, such as an individual's CYP2D6 subtype, body weight, as well as the degree of tolerance to DXM, and the concomitant use of other CYP2D6 substrates, including antidepressants (fluoxetine, fluvoxamine, nefazodone, paroxetine, sertraline, venlafaxine), antipsychotics (clozapine, haloperidol, risperidone, thioridazine), $\beta$-blockers (atenolol, metoprolol, propranolol), antiarrhythmics, and opioid analgesics (codeine, tramadol, and methadone), which may decrease the rate of DXM metabolism, resulting in a DXM intoxication $(13,19,47,121,122)$. Due to DXM catabolism by repeated demethylation, which may lead to abnormal folate demands for methyl group transfer, a folate deficiency has been described in association with chronic DXM use $(26,39,122)$. In addition, dental caries cases were associated with the high syrup content of cough mixtures (26). Although DXM is not thought to have addictive properties, with chronic use, vulnerable individuals may rapidly develop tolerance, dependence, and withdrawal $(35,36,56,58,63,66,76)$. Interactions with other substances can often produce synergistic effects; in fact, OTC cough formulations frequently contain, in addition to DXM, other pharmaceutical agents such as chlorpheniramine, acetaminophen, or pseudoephedrine, exhibiting different effects. Indeed, individuals abusing with chlorpheniramine-containing DXM formulations may also exhibit anticholinergic signs and symptoms $(25,31,42,47,49,73,74,123)$. Conversely, the antipyretic and analgesic acetaminophen produces delayed hepatic injury $(29,62)$. Finally, interactions between DXM and selective serotonin reuptake inhibitors (SSRIs) or monoamine oxidase inhibitor (MAOI) might further increase the risk of a serotoninergic syndrome occurrence $(67,68,121,124)$.

Although widely used and generally considered safe, cases of antihistamine abuse and dependence have been recorded (125). These molecules were originally marketed for their antiallergy properties and are now made available as sleeping aids. Antihistamines' toxicity appears to be clinically related to both central and peripheral acetylcholine antagonism. In addition, specifically due to multiple potential mechanisms of action, DPH (e.g., the antihistamine moiety of DH) can acutely block the cell membrane pump mechanism of central 5-hydroxytryptophane and peripheral noradrenaline neurons, causing the euphoria reported by some users (Table 1). At high dosages, and taken together with other drugs (e.g., alcohol, cannabis, and stimulants), DPH and $\mathrm{DH}$ might be used to achieve a stimulant effect $(87,91,92,126,127)$. Reported cases of DPH dependence have resulted from long-term usage of large doses (often over 1,000 mg/day). Gradual tapering has been described to alleviate withdrawal symptoms (17, 125). Conversely, promethazine is used in cough syrups for its antihistaminic, antiemetic, and sedative effects, available with codeine in common cough suppressants (128); its abuse potential appears related to its calming and sedating effect and enhancement of other coingested substances (Table 1). A recreational use of promethazine mixed with a soft drink and/or alcohol ("purple drank") is currently popular among young people for its euphoric effects and easy accessibility $(19,20,129-131)$. Promethazine has been reported in SUD clients and is misused as a substitute for another drug or to increase the effects of inadequate dosing (i.e., to delay the onset of opioid withdrawal or to potentiate the sedating effect of benzodiazepines/Z-drugs) (13, 19, 20, 103, 129, 130, 132, 133). Overdose of promethazine is associated with an antimuscarinic delirium, agitation, and neuroleptic malignant syndrome (100, 102, 104, 133). Scott et al. (104) recorded a promethazineinduced delirium treated with physostigmine intravenously, which reversed both central and peripheral anticholinergic effects, similarly to a polydrug overdose due to the ingestion 
of DPH (94). Chlorpheniramine is used as a cheap sleep aid and/or as an anxiolytic due to its antimuscarinic properties; its abuse has been related to pleasurable feelings, which reinforces the repetitive use and the possibility of developing drug dependence (Table 1). It may, however, be associated with psychotic symptoms in predisposed individuals [e.g., people with mental illnesses or individuals concomitantly abusing other drugs; $(42,43,114,115)]$.

Codeine was reported within the misusing scenario of codeine-based cough and cold medicines and/or coingested with other substances, e.g., DXM, DPH, ephedrine, pseudoephedrine, methyl ephedrine, chlorpheniramine, promethazine, caffeine $(26,27,34,78-80,100,114,134)$. Codeine is a natural isomer of methylated morphine and, similarly to DXM, is a prodrug, requiring metabolic activation by $\mathrm{O}$-demethylation to morphine by CYP2D6. Thus, codeine-related effects are associated with CYP2D6 metabolism, e.g., ultrarapid CYP2D6 metabolizers produce an unexpectedly large amount of morphine, with resulting life-threatening opioid toxicity. Its recreational use is related to the agonism at mu receptors and the subjective effects of euphoria, elation, analgesia, and "liking" $(114,121)$. Codeine toxicity is characterized by respiratory depression and extreme somnolence progressing to stupor or coma (79); in severe overdosage cases, death may occur (121) (Supplementary Table $\mathbf{1}$ and Table 1). Idiosyncratic codeine administration procedures have been recorded, e.g., a misuser learned online how the codeine base might be extracted through a process called cold water extraction (CWE) to be then injected. Regular use of codeine is described here together with the development of both tolerance (135) and dependence $(80,114)$.

Decongestants, here recorded as being abused, both alone and with coingestants, were ephedrine and its stereoisomer pseudoephedrine $(78,79,106-109,111,112)$, which are sympathomimetic agents $(136,137)$ exerting a stimulating action on both alpha- and beta-adrenergic receptors $(136,137)$ (Supplementary Table 1 and Table 1). Indeed, ephedrine has been reported to obtain weight loss or to enhance athletic performance; both pseudoephedrine and ephedrine have been recorded as used illicitly in the production of methamphetamine $(136,138)$. The abuse was here associated with high dosage (106$109)$ and IV administration $(108,111,112)$. Dependence issues have been recorded (106-109).

\section{LIMITATIONS}

One of the difficulties regarding the literature on prescription drug misuse is both its heterogeneity and the issues in identifying misusing practices; interpretation was easier for both those cases reported by healthcare professionals, whose intervention was needed, National/Regional Poison Data System information (100, 101), etc. According to UNODC, the misuse of medicines is defined as "the problematic consumption outside of acceptable medical practice or medical guidelines, when self-medicating at higher doses and for longer than is advisable, for intoxicating purposes and when risks and adverse consequences outweigh the benefit" (8-11). However, levels of terminology variability and inconsistency to describe the OTC phenomenon were identified as well; this use was referred to as non-medical use, problem use, harmful use, recreational use, self-medication, or inappropriate use, which calls into question whether there is a consensus on the negative consequences (i.e., problem, harm) of OTC use. Indeed, some of these terms may not even necessarily refer to the same issue (8).

\section{CONCLUSIONS}

The current systematic review showed that OTC misuse is an increasingly relevant health issue associated with potential harms, including drug-related toxicity, addiction, and fatalities. Nowadays, the CoViD-19 pandemic has likely facilitated the occurrence of these misusing practices, as more users turned from street drugs to prescription/OTC products $(14,15)$. Indeed, OTC drugs are both widely accessible and perceived because of their favorable legal status as relatively safe, hence accepted in a "pill-popping culture" (11). There is the need of both drafting ad hoc treatment guidelines and planning preventative measures. These measures should revolve around the implementation of a range of associated issues, including scheduling amendments, proper surveillance, enhanced detection of misuse in clinical and pharmacy practice, and promotion of public health awareness initiatives $(9,11,16,139-141)$. As an example, due to the recent rise in opioid abuse and related overdose deaths worldwide, efforts are focusing on strengthening public health surveillance and limiting opioid prescribing $(142,143)$. Specifically, as codeine-containing products misusing levels might be hampered by their widespread and easy availability, upscheduling and pharmacy-based interventions targeting users might limit the purchase of codeine products without a prescription. The recent introduction of new OTC combinations with non-opioid agents may provide a safer alternative to these widely misused products (144). In the case of the antidiarrheal loperamide, found to be misused at high dosages and associated with cardiotoxicity, to support its safe use, the Food and Drug Administration (FDA) approved changes to the packaging for tablet and capsule forms limiting each carton to no more than $48 \mathrm{mg}$ of loperamide and requiring the tablets and capsules to be packaged in individual doses (145). A range of professionals should be involved in tackling the OTC misusing issues, including (i) physicians, especially general practitioners (GP), who can help OTC misusers in early recognizing a drug-related problem and refer them to the appropriate service (e.g., mental or addiction services); they should also take note of rapid increases in the amount of medication needed or frequent, unscheduled refill requests and uncovering possible "doctor shopping" practices. Physicians will continue to have a role in educating users to ensure that they use medications appropriately, following the prescribed directions, while being aware of potential interactions with other licit/illicit drugs $(11,16,18,116,135,141)$. Conversely, pharmacists should be watchful for prescription falsifications or alterations, being at the frontline in recognizing prescription drug abuse issues. Moreover, prescription drug monitoring programs could 
assist healthcare professionals in identifying patients who are getting prescriptions from multiple sources $(11,13,16-18,141$, 144, 146). Finally, abuse prevention campaigns might provide valuable resources on raising awareness and preventing medicine abuse [https://stopmedicineabuse.org/; (144)].

\section{DATA AVAILABILITY STATEMENT}

The original contributions presented in the study are included in the article/Supplementary Material, further inquiries can be directed to the corresponding author/s.

\section{AUTHOR CONTRIBUTIONS}

FS, SC, and GM conceived the idea of this paper. AM, MCS, and AMo extracted the data. FS, MP, GM, AG, and MDG supervised

\section{REFERENCES}

1. FDA. Understanding Over-the-Counter Medicines. Food and Drug Administration (2018). Available from: https://www.fda.gov/drugs/buyingusing-medicine-safely/understanding-over-counter-medicines (accessed January 17, 2021).

2. MHRA. Guidance Medicines: Reclassify Your Product. Medicines and Healthcare products Regulatory Agency (2020). Available from: https:// www.gov.uk/guidance/medicines-reclassify-your-product\#: :text='Over \%2Dthe\%2Dcounter\%20,in\%20the $\% 20$ UK\%20medicines\%20regulations (accessed January 17, 2021).

3. Cooper RJ. 'I can't be an addict. I am' Over-the-counter medicine abuse: a qualitative study. BMJ Open. (2013) 3:e002913. doi: 10.1136/bmjopen-2013-002913

4. Hughes C. A retrospective evaluation of over-the-counter (OTC) drugrelated overdoses at Accident and Emergency Departments in Northern Ireland. In: Abstract at the BPC-Manchester, UK (September 1, 2003) (2003). p. R45-5.

5. Wazaify M, Shields E, Hughes CM, McElnay JC. Societal perspectives on over-the-counter (OTC) medicines. Fam Pract. (2005) 22:170-6. doi: $10.1093 /$ fampra/cmh723

6. Cooper RJ. Over-the-counter medicine abuse-a review of the literature. $J$ Subst Use. (2013) 18:82e107. doi: 10.3109/14659891.2011.615002

7. NIDA. Over-the-Counter Medicines DrugFacts. National Institute on Drug Abuse (2017). Available from: https://www.drugabuse.gov/publications/ drugfacts/over-counter-medicines (accessed January 10, 2021).

8. Barrett SP, Meisner JR, Stewart SH. What constitutes prescription drug misuse? Problems and pitfalls of current conceptualizations. Curr Drug Abuse Rev. (2008) 1:255-62. doi: 10.2174/18744737108010 30255

9. Casati A, Sedefor R, Pfeiffer-Gerschel T. Misuse of medicines in the European Union: a systematic review of the literature. Eur Addict Res. (2012) 18:228-45. doi: 10.1159/000337028

10. Novak SP, Håkansson A, Martinez-Raga J, Reimer J, Krotki K, Varughese S. Nonmedical use of prescription drugs in the European Union. BMC Psychiatry. (2016) 16:274. doi: 10.1186/s12888-016-0909-3

11. UNODC. The Non-Medical Use of Prescription Drugs. Policy direction issues. Discussion paper. United Nations OFFICE on DRUGS and Crime (2011). Available from: https://www.unodc.org/documents/ drug-prevention-and-treatment/nonmedical-use-prescription-drugs. pdf (accessed January 10, 2021).

12. Fingleton NA, Watson MC, Duncan EM, Matheson C. Non-prescription medicine misuse, abuse and dependence: a cross-sectional survey of the UK general population. J Public Health. (2016) 38:722-30. doi: 10.1093/pubmed/fdv204 all stages of the process and were consulted to resolve any possible disagreement. SC, AM, and JMC drafted the first version and revised it after contributions from FS, AG, and GM. All authors contributed to the article and approved the submitted version.

\section{FUNDING}

GM received funds from the European Project entitled Analysis, Knowledge dissemination, Justice implementation, and Special Testing of Novel Synthetic Opioids-JUST-2017-AG-DRUG.

\section{SUPPLEMENTARY MATERIAL}

The Supplementary Material for this article can be found online at: https://www.frontiersin.org/articles/10.3389/fpsyt. 2021.657397/full\#supplementary-material

13. Chiappini S, Guirguis A, Corkery JM, Schifano F. Understanding the use of prescription and OTC drugs in obtaining highs and the pharmacist role in preventing abuse. Pharm J. (2020) 305:7943. doi: 10.1211/PJ.2020.20208538

14. Chiappini S, Guirguis A, John A, Corkery JM, Schifano F. COVID-19: The hidden impact on mental health and drug addiction. Front Psychiatry. (2020) 11:767. doi: $10.3389 /$ fpsyt.2020.00767

15. Chiappini S, Schifano F. What about "Pharming"? Issues regarding the misuse of prescription and over-the-counter drugs. Brain Sci. (2020) 10:736. doi: 10.3390/brainsci10100736

16. Levine DA. "Pharming": the abuse of prescription and overthe-counter drugs in teens. Curr Opin Pediatr. (2007) 19:270-4 doi: 10.1097/MOP.0b013e32814b09cf

17. Gracious B, Abe N, Sundberg J. The importance of taking a history of overthe-counter medication use: a brief review and case illustration of "PRN" antihistamine dependence in a hospitalized adolescent. $J$ Child Adolesc Psychopharmacol. (2010) 20:521-4. doi: 10.1089/cap.2010.0031

18. NIDA. National Institute of Health (NIH). Research Report Series. Prescription Drug Abuse. National Institute on Drug Abuse (2011).

19. NIDA. Cough and Cold Medicines. National Institute on Drug Abuse (2020). Available from: https://teens.drugabuse.gov/drug-facts/cough-andcold-medicines (accessed January 10, 2021).

20. NIDA. Misuse of Prescription Drugs. Overview. National Institute on Drug Abuse (2020). Available from: https://www.drugabuse.gov/publications/ research-reports/misuse-prescription-drugs/overview (accessed January 10, 2021).

21. Moher D, Shamseer L, Clarke M, Ghersi D, Liberati A, Petticrew $\mathrm{M}$, et al. Preferred reporting items for systematic review and metaanalysis protocols (PRISMA-P) 2015 statement. Syst Rev. (2015) 4:1. doi: 10.1186/2046-4053-4-1

22. Bernardo WM. PRISMA statement and PROSPERO. Int Braz J Urol. (2017) 43:383-4. doi: 10.1590/s1677-5538.ibju.2017.03.02

23. Higgins JPT, Altman DG, Sterne JAC (editors). Chapter 8: assessing risk of bias in included studies. In: Cochrane Handbook for Systematic Reviews of Interventions version 5.2.0 (updated June 2017), Cochrane, 2017 (2017). Available from: www.training.cochrane.org/handbook (accessed December 20, 2020).

24. Forrester MB. Dextromethorphan abuse in Texas, 2000-2009. J Addict Dis. (2011) 30:243-7. doi: 10.1080/10550887.2011.581986

25. Ritter D, Ouellette L, Sheets JD, Riley B, Judge B, Cook A, et al. "Robotripping": Dextromethorphan toxicity and abuse. Am J Emerg Med. (2020) 38:839-41. doi: 10.1016/j.ajem.2019.10.001

26. Au WY, Tsang J, Cheng TS, Chow WS, Woo YC, Ma SK, et al. Cough mixture abuse as a novel cause of megaloblastic anaemia and peripheral neuropathy. Br J Haematol. (2003) 123:956-8. doi: 10.1046/j.1365-2141.2003. 04694.x 
27. Ghosh AK, Peh LH. Dextromethorphan: abusing the overused. Singapore Med, J. (2011) 52:134-5.

28. Hapangama A, Kuruppuarachchi K. Dextromethorphan abuse. React Wkly. (2011) 18:109-110. doi: 10.2165/00128415-201113640-00066

29. Kirages TJ, Sulé HP, Mycyk MB. Severe manifestations of coricidin intoxication. Am J Emerg Med. (2003) 21:473-5. doi: 10.1016/S0735-6757(03)00168-2

30. Logan BK. Combined dextromethorphan and chlorpheniramine intoxication in impaired drivers. J Forensic Sci. (2009) 54:1176-80. doi: 10.1111/j.1556-4029.2009.01127.x

31. Logan BK, Yeakel JK, Goldfogel G, Frost MP, Sandstrom G, Wickham DJ. Dextromethorphan abuse leading to assault, suicide, or homicide. J Forensic Sci. (2012) 57:1388-94. doi: 10.1111/j.1556-4029.2012.02133.x

32. Murray S, Brewerton T. Abuse of over-the-counter dextromethorphan by teenagers. South Med J. (1993) 86:1151-3. doi: 10.1097/00007611-199310000-00014

33. Nordt SP. "DXM": a new drug of abuse? Ann Emerg Med. (1998) 31:794-5. doi: 10.1016/S0196-0644(98)70250-1

34. Tsang JS, Au WY. Cough mixture abuse and rhabdomyolysis. Hong Kong Med J. (2012) 18:68-9.

35. Ziaee V, Hamed EA, Hoshmand A, Amini H, Kebriaeizadeh A, Saman K. Side effects of dextromethorphan abuse, a case series. Addicti Behav. (2005) 30:1607-13. doi: 10.1016/j.addbeh.2005.02.005

36. Akerman SC, Hammel JL, Brunette MF. Dextromethorphan abuse and dependence in adolescents. J Dual Diagn. (2010) 6:266-78. doi: 10.1080/15504263.2010.537515

37. Alam LY, Nelson A, Bastiampillai T. Cough syrup psychosis: Is it under-recognised? Aust N Z J Psychiatry. (2013) 47:1209-10. doi: $10.1177 / 0004867413495927$

38. Amaladoss A, Brien SO. Cough syrup psychosis. CJEM. (2011) 13:53-6. doi: 10.2310/8000.2011.100216

39. Au WY, Cheng TS, Siu TS, Tam S. Cerebellar degeneration and folate deficiency due to cough mixture abuse. Haematologica. (2005) 90(Suppl.):ECR28.

40. Bernstein LB, Albert D, Baguer C, Popiel M. Long-term dextromethorphan use and acute intoxication results in an episode of mania and autoenucleation. J Addict Med. (2020) 14:e133-5. doi: 10.1097/ADM.0000000000000568

41. Bostwick JM. Dextromethorphan-induced manic symptoms in a bipolar patient on lithium. Psychosomatics. (1996) 37:571-3. doi: 10.1016/S0033-3182(96)71523-2

42. Boyer EW. Dextromethorphan abuse. CMJ. (2008) 53:109-10. doi: $10.4038 / \mathrm{cmj} . v 53 \mathrm{i} 3.256$

43. Butwicka A, Krystyna S, Retka W, Wolańczyk T. Neuroleptic malignant syndrome in an adolescent with CYP2D6 deficiency. Eur J Pediatr. (2014) 173:1639-42. doi: 10.1007/s00431-013-2208-z

44. Cherkes JK, Friedman JH. Dextromethorphan-induced neurologic illness in a patient with negative toxicology findings. Neurology. (2006) 66:1952-3. doi: 10.1212/01.wnl.0000219805.24666.34

45. Craig DF. Psychosis with Vicks Formula 44-D abuse. CMAJ. (1992) 147:843-4

46. Desai S, Aldea D, Daneels E, Soliman M, Braksmajer AS, Kopes-Kerr CP. Chronic addiction to dextromethorphan cough syrup: a case report. J Am Board Fam Med. (2006) 19:320-3. doi: 10.3122/jabfm.19.3.320

47. Dilich A, Girgis C. Robo-tripping: a case of robitussin abuse in a methadone maintenance patient. Psychosomatics. (2017) 58:544-50. doi: 10.1016/j.psym.2017. 03.010

48. Fleming PM. Dependence on dextromethorphan hydrobromide. BMJ. (1986) 293:597. doi: 10.1136/bmj.293.6547.597

49. Ganetsky M, Babu KM, Boyer EW. Serotonin syndrome in dextromethorphan ingestion responsive to propofol therapy. Pediatr Emerg Care. (2007) 23:829-31. doi: 10.1097/PEC.0b013e31815a0667

50. Helfer J, Kim OM. Psychoactive abuse potential of Robitussin-DM. Am J Psychiatry. (1990) 147:672-3. doi: 10.1176/ajp.147.5.672b

51. Hendrickson RG, Cloutier RL. "Crystal Dex:" free-base dextromethorphan. J Emerg Med. (2007) 32:393-6. doi: 10.1016/j.jemermed.2007.03.010
52. Hinsberger A, Sharma V, Mazmanian D. Cognitive deterioration from longterm abuse of dextromethorphan: a case report. J Psychiatry Neurosci. (1994) 19:375-7.

53. Iaboni RP, Aronowitz JS. Dextromethotphan abuse in a dually diagnosis patient. J Nerv Ment Dis. (1995) 183:341-2. doi: 10.1097/00005053-199505000-00014

54. Jamison SC, Vasudeva S. A 60-year-old woman with agitation and psychosis following ingestion of dextromethorphan and opioid analgesics. J Psychopharmacol. (2009) 23:989-91. doi: 10.1177/0269881108092125

55. Kaplan B, Buchanan J, Krantz MJ. QTc prolongation due to dextromethorphan. Int $J$ Cardiol. (2011) 148:363-4. doi: $10.1016 /$ j.ijcard.2010.09.024

56. Roy AK III, Hsieh C, Crapanzano K. Dextromethorphan addiction mediated through the NMDA system: common pathways with alcohol? J Addict Med. (2015) 9:499-501. doi: 10.1097/ADM.0000000000000152

57. Kimber TE, Thompson PD. Segmental dystonia in the context of dextromethorphan abuse: a new cause of delayed onset drug-induced dystonia? Mov Disord Clin Pract. (2015) 2:299-300. doi: 10.1002/mdc3.12148

58. Linn KA, Long MT, Pagel PS. "Robo-tripping": dextromethorphan abuse and its anesthetic implications. Anesth Pain Med. (2014) 4:e20990. doi: 10.5812/aapm.20990

59. Majlesi N, Lee DC, Ali SS. Dextromethorphan abuse masquerading as a recurrent seizure disorder. Pediatr Emerg Care. (2011) 27:210-1. doi: 10.1097/PEC.0b013e31820d8dc1

60. Marsh LD, Key JD, Spratt E. Bulimia and dextromethorphan abuse: a case study. J Subst Abuse Treat. (1997) 14:373-6. doi: 10.1016/S0740-5472(97)00019-6

61. Martinak B, Bolis RA, Black JR, Fargason RE, Birur B. Dextromethorphan in cough syrup: the poor man's psychosis. Psychopharmacol Bull. (2017) 47:5963.

62. Medows M, Acosta C, Vega V. Pancreatitis and acute liver failure from Coricidin $\mathbb{R}$ HBP intoxication. Cureus. (2020) 12:e10202. doi: 10.7759/cureus.10202

63. Miller SC. Treatment of dextromethorphan dependence with naltrexone. Addict Disord Treatment. (2005) 4:145-8. doi: 10.1097/01.adt.0000186359.43340.76

64. Modi D, Bhalavat R, Patterson JC II. Suicidal and homicidal behaviors related to dextromethorphan abuse in a middle-aged woman. J Addict Med. (2013) 7:143-4. doi: 10.1097/ADM.0b013e318281a547

65. Monks S, Yen M, Myers J. Bromism: an overlooked and elusive toxidrome from chronic dextromethorphan abuse. Am J Emerg Med. (2020) 38:1696.e1. doi: 10.1016/j.ajem.2019.158491

66. Mutschler J, Koopmann A, Grosshans M, Hermann D, Mann K, Kiefer F. Dextromethorphan: Entzugs- und abhängigkeitssyndrom. Dtsch Arztebl. (2010) 7:537-40. doi: 10.3238/arztebl.2010.0537

67. Navarro A, Perry C, Bobo WV. A case of serotonin syndrome precipitated by abuse of the anticough remedy dextromethorphan in a bipolar patient treated with fluoxetine and lithium. Gen Hosp Psychiatry. (2006) 28:78-80. doi: 10.1016/j.genhosppsych.2005.06.008

68. Okland T, Shirazi M, Rylander M, Holland J. A case of aggressive psychosis in the setting of regular dextromethorphan abuse. Psychosomatics. (2016) 57:655-6. doi: 10.1016/j.psym.2016.06.002

69. Orrell MW. Dependence on dextromethorphan hydrobromide. BMJ. (1996) 312:44.

70. Osterhoudt KC, Miloradovich J. Snurfin' USA. Pediatr Emerg Care. (2010) 26:693-4. doi: 10.1097/PEC.0b013e3181f4f1e5

71. Polles A, Griffith JL. Dextromethorphan-induced mania. Psychosomatics. (1996) 37:71-4. doi: 10.1016/S0033-3182(96)71601-8

72. Roberge RJ, Hirani KH, Rowland PL III, Berkeley R, Krenzelok EP. Dextromethorphan and pseudoephedrine-induced agitated psychosis and ataxia: case report. J Emerg Med. (1999) 17:285-8. doi: 10.1016/S0736-4679(98)00193-0

73. Sharma A, Dewan V, Petty F. Acute psychosis with Coricidin cold medicine. Ann Pharmacother. (2005) 39:1577-8. doi: 10.1345/aph.1G193

74. Stanciu CN, Penders TM. Mania after misuse of dextromethorphan: a case report and brief review of "robotripping". J Addict Med. (2015) 9:159-60. doi: 10.1097/ADM.0000000000000104 
75. Walker J, Yatham LN. Benylin (dextromethorphan) abuse and mania. BMJ. (1993) 306:896. doi: 10.1136/bmj.306.6882.896

76. Wolfe TR, Caravati EM. Massive dextromethorphan ingestion and abuse. Am J Emerg Med. (1995) 13:174-6. doi: 10.1016/0735-6757(95)90088-8

77. Qiu YW, Lv XF, Jiang GH, Su HH, Ma XF, Tian JZ, et al. Potential gray matter unpruned in adolescents and young adults dependent on dextromethorphan-containing cough syrups: evidence from cortical and subcortical study. Brain Imaging Behav. (2017) 11:1470-8. doi: 10.1007/s11682-016-9628-0

78. Ishigooka J, Yoshida Y, Murasaki M. Abuse of "BRON": a Japanese OTC cough suppressant solution containing methylephedrine, codeine, caffeine and chlorpheniramine. Prog Neuropsychopharmacol Biol Psychiatry. (1991) 15:513-21. doi: 10.1016/0278-5846(91)90026-W

79. Murao S, Manabe $\mathrm{H}$, Yamashita $\mathrm{T}$, Sekikawa $\mathrm{T}$. Intoxication with over-the-counter antitussive medication containing dihydrocodeine and chlorpheniramine causes generalized convulsion and mixed acidosis. Intern Med. (2008) 47:1013-5. doi: 10.2169/internalmedicine.47.0925

80. Wong KM, Chak WL, Cheung CY, Chan YH, Choi KS, Chau KF, et al. Hypokalemic metabolic acidosis attributed to cough mixture abuse. Am J Kidney Dis. (2001) 38:390-4. doi: 10.1053/ajkd.2001.26107

81. Brown JH, Sigmundson HK. Delirium from misuse of dimenhydrinate. Can Med Assoc, J. (1969) 101:49-50. doi: 10.4039/Ent101109-1

82. Kaya FD. A patient with dimenhydrinate dependence: a case report. Klin Psikofarmakol Bul. (2014) 24:184-7. doi: 10.5455/bcp.20140131023347

83. Oliver M, Stenn PG. Is there a risk for dependency with therapeutic doses of dimenhydrinate? Psychosomatics. (1993) 34:459. doi: 10.1016/S0033-3182(93)71856-3

84. Wen X, Di Paola F, Chopra N. Psychotropics in your medicine cabinet: a case study of dimenhydrinate Use. J Addict Med. (2019) 13:412-4. doi: 10.1097/ADM.0000000000000511

85. White RF, Honer WG, Procyshyn RM, Vila-Rodriguez F. Dimenhydrinate use disorder with chronic psychosis. J Clin Psychopharmacol. (2015) 35:1057. doi: 10.1097/JCP.0000000000000238

86. Malcolm R, Miller WC. Dimenhydrinate (Dramamine) abuse: hallucinogenic experiences with a proprietary antihistamine. Am J Psychiatry. (1972) 128:1012-3. doi: 10.1176/ajp.128.8.1012

87. Prost E, Millson RC. Clozapine treatment of dimenhydrinate abuse. Am J Psychiatry. (2004) 161:1500. doi: 10.1176/appi.ajp.161.8.1500

88. Rowe C, Verjee Z, Koren G. Adolescent dimenhydrinate abuse: resurgence of an old problem. J Adolesc Health. (1997) 21:47-9. doi: 10.1016/S1054-139X(96)00291-1

89. Bonham C, Birkmayer F. Severe diphenhydramine dependence and withdrawal: case report. J Dual Diagn. (2009) 5:97-103. doi: 10.1080/15504260802620269

90. Chen TY, Yeh YW, Kuo SC, Chen CY, Lin TP, Chang CC. Diphenhydramine dependence through deep intramuscular injection resulting in myonecrosis and prolonged QT interval. J Clin Pharm Ther. (2014) 39:325-7. doi: $10.1111 /$ jcpt.12142

91. Cox D, Ahmed Z, McBride AJ. Diphenhydramine dependence. Addiction. (2001) 96:516-7.

92. Feldman MD, Behar M. A case of massive diphenhydramine abuse and withdrawal from use of the drug. JAMA. (1986) 255:3119-20. doi: 10.1001/jama.1986.03370220081028

93. Hermann DM, Bassetti CL. Reversible opsoclonus after diphenhydramine misuse. Eur Neurol. (2005) 53:46-7. doi: 10.1159/000084266

94. Phillips MA, Acquisto NM, Gorodetsky RM, Wiegand TJ. Use of a physostigmine continuous infusion for the treatment of severe and recurrent antimuscarinic toxicity in a mixed drug overdose. JMT. (2014) 10:205-9. doi: 10.1007/s13181-013-0330-y

95. Saran JS, Barbano RL, Schult R, Wiegand TJ, Selioutski O. Chronic diphenhydramine abuse and withdrawal: a diagnostic challenge. Neurol Clin Pract. (2017) 7:439-41. doi: 10.1212/CPJ.0000000000000304

96. Smith SG, Davis WM. Nonmedical use of butorphanol and diphenhydramine. JAMA. (1984) 252:1010. doi: 10.1001/jama.252.8.1010c

97. Sundararaghavan S, Suarez WA. Oral benadryl and central venous catheter abuse-a potentially "lethal combination". Pediatr Emerg Care. (2004) 20:6046. doi: 10.1097/01.pec.0000139743.38968.7f
98. Dinndorf PA, McCabe MA, Frierdich S. Risk of abuse of diphenhydramine in children and adolescents with chronic illnesses. J Pediatr. (1998) 133:293-5. doi: 10.1016/S0022-3476(98)70240-9

99. Tennant FS. Complications of methaqualone-diphenhydramine (Mandrax R) abuse. Br J Addict Alcohol Other Drugs. (1973) 68:327-30. doi: 10.1111/j.1360-0443.1973.tb01265.x

100. Tsay ME, Procopio G, Anderson BD, Klein-Schwartz W. Abuse and intentional misuse of promethazine reported to US poison centers: 2002 to 2012. J Addict Med. (2015) 9:233-7. doi: 10.1097/ADM.00000000000 00124

101. Jensen LL, Rømsing J, Dalhoff K. A Danish survey of antihistamine use and poisoning patterns. Basic Clin Pharmacol Toxicol. (2017) 120:64-70. doi: $10.1111 /$ bcpt. 12632

102. Page CB, Duffull SB, Whyte IM, Isbister GK. Promethazine overdose: clinical effects, predicting delirium and the effect of charcoal. QJM. (2009) 102:12331. doi: 10.1093/qjmed/hcn153

103. Clatts M, Giang le M, Goldsamt L, Colón-López V. Nonmedical use of promethazine hydrochloride among heroin injectors in Vietnam: unrecognized risks and unintended consequences. Subst Use Misuse. (2010) 45:515-27. doi: 10.3109/10826080903452520

104. Scott J, Pache D, Keane G, Buckle H, O’Brien N. Prolonged anticholinergic delirium following antihistamine overdose. Australas Psychiatry. (2007) 15:242-4. doi: 10.1080/10398560601147020

105. Tan CH, Kua EH, Lee EL. Cough mixture addiction-a case report. Singapore Med J. (1988) 29:186-7.

106. Alevizos B. Dependence and chronic psychosis with Dnor-pseudoephedrine. Eur Psychiatry. (2003) 18:423-5. doi: 10.1016/j.eurpsy.2003.05.001

107. Diaz MA, Wise TN, Semchyshyn GO. Self-medication with pseudoephedrine in a chronically depressed patient. Am J Psychiatry. (1979) 136:1217-8. doi: 10.1176/ajp.136.9.1217

108. Koksal A, Baybas S, Sozmen V, Koksal NS, Altunkaynak Y, Dirican A, et al. Chronic manganese toxicity due to substance abuse in Turkish patients. Neurol India. (2012) 60:224-7. doi: 10.4103/0028-3886.96407

109. Leighton KM. Paranoid psychosis after abuse of Actifed. Br Med J. (1982) 284:789-90. doi: 10.1136/bmj.284.6318.789-a

110. Pugh CR, Howie SM. Dependence on pseudoephedrine. Br J Psychiatry. (1986) 149:798. doi: 10.1192/bjp.149.6.798a

111. Sullivan G. Acute psychosis following intravenous abuse of pseudoephedrine: a case report. J Psychopharmacol. (1996) 10:324-5. doi: $10.1177 / 026988119601000413$

112. Sikk K, Taba P, Haldre S, Bergquist J, Nyholm D, Zjablov G, et al. Irreversible motor impairment in young addicts-ephedrone, manganism or both? Acta Neurol Scand. (2007) 115:385-9. doi: 10.1111/j.1600-0404.2007.00818.x

113. NIDA. Monitoring the Future Study: Trends in Prevalence of Various Drugs. Overview. National Institute on Drug Abuse (2020). Available from: https:// www.drugabuse.gov/drug-topics/trends-statistics/monitoring-future/ monitoring-future-study-trends-in-prevalence-various-drugs (accessed January 10, 2021).

114. Donuk T, Altlntoprak AE, Tekin H. Possible causal link between idiopathic intracranial hypertension and the misuse of codeine-based products. J Child Adolesc Psychopharmacol. (2016) 26:764-5. doi: 10.1089/cap.2015.0042

115. Das S, Palappallil DS, Malathesh BC, Chatterjee SS. Chlorpheniramine dependence presenting as mania. Asian J Psychiatr. (2017) 30:29-30. doi: 10.1016/j.ajp.2017.07.007

116. Inciardi JA, Surratt HL, Cicero TJ, Beard RA. Prescription opioid abuse and diversion in an urban community: the results of an ultrarapid assessment. Pain Med. (2009) 10:537-48. doi: 10.1111/j.1526-4637.2009.0 0603.x

117. Clinical guidelines on Drug Misuse and Dependence Update. Independent Expert Working Group (2017) Drug Misuse and Dependence: UK Guidelines on Clinical Management. London: Department of Health. (2017). Available from: https://assets.publishing.service.gov.uk/government/uploads/syst em/uploads/attachment_data/file/673978/clinical_guidelines_2017.pdf (accessed January 10, 2021).

118. Journey JD, Agrawal S, Stern E. Dextromethorphan toxicity. In: StatPearls. Treasure Island, FL: StatPearls Publishing (2020). 
119. Schifano F, Orsolini L, Papanti D, Corkery J. Novel psychoactive substances of interest for psychiatry. World Psychiatry. (2015) 14:15-26. doi: $10.1002 /$ wps.20174

120. Reissig CJ, Carter LP, Johnson MW, Mintzer MZ, Klinedinst MA, Griffiths RR. High doses of dextromethorphan, an NMDA antagonist, produce effects similar to classic hallucinogens. Psychopharmacol. (2012) 223:1-15. doi: 10.1007/s00213-012-2680-6

121. Burns JM, Boyer EW. Antitussives and substance abuse. Subst Abuse Rehabil. (2013) 4:75-82. doi: 10.2147/SAR.S36761

122. Zawertailo LA, Kaplan HL, Busto UE, Tyndale RF, Sellers EM. Psychotropic effects of dextromethorphan are altered by the CYP2D6 polymorphism: a pilot study. J Clin Psychopharmacol. (1998) 18:332-7. doi: 10.1097/00004714-199808000-00014

123. Carbonaro TM, Johnson MW, Griffiths RR. Subjective features of the psilocybin experience that may account for its self-administration by humans: a double-blind comparison of psilocybin and dextromethorphan. Psychopharmacol. (2020) 237:2293-304. doi: 10.1007/s00213-02005533-9

124. Monte AA, Chuang R, Bodmer M. Dextromethorphan, chlorphenamine and serotonin toxicity: case report and systematic literature review. Br J Clin Pharmacol. (2010) 70:794-8. doi: 10.1111/j.1365-2125.2010. 03747.x

125. Elwood WN. Sticky business: patterns of procurement and misuse of prescription cough syrup in Houston. J Psychoact Drugs. (2001) 33:121-33. doi: 10.1080/02791072.2001.10400477

126. Preston KL, Wolf B, Guarino JJ, Griffiths RR. Subjective and behavioral effects of diphenhydramine, lorazepam and methocarbamol: evaluation of abuse liability. J Pharmacol Exp Ther. (1992) 262:707-20.

127. Mumford GK, Silverman K, Griffiths RR. Reinforcing, subjective, and performance effects of lorazepam and diphenhydramine in humans. Exp Clin Psychopharmacol. (1996) 4:421-30. doi: 10.1037/1064-1297.4.4.421

128. Bergman J, Wallman P. Promethazine overdose: is it "Goodnight" after all?. NZ Med J. (1998) 111:246-8.

129. Chiappini S, Schifano F, Corkery JM, Guirguis A. Beyond the 'purple drank': Study of promethazine abuse according to the European Medicines Agency adverse drug reaction reports. J Psychopharmacol. (2021) 10:269881120959615. doi: 10.1177/0269881120959615

130. Miuli A, Stigliano G, Lalli A, Coladonato M, D’Angelo L, Esposito F, et al. "Purple Drank" (Codeine and Promethazine Cough Syrup): a systematic review of a social phenomenon with medical implications. J Psychoactive Drugs. (2020) 52:453-62. doi: 10.1080/02791072.2020.1797250

131. Williams JF, Lundahl LH. Focus on adolescent use of club drugs and "other" substances. Pediatr Clin North Am. (2019) 66:1121-34. doi: 10.1016/j.pcl.2019.08.013

132. Dahlman D, Abrahamsson T, Kral AH, Hakansson A. Nonmedical use of antihistaminergic anxiolytics and other prescription drugs among persons with opioid dependence. J Addict. (2016) 2016:9298571. doi: $10.1155 / 2016 / 9298571$

133. Shapiro BJ, Lynch KL, Toochinda T, Lutnick A, Cheng HY, Kral AH. Promethazine misuse among methadone maintenance patients and community-based injection drug users. J Addict Med. (2013) 7:96-101. doi: 10.1097/ADM.0b013e31827f9b43

134. Lee E, Cooper RJ. Codeine addiction and internet forum use and support: qualitative netnographic study. JMIRMent Health. (2019) 6:e12354. doi: $10.2196 / 12354$

135. Kimergård A, Foley M, Davey Z, Dunne J, Drummond C, Deluca P. Codeine use, dependence and help-seeking behaviour in the UK and Ireland: an online cross-sectional survey. QJM. (2017) 110:559-64. doi: $10.1093 /$ qjmed/hcx076
136. Gorodetsky R. Pseudoephedrine. In: Encyclopedia of Toxicology. 3rd ed. Elsevier, Academic Press (2014). p. 1123-5.

137. Pereira Limberger R, Bemvenuti Jacques AL, Schmitt GC, Dutra Arbo M. Pharmacological effects of ephedrine. In: Ramawat K, Mérillon JM, editors. Natural Products. Berlin, Heidelberg: Springer (2013).

138. Gov.uk. Pseudoephedrine and Ephedrine: Update on Managing Risk of Misuse (2015). Available from: https://www.gov.uk/drug-safety-update/ pseudoephedrine-and-ephedrine-update-on-managing-risk-of-misuse (accessed January 18, 2021).

139. Coombes H, Cooper RJ. Staff perceptions of prescription and over-thecounter drug dependence services in England: a qualitative study. Addict Sci Clin Pract. (2019) 14:41. doi: 10.1186/s13722-019-0170-4

140. Orriols L, Gaillard J, Lapeyre-Mestre M, Roussin A. Evaluation of abuse and dependence on drugs used for selfmedication: a pharmacoepidemiological pilot study based on community pharmacies in France. Drug Saf. (2009) 32:859-73. doi: 10.2165/11316590-000000000-00000

141. Lessenger JE, Feinberg SD. Abuse of prescription and over-thecounter medications. J Am Board Fam Med. (2008). 21:45-54. doi: 10.3122/jabfm.2008.01.070071. Erratum in: J Am Board Fam Med. (2008). 21:175.

142. Levy N, Mills P, Fawcett WJ. Avoiding an opioid crisis in the, U. K. BMJ. (2019) 364:11033. doi: 10.1136/bmj.11033

143. NIDA. Opioid Overdose Crisis. National Institute on Drug Abuse (2021). Available from: https://www.drugabuse.gov/drug-topics/opioids/ opioid-overdose-crisis (accessed March 04, 2021).

144. Van Hout MC, Norman I. Misuse of non-prescription codeine containing products: Recommendations for detection and reduction of risk in community pharmacies. Int J Drug Policy. (2016) 27:17-22. doi: 10.1016/j.drugpo.2015.09.007

145. FDA. DA Limits Packaging for Anti-diarrhea Medicine loperamide (Imodium) to Encourage Safe Use. Food and Drug Administration (2019). Available from: https://www.fda.gov/drugs/drug-safety-and-availability/fda-limitspackaging-anti-diarrhea-medicine-loperamide-imodium-encourage-safeuse (accessed March 04, 2021).

146. Wright J, Bond C, Robertson HD, Matheson C. Changes in over-the-counter drug misuse over 20 years: perceptions from Scottish pharmacists. J Public Health. (2016) 38:793-9. doi: 10.1093/pubmed/fdv169

Conflict of Interest: FS was a member of the UK Advisory Council on the Misuse of Drugs (ACMD; 2011-2019) and is currently a member of the EMA Advisory Board (Psychiatry). GM has been a consultant and/or a speaker and/or has received research grants from Angelini, Doc Generici, Janssen-Cilag, Lundbeck, Otsuka, Pfizer, Servier, Recordati. MDG has been a consultant and/or a speaker and/or has received research grants from Angelini, Janssen-Cilag, Lundbeck, Otsuka, Pfizer, Servier, Recordati. JMC is a member of the ACMD's Novel Psychoactive Substances and Technical Committees.

The remaining authors declare that the research was conducted in the absence of any commercial or financial relationships that could be construed as a potential conflict of interest.

Copyright (C) 2021 Schifano, Chiappini, Miuli, Mosca, Santovito, Corkery, Guirguis, Pettorruso, Di Giannantonio and Martinotti. This is an open-access article distributed under the terms of the Creative Commons Attribution License (CC BY). The use, distribution or reproduction in other forums is permitted, provided the original author(s) and the copyright owner(s) are credited and that the original publication in this journal is cited, in accordance with accepted academic practice. No use, distribution or reproduction is permitted which does not comply with these terms. 


\section{OPEN ACCESS}

Edited by:

Fabrizio Schifano, University of Hertfordshire,

United Kingdom

Reviewed by:

Stefania Chiappini,

University of Hertfordshire,

United Kingdom

Caroline Zangani,

University of Milan, Italy

*Correspondence:

Joëlle Micallef

joelle.micallef@ap-hm.fr

TORCID:

Thomas Soeiro

orcid.org/0000-0003-2604-9673

Vincent Pradel

orcid.org/0000-0003-2619-4432

Maryse Lapeyre-Mestre orcid.org/0000-0002-5494-5873

Joëlle Micallef

orcid.org/0000-0002-7172-7835

Specialty section:

This article was submitted to

Addictive Disorders,

a section of the journal

Frontiers in Psychiatry

Received: 10 December 2020 Accepted: 29 March 2021

Published: 17 May 2021

Citation:

Soeiro T, Lacroix C, Pradel V Lapeyre-Mestre M and Micallef $J$ (2021) Early Detection of Prescription Drug Abuse Using Doctor Shopping Monitoring From Claims Databases: Illustration From the Experience of the French Addictovigilance Network.

Front. Psychiatry 12:640120

doi: 10.3389/fpsyt.2021.640120
Early Detection of Prescription Drug Abuse Using Doctor Shopping Monitoring From Claims Databases: Illustration From the Experience of the French Addictovigilance Network

\author{
Thomas Soeiro ${ }^{1 \dagger}$, Clémence Lacroix ${ }^{1}$, Vincent Pradel ${ }^{1 \dagger}$, Maryse Lapeyre-Mestre ${ }^{2 \dagger}$ and \\ Joëlle Micallef ${ }^{1 *+}$ \\ ${ }^{1}$ Aix-Marseille Université, Inserm, UMR 1106, Hôpitaux Universitaires de Marseille, Service de Pharmacologie Clinique, \\ Centre d'évaluation et d'information sur la Pharmacodépendance - Addictovigilance, Marseille, France, ${ }^{2}$ Université Paul \\ Sabatier, Inserm, ClC 1436, Centre Hospitalier Universitaire de Toulouse, Service de Pharmacologie Clinique, Centre \\ d'évaluation et d'information sur la Pharmacodépendance - Addictovigilance, Toulouse, France
}

Opioid analgesics and maintenance treatments, benzodiazepines and z-drugs, and other sedatives and stimulants are increasingly being abused to induce psychoactive effects or alter the effects of other drugs, eventually leading to dependence. Awareness of prescription drug abuse has been increasing in the last two decades, and organizations such as the International Narcotics Control Board has predicted that, worldwide, prescription drug abuse may exceed the use of illicit drugs. Assessment of prescription drug abuse tackles an issue that is hidden by nature, which therefore requires a specific monitoring. The current best practice is to use multiple detection systems to assess prescription drug abuse by various populations in a timely, sensitive, and specific manner. In the early 2000's, we designed a method to detect and quantify doctor shopping for prescription drugs from the French National Health Data System, which is one of the world's largest claims database, and a first-class data source for pharmacoepidemiological studies. Doctor shopping is a well-known behavior that involves overlapping prescriptions from multiple prescribers for the same drug, to obtain higher doses than those prescribed by each prescriber on an individual basis. In addition, doctor shopping may play an important role in supplying the black market. The paper aims to review how doctor shopping monitoring can improve the early detection of prescription drug abuse within a multidimensional monitoring. The paper provides an in-depth overview of two decades of development and validation of the method as a complementary component of the multidimensional monitoring conducted by the French Addictovigilance Network. The process accounted for the relevant determinants of prescription drug abuse, such as pharmacological data (e.g., formulations and doses), chronological and geographical data (e.g., impact of measures and comparison 
between regions), and epidemiological and outcome data (e.g., profiles of patients and trajectories of care) for several pharmacological classes (e.g., opioids, benzodiazepines, antidepressants, and methylphenidate).

Keywords: doctor shopping, prescription drug abuse, claims database, signals detection, addictovigilance, opioids, benzodiazepines, methylphenidate

\section{INTRODUCTION}

Opioid analgesics and maintenance treatments, benzodiazepines and z-drugs, and other sedatives and stimulants are increasingly being abused to induce psychoactive effects or alter the effects of other drugs, eventually leading to dependence (1). Awareness of prescription drug abuse has been increasing in the last two decades, and organizations such as the International Narcotics Control Board has predicted that, worldwide, prescription drug abuse may exceed the use of illicit drugs (2). Prescription drug abuse is now qualified as an epidemic in economically developed countries, particularly in North America $(1,3,4)$.

Many studies pointed out an increasing trend of prescription drug abuse across European countries, highlighting the need for a specific monitoring (5-9). Several factors may explain this trend, such as a greater ease in obtaining prescription drugs than illicit drugs, a lower risk of arrest for trafficking, a higher social acceptability of their abuse, their higher purity, and their more predictable doses (6).

Assessment of prescription drug abuse tackles an issue that is hidden by nature, which therefore requires a specific monitoring. A single data source is rarely enough to assess such a complex phenomenon (10). The current best practice is to use multiple detection systems to assess prescription drug abuse by various populations in a timely, sensitive, and specific manner (11). By using various tools to mine epidemiological data, assess the pharmacological properties of the drugs, and assess the social contexts where the drugs are used, these systems demonstrated their usefulness to detect emerging trends earlier and intervene more quickly to protect the public from associated risks (12). Among these tools, assessing doctor shopping through overlapping prescriptions, multiple prescribers, or pharmacy shopping was implemented in several countries (13-19). Therefore, the paper aims to review how doctor shopping monitoring can improve the early detection of prescription drug abuse within a multidimensional monitoring. The paper provides an in-depth overview of two decades of development and validation of the method as a complementary component of the multidimensional monitoring conducted by the French Addictovigilance Network.

\section{RELEVANCE OF DOCTOR SHOPPING AS A PROXY FOR PRESCRIPTION DRUG ABUSE}

Drug-abusing patients may develop drug-seeking behavior to meet their need. Among them, doctor shopping has long been described, in several countries (e.g., North America, Europe, Asia, and Oceania) and for several pharmacological classes (e.g., opioids, stimulants, and benzodiazepines) (13-19). Doctor shopping involves overlapping prescriptions from multiple prescribers for the same drug, to obtain higher doses than those prescribed by each prescriber on an individual basis. Doctor shopping is based on circumventing the optimal one-to-one patient-prescriber relationship, and therefore on a lack of medical management, because one given prescriber does not know that other prescribers are also prescribing the same drug. The lack of medical management in addition to high doses increase the risks for adverse outcomes, such as high-risk use, overdose, and death $(13,20-24)$.

Among many diverted means for obtaining prescription drugs (e.g., friends or relatives, black market, or internet), doctor shopping is reported as one of the most frequent ones (25-27). In addition, obtaining prescription drugs from a dealer raises the question of how dealers obtain the prescription drugs they sell (28). Although the question is difficult to answer with a strong evidence, field studies suggest that doctor shopping may play an important role in supplying the black market (29-32). Notably, without regard to the final consumer (i.e., whether the patient himself or a subsequent purchaser), the concern for the lack of medical management remains, along with the risks associated with it.

\section{HOW TO QUANTIFY DOCTOR SHOPPING?}

Doctor shopping is difficult to monitor because the patient often attempts to hide the abuse and the prescribers may not even realize that they have been deceived. These observations underline the limitations of interviewing the prescribers or patients, and therefore, highlight the added value of claims databases to quantify doctor shopping objectively. Several teams from different countries have developed methods to detect doctor shopping in claims databases (13-19). The methods face two main challenges: a proper design of the method to accurately detect drug-abusing patients and the use of a data source that is representative of the population of interest.

\section{First Challenge: The Design of the Method}

The method must be both specific (i.e., must not red flag non-abusing patients) and sensible (i.e., must not miss real drug-abusing patients). Nevertheless, there is no standard definition of doctor shopping, and therefore, no gold standard method. Most studies assessing doctor shopping rely on the number of prescribers or pharmacies visited, without regard to successive and overlapping prescriptions (33). Such methods may overestimate abuse, because successive prescriptions from different prescribers may be legitimately needed, particularly in cancer and palliative care (34), or in similar situations when a 
general practitioner refers a regular patient to a specialist (35). Other situations involving successive prescribers may not be related to abuse or restricted to psychoactive prescription drugs, but rather related to prescriber factors (e.g., inconvenient hours or locations, long waiting times, or personal characteristics of the prescriber), illness factors (e.g., persistence of symptoms, lack of understanding, or lack of confidence in diagnosis or treatment), or psychological factors (e.g., anxiety leading to dose stockpiling) $(36,37)$.

Conversely, overlapping prescription is at the core of the safety concern, because it is the reason for the lack of medical management. Interestingly, a study compared the diagnostic odds ratios for opioid overdose of nine definitions of pharmacy shopping, using a multistate Medicaid claims database in the USA (38). The overdose rate was higher in patients with overlapping prescriptions than in patients with only pharmacy shopping. In addition, another study quantified episodes of multiple prescriber for benzodiazepines using a twoyear cohort in Japan. Consecutive overlapping prescriptions had the best accuracy to detect patients with potentially questionable prescribed quantities, and predict patients with episodes of multiple prescriber in the subsequent year (19).

In the early 2000's, we designed a method to detect and quantify doctor shopping for prescription drugs, accounting for overlapping prescriptions (14, 39-45) (Figure 1). To detect overlapping prescriptions, the method relies on periods of prescriptions, defined as the period between the first and last dispensing for each prescriber of each patient (i.e., the period during which a patient consults a prescriber). If there is a longer delay than a predefined threshold between two consecutive dispensings, the period of prescriptions is interrupted. During an interruption, a prescription from another prescriber is not considered as overlapping to avoid the overestimation of doctor shopping. If there are overlapping periods of prescriptions, there is a lack of medical management, and a share of the drug prescribed is considered to be obtained by doctor shopping. The method provides aggregated druglevel indicators (e.g., total quantity and proportion obtained by doctor shopping) and population-level indicators (e.g., number and proportion of patients with doctor shopping behavior), and individual patient-level indicators (e.g., individual quantity obtained by doctor shopping) (Figure 2). Taken together, these complementary indicators enable to assess the extent of abuse and abuse potential of prescription drugs, and characterize profiles of patients with doctor shopping behavior and their trajectories of care.

Notably, the method deliberately relies on a strict design to specifically detect overlapping prescriptions rather than the number of prescribers or pharmacies visited. In addition, the quantity obtained by doctor shopping is not the entire quantity received by a patient with doctor shopping behavior, but only the quantity received in addition to what is dispensed with only one prescriber. The underlying reason for this design is that the patient may legitimately need the drug for a medical use at the quantity prescribed by one prescriber. This design helps to rule out the hypothesis of pseudoaddiction [i.e., doctor shopping driven by insufficient dosing (46)], because it enables to discriminate patients who receive high doses in addition to a treatment considered legitimate $(14,45)$.

\section{Second Challenge: A Representative Data Source}

To enable an accurate quantification of doctor shopping, the database must be representative of the population of interest. In addition, the database must identify each health professional and health care consumer by a consistent pseudonym over time and across geography. Given that claims databases were initially designed for medicoadministrative purposes, it is far from trivial in practice. For example, in the USA, health insurance plans only cover residents by states or focus on a specific subset of the population (e.g., Medicaid covers low-income populations, while private insurances are employment-based). Notably, the use of a non-representative population may bias the results, because socioeconomic status is associated with abuse (47-49). In addition, some regulation [e.g., the 42 CFR part 2 in the USA, which aims to ensure confidentiality of records from federally funded drug and alcohol treatment centers (50)] may further complicate the use of claims databases.

In this regard, the French National Health Data System is a first-class data source for pharmacoepidemiological studies, as one of the world's largest claims database, whose representativeness is almost perfect (51-53). The National

FIGURE 1 | Method to detect and quantify doctor shopping for prescription drugs, accounting for overlapping prescriptions. *The quantity obtained by doctor
shopping is calculated as Qd-Qd/n, where Qd is the quantity dispensed, $\mathrm{n}$ is the number of overlapping periods of prescriptions, and Qd/n is the quantity that would
have been dispensed with only one prescriber.




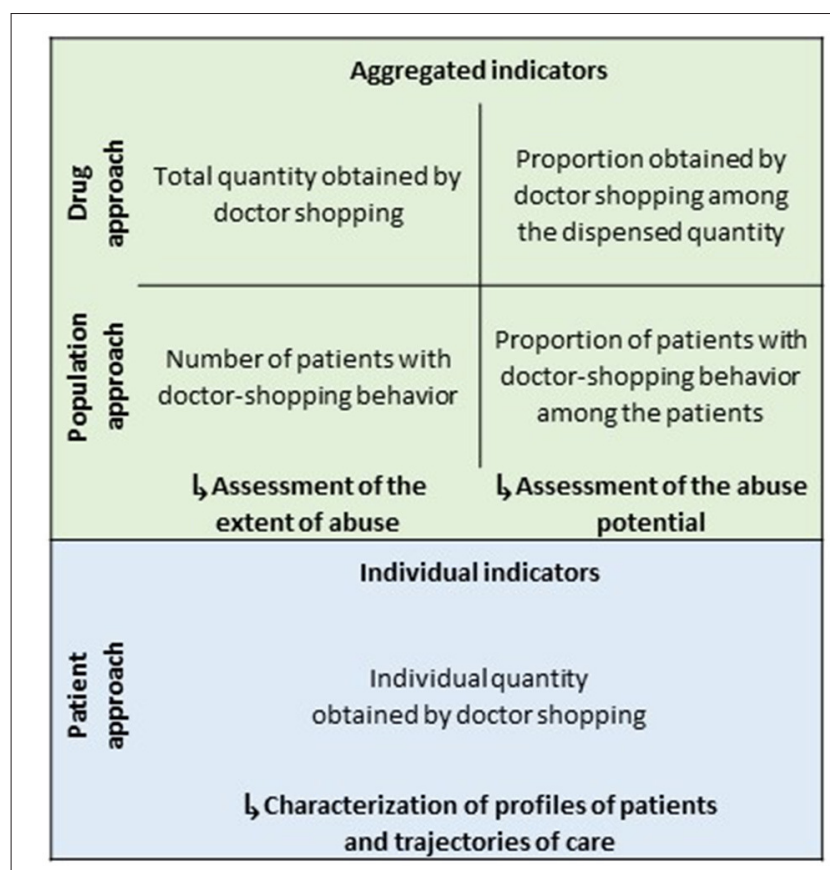

FIGURE 2 | Complementary indicators provided by the method.

Health Data System prospectively merges pseudonymized records of claims from all the French health insurance plans, the national hospital-discharge database, and the national death registry (54). Because the coverage by a health insurance plan is mandatory in France, the French National Health Data System covers almost $100 \%$ of the 67 million inhabitants, from birth to death, independently of the socioeconomic status and region of residence. In addition, each health professional and health care consumer is identified by a consistent pseudonym over time and across geography. As a result, the French National Health Data System enables a nationwide and exhaustive quantification of doctor shopping.

In the last two decades, the French National Health Data System has been extensively used for pharmacoepidemiological research, including some large-scale studies that have led to major public health interventions $(55,56)$. Among them, many studies have focused on psychoactive prescription drugs (57-63).

\section{VALIDATION OF DOCTOR SHOPPING AS A PHARMACOLOGICAL TOOL}

Before using doctor shopping as a proxy for prescription drug abuse, there is a need for an in-depth customized validation process within the health system of interest. The lack of a gold standard method makes a classical statistical validation process impossible (i.e., sensitivity, specificity, and predictive values). Therefore, an empirical approach is required to assess the external validity of the proxy in a given health system for several pharmacological classes. Such a process should rely on linking doctor shopping to relevant determinants of prescription drug abuse, such as pharmacological data (e.g., formulations and doses), chronological and geographical data (e.g., impact of measures and comparison between regions), and epidemiological and outcome data (e.g., profiles of patients and trajectories of care).

In the last two decades, we have conducted such an empirical validation of our method (14, 39-45) (Table 1). The process has provided solid evidence that the method is a relevant proxy for prescription drug abuse within the French health system, because it has always demonstrated an excellent external validity. In particular, the method demonstrated to be a useful pharmacological tool, able to provide detailed results by discriminating drugs, formulations, and doses.

\section{Detecting Prescription Drugs With a High Abuse Potential}

The method was first developed for buprenorphine maintenance treatment $(14,39)$, which was expected to have a high abuse potential in the real-life setting. In France, a wide access to maintenance treatments is ensured by an office-based setting for the majority of patients $(64,65)$. In parallel of a marked decrease in lethal heroin overdoses, a concern emerged along with observations of abuse (e.g., injection of crushed tablets, snorting, association with benzodiazepines such as flunitrazepam, and deaths) and an increasing buprenorphine black market (14). Interestingly, evidence of multiple prescribers for buprenorphine maintenance treatment was also described, but without quantifying the buprenorphine maintenance treatment involved, nor accounting for overlapping prescriptions (66).

A study was conducted among the 3,259 patients who received buprenorphine maintenance treatment in a population of two million inhabitants in South East France in 1999 and 2000. The method found that 225,351 defined daily doses (DDD) were obtained by doctor shopping, corresponding to $18.7 \%$ of the quantity dispensed (14). Doctor shopping was highly concentrated on a minority of patients (i.e., $8.5 \%$ of patients accounted for $45.4 \%$ of the quantity obtained by doctor shopping).

As a result, the health insurance implemented a prescription monitoring program for opioid maintenance therapies in 2004, for both public health and economic concerns. Patients who received $>32 \mathrm{mg} /$ day of buprenorphine maintenance treatment (i.e., twice the maximum recommended dose) were proposed a contract of care, including the choice of a single prescriber and pharmacist for buprenorphine maintenance treatment. Patients with particularly high doses who did not respond to the convocation, or did not respect their contract of care, could be prosecuted, or excluded from the health insurance plan. A second assessment of doctor shopping from 2000 to 2005 in the same population found that the prescription monitoring program led to a decrease in doctor shopping, without decreasing the access to buprenorphine maintenance treatment (39). 
TABLE 1 | Empirical validation of the method accounting for overlapping prescription, in the last two decades, in France.

\begin{tabular}{|c|c|c|c|c|c|}
\hline References & Date & Setting & $\begin{array}{l}\text { Prescription drugs } \\
\text { under study }\end{array}$ & $\begin{array}{l}\text { Number of patients } \\
\text { included }\end{array}$ & Main findings \\
\hline Pradel et al. (14) & 1999 and 2000 & $\begin{array}{l}\text { Two million } \\
\text { inhabitants in } \\
\text { South East France }\end{array}$ & $\begin{array}{l}\text { Buprenorphine } \\
\text { maintenance } \\
\text { treatment }\end{array}$ & 3,259 & $\begin{array}{l}225,351 \text { DDD were obtained by doctor shopping, } \\
\text { corresponding to } 18.7 \% \text { of the quantity dispensed. } \\
\text { Doctor shopping was highly concentrated on a minority } \\
\text { of patients (i.e., } 8.5 \% \text { of patients accounted for } 45.4 \% \text { of } \\
\text { the quantity obtained by doctor shopping). }\end{array}$ \\
\hline Pradel et al. (39) & 2000 to 2005 & $\begin{array}{l}\text { Two million } \\
\text { inhabitants in } \\
\text { South East France }\end{array}$ & $\begin{array}{l}\text { Buprenorphine } \\
\text { maintenance } \\
\text { treatment }\end{array}$ & $\begin{array}{l}>2,600 \text { each } \\
\text { semester }\end{array}$ & $\begin{array}{l}\text { Doctor shopping increased from } 2000 \text { (i.e., } 14.9 \% \text { of the } \\
\text { quantity dispensed) to } 2004 \text { (i.e., } 21.7 \% \text { of the quantity } \\
\text { dispensed), and decreased in } 2005 \text { (i.e., } 16.9 \% \text { of the } \\
\text { quantity dispensed) following the implementation of a } \\
\text { prescription monitoring program. } \\
\text { The number of patients remained stable from } 2000 \\
\text { to } 2005 \text {. }\end{array}$ \\
\hline Pradel et al. (40) & 2003 & $\begin{array}{l}\text { One million } \\
\text { inhabitants in } \\
\text { South West } \\
\text { France }\end{array}$ & Benzodiazepines & 128,230 & $\begin{array}{l}\text { Benzodiazepines were ranked according to their abuse } \\
\text { potential in real-life setting. } \\
\text { The proportion obtained by doctor shopping was the } \\
\text { highest for flunitrazepam } 1 \mathrm{mg} \text { (i.e., } 42.8 \% \text { of the quantity } \\
\text { dispensed), then for diazepam } 10 \mathrm{mg} \text { (i.e., } 3.2 \% \text { of the } \\
\text { quantity dispensed), and clorazepate } 50 \mathrm{mg} \text { (i.e., } 2.7 \% \text { of } \\
\text { the quantity dispensed). }\end{array}$ \\
\hline Rouby et al. (41) & 2005 & $\begin{array}{l}\text { Five million } \\
\text { inhabitants in } \\
\text { South East France }\end{array}$ & $\begin{array}{l}\text { Antidepressants and } \\
\text { benzodiazepines as } \\
\text { comparator }\end{array}$ & 410,525 & $\begin{array}{l}\text { Tianeptine ranked first among antidepressants for the } \\
\text { proportion obtained by doctor shopping (i.e., } 2.0 \% \text { of the } \\
\text { quantity dispensed), and was close to benzodiazepines } \\
\text { with a well-known abuse potential in real-life setting. }\end{array}$ \\
\hline Nordmann et al. (42) & 2008 & $\begin{array}{l}14 \text { million } \\
\text { inhabitants in three } \\
\text { regions in South } \\
\text { France (i.e., } \\
\text { Provence-Alpes- } \\
\text { Côte d'Azur, } \\
\text { Rhône-Alpes, and } \\
\text { Midi-Pyrénées) }\end{array}$ & Opioids & $\begin{array}{l}885,941 \text { in } \\
\text { Provence-Alpes-Côte } \\
\text { d'Azur } \\
945,102 \text { in } \\
\text { Rhône-Alpes } \\
\text { 386,834 } \\
\text { in Midi-Pyrénées }\end{array}$ & $\begin{array}{l}\text { The quantity obtained by doctor shopping in } \\
\text { Provence-Alpes-Côte d'Azur (i.e., } 213 \text { DDD/1,000 } \\
\text { inhabitants) was two-fold higher than in Rhône-Alpes } \\
\text { (i.e., } 115 \text { DDD/1,000 inhabitants) and in Midi-Pyrénées } \\
\text { (i.e., } 106 \text { DDD/1,000 inhabitants). } \\
\text { A signal emerged for oxycodone in Midi-Pyrénées. }\end{array}$ \\
\hline Ponté et al. (43) & 2013 & $\begin{array}{l}14 \text { million } \\
\text { inhabitants in } \\
\text { South France }\end{array}$ & $\begin{array}{l}\text { Opioids and } \\
\text { benzodiazepines as } \\
\text { comparator }\end{array}$ & $1,257,246$ & $\begin{array}{l}\text { The proportion obtained by doctor shopping was the } \\
\text { highest for the highest doses of morphine (i.e., } 8.4 \% \text { of } \\
\text { the quantity dispensed for morphine } 200 \mathrm{mg} \text { ) and } \\
\text { oxycodone (i.e., } 2.8 \% \text { of the quantity dispensed for } \\
\text { oxycodone } 80 \mathrm{mg} \text { ), and for nasal and transmucosal } \\
\text { fentanyl (i.e., respectively } 4.1 \text { and } 3.3 \% \text { of the quantity } \\
\text { dispensed). }\end{array}$ \\
\hline Soeiro et al. (44) & 2010 and 2016 & $\begin{array}{l}67 \text { million } \\
\text { inhabitants in } \\
\text { France }\end{array}$ & Oxycodone & $\begin{array}{l}67,838 \text { in } 2010 \\
212,753 \text { in } 2016\end{array}$ & $\begin{array}{l}\text { There was a three-fold increase in doctor shopping in } \\
\text { line with population exposure. } \\
\text { The quantity obtained by doctor shopping increased with } \\
\text { the dose for both immediate-release and } \\
\text { extended-release tablets. }\end{array}$ \\
\hline Soeiro et al. (45) & 2016 & $\begin{array}{l}67 \text { million } \\
\text { inhabitants in } \\
\text { France }\end{array}$ & Methylphenidate & 63,739 & $\begin{array}{l}\text { Patients with heavy doctor shopping behavior were } \\
\text { older, received more concomitant dispensing of } \\
\text { antipsychotics and opioid maintenance treatments, and } \\
\text { had more prescribers. }\end{array}$ \\
\hline
\end{tabular}

\section{Ranking Prescription Drugs Within a Pharmacological Class Known for Abuse}

The method demonstrated its ability to rank prescription drugs according to their abuse potential in the real-life setting. A study was conducted among the 128,230 patients who received benzodiazepine in a population of one million inhabitants in South West France in 2003. The method found a much higher proportion obtained by doctor shopping for flunitrazepam $1 \mathrm{mg}$ (i.e., $42.8 \%$ of the quantity dispensed), then for diazepam $10 \mathrm{mg}$ (i.e., $3.2 \%$ of the quantity dispensed), and clorazepate $50 \mathrm{mg}$ (i.e., $2.7 \%$ of the quantity dispensed) (40) (Figure 3 ).

Interestingly, although flunitrazepam has pharmacological characteristics prone to abuse [e.g., rapid onset of action, liposolubility, and additive effects with alcohol $(67,68)]$, there is no evidence of any important experimental difference for its abuse potential compared to other benzodiazepines 


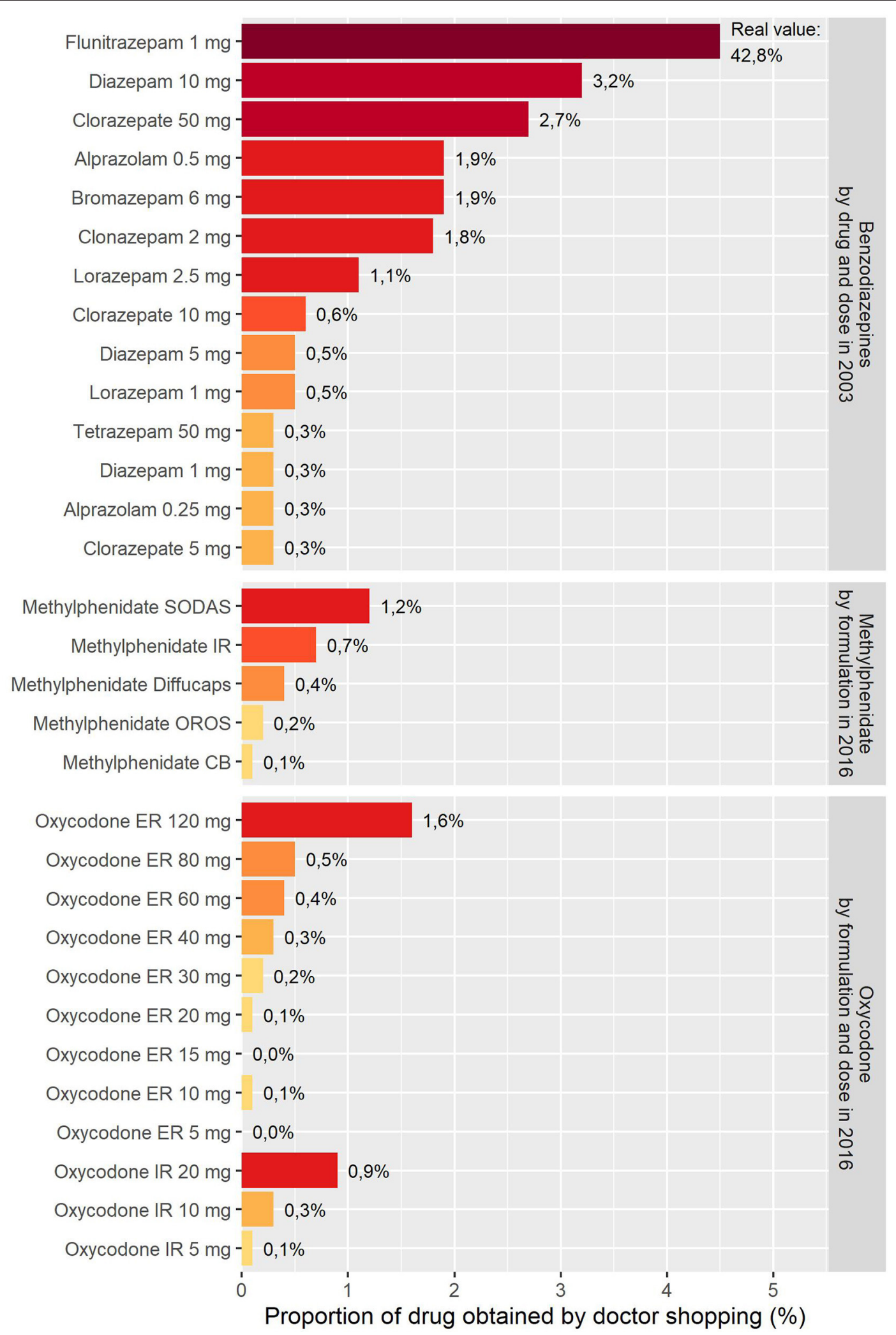

FIGURE 3 | Validation of doctor shopping as a pharmacological tool through its ability to rank prescription drugs within a pharmacological class known for abuse (e.g., benzodiazepines) and recover pharmacological determinants of abuse (e.g., formulation for methylphenidate and dose for oxycodone). SODAS: Spheroidal Oral Drug Absorption System; IR: Immediate-release; OROS: Osmotic-Controlled Release Oral Delivery System; CB: Coated beads. See Table 1 in Soeiro et al. (45) for details on formulations. 
(69). Nevertheless, a review of the literature found that the two benzodiazepines with the highest abuse potential are flunitrazepam and diazepam (70), particularly in opioid-abusing patients, many of whom reported a preference for flunitrazepam over other benzodiazepines (69).

\section{Discriminating Prescription Drugs Within a Pharmacological Class Not Known for Abuse}

The method also demonstrated its ability to discriminate prescription drugs by specifically detecting tianeptine among antidepressants (41). Back then, tianeptine was thought to have no abuse potential, as mentioned in the French summary of product characteristics before 2005, because there was no evidence of such a risk on the data available before approval (71). Nevertheless, the first reports of abuse with tianeptine emerged in the literature (72-75).

A study was conducted among the 410,525 patients who received an antidepressant in a population of five million inhabitants in South East France in 2005. Tianeptine ranked first among the antidepressants for the proportion obtained by doctor shopping (i.e., 2.0\% of the quantity dispensed), and was close to benzodiazepines with a well-known abuse potential in the reallife setting (41). In addition to reports from other data sources, these findings led to a stricter regulation of tianeptine in France.

Interestingly, tianeptine is a selective serotonin reuptake enhancer and an opioid agonist (76), with a chemical structure close to amineptine, which was withdrawn in several countries because of the abuse associated with hepatitis $(77,78)$. In addition, psychostimulant effects of tianeptine appear at high doses (75). These pharmacological properties makes tianeptine an atypical antidepressant, and may account for its abuse potential.

\section{Recovering Pharmacological Determinants of Abuse}

The method finally demonstrated its ability to recover pharmacological determinants of abuse, such as a preference for specific formulations and high doses for several pharmacological classes (e.g., benzodiazepines, opioids, and methylphenidate) (40, 42-45).

The effect of formulation is especially notable for methylphenidate, which was available in five formulations, using different extended-release technologies and ratio of immediaterelease/extended-release methylphenidate in France in 2016. On the same year, a study was conducted among the 63,739 patients who received methylphenidate in the 67 million inhabitants in France. Patients with doctor shopping behavior preferred formulations with a higher ratio of immediate-release/extendedrelease methylphenidate (e.g., methylphenidate with Spheroidal Oral Drug Absorption System and methylphenidate immediaterelease) over methylphenidate with Osmotic-Controlled Release Oral Delivery System (OROS) (45) (Figure 3). Given that the use of intravenous route for methylphenidate is frequent in France (79-81), this pattern also suggests that a part of methylphenidate obtained by doctor shopping may be used by intravenous route, because methylphenidate OROS is the least preferred drug for intravenous route in drug-abusing patients (82). Interestingly, OROS increases the time for preparing due to the viscosity of the preparation, which may be the reason for this preference (83).

Similarly, the effect of dose is especially notable for oxycodone, which was available in 12 doses from 5 to $120 \mathrm{mg}$ in France in 2016. A study was conducted in 2016 among the 212,753 patients who received oxycodone in the 67 million inhabitants in France. There was a dose-response-like relationship between dose and doctor shopping (i.e., the quantity obtained by doctor shopping increased with the dose for both immediate- and extendedrelease tablets) (44) (Figure 3). Interestingly, as soon as 2008, the method detected a first signal for oxycodone, particularly in one region (81), although no oxycodone abuse had been detected in France back then. This finding underlines the usefulness of local monitoring to assess the geographical specificities of abuse, which may help to target public health interventions (84).

\section{ADDED VALUE OF DOCTOR SHOPPING MONITORING TO IMPROVE THE EARLY DETECTION OF PRESCRIPTION DRUG ABUSE WITHIN A MULTIDIMENSIONAL MONITORING}

In order to face the challenges of monitoring prescription drug abuse, several authors and health authorities advocate for a multidimensional proactive post-marketing monitoring (10-12). Such a multidimensional monitoring is already operational in France through the French Addictovigilance Network (85-90). In addition to a spontaneous notification by health professionals and pharmacoepidemiological studies from claims databases (91, 92), multiple ad hoc studies have been conducted nationwide, such as: the OSIAP program, to detect forged prescription (93, 94); the OPPIDUM program, to detect psychoactive drug use in drug-abusing patients $(95,96)$; the DRAMES program, to detect deaths related to psychoactive drugs; the DTA program, to detect deaths related to analgesic prescription drugs; or the chemical submission program, to detect psychoactive drugs administered without the victim's knowledge (97).

The multidimensional monitoring conducted by the French Addictovigilance Network enables the detection of signals by crossing complementary data sources, which overcomes the limitation of each data source taken individually (Figure 4). In addition to the already existing programs of the French Addictovigilance Network, the added value of doctor shopping monitoring is its ability to exhaustively detect drug-abusing patients in the general population, and for all the marketed prescription drugs. This ability in not only theoretical, as demonstrated by a nationwide quantification of doctor shopping recently conducted in France for 220 psychoactive prescription drugs from many pharmacological classes (e.g., opioids, benzodiazepines, stimulants, antihistamines, gabapentinoids, antidepressants, and antipsychotics) (98). Given its automatic nature, the method can be implemented routinely, with minimal costs and limited workforce. Interestingly, doctor shopping monitoring is not impaired by under-declaration. Such features 


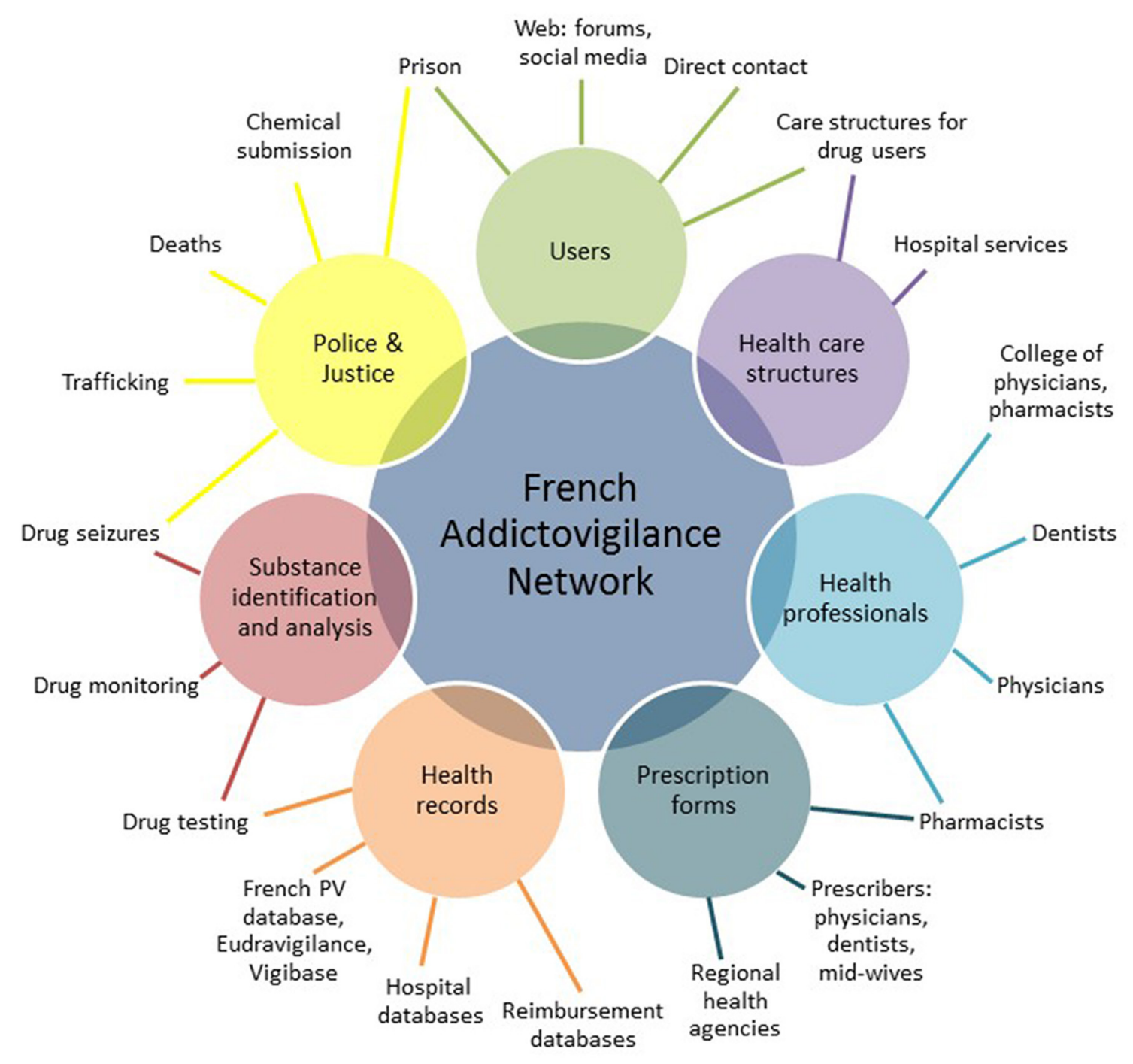

FIGURE 4 | Multidimensional monitoring conducted by the French Addictovigilance Network to detect signals by crossing complementary data sources.

make doctor shopping monitoring a complementary tool, which is even more topical in the big-data era to assess prescription drug abuse and detect emerging trends in the field of addictovigilance as early as possible (99).

For example, the monitoring of tramadol conducted by the French Addictovigilance Network detected an increasing abuse $(100,101)$. Beside a regular increase in spontaneous reports, tramadol has been used in combination or in alternation with other opioids in drug-abusing patients according to the OPPIDUM program; has ranked first among analgesics for deaths in the DTA program; and has increased for falsified prescriptions in OSIAP. These converging data are further strengthened and complemented by the nationwide quantification of doctor shopping in France (98). Notably, tramadol ranked ninth among 220 psychoactive prescription drugs for the quantity obtained by doctor shopping (i.e., 755,333 DDD). From 2010 to 2016, tramadol was one of the few opioids for which both quantity and proportion obtained by doctor shopping increased (i.e., $+12 \%$ and $+5 \%$, respectively). In the population approach, tramadol ranked first for the number of patients with doctor shopping behavior (i.e., 44,088 patients). Interestingly, tramadol is an atypical opioid analgesic that also inhibits the reuptake of serotonin and norepinephrine (102). In addition, O-desmethyltramadol, which is produced by the polymorphic cytochrome P450 2D6, has a 200 to 500 higher affinity for $\mu$-opioid receptor than tramadol (103). In light of the pharmacological properties of tramadol and international data (104-106), these increasing trends are strong signals in the French context.

\section{DISCUSSION}

The paper aims to review how doctor shopping monitoring can improve the early detection of prescription drug abuse within a 
multidimensional monitoring. The paper provides an in-depth overview of two decades of development and validation of the method as a complementary component of the multidimensional monitoring conducted by the French Addictovigilance Network. In this context, doctor shopping monitoring has demonstrated its added value to improve the early detection of prescription drug abuse. Notably, the monitoring must also include a strong pharmacological expertise, which is essential to both analyze signals and interpret pharmacoepidemiological data.

While the method has been developed and validated in France, the rationale is transposable in other health systems with available claims databases. In practice, given the increasing availability of claims databases in several countries, the main issue is to integrate doctor shopping monitoring within a multidimensional monitoring. In addition, the method must undergo an in-depth customized validation process, accounting for the specificities of the targeted health system (e.g., availability of prescription drugs and illicit alternatives, cost of prescription drugs and visits, prescription and control methods, and risks involved for fraud).

Such pharmacoepidemiological monitoring is intended to develop in the big-data era. Interestingly, it is nowadays technically possible to implement a real-time doctor shopping monitoring, assuming that a quick access to data is available, which is currently the bottleneck.

Finally, as a public health mission, monitoring prescription drug abuse must rely on free from conflict-of-interest organizations to prevent private interest from interfering, as it was the case in the opioid crisis $(107,108)$. This is even

\section{REFERENCES}

1. Hernandez SH, Nelson LS. Prescription drug abuse: insight into the epidemic. Clin Pharmacol Ther. (2010) 88:307-17. doi: 10.1038/clpt.2010.154

2. Zarocostas J. Misuse of prescription drugs could soon exceed that of illicit narcotics, uN panel warns. BMJ. (2007) 334:444. doi: 10.1136/bmj.39140.394410.DB

3. Wright ER, Kooreman HE, Greene MS, Chambers RA, Banerjee A, Wilson J. The iatrogenic epidemic of prescription drug abuse: county-level determinants of opioid availability and abuse. Drug Alcohol Dependence. (2014) 138:209-15. doi: 10.1016/j.drugalcdep.2014.03.002

4. McHugh RK, Nielsen S, Weiss RD. Prescription drug abuse: from epidemiology to public policy. I Subst Abuse Treat. (2015) 48:17. doi: 10.1016/j.jsat.2014.08.004

5. Griffiths P, Mounteney J, Lopez D, Zobel F, Götz W. Addiction research centres and the nurturing of creativity. Monitoring the European drug situation: the ongoing challenge for the European monitoring centre for drugs and drug addiction (EMCDDA. Addiction. (2012) 107:2548. doi: $10.1111 / j .1360-0443.2011 .03369 . x$

6. Casati A, Sedefov R, Pfeiffer-Gerschel T. Misuse of medicines in the European union: a systematic review of the literature. Eur Addict Res. (2012) 18:228-45. doi: 10.1159/000337028

7. Lapeyre-Mestre M, Dupui M. Drug abuse monitoring: which pharmacoepidemiological resources at the european level? Therapies. (2015) 70:157-65. doi: 10.2515/therapie/2015010

8. Chiappini S, Schifano F. What about "Pharming"? Issues regarding the misuse of prescription and over-the-counter drugs. Brain Sci. (2020) 10:736. doi: 10.3390/brainsci10100736

9. Chiappini S, Guirguis A, Corkery JM, Schifano F. Misuse of prescription and over-the-counter drugs to obtain illicit highs: how pharmacists can prevent abuse. Pharmaceutical J. (2020). Available online at: more necessary given that such monitoring may lead to the reconsideration of the safety of some prescription drugs in the real-life setting, and trigger regulatory measures. Among them, prescription monitoring programs are efficient to mitigate doctor shopping and its consequences $(39,109,110)$. Nevertheless, the consequences of such regulatory measures must be globally assessed, because hardening the access to prescription drugs may lead to switching to illicit drugs. The challenge is to develop methods that maximize the detection and prevention of prescription drug abuse, while minimizing any adverse impact on legitimate medical treatments.

\section{CONCLUSION}

To conclude, doctor shopping monitoring is a useful component for an efficient multidimensional monitoring to improve the early detection of prescription drug abuse in the field of addictovigilance.

\section{AUTHOR CONTRIBUTIONS}

TS wrote the manuscript. CL, VP, ML-M, and JM reviewed the manuscript. All authors contributed to the article and approved the submitted version.

\section{ACKNOWLEDGMENTS}

The authors would like to thank Véronique Channaut Soeiro for her careful proofreading of the manuscript.

pharmaceutical-journal.com/research/perspective-article/misuse-ofprescription-and-over-the-counter-drugs-to-obtain-illicit-highs-howpharmacists-can-prevent-abuse/20208538.article (accessed February 17, 2021).

10. Schifano F, Papanti GD, Orsolini L, Corkery JM. The consequences of drug misuse on post-marketing surveillance. Expert Rev Clin Pharmacol. (2016) 9:867-71. doi: 10.1080/17512433.2016.1178571

11. Dart RC. Monitoring risk: post marketing surveillance and signal detection. Drug Alcohol Depend. (2009) 105:S2632. doi: 10.1016/j.drugalcdep.2009.08.011

12. Throckmorton DC, Gottlieb S, Woodcock J. The fDA and the next wave of drug abuse - proactive pharmacovigilance. N Engl J Med. (2018) 379:2057. doi: 10.1056/NEJMp1806486

13. Martyres RF, Clode D, Burns JM. Seeking drugs or seeking help? Escalating « doctor shopping 》 by young heroin users before fatal overdose. Med J Aust. (2004) 180:211-4. doi: 10.5694/j.1326-5377.2004.tb05887.x

14. Pradel V, Thirion X, Ronfle E, Masut A, Micallef J, Bégaud B. Assessment of doctor-shopping for high dosage buprenorphine maintenance treatment in a French region: development of a new method for prescription database. Pharmacoepidemiol Drug Safety. (2004) 13:473-81. doi: 10.1002/pds.892

15. Cepeda MS, Fife D, Chow W, Mastrogiovanni G, Henderson SC. Assessing opioid shopping behaviour: a large cohort study from a medication dispensing database in the US. Drug Saf. (2012) 35:32534. doi: 10.2165/11596600-000000000-00000

16. Chen C-Y, Yeh H-H, Fang S-Y, Wu EC-H, Chang I-S, Lin KM. Overlapping prescriptions of stimulants for children and adolescents with attention-Deficit hyperactivity disorder. PS. (2012). 63:1011-8. doi: 10.1176/appi.ps.201100473

17. Lu T-H, Lee Y-Y, Lee H-C, Lin Y-M. Doctor shopping behavior for zolpidem among insomnia patients in Taiwan: a nationwide population-based study. Sleep. (2015) 38:1039-44. doi: 10.5665/sleep.4806 
18. Rasmussen L, Zoëga H, Hallas J, Pottegård A. Deviant patterns of methylphenidate use in adults: a Danish nationwide registry-based drug utilization study: deviant use of methylphenidate in Denmark. Pharmacoepidemiol Drug Saf. (2015) 24:1189-96. doi: 10.1002/pds.3852

19. Okumura Y, Shimizu S, Matsumoto T. Prevalence, prescribed quantities, and trajectory of multiple prescriber episodes for benzodiazepines: a 2-year cohort study. Drug Alcohol Depend. (2016) 158:118-25. doi: 10.1016/j.drugalcdep.2015.11.010

20. Hall AJ. Patterns of abuse among unintentional pharmaceutical overdose fatalities. JAMA. (2008) 300:2613. doi: 10.1001/jama.2008.802

21. Peirce GL, Smith MJ, Abate MA, Halverson J. Doctor and pharmacy shopping for controlled substances. Med Care. (2012) 50:494-500. doi: 10.1097/MLR.0b013e31824ebd81

22. Jena AB, Goldman D, Weaver L, Karaca-Mandic P. Opioid prescribing by multiple providers in medicare: retrospective observational study of insurance claims. BMJ. (2014) 348:g1393. doi: 10.1136/bmj.g1393

23. Baumblatt JAG, Wiedeman C, Dunn JR, Schaffner W, Paulozzi LJ, Jones TF. High-Risk use by patients prescribed opioids for pain and its role in overdose deaths. JAMA Internal Med. (2014) 174:796. doi: 10.1001/jamainternmed.2013.12711

24. Young SG, Hayes CJ, Aram J, Tait MA. Doctor hopping and doctor shopping for prescription opioids associated with increased odds of high-risk use. Pharmacoepidemiol Drug Saf. (2019) 28:1117-24. doi: 10.1002/pds.4838

25. Davis WR, Johnson BD. Prescription opioid use, misuse, and diversion among street drug users in New York city. Drug Alcohol Depend. (2008) 92:267-76. doi: 10.1016/j.drugalcdep.2007.08.008

26. Fischer B, Bibby M, Bouchard M. The global diversion of pharmaceutical drugs non-medical use and diversion of psychotropic prescription drugs in North America: a review of sourcing routes and control measures: diversion of psychotropic prescription drugs. Addiction. (2010) 105:206270. doi: 10.1111/j.1360-0443.2010.03092.x

27. Jones CM, Paulozzi LJ, Mack KA. Sources of prescription opioid pain relievers by frequency of past-Year nonmedical use: united states, 2008-2011. JAMA Intern Med. (2014) 174:802. doi: 10.1001/jamainternmed.2013.12809

28. Simeone R. Doctor shopping behavior and the diversion of prescription opioids. Subst Abuse Res Treat. (2017) 11:117822181769607. doi: 10.1177/1178221817696077

29. Inciardi JA, Surratt HL, Kurtz SP, Cicero TJ. Mechanisms of prescription drug diversion among drug-Involved club- and street-based populations. Pain Med. (2007) 8:171-83. doi: 10.1111/j.1526-4637.2006.00255.x

30. Rigg KK, March SJ, Inciardi JA. Prescription drug abuse \& diversion: role of the pain clinic. J Drug Issues. (2010) 40:681-701. doi: 10.1177/002204261004000307

31. Rigg KK, Kurtz SP, Surratt HL. Patterns of prescription medication diversion among drug dealers. Drugs Educ Prev Policy. (2012) 19:14555. doi: 10.3109/09687637.2011.631197

32. Hulme S, Hughes CE, Nielsen S. Drug sourcing and motivations among a sample of people involved in the supply of pharmaceutical drugs in Australia. Int J Drug Policy. (2019) 66:38-47. doi: 10.1016/j.drugpo.2019.01.022

33. Biernikiewicz M, Taieb V, Toumi M. Characteristics of doctor-shoppers: a systematic literature review. J Mark Access Health Policy. (2019) 7:1595953. doi: 10.1080/20016689.2019.1595953

34. Neutel CI, Skurtveit S, Berg C, Sakshaug S. Multiple prescribers in older frequent opioid users-does it mean abuse? J Popul Ther Clin Pharmacol. (2013) 20:e397-405

35. Adewumi AD, Maravilla JC, Alati R, Hollingworth SA, Hu X, Loveday B, et al. Multiple opioid prescribers: a genuine quest for treatment rather than aberrant behaviour. A two-decade population-based study. Addict Behav. (2020) 108:106458. doi: 10.1016/j.addbeh.2020.106458

36. Kasteler J, Kane RL, Olsen DM, Thetford C. Issues underlying prevalence of $\ll$ doctor-shopping 》 behavior. J Health Soc Behav. (1976) 17:32939. doi: $10.2307 / 2136711$

37. Sansone RA, Sansone LA. Doctor shopping: a phenomenon of many themes. Innov Clin Neurosci. (2012) 9:42-6.

38. Yang Z, Wilsey B, Bohm M, Weyrich M, Roy K, Ritley D, et al. Defining risk of prescription opioid overdose: pharmacy shopping and overlapping prescriptions among long-Term opioid users in medicaid. J Pain. (2015) 16:445-53. doi: 10.1016/j.jpain.2015.01.475
39. Pradel V, Frauger E, Thirion X, Ronfle E, Lapierre V, Masut A, et al. Impact of a prescription monitoring program on doctor-shopping for high dosage buprenorphine. Pharmacoepidemiol Drug Saf. (2009) 18:3643. doi: $10.1002 /$ pds. 1681

40. Pradel V, Delga C, Rouby F, Micallef J, Lapeyre-Mestre M. Assessment of abuse potential of benzodiazepines from a prescription database using 'Doctor shopping' as an indicator. CNS Drugs. (2010) 24:61120. doi: 10.2165/11531570-000000000-00000

41. Rouby F, Pradel V, Frauger E, Pauly V, Natali F, Reggio P, et al. Assessment of abuse of tianeptine from a reimbursement database using "doctor-shopping" as an indicator: assessment of abuse liability of tianeptine. Fundam Clin Pharmacol. (2012) 26:286-94. doi: 10.1111/j.1472-8206.2010.00906.x

42. Nordmann S, Pradel V, Lapeyre-Mestre M, Frauger E, Pauly V, Thirion X, et al. Doctor shopping reveals geographical variations in opioid abuse. Pain Physician. (2013) 16:89-100. doi: 10.36076/ppj.2013/16/89

43. Ponté C, Lepelley M, Boucherie Q, Mallaret M, Lapeyre Mestre M, Pradel V, et al. Doctor shopping of opioid analgesics relative to benzodiazepines: a pharmacoepidemiological study among 11.7 million inhabitants in the french countries. Drug Alcohol Depend. (2018) 187:8894. doi: 10.1016/j.drugalcdep.2018.01.036

44. Soeiro T, Pradel V, Lapeyre-Mestre M, Micallef J. Evolution of doctor shopping for oxycodone in the 67 million inhabitants in France as a proxy for potential misuse or abuse. Pain. (2020) 162:7707. doi: 10.1097/j.pain.0000000000002093

45. Soeiro T, Frauger É, Pradel V, Micallef J. Doctor shopping for methylphenidate as a proxy for misuse and potential abuse in the 67 million inhabitants in france. Fundam Clin Pharmacol. (2020). doi: 10.1111/fcp.12612. [Epub ahead of print].

46. Weissman DE, Haddox DJ. Opioid pseudoaddiction - an iatrogenic syndrome. Pain. (1989) 36:363-6. doi: 10.1016/0304-3959(89) 90097-3

47. Stewart TD, Reed MB. Lifetime nonmedical use of prescription medications and socioeconomic status among young adults in the United States. Am J Drug Alcohol Abuse. (2015) 41:458-64. doi: 10.3109/00952990.2015.1060242

48. Pear VA, Ponicki WR, Gaidus A, Keyes KM, Martins SS, Fink DS, et al. Urban-rural variation in the socioeconomic determinants of opioid overdose. Drug Alcohol Depend. (2019) 195:66-73. doi: 10.1016/j.drugalcdep.2018.11.024

49. Chen T-C, Chen L-C, Kerry M, Knaggs RD. Prescription opioids: regional variation and socioeconomic status - evidence from primary care in England. Int J Drug Policy. (2019) 64:87-94. doi: 10.1016/j.drugpo.2018.10.013

50. McCarty D, Rieckmann T, Baker RL, McConnell KJ. The perceived impact of 42 CFR part 2 on coordination and integration of care: a qualitative analysis. PS. (2017) 68:245-9. doi: 10.1176/appi.ps.201600138

51. Moulis G, Lapeyre-Mestre M, Palmaro A, Pugnet G, Montastruc J-L, Sailler L. French health insurance databases: what interest for medical research? La Revue de Médecine Interne. (2015) 36:411-7. doi: 10.1016/j.revmed.2014.11.009

52. Palmaro A, Moulis G, Despas F, Dupouy J, Lapeyre-Mestre M. Overview of drug data within french health insurance databases and implications for pharmacoepidemiological studies. Fundam Clin Pharmacol. (2016) 30:61624. doi: $10.1111 /$ fcp. 12214

53. Système National des Données de Santé. Système National des Données de Santé. Available online at: https://www.snds.gouv.fr (accessed March 24, 2021).

54. Tuppin P, Rudant J, Constantinou P, Gastaldi-Ménager C, Rachas A, de Roquefeuil L, et al. Value of a national administrative database to guide public decisions: from the système national d'information interrégimes de l'Assurance maladie (SNIIRAM) to the système national des données de santé (SNDS) in France. Revue d'Épidémiol Santé Publique. (2017) 65:S14967. doi: 10.1016/j.respe.2017.05.004

55. Martin-Latry K, Bégaud B. Pharmacoepidemiological research using french reimbursement databases: yes we can!: pharmacoepidemiological research in France. Pharmacoepidemiol Drug Saf. (2010) 19:256-65. doi: 10.1002/pds.1912

56. Bezin J, Duong M, Lassalle R, Droz C, Pariente A, Blin P, et al. The national healthcare system claims databases in France, sNIIRAM and eGB: 
powerful tools for pharmacoepidemiology. Pharmacoepidemiol Drug Saf. (2017) 26:954-62. doi: 10.1002/pds.4233

57. Mancini J, Thirion X, Masut A, Saillard C, Pradel V, Romain F, et al. Anxiolytics, hypnotics, and antidepressants dispensed to adolescents in a French region in 2002. Pharmacoepidemiol Drug Saf. (2006) 15:494503. doi: $10.1002 / \mathrm{pds} .1258$

58. Pariente A, Pinet $\mathrm{M}$, Moride $\mathrm{Y}$, Merlière $\mathrm{Y}$, Moore $\mathrm{N}$, FourrierRéglat A. Factors associated with persistence of cholinesterase inhibitor treatments in the elderly. Pharmacoepidemiol Drug Saf. (2010) 19:6806. doi: $10.1002 /$ pds.1933

59. Fuzier R, Serres I, Bourrel R, Palmaro A, Montastruc J-L, Lapeyre-Mestre M. Analgesic drug consumption increases after knee arthroplasty: a pharmacoepidemiological study investigating postoperative pain. Pain. (2014) 155:1339-45. doi: 10.1016/j.pain. 2014.04.010

60. Beziz D, Colas S, Collin C, Dray-Spira R, Zureik M. Association between exposure to benzodiazepines and related drugs and survivorship of total hip replacement in arthritis: a population-based cohort study of 246,940 patients. Rudan J, éditeur. PLoS ONE. (2016) 11:e0155783. doi: 10.1371/journal.pone. 0155783

61. Fagot J-P, Cuerq A, Samson S, Fagot-Campagna A. Cohort of one million patients initiating antidepressant treatment in france: 12-month follow-up. Int J Clin Pract. (2016) 70:744-51. doi: 10.1111/ijcp.12850

62. Bénard-Laribière A, Pambrun E, Sutter-Dallay A-L, Gautier S, HuraultDelarue C, Damase-Michel C, et al. Patterns of antidepressant use during pregnancy: a nationwide population-based cohort study: patterns of antidepressant use in pregnancy. Br J Clin Pharmacol. (2018) 84:176475. doi: 10.1111/bcp. 13608

63. Blotière P-O, Raguideau F, Weill A, Elefant E, Perthus I, Goulet $\mathrm{V}$, et al. Risks of 23 specific malformations associated with prenatal exposure to 10 antiepileptic drugs. Neurology. (2019) 93:e167-80. doi: 10.1212/WNL.0000000000007696

64. Thirion X, Lapierre V, Micallef J, Ronflé E, Masut A, Pradel $\mathrm{V}$, et al. Buprenorphine prescription by general practitioners in a French region. Drug Alcohol Depend. (2002) 65:197204. doi: 10.1016/S0376-8716(01)00161-2

65. Dupouy J, Maumus-Robert S, Mansiaux Y, Pariente A, Lapeyre-Mestre M. Primary care of opioid use disorder: the end of "the French model"? Eur Addict Res. (2020) 26:346-54. doi: 10.1159/000506630

66. Lapeyre-Mestre M, Llau M-E, Gony M, Navel A-M, Bez J, Grau M, et al. Opiate maintenance with buprenorphine in ambulatory care: a $24-$ week follow-up study of new users. Drug Alcohol Depend. (2003) 72:297303. doi: 10.1016/j.drugalcdep.2003.08.005

67. Simmons MM, Cupp MJ. Use and abuse of flunitrazepam. Ann Pharmacother. (1998) 32:117-9. doi: 10.1345/aph.17027

68. Druid H, Holmgren P, Ahlner J. Flunitrazepam: an evaluation of use, abuse and toxicity. Forensic Sci Int. (2001) 122:13641. doi: 10.1016/S0379-0738(01)00481-9

69. Woods JH, Winger G. Abuse liability of flunitrazepam. J Clin Psychopharmacol. (1997) 17:1S-57S. doi: 10.1097/00004714-199706001-00001

70. Griffiths RR, Johnson MW. Relative abuse liability of hypnotic drugs: a conceptual framework and algorithm for differentiating among compounds. J Clin Psychiatry. (2005) 66(Suppl. 9):31-41.

71. Wagstaff AJ, Ormrod D, Spencer CM. Tianeptine: a review of its use in depressive disorders. CNS Drugs. (2001) 15:23159. doi: 10.2165/00023210-200115030-00006

72. Vandel P, Regina W, Bonin B, Sechter D, Bizouard P. Abuse of tianeptine. A case report. Encephale. (1999) 25:672-3.

73. Guillem E, Lépine J-P. Does addiction to antidepressants exist? About a case of one addiction to tianeptine. Encephale. (2003) 29:456-9.

74. Leterme L, Singlan Y-S, Auclair V, Le Boisselier R, Frimas V. Misuse of tianeptine: five cases of abuse. Ann Med Interne. (2003) 154:S58-63.

75. Kisa C, Bulbul DO, Aydemir C, Goka E. Is it possible to be dependent to tianeptine, an antidepressant? A case report. Prog NeuroPsychopharmacol Biol Psychiatry. (2007) 31:776-8. doi: 10.1016/j.pnpbp. 2007.01.002
76. Gassaway MM, Rives M-L, Kruegel AC, Javitch JA, Sames D. The atypical antidepressant and neurorestorative agent tianeptine is a $\mu$-opioid receptor agonist. Transl Psychiatry. (2014) 4:e411. doi: 10.1038/tp.2014.30

77. Castot A, Benzaken C, Wagniart F, Efthymiou ML. Amineptin abuse. Analysis of 155 cases. An evaluation of the official cooperative survey of the regional centers of pharmacovigilance. Therapie. (1990) 45:399-405.

78. Haddad P. Do antidepressants have any potential to cause addiction? J Psychopharmacol. (1999) 13:300-7. doi: 10.1177/026988119901300321

79. Micallef J, Frauger E, Palmaro A, Boucherie Q, Mestre ML. Example of an investigation of an "Emergent" phenomenon in addiction vigilance: the case of methylphenidate. Therapies. (2015) 70:197-202. doi: 10.2515/therapie/2015013

80. Frauger E, Amaslidou D, Spadari M, Allaria-Lapierre V, Braunstein D, Sciortino V, et al. Patterns of methylphenidate use and assessment of its abuse among the general population and individuals with drug dependence. Eur Addict Res. (2016) 22:119-26. doi: 10.1159/000439273

81. Nordmann S, Nefau T, Micallef J, Duplessy C, Catusse J-C, Frauger E. Analyse des seringues usagées : un reflet de la consommation de substances psychoactives par les usagers de drogues par voie intraveineuse? Thérapie. (in press). doi: 10.2515/therapie/2015054

82. Bjarnadottir GD, Magnusson A, Rafnar BO, Sigurdsson E, Steingrimsson $\mathrm{S}$, Johannsson $\mathrm{M}$, et al. Intravenous use of prescription psychostimulants; a Comparison of the pattern and subjective experience between different methylphenidate preparations, amphetamine and cocaine. Eur Addict Res. (2016) 22:259-67. doi: 10.1159/000446428

83. Bjarnadottir GD, Johannsson M, Magnusson A, Rafnar BO, Sigurdsson E, Steingrimsson S, et al. Methylphenidate disintegration from oral formulations for intravenous use by experienced substance users. Drug Alcohol Depend. (2017) 178:165-9. doi: 10.1016/j.drugalcdep.2017.04.028

84. Brownstein JS, Green TC, Cassidy TA, Butler SF. Geographic information systems and pharmacoepidemiology: using spatial cluster detection to monitor local patterns of prescription opioid abuse. Pharmacoepidem Drug Saf. (2010) 19:627-37. doi: 10.1002/pds.1939

85. Baumevieille M, Daveluy A, Maurain C, Bégaud B, Haramburu F. Medicines submitted to narcotics regulations in france, 1992-2007. Fundam Clin Pharmacol. (2009) 23:345-9. doi: 10.1111/j.1472-8206.2009.00678.x

86. Jouanjus E, Gibaja V, Kahn J-P, Haramburu F, Daveluy A. Signal identification in addictovigilance: the functioning of the French system. Therapies. (2015) 70:123-31. doi: 10.2515/therapie/2015011

87. Peyrière H, Eiden C, Mallaret M, Victorri-Vigneau C. Medical complications of psychoactive substances with abuse risks: detection and assessment by the network of french addictovigilance centres. Therapies. (2016) 71:56373. doi: 10.1016/j.therap.2016.07.001

88. Gentile G, Jego M, Spadari M, Griffiths K, Jouanjus E, Micallef J. Identification and tracking of addictovigilance signals in general practice: which interactions between the general practitioners and the french addictovigilance network? Fundam Clin Pharmacol. (2018) 32:64351. doi: $10.1111 /$ fcp. 12401

89. Micallef J, Jouanjus É, Mallaret M, Lapeyre Mestre M. Détection des signaux du réseau français d'addictovigilance : méthodes innovantes d'investigation, illustrations et utilité pour la santé publique. Therapies. (2019) 74:57990. doi: 10.1016/j.therap.2019.09.005

90. Lapeyre-Mestre M, Boucher A, Daveluy A, Gibaja V, Jouanjus E, Mallaret $M$, et al. Addictovigilance contribution during COVID-19 epidemic and lockdown in France. Therapies. (2020) 75:343-54. doi: 10.1016/j.therap.2020.06.006

91. Micallef J, Pradel V, Thirion X, Jolliet P, Lapeyre-Mestre M. Utilisation des bases de données de l'Assurance maladie par les centres d'Evaluation et d'Information sur la pharmacodépendance : exemples, intérêts et perspectives. Therapies. (2004) 59:581-8. doi: 10.2515/therapie:2004100

92. Dupui M, Micallef J, Lapeyre-Mestre M. Interest of large electronic health care databases in addictovigilance: lessons from 15 years of pharmacoepidemiological contribution. Therapies. (2019) 74:307-14. doi: 10.1016/j.therap.2018.09.078

93. Boeuf O, Lapeyre-Mestre M. Survey of forged prescriptions to investigate risk of psychoactive medications abuse in France: results of OSIAP survey. Drug Saf. (2007) 30:265-76. doi: 10.2165/00002018-200730030-00007 
94. Jouanjus E, Guernec G, Lapeyre-Mestre M, the French Addictovigilance Network. Medical prescriptions falsified by the patients: a 12 -year national monitoring to assess prescription drug diversion. Fundam Clin Pharmacol. (2018) 32:306-22. doi: 10.1111/fcp.12356

95. Frauger E, Moracchini C, Le Boisselier R, Braunstein D, Thirion X, Micallef J, et al. OPPIDUM surveillance program: 20 years of information on drug abuse in france. Fundam Clin Pharmacol. (2013) 27:67282. doi: $10.1111 /$ fcp. 12024

96. Frauger E, Pochard L, Boucherie Q, Giocanti A, Chevallier C, Daveluy A, et al. Dispositif pharmacoépidémiologique de surveillance des substances psychoactives : intérêts du programme national oPPIDUM du réseau français d'addictovigilance. Therapies. (2017) 72:491-501. doi: 10.1016/j.therap.2017.01.010

97. Djezzar S, Burin E, Dally S, The French Network of Centers for Evaluation and Information on Pharmacodependence. Chemical submission: results of 4-year French inquiry. Int J Legal Med. (2009) 123:213-9. doi: 10.1007/s00414-008-0291-x

98. Micallef J. Monitoring prescription drug abuse using doctor shopping behavior (MEGADOSE). (2020). Available online at: http://www.encepp.eu/encepp/ viewResource.htm?id=32867 (accessed March 24, 2021).

99. Rudorfer MV. Psychopharmacology in the age of "Big data": the promises and limitations of electronic prescription records. CNS Drugs. (2017) 31:4179. doi: 10.1007/s40263-017-0419-y

100. Roussin A, Doazan-d'Ouince O, Géniaux H, Halberer C. Evaluation of abuse and dependence in addiction monitoring systems: tramadol as an example. Therapies. (2015) 70:213-21. doi: 10.2515/therapie/ 2015014

101. Roussin A, Lapeyre-Mestre M. Évolution des données d'addictovigilance du tramadol en France : un signal d'augmentation des usages problématiques ! Therapies. (2018) 73:569. doi: 10.1016/j.therap. 2018.09.005

102. Rickli A, Liakoni E, Hoener MC, Liechti ME. Opioid-induced inhibition of the human 5-HT and noradrenaline transporters in vitro: link to clinical reports of serotonin syndrome: opioids and 5-HT. Br J Pharmacol. (2018) 175:532-43. doi: 10.1111/bph.14105

103. Gillen C, Haurand M, Kobelt DJ, Wnendt S. Affinity, potency and efficacy of tramadol and its metabolites at the cloned human $\mu$-opioid receptor. Naunyn-Schmiedebergs Arch Pharmacol. (2000) 362:116-21. doi: 10.1007/s002100000266

104. Ojanperä I, Kriikku P, Vuori E. Fatal toxicity index of medicinal drugs based on a comprehensive toxicology database. Int J Legal Med. (2016) 130:1209-16. doi: 10.1007/s00414-016-1358-8

105. Chen T-C, Chen L-C, Knaggs RD. A 15-year overview of increasing tramadol utilisation and associated mortality and the impact of tramadol classification in the united kingdom. Pharmacoepidemiol Drug Saf. (2018) 27:487-94. doi: 10.1002/pds.4320

106. Salm-Reifferscheidt L. Tramadol: Africa's opioid crisis. Lancet. (2018) 391:1982-3. doi: 10.1016/S0140-6736(18)31073-0

107. Hadland SE, Rivera-Aguirre A, Marshall BDL, Cerdá M. Association of pharmaceutical industry marketing of opioid products with mortality from opioid-Related overdoses. JAMA Netw Open. (2019) 2:e186007. doi: 10.1001/jamanetworkopen.2018.6007

108. Dyer O. WHO retracts opioid guidelines after accepting that industry had an influence. BMJ. (2020) 368:m105. doi: 10.1136/bmj.m105

109. Reifler LM, Droz D, Bailey JE, Schnoll SH, Fant R, Dart $\mathrm{RC}$, et al. Do prescription monitoring programs impact state trends in opioid abuse/misuse? Pain Med. (2012) 13:43442. doi: 10.1111/j.1526-4637.2012.01327.x

110. Katz N, Panas L, Kim M, Audet AD, Bilansky A, Eadie J, et al. Usefulness of prescription monitoring programs for surveillance-analysis of schedule II opioid prescription data in Massachusetts, 1996-2006. Pharmacoepidemiol Drug Saf. (2010) 19:115-23. doi: 10.1002/pds.1878

Conflict of Interest: The authors declare that the research was conducted in the absence of any commercial or financial relationships that could be construed as a potential conflict of interest.

Copyright (c) 2021 Soeiro, Lacroix, Pradel, Lapeyre-Mestre and Micallef. This is an open-access article distributed under the terms of the Creative Commons Attribution License (CC BY). The use, distribution or reproduction in other forums is permitted, provided the original author(s) and the copyright owner(s) are credited and that the original publication in this journal is cited, in accordance with accepted academic practice. No use, distribution or reproduction is permitted which does not comply with these terms. 


\section{OPEN ACCESS}

Edited by:

Giovanni Martinotti,

University of Studies G. d'Annunzio

Chieti and Pescara, Italy

Reviewed by:

Mauro Ceccanti,

Sapienza University of Rome, Italy Maria Chiara Alessi, University of Studies G. d'Annunzio

Chieti and Pescara, Italy

*Correspondence:

Georgios Papazisis papazisg@auth.gr orcid.org/0000-0003-1641-9095

Specialty section: This article was submitted to

Addictive Disorders,

a section of the journal

Frontiers in Psychiatry

Received: 11 December 2020 Accepted: 02 March 2021

Published: 20 May 2021

Citation: Papazisis G, Spachos D, Siafis S, Pandria N, Deligianni E, Tsakiridis I and Goulas A (2021) Assessment of the Safety Signal for the Abuse Potential of Pregabalin and Gabapentin Using the FAERS Database and Big Data Search Analytics.

Front. Psychiatry 12:640264. doi: 10.3389/fpsyt.2021.640264

\section{Assessment of the Safety Signal for the Abuse Potential of Pregabalin and Gabapentin Using the FAERS Database and Big Data Search Analytics}

\author{
Georgios Papazisis ${ }^{1 *}$, Dimitrios Spachos ${ }^{2}$, Spyridon Siafis ${ }^{1}$, Niki Pandria ${ }^{2}$, \\ Eleni Deligianni ${ }^{1}$, Ioannis Tsakiridis ${ }^{3}$ and Antonios Goulas ${ }^{4}$
}

\begin{abstract}
'Department of Clinical Pharmacology, School of Medicine, Aristotle University of Thessaloniki, Thessaloniki, Greece, ${ }^{2}$ Laboratory of Medical Physics, School of Medicine, Aristotle University of Thessaloniki, Thessaloniki, Greece, ${ }^{3}$ Department of Obstetrics and Gynaecology, School of Medicine, Aristotle University of Thessaloniki, Thessaloniki, Greece, ${ }^{4}$ First Department of Clinical Pharmacology, School of Medicine, Aristotle University of Thessaloniki, Thessaloniki, Greece
\end{abstract}

Introduction: The latest decade, an emerging issue has been the abuse potential of the gabapentinoids pregabalin and gabapentin. The aim of our study was to assess this safety signal combining two different methods of surveillance: search analytics big data and the FDA spontaneous reporting system database.

Methods: Analysis of big data and the FAERS was used to detect pregabalin's and gabapentin's abuse potential in comparison with two controls, clonazepam and levetiracetam, and further, the correlation between these domains was investigated. Data from the United States between 2007 and 2020Q2 were analyzed.

Results: The FAERS analysis revealed the following pattern of signals: clonazepam $>$ pregabalin $\geq$ gabapentin $>$ levetiracetam, for both the primary term "drug abuse and dependence" and the secondary terms (withdrawal, tolerance, overdose). The Google domain pattern was slightly different: clonazepam $\geq$ gabapentin $\geq$ pregabalin $\geq$ levetiracetam. A monotonic correlation was found between FAERS and Google searches for gabapentin $(r=0.558 ; p<0.001)$, pregabalin $(r=0.587 ; p<0.001)$, and clonazepam $(r=0.295 ; p=0.030)$.

Conclusion: Our results revealed that there is preliminary evidence of a safety signal for the abuse potential of pregabalin and gabapentin. Analysis of the FAERS database, supplemented by big data search analytics, suggests that there is potential of using these methods as a supplementary tool to detect drug abuse-related safety signals in pharmacovigilance.

Keywords: pregabalin, gabapentin, big data, Google search analytics, disproportionality analysis, abuse potential, safety signal, FAERS database 


\section{INTRODUCTION}

Gabapentinoids (pregabalin and gabapentin) are a class of drugs that have been widely used-prescribed for neuropathic pain, epilepsy, anxiety, and other psychiatric disorders, while pregabalin showed promise as a treatment for alcohol dependence $(1,2)$. Gabapentin and pregabalin have a similar structure and are derivatives of the inhibitory neurotransmitter GABA. Their proposed mechanism of action is the inhibition of calcium currents via high-voltage-activated channels containing the a2d-1 subunit (3). Since their first approval, both gabapentinoids are widely prescribed medications in the United States $(4,5)$.

The latest decade, an emerging issue has been the abuse potential of both pregabalin and gabapentin. An increase in non-medical use of gabapentinoids for recreational purposes has been reported, especially in Europe $(6,7)$. Higher doses of gabapentinoids use have been characterized by causing euphoria effects and a range of experiences such as relaxation, improved sociability, and sedative and psychedelic-like effects (8). From the EudraVigilance database review on gabapentinoids, fatalities were also reported associated with pregabalin and gabapentin use and in most of the cases in combination with opioids (9). Pharmacovigilance data from the Food and Drug Administration Adverse Event Reporting System (FAERS) have shown adverse drug events from gabapentinoid abuse with a higher prevalence in young and male individuals (10). Both pregabalin and gabapentin from 1st April 2019 have been classified as Schedule 3 controlled drugs under the Misuse of Drugs Regulations 2001, and Class C of the Misuse of Drugs Act 1971 in the UK. On the other hand, in the US, pregabalin is a Schedule 5 controlled substance while gabapentin is a controlled substance only in some States. In Australia, pregabalin and gabapentin are classified as Schedule 4 (prescription only) medications; therefore there are no special control measures on supply or possession yet (11).

Considering the abovementioned data on the relative wellestablished abuse potential profile of the gabapentinoids, the aim of our study was (i). to detect pregabalin's and gabapentin's abuse potential in comparison with two controls, clonazepam and levetiracetam and (ii). to investigate the correlation between the search analytics and the FAERS domain. Our group has recently published the methodology of combining these pharmacovigilance domains in order to detect safety signals $(12,13)$.

\section{METHODS}

\section{Data Sources}

Following the methodology of our previous analysis that investigated mirtazapine's abuse liability (12), herein, we investigated the abuse liability of the gabapentinoids combining pharmacovigilance and search analytics data from the United States between 2007 and 2020Q2. Clonazepam, a frequently used benzodiazepine with a well-known abuse potential profile, was used as a positive control $(12,14)$, while levetiracetam (a well-known antiepileptic with a low abuse potential) (15) served as negative control.
TABLE 1 | Drug names and drug abuse-related terms.

\begin{tabular}{|c|c|c|}
\hline & Google & FAERS \\
\hline Drugs & $\begin{array}{l}\text { - Clonazepam, Klonopin } \\
\text { - Gabapentin, Neurontin } \\
\text { - Pregabalin, Lyrica } \\
\text { - Levetiracetam, Kepra }\end{array}$ & $\begin{array}{l}\text { - Clonazepam } \\
\text { - Gabapentin } \\
\text { - Pregabalin } \\
\text { - Levetiracetam }\end{array}$ \\
\hline \multirow[t]{5}{*}{$\begin{array}{l}\text { Drug abuse-related } \\
\text { terms }\end{array}$} & $\{$ Abuse, dependence\} & $\begin{array}{l}\text { Drug abuse and } \\
\text { dependence (SMQ narrow } \\
\text { scope) }\end{array}$ \\
\hline & $\{$ Withdrawal\} & $\begin{array}{l}\text { Drug withdrawal (SMQ } \\
\text { narrow scope) }\end{array}$ \\
\hline & \{Overdose\} & $\begin{array}{l}\text { Tolerance [drug tolerance } \\
(\mathrm{PT}) \text { and drug tolerance } \\
\text { increased (PT)] }\end{array}$ \\
\hline & $\{$ Tolerance\} & $\begin{array}{l}\text { Overdose [overdose (PT) } \\
\text { and intentional overdose } \\
(\mathrm{PT})]\end{array}$ \\
\hline & $\{H i g h\}$ & $\begin{array}{l}\text { Euphoria [euphoric mood } \\
(\mathrm{PT}) \text {, feeling abnormal } \\
(\mathrm{PT}) \text {, feeling drunk (PT), } \\
\text { feeling of relaxation (PT), } \\
\text { dizziness (PT), thinking } \\
\text { abnormal (PT), } \\
\text { hallucination (PT), } \\
\text { inappropriate affect (PT)] }\end{array}$ \\
\hline
\end{tabular}

In the FAERS database, the drugs are registered with their generic names; in Google search analytics, a brand name was also used. Drug-abuse-related MedDRA terms were selected in FAERS, and similar abuse-related search terms in the search analytics domain (SMQ, standardized MedDRA query; PT, preferred term).

\section{FAERS}

The pharmacovigilance database of the FAERS consists of individual safety reports originated mainly from the United States. The structure and data mining algorithms of FAERS have been described elsewhere (16). Briefly, reports can be submitted by patients, the pharmaceutical industry, and healthcare professionals, while adverse events are classified with MedDRA terminology $(16,17)$. The freely available pharmacovigilance tool OpenVigil-2.1-MedDRA (available at http://openvigil.sourceforge.net/) was used in order to access cleaned FAERS data, by removing duplicates and normalizing drug names to the generic name of the drug (18). Similar to our previous analysis, higher level terms were used, whenever possible, to classify reports with drug-abuse-related adverse events (12). The narrow scope of the Standardized MedDRA Query (SMQ) "drug abuse and dependence" was used as the primary term, and other terms related to drug abuse, including overdose, tolerance, withdrawal, and euphoria-related events, were used as secondary terms (Table 1) $(12,19)$. Disproportionality analysis was conducted for the aggregated period of 2007-2020Q2 for both the primary and secondary terms, while correlation analyses were conducted using quarterly data of the primary term.

\section{Google Analytics}

The Google search engine receives more than 5 billion of queries per day (20). Although it does not provide detailed analytics, 
some indicators, such as the interest over time, are publicly accessible. Usually, search queries contain terms related to the generic and brand names of the drug, combined together with some additional terms (e.g., "Can you get high of...?"). We combined analytics data retrieved using both the generic name and a common brand name of each drug (Table 1).

An important aspect for retrieving analytics data from the Google search engine is the context. We can define the search context by limiting the returned results per category. The widest category is the "general search term," where Google returns analytics from searches in all categories. However, since we were studying a very specific area of interest, we could also restrict our results in a more specific category (e.g., "medication"). Google is using search semantics to classify each search query and is expected that the more specific category will provide more accurate results. However, depending on the search popularity of some terms, there may not be enough results inside the category context, because Google returns only results that can be considered as big data volumes. In our study, we used only the "prescription drug" category for the extraction of our data.

Next, we defined a set of six abuse-related search terms, similar to the MedDRA abuse-related terms: \{"abuse," "dependence," "overdose," "withdrawal," "tolerance," and "high"\}. Table 1 indicates the relationship of the terms between the FAERS and the Google domains. We used the term "high" as the corresponding term of "euphoria," as the second did not have enough data.

By default, Google does not return results for searches with terms and queries made by a few people. Moreover special characters (i.e., queries with apostrophes) were filtered-this is a way of normalization that is also made by default. It is also important that Google's tools eliminate repeated searches from the same person over a short period of time. We identified queries containing combinations of the drug names and the abuse-related terms from the set we defined in a previous step. Finally, we filtered the results manually, by dropping out queries unrelated to abuse. For example, while the search query "clonazepam and high blood pressure" contains both the terms "clonazepam" and "high," it is not related to abuse. Instead, the query "can you get high of pregabalin" is related to abuse and, thus, included to our search results.

\section{Statistical Analysis}

The search interest over time is measured by the search popularity score (SPS) in the Google domain. We used the SPS score to collect metrics related to abuse liability. In the FAERS domain, we used the reporting odds ratio (ROR) for abuserelated adverse events. This methodology of analysis was recently published from our group (12).

\section{Search Interest Over Time}

Google reports top searches for every search query. These are terms (queries) that are most frequently searched with the main term in the same search session and within the selected category, country, or region (21).

The most popular queries are sorted by SPS. The value of SPS is between 0 and 100. The most popular term (in our case the main drug name, e.g., "Lyrica") has a normalized score of
100 , which is the maximum score. All other queries have a score under this value. This indicator represents the total number of searches divided by the total number of related searches on the specific country or region at the given time range. This is the default method used by Google in a tool called "Google Trends," to compare relative popularity between topics. For example, an SPS of 50 is assigned to a query that has been searched half as often as the top query. Queries with a search rate $<1 \%$ are not reported and are signed with a 0 SPS which is neither a percentage value nor an absolute value of searches. Combining more than one term or queries, the value can be above 100. Considering the large number of queries, we can safely assume that all referred statistics come from big data volumes.

We obtained the monthly SPS for all abuse-related terms for each drug. We developed timelines representing the cumulative search interest over time for the abuse-related terms beginning at 2007Q1 and ending at 2020Q2.

\section{Disproportionality Analysis}

Disproportionality analysis was conducted to investigate the association between abuse-related events and the tested drugs in comparison to all other drugs and all other events in the FAERS database. The reporting odds ratio (ROR) was used to quantify this association, and a larger ROR demonstrates a more frequent co-reporting of the tested drug and the selected term as well as a stronger safety signal. We detected safety signals when the number of reports with the combination of the tested drug and selected event was $>3$ and the lower boundary of the $95 \%$ confidence interval of ROR was $>1$ (16). The disproportionality analysis and RORs were calculated using the OpenVigil2.1MedDRA (18).

\section{Correlation Between FAERS and Search Analytics Domains}

A correlation coefficient is a statistical metric that measures the probability of two variables to change together. It describes both the strength and the direction of the relationship. The Pearson correlation coefficient is the most well-known metric, which evaluates the linear relationship between two variables. The Spearman correlation coefficient evaluates the monotonic relationship between two continuous or ordinal variables. The difference is that, in a monotonic relationship, the variables tend to change in the same direction, increasing or decreasing their values, but not necessarily at a constant rate, as in a linear relationship. Unlike Pearson's correlation, Spearman's method does not require normality of the variables and, thus, it is a non-parametric statistic.

\section{RESULTS}

\section{Google Search Analytics}

According to the analysis for the cumulative period, the overall abuse-related terms had an average SPS of 8 for Levetiracetam, 11.25 for pregabalin, 22.5 for gabapentin, and 45.5 for Clonazepam (Figure 1). Considering that Google is receiving billion queries per day, even low values of SPS in the given time 


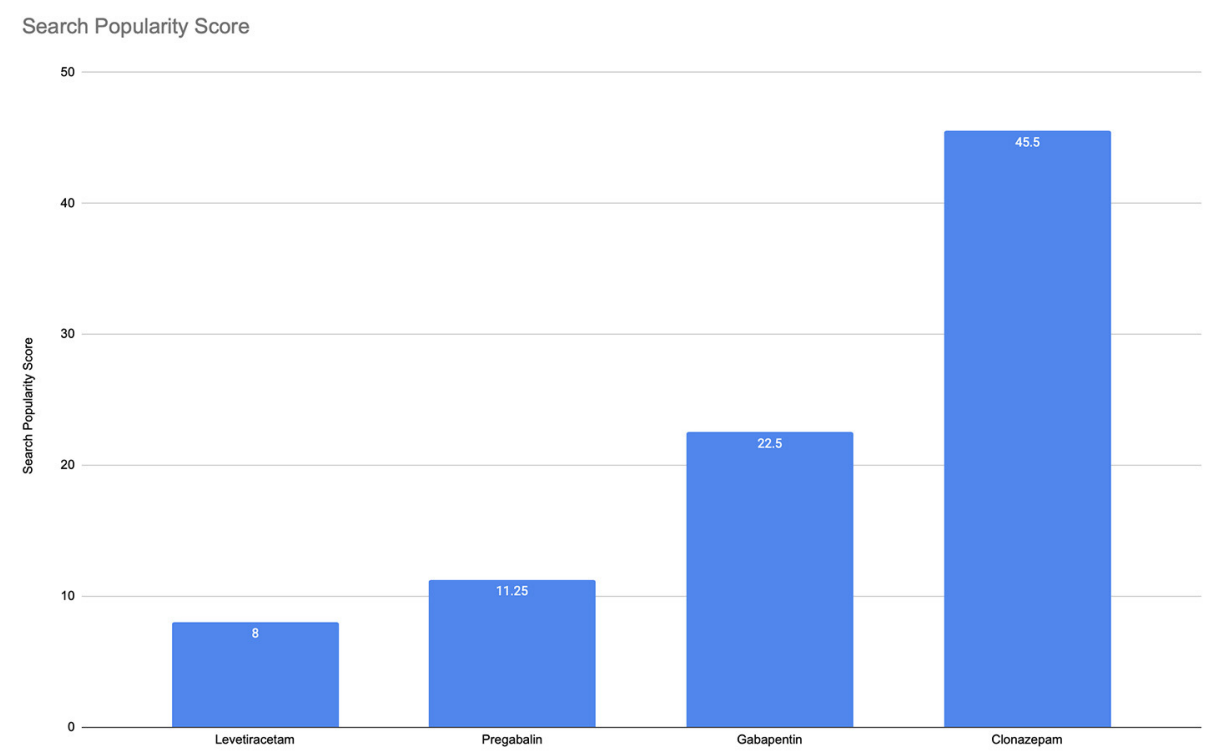

FIGURE 1 | Search Popularity Score (SPS) in the Google analytics domain for the four drugs.

range represent millions of queries about a topic (22). A nonformal interpretation of these numbers could be as follows: e.g., for pregabalin, for every 100 search queries related to pregabalin, there are 11.25 more queries (on top of the 100) related to pregabalin and abuse related terms.

Figure 2 shows the search interest over time for pregabalin, gabapentin, and clonazepam. The search volume for levetiracetam was significantly low, and thus, there were not enough data to be reported by the Google engine. While this may sound as a serious limiting condition, instead it ensures that the reported data are accurate and cannot be affected or modified by a small number of people who perform search queries producing "fake" trends.

The median values of search analytics over time were 82.5, IQR [53.25, 128] for pregabalin, 37, IQR [16.25, 47] for gabapentin, and 203.5, IQR [145.25, 258] for clonazepam.

\section{Disproportionality Analysis}

During the period of 2007-2020Q2, there were in total 7430750 reports submitted in FAERS. The total number of reports $(\mathrm{N})$ was larger for pregabalin $(N=107,905)$ and gabapentin $(N=$ $102,386)$, and about half for each of the controls, clonazepam $(N$ $=55,856)$, and levetiracetam $(N=43,842)$. For the primary term "drug abuse and dependence" $(N=118,980)$, safety signals were identified for both gabapentinoids (pregabalin: ROR $2.7895 \% \mathrm{CI}$ [2.70-2.86]; gabapentin ROR 1.83 95\% CI [1.76-1.90]), while the positive control clonazepam had the largest signal (ROR $4.4795 \%$ CI [4.32-4.62]), and the negative control levetiracetam had a very weak signal (ROR $1.1095 \%$ CI [1.02-1.18]). The secondary terms followed the same pattern of signals (clonazepam $>$ pregabalin $\geq$ gabapentin $>$ levetiracetam), except for euphoria-related terms, for which pregabalin had the largest ROR and overdoserelated terms, for which the gabapentinoids and levetiracetam demonstrated similar signals (Table 2). Figure 3 shows the number of reported adverse events related to abuse terms in the FAERS database.

\section{Correlation Between FAERS and Search Analytics Domains}

A monotonic correlation was found between FAERS and Google searches for clonazepam $(r=0.295 ; p=0.030$, Figure 4A), gabapentin $(r=0.558 ; p<0.001$, Figure 4B), and pregabalin $(r=$ 0.587; $p<0.001$, Figure 4C). Since Google reports only volumes with a significant number of searches, which can be considered as big data volumes, we were not able to collect the amount of data required for analysis for levetiracetam.

\section{DISCUSSION}

Based on extensive literature search, this is the first study investigating the abuse potential of pregabalin and gabapentin using two different pharmacovigilance methods: disproportionality analysis in the FAERS and Google search analytics. A positive control and a negative control were used, the benzodiazepine clonazepam, with a well-known abuse profile and the antiepileptic levetiracetam, with a previously unreported abuse potential, respectively.

\section{Signals in the FAERS Database}

Our disproportionality analysis of the FAERS revealed the following pattern of signals: clonazepam $>$ pregabalin $\geq$ gabapentin $>$ levetiracetam, both for the primary term "drug abuse and dependence" and the secondary terms (withdrawal, tolerance, overdose). Our results confirm previous findings from the pharmacovigilance domain that highlight the abuse potential of pregabalin. According to the review of the 


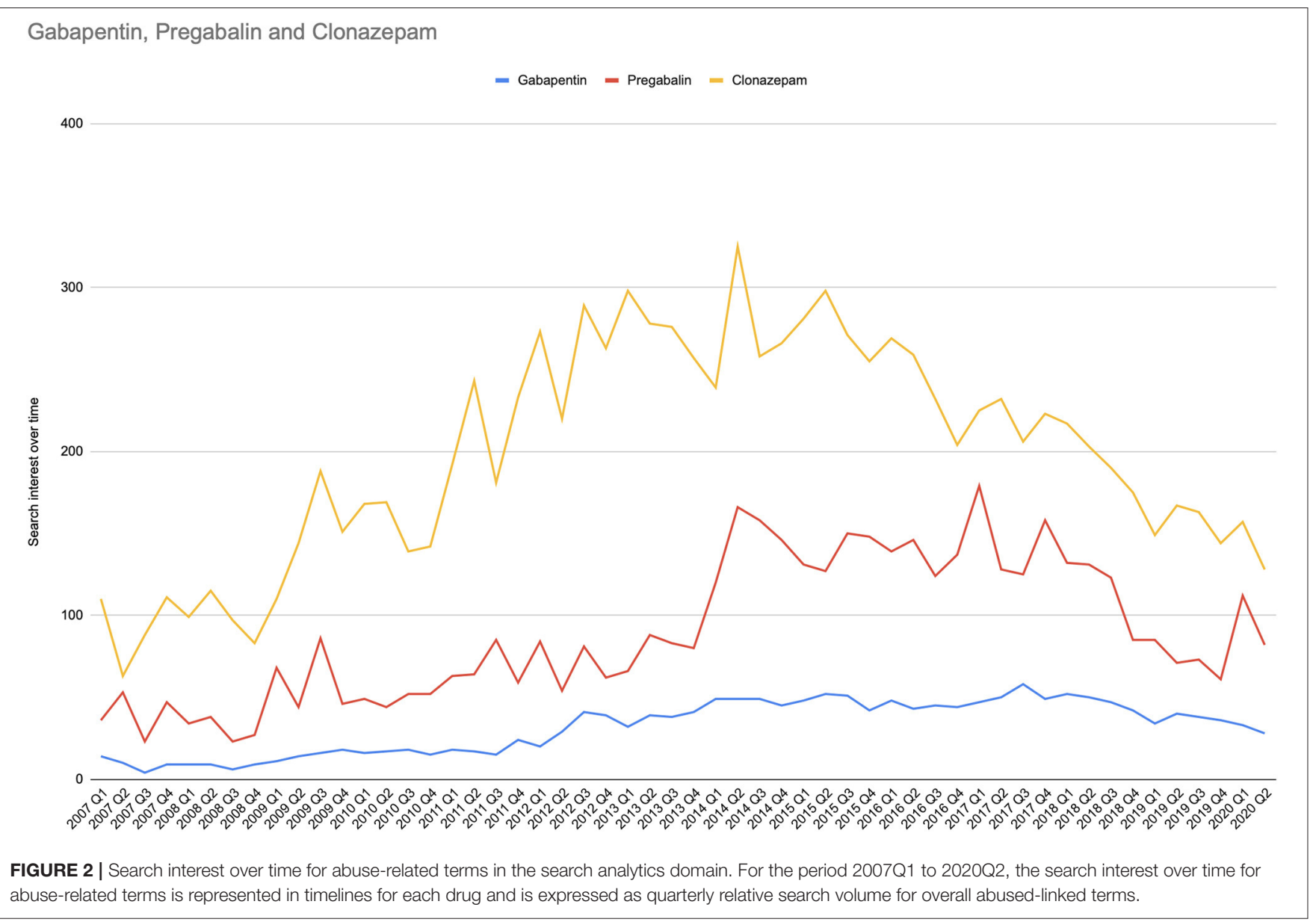

TABLE 2 | Number of reports and ROR \& 95\% Cl related to drug abuse per drug.

\begin{tabular}{|c|c|c|c|c|}
\hline & $\begin{array}{c}\text { Pregabalin } \\
(N=107,905)\end{array}$ & $\begin{array}{c}\text { Gabapentin } \\
(N=102,386)\end{array}$ & $\begin{array}{l}\text { Levetiracetam } \\
(N=43,842)\end{array}$ & $\begin{array}{l}\text { Clonazepam } \\
(N=55,856)\end{array}$ \\
\hline $\begin{array}{l}\text { Drug abuse and } \\
\text { dependence }(N= \\
118,980)\end{array}$ & $\begin{array}{c}\text { ROR } 2.7895 \% \mathrm{Cl} \\
{[2.70-2.86] ; N=4,558}\end{array}$ & $\begin{array}{c}\text { ROR } 1.8395 \% \mathrm{Cl} \\
{[1.76-1.90] ; N=2,924}\end{array}$ & $\begin{array}{c}\text { ROR } 1.1095 \% \mathrm{Cl} \\
{[1.02-1.18] ; N=767}\end{array}$ & $\begin{array}{c}\text { ROR } 4.4795 \% \mathrm{Cl} \\
{[4.32-4.62] ; N=3,700}\end{array}$ \\
\hline $\begin{array}{l}\text { Drug withdrawal }(N= \\
28,149)\end{array}$ & $\begin{array}{c}\text { ROR 3.76 95\% Cl } \\
{[3.56-3.96] ; N=1,463}\end{array}$ & $\begin{array}{c}\text { ROR 2.09 95\% Cl } \\
{[1.95-2.25] ; N=796}\end{array}$ & $\begin{array}{c}\text { ROR 1.54 } 95 \% \text { Cl } \\
{[1.36-1.74] ; N=254}\end{array}$ & $\begin{array}{c}\text { ROR 4.81 95\% Cl } \\
{[4.51-5.13] ; N=976}\end{array}$ \\
\hline $\begin{array}{l}\text { Drug tolerance }(N= \\
1,965)\end{array}$ & $\begin{array}{c}\text { ROR } 4.7395 \% \mathrm{Cl} \\
{[3.96-5.66] ; \mathrm{N}=128}\end{array}$ & $\begin{array}{c}\text { ROR } 3.7695 \% \mathrm{Cl} \\
{[3.07-4.61] ; N=98}\end{array}$ & $\begin{array}{l}\text { ROR 0.78 95\% Cl } \\
{[0.40-1.49] ; N=9}\end{array}$ & $\begin{array}{c}\text { ROR } 6.9495 \% \mathrm{Cl} \\
{[5.66-8.51] ; N=98}\end{array}$ \\
\hline $\begin{array}{l}\text { Euphoria-related events ( } N \\
=280,097)\end{array}$ & $\begin{array}{c}\text { ROR } 2.8795 \% \mathrm{Cl} \\
{[2.81-2.93] ; N=} \\
10,664\end{array}$ & $\begin{array}{c}\text { ROR } 2.0995 \% \mathrm{Cl} \\
{[2.04-2.14] ; N=7,644}\end{array}$ & $\begin{array}{c}\text { ROR } 1.2795 \% \mathrm{Cl} \\
{[1.22-1.33] ; N=2,077}\end{array}$ & $\begin{array}{c}\text { ROR } 2.4195 \% \mathrm{Cl} \\
{[2.33-2.48] ; N=4,765}\end{array}$ \\
\hline
\end{tabular}

Each drug has been compared with all other drugs in the FAERS database. The study population consisted of 6993352 reports.

EudraVigilance database, adverse drug reactions were more frequently reported for pregabalin use compared to gabapentin (23). Pharmacovigilance data from FAERS have also shown adverse drug events from pregabalin use and in general gabapentinoid abuse with a prevalence in young and male individuals (10). In contrast, from the EudraVigilance database review, there were adverse drug reaction reports related to abuse/dependence and misuse of pregabalin and gabapentin with a prevalence in female adults (9). The last decade, apart from gabapentinoid abuse there has also been reported extended misuse, with a greater potential of misuse for pregabalin (9). The misuse of pregabalin has been strongly linked to its 


\section{FAERS}

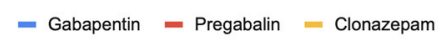

500

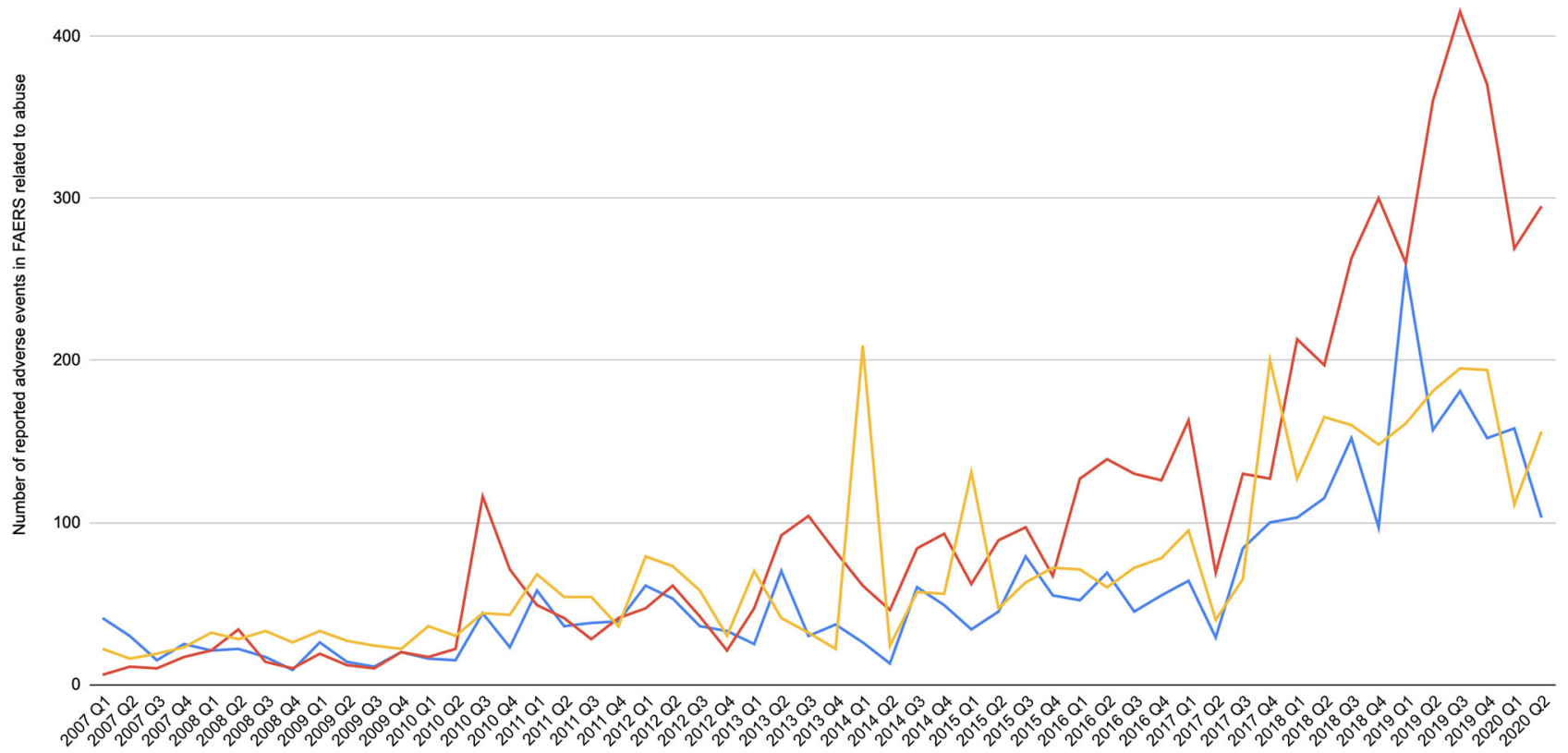

FIGURE 3 | Number of reported adverse events in the FAERS database related to abuse.
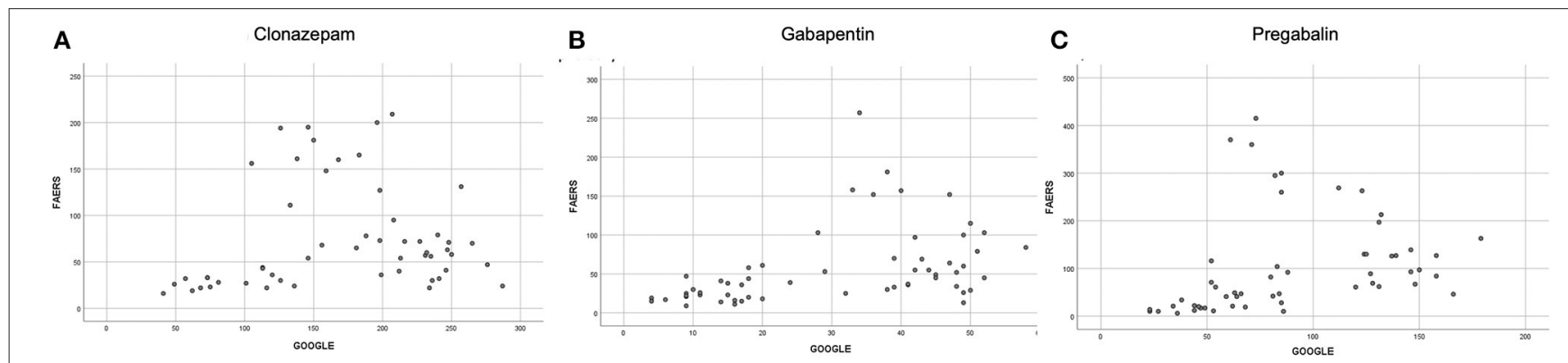

FIGURE 4 | Correlation between FAERS and Google searches for (A) clonazepam ( $r=0.295 ; p=0.030)$; (B) gabapentin $(r=0.558 ; p<0.001)$; (C) pregabalin $(r=$ $0.587 ; p<0.001)$

strong sedative and psychedelic effects. It has been stated that pregabalin misuse is more likely to occur in new users (24). Besides being considered as less powerful than pregabalin, gabapentin misuse was also associated with similar psychedelic effects. A few substances have been reported for misuse in combination with gabapentin, such as cannabis, alcohol, selective serotonin reuptake inhibitors (SSRIs), LSD, amphetamine, and gamma-hydroxybutyrate $(\mathrm{GHB})(8,25)$. There is agreement from other studies that the majority of individuals that have been reported for pregabalin abuse have a history of other substance and medication abuse as well $(11,26)$. The differences in the pharmacokinetic and pharmacodynamic profile of the gabapentinoids should be carefully examined in order to understand pregabalin's higher abuse potential compared to gabapentin $(11,25)$.

\section{Signals in the Google Analytics Domain}

The Google search analytics data are big data. Their volume, velocity, and variety are far beyond any other dataset of collected data, such as the adverse event reports. While they 
cannot be considered as a safe source for safety signals, their recognition of the potential is rising (27), and their use in pharmacovigilance is emerging. A recently published study of the French Addictovigilance Network combined Google Trends with the analysis of the global database of individual case safety reports (VigiBase) (28). Our team has recently published this method of combining different data sources of drug safety surveillance, Google search analytics, and disproportionality analysis of the US FAERS database (12) to detect safety signals. Data from this timeline series from 2004Q1 to 2017Q2 revealed a consistent association of abuse-related searches in the Google search engine with the antidepressant mirtazapine, and a similar pattern of association between abuse-related events and the drug was found in FAERS. The results of this previous study already suggested that search analytics and disproportionality analysis of FAERS may be used combined as a supplementary pharmacovigilance tool. Signals of gabapentinoid abuse found agreed with the signals for the positive and negative control drugs (clonazepam and levetiracetam). The generic pattern for FAERS was clonazepam $\geq$ pregabalin $\geq$ gabapentin $\geq$ levetiracetam. The Google domain pattern was slightly different: clonazepam $\geq$ gabapentin $\geq$ pregabalin $\geq$ levetiracetam. This difference can be explained by the fact that gabapentin was first approved for use in 1993 and in 2018 it was the eleventh most commonly prescribed medication in the United States, with more than 46 million prescriptions in 2018 and an increasing number of prescription over time (5). On the other hand, pregabalin (FDA approved in 2004) had an estimated number of 11.5 million prescriptions in 2018 in the United States being in ranking 70th among the most commonly prescribed medication (4). It should also be noted that disproportionality analysis cannot quantify the true risk, which should also be the case for the Google domain (29).

\section{Correlation Between the Domains}

A significant monotonic correlation was found between FAERS and Google searches for gabapentin $(r=0.558 ; p<0.001)$, pregabalin $(r=0.587 ; p<0.001)$, and clonazepam $(r=0.295 ; p$ $=0.030)$. This relationship between two totally different domains indicates that when one of the values changes in one domain, there is a significant probability to change in the same way in the other domain. Thus, changes of abuse-related searches on Google for pregabalin, gabapentin, or clonazepam are accompanied by analogous changes of abuse-related events in FAERS and

\section{REFERENCES}

1. Di Nicola M, Martinotti G, Tedeschi D, Frustaci A, Mazza M, Sarchiapone $\mathrm{M}$, et al. Pregabalin in outpatient detoxification of subjects with mildto-moderate alcohol withdrawal syndrome. Hum Psychopharmacol. (2010) 25:268-75. doi: 10.1002/hup.1098

2. Martinotti G, Nicola M, Tedeschi D, Mazza M, Janiri L, Bria P. Efficacy and safety of pregabalin in alcohol dependence. Adv Ther. (2008) 25:60818. doi: 10.1007/s12325-008-0066-2

3. Tzellos TG, Papazisis G, Toulis KA, Sardeli C, Kouvelas D. A2 $\delta$ ligands gabapentin and pregabalin: future implications in daily clinical practice. Hippokratia. (2010) 14:71-5.

4. ClinCalk. Pregabalin Drug Usage Statistics, United States, 2008 - 2018 (2020). vice versa. There is no causality on this fact but, rather, a similar behavior of two data domains. Interestingly, there were not enough big data volumes for levetiracetam to develop the timelines and, thus, no comparison could be made.

\section{Study Limitations}

Our study has some methodological considerations and limitations. Disproportionality analysis cannot differentiate between recreational, self-treatment, or mixed type of abuse; however, it is a suitable tool to quantitate signals of abuse of known and novel psychoactive substances. Further, the causal relationship between drugs and the adverse event (abuse) cannot be verified without a clinically performed causality assessment, while confounders as comorbidity and concomitant drugs cannot also be assessed properly. Regarding search analytics, since Google only reports large datasets, terms such as dependence, tolerance, and misuse have not provided substantial numbers and were not included in the analysis. In addition, the algorithms and their updates utilized by Google to analyze data are not publicly available. Finally, there were not enough data volumes before 2007 .

\section{CONCLUSION}

Concluding, the present study revealed a safety signal for the abuse potential of pregabalin and gabapentin using two different methods of surveillance, the FAERS database analysis and big data search analytics. We suggest that these methods can be used in combination as a supplementary pharmacovigilance tool to detect drug safety signals.

\section{DATA AVAILABILITY STATEMENT}

Publicly available datasets were analyzed in this study. This data can be found at: http://openvigil.sourceforge.net/.

\section{AUTHOR CONTRIBUTIONS}

GP: project development and manuscript writing and editing. DS and SS: data collection, data analysis, and manuscript drafting. NP and IT: data analysis and manuscript editing. AG: review of the final manuscript. All authors contributed to the article and approved the submitted version. (2020). Available online at: https://clincalc.com/DrugStats/Drugs/Gabapentin (accessed December 8, 2020).

6. European Monitoring Centre for Drugs and Drug Addiction. European Drug Report 2014: Trends and Developments (2014).

7. Martinotti G, Lupi M, Sarchione F, Santacroce R, Salone A, Berardis $\mathrm{D}$, et al. The potential of pregabalin in neurology, psychiatry and addiction: a qualitative overview. Curr Pharm Des. (2013) 19:636774. doi: 10.2174/13816128113199990425

8. Schifano F. Misuse and abuse of pregabalin and gabapentin: cause for concern? CNS Drugs. (2014) 28:491-6. doi: 10.1007/s40263-014-0164-4

9. Chiappini S, Schifano F. A decade of gabapentinoid misuse: an analysis of the European Medicines Agency's 'suspected adverse drug reactions' 
database. CNS Drugs. (2016) 30:647-54. doi: 10.1007/s40263-016-0 359-y

10. Evoy KE, Covvey JR, Peckham AM, Ochs L, Hultgren KE. Reports of gabapentin and pregabalin abuse, misuse, dependence, or overdose: an analysis of the Food And Drug Administration Adverse Events Reporting System (FAERS). Res Soc Adm Pharm. (2019) 15:953-8. doi: 10.1016/j.sapharm.2018.06.018

11. Papazisis G, Tzachanis D. Pregabalin's abuse potential: a mini review focusing on the pharmacological profile. Int J Clin Pharmacol Ther. (2014) 52:70916. doi: $10.5414 / C P 202118$

12. Spachos D, Siafis S, Bamidis P, Kouvelas D, Papazisis G. Combining big data search analytics and the FDA Adverse Event Reporting System database to detect a potential safety signal of mirtazapine abuse. Health Informatics $J$. (2020) 26:2265-79. doi: 10.1177/1460458219901232

13. Spachos D, Siafis S, Bamidis P, Kouvelas D, Papazisis G. Detecting a potential signal of quetiapine abuse using the faers database and big data search analytics. Drug Saf. (2018) 41:1236.

14. Bossard JB, Ponté C, Dupouy J, Lapeyre-Mestre M, Jouanjus E. Disproportionality analysis for the assessment of abuse and dependence potential of pregabalin in the French pharmacovigilance database. Clin Drug Investig. (2016) 36:735-42. doi: 10.1007/s40261-016-0421-z

15. Schoedel KA, Stockis A, Sellers EM. Human abuse potential of brivaracetam in healthy recreational central nervous system depressant users. Epilepsy Behav. (2018) 78:194-201. doi: 10.1016/j.yebeh.2017.09.008

16. Sakaeda T, Tamon A, Kadoyama K, Okuno Y. Data mining of the public version of the FDA adverse event reporting system. Int J Med Sci. (2013) 10:796-803. doi: 10.7150/ijms.6048

17. Harrison J, Mozzicato P. MedDRA $®$ : the tale of a terminology: side effects of drugs essay. Side Effects Drugs Ann. (2009) 31: xxxiii-xli. doi: 10.1016/S0378-6080(09)03160-2

18. Böhm R, Von Hehn L, Herdegen T, Klein HJ, Bruhn O, Petri H, et al. OpenVigil FDA - Inspection of U.S. American adverse drug events pharmacovigilance data and novel clinical applications. PLoS ONE. (2016) 11: e0157753. doi: 10.1371/journal.pone.0157753

19. Brooks SK, Webster RK, Smith LE, Woodland L, Wessely S, Greenberg N, et al. The psychological impact of quarantine and how to reduce it: rapid review of the evidence. Lancet. (2020) 395:912-20. doi: 10.1016/S0140-6736(20)30460-8

20. Berners-Lee T. Google Search Statistics. Internet Live Stats. (2017). Available online at: http://www.internetlivestats.com/google-search-statistics (accessed December 10, 2020).

21. support.google.com. The Homepage Explained Title. Available online at: https://support.google.com/trends/answer/6248105?hl=en\&ref_topic= 6248052
22. Van Puijenbroek EP, Bate A, Leufkens HGM, Lindquist M, Orre R, Egberts ACG. A comparison of measures of disproportionality for signal detection is spontaneous reporting systems for adverse drug reactions. Pharmacoepidemiol Drug Saf. (2002) 11:3-10. doi: 10.1002/ pds.668

23. Schifano F, Chiappini S, Corkery JM, Guirguis A. An insight into Zdrug abuse and dependence: an examination of reports to the European Medicines Agency Database of suspected adverse drug reactions. Int J Neuropsychopharmacol. (2019) 22:270-7. doi: 10.1093/ijnp/ pyz007

24. Driot D, Jouanjus E, Oustric S, Dupouy J, Lapeyre-Mestre M. Patterns of gabapentin and pregabalin use and misuse: results of a populationbased cohort study in France. Br J Clin Pharmacol. (2019) 85:12609. doi: 10.1111/bcp. 13892

25. Martinotti G, Papazisis G, Santacroce R, Kouvelas D, Cinosi E, Lupi M, di Giannantonio M. Pregabalin abuse and addiction. In: Preedy VR, editor. Neuropathology of Drug Addictions and Substance Misuse. London, UK: Academic Press (2016). p. 945-51. doi: 10.1016/B978-0-12-800634-4.00093-7

26. Papazisis G, Garyfallos G, Sardeli C, Kouvelas D. Pregabalin abuse after past substanceseeking behavior. Int J Clin Pharmacol Ther. (2013) 51:4412. doi: 10.5414/CP201881

27. Kruse CS, Goswamy R, Raval Y, Marawi S. Challenges and opportunities of big data in health care: a systematic review. JMIR Med Informatics. (2016) 4:e38. doi: 10.2196/medinform.5359

28. Ponté C, Pi C, Palmaro A, Jouanjus E, Lapeyre-Mestre M. Early signal of diverted use of tropicamide eye drops in France. Br J Clin Pharmacol. (2017) 83:1791-800. doi: 10.1111/bcp.13272

29. Montastruc JL, Sommet A, Bagheri H, Lapeyre-Mestre M. Benefits and strengths of the disproportionality analysis for identification of adverse drug reactions in a pharmacovigilance database. Br J Clin Pharmacol. (2011) 72:905-8. doi: 10.1111/j.1365-2125.2011.04037.x

Conflict of Interest: The authors declare that the research was conducted in the absence of any commercial or financial relationships that could be construed as a potential conflict of interest.

Copyright (c) 2021 Papazisis, Spachos, Siafis, Pandria, Deligianni, Tsakiridis and Goulas. This is an open-access article distributed under the terms of the Creative Commons Attribution License (CC BY). The use, distribution or reproduction in other forums is permitted, provided the original author(s) and the copyright owner(s) are credited and that the original publication in this journal is cited, in accordance with accepted academic practice. No use, distribution or reproduction is permitted which does not comply with these terms. 


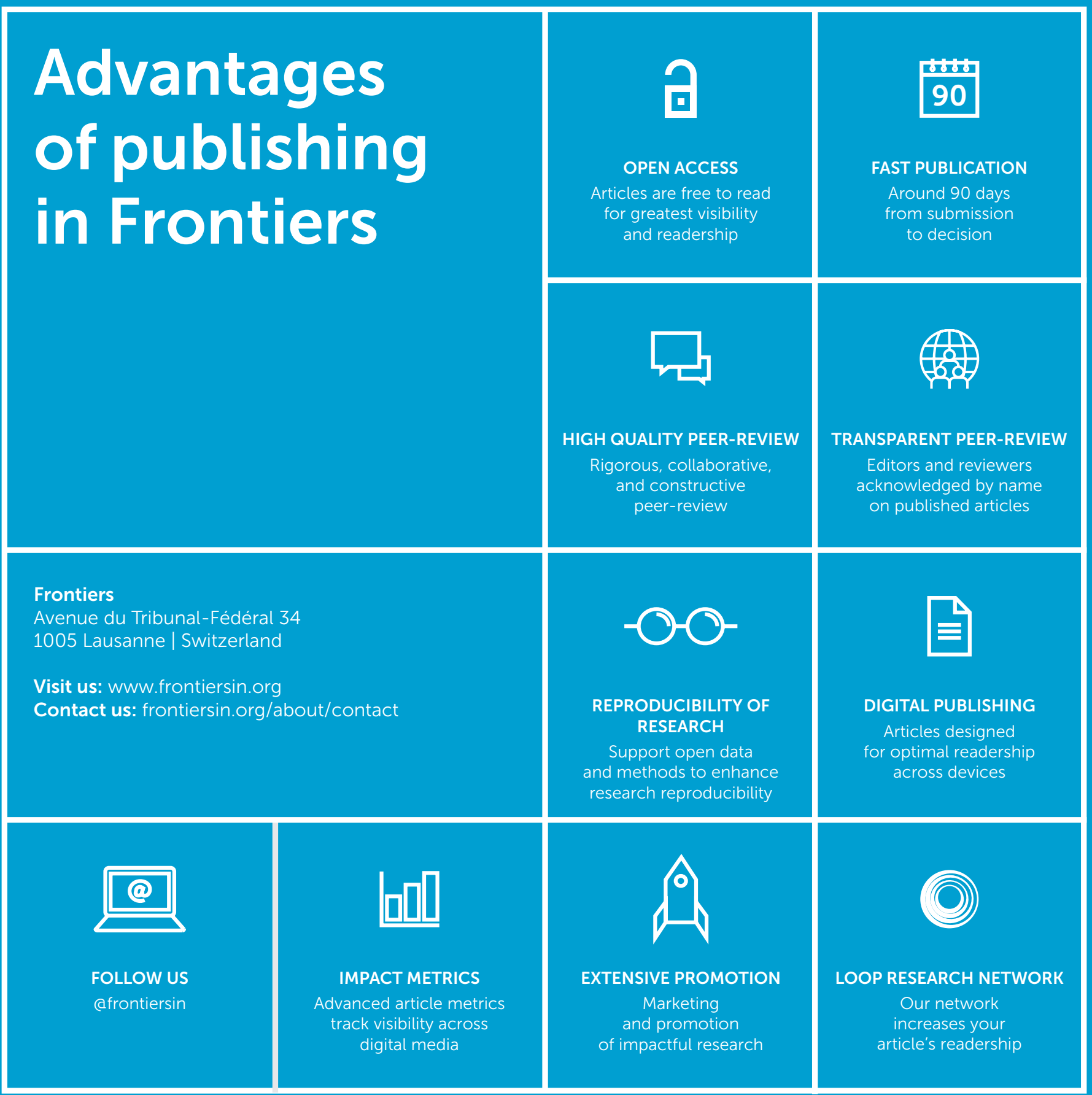

